\title{
The null Penrose inequality and the shell version in Minkowski
}

Instituto Universitario de Física Fundamental y Matemáticas Universidad de Salamanca

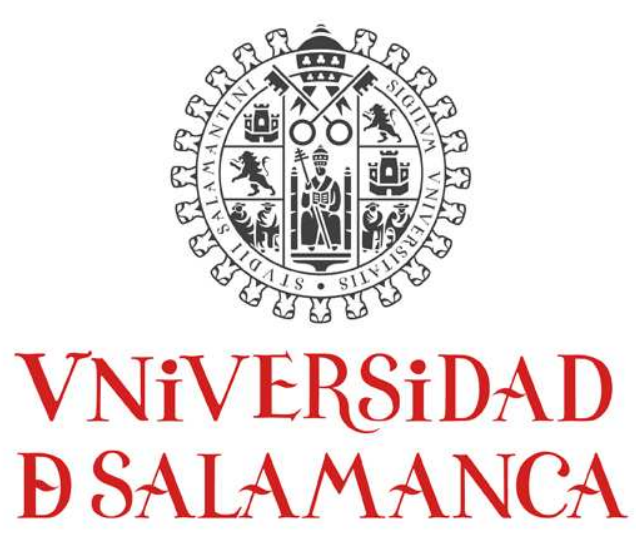

CAMPUS OF INTERNATIONAL EXCELLENCE

MEMORIA PARA OPTAR AL TÍTULO DE DOCTOR

Alberto Eduardo Soria Marina

PhD Thesis, 2017 

D. Marc Mars Lloret, Profesor Titular de la Universidad de Salamanca

\section{CERTIFICA:}

Que el trabajo de investigación que se recoge en la siguiente memoria titulado "The null Penrose inequality and the shell version in Minkowski", presentada por D. Alberto Eduardo Soria Marina para optar al título de doctor, ha sido realizada en su totalidad bajo su dirección y autoriza su presentación.

Salamanca, 29 de mayo de 2017.

D. Marc Mars Lloret

Profesor Titular de Universidad

Universidad de Salamanca 



\title{
The null Penrose inequality and the shell version in Minkowski
}

\author{
Alberto Eduardo Soria Marina
}

2017 

A mis padres Tomás y Elvira.

A mi hermana Lorena. 

„Wir müssen wissen

—wir werden wissen".

David Hilbert 



\section{Agradecimientos}

Querría dedicar las primeras líneas de estos agradecimientos a mi director de tesis, el profesor Marc Mars Lloret, por su continuo empeño y dedicación conmigo y con este trabajo. Gracias, Marc, por haber podido contar contigo en cada instante de estos años que he pasado en la bonita ciudad de Salamanca. Infinitas gracias por tu orientación, por tus bellas y útiles ideas, y tu continua preocupación por hacer las cosas bien. Gracias por tenderme la mano ante un problema tan hermoso y tan complejo. Sin tu ayuda esta tesis nunca habría podido realizarse.

Agradezco a todos los profesores del departamento de Física Fundamental de la USAL el gran trato que me han dado todos estos años, y la curiosidad mostrada ante el desarrollo de mi trabajo. Estoy asimismo agradecido a los profesores del departamento de Geometría y Topología de la UGR por las enseñanzas recibidas en la primera etapa formativa del doctorado, y en especial al profesor Miguel Sánchez Caja, mi tutor en tal época.

Me gustaría también agradecer a los compañeros de doctorado que he tenido su compañía y amistad. Las incontables charlas con ellos, el intercambio de saber, las comidas, y el buen sentido del humor que juntos hemos compartido han contribuido claramente a que la vivencia en esta universidad haya sido excelente.

Deseo agradecer de manera muy especial a mis padres y a mi hermana el que me hayan sabido querer y aupar cuando más lo necesitaba. Gracias por vuestro sacrificio y amor incondicional, sin los cuales nunca habría podido llegar hasta donde ahora mismo estoy.

Finalmente, agradezco al MICINN por el apoyo económico prestado. 

2 Fundamentals for the Penrose inequality

2.1 Basic Definitions . . . . . . . . . . . . . . . . . . . . 19

2.2 Asymptotically flat spacetimes . . . . . . . . . . . . . . . . . 22

2.3 The ADM and Bondi energy-momentum four vectors in AF spacetimes 24

2.4 Black holes and the weak cosmic censorship conjecture . . . . . . . . 26

2.5 The Penrose inequality conjecture. Heuristics . . . . . . . . . . . . . 27

2.6 Hypersurfaces and shells ... . . . . . . . . . . . . . . . . . 31

2.7 Penrose construction in Minkowski. The shell Penrose inequality . . . . 38

3 Projection along the Killing in static spacetimes 47

3.1 Introduction ......................... 47

3.2 The metric determined by the Killing . . . . . . . . . . . . . . . . . . 48

3.3 Geometry of the intial surface in terms of the projected one . . . . . . 49

3.3.1 A critical revision of Gibbons' argument . . . . . . . . . . . 58

3.3.2 The projection along the Killing direction in Minkowski. Applications to the shell Penrose inequality. . . . . . . . . . . 65

4 Projection along the past null cone $\Omega$ in Minkowski 69 
4.1 Introduction . . . . . . . . . . . . . . . . . . 69

4.2 Notation and basic definitions . . . . . . . . . . . . . . 70

4.3 Shell Penrose inequality in the Minkowski spacetime in terms of the geometry of convex surfaces . . . . . . . . . . . . . . 71

4.4 The shell Penrose inequality in Minkowski in terms of the support function 77 4.4.1 Spherically symmetric case . . . . . . . . . . . . . . . 79

4.4.2 Penrose inequality in terms of the support function in spacetime dimension four . . . . . . . . . . . . . . . 81

4.5 Dragging the surface along its past null cone . . . . . . . . . . . . 86

5 Normal graphs in Euclidean space. Applications to the shell Penrose inequality

5.1 Introduction .......................... 97

5.2 Geometry of normal graphs on hypersurfaces in the Euclidean space . . 98

5.3 Matching the two different projections . . . . . . . . . . . . . . . 103

6 The Hawking energy along null AF hypersurfaces 111

6.1 Introduction . . . . . . . . . . . . . . . . . . . . 111

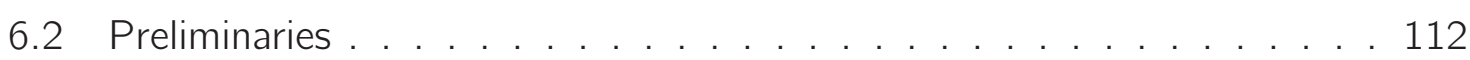

6.3 Null asymptotic flatness of $\Omega$ and asymptotic behaviour . . . . . . . . 117

6.4 Background foliation approaching large spheres . . . . . . . . . . . . . 124

6.5 Limit of the Hawking energy for affine foliations . . . . . . . . . . . . . 125

6.6 Limit of the Hawking energy for non-affine foliations . . . . . . . . . . 133

6.7 The large sphere equation and the Bondi energy-momentum . . . . . . 138

7 On the Penrose inequality along null hypersurfaces 147

7.1 Introduction . . . . . . . . . . . . . . . . . . . . . . . . . . . . . . . . .

7.2 A functional on two-surfaces . . . . . . . . . . . . . . . . 148

7.3 GAB foliations and a Penrose type inequality ... . . . . . . . . 153

7.4 On the inequality $D\left(S_{\lambda}, \ell\right) \leq \lim _{\lambda \rightarrow \infty} D\left(S_{\lambda}, \ell\right) \ldots \ldots . . . . . . . .159$

7.4.1 On the monotonicity of $F\left(S_{\lambda}\right)$ for large $\lambda \ldots . . . . . .161$

7.5 Renormalized area method for the Penrose inequality . . . . . . . . . 163 
7.6 Shear-free vacuum case . . . . . . . . . . . . . . . . . . . . 167

7.7 Renormalized area method for the shell Penrose inequality in $\mathcal{M}^{1,3} \ldots 171$

7.8 GAB foliations in $\mathcal{M}^{1,3}$. Applications to the shell Penrose inequality . . 175

7.9 An upper bound for the area of $S_{\lambda}$ along $\Omega \ldots \ldots \ldots$

8 Conclusions 181

$\begin{array}{ll}\text { Appendices } & 183\end{array}$

A Geometry of non-degenerate submanifolds 185

A.1 Induced connection and second fundamental form . . . . . . . . . 185

A.2 Induced Hessian . . . . . . . . . . . . . . . . . . . . . . . . . . . 188

A.3 Gauss, Ricci and Codazzi identities . . . . . . . . . . . . . . . . . . 189

B Geometry of null hypersurfaces 195

B.1 Hypersurfaces and null geodesics . . . . . . . . . . . . . . . 195

B.2 Quotient structure on a null hypersurface . . . . . . . . . . . . 197

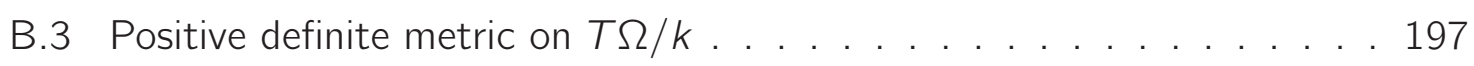

B.4 Second fundamental form on $T \Omega / k \ldots \ldots \ldots \ldots$

B.5 Evolution equations on $\Omega$. Ricatti and Raychaudhuri equation . . . . 200

B.6 Flow of surfaces along $\Omega$. Evolution equations . . . . . . . . . . . 204

C Geometry of convex bodies. The support function 211

C.1 Mapping a convex body onto the unit sphere . . . . . . . . . 211

C.2 Convex functions. The support function . . . . . . . . . . . 215

C.3 Geometry of Euclidean hypersurfaces in terms of the support function . 221

C.4 The support function of the sphere . . . . . . . . . . . . 230 



\section{Introduction}

The theory of General Relativity (GR) was developed by Einstein and completed in 1915 and it is, so far, the most accurate theory to describe gravitational physics. It generalizes the Special theory of Relativity, published in 1905. Einstein understood that in presence of gravitational fields, the spacetime is a four dimensional differentiable manifold endowed with a metric tensor of signature $(1,3)$ solving the so-called Einstein field equations of General Relativity, which are fundamental to study the gravitational interaction. Einstein's theory makes remarkable statements concerning the structure of space and time and the nature of the gravitational field. The theory uses complex and powerful mathematical tools.

The influence of Riemann's ideas was essential for the foundation of GR. Minkowski's and Ricci's work endowed Einstein's special and general theory of relativity with a solid geometrical structure, and contributed to set the pillars of both theories. In 1906 Einstein published the Equivalence Principle and from 1907 to 1911 Einstein's work was directed to the obtention of a field law for gravitation. With the help of Grossmann for a better understanding of the arising mathematical difficulties, Einstein's main achievement in the period going from 1912 to 1914 was the Principle of General Covariance. After a long and difficult process, including a final race with Hilbert, Einstein published his famous field equations of gravity in his article "Die Feldgleichungen der Gravitation" ("The field equations of Gravitation") the 25th of November of 1915 in the Prussian Academy of Sciences [31].

GR theory can be applied to numerous areas of physics. Among others, it allows us to study the evolution of the universe as a whole. The theory can also be used to study the behaviour of highly energetic, compact astronomical objects such as quasars and compact X-ray sources. It is also the best theory to study the gravitational collapse of a massive object and it predicts accurately the behaviour of moving bodies in strong gravitational fields. Einstein's theory also predicted in 1916 the existence of gravitational waves, ripples of the distorted spacetime traveling at the speed of light and caused by 
massive accelerating objects. A major confirmation of the theory has been the recent detection of gravitational waves by the observatory LIGO on the 14th of September 2015 [62]. Other GR predictions have also been found to be in excellent agreement with experiments and observations. It is also believed that General Relativity will play a capital role in the future development of a quantum theory of gravitation.

The subject of this thesis is the so-called Penrose inequality conjecture. In order to describe the general context in which it arises we start with some general remarks on gravitational collapse.

To study the process of collapse, the spacetime is often divided in two well differentiated regions, the interior of the massive body and its exterior. The (exterior) metric generated by the isolated massive object is modeled by a so called asymptotically flat vacuum solution (a precise definition will be given in Chapter 21). Asymptotically flat (AF) spacetimes require restrictions "far away" from the sources, specifically the metric and a finite number of its derivatives are assumed to approach the Minkowski values at an appropriate rate in the far away region. The limit can be taken along null directions (null infinity) or spacelike directions (spacelike infinity). As a consequence, several notions of asymptotic flatness exist.

In 1939 Oppenheimer, Snyder, and Volkoff [81, 82] studied for the first time a particular a process of gravitational collapse under the assumption of spherical symmetry. This led eventually to the concepts of "white dwarfs", "neutron stars" (both as stellar remnants), and of "black holes" (solutions of the vacuum field equations), as possible outcomes of such collapsing process. When a massive star exhausts its nuclear fuel, it leaves behind a small, dense remnant core. If the core mass is greater that two to three solar masses it is believed to enter the stage of continual gravitational collapse without any final equilibrium state. The radius shrinks and the star reaches higher densities. One of the most important problems that the General Relativity attempts to solve is to predict and to explain the final state of such an object. This is a central question in relativistic astrophysics and gravitation theory today.

The discovery of the Schwarzschild vacuum solution to the Einstein field equations in 1915, combined with the work of Oppenheimer, Snyder, and Volkoff on gravitational collapse, allows one to understand the whole process of collapse of a spherically symmetric non-rotating star and the consequent "formation" of a Schwarzshild black hole and a singularity. In 1915 Karl Schwarzschild solved the Einstein vacuum field equations for an uncharged spherically symmetric non-rotating single mass. The work was published in 1916 with the title „Über das Gravitationsfeld eines Massenpunktes nach der Einsteinschen Theorie" ( "On the Gravitational Field of a Point-Mass, according to Einstein's Theory"). Concerning Oppenheimer, Snyder, and Volkoff's work, the amount of mass that determines if the final state of collapse is a neutron star or a black hole is known as the Tolman-Oppenheimer-Volkoff (TOV) limit. It is estimated to be around two to three solar masses. 
The natural philosopher and geologist John Michell speculated in 1783 about the existence of massive bodies generating such a strong gravitational field that not even light could escape, and Laplace published the same idea in his masterpiece "Exposition du Système du Monde" ("The system of the world") in 1796. However, Einstein's General Relativity proved the theoretical existence of black holes for the first time. In 1964, the journalist Ann Ewing wrote an article about this topic titled "'Black holes' in space". Three years later Wheeler popularized the term "black hole". Nowadays, existence of black holes is accepted by most of astronomers. In fact it is widely believed that most of the galaxies have a supermassive black hole in their center.

Black holes are an important outcome of gravitational collapse. Another, and in fact an even more generic one is the existence of singularities. Indeed, there exist theorems in GR asserting that under quite general circumstances when the collapse has gone past a certain point the formation of a singularity is inevitable (see for instance [44, 98]). The concept of singularity in GR is not trivial since, by definition, a singularity cannot be part of the manifold. The natural idea that some physical or geometrical quantity must blow up at the singularity turns out to be inadequate as there exist spacetimes with regular (or even vanishing) curvature invariants which are nevertheless singular. To avoid this difficulty, the most commonly accepted way to define singularities is by using spacetime curves as pointers to them. The curves represent real or hypothetical particles that are travelling across the spacetime and suddenly disappear in a finite time (this may happen both to the future or to the past of the trajectory). The basic definition of singularity is the existence of incomplete and inextendible non-necessarily geodesic curves in the spacetime. For this definition to make sense it is necessary that the spacetime itself is assumed to be inextendible. The incomplete curves are usually assumed to have a causal character, although spacelike curves can be also used to define singularities. However, all singularity theorems intend to prove the geodesic incompleteness of the spacetime, which is a stronger condition.

The theory of singularities was mostly developed in the 60s. The first singularity theorem was published by Penrose in 1965 [83], who proved null geodesic incompleteness under fairly general initial conditions describing states of collapse. This was signaled by the presence of a closed trapped surface. The use of such surfaces helped to prove many other important singularity theorems, including the most important one due to Hawking and Penrose in 1970 [45]. These theorems are remarkable since they can be applied to cosmological situations, to star or galaxy collapse and to collision of gravitational waves.

A fundamental issue is whether singularities and black holes always come together when gravitational collapse occurs. More specifically, in a gravitational collapse setup, one possibility is that an event horizon starts developing at a sufficiently early phase such that the collapsing star and the eventual singularity gets hidden inside the event horizon. In this case not even light can escape and reach a far-away observer in the 
exterior region. The spacetime contains a non empty "region of no escape", which is causally disconnected from future null infinity. A spacetime satisfying this property is called a black hole. The singularity theorems of Hawking and Penrose do not state that a shielded singularity forms necessarily as a consequence of gravitational collapse. On the other hand, if the formation of the event horizon gets delayed sufficiently (or does not form at all) during the collapse, the result is a naked singularity, and radiation from the singularity could escape to infinity. This might happen for instance if the formation of the singularity at the center of the collapsing spherical star lies to the causal past of the instant when the star surface enters its Schwarzschild radius.

Naked singularities have been found to be possible, e.g. in the collapse of spherically symmetric inhomogeneous dust, depending on the nature of the initial data. However it is widely believed that the symmetry assumption plays an important role for the existence of such models. The outcome of collapse is crucial to the problem of asymptotic predictability. The difference between the two types of singularities is very significant. In a spacetime that develops a naked singularity, there would be a total loss of predictability in the future of the singular point. In the case of the shielded singularity, predictability would be preserved at least in the spacetime region outside the horizon. This issue has great importance for black hole astrophysics and for the theory of black holes. The validity of many theorems on black hole dynamics depends on the assumption of absence of naked singularities.

The conjecture that states that, generically, the singularities of gravitational collapse are contained in black holes was first proposed by Penrose in 1969 and is known as the weak cosmic censorship conjecture [84]. In physical terms, the main idea behind this conjecture is that any observer that is sufficiently far away from a collapsing object will neither encounter any singularities nor effects coming from them. In other words, the weak cosmic censorship conjectures that distant observers can live out their lives free from the effects of any catastrophic events happening in the collapsing area of spacetime. Consequently, if singularities arise, they cannot be seen from infinity. As already mentioned, the outcome of the collapse is not always a black hole and a naked singularity can occur in some situations. However the most important question is the genericity and stability of such naked singularities arising from initial regular data. The weak cosmic censorship would hold in case the initial data subspace giving rise to naked singularities has zero measure in a suitable sense. In other words, the weak cosmic censorship allows naked singularities, as long as they are not generic. Another related conjecture, called strong cosmic censorship conjecture, also formulated by Penrose in 1979 [86], states that, generically, timelike singularities never occur, so that even an observer who falls into a black hole will never "see" the singularity.

So far no version of weak cosmic censorship has been proved in full generality. One of the main problems is that the event horizon is a feature that depends on the whole future behaviour of the solution of the field equations over an infinite period of time. 


\section{Introduction}

Any proof of the weak cosmic censorship requires a much deeper knowledge of general global properties of the Einstein equations than we have today. One possible way to approach the problem is to study the stability of particularly relevant spacetimes.

The idea is that proving the (non-linear) stability of either singularity-free or black hole spacetimes would provide us with a large class of spacetimes with the same properties, which in turn would give support to the weak cosmic censorship conjecture. The global non-linear stability problem is formulated from initial data on a spacelike hypersurface which is a small perturbation of a spacelike slice of the spacetime under consideration. The initial data set contains the geometric information of the initial spacelike hypersurface and its initial content of matter. The fundamental existence result is due to Choquet-Bruhat [17] and asserts that for a suitable matter model (including vacuum), each initial data set has a unique maximal, globally hyperbolic Cauchy development (intuitively, the maximal part of the spacetime uniquely determined by the prescribed initial data).

The only non-linear stability result known so far is the Christodoulou and Kleinerman global stability of the Minkowski spacetime [18]. The initial spacelike slice is a constant time hyperplane in Minkowski space. Christodoulou and Kleinerman proved that for small but non-linear perturbations of this Minkowski data, the Cauchy development has qualitative behaviour similar to Minkowski spacetime. In particular, the causal past of future null infinity is the whole spacetime. This means that all spacetimes arising from this perturbed data do not collapse nor form black holes, and consequently the weak cosmic censorship conjecture holds for all of them.

The problem of stability of explicit black hole spacetimes is a formidable problem and it is natural to consider linear perturbations first, i.e. the study of the convergence or divergence of the evolution of scalar, vector and tensor fields in those black hole spacetimes. If the fields do not decay at infinity, non-linear effects are expected to enhance this and we can expect that the solution is not (non-linearly) stable. On the other hand, linear stability would support the validity of the weak cosmic censorship conjecture.

In the four dimensional case, the perturbation analysis of the Schwarzschild metric was initiated by Regge and Wheeler [90]. Later on, Vishveshwara [105] proved linear stability to axial ("odd parity") perturbations in 1970. Shortly afterwards, Price [88, 89] gave a partial result concerning the stability of the Schwarzschild black hole, with precise information about the decay properties of the perturbations. The work of Zerilli [113] and Moncrief [79] also contributed to determine the modal (i.e. in terms of its decomposition in spherical harmonics) linear stability of gravitational perturbations for the Schwarzshild black hole. The study of black hole non-modal stability was initiated by Kay and Wald [56], giving a proof of the boundedness of perturbations at asymptotically late times of the Schwarzshild black hole. This area of research has been very active in the last decade or so, with the culminating breakthrough of Dafermos-Holzegel- 
Rodnianski [25] in 2016, in which the linear stability was fully proven. Very recently, Hung, Keller and Wang [53] have found an alternative, complementary and simpler proof for the linear stability of the Schwarzshild black hole.

The stability of a Kerr black hole is much more difficult to prove. Whiting [112] proved in 1989 the modal stability for the Kerr black hole using the Teukolsky equation. An analogue theorem to the one proved by Kay and Wald, applicable to a large class of backgrounds (including the slow rotating Kerr black hole) was proved by Dafermos and Rodniansky [27]. The same authors also provided in [26] the essential elements for the proof of decay of solutions of the scalar wave equation in general subextremal Kerr backgrounds. This finally allowed Dafermos, Rodnianski and Shlapentokh-Rothman [28] to prove in 2014 the linear stability for scalar fields on such backgrounds. Extremal black holes have also been object of study because they lie at the boundary between black holes and naked singularities and hence are believed to be good indicators to test the weak cosmic censorship. In [2, 4], Aretakis exhibited instability properties of a general class of extremal black holes (including the Kerr extremal black hole) with respect to scalar perturbations. These instabilities are determined by local properties of extremal horizons and hence do not depend on global aspects of the spacetime. On the other hand, Dain and Gentile de Austria [30] found a positive definite and conserved energy for axially symmetric linear gravitational perturbations of the extreme Kerr black hole, which gives support to its linear stability against axial perturbations. The existence of such a conserved quantity does not contradict Aretakis instability because the former is only defined in the black hole exterior region and does not involve any transverse derivative at the horizon.

So far, the tests of stability of black holes to general, linear perturbations have provided support to the weak cosmic censorship. However, there have also been attempts to disprove Penrose's conjecture. The setup that Penrose used to test the validity of the conjecture was a shell of null dust moving inwards at the speed of light in a flat spacetime. Penrose devised this construction as a potential counterexample to weak cosmic censorship. Several attempts to find counterexamples using time symmetric initial data have also been considered. The two following properties are crucial in order to put forward the counterexamples. The first is that if weak cosmic censorship holds, then every trapped surface must lie in the black hole region (a trapped surface $S$ is a compact, two dimensional surface satisfying that the convergence of both the outgoing and ingoing null geodesics normal to $S$ is everywhere negative). The second is that if the weak cosmic censorship holds, and if the matter satisfies the null energy condition (i.e. if $T(k, k) \geq 0$ for all null $k$ ), then the area of the event horizon of a black hole cannot decrease with time.

As we will see with detail in the Chapter 2, the weak cosmic censorship conjecture together with additional physically reasonable assumptions imply the inequality that is the core of this thesis: the Penrose inequality. This inequality involves the concept of 
total mass/energy of the spacetime, and the area of surfaces related to quasi-local black holes. The total energy is defined in terms of integrals in the corresponding asymptotic region, where the metric is close to Minkowski, and its definition depends on the chosen infinity, spatial or null. In any case, both energies are defined as components of two respective vectors: the ADM energy-momentum vector where the integral is taken at spatial infinity, and the Bondi energy-momentum vector where the integral is taken at null infinity. Both transform as Lorentz vectors under suitable transformations, and their Lorentz lengths are called masses (ADM mass and Bondi mass respectively). The ADM mass is a conserved quantity upon evolution and the Bondi mass is monotonically decreasing in advanced time. More information about such energy-momentum vectors can be found e.g. in [106]. These different masses give rise to different versions of the Penrose inequality.

In this thesis, we will mainly focus our attention in the so-called null Penrose inequality, which as its name indicates, is related to the Bondi energy-momentum vector of the spacetime. However in this introduction, and for the sake of completeness we also discuss briefly its "spacelike version". In 1973, Penrose [85] came to the conclusion that the ADM mass of an asymptotically flat spacetime should be at least the mass of the black holes that it contains, if the energy density is non-negative everywhere. Penrose's heuristic argument (partly based in the assumption of weak cosmic censorship and that will be exposed in the next chapter in full detail) drove him to formulate the following so-called Penrose inequality conjecture (precise definitions will be given later):

Conjecture 1.0.1 (The Penrose inequality for the ADM mass in asymptotically flat spacetimes). Let $(M, g)$ be a four dimensional spacetime satisfying the DEC (dominant energy condition), admitting an asymptotically flat spacelike hypersurface $(\Sigma, \gamma, K)$. Assume that $\Sigma$ contains a closed (compact and without boundary) WOTS (weakly outer trapped surface) $S$. Then

$$
M_{A D M} \geq \sqrt{\frac{\left|\mathcal{S}_{\min }(S)\right|}{16 \pi}}
$$

where $M_{A D M}$ is the $A D M$ mass of the spacetime, and $\mathcal{S}_{\min }(S)$ the minimal area enclosure of $S$ (i.e. the outermost of all surfaces in $\Sigma$ that enclose $S$ and have less or equal area than any other surface enclosing S). Moreover, if the inequality becomes equality, $(\Sigma, \gamma, K)$ can be isometrically embedded in the Schwarzschild spacetime.

Although the heuristic argument by Penrose was originally formulated in a four dimensional context, the Penrose inequality for the ADM mass in asymptotically flat spacetimes can also be formulated in arbitrary dimensions. The form of the inequality in a $(n+1)$-dimensional spacetime is

$$
M_{A D M} \geq \frac{1}{2}\left(\frac{\left|\mathcal{S}_{\min }(S)\right|}{\omega_{n-1}}\right)^{\frac{n-2}{n-1}}
$$


where $\omega_{n-1}$ is the area of the unit $(n-1)$-sphere. Studying the Penrose inequality is an interesting problem because its validity would provide a strengthening of the positive mass theorem, and in the context of gravitational collapse it would give indirect support to the validity of weak cosmic censorship.

We next describe briefly the main results known concerning the validity of the Penrose inequality. More information and references can be found for instance in [69].

Malec and Ó Murchadha [68] proved the so called Penrose inequality in spherical symmetry. They studied three-dimensional spherically symmetric asymptotically flat spacelike hypersurfaces $\Sigma$, satisfying the dominant energy condition. Moreover, the hypersurfaces were assumed to be maximal, i.e. with second fundamental form of vanishing trace. In this setup they proved that

$$
E_{A D M} \geq \sqrt{\frac{|S|}{16 \pi}},
$$

where $E_{A D M}$ is the ADM energy of $\Sigma$, and $S$ is the outermost trapped surface whose exterior does not contain any other trapped surface. This inequality is weaker than the Penrose inequality since it involves the ADM energy instead of the mass. In 1996 Hayward [47] proved the inequality in full generality, i.e. for non-maximal hypersurfaces, using properties that he obtained in [46] of the so-called Misner-Sharp quasi-local energy [78], which coincides with the Hawking energy when particularized to spherical symmetry.

The Riemannian Penrose inequality is formulated in asymptotically flat Riemannian three manifolds with non-negative scalar curvature and states that the area of the outermost minimal surface $S$ (i.e. the minimal surface that encloses any other minimal surface in $\Sigma$ ) is bounded by the ADM mass by

$$
M_{A D M} \geq \sqrt{\frac{|S|}{16 \pi}} .
$$

This version of the inequality corresponds to the case where the hypersurface $\Sigma$ is time-symmetric (i.e. with vanishing second fundamental form) because in this setup the dominant energy condition transforms into the condition of non-negative scalar curvature and $\mathcal{S}_{\min }(S)$ is necessarily the outermost minimal surface. The Riemann Penrose inequality for three dimensional hypersurfaces was proved by Huisken \& IImanen [52] for a connected $S$, and by Bray [12] for arbitrary $S$. Bray and Lee [14] proved the conjecture for hypersurfaces with dimension at most seven. The Riemannian Penrose inequality has also been proven in arbitrary dimension for graphs in the Euclidean space [57, 50]. Given the importance of the breakthroughs of Huisken \& IImanen and Bray we describe very briefly the main ideas in their proofs.

The method used by Huisken and IImanen [52] to prove the inequality is based on an earlier heuristic argument due to Geroch [33] and improved later on by Jang \& Wald 
[55], which uses the monotonicity of the Hawking energy under inverse mean curvature flow. Moreover, the Hawking energy tends to $E_{A D M}$ when the flow approaches large spheres at infinity. If the initial surface $\partial \Sigma$ is minimal, an immediate combination of these properties yields the Penrose inequality. The complication is that in general the inverse mean curvature flow develops singularities. This issue was handled by Huisken and Ilmanen by putting forward a weak formulation of the inverse mean curvature flow and proving both existence of this generalized flow and that all the necessary monotonicity and asymptotic requirements are satisfied. The method also allowed Huisken and IImanen to prove the rigidity part of the Penrose inequality.

Bray [12] proved the Riemannian Penrose inequality in full generality in 2001. His method was based on the existence of a conformal flow of metrics on $\Sigma$, which interpolates between the initial metric and the Schwarzschild metric. The crucial facts are that the ADM mass of the metrics does not increase along the flow, and that the metric outside the outermost minimal area enclosure of $\partial \Sigma$ tends to the Schwarzschild metric of mass at least $\sqrt{\frac{|\partial \Sigma|}{16 \pi}}$. Bray's method can be extended up to dimension seven (Bray and Lee [14]), whereas Huisken and IImanen's can only be applied to three-dimensional manifolds. It should be emphasized that for the rigidity part Bray and Lee had to assume that the manifold is spin (which holds automatically in three-dimensions).

The Penrose inequality for asymptotically hyperbolic manifolds has also attracted considerable attention. Asymptotically hyperbolic Riemannian manifolds arise as slices of asymptotically flat vacuum spacetimes, and whose second fundamental form is proportional to its metric (umbilical initial data). Such slices cannot reach spatial infinity, and they are usually called asymptotically hyperbolic or hyperboloidal. In fact, they approach null infinity. Asymptotically hyperbolic manifolds also arise as time-symmetric hypersurfaces in spacetimes solving the vacuum field equations with negative cosmological constant.

The Penrose inequality is different in each context. In the umbilical case, where the second fundamental form is a constant $\lambda$ times the metric, the surface $S$ of interest is the outermost surface with mean curvature $H_{S}=2|\lambda|$. In the time-symmetric and $\Lambda<0$ case, the surface to consider is outermost minimal. In both cases the scalar curvature of the hypersurface satisfies Scal ${ }_{\gamma}>-6 \lambda^{2}$, where in the time-symmetric case $\lambda$ is defined by $\Lambda=-3 \lambda^{2}$. The appropriate asymptotic behaviour of the hypersurface and the definition of mass (see e.g. [22]) are more difficult than in the euclidean case. When the boundary at infinity has spherical topology, the definition of mass was first given in [107], and the Penrose inequality reads

$$
M \geq \sqrt{\frac{|S|}{16 \pi}}+\frac{\lambda^{2}}{2}(1-\epsilon)\left(\frac{|S|}{4 \pi}\right)^{\frac{3}{2}},
$$

where $M$ is the mass of the asymptotically hyperbolic manifold, and $\epsilon=0,1$ depending on whether we are in the time-symmetric case, or in the umbilical case. 
Several authors have worked on this problem. In [80] Neves showed that in the umbilical version of (1.2), the inverse mean curvature flow along the hyperboloid does not have the necessary convergence properties. More specifically, the method requires that the limit along the flow of the Hawking energy is not larger than the total mass $M$ of the manifold, and Neves showed that this cannot be guaranteed in general.

A method to prove the validity of the non-time symmetric Penrose inequality in the AF case was put forward by Bray and Khuri in [13], where the inverse mean curvature flow was used in combination with the generalized Jang equation to get a system of PDEs which, provided existence of solutions with suitable boundary and asymptotic behaviour holds, would imply the Penrose conjecture for a single black hole. Using an analogous reasoning, Ye Sle Cha, Khuri and Sakovich [16] showed that the problem in the asymptotically hyperboloidal case could be reduced to the asymptotically flat one provided a suitably modified system of PDE also admits solutions. In [29], Dahl, Gicquaud and Sakovich proved the validity of Penrose-type inequalities in arbitrary dimension for asymptotically hyperbolic graphs over the hyperbolic space $\mathbb{H}^{n}$ with a minimal horizon $\Gamma$, and whose scalar curvature satisfies Scal $\geq-n(n-1)$. In the particular case where $\Gamma \subset \mathbb{H}^{n}$ is star-shaped and mean convex, the time symmetric version of the Penrose inequality (1.2) has been proved by Lopes de Lima and Girão [65]. The same authors have also proved in [64] the umbilical version of (1.2) in the graph case where the horizon $\Gamma$ satisfies suitable restrictions.

After this brief description of the known results in the spacelike case, we next discuss the null Penrose inequality, which is the main topic of this thesis. This inequality involves spacetimes that admit a null hypersurface $\Omega$ extending smoothly to past null infinity. The precise form for the Penrose conjecture in this setting is

Conjecture 1.0.2 (The Penrose inequality for the Bondi mass in asymptotically flat spacetimes). Let $(M, g)$ be a four dimensional spacetime, asymptotically flat at null infinity, satisfying the dominant energy condition, and admitting a null hypersurface $\Omega$ that extends smoothly to past null infinity. Assume that $\Omega$ has an embedded closed weakly outer trapped surface $S$. Then

$$
M_{B} \geq \sqrt{\frac{|S|}{16 \pi}}
$$

where $M_{B}$ is the Bondi mass of the spacetime determined by the cut of $\Omega$ with past null infinity. Moreover, the inequality becomes equality if and only if $\Omega$ is isometric (intrinsically and extrinsically) to a spherically symmetric null hypersurface in a Schwarzshild spacetime.

As already mentioned, the original construction that led Penrose to formulate his inequality in 1973 was a collapsing shell of null dust in the Minkowski spacetime which, after passing, leaves two well defined regions separated by the null hypersurface along 
which the shell propagates. The inner region is isometric to the Minkowski spacetime while the exterior region is no longer flat. Once a cross section $S$ of $\Omega$ is selected, the matter content of the shell can be adjusted so that $S$ becomes a WOTS with respect to the outer geometry. The crucial advantage of the construction is that the inequality (1.3) can be rewritten solely in terms of the inner geometry (details of the construction will be given in the following chapter). Specifically, the equivalent form of (1.3) in arbitrary dimension in terms of the Minkowski geometry reads

$$
\int_{S} \theta_{\ell} \boldsymbol{\eta}_{S} \geq(n-1)\left(\omega_{n-1}\right)^{\frac{1}{n-1}}|S|^{\frac{n-2}{n-1}}
$$

where $\theta_{\ell}$ is the outer null expansion of $S$, and $\omega_{n-1}$ is the area of the $(n-1)$-sphere. We will refer to inequality (1.4) as the shell Penrose inequality.

A proof of the general null Penrose inequality (1.3) was claimed by Ludvigsen \& Vickers [67]. However, a gap was found by Bergqvist [8] who, at the same time, substantially streamlined the argument. Ludvigsen \& Vickers and Bergqvist's argument was based on two facts. The first one was the existence of a quasi-local object defined on surfaces which enjoyed monotonicity properties along past directed null geodesic foliations. This functional was introduced by Bergqvist [8] and we refer to it as Bergqvist mass in this thesis (also in [69, 74]) The second fact was a suitable upper bound for the area of the weakly outer trapped surface $S_{0}$. Establishing this bound involved that the geodesic null foliation $\left\{S_{r}\right\}$ of $\Omega$ starting at $S_{0}$ satisfied two additional properties. The first one was that the future null expansion $\theta_{k}$ of $S_{r}$ along the future null generator $k$ tangent to $\Omega$ admits an expansion of the form

$$
\theta_{k}=\frac{-2}{r}+O\left(\frac{1}{r^{3}}\right)
$$

i.e. with vanishing coefficient in the term $r^{-2}$. The second one was that the rescaled metric $r^{-2} \gamma(r)$ (where $\gamma(r)$ is the induced metric of $S_{r}$ ) approaches a round metric on the sphere when $r \rightarrow+\infty$. The main result by Ludvigsen \& Vickers is that under these circumstances the Penrose inequality (1.3) follows. Ludvigsen \& Vickers took for granted that a geodesic foliation $\left\{S_{r}\right\}$ satisfying these two properties always exists. Bergqvist noted that under the assumption (1.5) it was not at all clear that the condition that the metric $r^{-2} \gamma(r)$ approaches a round sphere needs to be satisfied. This was the gap in the original paper [67]. Despite this, the ideas of Ludvigsen \& Vickers and specially Bergqvist have been a relevant source of inspiration for this thesis, as it will become clear later.

Concerning successful proofs of the null Penrose inequality in particular situations, the only case fully addressed is when the null hypersurface $\Omega$ is shear-free in a vacuum spacetime. This result was proved by Sauter [93], in his Ph.D. thesis. More recently, Tafel [99] has proved the null Penrose inequality for a cross section of the event horizon 
in black hole spacetimes under asymptotic conditions to the future weaker than stationarity. Finally, Alexakis [1] has proved the null Penrose inequality for vacuum spacetimes that are small but non-linear perturbations of Schwarzshild.

Concerning the shell Penrose inequality (1.4), the first case that was solved involved surfaces $S$ lying on a constant time hyperplane $\left\{t=t_{0}\right\}$, and was proved by Gibbons in [36, 37], who showed that the inequality reduces to the classic Minkowski inequality (see e.g. [10]) between the total mean curvature and the area of convex surfaces in Euclidean space. The second case refers to surfaces $S$ contained in the past null cone of a point and leads to a non-trivial inequality for functions on the sphere [85, 5]. In spacetime dimension four, its validity was proved by Tod [101] using the Sobolev inequality in $\mathbb{R}^{4}$. In fact, the four dimensional spherical case can also be viewed as a particular case of a Penrose inequality for spacetimes admitting shear-free null hypersurfaces extending from the trapped surface to past null infinity proved by Sauter [93].

Regarding the general case (i.e. when the surface $S$ does not lie necessarily in a hyperplane), Gibbons claimed [37] to have a general proof. However, the argument contains a serious gap. His strategy in [37] was to project $S$ along the time translation Killing vector of the Minkowski spacetime onto a constant time hyperplane. The main idea was to rewrite the shell Penrose inequality in terms of the geometry of the projected surface as a hypersurface of the Euclidean space. Gibbons computed an (erroneous) expression for the mean curvature of the projected surface. This gap was noticed for the first time by Mars in [69].

In spacetime dimension four, Wang [108] has proved the inequality for surfaces lying on a spacelike hyperboloid of Minkowski with the properties of being mean convex and star-shaped. Recently Brendle and Wang [15] have proved the inequality for another large class of surfaces, namely those lying on a timelike cylinder over a convex surface. These cylinders are called convex static timelike hypersurfaces in [15]. In fact, the case analyzed by the authors refers to a generalization of inequality (1.4) conjectured for the Schwarzschild spacetime, but the argument applies to the Minkowski situation as well. The main idea behind their result consists again in performing a projection of $S$ along the time translation $\xi$ onto a constant time hyperplane $\Sigma_{t_{0}}$. By relating the geometry of $S$ to the geometry of the projected surface $\bar{S}$ on $\Sigma_{t_{0}}$, inequality (1.4) becomes a consequence of the standard Minkowski inequality in Euclidean space provided $\bar{S}$ is convex.

In the remaining of this Introduction, we will give a general idea of the structure of the thesis and will describe its main results.

In Chapter 2 we describe the heuristic argument by Penrose to support his conjecture and present the calculations involved in the derivation of the shell version of the inequality. In addition we give the main definitions used throughout this thesis. The standard heuristic argument is formulated for AF spacetimes, so we start by introducing 
this concept and the associated notion of energy-momentum four vector. As already mentioned, the Penrose inequality can be considered as a strengthening of the Positive mass theorem, which we state in Theorem 2.3.2. At this point, and after defining the concept of black hole and stating the weak cosmic censorship conjecture, we have the necessary ingredients to describe in detail Penrose's heuristic argument. We start with the original formulation in terms of the ADM mass and then describe the modifications that are required for the null case. In the second part of Chapter 2 we justify the relation between the null Penrose inequality (1.3) and its shell version (1.4) in Minkowski by using the theory of shells of general character introduced by Mars [71] and that we also summarize in the second part of Chapter 2.

As already mentioned, Gibbons [37] attempted the projection of spacelike surfaces in Minkowski onto a constant time hyperplane in order to rewrite and prove the shell Penrose inequality in terms of the projected geometry. Although the computations were wrong, the idea is nevertheless worth exploring. In fact the natural setup to do this is the class of spacetimes which admit both a timelike Killing along which one can project and also geometrically privileged spacelike hypersurfaces onto which one can project. Since the shell Penrose inequality can in principle be formulated for shells propagating in other backgrounds than Minkowski (e.g. in the Schwarzshild spacetime, or others) this more general setup may also have applications for the Penrose inequality.

Chapter 3 begins by recalling the concept of staticity, and studies in detail the projection of embedded spacelike surfaces along the static Killing field onto constant time hypersurfaces. Projections of this sort in the Minkowski spacetime have also been studied in connection to a new definition of quasi-local mass by Wang and Yau [109, 110]. Partial results in the general static case have also appeared in [13]. However, to the best of our knowledge, no systematic or exhaustive account of the relation between all the intrinsic and extrinsic geometric properties of $S$ and its projection $\bar{S}$ has appeared in the literature, neither in the Minkowski nor in the general static case. We devote most of Chapter 3 to this task. With this machinery at hand we can describe why Gibbons argument is erroneous. This is also discussed in full detail in Chapter 3, which we conclude by giving an alternative proof of Brendle and Wang's main result [15] mentioned before. In fact our proof of this result was simultaneous and independent of Brendle and Wang's, and used the projection identities described in Chapter 3, which were published in 75 .

Chapter 4 is devoted to the study of the projection of the surface $S$ along its past null cone $\Omega$ onto constant time hyperplanes in the Minkowski background. This projection was used for the first time in [74] to tackle the shell Penrose inequality, and allows us to rewrite the original shell Penrose inequality in terms of the time height function $\tau=\left.t\right|_{S}-t_{0}$ and the geometry of a convex Euclidean surface, specifically the one obtained by intersecting the past null cone $\Omega$ of $S$ with a constant time hyperplane $\left\{t=t_{0}\right\}$. This alternative form of the shell Penrose inequality is given in Theorem 
4.3.5. A powerful Sobolev type inequality on the sphere due to Beckner [7] allows us to prove the validity of this inequality in the case when the surface $S$ lies in the past null cone of a point (Theorem 4.4.4). This generalizes to arbitrary dimension the result by Tod [101] in spacetime dimension four and shows that a conjecture put forward by this author regarding the optimal form of the inequality is in fact true. The geometry of convex, compact hypersurfaces in Euclidean space can be fully described in terms of a single function $h$ on the unit sphere. This function is called the "support function" and plays an important role in this thesis. In spacetime dimension four, the support function was already used in [103] in a related but different context. One of our main results is Theorem 4.4.2, where we write down the Penrose inequality in Minkowski as an inequality involving two smooth functions on the $n$-dimensional sphere. Inspired by the argument by Ludvigsen \& Vickers [67] and Bergqvist [8], we are able to prove (Theorem 4.5.3) the validity of this inequality in four spacetime dimensions for a large class of surfaces which can be explicitly characterized and which contains an open set of surfaces. However, when applied to surfaces lying on the past null cone of a point, the only case covered by this theorem is when $S$ is a round sphere. Thus, the cases covered by Theorem 4.4.4 and by Theorem 4.5.3 are essentially complementary, which indicates that any attempt of proving the shell Penrose inequality in Minkowski in the general case will probably require a combination of both methods.

In Chapter 5 we consider the $(n+2)$-dimensional Minkowski spacetime, with $n \geq 2$, and three different surfaces: the initial surface $S$ embedded in the null hypersurface $\Omega$, the euclidean surface $\widehat{S}_{0}$, obtained as the intersection of the past null cone $\Omega$ of $S$ and the constant time hyperplane $\left\{t=t_{0}\right\}$, and the euclidean surface $\bar{S}$, which is the projection of $S$ along the time translation $\xi$ onto the constant time hyperplane $\Sigma_{t_{0}}$. Brendle and Wang's main result in [15] (Theorem 3.3.7 in this thesis) showed that the convexity of $\bar{S}$ was sufficient for the validity of the shell Penrose inequality for $S$. The convexity of $\bar{S}$ can also be codified in the geometry of $\widehat{S}_{0}$ and in the time height function $\tau$ of $S$, which measures the "distance" that separates $S$ from the constant time hyperplane $\left\{t=t_{0}\right\}$. This codification is made in Theorem 5.3.1, where we obtain an explicit differential inequality for $\tau$ and the geometry of $\widehat{S}_{0}$ which implies the validity of the shell Penrose inequality for $S$. This requires analyzing the geometry of $\bar{S}$ as a graph over $\widehat{S}_{0}$. Most of our work consists in relating the induced metric and second fundamental forms of $\bar{S}$ to those of $\widehat{S}_{0}$. This is a calculation purely in Euclidean space which had not been considered before. This chapter is concluded with two examples in order to give an idea of the range of applicability of the result.

Chapter 6 is devoted to studying the Hawking energy along null hypersurfaces. The Hawking energy is known to approach the total energy of the spacetime when the surfaces approach round spheres both in the asymptotically flat [41, 52] and asymptotically hyperboloidal case [80]. The same is true for surfaces along null hypersurfaces [6, 87]. However, understanding the behaviour of the Hawking energy at infinity when the condition of round spheres is not imposed is much more subtle. The aim of Chapter 
[6 is to carry out such an analysis for surfaces embedded in a asymptotically flat null hypersurface. This problem is interesting for several reasons. First of all, it is relevant in order to help clarifying the physical meaning of the Hawking energy, which is related to an energy in some circumstances but not in others. From a more practical point of view, the Hawking energy has become a very valuable tool for various problems in geometric analysis. The underlying reason is that the Hawking energy enjoys interesting monotonicity properties for specific flows of surfaces. In order to become truly useful, this monotonicity needs to be complemented with a good behaviour of the Hawking energy at infinity, so that its asymptotic value can be related to the ADM (or Bondi) energies of the spacetime. Whenever the flow can be proved to approach large round spheres, the results above suffice, but often this is not the case and understanding the behaviour of the Hawking energy at infinity under general circumstances becomes a useful piece of information.

Studying the limit of the Hawking energy along flows in null hypersurfaces is particularly interesting because it allows for a very neat description of spacelike surfaces embedded in the null hypersurfaces as graphs with respect to a background foliation that can be chosen conveniently. We exploit this fact in order to obtain an explicit and simple expression for the limit of the Hawking energy at infinity for a very general flow of spacelike surfaces (see Theorem 6.6.2 in Chapter 6). In addition to this theorem we also find an interesting covariance property of the integrand in (7.57) under changes of background foliation. This is part of the content of Theorems 6.5.5, 6.5.6, and 6.6.1 in Chapter 6.

Chapter 7 is devoted to the use of flows of general character along null hypersurfaces in AF spacetimes satisfying the dominant energy condition with the purpose of addressing the null Penrose inequality. Motivated by Ludvigsen \& Vickers and Bergqvist ideas [67, 8], in the first part of the chapter we consider the setup where (1.5) is kept and we relax the condition of approaching large spheres. Geodesic foliations with this property are named Geodesic Asymptotically Bondi in this work (or GAB for short). A motivation for this name will be given later. GAB foliations turn out to always exist and be (geometrically) unique given any cross section $S_{0}$ in a past asymptotically flat null hypersurface. Our main result in this setting is a Penrose-type inequality which relates the area of any weakly outer trapped surface $S_{0}$ and the limit at infinity of the Hawking energy along the GAB foliation associated to $S_{0}$. This is the content of Theorem 7.3.8. In combination with the study of the limit of the Hawking energy along general foliations of asymptotically flat null hypersurfaces $\Omega$ carried out in Chapter 6 (and published in [76]), this theorem provides an interesting Penrose-type inequality with potentially useful applications. This theorem immediately extends Ludvigsen \& Vickers and Bergqvist result because when the flow approaches large spheres one automatically has that the limit of the Hawking energy along the flow is the Bondi energy of the cut at $\mathscr{I}^{-}$defined by $\Omega$ and measured by the observer defined by the flow. 
The second method we analyze is complementary to the previous one. Here we relax condition (1.5) and keep the assumption that the flow of surfaces along the null hypersurfaces approaches round spheres. In this context, one of our main achievements is Theorem 7.5.5, where we find two sufficient conditions that imply the validity of the null Penrose inequality. The method that has allowed us to obtain these conditions has been called Renormalized Area Method (a motivation for this name will be given later), and uses monotonic properties and limits of a suitable functional on spacelike surfaces which we introduce.

In this chapter we also study applications of the two methods. The Renormalized Area Method is applied to two particular but relevant cases, namely the case when $\Omega$ is shear-free and vacuum (where, as mentioned, the null Penrose inequality is known to hold by other methods [93]) and the case of null shells propagating in the Minkowski spacetime. The latter will allow us in particular to provide a link between the analysis here and the one in Chapter 4. Concerning the application of Theorem 7.3.8 to the Minkowski setting, we derive a shell Penrose type inequality (Theorem 7.8.2) valid for any closed spacelike surface in Minkowski for which its outer past null cone extends smoothly to past null infinity.

Some known results that are needed in this thesis are presented in three different Appendices. Appendix $\mathrm{A}$ is devoted to the study of the geometric relations between an embedded non degenerate pseudo-Riemannian manifold $N$ in an ambient one $M$. In this Appendix we find the relation between the second fundamental form vectors of more than two successively embedded manifolds, as well as the equation relating the Hessian of $N$ and $M$. We also state and prove the Gauss, Ricci and Codazzi identities.

We devote Appendix $B$ to study the geometry of null hypersurfaces $\Omega$. We start by introducing the standard quotient structure that allows to define a positive definite metric and a second fundamental form. We then devote most of the appendix to derive the evolution equations of various geometric quantities associated to $\Omega$ and to foliations of $\Omega$.

Appendix $\mathrm{C}$ is devoted to the study of the relation of the geometry of a convex body $C$ in $\mathbb{R}^{n}$ with the geometry of the standard sphere. In particular we define the support function and present its main properties. Our main objective is to find explicit expressions for the first and second fundamental forms of $C$ in terms of the support function and the geometry of the standard sphere. This is relevant for Chapter 4 in order to find an alternative form of the shell Penrose inequality.

To summarize, in this thesis we have studied the null Penrose inequality in detail. We have been able to prove the inequality in some cases and give a full proof of a Penrose type inequality. The general methods presented here open up new possibilities to address the problem. In fact, a recent approach by Roesch [92] uses as a key tool the main result of [76] corresponding in this thesis to Theorem 6.6.2 in Chapter 6. 
Exploiting the limit of the Hawking energy along $\Omega$ Roesch is able to prove the null Penrose inequality provided $\Omega$ admits a geodesic foliation satisfying certain restrictions. 



\section{Fundamentals for the Penrose inequality}

\subsection{Basic Definitions}

All manifolds in this thesis will be smooth and Hausdorff. Let $(M, g)$ be an $m$-dimensional oriented pseudo-Riemannian manifold of arbitrary signature. Tensors in $M$ carry Greek indices and we denote by $\nabla$ the Levi-Civita covariant derivative of $M$. Our sign convention for the curvature tensor is

$$
R(X, Y) Z:=\nabla_{X} \nabla_{Y} Z-\nabla_{Y} \nabla_{X} Z-\nabla_{[X, Y]} Z
$$

where $X, Y, Z$ are vector fields on $M$. The Riemann tensor $\operatorname{Riem}^{g}$ of $(M, g)$ is defined in terms of the curvature tensor by $\operatorname{Riem}^{g}(X, Y, Z, T):=g(T, R(Z, T) Y)$. Contracting the first and third indices of Riem ${ }^{g}$, we obtain the Ricci curvature tensor $\operatorname{Ric}^{g}(X, Y)=$ $\operatorname{tr}_{g}\left(\operatorname{Riem}^{g}(\cdot, X, \cdot, Y)\right)$, and its trace is the scalar curvature Scal ${ }^{g}$. The Einstein tensor $\operatorname{Ein}^{g}$ of $(M, g)$ reads

$$
\operatorname{Ein}^{g}=\operatorname{Ric}^{g}-\frac{1}{2} \operatorname{Scal}^{g} g
$$

Embedded submanifolds will play a relevant role in this work, and we devote Appendix A to describe their geometry. The basic objects are as follows: let $N$ be an $n$-dimensional submanifold embedded in $(M, g)$ with non-degenerate first fundamental form $\gamma$. The Gauss formula (cf. Appendix $\mathbb{A}$ ) relates the ambient connection $\nabla$ with the induced connection $\nabla^{N}$ by the formula

$$
\nabla_{X} Y=\nabla_{X}^{N} Y+\left(\nabla_{X} Y\right)^{\perp}
$$

for any vector fields $X, Y$ tangent to $N$, where ' ${ }^{\prime}$ ' is the operator that gives the orthogonal component to $N$ of a vector. The second term of the right-hand side of (2.1) defines the so called second fundamental form vector. 
Definition 2.1.1 (Second fundamental form vector). The second fundamental form vector of $N$ as an embedded manifold in $M$ is the $(2,1)$-tensor

$$
\vec{K}(X, Y)=-\left(\nabla_{X} Y\right)^{\perp} .
$$

The second fundamental form vector is by construction, orthogonal to $N$ (see Figure 2.1). In Appendix $\mathrm{A}$ we show that $\vec{K}$ is also symmetric in $X, Y$.

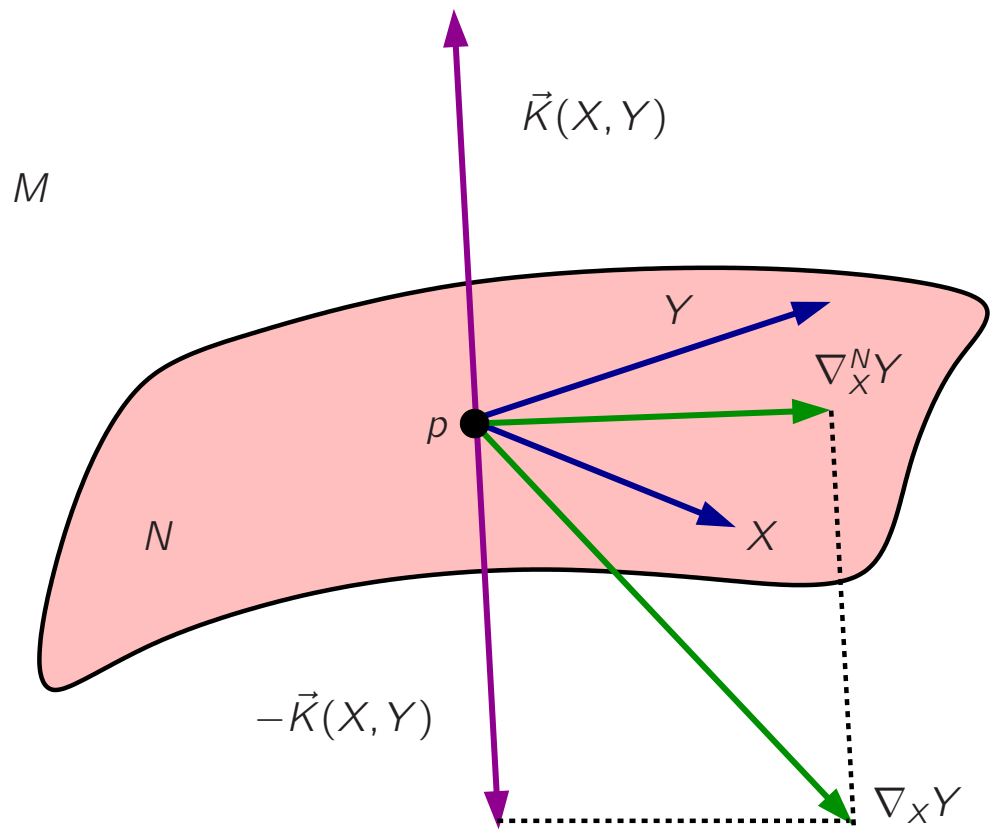

Figure 2.1: Schematic representation of the Gauss formula. The difference between the induced connection $\nabla^{N}$ on a non-degenerate embedded manifold $N$ and the ambient connection $\nabla$ of a pseudo-Riemannian manifold $M$ is the second fundamental form vector of $N$, namely $\nabla_{X} Y=\nabla_{X}^{N} Y-\vec{K}(X, Y)$.

Definition 2.1.2 (Mean curvature vector). The trace $\vec{H}$ of the second fundamental form vector with respect to the induced metric on $N$ is the mean curvature vector, i.e.

$$
\vec{H}:=\operatorname{tr}_{N} \vec{K}
$$

Let us consider a normal vector field $\nu$ to $N$, i.e. a vector $\nu$ satisfying $\nu(p) \in T_{p} N^{\perp}$ $\forall p \in N$.

Definition 2.1.3 (Second fundamental form tensor along $\nu$ ). The second fundamental form tensor along $\nu$ is defined as

$$
K^{\nu}(X, Y) \equiv\langle\nu, \vec{K}(X, Y)\rangle_{g}=-\left\langle\nu, \nabla_{X} Y\right\rangle_{g}=\left\langle\nabla_{X} \nu, Y\right\rangle_{g}, \quad \text { with } \quad X, Y \in \Gamma(T N)
$$




\section{Fundamentals for the Penrose inequality}

The second fundamental form tensor is symmetric, i.e. $K^{\nu}(X, Y)=K^{\nu}(Y, X)$, as a consequence of the symmetry of $\vec{K}$.

Definition 2.1.4 (Expansion along $\nu$ ). The expansion of $N$ along $\nu$, denoted by $\theta_{\nu}$, is the function

$$
\theta_{\nu}=\operatorname{tr}_{N}\left(K^{\nu}\right)
$$

Definition 2.1.5 (Volume element). The volume element $\boldsymbol{\eta}_{M}$ of $(M, g)$ is the $m$-form

$$
\left(\boldsymbol{\eta}_{M}\right)_{\alpha_{1} \ldots \alpha_{n}}=\sqrt{|\operatorname{det} g|} \epsilon_{\alpha_{1} \ldots \alpha_{n}}
$$

in any coordinate chart of the associated oriented atlas, where $\epsilon_{\alpha_{1} \ldots \alpha_{n}}$ is the Levi-Civita symbol and det $g$ is the determinant of $g$ in this chart.

Everything we have said is so far valid in any signature. To define the notion of spacetime we need the concept of time orientability and time-orientation:

Definition 2.1.6. A Lorentzian manifold $(M, g)$ is time-orientable if and only if there exists a vector field $u \in \mathfrak{X}(M)$ which is timelike everywhere on $M$. In this case a time-orientation is a choice of a timelike vector field $u$ which is declared to be future directed.

Definition 2.1.7 (Spacetime). A spacetime $(M, g)$ is an m-dimensional Lorentzian manifold, connected and orientable, endowed with a time orientation.

The metric $g$ satisfies the Einstein field equations, which take the form

$$
\operatorname{Ein}^{g}+\wedge g=\chi T, \quad \chi=\frac{8 \pi G}{c^{4}},
$$

where $G$ is the Newton gravitational constant, $c$ is the speed of light in vacuum, $T$ is the energy-momentum tensor of all non-gravitational fields and $\Lambda$ is the cosmological constant.

The energy conditions are usually defined in terms of the energy-momentum tensor $T$. We may express them in terms of the Einstein tensor Ein $^{g}$ because in our setting the cosmological constant $\Lambda$ vanishes. By defining the energy conditions directly in terms of Ein $^{g}$ we can forget altogether about the Einstein field equations so that all our results become applicable to the geometric theory of gravity. The energy conditions we use are

Definition 2.1.8. Let $(M, g)$ be a spacetime. Then

- $(M, g)$ satisfies the null energy condition (NEC) if the Einstein tensor Ein ${ }^{g}$ satisfies $\operatorname{Ein}^{g}(k, k) \geq 0$ for any null vector $k \in \mathfrak{X}(M)$. 


\subsection{Asymptotically flat spacetimes}

- $(M, g)$ satisfies the dominant energy condition (DEC) if the Einstein tensor Ein ${ }^{g}$ satisfies that - $\left.\operatorname{Ein}^{g \mu}{ }_{\nu} X^{\nu}\right|_{p}$ is a future directed causal vector for any future directed causal vector $X \in \mathfrak{X}(M)$ and all $p \in M$ (or equivalently when $\operatorname{Ein}_{\mu \nu}^{g} X^{\mu} Y^{\nu} \geq 0$ for all future causal vectors)

Remark 2.1.9. Note that the NEC is obviously weaker than the DEC.

In physical terms the DEC means that any observer measures a positive energy density for the field and the energy flows at a speed no larger than $c$.

Let $S$ be a compact, embedded, oriented, codimension-two surface in an $m$-dimensional spacetime $(M, g)$. The mean curvature vector $\vec{H}$ of $S$ plays an important role since the surface $S$ can be classified according to its causal character. Since the surface has codimension two, we can consider a future oriented null basis $\{k, \ell\}$ of vectors normal to $S$ and normalized so that $\langle k, \ell\rangle=-2$. In this case the mean curvature vector decomposes as

$$
\vec{H}=-\frac{1}{2}\left(\theta_{\ell} k+\theta_{k} \ell\right) \text {. }
$$

Definition 2.1.10. A closed surface is a:

- Future trapped surface if $\theta_{k}<0$ and $\theta_{\ell}<0$. Or equivalently, if $\vec{H}$ is timelike and future directed.

- Weakly future trapped surface if $\theta_{k} \leq 0$ and $\theta_{\ell} \leq 0$. Or equivalently, if $\vec{H}$ is causal and future directed.

- Marginally future trapped surface if either, $\theta_{k}=0$ and $\theta_{\ell} \leq 0$ everywhere, or, $\theta_{k} \leq 0$ and $\theta_{\ell}=0$ everywhere. Equivalently, if $\vec{H}$ is future directed and either proportional to $k$ or proportional to $\ell$ everywhere.

In spacetime dimension four, the Hawking energy is a functional acting on closed spacelike surfaces $S$ with spherical topology and reads

Definition 2.1.11 (Hawking energy). The Hawking energy of $S$ is defined by

$$
m_{H}(S)=\sqrt{\frac{|S|}{16 \pi}}\left(1-\frac{1}{16 \pi} \int_{S} \vec{H}^{2} \boldsymbol{\eta}_{S}\right),
$$

where $\vec{H}$ is the mean curvature of $S$ and $|S|$ is the area of $S$.

\subsection{Asymptotically flat spacetimes}

The notion of asymptotically flat (AF) spacetime is suitable to describe isolated objects. There are two different types of AF spacetimes depending on the infinity considered. We start with asymptotic flatness at spatial infinity. 


\section{Fundamentals for the Penrose inequality}

Definition 2.2.1. An asymptotically flat end of a spacelike hypersurface $\Sigma$ with first fundamental form $g$ and second fundamental form $K$ is a subset $\Sigma_{0}^{\infty} \subset \Sigma$ which is diffeomorphic to $\mathbb{R}^{3} \backslash \overline{B_{R}}$, where $B_{R}$ is an open ball of radius $R$. Moreover, in the Cartesian coordinates $\left\{x^{i}\right\}$ induced by the diffeomorphism, the following decay conditions hold

$$
\mathrm{Scal}^{g}=O\left(r^{-4}\right), \quad \nabla_{b}^{\Sigma}\left(K^{b}{ }_{a}-\left(\operatorname{tr}_{g} K\right) \delta^{b}{ }_{a}\right)=O\left(r^{-4}\right),
$$

where $r=|x|=\sqrt{x^{a} x^{b} \delta_{a b}}$, Scal ${ }^{g}$ is the scalar curvature of $g$ and $\nabla^{\Sigma}$ is the Levi-Civita connection on $\Sigma$.

Definition 2.2.2 (AF spacelike hypersurface). A spacelike hypersurface $\Sigma$ with first fundamental form $g$ and second fundamental form $K$, possibly with boundary, is asymptotically flat if $\Sigma=\mathcal{K} \cup \Sigma^{\infty}$, where $\mathcal{K}$ is a compact set and $\Sigma^{\infty}=\bigcup_{i} \Sigma_{i}^{\infty}$ is a finite union of asymptotically flat ends $\sum_{i}^{\infty}$.

Definition 2.2.3 (AF spacetime at spatial infinity). A spacetime is asymptotically flat at spatial infinity if it admits an AF spacelike hypersurface.

We next characterize AF spacetimes at null infinity. This requires using basic notions of causality. Let $p$ and $q$ be two different points in the spacetime $M$. We say that

- $p$ chronologically precedes $q$ (denoted by $p<<q$ ) if there exists a future-directed timelike curve from $p$ to $q$.

- $p$ strictly causally precedes $q$ (denoted by $p<q$ ) if there exists a future-directed causal curve from $p$ to $q$.

- $p$ causally precedes $q$ (denoted by $p \leq q$ ) if $p$ strictly causally precedes $q$ or $p=q$.

These notions allow one to define the chronological future and past and the causal future and past of a point:

- The chronological future of $p$, denoted by $I^{+}(p)$, is the set of all points $q$ in $M$ such that $p$ chronologically precedes $q: I^{+}(p)=\{q \in M: p<<q\}$.

- The chronological past of $p$, denoted by $I^{-}(p)$, is the set of all points $q$ in $M$ such that $q$ chronologically precedes $p: I^{-}(p)=\{q \in M: q<<p\}$.

- The causal future of $p$, denoted by $J^{+}(p)$, is the set of all points $q$ in $M$ such that $p$ causally precedes $q$ : $J^{+}(p)=\{q \in M: p \leq q\}$.

- The causal past of $p$, denoted by $J^{-}(p)$, is the set of all points $q$ in $M$ such that $q$ causally precedes $p: J^{-}(p)=\{q \in M: q \leq p\}$. 


\subsection{Energy-momentum vectors in AF spacetimes}

In order to define the concept of asymptotically flat spacetime at null infinity, we consider a compactification of the spacetime (see e.g. [106] for definitions), where spatial infinity will be denoted by $i^{0}$, and the hypersurfaces representing past and future null infinity will be denoted by $\mathscr{I}^{-}$and $\mathscr{I}^{+}$respectively.

Definition 2.2.4 (AF spacetime at null infinity). A spacetime $(M, g)$ is said to be asymptotically flat at null infinity if there exits a manifold $\bar{M}$ with boundary, with smooth metric $\bar{g}$, and a conformal isometry of $M$ onto the interior of $\bar{M}$ with conformal factor $\Omega$. By defining $\mathscr{I}$ as the boundary of $\bar{M}$, the following conditions are required:

(i) $\mathscr{I}$ can be written as the disjoint union of two pieces $\mathscr{I}^{+}$and $\mathscr{I}^{-}$, such that $\mathscr{I}^{+} \cap J^{-}[\operatorname{int}(M)]=\emptyset$ and $\mathscr{I}^{-} \cap J^{+}[\operatorname{int}(M)]=\emptyset$.

(ii) $\Omega$ can be extended to a $C^{\infty}$ function on all of $\bar{M}$. On $\mathscr{I}^{+}$and $\mathscr{I}^{-}$we have $\Omega=0$ and $d \Omega \neq 0$.

(iii) $\mathscr{I}^{+}$and $\mathscr{I}^{-}$each have the topology $\mathbb{S}^{2} \times \mathbb{R}$.

(iv) There exists a smooth function $f$ defined on $\bar{M}$, with $f>0$ on $M \cup \mathscr{I}^{+} \cup \mathscr{I}^{-}$and satisfying $\bar{\nabla}_{\alpha}\left(f^{4} n^{\alpha}\right)=0$ on $\mathscr{I}^{+} \cup \mathscr{I}^{-}$(where $\bar{\nabla}_{\alpha}$ is the connection associated with $\bar{g}$ ), the vector field $f^{-1} n^{\alpha}$ is complete on $\mathscr{I}^{+} \cup \mathscr{I}^{-}$.

Both types of asymptoticity are combined in the following definition:

Definition 2.2.5 (AF spacetime). A spacetime $(M, g)$ is said to be asymptotically flat if is is AF at null infinity and it admits an AF spacelike hypersurface

\subsection{The ADM and Bondi energy-momentum four vec- tors in AF spacetimes}

The concept of energy in General Relativity is a delicate issue. In particular there is no notion of energy density of the gravitational field, However, there exists a useful notion of the total energy of an isolated system which is described by the total energymomentum four-vector $P$. This vector is defined in an abstract Minkowski spacetime with metric $\eta$. The time component of this vector is the energy $E$ of the spacetime, and the rest of the components define an spacelike three-momentum $p$. Its Minkowskian length is the total mass of the spacetime.

In an AF spacetime, two different types of energy-momentum four vector can be defined depending the infinity that we are considering. The ADM (Arnowitt, Deser, Misner) four-momentum $P_{A D M}=\left(E_{A D M}, p_{A D M}\right)$ is associated to spacetimes that are $A F$ at spatial infinity $i^{0}$, and the Bondi energy-momentum four-vector $P_{B}=\left(E_{B}, p_{B}\right)$ 


\section{Fundamentals for the Penrose inequality}

is measured at a cut $S^{\infty}$ of null infinity $\mathscr{I}$, where $S^{\infty}$ is a spacelike cross section of the null hypersurface $\mathscr{I}$. The Bondi four-momentum depends on the cut and approaches the ADM four-momentum at $i^{0}$ under suitable conditions [114].

The expression for the total energy-momentum $P_{A D M}$ is given in terms of the geometry of an AF hypersurface $(\Sigma, g, K)$ and was put forward by Arnowitt, Deser and Misner in 1962 [3]:

Definition 2.3.1. Consider a spacelike hypersurface $(\Sigma, g, K)$ with a selected asymptotically flat end $\Sigma_{0}^{\infty}$. Then, the ADM energy-momentum $P_{A D M}$ associated to $\Sigma_{0}^{\infty}$ is the spacetime vector with components

$$
\begin{aligned}
& P_{A D M 0}=E_{A D M}=\lim _{r \rightarrow \infty} \frac{1}{16 \pi} \int_{S_{r}}\left(\partial_{b} g_{a b}-\partial_{a} g_{b b}\right) m^{a} \boldsymbol{\eta}_{\boldsymbol{S}}, \\
& P_{A D M a}=p_{A D M a}=\lim _{r \rightarrow \infty} \frac{1}{8 \pi} \int_{S_{r}}\left(K_{a b}-g_{a b} t r_{g} K\right) m^{b} \boldsymbol{\eta}_{\boldsymbol{S}},
\end{aligned}
$$

where $\left\{x^{a}\right\}$ are the Cartesian coordinates induced by the diffeomorphism which defines the asymptotically flat end, $S_{r}$ is the surface at constant $r, m^{a}$ is the outward unit normal of $S_{r}$ and $\boldsymbol{\eta}_{\boldsymbol{S}}$ its volume form. $E_{A D M}$ is called the $A D M$ energy and $p_{A D M}$ the ADM spatial momentum vector.

The notion of ADM mass (the Minkowskian length of $P_{A D M}$ ) is independent of the choice of coordinates as long as the decay conditions (2.4) are satisfied. One of the most important theorems concerning the ADM energy-momentum is the Positive mass theorem (PMT) by Schoen and Yau [95].

Theorem 2.3.2 (Positive mass theorem (PMT), Schoen \& Yau, 1981). Let $(\Sigma, g, K)$ be an asymptotically flat spacelike hypersurface without boundary and satisfying the $D E C$. Then the total ADM energy-momentum $P_{A D M}$ is a future directed causal vector. Furthermore, $P_{A D M}=0$ if and only if $\Sigma$ is a slice of the Minkowski spacetime.

The PMT was proved by Schoen and Yau for manifolds of dimension less or equal than seven, and by Witten [111] for spin manifolds of arbitrary dimension using spinor techniques.

Concerning the Bondi energy, the first definition was given in coordinates and was introduced by Bondi, van der Burg and Metzner in 1962 [9]. Later Geroch and Winicour [35] gave an alternative form in terms of asymptotic time translations, i.e. vector fields that tend to a Killing vector near infinity and satisfy $\nabla_{\alpha} \xi^{\alpha}=0$. Specifically, let $\left\{S_{\alpha}\right\}$ be a one-parameter family of topological spheres, which in the associated compactified spacetime approach a cross section $S^{\infty}$ of $\mathscr{I}^{-}$. Then, the quantity

$$
E_{B}^{\xi}=-\lim _{S_{\alpha} \rightarrow S_{\infty}} \frac{1}{8 \pi} \int_{S_{\alpha}} \epsilon_{\alpha \beta \gamma \delta} \nabla^{\gamma} \xi^{\delta}
$$


is independent of how $S_{\alpha}$ approaches $S_{\infty}$. We refer to $E_{B}^{\xi}$ as the energy measured by the observer $\xi$. Formula (2.5) reduces to the Komar mass in stationary spacetimes.

In practice, there is a very effective way to compute the Bondi energy of a spacetime admitting a null hypersurface $\Omega$ that extends smoothly to null infinity, and is based on the use of the Hawking mass functional $m_{H}$ (see Definition 2.3 in Chapter 2) evaluated on flows $\left\{S_{s}\right\}$ approaching large spheres along $\Omega$. Specifically

$$
E_{B}^{u}=\lim _{s \rightarrow \infty} m_{H}\left(S_{s}\right)
$$

where $u$ is the observer determined by the foliation $\left\{S_{s}\right\}$ (for more information, see Chapter (6). This is actually the method used in this thesis to compute the Bondi energy. As in the ADM case, the Bondi energy is the time component of an energymomentum four vector $P_{B}$, i.e. $E_{B}^{u}=-\eta\left(P_{B}, u\right)$, where $\eta$ the Minkowski metric in the abstract space where the observer $u$ is defined. In analogy with the ADM case, the Bondi mass $M_{B}$ is the Lorentzian length of $P_{B}$.

The ADM energy represents the total energy available in the spacetime, whereas the Bondi energy is interpreted as the energy remaining in the spacetime at the "retarded time" given by the cross section $S_{\infty}$ of $\mathscr{I}^{+}$, after emission of gravitational radiation. There is also a theorem of positivity of the Bondi energy (Horowitz and Perry 1982 [49]; Schoen and Yau 1982 [96]; Ludvigsen \& Vickers 1982 [66]; Reula and Tod 1984 [91]).

\subsection{Black holes and the weak cosmic censorship con- jecture}

The heuristic argument put forward by Penrose to formulate the Penrose inequality conjecture is based on the weak cosmic censorship conjecture. As already mentioned in the Introduction, this conjecture states roughly that singularities are always hidden within a black hole.

A spacetime $(M, g)$ is a black hole if it is asymptotically flat at null infinity and if there are events causally disconnected from the asymptotic region, i.e. no causal curves starting at the event in question ever reach the asymptotic region. The black hole region $\mathcal{B}$ of the spacetime is defined by

$$
\mathcal{B}=M \backslash I^{-}\left(\mathscr{I}^{+}\right)
$$

where the chronological past ${I^{-}}^{-}$is considered in the conformally completed spacetime. The event horizon $\mathcal{H}$ of the black hole is defined to be the boundary of $\mathcal{B}$ in $M$

$$
\mathcal{H}=\partial \mathcal{B}
$$




\section{Fundamentals for the Penrose inequality}

The event horizon $\mathcal{H}$ is the topological boundary of the black hole region. $\mathcal{H}$ is a (Lipschitz) null hypersurface ruled by future inextendible null geodesics [44].

A more precise statement of the weak cosmic censorship conjecture is as follows:

Weak cosmic censorship conjecture: Let $(\Sigma, g, K ; \psi)$ be an asymptotically flat initial data set of the Einstein field equations with suitable matter (where $\psi$ represents the matter fields). Then, generically, its maximal Cauchy evolution is asymptotically flat at future null infinity, with complete $\mathscr{I}^{+}$.

The notions of suitable and generic are kept deliberately vague since their precise meaning will here to be adjusted to the actual theorem one can eventually prove. However, roughly speaking, suitable matter should mean that the initial value formulation for the coupled Einstein-matter field equations is well posed and the stress-energy tensor satisfies suitable energy conditions. The genericity condition should mean that initial configurations leading to naked singularities have empty interior in a suitable topology in the space of initial data sets.

\subsection{The Penrose inequality conjecture. Heuristics}

This section is devoted to describe the heuristics behind the Penrose inequality. In the following section we will concentrate on the study of hypersurfaces and shells in spacetimes, with the aim of writing down the shell Penrose inequality.

The Penrose inequality conjecture is a strengthening of the positive energy theorem when black holes are present. In spacetimes satisfying the null energy condition (NEC), i.e. spacetimes where $\operatorname{Ein}^{g}(k, k) \geq 0$ for all null vectors $k$, the event horizon satisfies the area theorem, which essentially says that in such spacetimes the area of the cross sections of the event horizon grows with time [42, 43, 20]. The precise formulation of the area theorem reads as follows:

Theorem 2.5.1 (Black hole area theorem [20]). Let $(M, g)$ be a black hole spacetime satisfying the NEC. Let $\Sigma_{1}$ and $\Sigma_{2}$ be achronal hypersurfaces (no timelike curve intersects them twice), and spacelike and define $\mathcal{H}_{\Sigma_{a}}:=\mathcal{H} \cap \Sigma_{a}(a=1,2)$ sections of the event horizon. If $\mathcal{H}_{\Sigma_{1}}$ lies in the past of $\mathcal{H}_{\Sigma_{2}}$, then $\left|\mathcal{H}_{\Sigma_{1}}\right| \leq\left|\mathcal{H}_{\Sigma_{2}}\right|$.

In physical terms, it is expected that any black hole spacetime must settle down to an asymptotic stationary state in the distant future. All matter fields (except electromagnetic ones or, perhaps, other fields with globally conserved charges) are expected to be radiated away or fall into the black hole region. The asymptotic spacetime is then expected to be electrovacuum and stationary. The black hole uniqueness theorem (see e.g. [19, 48]) can be applied to conclude that the exterior region of the asymptotic black hole is isometric to the exterior region of a Kerr-Newman black hole. 
Let us recall a few known properties of the Kerr-Newman black hole spacetime $\left(M, g_{m, a, q}\right)$. The metric is described by three parameters $m, a$ and $q$ (mass, angular momentum parameter and charge) satisfying

$$
m \geq \sqrt{a^{2}+q^{2}}
$$

When $a=q=0$, we have the Kruskal spacetime. All sections $S$ of the event horizon $\mathcal{H}$ are isometric to each other and have area

$$
|S|=8 \pi m\left(m+\sqrt{m^{2}-a^{2}-q^{2}}\right)-4 \pi q^{2} \leq 16 \pi m^{2} .
$$

As already mentioned, the paradigm described above can be rephrased by saying that for any dynamic black hole spacetime, there exist $m, a, q \in \mathbb{R}$ satisfying $\sqrt{a^{2}+q^{2}} \leq$ $m \neq 0$ such that the exterior region of the black hole approaches (in a suitable sense) the spacetime $\left(M, g_{m, a, q}\right)$ when $t \rightarrow+\infty$.

The Bondi-mass $M_{B}:=\left(E_{B}^{2}-\vec{P}_{B}^{2}\right)^{\frac{1}{2}}$ decreases to the future and its limit at $i_{0}$ is the $A D M$ mass $M_{A D M}:=\left(E_{A D M}^{2}-\vec{P}_{A D M}^{2}\right)^{\frac{1}{2}}$. This property physically means that the gravitational radiation can only extract energy from the spacetime. Moreover the Bondi mass of the Kerr-Newman spacetime is $m$. Using these facts, Penrose obtained the following chain of inequalities:

$$
\left|\mathcal{H}_{\Sigma}\right| \leq\left|\mathcal{H}_{\Sigma_{\infty}}\right| \leq 16 \pi m^{2} \leq 16 \pi M_{B}^{2} \leq 16 \pi M_{A D M}^{2}
$$

where the first inequality is a consequence of the area theorem, the second is a property of the Kerr-Newman spacetime, and the two last ones are consequences of the properties of the total energy-momentum in an asymptotically flat spacetime.

The resulting inequality $\left|\mathcal{H}_{\Sigma}\right| \leq 16 \pi M_{A D M}^{2}$ involves no future asymptotic properties, but still involves the event horizon, which is a global concept in the spacetime. A priori one does not know if the event horizon exists or where it lies. The second main observation of Penrose was that the paradigm of gravitational collapse also implied an inequality "local in time" as follows.

Strong gravitational fields can be detected by weakly future trapped surfaces. Consider an AF spacetime $(M, g)$ satisfying the DEC. Assume that the spacetime contains a weakly future trapped surface $S$ embedded in an asymptotically flat spacelike hypersurface $\Sigma$ with one end. Under these hypotheses it follows [83, 40] (see also [98] for a nice review) that the spacetime $(M, g)$ has a singularity (in the sense that there is an inextendible, incomplete causal geodesic and the spacetime itself is inextendible). Under weak cosmic censorship, this singularity is shielded from infinity by an event horizon and $M$ is necessarily a black hole. A fundamental property of the black hole spacetimes is that any weakly future trapped surface $S$ is necessarily contained in the black hole region [106, 21]. Consider the spacelike hypersurface $\Sigma$ that contains $S$. This hypersurface 


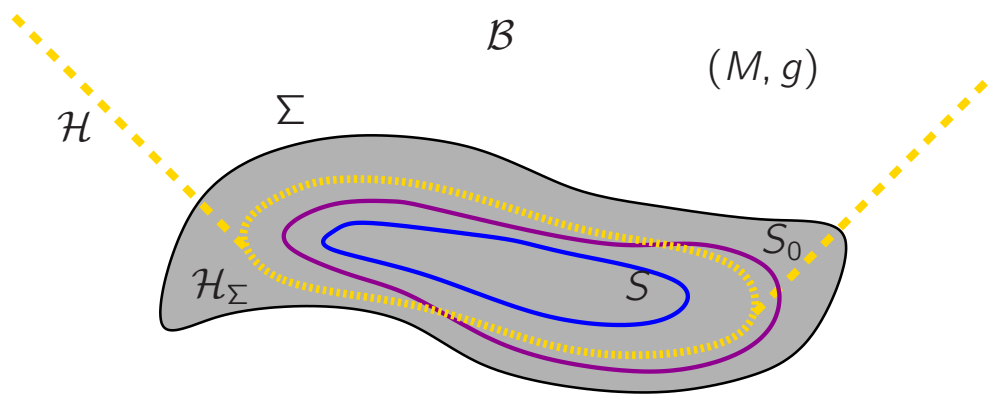

Figure 2.2: When the spacetime $(M, g)$ satisfies the dominant energy condition and contains a weakly outer trapped surface $S$, it necessarily contains a singularity. Under the weak cosmic censorship the singularity must lie in a black hole region $\mathcal{B}$, and $(M, g)$ is a black hole. The surface $S$ must lie in $\mathcal{B}$. The surface $S_{0}$ that minimizes area among all those surfaces in a spacelike hypersurfaces $\Sigma$ enclosing $S$ satisfies $\left|S_{0}\right| \leq\left|\mathcal{H}_{\Sigma}\right|$ (where $\mathcal{H}_{\Sigma}$ is the intersection of the event horizon with $\Sigma$ ) and does not have to lie necessarily within $\mathcal{B}$. Under the standard paradigm of gravitational collapse (including weak cosmic censorship) the Penrose inequalities $\left|S_{0}\right| \leq 16 \pi M_{B}^{2} \leq 16 \pi M_{A D M}^{2}$ follow.

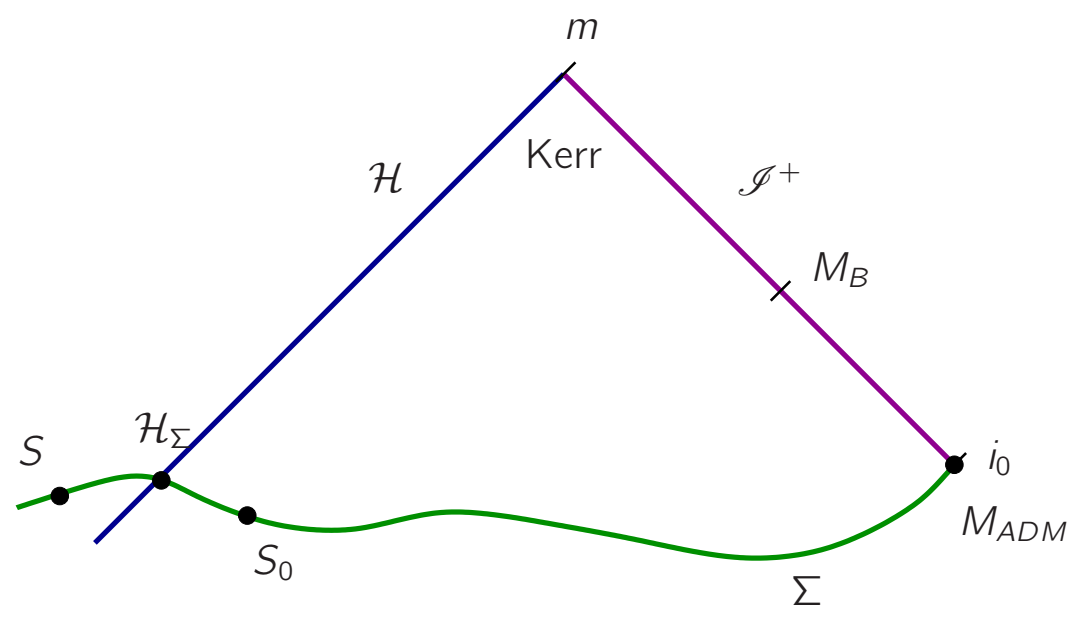

Figure 2.3: Equivalent representation to Figure 2.2, where the standard paradigm of gravitational collapse is represented. The spacetime becomes asymptotically stationary as time goes to infinity and it is therefore expected to tend to Kerr-Newman. The area of the cross sections $\mathcal{H}_{\Sigma}$ of the event horizon $\mathcal{H}$ grows with time, and in the limit $\left|\mathcal{H}_{\Sigma_{\infty}}\right| \leq 16 \pi \mathrm{m}^{2}$, with $m$ the mass of the Kerr-Newman spacetime. The relation between the masses $m \leq M_{B} \leq M_{A D M}$ implies $\left|\mathcal{H}_{\Sigma}\right| \leq 16 \pi M_{B}^{2} \leq 16 \pi M_{A D M}^{2}$, and since $S_{0}$ minimizes area among all those surfaces in the spacelike hypersurfaces $\Sigma$ enclosing $S$ (in particular $\left|S_{0}\right| \leq\left|\mathcal{H}_{\Sigma}\right|$ ), the Penrose inequalities $\left|S_{0}\right| \leq 16 \pi M_{B}^{2} \leq 16 \pi M_{A D M}^{2}$ follow. 
intersects the event horizon in a cross section $\mathcal{H}_{\Sigma}$. If the area $|S|$ were smaller than the area of $\mathcal{H}_{\Sigma}$, we would have

$$
|S| \leq\left|\mathcal{H}_{\Sigma}\right| \leq 16 \pi M_{B}^{2} \leq 16 \pi M_{A D M}^{2}
$$

i.e. $|S| \leq 16 \pi M_{A D M}^{2}$, which already involves only the geometry of the spacelike hypersurface $\Sigma$. Although $\mathcal{H}_{\Sigma}$ encloses $S$, it is not necessarily true that $|S| \leq\left|\mathcal{H}_{\Sigma}\right|$, because from the fact that $\mathcal{H} \cap \Sigma$ encloses $S$ does not follow that its area is necessarily larger. This problem can be avoided by considering the minimal area enclosure of $S$, i.e. the outermost of all surfaces which enclose $S$ and have less or equal area than any other surface enclosing $S$. The minimal area enclosure of $S$ always exists provided the dimension of the spacetime is $n \leq 8$ and $S$ bounds an exterior domain. We will denote by $\mathcal{S}_{\text {min }}(S)$ the minimal surface enclosure of $S$. The spacelike cut $\mathcal{H} \cap \Sigma$ of the event horizon also encloses $S$, so we have $\left|\mathcal{S}_{\min }(S)\right| \leq|\mathcal{H} \cap \Sigma|$. From (2.6), it follows

$$
M_{A D M} \geq \sqrt{\frac{\left|\mathcal{S}_{\min }(S)\right|}{16 \pi}},
$$

and considering the supremum among all weakly outer trapped surfaces, we finally obtain

$$
M_{A D M} \geq \sup \sqrt{\frac{\left|\mathcal{S}_{\min }(S)\right|}{16 \pi}} .
$$

Defining $S_{0}$, we arrive at the two inequalities

$$
\left|S_{0}\right| \leq 16 \pi M_{B}^{2} \quad \text { and } \quad\left|S_{0}\right| \leq 16 \pi M_{A D M}^{2}
$$

These inequalities do not require to know anything about the future of the spacetime. Inequalities (2.7) are called Penrose inequalities and are the main theme of this work (variations of these inequalities are also sometimes considered, while keeping the name of Penrose inequalities). Proving them would give strong indirect support to the general paradigm of gravitational collapse and would also be a strengthening of the positive energy theorem. In this thesis we concentrate on the so-called null Penrose inequality.

For this case, assume that $(M, g)$ admits a null hypersurface $\Omega$ containing a weakly future trapped surface $S$ and extending smoothly all the way to past null infinity $\mathscr{I}^{-}$. In this case, any spacelike surface $S^{\prime}$ lying to the past of $S$ along $\Omega$ satisfies $|S| \leq\left|S^{\prime}\right|$. The Bondi energy $E_{B}(\Omega)$ and mass $M_{B}(\Omega)$ of $\Omega$ can be defined. Following the above reasoning, under weak cosmic censorship $(M, g)$ must be necessarily a black hole and $S$ must be completely contained in the black hole region, and this necessarily implies that the intersection $\mathcal{H}_{\Omega}$ of the event horizon $\mathcal{H}$ with $\Omega$ must lie completely in the causal past of $S$. This implies $\left|\mathcal{H}_{\Omega}\right| \geq|S|$, i.e. the area $S$ is smaller than the area of the section of the event horizon lying on $\Omega$. Thus, we can work with $S$ and there is no need to take the minimal area enclosure as in the spacelike case above. Applying the same reasoning as above, it follows

$$
|S| \leq\left|\mathcal{H}_{\Omega}\right| \leq\left|\mathcal{H}_{\Omega_{\infty}}\right| \leq 16 \pi m^{2} .
$$




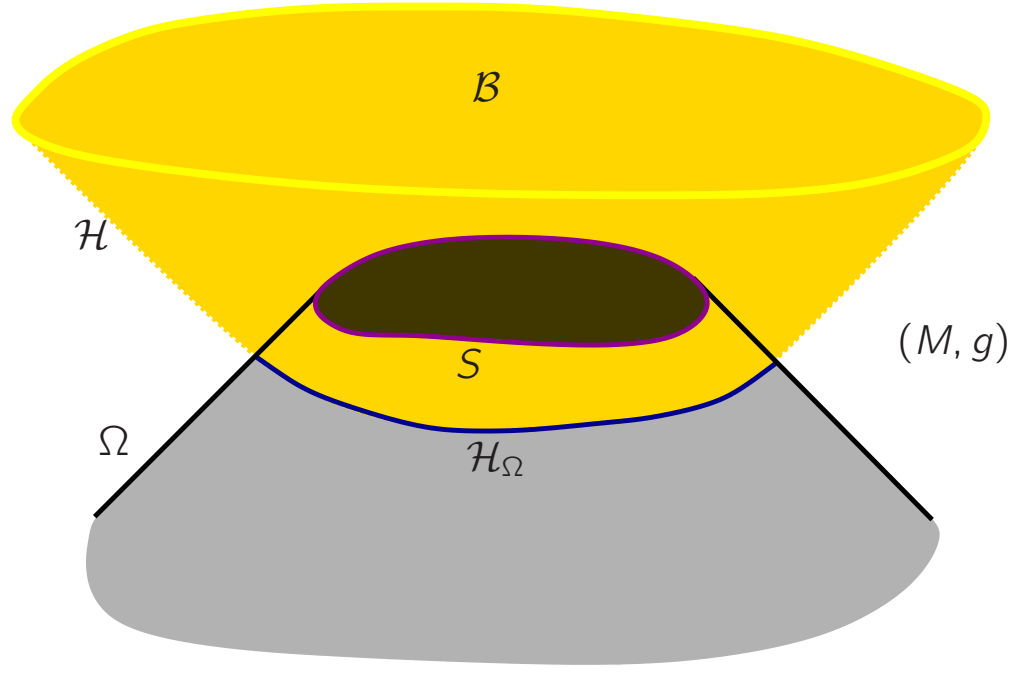

Figure 2.4: Analogous case to Figure 2.2, where now the hypersurface $\Omega$ is null and contains a weakly future trapped surface $S$. Following the same argument as in Figure 2.2. $S$ lies within the black hole region $\mathcal{B}$ under the standard paradigm of gravitational collapse. Since $|S| \leq\left|\mathcal{H}_{\Omega}\right|$, there is no need to consider the minimal area enclosure of $S$ as in the spacelike case in Figure 2.2. In these circumstances the paradigm of gravitational collapse implies the null Penrose inequality $|S| \leq 16 \pi E_{B}^{2}(\Omega)$.

The mass $m$ of the Kerr-Newman spacetime satisfies $m \leq M_{A D M}$. The spacetime $(M, g)$ is determined from the initial data on $\Omega$ and on $\mathscr{I}^{-}$. Now we use the fact that the spacetime $(M, g)$ can be changed without modifying the data on $\Omega$ (in particular without changing the Bondi mass $M_{B}(\Omega)$ ). We are free to impose the condition that there is no incoming radiation at $\mathscr{I}^{-}$. In this case, the Bondi mass $M_{B}(\Omega)$ defined by the cut of the hypersurface $\Omega$ and past null infinity equals the total ADM mass and we can conclude

$$
|S| \leq 16 \pi M_{B}^{2}(\Omega) \leq 16 \pi E_{B}^{2}(\Omega) .
$$

The inequality $|S| \leq 16 \pi E_{B}^{2}(\Omega)$ is the so called null Penrose inequality and involves only the geometry along $\Omega$.

\subsection{Hypersurfaces and shells}

A particular but very interesting case of the null Penrose inequality is the original construction used by Penrose [85] in 1973, which consisted of a shell of collapsing dust moving inwards in the Minkowski spacetime. The shell separates the spacetime into two components. The interior one has a Minkowski metric and the outer part is no longer flat. The two components are joined by a null hypersurface. One of the main 


\subsection{Hypersurfaces and shells}

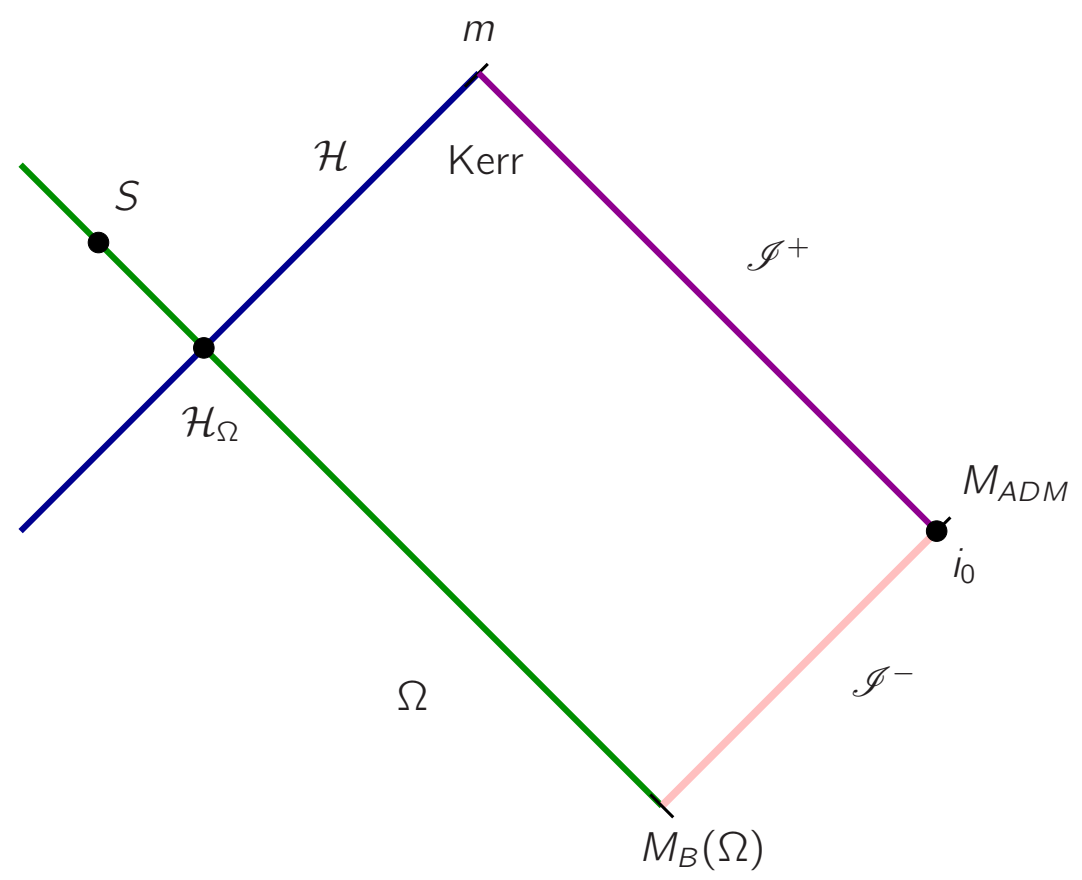

Figure 2.5: Analogous representation as in Figure 2.3 in the case where $\Omega$ is null. The area of the cross sections $\mathcal{H}_{\Omega}$ of the event horizon $\mathcal{H}$ grows with time, and in the limit $\left|\mathcal{H}_{\Omega_{\infty}}\right| \leq 16 \pi \mathrm{m}^{2}$, with $m$ the mass of the Kerr-Newman spacetime. Since $m \leq M_{A D M}$ and $\mathcal{H}_{\Omega}$ lies in the causal past of $S$, then $|S| \leq\left|\mathcal{H}_{\Omega}\right| \leq 16 \pi M_{A D M}^{2}$. We are allowed to change the spacetime to the future of $\Omega$ by assuming that there is no incoming gravitational radiation at $\mathscr{I}^{-}$, and hence $M_{A D M}=M_{B}(\Omega)$, which implies the null Penrose inequality $|S| \leq 16 \pi E_{B}^{2}(\Omega)$.

aims in this chapter is to recover the particular expression that Penrose found for his inequality in this shell setup. To that purpose we find it convenient to study in a more general setting the geometry of embedded hypersurfaces of arbitrary causal character that will separate two different spacetimes matched across their boundaries. This geometry is relevant because it determines the expression that the energy-momentum tensor of the shell concentrated on the hypersurface has, as well as the corresponding shell field equations (see [71]). This will allow us to particularize to the case when the interior spacetime is Minkowski and the exterior is a non-flat spacetime generated by the incoming null shell of dust as in Penrose's construction, and will allow us to rewrite the original null Penrose inequality as a geometric inequality for surfaces embedded in the Minkowski spacetime. The exposition here is based on the results by Mars presented in [70].

Let $\Phi: \Omega \rightarrow M$ be the embedding of a hypersurface $\Omega$ of arbitrary causal character into the spacetime. In order to describe the intrinsic and extrinsic geometry of $\Omega$, is is convenient to introduce an additional structure, namely a spacetime vector field along 


\section{Fundamentals for the Penrose inequality}

$\Omega$ which is transverse to $\Omega$ everywhere. Such vector field, called rigging, was first introduced by Schouten [97]:

Definition 2.6.1. (Schouten [97]) A rigging $\ell$ is a smooth section $\ell \in \Gamma\left(T_{\Omega} M\right)$ satisfying $\left.\ell\right|_{p} \notin T_{p} \Omega$ for all $p \in \Omega$.

We can define the scalar $\ell^{(2)}=g(\ell, \ell)$ and the one-form $\ell:=g(\ell, \cdot)$. Consider a basis $\left\{e_{a}\right\}$ tangent to $\Omega$. Pulling $\boldsymbol{\ell}$ back to $\Omega$, we obtain a one form $\ell_{a}=g\left(\ell, e_{a}\right)$. We denote by $\gamma$ the pullback on $\Omega$ of the ambient metric $g$. Note that $\gamma$ is degenerate whenever $\Omega$ is a null hypersurface. However, the square matrix

$$
\mathbb{A} \equiv\left(\begin{array}{c|c}
\gamma_{a b} & \ell_{a} \\
\hline \ell_{b} & \ell^{(2)}
\end{array}\right)
$$

always has Lorentzian signature at every point $p \in \Omega$ (because this is simply the matrix representation of the ambient metric $g$ in the basis $\left.\left\{e_{a}, \ell\right\}\right)$. This suggested the following definition [71] where everything refers to $\Omega$ as an abstract manifold, not embedded in any ambient spacetime:

Definition 2.6.2. A smooth three-dimensional manifold $\Omega$, a symmetric tensor $\gamma_{a b}, a$ one-form $\ell_{a}$ and a scalar $\ell^{(2)}$ define a hypersurface metric data set provided the square matrix $\mathbb{A}$ has Lorentzian signature at every point $p \in \Omega$.

In this case the inverse of $\mathbb{A}$ exists and a symmetric tensor $P^{a b}$, a vector $n^{a}$ and a scalar $n^{(2)}$ can be defined by means of

$$
\mathbb{A}^{-1}:=\left(\begin{array}{c|c}
P^{a b} & n^{a} \\
\hline n^{b} & n^{(2)}
\end{array}\right) .
$$

In [71] Mars also introduced the following definition:

Definition 2.6.3 (Hypersurface data). A five-tuple $\left\{\Omega, \gamma_{a b}, \ell_{a}, \ell^{(2)}, Y_{a b}\right\}$ where $\left\{\Omega, \gamma_{a b}\right.$, $\left.\ell_{a}, \ell^{(2)}\right\}$ is hypersurface metric data and $Y_{a b}$ is a symmetric tensor is called hypersurface data.

This abstract definition makes sense insofar it can be connected to the intrinsic and extrinsic geometry of $\Omega$ when this is embedded in a spacetime. The following definition summarizes this connection:

Definition 2.6.4 (Embedding of hypersurface data). Let $\left\{\Omega, \gamma_{a b}, \ell_{a}, \ell^{(2)}, Y_{a b}\right\}$ be hypersurface data. This data is embedded in a spacetime $(M, g)$ if there exists an embedding $\Phi: \Omega \rightarrow M$ and a choice of rigging $\ell$ such that, with $\ell:=g(\ell, \cdot)$,

$$
\Phi^{*}(g)=\gamma, \quad \Phi^{*}(g(\ell, \cdot))_{a} \equiv \ell_{a}, \quad g(\ell, \ell)=\ell^{(2)}, \quad \frac{1}{2} \Phi^{*}\left(£_{\ell} g\right)=Y .
$$




\subsection{Hypersurfaces and shells}

There is a natural way of defining a connection on the hypersurface $\Omega$. Given two vectors $X, Y \in \mathfrak{X}(\Omega)$ we define

$$
\begin{aligned}
\bar{\nabla}_{X} Y & :=\left(\nabla_{X} Y\right)^{\|_{\ell}}, \\
K(X, Y) & :=-\left(\nabla_{X} Y\right)^{\perp_{\ell}},
\end{aligned}
$$

where $\|_{\ell}$ denotes the tangent component to $\Omega$, and ${ }^{\perp_{\ell}}$ the parallel component to $\ell$ in the direct sum decomposition $T_{p} M=T_{p} \Omega \oplus<\ell_{p}>$. It is immediate to check [72] that $\bar{\nabla}$ defines a torsion-free covariant derivative on $\Omega$ and that $K(X, Y)$ is a symmetric tensor. As shown in [72], the Christoffel symbols $\bar{\Gamma}_{b c}^{a}$ associated to $\bar{\nabla}$ are defined by

$$
\nabla_{e_{a}} e_{b}=-K_{a b} \ell+\bar{\Gamma}_{b a}^{c} e_{c} .
$$

In any coordinate basis of $\Omega, \bar{\Gamma}_{a b}^{c}$ takes the following explicit form

$$
\bar{\Gamma}_{a b}^{c}=\frac{1}{2} P^{c d}\left(\partial_{a} \gamma_{b d}+\partial_{b} \gamma_{a d}-\partial_{d} \gamma_{a b}\right)+n^{c}\left(-Y_{a b}+\frac{1}{2}\left(\partial_{a} l_{b}+\partial_{b} l_{a}\right)\right) .
$$

Note that this expression makes sense even when $\Omega$ is not embedded. Thus the connection $\bar{\nabla}$ exists already at the abstract hypersurface data level.

When the hypersurface data is embedded in the spacetime $(M, g)$, we can consider the scalar $\rho_{\ell}$ and the one-form $J_{a}$ on $\Omega$ defined as

$$
\rho_{\ell}:=-\operatorname{Ein}^{g}(n, \ell), \quad J_{a}:=-\operatorname{Ein}^{g}\left(n, e_{a}\right) .
$$

The scalar $\rho_{\ell}$ is called [71] the "energy along $\ell$ " and $J_{a}$ the "energy flux". Both depend on the choice of the rigging. The definition of matter-hypersurface data was also put forward in [71]:

Definition 2.6.5 (Embedded matter-hypersurface data). A 7-tuple $\left\{\Omega, \gamma_{a b}, \ell_{a}, \ell^{(2)}, Y_{a b}\right.$, $\left.\rho_{\ell}, J_{a}\right\}$ defines embedded matter-hypersurface data provided the hypersurface data $\left\{\Omega, \gamma_{a b}, \ell_{a}, \ell^{(2)}, Y_{a b}\right\}$ is embedded with embedding $\Phi$ and rigging $\ell$, and the scalar $\rho_{\ell}$ and one-form $J_{a}$ on $\Omega$ satisfy

$$
\rho_{\ell}=-\Phi^{*}\left(\operatorname{Ein}^{g}(\ell, n)\right), \quad \boldsymbol{J}=-\Phi^{*}\left(\operatorname{Ein}^{g}(\cdot, n)\right),
$$

with $n$ being the vector normal to $\Omega$ satisfying $g(n, \ell)=1$.

With these notions at hand one can define the notion of shell. Shells arise naturally in the matching theory of two spacetimes. Consider two different spacetimes $\left(M^{ \pm}, g^{ \pm}\right)$ with diffeomorphic boundaries $\partial M^{ \pm}$. Let $\Omega$ be diffeomorphic to $\partial M^{+}$(or $\partial M^{-}$), and $\Phi^{ \pm}: \Omega \rightarrow M^{ \pm}$be embeddings such that $\Phi^{ \pm}(\Omega)=\partial M^{ \pm}$. The map $\Phi^{+} \circ\left(\Phi^{-}\right)^{-1}$ establishes a diffeomorphism between $\partial M^{+}$and $\partial M^{-}$. The two spacetimes can be joined accross the boundaries to produce a spacetime with continuous metric provided additional conditions are satisfied. Clarke and Dray [24] studied the case of the boundaries 


\section{Fundamentals for the Penrose inequality}

with constant signature case (including null), and they set the necessary and sufficient conditions to match both spacetimes. These conditions are that the induced first fundamental form on $\Omega$ from both embeddings $\Phi^{ \pm}$coincide. In [72] the arguments were extended to the case of arbitrary causal character. However, in the case when the boundary has null points, it turns out that the equality of the first fundamental form is a necessary condition but it fails in general to be also sufficient. The necessary and sufficient conditions were given in [73]. With the notation introduced above we can state this theorem as follows:

Theorem 2.6.6 ([24], [72], [73]). Consider two $(m+1)$-dimensional spacetimes $\left(M^{ \pm}, g^{ \pm}\right)$ with boundaries $\partial M^{ \pm}$. They can be matched across their boundaries to produce a spacetime $(M, g)$ with continuous metric (in a suitable differentiable atlas) if and only if:

- There exists hypersurface metric data $\left\{\Omega, \gamma_{a b}, \ell_{a}, \ell^{(2)}\right\}$ which can be embedded both in $\left(M^{+}, g^{+}\right)$and in $\left(M^{-}, g^{-}\right)$with respective embedding and riggings $\Phi^{ \pm}$ and $\ell^{ \pm}$. Moreover, the embeddings satisfy $\Phi^{ \pm}(\Omega)=\partial \Omega^{ \pm}$.

- The rigging vectors $\ell^{ \pm}$point, respectively, inside and outside of $M^{ \pm}$.

When the requirements of this theorem are translated into hypersurface data level, it is necessary to consider two hypersurface data $\left\{\Omega, \gamma_{a b}, \ell_{a}, \ell^{(2)}, Y_{a b}^{ \pm}\right\}$which differ at most on the transverse tensor $Y_{a b}^{ \pm}$, i.e. such that they define the same hypersurface metric data. The first case that was studied was the non-null one. In this setting and when $\left[K_{a b}\right] \equiv K_{a b}^{+}-K_{a b}^{-}=0$, there is a subatlas of the matched spacetime $(M, g)$ where the metric $g$ is $C^{1}$ [11, 54]. The Riemann tensor of $(M, g)$ may be discontinuous at $\Omega$ but it is otherwise regular everywhere. In physical terms this means that there is no matter-energy or gravitational field concentrated on the matching hypersurface. On the other hand, if $\left[K_{a b}\right] \neq 0$, the Riemann tensor has a Dirac delta function supported on $\Omega$ when it is considered as a tensor distribution in $(M, g)$ (see [61, 100, 34, 72, 60]). The interpretation of this phenomena is that there is a layer of energy and momentum concentrated on the hypersurface $\Omega$ (a "shell" of matter-energy). The Dirac delta part of the Einstein tensor (still in the nowhere null case) reads in this case

$$
\operatorname{Ein}^{g \mu \nu}=\tau^{a b} e_{a}^{\mu} e_{b}^{\nu} \boldsymbol{\delta}_{\Omega}, \quad \text { with } \quad \tau_{a b}=-\left(\left[K_{a b}\right]-[K] \gamma_{a b}\right), \quad \boldsymbol{\delta}_{\Omega}: \text { Dirac delta on }(\Omega, \gamma),
$$

where the Dirac delta distribution is defined by integration with the volume form of the induced metric on the shell. The (distributional) conservation equations $\operatorname{div}_{g} \operatorname{Ein}^{g}=0$ imply

$$
\left(K_{a b}^{+}+K_{a b}^{-}\right) \tau^{a b}=2\left[\operatorname{Ein}_{\mu \nu}^{g} n^{\mu} n^{\nu}\right], \quad \bar{\nabla}_{b} \tau_{a}^{b}=\left[J_{a}\right],
$$

which are the Israel field equations for the shell [54, 58, 59]. These field equations were extended to the null case by Barrabès and Israel [5] by taking limits where the spacelike/timelike matching hypersurface becomes null. 


\subsection{Hypersurfaces and shells}

In the spacelike case, the Israel equations can also be derived from the usual constraint equations

$$
\begin{aligned}
2 \rho & :=\left.2 \operatorname{Ein}_{\alpha \beta}^{g} n^{\alpha} n^{\beta}\right|_{\Omega}=\mathrm{Scal}_{\gamma}-K_{a b} K^{a b}+K^{2}, \quad K:=\gamma^{a b} K_{a b}, \\
-J_{a} & :=\left.\operatorname{Ein}_{\alpha \beta}^{g} e_{a}^{\alpha} n^{\beta}\right|_{\Omega}=D_{b}\left(K^{b}{ }_{a}-K \delta^{b}{ }_{a}\right),
\end{aligned}
$$

where $D$ is the Levi-Civita covariant derivative of $(\Omega, \gamma)$ and $\mathrm{Scal}_{\gamma}$ its curvature scalar, $n^{\alpha}$ the unit normal used to define $K_{a b}$ and $e_{a}^{\alpha}$ is the push-forward to $M$ of the coordinate vector $\partial_{a}$ in $\Omega$. The Israel equations follow by simply taking the difference of both equations at each side of the matching hypersurface, and using the fact that the induced metric and the corresponding Levi-Civita connection do not jump across the shell. Exploiting a similar procedure Mars obtained the shell equations for matching hypersurfaces of arbitrary causal character in [71] by taking differences of the constraint equations on two hypersurface data of the form $\left(\left\{\Omega, \gamma_{a b}, \ell_{a}, \ell^{(2)}, Y_{a b}^{ \pm}\right\}\right)$. With this approach there is no need to introduce spacetime distributions nor specific atlas, and it is conceptually simpler. The method does not require either the existence of the spacetime (although our presentation here has been restricted for simplicity to the embedded case from the outset, see Definition 2.6.5), so we prefer to use this more general framework to obtain the expression of the shell Penrose inequality.

A disadvantage of the connection $\bar{\nabla}$ described above is that it depends on the extrinsic curvature of $\Omega$ via $Y_{a b}$. In [71] a new connection was introduced that depends only on the metric hypersurface data. Its explicit definition is

$$
\stackrel{\circ}{\Gamma}_{a b}^{c}=\frac{1}{2} P^{c d}\left(\partial_{a} \gamma_{b d}+\partial_{b} \gamma_{a d}-\partial_{d} \gamma_{a b}\right)+\frac{1}{2} n^{c}\left(\partial_{a} l_{b}+\partial_{b} l_{a}\right)
$$

This connection is called metric hypersurface connection. It is torsion-free and it relates to the induced connection $\bar{\Gamma}_{a b}^{c}$ by

$$
\bar{\Gamma}_{a b}^{c}=\check{\Gamma}_{a b}^{c}-n^{a} Y_{b c} .
$$

In terms of the embedded matter hypersurface data $\left\{\Omega, \gamma_{a b}, \ell_{a}, \ell^{(2)}, Y_{a b}, \rho_{\ell}, J_{a}\right\}$, the constraint field equations are expressed as [71]

$$
\begin{aligned}
\rho_{\ell} & =\frac{1}{2} \operatorname{Riem}_{b c d}^{c} P^{b d}+\frac{1}{2} \ell_{a} \operatorname{Riem}_{b c d}^{a} P^{b d} n^{c}+\stackrel{\circ}{\nabla}\left(\left(P^{b d} n^{c}-P^{b c} n^{d}\right) Y_{b c}\right) \\
& +\frac{1}{2} n^{(2)} P^{b d} P^{a c}\left(Y_{b c} Y_{d a}-Y_{b d} Y_{c a}\right) \\
& +\frac{1}{2}\left(P^{b d} n^{c}-P^{b c} n^{d}\right)\left[\ell^{(2)} \stackrel{\circ}{\nabla}_{d} U_{b c}+\left(U_{b c}+n^{(2)} Y_{b c}\right) \partial_{d} \ell^{(2)}+2 Y_{b c}\left(F_{d f}-Y_{d f}\right) n^{f}\right],
\end{aligned}
$$




\section{Fundamentals for the Penrose inequality}

$$
\begin{aligned}
J_{c}= & \ell_{a} \operatorname{Riem}_{b c d}^{a} n^{b} n^{d}-\stackrel{\circ}{\nabla}_{f}\left[\left(n^{(2)} P^{b d}-n^{b} n^{d}\right)\left(\delta_{d}^{f} Y_{b c}-\delta_{c}^{f} Y_{b d}\right)\right] \\
- & \left(P^{b d}-\ell^{(2)} n^{b} n^{d}\right)\left(\stackrel{\circ}{\nabla}_{d} U_{b c}-\stackrel{\circ}{\nabla}_{c} U_{b d}\right) \\
- & \left(n^{(2)} P^{b d}-n^{b} n^{d}\right) \times \\
& {\left[\frac{1}{2}\left(U_{b c}+n^{(2)} Y_{b c}\right) \partial_{d} \ell^{(2)}-\frac{1}{2}\left(U_{b d}+n^{(2)} Y_{b d}\right) \partial_{c} \ell^{(2)}+\left(Y_{b c} F_{d f}-Y_{b d} F_{c f}\right) n^{f}\right] } \\
- & \left(P^{b d} n^{f}-P^{b f} n^{d}\right) Y_{b d} U_{c f}-P^{b d} n^{f}\left(U_{b c} F_{d f}-U_{b d} F_{c f}\right),
\end{aligned}
$$

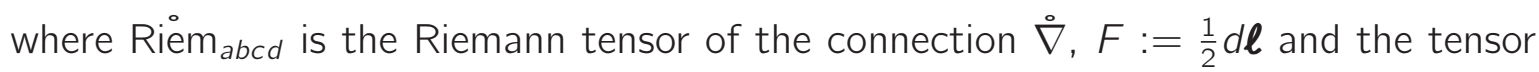
$U_{a b}$ is defined as

$$
U_{a b}:=\frac{1}{2}\left(£_{n} \gamma_{a b}+\ell_{a} \partial_{b} n^{(2)}+\ell_{b} \partial_{a} n^{(2)}\right)
$$

Assume we are given two embedded matter-hypersurface data $\left\{\Omega, \gamma_{a b}, \ell_{a}, \ell^{(2)}, Y_{a b}^{ \pm}, \rho_{\ell}^{ \pm}\right.$, $\left.J_{a}^{ \pm}\right\}$and let us define $V_{a b}:=Y_{a b}^{+}-Y_{a b}^{-}$. Define also the jump in the "energy density" and in the "energy flux" as $\left[\rho_{\ell}\right]:=\rho_{\ell}^{+}-\rho_{\ell}^{-}$and $\left[J_{a}\right]:=J_{a}^{+}-J_{a}^{-}$. These jumps, when computed using (2.10) and (2.11) involve naturally a two contravariant tensor $\tau(V)^{a b}$ defined as

$\tau(V)^{a b}=\left(n^{a} P^{b c}+n^{b} P^{a c}\right) n^{d} V_{c d}-\left(n^{(2)} P^{a c} P^{b d}+P^{a b} n^{c} n^{d}\right) V_{c d}+\left(n^{(2)} P^{a b}-n^{a} n^{b}\right) P^{c d} V_{c d}$

Thus, hypersurface data admits a natural linear map from symmetric two-covariant tensors into symmetric two-contravariant tensors. For the sake of simplicity, we will use $\tau^{a b}$ instead of $\tau(V)^{a b}$ when no confusion arises.

The tensor $\tau(V)^{a b}$ has the symmetries of an energy-momentum tensor and recovers the standard definition of the energy-momentum tensor on the shell in the case where there are no null points. When two spacetimes are matched across null hypersurfaces, $\tau(V)^{a b}$ also recovers the definition for the energy-momentum tensor put forward by Barrabès and Israel (see (31) in [5]). In the particular case where the matching hypersurface has a general causal character, the tensor $\tau^{a b} e_{a}^{\mu} e_{b}^{\nu}$ agrees with the Dirac delta part of the Einstein tensor of the matched spacetime $(M, g)$ obtained in [72] (see expression (71) in that paper). These considerations lead to the following definition [71]:

Definition 2.6.7 (Shell). A shell is a pair of matter-hypersurface data of the form $\left\{\Omega, \gamma_{a b}, \ell_{a}, \ell^{(2)}, Y_{a b}^{ \pm}, \rho_{\ell}^{ \pm}, J_{a}^{ \pm}\right\}$. The energy-momentum tensor on the shell is the symmetric 2-covariant tensor $\tau(V)$ ab defined above, where $V_{a b}:=Y_{a b}^{+}-Y_{a b}^{-}$.

The shell field equations are the following pair of partial differential equations [71] :

Definition 2.6.8 (Shell field equations). Consider the shell $\left\{\Omega, \gamma_{a b}, \ell_{a}, \ell^{(2)}, Y_{a b}^{ \pm}, \rho_{\ell}^{ \pm}, J_{a}^{ \pm}\right\}$. 
2.7. Penrose construction in Minkowski. The shell Penrose inequality

The shell field equations are the pair of partial differential equations

$$
\begin{aligned}
& \stackrel{\circ}{a}_{a}\left(\tau^{a b} I_{b}\right)+\tau^{a b} I_{b}\left(\frac{1}{2} n^{(2)} \partial_{a} \ell^{(2)}+F_{a c} n^{c}\right)-\frac{1}{2} \tau^{a b}\left(Y_{a b}^{+}+Y_{a b}^{-}\right)=\left[\rho_{\ell}\right], \\
& \stackrel{\circ}{\nabla}_{b} \tau_{a}^{b}+\tau_{a}^{b}\left(\frac{1}{2} n^{(2)} \partial_{b} \ell^{(2)}+F_{b c} n^{c}\right)+\tau^{b c} \ell_{c} U_{b a}=\left[J_{a}\right] .
\end{aligned}
$$

\subsection{Penrose construction in Minkowski. The shell Pen- rose inequality}

Penrose's original setup to test the validity of cosmic censorship used a shell of matter propagating in a flat spacetime at the speed of light. The shell is assumed to have the topology $\mathbb{S}^{2} \times \mathbb{R}$ and the matter within the shell is made of null dust (the particles are massless and all pressures vanish). In order to have a flat metric inside the shell, it is necessary that no point in the interior lies in the causal future of the shell. In other words, if a Minkowskian time $t$ is chosen inside the shell, the null hypersurface $\Omega$ must not develop self-intersections to the past of some $t=t_{0}$. Thus, the setup requires that the null hypersurface has one cross section $S_{t_{0}} \equiv \Omega \cap\left\{t=t_{0}\right\}$ which is convex as an euclidean surface of the hyperplane $\left\{t=t_{0}\right\}$. This property is then true for all $t \leq t_{0}$, which means that the null hypersurface $\Omega$ extends to past null infinity smoothly without developing caustics. On the other hand $\Omega$ will become singular to the future at the first focal point of the incoming null geodesics. The collapsing dust may emit gravitational waves which alter the metric of the spacetime outside the shell, so when the shell has passed the metric is no longer flat.

Let $k$ be the future directed null tangent to $\Omega$ normalized by $k(t)=1$. Consider any closed, spacelike surface $S$ embedded in $\Omega$ and let $\ell$ be its future null normal satisfying $\langle k, \ell\rangle=-2$. The energy momentum of the spacetime is a distribution supported on $\Omega$ which reads $T_{\alpha \beta}=8 \pi \rho k_{\alpha} k_{\beta} \delta$, where $\rho$ is the energy density of the shell and the Dirac $\delta$ is defined with respect to the volume form induced by the normal $k$ to $\Omega$. The null expansion $\theta_{\ell}$ jumps across $\Omega$, and this jump can be determined using the Raychaudhuri equation and some properties of the Dirac delta $\boldsymbol{\delta}$, see e.g. [69]. However we compute this jump using the formulation presented above.

For an arbitrary spacelike surface embedded in $\Omega$, its expansion along $k$ coincides with the null expansion of the hypersurface. This means that the expansion does not depend on how the surface is embedded in the null hypersurface and only depends on the point where this expansion is considered (see Appendix $(\mathrm{B})$ ). We will prove later that $\theta_{k}=-\operatorname{tr}_{p}\left(£_{n} \gamma\right)$, i.e. that $\theta_{k}$ depends only on the metric hypersurface data of $\Omega$. As a consequence, the incoming null expansion $\theta_{k}$ is continuous across the shell. On $S_{t_{0}}$, the null expansion $\theta_{k}$ coincides with the mean curvature of $S_{t_{0}}$ as a surface in Euclidean space with respect to the inner normal and it is therefore non positive (because $S_{t_{0}}$ 


\section{Fundamentals for the Penrose inequality}

is convex) and not everywhere zero (since $S_{t_{0}}$ is closed). The Raychaudhuri equation then implies that $\theta_{k} \leq 0$ everywhere on $\Omega$ to the past of $S_{t_{0}}$. Then, after the shell has passed, a spacelike surface $S \subset \Omega$ is marginally outer trapped (i.e. $\theta_{\ell}^{+}=0$ ) if and only if it is marginally future trapped $\left(\theta_{\ell}^{+}=0\right.$ and $\left.\theta_{k} \leq 0\right)$.

Consider a general asymptotically flat four dimensional spacetime $(M, g)$ admitting a null shell $\Omega$ extending smoothly to past null infinity $\mathscr{I}^{-}$, and satisfying the DEC (we will return to the Minkowski spacetime later). Let us assume that there exists a compact cross section $S$ satisfying $\theta_{\ell}^{+}=0$ (i.e. $S$ is a MOTS after the shell has passed). We do not assume yet that the shell is made of any specific type of matter (we will later prove that in the case of shells of null dust, given any cross section $S \subset \Omega$ the shell can always be chosen so that $S$ is a MOTS with respect to the outer geometry). Using the standard heuristic argument described in Section 2.5, it follows

$$
E_{B}^{+} \geq \sqrt{\frac{|\mathcal{H} \cap \Omega|}{16 \pi}} \geq \sqrt{\frac{|S|}{16 \pi}}
$$

where $E_{B}^{+}$is the Bondi energy of past null infinity at the cut defined by $\Omega$ in the exterior spacetime. The main idea of Penrose's construction for shells is that this inequality can be expressed fully in terms of the interior geometry by using the shell properties. For instance, knowing the Bondi energy of the interior spacetime and its jump across the shell, then $E_{B}^{+}$will be fully determined. As mentioned before, the Bondi energy can be computed using limits at infinity of the Hawking energy along a suitable foliation.

Select a nowhere zero null vector field $k$ tangent to $\Omega$. The level sets of the function $\lambda: \Omega \rightarrow \mathbb{R}$ solution of the equation $k(\lambda)=-1$ with initial value $\left.\lambda\right|_{s_{0}}=0$ define a foliation of surfaces $\left\{S_{\lambda}\right\}$, along $\Omega$ with $S_{\lambda_{0}}=\left\{p \in \Omega: \lambda(p)=\lambda_{0}\right\}$. In adapted coordinates $k=-\partial_{\lambda}$. Assume that $\left\{S_{\lambda}\right\}$ tends to large spheres (a precise definition will be given in Chapter 6 ). Let $M_{H}^{+}\left(S_{\lambda}\right)$ be the Hawking mass of $S_{\lambda}$ as a surface embedded in the outer spacetime. Since $\left\{S_{\lambda}\right\}$ tends to large spheres, the limit of $M_{H}^{+}\left(S_{\lambda}\right)$ is the Bondi energy $E_{B}^{+}$of the cut defined by the hypersurface $\Omega$ and past null infinity (see [87, 6] and Chapter 6). Likewise $M_{H}^{-}\left(S_{\lambda}\right)$ tends to the Bondi energy $E_{B}^{-}$of the interior spacetime. Hence

$$
\lim _{\lambda \rightarrow \infty}\left[M_{H}\left(S_{\lambda}\right)\right]=\left[E_{B}\right]
$$

It is clear that in order to compute the left-hand side we need to evaluate the jump of the Hawking energy across the leaves of the foliation $S_{\lambda}$ of $\Omega$. From (2.3) we have

$$
\left[M_{H}\left(S_{\lambda}\right)\right]=-\frac{1}{16 \pi} \sqrt{\frac{\left|S_{\lambda}\right|}{16 \pi}} \int_{S_{\lambda}}\left[\vec{H}^{2}\right] \eta_{S_{\lambda}},
$$

where $\left[\vec{H}^{2}\right]=\left(\vec{H}^{+}\right)^{2}-\left(\vec{H}^{-}\right)^{2}$. Consider the null vector $\ell$ orthogonal to each $S_{\lambda}$ and normalized by $g(k, \ell)=-2$ and choose this vector field along $\Omega$ as rigging vector. Decomposing $\vec{H}$ in the null basis $\{k, \ell\}$ one has $\vec{H}=-\frac{1}{2} \theta_{\ell} k-\frac{1}{2} \theta_{k} \ell$, and hence

$$
\left[\vec{H}^{2}\right]=\left[-\theta_{k} \theta_{\ell}\right]
$$




\subsection{Penrose construction in Minkowski. The shell Penrose inequality}

The first fundamental form $\gamma_{a b}$ of $\Omega$ and the choice of the rigging $\ell$ determine the matrix $\mathbb{A}$ given in (2.8), and hence the vector field $n$ is also uniquely determined. Since $n$ is normal to $\Omega$ and it satisfies $g(n, \ell)=1$, it is clear that $n=-\frac{1}{2} k$. Fix any leaf $S_{\lambda}$ of the foliation, and consider its second fundamental form $K^{n}$ along $n$. We first show that the scalar $\theta_{k}=-2 \theta_{n}=-2\left(\tilde{\gamma}^{-1}\right)^{A B} K_{A B}^{n}$ does not jump across the hypersurface $\Omega$. More precisely, we want to prove that $\theta_{k}=-\operatorname{tr}_{P}\left(£_{n} \gamma\right)$ which only depends on metric hypersurface data. Given that in this case $n^{(2)}=0$, (2.12) becomes $U_{a b}=\frac{1}{2} £_{n} \gamma_{a b}$. We prove first that $U(X, Y)=K^{n}(X, Y)$, where $X, Y$ are any vector fields tangent to $S_{\lambda}$. To this purpose it is necessary to evaluate $U_{a b}$ at $X, Y$. We can extend $X$ and $Y$ along $n$ so that $[n, X]=[n, Y]=0$. Then

$$
\begin{aligned}
£_{n}(\gamma(X, Y)) & =\nabla_{n}(\langle X, Y\rangle)=\left\langle\nabla_{n} X, Y\right\rangle+\left\langle X, \nabla_{n} Y\right\rangle=\left\langle\nabla_{X} n, Y\right\rangle+\left\langle X, \nabla_{Y} n\right\rangle \\
& =2 K^{n}(X, Y) .
\end{aligned}
$$

Besides

$$
£_{n}(\gamma(X, Y))=\left(£_{n} \gamma\right)(X, Y)+\gamma\left(£_{n} X, Y\right)+\gamma\left(X, £_{n} Y\right)=\left(£_{n} \gamma\right)(X, Y),
$$

and we conclude $\left(£_{n} \gamma\right)(X, Y)=2 K^{n}(X, Y)$, which implies $U_{A B}=K_{A B}^{n}$. The following step is to prove that $\operatorname{tr}_{P} U=\operatorname{tr}_{\tilde{\gamma}} K^{n}$, so it is necessary to compute $P^{a b}$. In this setting where the rigging is null and satisfies $g(k, \ell)=-2$, we have that $\ell^{(2)}=\langle\ell, \ell\rangle=0$. Consider the basis $\left\{e_{a}\right\}=\left.\left\{k, X_{A}\right\}\right|_{p}$ tangent to $\Omega$ so that $\left\{X_{A}\right\}$ are tangent to $S_{\lambda} \subset \Omega$. In this case $\ell_{0}=\langle\ell, k\rangle=-2$ and $\ell_{A}=\left\langle\ell, X_{A}\right\rangle=0$ (we will simply write $\ell_{a}=(-2,0,0$ ) in the corresponding dual basis). Denoting by $\tilde{\gamma}_{A B}=\left\langle X_{A}, X_{B}\right\rangle$ the metric of $S_{\lambda}$, then the first fundamental form $\gamma_{a b}$ of the null hypersurface $\Omega$ reads

$$
\gamma_{a b} \equiv\left(\begin{array}{c|c}
0 & 0 \\
\hline 0 & \tilde{\gamma}_{A B}
\end{array}\right) .
$$

Since we know $\ell_{a}, \ell^{(2)}$ and $\gamma_{a b}$, the corresponding tensors $P^{a b}, n^{a}$ and $n^{(2)}$ can be computed by inverting the matrix $\mathbb{A}$ given in (2.8). The result is

$$
P^{a b}:=\left(\begin{array}{c|c}
0 & 0 \\
\hline 0 & \left(\tilde{\gamma}^{-1}\right)^{A B}
\end{array}\right), \quad n^{a}=\left(-\frac{1}{2}, 0,0\right), \quad n^{(2)}=0 .
$$

It becomes now clear that

$$
\theta_{k}=-2 \theta_{n}=-2\left(\tilde{\gamma}^{-1}\right)^{A B} K_{A B}^{n}=-2 P^{a b} U_{a b}=-2 \operatorname{tr}_{P} U=-\operatorname{tr}_{P}\left(£_{n} \gamma\right) .
$$

This shows that $\theta_{k}$ does not jump across the shell and we simply write $\theta_{k}:=\theta_{k}^{+}=\theta_{k}^{-}$ from now on. Consequently

$$
\left[\vec{H}^{2}\right]=-\theta_{k}\left[\theta_{\ell}\right]
$$

For later use we show that $U_{a b} n^{b}=0$. Indeed, let $T$ be any vector tangent to $\Omega$ and compute

$$
2 U_{a b} n^{a} T^{b}=\left(£_{n} \gamma\right)(n, T)=n(\gamma(n, T))-\gamma\left(£_{n} n, T\right)-\gamma\left(n, £_{n} T\right)=0,
$$




\section{Fundamentals for the Penrose inequality}

where we used $\gamma(n, \cdot) \equiv 0$, and $[n, n]=0$.

A fundamental step in the shell construction is to study the relation between $\left[\theta_{\ell}\right]$ and the energy-momentum tensor of the shell. This requires studying the transverse tensor $Y$. In our construction the embedding $\Phi: \Omega \rightarrow M$ and the transverse null vector $\ell$ that plays the role of the rigging are given, and consequently the symmetric tensor $Y$ is uniquely defined. In order to compute it, consider $X, Y$ tangent vectors to the cross section $S_{\lambda} \subset \Omega$, and define the function $Q_{k}$ by $\nabla_{k} k=Q_{k} k$ (recall that $k$ is autoparallel, being null and hypersurface orthogonal). Then

$$
Y(k, k)=\frac{1}{2}\left(\nabla_{\alpha} \ell_{\beta}+\nabla_{\beta} \ell_{\alpha}\right) k^{\alpha} k^{\beta}=\left\langle\nabla_{k} \ell, k\right\rangle=-\left\langle\ell, \nabla_{k} k\right\rangle=-\left\langle\ell, Q_{k} k\right\rangle=2 Q_{k} .
$$

In addition

$$
\begin{aligned}
Y(k, X) & =\frac{1}{2}\left(\nabla_{\alpha} \ell_{\beta}+\nabla_{\beta} \ell_{\alpha}\right) k^{\alpha} X^{\beta}=\frac{1}{2}\left(\left\langle\nabla_{k} \ell, X\right\rangle+\left\langle\nabla_{X} \ell, k\right\rangle\right) \\
& =\frac{1}{2}\left(-\left\langle\ell, \nabla_{k} X\right\rangle-\left\langle\ell, \nabla_{X} k\right\rangle\right)=-2 s_{\ell}(X),
\end{aligned}
$$

where $s_{\ell}(X):=\frac{1}{2}\left\langle\nabla_{X} k, \ell\right\rangle$ is the connection one-form of $S_{\lambda}$ (see Appendix B) (note that we have used the property $[X, k]=0$ ). Finally

$$
Y(X, Y)=\frac{1}{2}\left(\nabla_{\alpha} \ell_{\beta}+\nabla_{\beta} \ell_{\alpha}\right) X^{\alpha} Y^{\beta}=\frac{1}{2}\left(\left\langle\nabla_{X} \ell, Y\right\rangle+\left\langle\nabla_{Y} \ell, X\right\rangle\right)=K^{\ell}(X, Y),
$$

where $K^{\ell}(X, Y)=\left\langle\nabla_{X} \ell, Y\right\rangle$ is the second fundamental form along $\ell$ of $S_{\lambda}$ (see Appendix B). In summary, the tensor $Y$ has the form, in matrix notation

$$
Y_{a b} \equiv\left(\begin{array}{c|c}
2 Q_{k} & -2\left(s_{\ell}\right)_{A} \\
\hline-2\left(s_{\ell}\right)_{B} & K_{A B}^{\ell}
\end{array}\right) .
$$

This together with the value of $P^{a b}, n^{a}$ and $n^{(2)}$ given in (2.9) fully determines the shell energy-momentum acting on the tensor $Y$. In this null case (2.13) takes the form

$$
\tau(Y)=X^{Y} \otimes n+n \otimes X^{Y}+Q^{Y} P
$$

where $Q^{Y}:=Y(n, n)$, and $X^{Y}:=P(Y(n, \cdot), \cdot)-\frac{1}{2}\left(\operatorname{tr}_{P} Y\right) n$. Observe that if

$$
\left[Q^{Y}\right]=0, \quad P([Y](n, \cdot), \cdot)=0
$$

then the energy momentum tensor on the shell will be of the form

$$
\tau([Y])=-\left[\operatorname{tr}_{P} Y\right] n \otimes n
$$

i.e. it will correspond to the energy momentum tensor of null dust. Note that $\operatorname{tr}_{P} Y=$ $P^{a b} Y_{a b}=P^{A B} Y_{A B}=\left(\tilde{\gamma}^{-1}\right)^{A B} K_{A B}^{\ell}=\theta_{\ell}$, so an alternative form of (2.20) is

$$
\tau([Y])=-\left[\theta_{\ell}\right] n \otimes n
$$




\subsection{Penrose construction in Minkowski. The shell Penrose inequality}

If we choose the jump so that

$$
\left[Y_{a b}\right]=Y_{a b}^{+}-Y_{a b}^{-}=-(8 \pi \rho) \gamma_{a b},
$$

with $\rho: \Omega \rightarrow \mathbb{R}$, then conditions (2.19) are automatically satisfied and we are in the case of null dust. Substituting $V_{a b}=\left[Y_{a b}\right]=-(8 \pi \rho) \gamma_{a b}$ in (2.13) it follows

$$
\tau([Y])^{a b}=(16 \pi \rho) n^{a} n^{b}
$$

after using that

$(P \cdot[Y])_{c}^{a}=P^{a b}\left[Y_{b c}\right] \equiv\left(\begin{array}{c|c}0 & 0 \\ \hline 0 & \left(\tilde{\gamma}^{-1}\right)^{A B}\end{array}\right) \cdot\left(\begin{array}{c|c}0 & 0 \\ \hline 0 & -(8 \pi \rho) \tilde{\gamma}_{B C}\end{array}\right)=\left(\begin{array}{c|c}0 & 0 \\ \hline 0 & -(8 \pi \rho) \delta^{A}{ }_{C}\end{array}\right)$.

From (2.21) and (2.22) it follows that the jump of transverse null expansion $\theta_{\ell}$ is directly related to the energy density $\rho$ of the null dust:

$$
\left[\theta_{\ell}\right]=\theta_{\ell}^{+}-\theta_{\ell}^{-}=-16 \pi \rho .
$$

Substituting this in (2.18), we find

$$
\left[M_{H}\left(S_{\lambda}\right)\right]=-\sqrt{\frac{\left|S_{\lambda}\right|}{16 \pi}} \int_{S_{\lambda}} \theta_{k} \rho \boldsymbol{\eta}_{S_{\lambda}} .
$$

To compute the limit of the right-hand side of (2.24) evaluated on this foliation, we need to find the asymptotic expansions of $\theta_{k}, \boldsymbol{\eta}_{S_{\lambda}}$ and $\rho$ at $\lambda=\infty$. As we will show in Chapter 6 , the expansion of $\theta_{k}$ has the form

$$
\theta_{k}=\frac{-2}{\lambda}+\frac{\theta_{k}^{(1)}}{\lambda^{2}}+o\left(\lambda^{-2}\right)
$$

From equation (B.40) in Appendix B, the volume form admits the expression $\eta_{S_{\lambda}}=$ $\left(\lambda^{2}+o\left(\lambda^{2}\right)\right) \boldsymbol{\eta}_{\boldsymbol{q}}$, where $\boldsymbol{\eta}_{\mathfrak{q}}$ is the volume form of the standard two-sphere. It just remains to compute the asymptotic expansion of $\rho$. To do so, we need to know how $\rho$ evolves along the null direction $k$. This is equivalent to computing the expressions for the shell field equations (2.14) and (2.15). Since we already know the expression for $U_{a b}$, we just need to calculate $F_{a b}$. The simplest way to to this is by restricting to the case where the basis $\left\{e_{a}\right\}=\left\{k, X_{A}\right\}$ is a coordinate basis, i.e. there exist adapted coordinates $\left\{\lambda, x^{A}\right\}$ such that $k=-\partial_{\lambda}$ and $X_{A}=\partial_{x^{A}}$. Given that the components of $\ell_{a}$ are constant, we have

$$
F_{a b}=\frac{1}{2}(d \boldsymbol{l})_{a b}=\frac{1}{2}\left(\partial_{a} \ell_{b}-\partial_{b} \ell_{a}\right)=0,
$$

which is of course independent of the choice of basis.

In order to write down the shell equations we need to make a choice of energymomentum tensor of the spacetime left after the shell has passed. The simplest case 


\section{Fundamentals for the Penrose inequality}

(and the corresponding to the original Penrose construction, too) is $\left[\rho_{\ell}\right]=\left[J_{a}\right]=0$, which we assume from now on. Inserting the expression (2.26) for $F_{a b}$, the first term in (2.14) becomes

$$
\begin{aligned}
\stackrel{\circ}{\nabla}_{a}\left(\tau^{a b} I_{b}\right) & =\stackrel{\circ}{\nabla}_{a}\left((16 \pi \rho) n^{a} n^{b} I_{b}\right)=\stackrel{\circ}{\nabla}_{a}\left((16 \pi \rho) n^{a}\right)=16 \pi\left(n(\rho)+\rho \stackrel{\circ}{\nabla}_{a} n^{a}\right) \\
& =16 \pi\left(n(\rho)+\rho \theta_{n}\right) .
\end{aligned}
$$

The second term in (2.14) clearly vanishes, and for the third term we have

$$
\tau^{a b}\left(Y_{a b}^{+}+Y_{a b}^{-}\right)=(16 \pi \rho) n^{a} n^{b}\left(2 Y_{a b}^{-}-8 \pi \rho \gamma_{a b}\right)=(32 \pi \rho) Y^{-}(n, n)=-(32 \pi \rho) Q_{n}
$$

Consequently equation (2.14) turns into

$$
n(\rho)+\rho\left(\theta_{n}+Q_{n}\right)=0
$$

For the second equation (2.15), the first term vanishes since

$$
\tau^{b}{ }_{a}=\tau^{b c} \gamma_{c a}=16 \pi \rho n^{b}\left(n^{c} \gamma_{c a}\right)=0
$$

The second term clearly vanishes and the third term also becomes zero since $\tau^{b c} U_{b a}=$ $(16 \pi \rho) n^{b} n^{c} U_{b a}$, because $U_{b a} n^{b}=0$. So, the only shell equation for null dust is (2.27) above.

Let us now return to the original Penrose construction where $\left(\mathrm{M}^{-}, \mathrm{g}^{-}\right)$is Minkowski. Recall that a Minkowskian time $t$ had been chosen, and consequently a unit Killing vector $\xi$ defined by $\boldsymbol{\xi}=-d t$ existed. Also, the null vector $k$ was normalized so that $\langle k, \xi\rangle=-1$. In this situation, the corresponding foliation $\left\{S_{\lambda}\right\}$ automatically tends to large spheres, and the particularization of formula (2.24) to this situation gives

$$
\lim _{\lambda \rightarrow \infty}\left[M_{H}\left(S_{\lambda}\right)\right]=E_{B}
$$

where we have used that the Bondi energy of the Minkowski spacetime is zero.

The normalization of $k$ implies that the null geodesics along $\Omega$ generated by $k$ are affinely parametrized in the Minkowski spacetime. Indeed, by multiplying the geodesic equation $\nabla_{k} k=Q_{k} k$ with $\xi$, one finds $-Q_{k}=\left\langle\nabla_{k} k, \xi\right\rangle=-\left\langle k, \nabla_{k} \xi\right\rangle=0$, where the last equality follows because $\xi$ is covariantly constant. The geodesics are also affinely parametrized in the outer spacetime for null dust (which recall satisfies $\left[Q_{n}\right]=0$ ). Hence equation (2.27) simplifies to

$$
k(\rho)+\rho \theta_{k}=0
$$

This equation will be used to show that the integral of the energy density $\rho$ on each leaf $S_{\lambda}$ of the foliation is independent of $\lambda$ and in fact takes the value $E_{B}$, and also to 


\subsection{Penrose construction in Minkowski. The shell Penrose inequality}

determine the expression for the asymptotic expansion of $\rho$ at $\lambda=\infty$. For the latter, choose $s_{0}>0$ and $\lambda>s_{0}$. Since $k=-\partial_{\lambda}$, integrating (2.29) in $\left[s_{0}, \lambda\right]$ gives

$$
\rho(\lambda)=\rho\left(s_{0}\right) \exp \left(\int_{s=s_{0}}^{\lambda} \theta_{k}(s) d s\right) .
$$

Substituting (2.25) it follows

$$
\begin{aligned}
\rho(\lambda) & =\rho\left(s_{0}\right) \exp \left(\int_{s=s_{0}}^{\lambda} \frac{-2}{s} d s\right) \exp \left(\int_{s=s_{0}}^{\lambda}\left(\frac{\theta_{k}^{(1)}}{s^{2}}+o\left(s^{-2}\right)\right) d s\right) \\
& =\rho\left(s_{0}\right) \frac{s_{0}^{2}}{\lambda^{2}} \exp \left(\int_{s=s_{0}}^{\lambda}\left(\frac{\theta_{k}^{(1)}}{s^{2}}+o\left(s^{-2}\right)\right) d s\right) .
\end{aligned}
$$

We next use the property that for every continuous function $f \in O\left(s^{-2}\right)$, there is a constant $C\left(s_{0}\right)$ such that $\int_{s=s_{0}}^{\lambda} f(s) d s=C\left(s_{0}\right)+o\left(\lambda^{-1}\right)$. To show this we write $\int_{s=s_{0}}^{\lambda} f(s) d s$ as

$$
\int_{s=s_{0}}^{\lambda} f(s) d s=\int_{s=s_{0}}^{\infty} f(s) d s-\int_{\lambda}^{\infty} f(s) d s:=C\left(s_{0}\right)-\int_{\lambda}^{\infty} f(s) d s,
$$

where both integrals extending to infinity are finite because a function $f \in O\left(s^{-2}\right)$ is automatically integrable. It just remains to prove that $\int_{\lambda}^{\infty} f(s) d s \in o\left(\lambda^{-1}\right)$, i.e. $\lim _{\lambda \rightarrow \infty} \lambda \int_{\lambda}^{\infty} f(s) d s=0$. Applying the L'Hôpital rule gives

$$
\lim _{\lambda \rightarrow \infty} \lambda \int_{\lambda}^{\infty} f(s) d s=\lim _{\lambda \rightarrow \infty} \frac{\int_{\lambda}^{\infty} f(s) d s}{\frac{1}{\lambda}}=\lim _{\lambda \rightarrow \infty} \frac{-f(\lambda)}{-\frac{1}{\lambda^{2}}}=0,
$$

where the last equality follows from $f \in O\left(\lambda^{-2}\right)$. Applying the result to the exponential in (2.30),

$$
\begin{aligned}
\exp \left(\int_{s=s_{0}}^{\lambda}\left(\frac{\theta_{k}^{(1)}}{s^{2}}+o\left(s^{-2}\right)\right) d s\right) & =\underbrace{\exp \left(-\frac{\theta_{k}^{(1)}}{\lambda}+\frac{\theta_{k}^{(1)}}{s_{0}}+C\left(s_{0}\right)+o\left(\lambda^{-1}\right)\right)}_{:=\alpha\left(s_{0}\right)} \\
& =\underbrace{\exp \left(C\left(s_{0}\right)+\frac{\theta_{k}^{(1)}}{s_{0}}\right)} \exp \left(-\frac{\theta_{k}^{(1)}}{\lambda}+o\left(\lambda^{-1}\right)\right) \\
& =\alpha\left(s_{0}\right)(1+o(1))=\alpha\left(s_{0}\right)+o(1),
\end{aligned}
$$

and we conclude

$$
\rho=\frac{\hat{\rho}}{\lambda^{2}}+o\left(\lambda^{-2}\right)
$$




\section{Fundamentals for the Penrose inequality}

for some $\hat{\rho}$ independent of $\lambda$. We can now substitute the expansions of $\theta_{k}, \boldsymbol{\eta}_{S_{\lambda}}$ and $\rho$ in the right-hand side of (2.24) to compute its limit. Since $\sqrt{\left|S_{\lambda}\right|}=\sqrt{4 \pi} \lambda+O(\lambda)$, it follows

$$
\begin{aligned}
& \lim _{\lambda \rightarrow \infty}\left(-\sqrt{\frac{\left|S_{\lambda}\right|}{16 \pi}} \int_{S_{\lambda}} \theta_{k} \rho \boldsymbol{\eta}_{S_{\lambda}}\right) \\
= & \lim _{\lambda \rightarrow \infty} \frac{-1}{\sqrt{16 \pi}}(\sqrt{4 \pi} \lambda+o(\lambda)) \int_{\mathbb{S}^{2}}\left(\frac{-2}{\lambda}+o\left(\lambda^{-1}\right)\right)\left(\frac{\hat{\rho}}{\lambda^{2}}+o\left(\lambda^{-2}\right)\right)\left(\lambda^{2}+o\left(\lambda^{2}\right)\right) \boldsymbol{\eta}_{\tilde{q}}= \\
= & \int_{\mathbb{S}^{2}} \hat{\rho} \boldsymbol{\eta}_{\dot{q}} .
\end{aligned}
$$

We still need to relate this integral at infinity with integrals along $\left\{S_{\lambda}\right\}$. This is achieved from the following two observations. First

$$
\lim _{\lambda \rightarrow \infty} \int_{S_{\lambda}} \rho \boldsymbol{\eta}_{S_{\lambda}}=\lim _{\lambda \rightarrow \infty} \int_{\mathbb{S}^{2}}\left(\frac{\hat{\rho}}{\lambda^{2}}+o\left(\lambda^{-2}\right)\right)\left(\lambda^{2}+o\left(\lambda^{2}\right)\right) \boldsymbol{\eta}_{\tilde{q}}=\int_{\mathbb{S}^{2}} \hat{\rho} \boldsymbol{\eta}_{\tilde{q}},
$$

and second, $\int_{S_{\lambda}} \rho \boldsymbol{\eta}_{S_{\lambda}}$ is in fact a constant independent of $\lambda$ because

$$
k\left(\int_{S_{\lambda}} \rho \boldsymbol{\eta}_{S_{\lambda}}\right)=\int_{S_{\lambda}} k(\rho) \boldsymbol{\eta}_{S_{\lambda}}+\int_{S_{\lambda}} \rho k\left(\boldsymbol{\eta}_{S_{\lambda}}\right)=\int_{S_{\lambda}}\left(-\rho \theta_{k}\right) \boldsymbol{\eta}_{S_{\lambda}}+\int_{S_{\lambda}}\left(\rho \theta_{k}\right) \boldsymbol{\eta}_{S_{\lambda}}=0,
$$

where we have used (2.29) and the expression for the Lie derivative of a volume form along $k$ (see (B.41) in Appendix $(\mathrm{B})$. Putting together (2.28), (2.24), (2.31) and (2.32) yields

$$
\begin{aligned}
E_{B} & =\lim _{\lambda \rightarrow \infty}\left[M_{H}\left(S_{\lambda}\right)\right]=\lim _{\lambda \rightarrow \infty}\left(-\sqrt{\frac{\left|S_{\lambda}\right|}{16 \pi}} \int_{S_{\lambda}} \theta_{k} \rho \boldsymbol{\eta}_{S_{\lambda}}\right)=\int_{\mathbb{S}^{2}} \hat{\rho} \boldsymbol{\eta}_{\mathfrak{q}}=\lim _{\lambda \rightarrow \infty} \int_{S_{\lambda}} \rho \boldsymbol{\eta}_{S_{\lambda}} \\
& =\int_{S_{\lambda}} \rho \boldsymbol{\eta}_{S_{\lambda}} \forall \lambda .
\end{aligned}
$$

So far, we have used the evolution equation (2.29), but we still have not fixed any initial condition for $\rho$. It remains to show that the initial data for $\rho$ can be chosen so that the initial surface $S$ is a MOTS in the exterior geometry. Indeed, from the jump equation (2.23).

$$
\left.\left[\theta_{\ell}\right]\right|_{s}=\left.\left(\theta_{\ell}^{+}\right)\right|_{s}-\left.\left(\theta_{\ell}^{-}\right)\right|_{s}=-\left.16 \pi \rho\right|_{s},
$$

$S$ will be a MOTS in the outer spacetime $\left(\left.\left(\theta_{\ell}^{+}\right)\right|_{S}=0\right)$ if and only if

$$
\left.\rho\right|_{S}=\left.\frac{1}{16 \pi}\left(\theta_{\ell}^{-}\right)\right|_{s}
$$

Combining with (2.33), we have

$$
\int_{S} \theta_{\ell}^{-} \boldsymbol{\eta}_{S}=16 \pi E_{B}
$$


and the Penrose inequality (2.16) finally becomes

$$
\int_{S} \theta_{\ell}^{-} \boldsymbol{\eta}_{S} \geq \sqrt{16 \pi|S|}
$$

This inequality has the remarkable property of making no reference to the exterior geometry at all. Since the density $\rho$ is freely specifiable, this inequality should hold for any closed spacelike surfaces $S$ in Minkowski spacetime for which the null hypersurface $\Omega$ generated by past directed and outer null geodesics orthogonal to $S$ remains regular everywhere. We emphasize that analogous shell Penrose inequalities (also called Gibbons-Penrose inequalities in the literature) exist in arbitrary spacetime dimension. 


\section{Projection along the Killing in static spacetimes}

\subsection{Introduction}

After having explained in detail the Penrose inequality conjecture and introduced the fundamental notions we will use, we start with the presentation of the original results in this thesis.

In this chapter we approach the shell Penrose inequality by means of a vertical projection along the time translation Killing vector. As explained in the introduction, this was the method proposed by Gibbons to address the problem. Although his implementation was wrong, the idea is nevertheless interesting and worth exploring. In view of potential applications of the method in more general settings than in Minkowski, we analyze in detail the projection of general codimension two spacelike surfaces embedded in a static spacetime. The projection is performed along the static Killing and onto a hypersurface orthogonal to this Killing. We study the relationship between the intrinsic and extrinsic geometry of the original surface with the projected one. In particular, we find explicit expressions for the relation between the induced metrics, the second fundamental forms and the connection of the normal bundle between the two surfaces. Definitions of these concepts and basic results concerning the geometry of embedded submanifolds are summarized in Appendix A.

Although our main interest to find these geometric relations is with regard to the shell Penrose inequality, the results are of independent interest. The computations are performed in the static setup but, for the sake of generality we also give the expressions in the case when the ambient manifold is of euclidean signature (and admits a hypersurface orthogonal nowhere zero Killing vector). The results in this chapter have been published in [75].

In connection to the shell Penrose inequality, we first explain where and for which reason the calculation by Gibbons fails. This is done in Subsection 3.3.1. In Subsection 
3.3.2 we apply our result to give an independent proof of the theorem by Brendle and Wang [15] mentioned in the introduction and which gives sufficient conditions for the validity of the shell Penrose inequality in terms of the convexity of the projected surface. As already stated, our proof of this theorem was obtained at about the same time as Brendle and Wang and in a completely independent way.

\subsection{The metric determined by the Killing}

Throughout this thesis and unless otherwise stated, $(M, g)$ denotes an $(n+2)$-dimensional spacetime. We always take $n \geq 2$. On a manifold with metric $\gamma$, we denote by $\gamma(\cdot, \cdot)$ the scalar product with this metric. When the scalar product is with respect to the spacetime metric $g$ we simply write $\langle\cdot, \cdot\rangle$.

Definition 3.2.1 (Killing vector field). Let $\xi$ be a vector field in a spacetime $(M, g)$. $\xi$ is a Killing vector field if it satisfies the relation

$$
£_{\xi} g=0 .
$$

This condition is equivalent to:

$$
\nabla_{\alpha} \xi_{\beta}+\nabla_{\beta} \xi_{\alpha}=0
$$

Definition 3.2.2 (Stationary spacetime). Let $(M, g)$ be a spacetime admitting a Killing vector field $\xi$. $(M, g)$ is stationary if $\xi$ is timelike.

Definition 3.2.3 (Static spacetime). A spacetime $(M, g)$ is static if it is stationary, and in addition there exists a spacelike hypersurface $\Sigma$ which is orthogonal to the integral curves of the corresponding Killing vector $\xi$ through each point. Such $\xi$ is called static. By the Fröbenius theorem, a stationary spacetime with Killing vector $\xi$ is static if and only if

$$
\xi_{[\alpha} \nabla_{\beta} \xi_{\gamma]}=0
$$

The following theorem is well-known. We include its proof for completeness.

Theorem 3.2.4. Let $(M, g)$ be a spacetime that admits a static Killing vector field $\xi$ with the norm $\xi^{\alpha} \xi_{\alpha}=-V^{2}$, and $V$ a strictly positive function. Then there exists locally in $M$ a differentiable function $t$ so that

$$
\xi_{\alpha}=-V^{2} \nabla_{\alpha} t
$$

and a coordinate system where the metric is expressed as

$$
g=-V^{2} d t^{2}+h_{i j} d x^{i} d x^{j}
$$

with $h_{i j}$ a positive definite metric. 


\section{Projection along the Killing in static spacetimes}

Remark 3.2.5. A function $t$ is locally defined in $M$ when it is defined in some (nonempty) open neighbourhood $U(p)$ around any point $p$.

Proof. We denote by $\boldsymbol{\xi}$ the one form obtained from $\xi$ by lowering indices. By the Poincaré lemma $d t=\frac{\xi}{-V^{2}}$ will have a local solution if and only if $d\left(\frac{\xi}{-V^{2}}\right)=0$, i.e. if and only if

$$
\nabla_{\nu}\left(\frac{\xi_{\mu}}{-V^{2}}\right)-\nabla_{\mu}\left(\frac{\xi_{\nu}}{-V^{2}}\right)=0
$$

or equivalently

$$
V^{2} \nabla_{[\mu} \xi_{\nu]}+\xi_{[\mu} \nabla_{\nu]} V^{2}=0
$$

Expanding $\xi_{[\mu} \nabla_{\nu} \xi_{\alpha]}=0$ and multiplying it by $\xi^{\mu}$, we obtain

$$
0=6 \xi^{\mu} \xi_{[\mu} \nabla_{\nu} \xi_{\alpha]}=2 V^{2} \nabla_{[\alpha} \xi_{\nu]}+2 \xi_{\nu} \xi^{\mu} \nabla_{\alpha} \xi_{\mu}-2 \xi_{\alpha} \xi^{\mu} \nabla_{\nu} \xi_{\mu},
$$

where we have used the Killing equation. Inserting $\nabla_{\alpha} V^{2}=-2 \xi^{\mu} \nabla_{\alpha} \xi_{\mu}$ yields (3.4), as required.

Let $\Sigma_{t}$ be the level sets of $t$, and fix $t_{0}=t(p)$ for any choice $p \in M$. Let $\left\{x^{i}\right\}$ be a coordinate system in $\Sigma_{t_{0}}$, and extend it off $\Sigma_{t_{0}}$ by means of $\xi\left(x^{i}\right)=0$. It is clear that $\left(t, x^{i}\right)$ forms a system of coordinates in a neighbourhood of $p$. From $\xi\left(x^{i}\right)=0$ we have that $\xi$ is parallel to $\partial_{t}$. Moreover, the conditions $\langle\xi, \xi\rangle=-V^{2}$ and $\boldsymbol{\xi}=-V^{2} d t$ yield that

$$
\xi=\partial_{t},
$$

so that the metric of $M$ locally reads

$$
g=-V^{2} d t^{2}+h_{i j} d x^{i} d x^{j},
$$

where $h_{i j}=\left\langle\partial_{x^{i}}, \partial_{x^{j}}\right\rangle$, with $\left(\Sigma_{t}, g_{\mid \Sigma_{t}}=h\right)$ a Riemannian hypersurface. Obviously $V$ and $h_{i j}$ are independent of $t$ because $\xi=\partial_{t}$ is a Killing vector.

\subsection{Geometry of the intial surface in terms of the pro- jected one}

From now on we are in the setup of Theorem 3.2.4. Consider a codimension-two spacelike surface $S$ in $M$. Since all calculations are local we can assume without loss of generality that $S$ is embedded, and as before let $t: M \rightarrow \mathbb{R}$ be defined by $\boldsymbol{\xi}=-V^{2} d t$. Choose any $t_{0} \in \mathbb{R}$ and let $\Sigma_{t_{0}}=\left\{t=t_{0}\right\}$. The projection $\bar{S}$ of $S$ onto $\Sigma_{t_{0}}$ along the orbits of $\xi$ defines a codimension-two surface which again can be taken to be 


\subsection{Geometry of the intial surface in terms of the projected one}

embedded (after restricting $S$ if necessary). Thus, we have a diffeomorphism $\pi: S \rightarrow \bar{S}$ defined by projection along $\xi$ (see Figure 3.11). The induced metrics and covariant derivative on $S$ (resp. $\bar{S}$ ) are denoted as $\gamma$ and $D$ (resp. $\bar{\gamma}$ and $\bar{D})$. The function $\tau:=\left.t\right|_{S}-t_{0}$ and $V_{S}:=\left.V\right|_{S}$ will play an important role in relating the geometry of the two surfaces. Scalar functions on $S$ will be transferred to $\bar{S}$ by means of $\pi$ while keeping their names. The precise meaning will follow from the context. Here and in the following $|D f|_{\gamma}^{2}=\gamma^{A B} f_{, A} f_{, B}$ for any function $f: S \rightarrow \mathbb{R}$, and likewise $|D f|_{\gamma}^{2}=\bar{\gamma}^{A B} f_{, A} f_{, B}$.

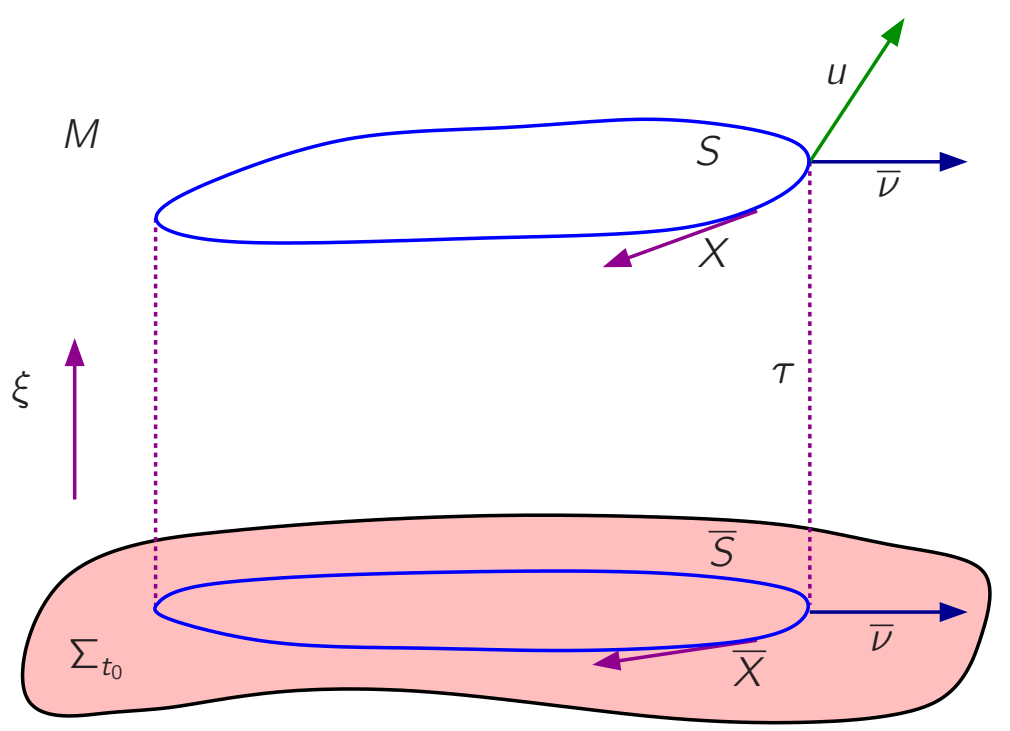

Figure 3.1: The spacetime convex surface $S$ is projected along the Killing $\xi$ onto the constant time hyperplane $\Sigma_{t_{0}}=\left\{t=t_{0}\right\}$ in the static spacetime $M$. S lies at a time height distance $\tau$ from $\Sigma_{t_{0}}$. The tangent vector $X$ to $S$ is projected orthogonally to the tangent vector $\bar{X}$. For the normals, the vector $\bar{\nu}$ on $\bar{S}$ is Lie-dragged to $S$ along $\xi$ and $u$ is the normalized normal component of $\xi$ to $S$.

For any vector field $X \in \mathfrak{X}(S)$ we denote its projection $d \pi(X) \in \mathfrak{X}(\bar{S})$ as $\bar{X}$. Given any such vector $\bar{X}$ we extend it along the orbits of the Killing vector by Lie transport along $\xi$, i.e. solving $[\xi, \bar{X}]=0$. Again we keep the same name for the extension. Note that $\bar{X}$ is everywhere orthogonal to $\xi$. With these definitions it is straightforward that, at any $p \in S$,

$$
\left.X\right|_{p}=\left.\bar{X}(\tau) \xi\right|_{p}+\left.\bar{X}\right|_{p}
$$

As a consequence, the metrics $\gamma$ and $\bar{\gamma}$ are related by

$$
\left.\gamma(X, Y)\right|_{p}=\left.\langle\bar{X}(\tau) \xi+\bar{X}, \bar{Y}(\tau) \xi+\bar{Y}\rangle\right|_{p}=\left.\left(\pi^{*}(\bar{\gamma})-\left.V_{S}^{2} d \tau \otimes d \tau\right|_{p}\right)(X, Y)\right|_{p},
$$

where we have used $\left.d \pi\right|_{p}(X)=\left.\bar{X}\right|_{\pi(p)}$ and $\bar{X}(\tau)=d \tau(X)$. So, we conclude

$$
\gamma=\pi^{\star}(\bar{\gamma})-V_{S}^{2} d \tau \otimes d \tau .
$$




\section{Projection along the Killing in static spacetimes}

The inverse metrics are then related by

$$
\bar{\gamma}^{-1}=d \pi\left(\gamma^{-1}\right)-\frac{V_{S}^{2}}{W^{2}} \operatorname{grad}_{\bar{\gamma}}(\tau) \otimes \operatorname{grad}_{\bar{\gamma}}(\tau), \quad W:=\sqrt{1-V_{S}^{2}|d \tau|_{\bar{\gamma}}^{2}},
$$

which has, as immediate consequences,

$$
d \pi\left(\operatorname{grad}_{\gamma}(\tau)\right)=\frac{1}{W^{2}} \operatorname{grad}_{\bar{\gamma}}(\tau), \quad|d \tau|_{\gamma}^{2}=\frac{|d \tau|_{\bar{\gamma}}^{2}}{W^{2}}, \quad W=\frac{1}{\sqrt{1+V_{S}^{2}|d \tau|_{\gamma}^{2}}} .
$$

The bound $1-V_{S}^{2}|d \tau|_{\gamma}^{2}>0$ (necessary for $W$ to be real) is a consequence of $S$ being spacelike everywhere. It is also immediate to show that the respective volume forms $\boldsymbol{\eta}_{S}$ and $\boldsymbol{\eta}_{\bar{S}}$ are related by

$$
\eta_{s}=W \eta_{\bar{s}}
$$

In order to study the relation between the extrinsic geometries of $S$ and $\bar{S}$ it is useful to choose a basis of the normal bundle of each surface. Concerning $\bar{S}$, the natural choice is $\left\{\bar{\nu},\left.V_{S}^{-1} \xi\right|_{S}\right\}$, where $\bar{\nu}$ is a unit normal of $\bar{S}$ as a hypersurface in $\Sigma_{t_{0}}$. We denote by $\bar{K}$ the second fundamental form of $\bar{S}$ along $\bar{\nu}$. Concerning $S$, the Lie constant extension $\bar{\nu}$ along the Killing $\xi$ defines a spacelike and unit normal to $S$, still denoted by $\bar{\nu}$. For the second vector, note that $\left.\xi\right|_{S}$ is nowhere tangent to $S$ and hence its normal component $\xi^{\perp}$ in the orthogonal decomposition $T_{p} M=T_{p} S \oplus N_{p} S$ is nowhere zero and, in fact, timelike. From $\boldsymbol{\xi}=-V^{2} d t$ we have, for any $X \in T_{p} S,\left\langle\left.\xi\right|_{S}, X\right\rangle=-V_{S}^{2} d \tau(X)$ which means that the tangential component of $\left.\xi\right|_{S}$ is $-V_{S}^{2} \operatorname{grad}_{\gamma}(\tau)$, or equivalently $\xi^{\perp}=\left.\xi\right|_{S}+V_{S}^{2} \operatorname{grad}_{\gamma}(\tau)$. Following [110] we denote by $u$ the future directed unit vector tangent to $\xi^{\perp}$. Its explicit form is

$$
u=\frac{W}{V_{S}}\left(\left.\xi\right|_{S}+V_{S}^{2} \operatorname{grad}_{\gamma}(\tau)\right),
$$

as a consequence of $u$ being unit and orthogonal to $\operatorname{grad}_{\gamma}(\tau)$ and the property $\langle\xi, \xi\rangle=$ $-V^{2}$. We note that $\{\bar{\nu}, u\}$ defines an orthonormal basis of the normal bundle of $S$.

The extrinsic geometry of $S$ is encoded into its second fundamental form vector $K$ and the connection of the normal bundle $\boldsymbol{\alpha}$. For the basis above, this geometric information is in turn given by the two symmetric tensors $K^{u}:=\langle K, u\rangle, K^{\bar{\nu}}:=\langle K, \bar{\nu}\rangle$ and the oneform $\boldsymbol{\alpha}_{\bar{\nu}}(X):=\left\langle\nabla_{X}^{M} \bar{\nu}, u\right\rangle, X \in \mathfrak{X}(S)$. The following proposition relates these objects with the geometry of the projected surface:

Proposition 3.3.1. With the notation above,

$$
\begin{aligned}
K^{\bar{\nu}} & =\pi^{*}(\bar{K})-\left.V_{S} \bar{\nu}(V)\right|_{S} d \tau \otimes d \tau, \\
K^{u} & =\frac{1}{W}\left(d V_{S} \otimes d \tau+d \tau \otimes d V_{S}+V_{S} \pi^{*}\left(\operatorname{Hess}_{\bar{\gamma}}(\tau)\right)\right) \\
& -\frac{V_{S}^{2}}{W} d V_{S}\left(\operatorname{grad}_{\bar{\gamma}}(\tau)\right) d \tau \otimes d \tau, \quad W=\sqrt{1-V_{S}^{2}|d \tau|_{\bar{\gamma}}^{2}}, \\
\boldsymbol{\alpha}_{\bar{\nu}} & =\frac{1}{W}\left(V_{S} \pi^{\star}\left(\bar{K}\left(\operatorname{grad}_{\bar{\gamma}}(\tau), \cdot\right)\right)-\left.\bar{\nu}(V)\right|_{S} d \tau\right) .
\end{aligned}
$$




\subsection{Geometry of the intial surface in terms of the projected one}

Proof. Inserting (3.5) in the defining expression $K^{\bar{\nu}}(X, Y)=\left\langle\nabla_{X}^{M} \bar{\nu}, Y\right\rangle$ gives, after using $\bar{X}(\tau)=d \tau(X)$,

$$
K^{\bar{\nu}}(X, Y)=d \tau(Y)\left\langle\nabla_{X}^{M} \bar{\nu}, \xi\right\rangle+d \tau(X)\left\langle\nabla_{\xi}^{M} \bar{\nu}, \bar{Y}\right\rangle+\left\langle\nabla_{\bar{X}}^{M} \bar{\nu}, \bar{Y}\right\rangle
$$

Now, $\left\langle\nabla_{X}^{M} \bar{\nu}, \xi\right\rangle=\bar{X}(\tau)\left\langle\nabla_{\xi}^{M} \bar{\nu}, \xi\right\rangle+\left\langle\nabla_{\bar{X}}^{M} \bar{\nu}, \xi\right\rangle=\bar{X}(\tau)\left\langle\nabla_{\xi}^{M} \bar{\nu}, \xi\right\rangle$, the second equality following from $\Sigma_{t_{0}}$ being totally geodesic. To elaborate this further, we note that $d \boldsymbol{\xi}=2 V^{-1} d V \wedge \boldsymbol{\xi}$ as a consequence of $\boldsymbol{\xi}=-V^{2} d t$. Hence

$$
\nabla_{\bar{\nu}}^{M} \boldsymbol{\xi}=\frac{1}{2} d \boldsymbol{\xi}(\bar{\nu}, \cdot)=\frac{\left.\bar{\nu}(V)\right|_{S}}{V_{S}} \boldsymbol{\xi}
$$

where in the first equality we used the Killing equations and in the second the orthogonality of $\bar{\nu}$ and $\xi$. Raising indices and recalling that $[\xi, \bar{\nu}]=0$ we conclude

$$
\nabla_{\xi}^{M} \bar{\nu}=\nabla_{\bar{\nu}}^{M} \xi=\frac{\left.\bar{\nu}(V)\right|_{S}}{V_{S}} \xi
$$

and therefore

$$
\left\langle\nabla_{X}^{M} \bar{\nu}, \xi\right\rangle=-\left.V_{S} \bar{\nu}(V)\right|_{S} d \tau(X)
$$

Using these expressions, the first term in (3.14) becomes $-\left.V_{S} \bar{\nu}(V)\right|_{S}(d \tau \otimes d \tau)(X, Y)$, while the second term vanishes. Finally, the last term gives the second fundamental form of $\bar{S}$ and (3.11) follows (to our knowledge, this identity appeared for the first time in [15]).

Concerning $K^{u}$, its symmetry properties allows us to write $K^{u}(X, Y)=\frac{1}{2}\left(\left\langle\nabla_{X}^{M} u, Y\right\rangle+\right.$ $\left.\left\langle\nabla_{Y}^{M} u, X\right\rangle\right)$, which after inserting (3.10) yields

$$
\begin{aligned}
K^{u}(X, Y)= & \frac{W}{2 V_{S}}\left(\left\langle\nabla_{X}^{M} \xi, Y\right\rangle+\left\langle\nabla_{Y}^{M} \xi, X\right\rangle+\right. \\
& \left.+\left\langle\nabla_{X}^{M}\left(V_{S}^{2} \operatorname{grad}_{\gamma}(\tau)\right), Y\right\rangle+\left\langle\nabla_{Y}^{M}\left(V_{S}^{2} \operatorname{grad}_{\gamma}(\tau)\right), X\right\rangle\right) .
\end{aligned}
$$

The Killing equations imply that the first two terms cancel each other. Now, the tangential projection to $S$ of a spacetime covariant derivative coincides with the intrinsic covariant derivative on $S$. More precisely, for any vector fields $X, Y, Z$ tangent to $S$ we have $\left\langle X, \nabla_{Y} Z\right\rangle=\left\langle X, D_{Y} Z\right\rangle_{\gamma}$. Thus, the third term in (3.16) becomes

$$
\begin{aligned}
\left\langle Y, \nabla_{X}\left(V_{S}^{2} \operatorname{grad}_{\gamma} \tau\right)\right\rangle & =2 V_{S} X\left(V_{S}\right)\left\langle Y, \operatorname{grad}_{\gamma} \tau\right\rangle+V_{S}^{2}\left\langle Y, D_{X} \operatorname{grad}_{\gamma} \tau\right\rangle \\
& =2 V_{S}\left(d V_{S} \otimes d \tau\right)(X, Y)+V_{S}^{2} \operatorname{Hess}_{\gamma} \tau(X, Y)
\end{aligned}
$$

where we used the definition of Hessian $\operatorname{Hess}_{\gamma} \tau(X, Y)=\left\langle Y, D_{X} \operatorname{grad}_{\gamma} \tau\right\rangle$. The same reasoning is valid for the fourth term. It follows immediately

$$
K^{u}=W\left(d V_{S} \otimes d \tau+d \tau \otimes d V_{S}+V_{S} \operatorname{Hess}_{\gamma}(\tau)\right)
$$




\section{Projection along the Killing in static spacetimes}

In order to rewrite this in terms of the projected geometry, we need to find the relation between the Hessians of $\tau$ on each one of the surfaces. To that aim, recall that the difference between connections $D$ and $\bar{D}$ on a given manifold defines a type $(1,2)$ tensor $\mathcal{Z}$ such that the following identity holds for any one-form $\boldsymbol{\omega}$ (see e.g. [106]):

$$
(D \boldsymbol{\omega})(X, Y)-(\bar{D} \boldsymbol{\omega})(X, Y)=-\mathcal{Z}(\boldsymbol{\omega}, X, Y)
$$

In our context, we can use $\pi^{\star}(\bar{\gamma})$ on $S$ and the corresponding connection $\bar{D}$ it defines. Given the relation (3.6), a straightforward computation gives

$$
\begin{aligned}
\mathcal{Z}(d \tau, \cdot, \cdot)= & -V_{S}|d \tau|_{\gamma}^{2}\left(d V_{S} \otimes d \tau+d \tau \otimes d V_{S}+\right. \\
& \left.+V_{S} \pi^{*}\left(\operatorname{Hess}_{\bar{\gamma}}(\tau)\right)\right)+V_{S} d V_{S}\left(\operatorname{grad}_{\gamma}(\tau)\right) d \tau \otimes d \tau
\end{aligned}
$$

Inserting this into (3.19) with $\boldsymbol{\omega}=d \tau$ and using (3.8) it follows

$$
\begin{aligned}
W^{2} \operatorname{Hess}_{\gamma_{0}}(\tau)= & \pi^{*}\left(\operatorname{HesS}_{\bar{\gamma}}(\tau)\right)+V_{S}|d \tau| \frac{2}{\gamma}\left(d \tau \otimes d V_{S}+d V_{S} \otimes d \tau\right)- \\
& -V_{S} d V_{S}\left(\operatorname{grad}_{\bar{\gamma}}(\tau)\right) d \tau \otimes d \tau
\end{aligned}
$$

Combining this and (3.18) gives (3.12) at once.

It only remains to compute the connection 1 -form $\boldsymbol{\alpha}_{\bar{\nu}}(X)=\left\langle\nabla_{X}^{M} \bar{\nu}, u\right\rangle$. Substituting (3.10) one finds

$$
\begin{aligned}
\boldsymbol{\alpha}_{\bar{\nu}}(X) & =\frac{W}{V_{S}}\left\langle\nabla_{X}^{M} \bar{\nu}, \xi+V_{S}^{2} \operatorname{grad}_{\gamma}(\tau)\right\rangle= \\
& =\frac{W}{V_{S}}\left\langle\nabla_{X}^{M} \bar{\nu}, \xi\right\rangle+W V_{S} K^{\bar{\nu}}\left(\operatorname{grad}_{\gamma}(\tau), X\right)= \\
& =-\left.W \bar{\nu}(V)\right|_{S} d \tau(X)+W V_{S} K^{\bar{\nu}}\left(\operatorname{grad}_{\gamma}(\tau), X\right)
\end{aligned}
$$

where in the last equality we used (3.15). Replacing (3.11) and using the first and second relation in (3.8) and the definition of $W$, then

$$
\begin{aligned}
\boldsymbol{\alpha}_{\bar{\nu}}(X)= & -\left.W \bar{\nu}(V)\right|_{S} d \tau(X)+ \\
& +W V_{S}\left(\pi^{*}(\bar{K})\left(\operatorname{grad}_{\gamma}(\tau), X\right)-\left.V_{S} \bar{\nu}(V)\right|_{S}|d \tau|_{\gamma}^{2} d \tau(X)\right)= \\
= & -\left.W \bar{\nu}(V)\right|_{S} d \tau(X)+\frac{V_{S}}{W}\left(\bar{K}\left(\operatorname{grad}_{\bar{\gamma}}(\tau), d \pi(X)\right)-\left.V \bar{\nu}(V)\right|_{S}|d \tau|_{\bar{\gamma}}^{2} d \tau(X)\right)= \\
= & \frac{V_{S}}{W} \bar{K}\left(\operatorname{grad}_{\bar{\gamma}}(\tau), d \pi(X)\right)-\left.\left(W+\frac{V_{S}^{2}|d \tau| \frac{2}{\gamma}}{W}\right) \bar{\nu}(V)\right|_{S} d \tau(X)= \\
= & \frac{1}{W}\left(V_{S} \bar{K}\left(\operatorname{grad}_{\bar{\gamma}}(\tau), d \pi(X)\right)-\left.\bar{\nu}(V)\right|_{S} d \tau(X)\right),
\end{aligned}
$$

as claimed. 


\subsection{Geometry of the intial surface in terms of the projected one}

Remark 3.3.2. Although we have assumed $\xi$ to be timelike, all the calculations above are similar when $\xi$ is spacelike and nowhere zero. In particular the geometric relations between $S$ and its projection $\bar{S}$ in a purely Riemannian context where $\langle\xi, \xi\rangle=V^{2}$ and $\boldsymbol{\xi}=V^{2} d t$ are

$$
\begin{aligned}
\gamma & =\pi^{\star}(\bar{\gamma})+V_{S}^{2} d \tau \otimes d \tau, \\
\eta_{S} & =W \eta_{\bar{S}} \quad W=\sqrt{1+V_{S}^{2}|d \tau|_{\bar{\gamma}}^{2}}, \\
K^{\bar{\nu}} & =\pi^{*}(\bar{K})+\left.V_{S} \bar{\nu}(V)\right|_{S} d \tau \otimes d \tau, \\
K^{u} & =-\frac{1}{W}\left(d V_{S} \otimes d \tau+d \tau \otimes d V_{S}+V_{S} \pi^{*}\left(\operatorname{Hess}_{\bar{\gamma}}(\tau)\right)\right)-\frac{V_{S}^{2}}{W} d V_{S}\left(\operatorname{grad}_{\bar{\gamma}}(\tau)\right) d \tau \otimes d \tau, \\
\boldsymbol{\alpha}_{\bar{\nu}} & =\frac{1}{W}\left(-V_{S} \pi^{\star}\left(\bar{K}\left(\operatorname{grad}_{\bar{\gamma}}(\tau), \cdot\right)\right)+\left.\bar{\nu}(V)\right|_{S} d \tau\right),
\end{aligned}
$$

where this time the unit vector $u$ reads

$$
u=\frac{W}{V_{S}}\left(\left.\xi\right|_{S}-V_{S}^{2} \operatorname{grad}_{\gamma}(\tau)\right)
$$

Remark 3.3.3. Note that the expressions above contain all the information needed to relate any geometric quantity on $S$ with geometric information on its projection $\bar{S}$. For instance, the mean curvature vector of $S$ can be related to the projected geometry simply taking the trace in $K=K^{\bar{\nu}} \bar{\nu}-K^{u} u$ with the metric $\gamma^{-1}$ and using (3.6) together with the results in Proposition 3.3.1. If we choose any other null basis $\{k, \ell\}$ of the normal bundle normalized so that $\langle k, \ell\rangle=-2$, it must be necessarily of the form

$$
k=f(-\bar{\nu}+u), \quad \ell=f^{-1}(\bar{\nu}+u)
$$

where $f: S \rightarrow \mathbb{R} \backslash\{0\}$ is smooth.

The null second fundamental forms $K^{k}, K^{\ell}$ of $S$ along the basis of null normals $\{k, \ell\}$ can be obtained directly from Proposition 3.3.1 using the decomposition (3.20). Namely, if we consider a linear combination $\omega=a \bar{\nu}+b u$ of the vectors $\{\bar{\nu}, u\}$ we have

$$
K_{A B}^{\omega}=\left\langle\nabla_{X_{A}}(a \bar{\nu}+b u), X_{B}\right\rangle=a\left\langle\nabla_{X_{A}} \bar{\nu}, X_{B}\right\rangle+b\left\langle\nabla_{X_{A}} u, X_{B}\right\rangle=a K_{A B}^{\bar{\nu}}+b K_{A B}^{u}
$$

Hence using (3.20) and Proposition 3.3.1, we obtain

$$
\begin{aligned}
K_{A B}^{k}= & f\left(K_{A B}^{u}-K_{A B}^{\bar{\nu}}\right) \\
= & f\left(\frac{1}{W}\left(d V_{S} \otimes d \tau+d \tau \otimes d V_{S}\right)+\frac{V_{S}}{W} \pi^{*}\left(\operatorname{Hess}_{\bar{\gamma}}(\tau)\right)-\pi^{*}(\bar{K})\right. \\
& \left.+\left(\left.V_{S} \bar{\nu}(V)\right|_{S}-\frac{V_{S}^{2}}{W} d V_{S}\left(\operatorname{grad}_{\bar{\gamma}}(\tau)\right)\right) d \tau \otimes d \tau\right),
\end{aligned}
$$




\section{Projection along the Killing in static spacetimes}

and

$$
\begin{aligned}
K_{A B}^{\ell}= & f^{-1}\left(K_{A B}^{u}+K_{A B}^{\bar{\nu}}\right) \\
= & f^{-1}\left(\frac{1}{W}\left(d V_{S} \otimes d \tau+d \tau \otimes d V_{S}\right)+\frac{V_{S}}{W} \pi^{*}\left(\operatorname{Hess}_{\bar{\gamma}}(\tau)\right)+\pi^{*}(\bar{K})\right. \\
& \left.-\left(\left.V_{S} \bar{\nu}(V)\right|_{S}+\frac{V_{S}^{2}}{W} d V_{S}\left(\operatorname{grad}_{\bar{\gamma}}(\tau)\right)\right) d \tau \otimes d \tau\right) .
\end{aligned}
$$

We can now take the trace of (3.21) with the contravariant form of the metric $\gamma$ to obtain

$$
\begin{aligned}
f \theta_{\ell}= & \frac{2}{W} d V_{S}\left(\operatorname{grad}_{\gamma}(\tau)\right)+\operatorname{tr}_{\gamma}\left(\frac{V_{S}}{W} \pi^{*}\left(\operatorname{Hess}_{\bar{\gamma}}(\tau)\right)+\pi^{*}(\bar{K})\right) \\
& -\left(\left.V_{S} \bar{\nu}(V)\right|_{S}+\frac{V_{S}^{2}}{W} d V_{S}\left(\operatorname{grad}_{\bar{\gamma}}(\tau)\right)\right)|d \tau|_{\gamma}^{2} .
\end{aligned}
$$

Using now the first and second relations in (3.8), and expression (3.7), we obtain

$$
\begin{aligned}
f \theta_{\ell}= & \frac{2}{W^{3}} d V_{S}\left(\operatorname{grad}_{\bar{\gamma}}(\tau)\right)+\frac{V_{S}}{W}\left(\triangle_{\bar{\gamma}} \tau\right)+\bar{H}+\frac{V_{S}^{3}}{W^{3}}\left(\operatorname{Hess}_{\bar{\gamma}}(\tau)\right)\left(\operatorname{grad}_{\bar{\gamma}}(\tau), \operatorname{grad}_{\bar{\gamma}}(\tau)\right) \\
& +\frac{V_{S}^{2}}{W^{2}} \bar{K}\left(\operatorname{grad}_{\bar{\gamma}}(\tau), \operatorname{grad}_{\bar{\gamma}}(\tau)\right)-\left.\frac{V_{S}}{W^{2}} \bar{\nu}(V)\right|_{S}|d \tau|_{\bar{\gamma}}^{2}-\frac{V_{S}^{2}}{W^{3}} d V_{S}\left(\operatorname{grad}_{\bar{\gamma}}(\tau)\right)|d \tau|_{\bar{\gamma}}^{2}
\end{aligned}
$$

It is convenient to organize the terms in the following form:

$$
f \theta_{\ell}=\bar{H}+\frac{V_{S}^{2}}{W^{2}}\left(\bar{K}\left(\operatorname{grad}_{\bar{\gamma}}(\tau), \operatorname{grad}_{\bar{\gamma}}(\tau)\right)-\frac{\left.\bar{\nu}(V)\right|_{S}}{V_{S}}|d \tau|_{\bar{\gamma}}^{2}\right)+\equiv,
$$

where

$$
\begin{aligned}
\equiv= & \frac{2}{W^{3}} d V_{S}\left(\operatorname{grad}_{\bar{\gamma}}(\tau)\right)+\frac{V_{S}}{W}\left(\triangle_{\bar{\gamma}} \tau\right)+\frac{V_{S}^{3}}{W^{3}}\left(\operatorname{HesS}_{\bar{\gamma}}(\tau)\right)\left(\operatorname{grad}_{\bar{\gamma}}(\tau), \operatorname{grad}_{\bar{\gamma}}(\tau)\right) \\
& -\frac{V_{S}^{2}}{W^{3}} d V_{S}\left(\operatorname{grad}_{\bar{\gamma}}(\tau)\right)|d \tau|_{\bar{\gamma}}^{2}
\end{aligned}
$$

We next prove that $\equiv$ is proportional to a divergence. We use here index notation for convenience. We want to show that

$$
\equiv=\frac{1}{V_{S}} \bar{\nabla}_{A}\left(\frac{V_{S}^{2}}{W} \bar{\nabla}^{A} \tau\right)
$$

Expanding the right-hand side

$$
\frac{1}{V_{S}} \bar{\nabla}_{A}\left(\frac{V_{S}^{2}}{W} \bar{\nabla}^{A} \tau\right)=\frac{1}{V_{S}} \bar{\nabla}_{A}\left(\frac{V_{S}^{2}}{W}\right) \bar{\nabla}^{A} \tau+\frac{V_{S}}{W} \triangle_{\bar{\gamma}} \tau
$$




\subsection{Geometry of the intial surface in terms of the projected one}

The expression $W=\sqrt{1-V_{S}^{2}|d \tau|^{2}}$ implies

$$
\bar{\nabla}_{A} W=\frac{1}{W}\left(-V_{S} V_{S, A}|d \tau|_{\bar{\gamma}}^{2}-V_{S}^{2}\left(\bar{\nabla}_{A} \bar{\nabla}^{L} \tau\right) \tau_{, L}\right)
$$

so that (3.23) becomes

$$
\frac{V_{S}}{W} \triangle_{\bar{\gamma}} \tau+\frac{2}{W} V_{S, A} \bar{\tau}^{, A}+\frac{V_{S}^{2}}{W^{3}} V_{S, A} \bar{\tau}^{, A}|d \tau|_{\bar{\gamma}}^{2}+\frac{V_{S}^{3}}{W^{3}}\left(\bar{\nabla}_{A} \bar{\nabla}_{B} \tau\right) \bar{\tau}^{, A} \bar{\tau}^{, B},
$$

which is exactly (3.22) after using the definition of $W$.

In summary, we have obtained an expression for $\theta_{\ell}$ in terms of the geometry of the projected surface:

$\theta_{\ell}=\frac{1}{f}\left(\bar{H}+\frac{V_{S}^{2}}{W^{2}}\left(\bar{K}\left(\operatorname{grad}_{\bar{\gamma}}(\tau), \operatorname{grad}_{\bar{\gamma}}(\tau)\right)-\frac{\left.\bar{\nu}(V)\right|_{S}}{V_{S}}|d \tau|_{\bar{\gamma}}^{2}\right)+\frac{1}{V_{S}} \bar{\nabla}_{A}\left(\frac{V_{S}^{2}}{W} \bar{\nabla}^{A} \tau\right)\right)$.

Observe that

$$
\begin{aligned}
\langle k, \xi\rangle=f\langle u-\bar{\nu}, \xi\rangle=f\langle u, \xi\rangle=\frac{f W}{V_{S}}\left\langle\xi+V_{S}^{2} \operatorname{grad}_{\gamma}(\tau), \xi\right\rangle & =\frac{f W}{V_{S}}\left(-V_{S}^{2}-V_{S}^{4}|d \tau|_{\gamma}^{2}\right) \\
& =-f \frac{V_{S}}{W},
\end{aligned}
$$

where we have used that $\bar{\nu}$ is orthogonal to $\xi$, the definition of $W$ and that

$$
\left\langle\operatorname{grad}_{\gamma}(\tau), \xi\right\rangle=\left\langle\operatorname{grad}_{\gamma}(\tau), \xi^{\perp}-V_{S}^{2} \operatorname{grad}_{\gamma}(\tau)\right\rangle=-V_{S}^{2}|d \tau|_{\gamma}^{2}
$$

Hence, if we normalize $k$ so that $\langle k, \xi\rangle=-1$, the value of $f$ (that we will call in this case $f^{\star}$ ) becomes

$$
f^{\star}=\frac{W}{V_{S}} .
$$

This value is special when computing the total null extrinsic curvature $\int_{S} \theta_{\ell} \boldsymbol{\eta}_{S}$, because the divergence term of $\theta_{\ell}$ vanishes upon integration (recall the relation between volume forms (3.9) $)$. In other words, for $f=f^{\star}$ the total null extrinsic curvature becomes

$$
\int_{S} \theta_{\ell} \boldsymbol{\eta}_{S}=\int_{\bar{S}}\left(\bar{H} V_{S}+\frac{V_{S}^{3}}{W^{2}}\left(\bar{K}\left(\operatorname{grad}_{\bar{\gamma}}(\tau), \operatorname{grad}_{\bar{\gamma}}(\tau)\right)-\frac{\left.\bar{\nu}(V)\right|_{S}}{V_{S}}|d \tau|_{\bar{\gamma}}^{2}\right)\right) \boldsymbol{\eta}_{\bar{S}} .
$$

Concerning the connection one-form, its behaviour under change of basis is not tensorial (being a connection), so it is worth giving its explicit expression in the null basis $\{k, \ell\}$. With the usual definition of connection one-form in this basis given by $s(X):=\frac{1}{2}\left\langle\nabla_{X}^{M} k, \ell\right\rangle$ we have

$$
\boldsymbol{s}(X)=\frac{1}{2}\left\langle\nabla_{X}^{M} k, \ell\right\rangle=\frac{1}{2}\left\langle\nabla_{X}^{M}(-f \bar{\nu}+f u), f^{-1} \bar{\nu}+f^{-1} u\right\rangle=-\frac{X(f)}{f}-\boldsymbol{\alpha}_{\bar{\nu}}(X),
$$




\section{Projection along the Killing in static spacetimes}

and hence

$$
\boldsymbol{s}=-\frac{d f}{f}+\frac{1}{W}\left(\left.\bar{\nu}(V)\right|_{S} d \tau-V_{S} \pi^{\star}\left(\bar{K}\left(\operatorname{grad}_{\bar{\gamma}}(\tau), \cdot\right)\right)\right)
$$

The following lemma shows that the null expansions $K^{k}$ and $K^{\ell}$ of any spacelike surface $S$ in a strictly static spacetime are not independent to each other. In the case of the Minkowski spacetime this result was proved in [37].

Lemma 3.3.4 (Relationship between null extrinsic curvatures). Let $(M, g)$ be an $(n+2)$-dimensional static spacetime with static Killing vector $\xi$. Let $S$ be a spacelike surface in $(M, g)$. With the notation above, we have

$$
-\langle\xi, k\rangle K^{\ell}-\langle\xi, \ell\rangle K^{k}-2 V_{S}\left(d \tau \otimes d V_{S}+d V_{S} \otimes d \tau\right)-2 V_{S}^{2} H e s S_{\gamma} \tau=0,
$$

where $k$ and $\ell$ are a basis of null normal vectors to $S$ satisfying $\langle k, \ell\rangle=-2$, and where $V_{S}$ and $\tau$ are respectively, the restriction of $V$ and $t$ on $S$.

Proof. Since the relationship is local, it suffices to work on a suitably small neighbourhood $U_{p}$ of a point $p \in S$. We choose $U_{p}$ small enough so that $\xi_{\alpha}=-V^{2} \nabla_{\alpha} t$ on $U_{p}$ and work on $U_{p}$ from now on. Decomposing $\xi$ in tangential and normal components we have (cf. (3.10) )

$$
\left.\xi\right|_{S}=-\frac{1}{2}\langle\xi, k\rangle \ell-\frac{1}{2}\langle\xi, \ell\rangle k-V_{S}^{2} \operatorname{grad}_{\gamma} \tau \text {. }
$$

The Killing equation $\nabla_{\alpha} \xi_{\beta}+\nabla_{\beta} \xi_{\alpha}=0$ implies, on $U_{p}$, and for $X, Y \in \Gamma(T S)$

$$
\left\langle X, \nabla_{Y} \xi\right\rangle+\left\langle Y, \nabla_{X} \xi\right\rangle=0
$$

Let us work out the first term. Inserting the decomposition (3.26) and using the definition of null extrinsic curvature it follows

$$
\left\langle X, \nabla_{Y} \xi\right\rangle=-\frac{1}{2}\langle\xi, k\rangle K^{\ell}(X, Y)-\frac{1}{2}\langle\xi, \ell\rangle K^{k}(X, Y)-\left\langle X, \nabla_{Y}\left(V_{S}^{2} \operatorname{grad}_{\gamma} \tau\right)\right\rangle .
$$

The third term of (3.28) is developed as in (3.17). Inserting this result into (3.28) we conclude

$$
\begin{aligned}
\left\langle X, \nabla_{Y} \xi\right\rangle= & -\frac{1}{2}\langle\xi, k\rangle K^{\ell}(X, Y)-\frac{1}{2}\langle\xi, \ell\rangle K^{k}(X, Y)-2 V_{S}\left(d \tau \otimes d V_{S}\right)(X, Y) \\
& -V_{S}^{2} \operatorname{Hess}_{\gamma} \tau(X, Y),
\end{aligned}
$$

which combined with (3.27) proves the Lemma.

Remark 3.3.5. If we choose a basis $\left\{X_{A}\right\}$ of the tangent space to $S$, the relation (3.25) can be expressed in index notation as

$$
-\langle\xi, k\rangle K_{A B}^{\ell}-\langle\xi, \ell\rangle K_{A B}^{k}-D_{A}\left(V_{S}^{2} D_{B} \tau\right)-D_{B}\left(V_{S}^{2} D_{A} \tau\right)=0
$$




\subsection{Geometry of the intial surface in terms of the projected one}

Corollary 3.3.6. Under the same assumptions as in the previous Lemma,

$$
\langle\xi, \ell\rangle\langle\xi, k\rangle=V_{S}^{2}\left(1+V_{S}^{2}|D \tau|_{\gamma}^{2}\right) .
$$

Proof. Squaring (3.26) it follows

$$
-V_{S}^{2}=\langle\xi, \xi\rangle=-\langle\xi, k\rangle\langle\xi, \ell\rangle+V_{S}^{4}\left\langle\operatorname{grad}_{\gamma} \tau, \operatorname{grad}_{\gamma} \tau\right\rangle=-\langle\xi, k\rangle\langle\xi, \ell\rangle+V_{S}^{4}|D \tau|_{\gamma}^{2} .
$$

The projection along the Killing was used by Gibbons [37] in Minkowski to address the shell version of the Penrose inequality. However, his proof contained serious mistakes. In the following section we analyze his procedure and correct the expressions. To this purpose we will use all the machinery developed in the first part of the chapter.

\subsubsection{A critical revision of Gibbons' argument}

In this subsection we discuss the gap in Gibbons' attempt [37] to prove the general inequality (1.4). Following the notation of the previous section, we will denote by $S$ the spacelike, spacetime convex surface involved in the inequality. However, in order to stick to the notation used by Gibbons in [37] as much as possible, here the future directed null normals are called $k$ and $L$ and satisfy the normalization $\langle k, \xi\rangle=-1$ and $\langle L, k\rangle=-1$.

The strategy in [37] was to project $S$ along $\xi^{\alpha}$ onto a constant time hyperplane orthogonal to $\xi^{\alpha}$. The projected surface is denoted by $\bar{S}$. The main idea was to rewrite the shell Penrose inequality (1.4)

$$
\int_{S} \rho \boldsymbol{\eta}_{S} \geq \frac{n}{4}\left(\omega_{n}\right)^{\frac{1}{n}}|S|^{\frac{n-1}{n}}
$$

in terms of the geometry of $\bar{S}$ as a hypersurface in Euclidean space, where $2 \rho:=\nabla_{\alpha} L^{\alpha}$ is the null expansion of $L$ (hence $\rho=\frac{1}{4} \theta_{\ell}$ when compared with the normalization we used in (3.3), since $\ell=2 L$ ), and $\boldsymbol{\eta}_{S}$ is the area element of $S$.

Gibbons' gap consists in his claim that

$$
\int_{S} \rho \boldsymbol{\eta}_{S}=\frac{1}{4} \int_{\bar{S}} \bar{H} \boldsymbol{\eta}_{\bar{S}}
$$

It is clear that (3.31) contradicts our result (3.24) that gives the value of the total null expansion along $L$ in a static spacetime. With the current normalization conditions $(V=1)$, instead of (3.31) we find

$$
\int_{S} \rho \boldsymbol{\eta}_{\boldsymbol{S}}=\int_{\bar{S}} \frac{1}{4}\left(\bar{H}+\frac{1}{W^{2}} \bar{K}\left(\operatorname{grad}_{\bar{\gamma}} \tau, \operatorname{grad}_{\bar{\gamma}} \tau\right)\right) \boldsymbol{\eta}_{\bar{S}} .
$$




\section{Projection along the Killing in static spacetimes}

The expression (3.32) differs from (3.31) since $\bar{K}\left(\operatorname{grad}_{\bar{\gamma}} \tau, \operatorname{grad}_{\bar{\gamma}} \tau\right) \neq 0$ in general.

Before discussing in detail the problem that led Gibbons to conclude (erroneously) that expression (3.31) holds, let us briefly explain how Gibbons' argument would have continued had (3.31) been valid. The area of $\bar{S}$ is not smaller than the area of $S$ (because of (3.9)) and hence inequality (3.30) would follow from (3.31) and the Minkowski-type inequality

$$
\int_{\bar{S}} \bar{H} \boldsymbol{\eta}_{\bar{S}} \geq n\left(\omega_{n}\right)^{\frac{1}{n}}|\bar{S}|^{\frac{n-1}{n}}
$$

that Trudinger [104] claimed to be applicable to general mean convex surfaces in Euclidean space (i.e. surfaces with non-negative mean curvature). Notice that Gibbons finds not only (3.31) but also (3.39) below from which $\bar{H} \geq 0$ follows from $\rho \geq 0$.

The original Minkowski inequality (3.33) (for convex bodies) proves that "among all convex bodies of given surface area the sphere has the smallest integral of mean curvature". Its proof can be found in [10] (see expression (4) in Section 56), and it can also be derived from the Aleksandrov-Fenchel inequalities discussed in [104].

Trudinger gave an argument to show (3.33) using an elliptic method. However, Guan and Li [39], showed this argument is incomplete and at present it is not known whether inequality (3.33) holds for general mean convex bodies. In [39] a parabolic argument is proposed which proves the inequality for mean convex starshaped domains in Euclidean space (a starshaped domain is defined by the property that it contains a point $x_{0}$ and all segments between $x_{0}$ and any other $x$ in the domain).

Let us now go into the projection method used by Gibbons and see where it fails. Gibbons first extends $k$ to an ingoing null hypersurface $\mathcal{N}$ by solving the affinely parametrized null geodesic $\nabla_{k} k=0$ with initial data $k$ on $S$. Similarly, $L$ is extended to a null vector field on the outgoing null hypersurface $\mathcal{L}$ passing through $S$ and with tangent vector $L$. These vector fields are then extended to a spacetime neighbourhood of $S$ by parallel transport along $\xi$ (see Figure 3.2). With this extension, we have $\langle k, \xi\rangle=-1$ everywhere. Defining $\beta$ on this neighbourhood by $\beta:=-\langle\xi, L\rangle$, the following vector field can be introduced:

$$
\hat{\nu}=\frac{1}{\sqrt{2 \beta}}(L-\beta k) .
$$

It follows immediately that $\hat{\nu}$ is everywhere normal to $\xi$. Moreover, this field is orthogonal to $\bar{S}$ and unit on this projected surface (in fact it agrees with the $\bar{\nu}$ we introduced in the previous section).

Gibbons used in [37] that the mean curvature $\bar{H}$ of the projected surface $\bar{S}$ can be expressed as $\bar{H}=\left.\nabla_{\alpha} \hat{\nu}^{\alpha}\right|_{\bar{S}}$. This is one of the key problems in his argument, because this expression is not generally satisfied. Let $\left\{\bar{X}_{A}\right\}$ be a tangent basis for the projected surface $\bar{S}$, so that we can write

$$
\bar{H}=\bar{\gamma}^{A B} K_{A B}^{\hat{\nu}}=\bar{\gamma}^{A B}\left\langle\nabla_{\bar{X}_{A}} \hat{\nu}, \bar{X}_{B}\right\rangle .
$$




\subsection{Geometry of the intial surface in terms of the projected one}

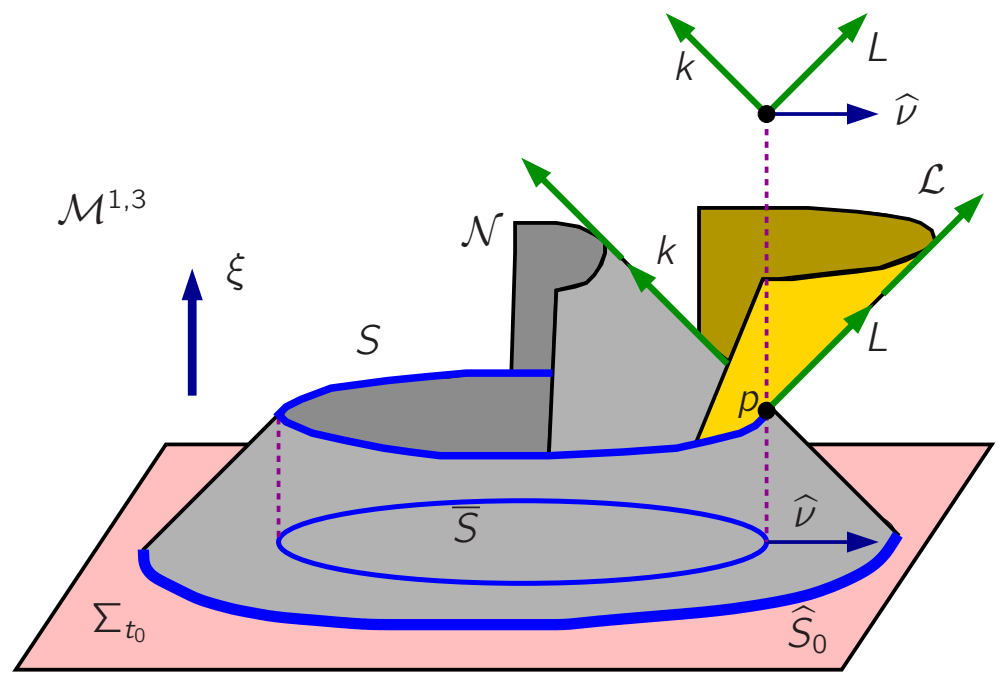

Figure 3.2: Two null hypersurfaces starting at $S$ are represented. Gibbons' construction uses a null hypersurface $\mathcal{N}$ generated by the geodesic null vector field $k$, and a null hypersurface $\mathcal{L}$ generated by the geodesic null vector $L . \quad k$ and $L$ are extended to a neighbourhood of $S$ by Lie-dragging them along the Killing $\xi$. The product of $k$ and $L$ is -1 when evaluated at points of $S$. The vector field $\widehat{\nu}=\frac{1}{\sqrt{2 \beta}}(L-\beta k)$, where $\beta:=-\langle\xi, L\rangle$, when evaluated on $\bar{S}$ agrees with its outer unit normal on $\Sigma_{t_{0}}$.

We want to relate this expression with $\left.\nabla_{\alpha} \hat{\nu}^{\alpha}\right|_{\bar{S}}$. In order to do so, let $B=\left\{e_{\alpha}\right\}$ be any basis of vectors of the Minkowski spacetime defined on points of $\bar{S}$. Then

$$
\left.\nabla_{\alpha} \hat{\nu}^{\alpha}\right|_{\bar{S}}=\eta^{\alpha \beta}\left\langle\nabla_{e_{\alpha}} \hat{\nu}, e_{\beta}\right\rangle
$$

Choosing the basis $B=\left\{\left.\xi\right|_{\bar{S}},\left.\hat{\nu}\right|_{\bar{S}}, \bar{X}_{A}\right\}$, the metric $\eta$ is

$$
\eta_{\alpha \beta} \equiv\left(\begin{array}{cc|c}
-1 & 0 & 0 \\
0 & 1 & 0 \\
\hline 0 & 0 & \bar{\gamma}_{A B}
\end{array}\right)
$$

so that inverting (3.35) we have

$$
\left.\nabla_{\alpha} \hat{\nu}^{\alpha}\right|_{\bar{S}}=-\left\langle\nabla_{\xi} \hat{\nu}, \xi\right\rangle+\left\langle\nabla_{\hat{\nu}} \hat{\nu}, \hat{\nu}\right\rangle+\bar{\gamma}^{A B}\left\langle\nabla_{\bar{X}_{A}} \hat{\nu}, \bar{X}_{B}\right\rangle
$$

The vectors $\xi$ and $\hat{\nu}$ are everywhere orthogonal and $\xi$ is covariantly constant. Thus $\left\langle\nabla_{\xi} \hat{\nu}, \xi\right\rangle=-\left\langle\hat{\nu}, \nabla_{\xi} \xi\right\rangle=0$, and the (correct) expression for the mean curvature in $(3.36)$ is

$$
\bar{H}=\left.\nabla_{\alpha} \hat{\nu}^{\alpha}\right|_{\hat{T}}-\left.\frac{1}{2} \nabla_{\hat{\nu}}\langle\hat{\nu}, \hat{\nu}\rangle\right|_{\hat{T}} .
$$

Thus Gibbons ignored the term $\nabla_{\hat{\nu}}\langle\hat{\nu}, \hat{\nu}\rangle$. This term vanishes whenever $\hat{\nu}$ has constant norm. However, the extension of $\hat{\nu}$ is already fixed and, as we shall see later in an 


\section{Projection along the Killing in static spacetimes}

example, it does not keep the norm of $\hat{\nu}$ constant. Gibbons still makes a second mistake when computing $\nabla_{\alpha} \hat{\nu}^{\alpha}$. The following expression is written down in [37]

$$
\bar{H}=\nabla_{\alpha} \hat{\nu}^{\alpha}=\nabla_{\alpha}\left(\frac{1}{\sqrt{2 \beta}} L^{\alpha}-\frac{\beta}{\sqrt{2 \beta}} k^{\alpha}\right)=\frac{1}{\sqrt{2 \beta}}\left(\nabla_{\alpha} L^{\alpha}\right)-\frac{\beta}{\sqrt{2 \beta}}\left(\nabla_{\alpha} k^{\alpha}\right),
$$

which implicitly assumes that the derivatives of $\beta$ along $k$ and $L$ are zero. Using the extension $\nabla_{L} L=0$ and that $\xi$ is covariantly constant it is immediate to check that $\nabla_{L} \beta=0$. However as our example below will show, it is not true that $\nabla_{k} \beta=0$. In summary, Gibbons finds the erroneous expression (cf. (5.11) in [37])

$$
\bar{H}=\sqrt{\frac{2}{\beta}} \rho+\sqrt{2 \beta} \mu,
$$

where $-2 \mu=\theta_{k}$. From here the argument is concluded by invoking the identity $2 \rho-$ $2 \beta \mu-\triangle_{\gamma} \tau=0$ so that

$$
\begin{aligned}
\int_{S} \rho \boldsymbol{\eta}_{S} & =\int_{S}\left(\frac{\beta}{\sqrt{2 \beta}} \bar{H}-\beta \mu\right) \boldsymbol{\eta}_{S}=\int_{S}\left(\frac{\beta}{\sqrt{2 \beta}} \bar{H}-\rho+\frac{1}{2} \triangle_{\gamma} \tau\right) \boldsymbol{\eta}_{S} \\
& =\int_{S}\left(\frac{\beta}{\sqrt{2 \beta}} \bar{H}-\rho\right) \boldsymbol{\eta}_{S},
\end{aligned}
$$

which leads to (3.31) by solving for $\int_{S} \rho \boldsymbol{\eta}_{S}$ and using $\boldsymbol{\eta}_{\bar{S}}=\sqrt{2 \beta} \boldsymbol{\eta}_{S}$.

In contrast, the correct expression for $\bar{H}$ is, combining (3.37) and (3.38)

$$
\bar{H}=\sqrt{\frac{2}{\beta}} \rho+\sqrt{2 \beta} \mu-\nabla_{k}\left(\sqrt{\frac{\beta}{2}}\right)-\left.\frac{1}{2} \nabla_{\hat{\nu}}\langle\hat{\nu}, \hat{\nu}\rangle\right|_{\bar{S}},
$$

where we have already used $\nabla_{L} \beta=0$. Expression (3.40) agrees with (3.39) only if the last two terms cancel each other. Neither $\nabla_{\hat{\nu}}\langle\hat{\nu}, \hat{\nu}\rangle$ nor the derivative of $\beta$ along $k$ need to vanish on $\bar{S}$. Even more, they need not, and in fact do not, cancel out in general. It is possible to derive general expressions both for $\nabla_{k} \beta$ and for $\nabla_{\hat{\nu}}\langle\hat{\nu}, \hat{\nu}\rangle$ on $\bar{S}$ (or $S$ ) which show that such cancellations do not occur. Instead of doing so, we find it more convenient to present an explicit example where the last two terms in (3.40) do not cancel each other. For completeness, we also evaluate $\bar{H}, \rho$ and $\mu$ explicitly on this example and show that (3.39) is not valid.

For the example, we use spherical coordinates $\{t, r, \theta, \phi\}$ in Minkowski, and consider the past null cone $\Omega_{p}$ of the origin $p\{t=0, r=0\}$. The defining equation for $\Omega_{p}$ is $t+r=0$, so the null tangent $k$ satisfying $\left\langle k, \partial_{t}\right\rangle=-1$ is

$$
k=\partial_{t}-\partial_{r} .
$$

In intrinsic coordinates $\{\hat{r}, \theta, \phi\}$ of $\Omega$ satisfying $k=-\partial_{\hat{r}}$ the first fundamental form is $\hat{\gamma}=\hat{r}^{2}\left(d \theta^{2}+\sin ^{2} \theta d \phi^{2}\right)$. Using the fundamental identity $£_{k} \hat{\gamma}=2 K^{k}$ (which we 


\subsection{Geometry of the intial surface in terms of the projected one}

prove explicitly in Appendix $(\mathrm{B})$, where $\left.K^{k}\right|_{q}$ is the extrinsic null curvature along $k$ of any spacelike surface $S_{q}$ embedded in the cone and that contains $q \in \Omega_{p}$, we find

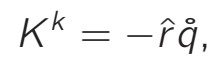

where $q$ is the standard metric on the sphere.

We consider an axially symmetric (with respect to the Killing vector $\partial_{\phi}$ ) spacelike surface $S$ embedded in $\Omega_{p}$. The embedding is then given by

$$
F:(\theta, \phi) \rightarrow(-R(\theta), R(\theta), \theta, \phi),
$$

where $R$ is a smooth, positive function (satisfying suitable regularity properties at the north and south poles, as usual). The induced metric $\gamma$ on $S$ is $\gamma=R^{2} \stackrel{\circ}{q}$.

Let us first compute $\beta=-\langle\xi, L\rangle$. Using formula (3.29) with the conditions $\langle\xi, k\rangle=$ -1 and $V=1$, and since $\ell=2 L$, we have

$$
\beta=\frac{1}{2}\left(1+|D \tau|_{\gamma}^{2}\right)
$$

where $\tau(\theta, \phi)=-R(\theta)$. Explicitly

$$
\beta=\frac{R^{2}+\left(R^{\prime}\right)^{2}}{2 R^{2}}
$$

where prime means derivative with respect to $\theta$. We next compute $\nabla_{k} \beta$. The analogous decomposition to (3.26) in the basis $\{k, L\}$ is

$$
\xi=\beta k+L-\operatorname{grad}_{\gamma} \tau
$$

so that

$$
\begin{aligned}
\nabla_{k} \beta & =-\left\langle\nabla_{k} \xi, L\right\rangle-\left\langle\xi, \nabla_{k} L\right\rangle=-\left\langle\xi, \nabla_{k} L\right\rangle=-\left\langle\xi, \nabla_{\frac{1}{\beta}\left(\xi-L+\operatorname{grad}_{\gamma} \tau\right)} L\right\rangle \\
& =\frac{-1}{\beta}\left\langle\xi, \nabla_{\operatorname{grad}_{\gamma} \tau} L\right\rangle,
\end{aligned}
$$

where in the last equality we used $\nabla_{\xi} L=\nabla_{L} L=0$, which hold directly from the way how $L$ has been extended. Inserting again (3.42) in the right-hand side

$$
\begin{aligned}
\nabla_{k} \beta & =\frac{-1}{\beta}\left\langle L+\beta k-\operatorname{grad}_{\gamma} \tau, \nabla_{\operatorname{grad}_{\gamma} \tau} L\right\rangle=-\left\langle k, \nabla_{\operatorname{grad}_{\gamma} \tau} L\right\rangle+\frac{1}{\beta}\left\langle\nabla_{\operatorname{grad}_{\gamma} \tau} L, \operatorname{grad}_{\gamma} \tau\right\rangle \\
& =s_{L}\left(\operatorname{grad}_{\gamma} \tau\right)+\frac{1}{\beta} K^{L}\left(\operatorname{grad}_{\gamma} \tau, \operatorname{grad}_{\gamma} \tau\right),
\end{aligned}
$$

where $s_{L}(X):=\left\langle\nabla_{X} k, L\right\rangle$ with the present normalization. Given that

$$
s_{L}(X)=\left\langle\nabla_{X} k, L\right\rangle=\left\langle\nabla_{X} k, \xi-\beta k+\operatorname{grad}_{\gamma} \tau\right\rangle=\left\langle\nabla_{X} k, \operatorname{grad}_{\gamma} \tau\right\rangle=K^{k}\left(X, \operatorname{grad}_{\gamma} \tau\right),
$$




\section{Projection along the Killing in static spacetimes}

(3.43) becomes

$$
\nabla_{k} \beta=K^{k}\left(\operatorname{grad}_{\gamma} \tau, \operatorname{grad}_{\gamma} \tau\right)+\frac{1}{\beta} K^{L}\left(\operatorname{grad}_{\gamma} \tau, \operatorname{grad}_{\gamma} \tau\right)
$$

We can use now the curvature equation (3.25), which in the present normalization is

$$
K^{L}+\beta K^{k}-\operatorname{Hess}_{\gamma} \tau=0
$$

and (3.44) simplifies to

$$
\nabla_{k} \beta=\frac{1}{\beta} \operatorname{Hess}_{\gamma} \tau\left(\operatorname{grad}_{\gamma} \tau, \operatorname{grad}_{\gamma} \tau\right)=\frac{1}{2 \beta} \gamma\left(\operatorname{grad}_{\gamma}\left(|D \tau|_{\gamma}^{2}\right), \operatorname{grad}_{\gamma} \tau\right)
$$

Inserting $|D \tau|_{\gamma}^{2}=\frac{\left(R^{\prime}\right)^{2}}{R^{2}}, \beta=\frac{R^{2}+\left(R^{\prime}\right)^{2}}{2 R^{2}}$ and $\operatorname{grad}_{\gamma} \tau=\frac{-R^{\prime}}{R^{2}} \partial_{\theta}$, we conclude

$$
\nabla_{k} \beta=\frac{2\left(R^{\prime}\right)^{2}\left(-R R^{\prime \prime}+\left(R^{\prime}\right)^{2}\right)}{R^{3}\left(R^{2}+\left(R^{\prime}\right)^{2}\right)},
$$

and the fourth term in the right hand-side of (3.40) is

$$
-\nabla_{k}\left(\sqrt{\frac{\beta}{2}}\right)=\frac{-1}{4} \sqrt{\frac{2}{\beta}}\left(\nabla_{k} \beta\right)=\frac{\left(R^{\prime}\right)^{2}\left(R^{\prime \prime} R-\left(R^{\prime}\right)^{2}\right)}{R^{2}\left(R^{2}+\left(R^{\prime}\right)^{2}\right)^{\frac{3}{2}}} .
$$

It remains to compute the last term of (3.40). We need to know the value of $\nabla_{\hat{\nu}}\langle\hat{\nu}, \hat{\nu}\rangle$, with $\hat{\nu}$ as in (3.34). Recall that the vector $k$ has been extended from $S$ by $\nabla_{k} k=0$ and then by $\nabla_{\xi} k=0$. Thus $k$ stays null in a whole spacetime neighbourhood of $p$. The same reasoning applies to $L$. Thus

$$
\langle\hat{\nu}, \hat{\nu}\rangle=\frac{1}{2 \beta}\left(\langle L, L\rangle+\beta^{2}\langle k, k\rangle-2 \beta\langle k, L\rangle\right)=-\langle k, L\rangle
$$

and we need to compute

$$
\nabla_{\hat{\nu}}\langle k, L\rangle=\nabla_{\frac{1}{\sqrt{2 \beta}} L-\frac{\beta}{\sqrt{2 \beta}} k}\langle k, L\rangle=\frac{1}{\sqrt{2 \beta}}\left\langle\nabla_{L} k, L\right\rangle-\frac{\beta}{\sqrt{2 \beta}}\left\langle\nabla_{k} L, k\right\rangle .
$$

Each term can be computed from (3.42) as follows

$$
\begin{aligned}
\left\langle\nabla_{L} k, L\right\rangle & =\left\langle\nabla_{\xi-\beta k+\operatorname{grad}_{\gamma} \tau} k, L\right\rangle=\left\langle\nabla_{\operatorname{grad}_{\gamma} \tau} k, L\right\rangle=\left\langle\nabla_{\operatorname{grad}_{\gamma} \tau} k, \xi-\beta k+\operatorname{grad}_{\gamma} \tau\right\rangle \\
& =\left\langle\nabla_{\operatorname{grad}_{\gamma} \tau} k, \operatorname{grad}_{\gamma} \tau\right\rangle=K^{k}\left(\operatorname{grad}_{\gamma} \tau, \operatorname{grad}_{\gamma} \tau\right), \\
\left\langle\nabla_{k} L, k\right\rangle & =\left\langle\nabla_{\frac{1}{\beta}\left(\xi-L+\operatorname{grad}_{\gamma} \tau\right)} L, k\right\rangle=\frac{1}{\beta}\left\langle\nabla_{\operatorname{grad}_{\gamma} \tau} L, k\right\rangle=\frac{-1}{\beta}\left\langle\nabla_{\operatorname{grad}_{\gamma} \tau} k, L\right\rangle \\
& =\frac{-1}{\beta} K^{k}\left(\operatorname{grad}_{\gamma} \tau, \operatorname{grad}_{\gamma} \tau\right),
\end{aligned}
$$




\subsection{Geometry of the intial surface in terms of the projected one}

and $(3.48)$ is finally

$$
\nabla_{\hat{\nu}}\langle k, L\rangle=\sqrt{\frac{2}{\beta}} K^{k}\left(\operatorname{grad}_{\gamma} \tau, \operatorname{grad}_{\gamma} \tau\right)=\frac{-2\left(R^{\prime}\right)^{2}}{R^{2} \sqrt{R^{2}+\left(R^{\prime}\right)^{2}}},
$$

where in the last equality we inserted $K^{k}=-R \stackrel{q}{\text { and }} \operatorname{grad}_{\gamma} \tau=\frac{-R^{\prime}}{R^{2}} \partial_{\theta}$. In summary, (3.47) becomes

$$
\left.\frac{-1}{2} \nabla_{\hat{\nu}}\langle\hat{\nu}, \hat{\nu}\rangle\right|_{r=R(\theta)}=\frac{-\left(R^{\prime}\right)^{2}}{R^{2} \sqrt{R^{2}+\left(R^{\prime}\right)^{2}}},
$$

which is not only nonzero but also does not cancel with (3.46) in general.

For completeness we compute now the null expansions. Since $\mu$ is a property of $\Omega_{p}$, it suffices to find the null expansion along $k$ of the past null cone $\Omega_{p}$. Let $q \in \Omega_{p}$. We

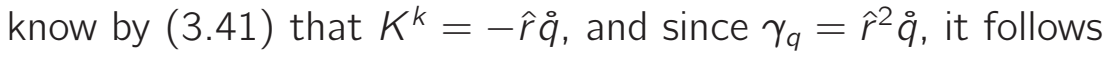

$$
\mu(q)=\frac{1}{\hat{r}(q)}
$$

To compute $\rho$, we will use the curvature equation (3.45), whose trace reads in this case as

$$
2 \rho-2 \beta \mu-\triangle_{\gamma} \tau=0
$$

The Laplacian of $\tau(\theta, \phi)=-R(\theta)$ is

$$
\triangle_{\gamma} \tau=\frac{-\cos \theta}{\sin \theta} \frac{R^{\prime}}{R^{2}}-\frac{R^{\prime \prime}}{R^{2}}
$$

Since $\beta=\frac{R^{2}+\left(R^{\prime}\right)^{2}}{2 R^{2}}$ and $\mu=\frac{1}{R}$, equation (3.50) implies

$$
\rho=\frac{-R R^{\prime \prime}+R^{2}+\left(R^{\prime}\right)^{2}}{2 R^{3}}-\frac{R^{\prime} \cos \theta}{2 R^{2} \sin \theta} .
$$

Using the values above together with (3.46) and (3.49) and substituting them in (3.40), we obtain

$$
\left.\bar{H}\right|_{\bar{S}}=\frac{1}{\sqrt{R^{2}+\left(R^{\prime}\right)^{2}}}\left(\frac{2 R^{2}+3\left(R^{\prime}\right)^{2}-R R^{\prime \prime}}{R^{2}+\left(R^{\prime}\right)^{2}}-\frac{R^{\prime}}{R} \frac{\cos \theta}{\sin \theta}\right) .
$$

On the other hand these expressions in the right-hand side of (3.39) gives

$$
\sqrt{\frac{2}{\beta}} \rho+\left.\sqrt{2 \beta} \mu\right|_{\bar{S}}=\frac{1}{\sqrt{R^{2}+\left(R^{\prime}\right)^{2}}}\left(\frac{2 R^{2}+2\left(R^{\prime}\right)^{2}-R R^{\prime \prime}}{R^{2}}-\frac{R^{\prime} \cos \theta}{R \sin \theta}\right),
$$

which is clearly different to the expression for $\left.\bar{H}\right|_{\bar{S}}$ in (3.51). This proves that (3.39) cannot be correct. If we instead perform the analogous substitution in (3.40) we find a consistent expression. 


\subsubsection{The projection along the Killing direction in Minkowski. Ap- plications to the shell Penrose inequality.}

Brendle and Wang have proved [15] a generalization of the shell Penrose inequality for the Schwarzschild spacetime. In the particular case of the Mikowski spacetime, the inequality holds for the class of surfaces whose projection along the Killing direction onto a constant time hypersurface is convex.

In the following theorem we quote Brendle and Wang result [15] and give a proof which in fact was obtained independently before their result was published. Our proof uses the machinery developed at the beginning of the chapter (and published in [75]), which allows us to express the shell Penrose inequality for the initial surface $S$ in Minkowski in terms of the projected geometry.

Theorem 3.3.7 (S. Brendle \& M.T. Wang). Let $\left(\mathcal{M}^{1, n+1}, \eta\right)$ be the $(n+2)$-dimensional Minkowski spacetime with $t$ a Minkowskian time defining a unit Killing $\boldsymbol{\xi}=-d t$. Let $S$ be a closed, connected, orientable and spacetime convex surface in $\left(\mathcal{M}^{1, n+1}, \eta\right)$ with contravariant metric $\gamma^{-1}$. Let $\pi: \mathcal{M}^{1, n+1} \rightarrow \Sigma_{t_{0}}$ be the orthogonal projection onto the hyperplane $\Sigma_{t_{0}}=\left\{t=t_{0}\right\}$ and define $\bar{S}=\pi(S)$. Denote by $\boldsymbol{\eta}_{\bar{S}}$ its volume form and by $\bar{K}$ its second fundamental form as a hypersurface of $(n+1)$-Euclidean space with respect to the outer unit normal. Then the shell Penrose inequality with respect to $\xi$ for $S$ is equivalent to

$$
\int_{\bar{S}} t r_{d \pi\left(\gamma^{-1}\right)} \bar{K} \eta_{\bar{S}} \geq n\left(\omega_{n}\right)^{\frac{1}{n}}|S|^{\frac{n-1}{n}}
$$

and holds if $\bar{S}$ is convex.

Proof. With the normalization we have been using up to now $(\langle k, \xi\rangle=-1$ and $\langle k, \ell\rangle=$ $-2)$ the shell Penrose inequality for arbitrary dimension reads

$$
\int_{S} \theta_{\ell} \boldsymbol{\eta}_{S} \geq n\left(\omega_{n}\right)^{\frac{1}{n}}|S|^{\frac{n-1}{n}}
$$

We can use now the expression (3.24) for the total null extrinsic curvature in static spacetimes for $f=f^{\star}$ (because $k$ has been chosen to satisfy $\langle k, \xi\rangle=-1$ ), and with $V=1$. This expression becomes in this case

$$
\int_{S} \theta_{\ell} \boldsymbol{\eta}_{\boldsymbol{S}}=\int_{\bar{S}}\left(\bar{H}+\frac{1}{W^{2}} \bar{K}\left(\operatorname{grad}_{\bar{\gamma}} \tau, \operatorname{grad}_{\bar{\gamma}} \tau\right)\right) \boldsymbol{\eta}_{\bar{S}}=\int_{\bar{S}} \operatorname{tr}_{d \pi\left(\gamma^{-1}\right)} \bar{K} \boldsymbol{\eta}_{\bar{S}}
$$

and the shell Penrose inequality can be rewritten as (3.52).

To conclude, we see that if $\bar{S}$ is convex it follows $\bar{K}\left(\operatorname{grad}_{\bar{\gamma}} \tau, \operatorname{grad}_{\bar{\gamma}} \tau\right) \geq 0$, and that implies

$$
\int_{S} \theta_{l} \boldsymbol{\eta}_{S} \geq \int_{\bar{S}} \bar{H} \boldsymbol{\eta}_{\bar{S}} \geq n\left(\omega_{n}\right)^{\frac{1}{n}}|\bar{S}|^{\frac{n-1}{n}} \geq n\left(\omega_{n}\right)^{\frac{1}{n}}|S|^{\frac{n-1}{n}}
$$




\subsection{Geometry of the intial surface in terms of the projected one}

where we have used the Minkowski inequality for convex surfaces in the Euclidean space and $|\bar{S}| \geq|S|$.

Remark 3.3.8. By definition, a spacetime convex surface $S$ embedded in a constant time hyperplane $\Sigma_{t_{0}}$ is a convex euclidean surface. When this happens, the vector $\ell$ decomposes as $\ell=\xi+\nu$ and then $\theta_{\ell}=\langle\vec{H}, \xi+\nu\rangle=\langle H \nu, \xi+\nu\rangle=H$ and consequently

$$
\int_{S} \theta_{\ell} \boldsymbol{\eta}_{S}=\int_{S} H \boldsymbol{\eta}_{S}
$$

and the shell Penrose inequality conjecture turns out to be in this case the Minkowski inequality for convex surfaces in the Euclidean space, and hence a true inequality. This result is also recovered in (3.53) by setting $\tau=0$. This particular case of the shell Penrose inequality was first proved by Gibbons [37]. Note that this shell Penrose inequality is with respect to the Killing orthogonal to the hyperplane $\Sigma_{t_{0}}$.

Although Gibbons used an alternative proof, this case is immediately covered by Theorem 3.3.7. In fact, this theorem also implies the validity of the shell Penrose inequality for $S$ with respect to any other time translation, as we show next.

Theorem 3.3.9. Let $S$ be a closed, connected and convex surface embedded in a spacelike hyperplane $\Sigma_{t_{0}^{\prime}}^{\prime} \hookrightarrow \mathcal{M}^{1,3}$. Let $\xi$ be any unit time translation (not necessarily orthogonal to $\Sigma_{t_{0}^{\prime}}^{\prime}$ ). Then the Penrose inequality with respect to $\xi$ holds for $S$.

Proof. Let $\nu^{\prime}$ be the outward normal to $S$ in $\Sigma_{t_{0}^{\prime}}^{\prime}$. Since a hyperplane is totally geodesic, the second fundamental form vector of $S$ is $K=K^{\nu^{\prime}} \nu^{\prime}$, where $K^{\nu^{\prime}}$ is positive semidefinite. Choose any hyperplane $\Sigma_{t_{0}}$ orthogonal to $\xi$ and define $\bar{S}$ as the orthogonal projection of $S$ onto $\Sigma_{t_{0}}$. To prove the theorem it suffices to show that $\bar{S}$ is convex, i.e. that its second fundamental form $\bar{K}$ with respect to the unit outer normal $\bar{\nu}$ in $\Sigma_{t_{0}}$ is non-negative. From Proposition 3.3.1 in Chapter 3 (formula (3.11), with $V=1$, as we are in Minkowski) we have

$$
K^{\bar{\nu}}=\pi^{\star}(\bar{K}),
$$

where $\pi: S \rightarrow \bar{S}$ is the projection along $\xi, \bar{\nu}$ is the parallel extension along $\xi$ of the normal vector $\bar{\nu}$ of $\bar{S}$ evaluated on $S$ and $K^{\bar{\nu}}:=\langle K, \bar{\nu}\rangle_{\eta}$. Thus, $\bar{K}$ is non-negative if and only if $\left\langle\nu^{\prime}, \bar{\nu}\right\rangle_{\eta}$ is non-negative. Now, both $\nu^{\prime}$ and $\bar{\nu}$ are normal to $S$, spacelike and unit. Since they belong to a two-dimensional Lorentzian space, $\left\langle\nu^{\prime}, \bar{\nu}\right\rangle_{\eta}$ vanishes nowhere, and, hence, has constant sign. For the choice $\xi=\xi^{\prime}$, i.e. the time translation normal to $\Sigma_{t_{0}^{\prime}}^{\prime}$, we obviously have $\nu^{\prime}=\bar{\nu}$ and the sign is positive. Since $\xi$ can be obtained from $\xi^{\prime}$ by a smooth deformation, and $\bar{\nu}$ also changes smoothly, it is impossible that the sign of $\left\langle\nu^{\prime}, \bar{\nu}\right\rangle_{\eta}$ changes from +1 to -1 , and the theorem is proved. 


\section{Projection along the Killing in static spacetimes}

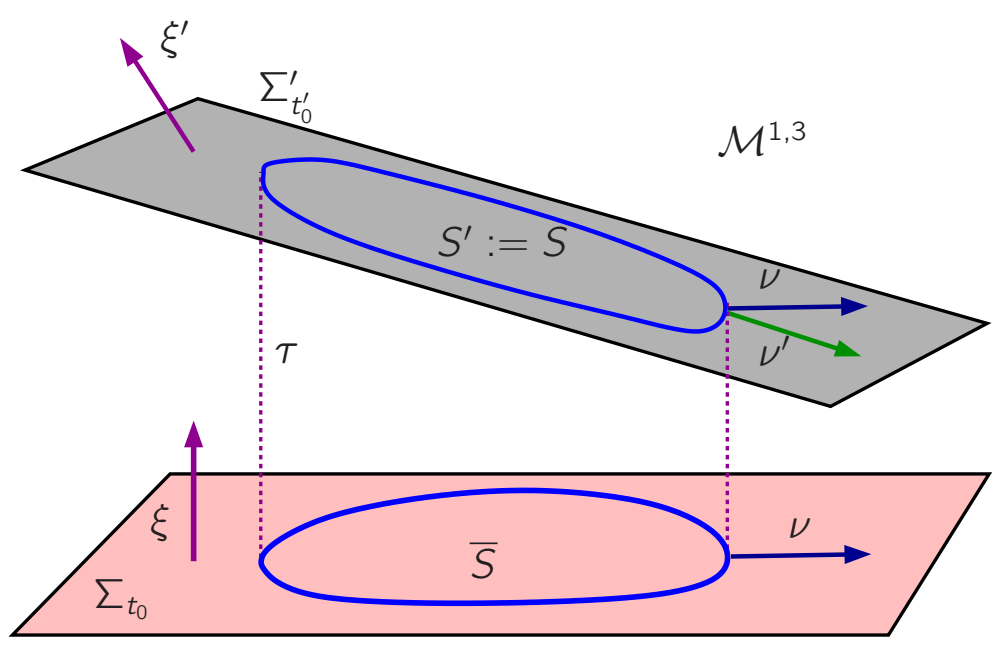

Figure 3.3: Particular case of the projection along the Killing in Figure 3.1 where $S^{\prime}:=S$ lies in a hyperplane orthogonal to a time translation $\xi^{\prime}$ different from $\xi$. In this case, if $S^{\prime}$ is convex, $\bar{S}$ is also convex and $S^{\prime}$ satisfies the shell Penrose inequality with respect to $\xi$. The shell Penrose inequality with respect to $\xi$ transforms in this context in a Minkowski type inequality for euclidean surfaces and reads $\int_{S^{\prime}} H^{\prime} f \boldsymbol{\eta}_{S^{\prime}} \geq \sqrt{16 \pi\left|S^{\prime}\right|}$, where $H^{\prime}$ is the mean curvature of $S^{\prime}$ as a surface of $\mathbb{R}^{3}$, and $f=\left\langle\nu^{\prime}, \xi\right\rangle-\left\langle\xi^{\prime}, \xi\right\rangle$.

This theorem implies a Minkowski type inequality for $S^{\prime}:=S$ as a convex surface of Euclidean space (see Figure 3.3). Indeed, the Killing vector $\xi$ can be decomposed as $\xi=\sqrt{1+|v|^{2}} \xi^{\prime}+v$ where $v$ is a translation of Euclidean space $\left(\mathbb{R}^{3}, g_{E}\right)$ (identified with the hyperplane $\Sigma_{t_{0}^{\prime}}^{\prime}$. With the definition of null vectors $k=\xi^{\prime}-\nu^{\prime}$ and $\ell=\xi^{\prime}+\nu^{\prime}$ on $S^{\prime}$ and, given that the mean curvature vector of $S^{\prime}$ is $H^{\prime} \nu^{\prime}$, where $H^{\prime}$ is the mean curvature of $S^{\prime} \hookrightarrow \mathbb{R}^{3}$, the Penrose inequality (2.34) with respect to $\xi$ becomes

$$
\int_{S^{\prime}} H^{\prime} f \eta_{S^{\prime}} \geq \sqrt{16 \pi\left|S^{\prime}\right|}
$$

where

$$
f=\sqrt{1+|v|^{2}}+g_{E}\left(\nu^{\prime}, v\right) .
$$

Obviously, when $v=0$ we recover the standard Minkowski inequality. The validity of this inequality suggests that it might be worth studying for which functions $f$ Minkowski type inequalities of the form (3.54) hold for arbitrary convex surfaces of Euclidean space. 



\section{Projection along the past null cone $\Omega$ in Minkowski}

\subsection{Introduction}

In the previous chapter we have studied the projection in the Killing direction onto time constant hyperplanes in static spacetimes. Another natural projection that turns out to be useful for the Penrose inequality is to drag the surface along its outward past null cone. This dragging will be studied in full detail in a subsequent chapter. Here we concentrate in the Minkowski case. Most of the content of this chapter was published in [74], where this projection was used for the first time to address the shell Penrose inequality.

Specifically, we consider spacelike closed surfaces $S$ in the Minkowski spacetime whose past null cone $\Omega$ extends smoothly all the way to past null infinity. Our aim in this chapter is to rewrite the shell Penrose inequality in Minkowski in terms of the geometry of the surface $\widehat{S}_{0}$ determined by the intersection of $\Omega$ with a constant time hyperplane $\left\{t=t_{0}\right\}$ lying to the past of $S$. The solution to the evolution equations of the quotient metric and second fundamental form (Ricatti equation) with which the null hypersurface $\Omega$ is endowed allows us to rewrite the geometry of any cross section of $\Omega$ (in particular $S$ ) in terms of the geometry of the euclidean surface $\widehat{S}_{0}$, which turns out to be convex. The fundamental results we need concerning the geometry of null hypersurfaces are explained in Appendix $B$. The expression we find for the shell Penrose inequality (Theorem 4.3.5) involves the time height function $\tau=\left.t\right|_{S}-t_{0}$ of $S$, that measures the "time distance" that separates $S$ from $\left\{t=t_{0}\right\}$. In the case that the surface lies in the past null cone of a point in Minkowski, the resulting inequality is proven to hold in arbitrary dimension. The key tool that allows us to prove this result is a Sobolev type inequality on the sphere due to Beckner [7]. Our result generalizes the validity for the shell Penrose inequality with spherical symmetry achieved by Tod [101] in the four dimensional Minkowski spacetime.

As explained in detail in Appendix [C, the geometry of a convex surface in Euclidean 
space can be fully encoded in terms of a single function. This function is called the support function of the surface. This allows us to find an alternative expression for the shell Penrose inequality in Minkowski which involves the support function $h$ of $\widehat{S}_{0}=$ $\Omega \cap\left\{t=t_{0}\right\}$ and the above mentioned time height function $\tau$, which is naturally associated to the initial surface $S$. Both $h$ and $\tau$ can be defined on the sphere using suitable pullbacks. The new expression for the shell Penrose inequality in terms of $h$ and $\tau$ involves the inverse of matrices of size $n \times n$. The special case $n=2$ (spacetime dimension four) is considered in Theorem 4.4.6, where the particular expression of the inequality with the explicitly computed inverses is obtained.

The final part of the chapter makes use of flows of surfaces beginning at $S$ and moving along $\Omega$ with the purpose of addressing the shell Penrose inequality in Minkowski. Null flows were already used by Ludvigsen \& Vickers [67], whose idea was later improved by Bergqvist [8]. Analyzing the monotonicity properties of what we call Bergqvist mass we find an inequality between $\tau$ and $h$ which is sufficient for the validity of the shell Penrose inequality. In particular we find that the class of surfaces which satisfy the Penrose inequality as a consequence of this method includes a non-empty open set. In contrast, the set of surfaces covered when applying Ludvigsen \& Vickers' and Bergqvist's method [67, 8] to the Minkowski spacetime turns out to be of measure zero.

\subsection{Notation and basic definitions}

For the sake of clarity let us start this chapter with a brief summary of the geometry of null hypersurfaces. Details can be found in Appendix $B$.

Let $\Omega$ be a null hypersurface of an $(n+2)$-dimensional spacetime $M$, and $k$ a future directed vector field tangent to $\Omega$ which is nowhere zero and null. This vector field is defined up to multiplication with a positive function $F: \Omega \rightarrow \mathbb{R}^{+}$. It is well-known (see e.g. [32]) that given any point $p \in \Omega$, an equivalence relation can be defined on $T_{p} \Omega$ by means of $X \sim Y$ if and only if $X-Y=c k$ with $c \in \mathbb{R}$. The equivalence class of $X \in T_{p} \Omega$ is denoted by $\bar{X}$ and the quotient space by $T_{p} \Omega / k$. The set $T \Omega / k=\bigcup_{p \in \Omega} T_{p} \Omega / k$, is endowed naturally with the structure of a vector bundle over $\Omega$ (with fibers of dimension $n)$ which is called quotient bundle.

Given $\bar{X}, \bar{Y} \in T_{p} \Omega / k$, it follows that $\gamma^{\Omega}(\bar{X}, \bar{Y}):=\langle X, Y\rangle$ is a positive definite metric on this quotient space. The tensor $K^{\Omega}(\bar{X}, \bar{Y}):=\left\langle\nabla_{X} k, Y\right\rangle$ is well-defined (i.e. independent of the representatives $X, Y \in T_{p} \Omega$ of $\bar{X}, \bar{Y}$ and of the extension of $Y$ to a neighbourhood of $p$ ). This tensor is symmetric and plays the role of a second fundamental form on $\Omega$. The Weingarten map, which we denote by $\boldsymbol{K}^{\Omega}$, is the endomorphism obtained from $K^{\Omega}$ by raising one index with the inverse of $\gamma^{\Omega}$. Finally, the trace of $K^{\Omega}$ with respect to $\gamma^{\Omega}$ is the null expansion $\theta_{k}$ of $\Omega$. Under a rescaling $k \longrightarrow F k$, these tensors transform as $K^{\Omega} \longrightarrow F K^{\Omega}, K^{\Omega} \longrightarrow F K^{\Omega}$ and $\theta_{k} \longrightarrow F \theta_{k}$. 


\section{Projection along the past null cone $\Omega$ in Minkowski}

A derivative of $T \Omega / k$ can be defined via $(\bar{X})^{\prime}:=\overline{\nabla_{k} X}$. Again this derivative is well-defined (i.e. independent of the representative chosen in the definition). Note, however, that it does depend on the choice of $k$. As usual, this derivative is extended to tensors in $T \Omega / k$ with the Leibniz rule. An important property of null hypersurfaces is that the quotient metric $\gamma^{\Omega}$, the quotient extrinsic curvature $K^{\Omega}$ and the ambient geometry $(M, g)$ are related by the following equations (see equations (B.6) and (B.12) in Appendix B]

$$
\begin{aligned}
& \left(\gamma^{\Omega}\right)^{\prime}=2 K^{\Omega}, \\
& \left(K^{\Omega}\right)^{\prime}+K^{\Omega} \circ K^{\Omega}+R-Q_{k} K^{\Omega}=0, \quad \text { (Ricatti equation) }
\end{aligned}
$$

where $\boldsymbol{K}^{\Omega} \circ \boldsymbol{K}^{\Omega}$ is the composition of endomorphisms, $R(\bar{X}):=\overline{R(X, k) k}$ and $Q_{k}$ is defined by $\nabla_{k} k=Q_{k} k$ (the integrals curves of $k$ are necessarily null geodesics but the parameter along them need not be affine).

In order to transform this system of equations into a system of ODE for tensor components, let us choose $k$ to be affinely parametrized, i.e. satisfying $\nabla_{k} k=0$. Let us also select $n$ vector fields $X_{A}(A, B, C=1, \cdots, n)$ tangent to $\Omega$ satisfying the properties (i) $\left[k, X_{A}\right]=0$ and (ii) $\left\{\left.k\right|_{p},\left.X_{A}\right|_{p}\right\}$ is a basis of $T_{p} \Omega$ at one point $p \in \Omega$. Denote by $\alpha_{p}(\sigma)$ an affinely parametrized null geodesic containing $p$ and with tangent vector $k$ (for later convenience we do not fix yet the origin of the affine parameter $\sigma$ ). Then $\left\{\left.\bar{X}_{A}\right|_{\alpha_{p}(\sigma)}\right\}$ is a basis of $T_{\alpha_{p}(\sigma)} \Omega / k$ and the tensor coefficients $\gamma_{A B}^{\Omega}(\sigma), K_{A B}^{\Omega}(\sigma)$ of $\left.\gamma^{\Omega}\right|_{\alpha_{p}(\sigma)}$ and $\left.K^{\Omega}\right|_{\alpha_{p}(\sigma)}$ in this basis satisfy the ODE (see equations (B.11) and (B.12) in Appendix (B)

$$
\begin{aligned}
& \frac{d\left(K^{\Omega}\right)_{B}^{A}}{d \sigma}=-\left(K^{\Omega}\right)_{C}^{A}\left(K^{\Omega}\right)_{B}^{C}-R_{B}^{A}, \\
& \frac{d\left(\gamma^{\Omega}\right)_{A B}}{d \sigma}=2\left(K^{\Omega}\right)_{A B},
\end{aligned}
$$

where $R_{B}^{A}$ are defined by $R\left(\bar{X}_{B}\right)=R_{B}^{A} \bar{X}_{A}$ and indices are lowered and raised with the metric $\left(\gamma^{\Omega}\right)_{A B}$ and its inverse $\left(\gamma^{\Omega}\right)^{A B}$.

\subsection{Shell Penrose inequality in the Minkowski space- time in terms of the geometry of convex surfaces}

We will restrict in this chapter to the $(n+2)$-dimensional Minkowski spacetime $\left(\mathcal{M}^{1, n+1}, \eta\right)(n \geq 2)$. Choose a Minkowskian coordinate system $\left(t, x^{\alpha}\right)$ and define $\xi=\partial_{t}$. Since this Killing vector is unit, we have $V=1$ in the notation of the previous chapter. The hyperplanes at constant $t=t_{0}$ will be denoted by $\Sigma_{t_{0}}$.

The main purpose of this section is to rewrite the shell Penrose inequality in terms of the geometry of the projected euclidean surfaces obtained by intersecting the past null 


\subsection{Shell Penrose inequality in $\mathcal{M}^{1, n+1}$ in terms of Euclidean geometry}

cone of the original surface (provided it satisfies suitable conditions of regularity) with a constant time hyperplane.

As already mentioned, the physical construction leading to the Penrose inequality involves null hypersurfaces which extend smoothly all the way to past null infinity. We introduce the following definition which captures this notion conveniently (recall that a null hypersurface is maximally extended if it cannot be extended to a larger smooth null hypersurface).

Definition 4.3.1 (Spacetime convex null hypersurface). Let $\Omega$ be a maximally extended null hypersurface in $\left(\mathcal{M}^{1, n+1}, \eta\right) . \Omega$ is spacetime convex if there exists $t_{0} \in \mathbb{R}$ for which the surface $\widehat{S}_{0}=\Omega \cap \Sigma_{t_{0}}$ is closed (i.e. smooth, compact and without boundary), connected and convex as a hypersurface of the euclidean geometry of $\Sigma_{t_{0}}$, and the null expansion of the future null generator $k$ of $\Omega$ evaluated at $\widehat{S}_{0}$ satisfies $\left.\theta_{k}\right|_{\widehat{S}_{0}}<0$ . $\Omega$ is called spacetime strictly convex if $\widehat{S}_{0}$ is strictly convex, namely with positive principal curvatures at every point.

Remark 4.3.2. Note that if the hypothesis $\left.\theta_{k}\right|_{\widehat{S}_{0}}<0$ is removed there would be two possible choices of hypersurface $\Omega$ : the future null cone of $\widehat{S}_{0}$ and the past null cone of $\widehat{S}_{0}$.

Remark 4.3.3. The idea of the definition is, obviously, that if the shape of the null hypersurface at some instant of Minkowskian time is convex, then the past directed outgoing null geodesics cannot develop caustics and hence the null hypersurface will extend smoothly to past null infinity. It is also clear that if $\Omega \cap \Sigma_{t_{0}}$ is closed and convex for some $t_{0}$, the same occurs for all $t \leq t_{0}$.

Given a spacetime convex null hypersurface $\Omega$, we always normalize the tangent null vector $k$ uniquely by the condition $\langle k, \xi\rangle=-1$. This vector field will also be normal to any spacelike surface embedded in $\Omega$. Since the Penrose inequality involves precisely this type of surfaces the following definition is useful:

Definition 4.3.4 (Spacetime convex surface). A spacelike surface $S$ embedded in $\left(\mathcal{M}^{1, n+1}, \eta\right)$ is called spacetime (strictly) convex if it can be embedded in a spacetime (strictly) convex null hypersurface $\Omega$ of $\left(\mathcal{M}^{1, n+1}, \eta\right)$.

It is intuitively obvious that a spacelike, closed and connected surface $S$ can be embedded at most in one spacetime convex null hypersurface $\Omega$. Thus, for any such surface we can define unambiguously a null basis $\{\ell, k\}$ of its normal bundle by the conditions that $k$ is tangent to the spacetime convex null hypersurface $\Omega$ containing $S$ and the normalization conditions $\langle k, \xi\rangle=-1,\langle\ell, k\rangle=-2$. We refer to $\ell$ as the outgoing null normal and to $k$ as ingoing null normal. The Penrose inequality (1.4) involves the null expansion $\theta_{\ell}$ with respect to the outer null normal. The idea we want to explore in this chapter is how this inequality can be related to the geometry of a convex hypersurface 


\section{Projection along the past null cone $\Omega$ in Minkowski}

of Euclidean space. The most natural convex surface arising in this setup is precisely the surface $\widehat{S}_{0}=\Omega \cap \Sigma_{t_{0}}$ (see Figure 4.1). On the other hand, any convex surface $\widehat{S}_{0} \hookrightarrow \Sigma_{t_{0}}$ defines uniquely a spacetime convex null hypersurface $\Omega$ and, then, any spacelike surface embedded in $\Omega$ is defined uniquely by the "time height" function over $\Sigma_{t_{0}}$, namely the function $\tau:=\left.t\right|_{S}-t_{0}$. This function is defined on $S$. However, and similarly as in the previous chapter, there is a canonical diffeomorphism $\phi: S \rightarrow \widehat{S}_{0}$ defined by the condition that $\phi(p)$ lies on the maximally extended null geodesic $\alpha_{p}$ passing through $p$ and with tangent vector $\left.k\right|_{p}$. This diffeomorphism allows us to transfer geometric information from $S$ onto $\widehat{S}_{0}$ and viceversa. In particular, we can define $\left(\phi^{-1}\right)^{\star}(\tau)$. Since no confusion will arise, we still denote this function by $\tau$. The precise meaning will be clear from the context.

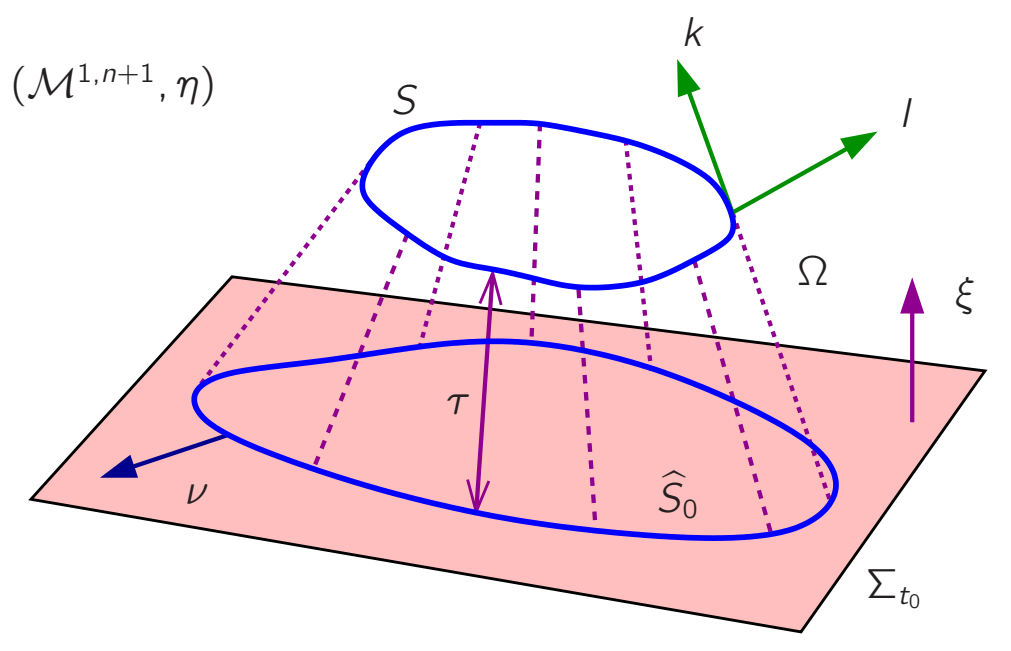

Figure 4.1: Schematic figure representing the construction above, where the spacetime convex surface $S$ is projected along $\Omega$ onto the constant time hyperplane $\Sigma_{t_{0}}=\{t=$ $\left.t_{0}\right\}$. The vectors on the normal bundle of $S$ are normalized so that $\langle k, \xi\rangle=-1$ and $\langle\ell, k\rangle=-2$. The vector field $\nu$ is unit, normal and pointing outside the surface $\widehat{S}_{0}$ within the hyperplane $\Sigma_{t_{0}}$.

The idea is thus to transform the Penrose inequality (1.4) into an inequality involving the geometry of $\widehat{S}_{0}$ as a hypersurface of Euclidean space $\left(\mathbb{R}^{n+1}, g_{E}\right)$ and the time height function $\tau$. The result is given in the following theorem [74] :

Theorem 4.3.5 (Shell Penrose inequality in Minkowski in terms of Euclidean geometry). Let $\left(\mathcal{M}^{1, n+1}, \eta\right)$ be the Minkowski spacetime with a selected Minkowskian coordinate system $\left(t, x^{\alpha}\right), \xi=\partial_{t}$. Let $(S, \gamma)$ be a spacetime convex surface in $\left(\mathcal{M}^{1, n+1}, \eta\right)$ and $\Omega$ the convex null hypersurface containing $S$. Consider the closed, convex surface 


\subsection{Shell Penrose inequality in $\mathcal{M}^{1, n+1}$ in terms of Euclidean geometry}

$\widehat{S}_{0}=\Omega \cap \Sigma_{t_{0}}$ as a hypersurface of Euclidean space $\left(\mathbb{R}^{n+1}, g_{E}\right)$ and let $\gamma_{0}$ be its induced metric, $\boldsymbol{\eta}_{\widehat{S}_{0}}$ its volume form, $K_{0}$ its second fundamental form with respect to the outer unit normal and $\boldsymbol{K}_{0}$ the associated Weingarten map. Then the shell Penrose inequality for $S$ can be rewritten as

$$
\begin{aligned}
& \int_{\widehat{S}_{0}}\left(1+\left[\left(\boldsymbol{I} \boldsymbol{d}-\tau \boldsymbol{K}_{0}\right)^{-2}\right]_{C}^{A}\left(\gamma_{0}^{-1}\right)^{C B} \tau_{, A} \tau_{, B}\right) \operatorname{tr}\left[\boldsymbol{K}_{0} \circ\left(\boldsymbol{I} \boldsymbol{d}-\tau \boldsymbol{K}_{0}\right)^{-1}\right] \Delta[\tau] \boldsymbol{\eta}_{\widehat{S}_{0}} \geqslant \\
& \geqslant n\left(\omega_{n}\right)^{\frac{1}{n}}\left(\int_{\widehat{S}_{0}} \Delta[\tau] \boldsymbol{\eta}_{\widehat{S}_{0}}\right)^{\frac{n-1}{n}},
\end{aligned}
$$

with $\omega_{n}$ the area of the $n$-sphere, and where $\mathbf{I d}$ is the identity endomorphism, $\tau=\left.t\right|_{s}-t_{0}$ and $\Delta[\tau]:=\operatorname{det}\left(\boldsymbol{I} \boldsymbol{d}-\tau \boldsymbol{K}_{0}\right)$.

Proof. Let us start by relating the integrals of $\theta_{\ell}$ and $\theta_{k}$. Taking the trace of (3.25) with respect to $\gamma$ (and using $V_{S}=1,\langle k, \xi\rangle=-1$ ) gives

$$
\theta_{\ell}-\langle\xi, I\rangle \theta_{k}-2 \triangle_{\gamma} \tau=0
$$

where $\triangle_{\gamma}=D_{A} D^{A}$ is the Laplacian of $(S, \gamma)$. Corollary (3.3.6) gives $-\langle\xi, \ell\rangle=1+|D \tau|_{\gamma}^{2}$ and the equation above becomes

$$
\theta_{\ell}+\left(1+|D \tau|_{\gamma}^{2}\right) \theta_{k}-2 \triangle_{\gamma} \tau=0
$$

Integrating on $S$ it follows

$$
\int_{S} \theta_{\ell} \boldsymbol{\eta}_{\boldsymbol{S}}=-\int_{S}\left(1+|D \tau|_{\gamma}^{2}\right) \theta_{k} \boldsymbol{\eta}_{\boldsymbol{S}}
$$

which gives the desired relationship.

The second step is to use the Ricatti equations on $\Omega$ in order to relate $\theta_{k}$ on $S$ with the extrinsic geometry of $\widehat{S}_{0}$. To that aim, we first note that the vector field $k$ on $\Omega$ satisfies $\nabla_{k} k=0$ (this is an immediate consequence of the fact that $\xi$ is covariantly constant and $\langle\xi, k\rangle=-1$ ). Thus, the Ricatti equations on $\Omega$ take the form (4.1) provided we have selected $n$ vector fields $\left\{X_{A}\right\}$ tangent to $\Omega$ and satisfying the requirements that (i) $\left[k, X_{A}\right]=0$ and (ii) $\left\{\left.k\right|_{p},\left.X_{A}\right|_{p}\right\}$ is a basis of $T_{p} \Omega, \forall p \in \Omega$ (more precisely $\left\{k, X_{A}\right\}$ is a basis of the tangent space of $\Omega$ on suitable open subsets, however this abuse of notation is standard and poses no complications). Without loss of generality we take $\left\{X_{A}\right\}$ tangent to $S$. Equations (4.1) still admit the freedom of choosing the initial value of the affine parameter $\sigma$ on each one of the null geodesics ruling $\Omega$. It turns out to be convenient to select $\sigma$ so that $\sigma=0$ on $\widehat{S}_{0}$. This determines $\sigma$ uniquely as a smooth function $\sigma: \Omega \rightarrow \mathbb{R}$ which assigns to each point $p \in \Omega$, the value of the affine parameter of the geodesic starting on $\widehat{S}_{0}$, with tangent vector $k$ and passing through p. Given that

$$
k(t)=\boldsymbol{d} t(k)=-\langle\xi, k\rangle=1
$$




\section{Projection along the past null cone $\Omega$ in Minkowski}

and $\left.t\right|_{\widehat{S}_{0}}=t_{0}$, it follows that $\sigma=\left.t\right|_{\Omega}-t_{0}$. In particular $\left.\sigma\right|_{S}=\tau$ (this is the main reason why this choice of the origin of the affine parameter $\sigma$ is convenient).

A crucial property of the geometry of a null hypersurface $\Omega$ (see Appendix $B$ ) is that, given any point $p \in \Omega$ and any embedded spacelike surface $S_{p}$ in $\Omega$ passing through $p$, the induced metric $\gamma_{S_{p}}$ of $S_{p}$ and the second fundamental form $K_{S_{p}}^{k}$ of $S_{p}$ along the null normal $\left.k\right|_{p}$ satisfy $\gamma_{S_{p}}(X, Y)=\gamma^{\Omega}(\bar{X}, \bar{Y})$ and $K_{S_{p}}^{k}(X, Y)=K^{\Omega}(\bar{X}, \bar{Y})$, where $X, Y \in T_{p} S_{p}$. In other words, the induced metric and the extrinsic geometry along $k$ of any embedded spacelike surface in $\Omega$ depends only on $p$ and not on the details of how $S_{p}$ is embedded in $\Omega$. Applying this result on $\widehat{S}_{0}$ we have, for any point $\hat{p} \in \widehat{S}_{0}$,

$$
\left.K^{\Omega}\left(\bar{X}_{A}, \bar{X}_{B}\right)\right|_{\hat{p}}=\left.K_{\hat{S}_{0}}^{k}\left(\hat{X}_{A}, \hat{X}_{B}\right)\right|_{\hat{p}}
$$

where $\left.\hat{X}_{A}\right|_{\hat{p}}$ is defined by the properties (i) $\left.\left.\hat{X}_{A}\right|_{\hat{p}} \in \bar{X}_{A}\right|_{\hat{p}}$ and (ii) $\left.\hat{X}_{A}\right|_{\hat{p}}$ is tangent to $\widehat{S}_{0}$ at $\hat{p}$ (it is immediate that these two properties define a unique $\hat{X}_{A}$ ). Now, the Jordan-Brouwer separation theorem (see e.g. [63]) states that any connected, closed hypersurface of Euclidean space separates $\mathbb{R}^{n}$ in two subsets, one with compact closure (called interior) and one with non-compact closure (called exterior). Let $\nu$ be the unit normal of $\widehat{S}_{0}$ pointing towards the exterior, and denote by $K_{0}$ the corresponding second fundamental form and by $K_{0}$ the associated Weingarten map. Let $\left(K_{0}\right)_{A B}$ be the components of $K_{0}$ in the basis $\left\{\hat{X}_{A}\right\}$. Since $\Sigma_{t_{0}}$ is totally geodesic and $\left.\langle k, \nu\rangle\right|_{\widehat{S}_{0}}=$ -1 (which follows from the fact that $k$ is ingoing, future directed, null and satisfies $\langle k, \xi\rangle=-1)$, we have

$$
\left.K_{\widehat{S}_{0}}^{k}\left(\hat{X}_{A}, \hat{X}_{B}\right)\right|_{\hat{p}}=-\left.\left(K_{0}\right)_{A B}\right|_{\hat{p}} .
$$

Expressions (4.5) and (4.6) provides us with initial data $\left.K_{A B}^{\Omega}\right|_{\sigma=0}=-\left(K_{0}\right)_{A B}$ for the Ricatti equation (4.1), which in the Minkowski spacetime simplifies to

$$
\begin{aligned}
& \frac{d\left(K^{\Omega}\right)_{B}^{A}}{d \sigma}=-\left(K^{\Omega}\right)_{C}^{A}\left(K^{\Omega}\right)_{B}^{C} \\
& \frac{d\left(\gamma^{\Omega}\right)_{A B}}{d \sigma}=2\left(K^{\Omega}\right)_{A B} .
\end{aligned}
$$

It is a matter of straightforward computation to check that the solution to these equations with initial data $\left.K_{A B}^{\Omega}\right|_{\sigma=0}=-\left(K_{0}\right)_{A B}$ is

$$
\begin{aligned}
& \left.\left(K^{\Omega}\right)_{B}^{A}\right|_{p}=-\left.\left(K_{0}\right)_{C}^{A}\right|_{\pi(p)}\left[\left(\boldsymbol{I} \boldsymbol{d}-\left.\sigma(p) \boldsymbol{K}_{0}\right|_{\pi(p)}\right)^{-1}\right]_{B}^{C}, \\
& \left.\left(\gamma^{\Omega}\right)_{A B}\right|_{p}=\left.\left(\gamma_{0}\right)_{A C}\right|_{\pi(p)}\left[\left(\boldsymbol{I} \boldsymbol{d}-\left.\sigma(p) \boldsymbol{K}_{0}\right|_{\pi(p)}\right)^{2}\right]_{B}^{C},
\end{aligned}
$$

where $\pi(p)$ is defined as the unique point on $\widehat{S}_{0}$ lying on the null geodesic $\alpha_{p}$. Now, the null expansion $\theta_{k}$ is related to $K^{\Omega}$ by

$$
\theta_{k}=\operatorname{tr}_{\gamma} K^{k}=\gamma^{A B}\left\langle\nabla_{X_{A}} k, X_{B}\right\rangle=\gamma^{\Omega}\left(\overline{\nabla_{X_{A}} k}, \overline{X_{B}}\right)=\left(\gamma^{\Omega}\right)^{A B} K_{A B}^{\Omega}=\left(K^{\Omega}\right)_{A}^{A} .
$$




\subsection{Shell Penrose inequality in $\mathcal{M}^{1, n+1}$ in terms of Euclidean geometry}

Evaluating (4.9) on $S$ (i.e. on $\sigma=\tau$ ) and taking the trace we find $\left.\theta_{k}\right|_{p}=-\left(K_{0}\right)^{A}{ }_{C}[(\boldsymbol{I} \boldsymbol{d}-$ $\left.\left.\tau K_{0}\right)^{-1}\right]\left._{A}^{C}\right|_{\pi(p)}$, or equivalently

$$
\theta_{k} \circ \phi^{-1}=-\operatorname{tr}\left[K_{0} \circ\left(\boldsymbol{I}-\tau \boldsymbol{K}_{0}\right)^{-1}\right]
$$

where $\phi:=\left.\pi\right|_{S}$ is the diffeomorphism between $S$ and $\widehat{S}_{0}$ introduced above. As we did in the previous chapter and in order to simplify the notation, we will from now on suppress all references to $\phi$ when transferring information from $S$ to $\widehat{S}_{0}$ via this diffeomorphism.

The remaining steps are to relate the volume forms of $S$ and $\widehat{S}_{0}$ and to determine $|D \tau|_{\gamma}^{2}$ (which appears in (4.4)). Both involve the metric $\gamma$ on S. Evaluating (4.10) on $S$ and using $\gamma_{A B}=\gamma_{A B}^{\Omega}$ it follows

$$
\gamma_{A B}=\left(\gamma_{0}\right)_{A C}\left[\left(\boldsymbol{I d}-\tau \boldsymbol{K}_{0}\right)^{2}\right]_{B}^{C}
$$

By construction, $\gamma_{A B}$ is positive definite, and hence invertible. Obviously this places restrictions on the range of variation of $\tau$ (which clearly come from the fact that $\Omega$ cannot be extended arbitrarily to the future as a smooth hypersurface). The precise range of variation of $\tau$ will be discussed below. Since $\gamma_{0}$ is positive definite, it follows that $\boldsymbol{I} \boldsymbol{d}-\tau \boldsymbol{K}_{0}$ is also invertible and

$$
\left(\gamma^{-1}\right)^{A B}=\left[\left(\boldsymbol{I} \boldsymbol{d}-\tau \boldsymbol{K}_{0}\right)^{-2}\right]_{C}^{A}\left(\gamma_{0}^{-1}\right)^{C B},
$$

which implies, in particular,

$$
|D \tau|_{\gamma}^{2}=\left[\left(\boldsymbol{I} \boldsymbol{d}-\tau \boldsymbol{K}_{0}\right)^{-2}\right]_{C}^{A}\left(\gamma_{0}^{-1}\right)^{C B} \tau_{, A} \tau_{, B}
$$

Taking determinants in (4.12) it follows that the volume forms of $S$ and $\widehat{S}_{0}$ are related by

$$
\boldsymbol{\eta}_{\boldsymbol{S}}=\Delta[\tau] \boldsymbol{\eta}_{\widehat{S}_{0}}
$$

where $\Delta[\tau]:=\operatorname{det}\left(\boldsymbol{I} \boldsymbol{d}-\tau \boldsymbol{K}_{0}\right)$. Inserting (4.11), (4.14) and (4.15) into (4.4) we find

$\int_{S} \theta_{\ell} \boldsymbol{\eta}_{\boldsymbol{S}}=\int_{\widehat{S}_{0}}\left(1+\left[\left(\boldsymbol{I} \boldsymbol{d}-\tau \boldsymbol{K}_{0}\right)^{-2}\right]_{C}^{A}\left(\gamma_{0}^{-1}\right)^{C B} \tau_{, A} \tau_{, B}\right) \operatorname{tr}\left[\boldsymbol{K}_{0} \circ\left(\boldsymbol{I} \boldsymbol{d}-\tau \boldsymbol{K}_{0}\right)^{-1}\right] \Delta[\tau] \boldsymbol{\eta}_{\widehat{\boldsymbol{S}}_{0}}$,

and the Penrose inequality (1.4) becomes (4.2), as claimed.

A natural question for Theorem 4.3.5 is what is the class of functions $\tau: \widehat{S}_{0} \rightarrow \mathbb{R}$ for which inequality (4.2) is conjectured. By construction, this amounts to knowing which is the range of variation of $\sigma$ in $\Omega$. Let $\left\{\kappa_{1}, \cdots, \kappa_{n}\right\}$ be the eigenvalues of $\boldsymbol{K}_{0}$, i.e. the principal curvatures of $\widehat{S}_{0}$ as a hypersurface in Euclidean space. $\widehat{S}_{0}$ being convex, all these curvatures are non-negative, and at least one of them is different from zero (because $\widehat{S_{0}}$ is closed). The eigenvalues of the endomorphism $I \boldsymbol{d}-\tau \boldsymbol{K}_{0}$ are obviously 


\section{Projection along the past null cone $\Omega$ in Minkowski}

$\left\{1-\tau \kappa_{1}, \cdots, 1-\tau \kappa_{n}\right\}$. Hence, this endomorphism is invertible as long as $\tau$ satisfies the bound

$$
\tau<\frac{1}{\max _{1 \leq A \leq n}\left\{\kappa_{A}\right\}} .
$$

Thus, the Penrose inequality, as written in inequality (4.2), is conjectured to hold for arbitrary smooth functions $\tau: \widehat{S}_{0} \rightarrow \mathbb{R}$ satisfying the pointwise bound (4.16). Incidentally, this statement also means that the range of variation of $\sigma$ on the null geodesic within $\Omega$ passing through $\hat{p} \in \widehat{S}_{0}$ is $\sigma \in\left(-\infty, \frac{1}{\max \left\{\kappa_{A} \mid \hat{p}\right\}}\right)$.

\subsection{The shell Penrose inequality in Minkowski in terms of the support function}

A remarkable property of convex hypersurfaces embedded in Euclidean space is that a single function determines all of its geometric properties, both intrinsic and extrinsic, in a very neat way. This function is called support function, and we have devoted Appendix [C to present its main properties. In the previous section we wrote the shell Penrose inequality in terms of the geometry of convex euclidean surfaces. The next step is to use the support function of this convex surfaces to obtain an alternative form of the shell Penrose inequality. This expression is interesting because it takes the form of an inequality for functions defined on the standard sphere.

The support function is defined as follows:

Definition 4.4.1 (Support function). Let $\widehat{S}_{0}$ be a closed, convex and connected hypersurface embedded in the Euclidean space $\left(\mathbb{R}^{n+1}, g_{E}\right)$. Let $x(p)$ be the position vector of $p \in \widehat{S}_{0}$. The support function $h: \widehat{S}_{0} \rightarrow \mathbb{R}$ is defined by $h(p)=\langle x(p), \nu(p)\rangle_{g_{E}}$ where $\nu(p)$ is the unit normal at $p$ pointing towards the exterior of $\widehat{S}_{0}$.

Closed, convex and connected hypersurfaces in $\left(\mathbb{R}^{n+1}, g_{E}\right)$ are always topologically $\mathbb{S}^{n}$. Moreover, if the surface is strictly convex the Gauss map $\nu: \widehat{S}_{0} \rightarrow \mathbb{S}^{n}$ is a diffeomorphism. We will restrict ourselves to the strictly convex case from now on. This entails no loss of generality for the Penrose inequality because any convex surface $\widehat{S_{0}}$ can be approximated by strictly convex surfaces (e.g. by mean curvature flow [51]). Let us denote by $\stackrel{\circ}{q}$ the pull-back on $\widehat{S}_{0}$ of the standard metric on the $n$-sphere and $\stackrel{\circ}{\nabla}$ the corresponding connection. Then, the induced metric $\gamma_{0}$ and second fundamental form $K_{0}$ of $\widehat{S}_{0} \hookrightarrow \mathbb{R}^{n+1}$ can be written in terms of the support function as follows (see formulas (C.19) and (C.20) in Appendix C):

$$
\begin{aligned}
\left(K_{0}\right)_{A B} & =\stackrel{\circ}{\nabla}_{A} \stackrel{\circ}{\nabla}_{B} h+\stackrel{\circ}{q}_{A B} h \\
\left(\gamma_{0}\right)_{A B} & =\left(\stackrel{\circ}{q}^{-1}\right)^{C D}\left(K_{0}\right)_{A C}\left(K_{0}\right)_{B D}
\end{aligned}
$$




\subsection{The shell Penrose inequality in Minkowski and the support function}

Combining these expressions with Theorem 4.3.5 it becomes possible to rewrite the shell Penrose inequality as an inequality on the sphere involving two smooth functions, namely $\tau$ and $h$. In this section we obtain the explicit form of this inequality. To that aim, it is convenient to introduce the endomorphism $B$ obtained by raising one index to $K_{0}$ with the spherical metric $\stackrel{q}{q}$, i.e. $B_{B}^{A}:=\left(\stackrel{q}{q}^{-1}\right)^{A C}\left(K_{0}\right)_{C B}$. It is immediate from (4.17) that $B$ is the inverse endomorphism of the Weingarten map $K_{0}$. Since $\widehat{S}_{0}$ is diffeomorphic to $\mathbb{S}^{n}$ via the Gauss map we can identify both manifolds and we can think of $\stackrel{q}{q}, h, B$ etc. as objects defined on $\mathbb{S}^{n}$. This applies in particular to the function $\tau: \widehat{S}_{0} \rightarrow \mathbb{R}$. With this notation, we can now state and prove the following theorem, which gives the shell Penrose inequality in terms of the support function.

Theorem 4.4.2 (Shell Penrose inequality in terms of the support function). Let $(S, \gamma)$ be a spacetime strictly convex surface in $\left(\mathcal{M}^{1, n+1}, \eta\right)$. With the same notation as in Theorem 4.3.5, let $h$ be the support function of $\widehat{S}_{0}$. Then the shell Penrose inequality takes the form

$$
\begin{aligned}
& \int_{\mathbb{S}^{n}}\left(1+\left[(\boldsymbol{B}-\tau \boldsymbol{l} \boldsymbol{d})^{-2}\right]_{C}^{A}\left(\stackrel{q}{q}^{-1}\right)^{C B} \tau_{, A} \tau_{, B}\right) \operatorname{tr}\left[(\boldsymbol{B}-\tau \boldsymbol{l} \boldsymbol{d})^{-1}\right] \operatorname{det}(\boldsymbol{B}-\tau \boldsymbol{l} \boldsymbol{d}) \boldsymbol{\eta}_{\dot{q}} \geqslant \\
& \geqslant n\left(\omega_{n}\right)^{\frac{1}{n}}\left(\int_{\mathbb{S}^{n}} \operatorname{det}(\boldsymbol{B}-\tau \boldsymbol{l} \boldsymbol{d}) \boldsymbol{\eta}_{\dot{q}}\right)^{\frac{n-1}{n}},
\end{aligned}
$$

where $\stackrel{q}{q}, \stackrel{\circ}{\nabla}, \boldsymbol{\eta}_{\mathfrak{q}}$ are the standard metric, connection and volume form on $\mathbb{S}^{n}$,

$$
B_{B}^{A}:=\left(\stackrel{\circ}{q}^{-1}\right)^{A C} \stackrel{\circ}{\nabla}_{C} \stackrel{\circ}{\nabla}_{B} h+\delta_{B}^{A} h,
$$

$h: \mathbb{S}^{n} \rightarrow \mathbb{R}$ is the support function of $\widehat{S}_{0} \hookrightarrow \mathbb{R}^{n+1}$ and $\tau: \mathbb{S}^{n} \rightarrow \mathbb{R}$ is the time height function of $S$.

Proof. From (4.17) it follows that $B$ determines the metric $\gamma_{0}$ via

$$
\left(\gamma_{0}\right)_{A B}=B_{A}^{C} B_{B}^{D} \stackrel{\circ}{q}_{C D}
$$

which implies

$$
\boldsymbol{\eta}_{\widehat{S}_{0}}=\operatorname{det}(\boldsymbol{B}) \boldsymbol{\eta}_{\dot{q}}
$$

Since $B$ is the inverse of $K_{0}$, we have

$$
\Delta[\tau] \boldsymbol{\eta}_{\widehat{S}_{0}}=\operatorname{det}\left(\boldsymbol{I} \boldsymbol{d}-\tau \boldsymbol{K}_{0}\right) \boldsymbol{\eta}_{\widehat{\boldsymbol{S}}_{0}}=\operatorname{det}\left(\boldsymbol{I} \boldsymbol{d}-\tau \boldsymbol{K}_{0}\right) \operatorname{det}(\boldsymbol{B}) \boldsymbol{\eta}_{\mathfrak{q}}=\operatorname{det}(\boldsymbol{B}-\tau \boldsymbol{I} \boldsymbol{d}) \boldsymbol{\eta}_{\dot{q}}
$$

Similarly,

$$
\operatorname{tr}\left[\boldsymbol{K}_{0} \circ\left(\boldsymbol{I} \boldsymbol{d}-\tau \boldsymbol{K}_{0}\right)^{-1}\right]=\operatorname{tr}\left[\boldsymbol{B}^{-1} \circ\left(\boldsymbol{I} \boldsymbol{d}-\tau \boldsymbol{B}^{-1}\right)^{-1}\right]=\operatorname{tr}\left[(\boldsymbol{B}-\tau \boldsymbol{I} \boldsymbol{d})^{-1}\right] .
$$




\section{Projection along the past null cone $\Omega$ in Minkowski}

It only remains to calculate $\left[\left(\boldsymbol{I d}-\tau \boldsymbol{K}_{0}\right)^{-2}\right]_{C}^{A}\left(\gamma_{0}^{-1}\right)^{C B}$. From (4.20) and using again the fact that $B$ is the inverse of $K_{0}$ we get

$$
\begin{aligned}
& {\left[\left(\boldsymbol{I} \boldsymbol{d}-\tau \boldsymbol{K}_{0}\right)^{-2}\right]_{C}^{A}\left(\gamma_{0}^{-1}\right)^{C B}=\left[\left(\boldsymbol{I} \boldsymbol{d}-\tau \boldsymbol{K}_{0}\right)^{-2}\right]_{C}^{A}\left(K_{0}\right)^{C}{ }_{D}\left(K_{0}\right)^{B}{ }_{F}\left(\stackrel{q}{ }^{-1}\right)^{D F}=} \\
& =\left[\left(\boldsymbol{I} \boldsymbol{d}-\tau \boldsymbol{K}_{0}\right)^{-2}\right]_{C}^{A}\left(K_{0}\right)_{D}^{C}\left(K_{0}\right)_{F}^{D}\left(\stackrel{q}{ }^{-1}\right)^{B F}
\end{aligned}
$$

where in the last equality we made use of the property that $\left(K_{0}\right)_{F}{ }_{F}\left(\stackrel{q}{q}^{-1}\right)^{D F}$ is symmetric (this follows from (4.17), which states in particular that this tensor is the inverse of the symmetric two-covariant tensor $\left.\left(K_{0}\right)_{B D}\right)$. Since $\left(\boldsymbol{I} \boldsymbol{d}-\tau \boldsymbol{K}_{0}\right)^{-1} \circ \boldsymbol{K}_{0}=(\boldsymbol{B}-\tau \boldsymbol{I} \boldsymbol{d})^{-1}$ it follows

$$
\begin{aligned}
{\left[\left(\boldsymbol{I} \boldsymbol{d}-\tau \boldsymbol{K}_{0}\right)^{-2}\right] \circ \boldsymbol{K}_{0} \circ \boldsymbol{K}_{0} } & =\left[\left(\boldsymbol{I} \boldsymbol{d}-\tau \boldsymbol{K}_{0}\right)^{-1}\right] \circ(\boldsymbol{B}-\tau \boldsymbol{I} \boldsymbol{d})^{-1} \circ \boldsymbol{K}_{0} \\
& =(\boldsymbol{B}-\tau \boldsymbol{I} \boldsymbol{d})^{-2}
\end{aligned}
$$

where in the second equality we have used the fact that $\left(\boldsymbol{I} \boldsymbol{d}-\tau \boldsymbol{K}_{0}\right)$ and $(\boldsymbol{B}-\tau \boldsymbol{I} \boldsymbol{d})$ commute. Using (4.24) in (4.23) yields

$$
\left[\left(\boldsymbol{I} \boldsymbol{d}-\tau \boldsymbol{K}_{0}\right)^{-2}\right]_{C}^{A}\left(\gamma_{0}^{-1}\right)^{C B}=\left[(B-\tau \boldsymbol{I} \boldsymbol{d})^{-2}\right]_{C}^{A}\left(\stackrel{\circ}{q}^{-1}\right)^{C B} .
$$

Substituting (4.21), (4.22) and (4.25) into inequality (4.2) proves the theorem.

In the following section we discuss the validity of the shell Penrose inequality when the incoming shell has spherical shape. Following [103] we refer to this situation as the "spherical case" (note however that the incoming shell need not carry a spherically symmetric matter distribution). In other words, we consider the case when the null hypersurface $\Omega$ is the past null cone of a point in Minkowski spacetime and $S$ is any surface embedded in $\Omega$. The explicit form of this inequality in spacetime dimension four appeared already in [85] and led to an inequality for positive functions on the sphere. This inequality turned out to be highly non-trivial. Tod in [101] was able to prove the inequality by using suitable functions on $\mathbb{R}^{4}$ and using the Sobolev inequality. In this section we show that the shell Penrose inequality for shells with spherical shape holds in any spacetime dimension.

\subsubsection{Spherically symmetric case}

Let us restrict ourselves to the case when $\Omega$ is the past null cone of a point (see Figure 4.2). As a consequence of Theorem 4.4.2, the Penrose inequality transforms in this case into an inequality for a single positive function on the sphere. Its validity will follow as a simple consequence of the Beckner inequality [7] which bounds from above the $L^{q}$ norm of a function on the sphere in terms of its $H^{2}$ norm. Specifically, 
Theorem 4.4.3 (Beckner, 1993). Let $F \in C^{1}\left(\mathbb{S}^{n}\right)$ and denote as before the standard metric, volume form and connection of the $n$-dimensional unit sphere by $\stackrel{q}{,} \boldsymbol{\eta}_{\mathfrak{q}}, \stackrel{\circ}{\nabla}$. Then

$$
\frac{q-2}{n} \int_{\mathbb{S}^{n}}|\stackrel{\circ}{\nabla} F|_{\dot{q}}^{2} \boldsymbol{\eta}_{\dot{q}}+\int_{\mathbb{S}^{n}}|F|^{2} \boldsymbol{\eta}_{\dot{q}} \geq\left(\omega_{n}\right)^{1-\frac{2}{q}}\left(\int_{\mathbb{S}^{n}}|F|^{q} \boldsymbol{\eta}_{\dot{q}}\right)^{\frac{2}{q}},
$$

where $2 \leq q<\infty$ if $n=1$ or $n=2$ and $2 \leq q \leq \frac{2 n}{n-2}$ if $n \geq 3$

The following theorem [74] settles the inequality when $\Omega$ is the past null cone of a point:

Theorem 4.4.4 (Shell Penrose inequality on a past null cone of a point). Consider a point $p \in \mathcal{M}^{1, n+1}(n \geqslant 2)$ and $\Omega_{p}$ the past null cone of $p$. Let $S$ be a closed spacelike surface embedded in $\Omega_{p}$. Then the Penrose inequality for $S$ reads

$$
\int_{\mathbb{S}^{n}}\left(r^{n-1}+r^{n-3}|\stackrel{\circ}{\nabla} r|_{\stackrel{q}{q}}^{2}\right) \boldsymbol{\eta}_{\dot{q}} \geq\left(\omega_{n}\right)^{\frac{1}{n}}\left(\int_{\mathbb{S}^{n}} r^{n} \boldsymbol{\eta}_{\dot{q}}\right)^{\frac{n-1}{n}}
$$

where $r=t(p)-\left.t\right|_{s}$. Moreover, this inequality holds true as a consequence of Beckner's theorem.

Proof. Select $t_{0}=t(p)-1$. Then, the function $\tau$ is written in terms of $r$ as $\tau=$ $\left.t\right|_{S}-t_{0}=\left.t\right|_{S}-t(p)+1=1-r$ and $\widehat{S}_{0}=\Omega_{p} \cap \Sigma_{t_{0}}$ is the $n$-dimensional unit sphere embedded in the Euclidean space. This surface has support function $h=1$, which implies $\left(K_{0}\right)_{A B}=\stackrel{\circ}{\nabla}_{A} \stackrel{\circ}{\nabla}_{B} h+\stackrel{\circ}{q}_{A B} h=\stackrel{\circ}{q}_{A B}$ (this simply states the well-known property that the unit sphere has all principal curvatures equal to one). Then $B_{B}^{A}=$ $\left(\stackrel{\circ}{q}^{-1}\right)^{A C}\left(K_{0}\right)_{C B}=\delta_{B}^{A}$ and $(B-\tau \boldsymbol{I} \boldsymbol{d})=(1-\tau) \boldsymbol{I} \boldsymbol{d}=r \boldsymbol{I d}$, from which

$$
\begin{aligned}
1+\left[(\mathbf{B}-\tau \boldsymbol{l} \boldsymbol{d})^{-2}\right]_{C}^{A}\left(\stackrel{\circ}{q}^{-1}\right)^{C B} \tau_{, A} \tau_{, B} & =1+\frac{1}{r^{2}}|\stackrel{\circ}{\nabla} r|_{\stackrel{q}{q}}^{2} \\
\operatorname{det}(\mathbf{B}-\tau \boldsymbol{l} \boldsymbol{d}) & =r^{n}, \\
\operatorname{tr}\left[(\mathbf{B}-\tau \boldsymbol{l} \boldsymbol{d})^{-1}\right] & =\frac{n}{r} .
\end{aligned}
$$

Substituting into (4.18) yields immediately (4.27). In order to show that this inequality is a particular case of the Beckner inequality, we define $q=\frac{2 n}{n-1}$ which clearly satisfies the bounds $2 \leq q \leq \infty$ if $n=2$ and $2 \leq q \leq \frac{2 n}{n-2}$ if $n \geq 3$. Introducing the function $F=r^{\frac{n-1}{2}}$, (4.27) becomes

$$
\left(\frac{2}{n-1}\right)^{2} \int_{\mathbb{S}^{n}}|\stackrel{\circ}{\nabla} F|_{\tilde{q}}^{2} \boldsymbol{\eta}_{\tilde{q}}+\int_{\mathbb{S}^{n}} F^{2} \boldsymbol{\eta}_{\tilde{q}} \geq\left(\omega_{n}\right)^{1-\frac{2}{q}}\left(\int_{\mathbb{S}^{n}} F^{q} \boldsymbol{\eta}_{\tilde{q}}\right)^{\frac{2}{q}} .
$$

Since $n \geq 2$, then $\frac{q-2}{n}=\frac{2}{n(n-1)} \leq\left(\frac{2}{n-1}\right)^{2}$ and inequality (4.28) is a particular case of (4.26). 


\section{Projection along the past null cone $\Omega$ in Minkowski}

Remark 4.4.5. As mentioned above, the case $n=2$ of this theorem was proved by Tod in [101] using the Sobolev inequality in $\mathbb{R}^{4}$. In a later paper, Tod proved [102] that the factor $\left(\frac{2}{n-1}\right)^{2}$ (i.e. 4 when $n=2$ ) in front of the gradient in (4.28) could be improved to $8 / 3$ by using the Sobolev inequality of $\mathbb{R}^{6}$ applied to suitable functions. Tod also conjectured that this factor could be improved to the value 1 . We note that Beckner's inequality implies in particular the validity of this conjecture by Tod.

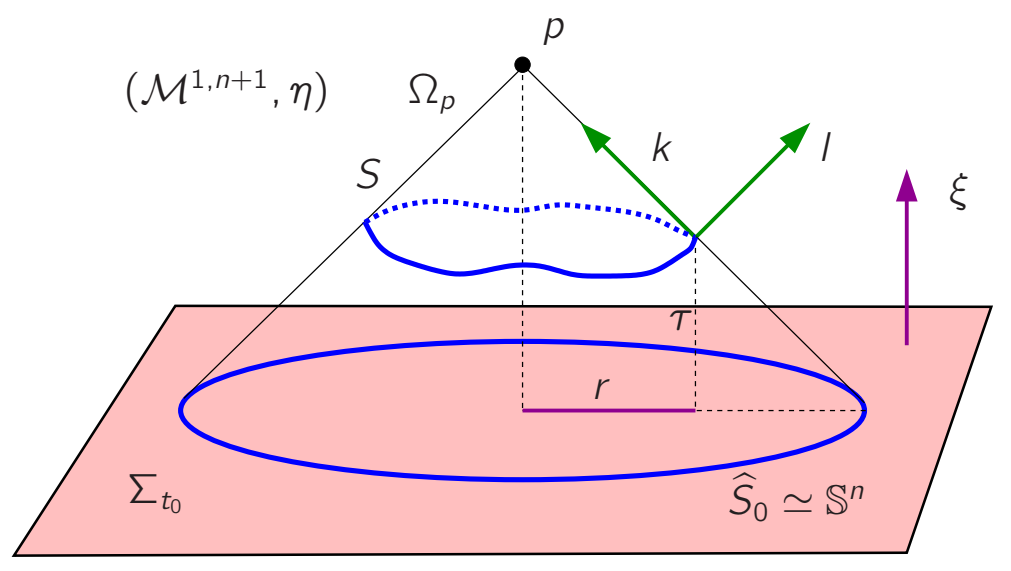

Figure 4.2: When the spacetime convex surface $S$ lies in the past null cone of a point $p \in \mathcal{M}^{1, n+1}$, its projection along $\Omega_{p}$ onto $\Sigma_{t_{0}}$ is always an $n$-sphere. The Penrose inequality transforms in this case into an inequality for a single positive function $r$.

\subsubsection{Penrose inequality in terms of the support function in space- time dimension four}

The general expression for the Penrose inequality in terms of the support function as written in Theorem 4.4 .2 involves the inverse of the endomorphism $\mathbf{B}-\tau \boldsymbol{l} \boldsymbol{d}$, where $B_{B}^{A}=\stackrel{\circ}{\nabla}^{A} \stackrel{\circ}{\nabla}_{B} h+\delta_{B}^{A} h$ (for notational simplicity in this section we will lower and raise all indices with the spherical metric $q$ and its inverse). Hence, the explicit form of the inequality in terms of the support function is rather involved. In this section we restrict ourselves to spacetime dimension four, where the expressions simplify notably. The reason is that, in this case, the endomorphism $B$ acts on a two-dimensional vector space where inverses are much simpler to calculate. In fact, we will exploit the fact that any endomorphism $\boldsymbol{A}: V_{2} \rightarrow V_{2}$ acting on a two-dimensional vector space $V_{2}$ satisfies the identity

$$
\boldsymbol{A}^{2}=\operatorname{tr}(\boldsymbol{A}) \boldsymbol{A}-\operatorname{det}(\boldsymbol{A}) \boldsymbol{I d} .
$$


This identity is a direct consequence of the expression of the minimal polynomial in terms of the eigenvalues of $\boldsymbol{A}$ and the fact that these eigenvalues can be expressed in terms of the trace and determinant of the endomorphism (alternatively, (4.29) can be proved by direct calculation in any basis). A simple consequence of (4.29) is that, whenever $\boldsymbol{A}$ is invertible

$$
\boldsymbol{A}^{-1}=-\frac{1}{\operatorname{det}(\boldsymbol{A})} \boldsymbol{A}+\frac{\operatorname{tr}(\boldsymbol{A})}{\operatorname{det}(\boldsymbol{A})} \boldsymbol{I} .
$$

Taking traces in (4.29) and (4.30) yields, respectively,

$$
\begin{aligned}
\operatorname{det}(\boldsymbol{A}) & =\frac{1}{2}\left[\operatorname{tr}(\boldsymbol{A})^{2}-\operatorname{tr}\left(\boldsymbol{A}^{2}\right)\right] \\
\operatorname{tr}\left(\boldsymbol{A}^{-1}\right) & =\frac{\operatorname{tr}(\boldsymbol{A})}{\operatorname{det}(\boldsymbol{A})} .
\end{aligned}
$$

Squaring (4.30) and using (4.29) and (4.31) we get an expression for $\boldsymbol{A}^{-2}$ which reads:

$$
\boldsymbol{A}^{-2}=-\frac{\operatorname{tr}(\boldsymbol{A})}{[\operatorname{det}(\boldsymbol{A})]^{2}} \boldsymbol{A}+\frac{\left[\operatorname{tr}(\boldsymbol{A})^{2}+\operatorname{tr}\left(\boldsymbol{A}^{2}\right)\right]}{2[\operatorname{det}(\boldsymbol{A})]^{2}} \boldsymbol{I} \boldsymbol{d} .
$$

Of particular interest below is the case when $A$ is of the form $\boldsymbol{A}=\boldsymbol{A}_{0}+f \boldsymbol{I} \boldsymbol{d}$ for some scalar $f$. Inserting this respectively into (4.31) and (4.33) gives, after a straightforward calculation,

$$
\begin{aligned}
\operatorname{det}\left(\boldsymbol{A}_{0}+f \boldsymbol{I} \boldsymbol{d}\right)= & \frac{1}{2}\left[\operatorname{tr}\left(\boldsymbol{A}_{0}\right)^{2}-\operatorname{tr}\left(\boldsymbol{A}_{0}^{2}\right)\right]+f \operatorname{tr}\left(\boldsymbol{A}_{0}\right)+f^{2}, \\
\left(\boldsymbol{A}_{0}+f \boldsymbol{I} \boldsymbol{d}\right)^{-2}= & -\underbrace{\frac{\operatorname{tr}\left(\boldsymbol{A}_{0}\right)+2 f}{\left[\operatorname{det}\left(\boldsymbol{A}_{0}+f \boldsymbol{I}\right)\right]^{2}}}_{:=W_{2}\left(\boldsymbol{A}_{0}, f\right)} \boldsymbol{A}_{0} \\
& +\underbrace{\frac{\frac{1}{2}\left[\operatorname{tr}\left(\boldsymbol{A}_{0}\right)^{2}+\operatorname{tr}\left(\boldsymbol{A}_{0}^{2}\right)\right]+2 f \operatorname{tr}\left(\boldsymbol{A}_{0}\right)+f^{2}}{\left[\operatorname{det}\left(\boldsymbol{A}_{0}+f \boldsymbol{I}\right)\right]^{2}}}_{:=W_{1}\left(\boldsymbol{A}_{0}, f\right)} \boldsymbol{I d} .
\end{aligned}
$$

Having noticed these algebraic identities, we can now write down the specific form of the shell Penrose inequality in terms of the support function in the case of four spacetime dimensions [74].

Theorem 4.4.6. Let $(S, \gamma)$ be a spacetime strictly convex surface in the Minkowski spacetime $\left(\mathcal{M}^{1,3}, \eta\right)$. With the same notation as in Theorem 4.4.2, the Penrose inequality can be written in the form

$$
\begin{aligned}
& \int_{\mathbb{S}^{2}}\left(1+W_{1}|\stackrel{\circ}{\nabla}|_{\stackrel{q}{q}}^{2}-W_{2}\left(\stackrel{\circ}{\nabla}^{A} \stackrel{\circ}{\nabla}^{B} h\right) \stackrel{\circ}{\nabla}_{A} \tau \stackrel{\circ}{\nabla}_{B} \tau\right)\left(\triangle_{\dot{q}} h+2(h-\tau)\right) \boldsymbol{\eta}_{\mathfrak{q}} \geqslant \\
& \geqslant \sqrt{16 \pi \int_{\mathbb{S}^{2}}\left((h-\tau)^{2}+\left(\triangle_{\dot{q}} h\right)(h-\tau)-\frac{1}{2}\left(h \triangle_{\dot{q}} h\right)\right) \boldsymbol{\eta}_{\dot{q}}}
\end{aligned}
$$




\section{Projection along the past null cone $\Omega$ in Minkowski}

where $\triangle_{\dot{q}}$ is the Laplacian of the unit 2-sphere and

$$
\begin{aligned}
& W_{1}:=\frac{(h-\tau)^{2}+2(h-\tau) \triangle_{\mathfrak{q}} h+\frac{1}{2}\left[\left(\triangle_{\mathfrak{q}} h\right)^{2}+\left(\stackrel{\circ}{\nabla}_{C} \stackrel{\circ}{\nabla}_{D} h\right)\left(\stackrel{\circ}{ }^{C} \stackrel{\circ}{\nabla}^{D} h\right)\right]}{\left[(h-\tau)^{2}+(h-\tau) \triangle_{\mathfrak{q}} h+\frac{1}{2}\left[\left(\triangle_{\mathfrak{q}} h\right)^{2}-\left(\stackrel{\circ}{\nabla}_{C} \stackrel{\circ}{\nabla}_{D} h\right)\left(\stackrel{\circ}{\nabla}^{C} \stackrel{\circ}{\nabla}^{D} h\right)\right]\right]^{2}}, \\
& W_{2}:=\frac{\triangle_{q} h+2(h-\tau)}{\left[(h-\tau)^{2}+(h-\tau) \triangle_{q} h+\frac{1}{2}\left[\left(\triangle_{q} h\right)^{2}-\left(\dot{\circ}_{C} \dot{\circ}_{D} h\right)\left(\dot{\circ}^{C} \dot{\nabla}^{D} h\right)\right]\right]^{2}}
\end{aligned}
$$

Proof. Define the endomorphism $A_{0}{ }_{B}=\stackrel{\circ}{\nabla}^{A} \stackrel{\circ}{\nabla}_{B} h$, so that

$$
\operatorname{tr}\left(\boldsymbol{A}_{0}\right)=\triangle_{\dot{q}} h, \quad \operatorname{tr}\left(\boldsymbol{A}_{0}^{2}\right)=\left(\stackrel{\circ}{\nabla}^{A} \stackrel{\circ}{\nabla}^{B} h\right)\left(\stackrel{\circ}{\nabla}_{A} \stackrel{\circ}{\nabla}_{B} h\right), \quad \boldsymbol{B}-\tau \boldsymbol{l} \boldsymbol{d}=\boldsymbol{A}_{0}+(\underbrace{h-\tau}_{:=f}) \boldsymbol{l} \boldsymbol{d} .
$$

Applying identity (4.34) with $f=h-\tau$ gives

$$
\operatorname{det}(\boldsymbol{B}-\tau \boldsymbol{I} \boldsymbol{d})=(h-\tau)^{2}+\triangle_{\mathfrak{q}} h(h-\tau)+\frac{1}{2}\left[\left(\triangle_{\mathfrak{q}} h\right)^{2}-\left(\stackrel{\circ}{\nabla}_{C} \stackrel{\circ}{\nabla}_{D} h\right)\left(\stackrel{\circ}{\nabla}^{C} \stackrel{\circ}{\nabla}^{D} h\right)\right] .
$$

Using (4.32) we have

$$
\operatorname{tr}\left[(\boldsymbol{B}-\tau \boldsymbol{I} \boldsymbol{d})^{-1}\right] \operatorname{det}(\boldsymbol{B}-\tau \boldsymbol{I} \boldsymbol{d})=\operatorname{tr}(\boldsymbol{B}-\tau \boldsymbol{I} \boldsymbol{d})=\triangle_{\dot{q}} h+2(h-\tau) .
$$

We still need to evaluate $(B-\tau \boldsymbol{l} \boldsymbol{d})^{-2}$ from (4.35). Using the definitions of $W_{1}:=$ $W_{1}\left(A_{0}, f\right)$ and $W_{2}:=W_{2}\left(A_{0}, f\right)$, we have

$$
\begin{aligned}
W_{1}:=W_{1}\left(A_{0}, f\right) & =\frac{\frac{1}{2}\left[\operatorname{tr}\left(\boldsymbol{A}_{0}\right)^{2}+\operatorname{tr}\left(\boldsymbol{A}_{0}^{2}\right)\right]+2 f \operatorname{tr}\left(\boldsymbol{A}_{0}\right)+f^{2}}{\left[\operatorname{det}\left(\boldsymbol{A}_{0}+f \boldsymbol{I}\right)\right]^{2}} \\
& =\frac{\frac{1}{2}\left[\left(\triangle_{\mathfrak{q}} h\right)^{2}+\left(\stackrel{\circ}{\nabla}_{C} \stackrel{\circ}{\nabla}_{D} h\right)\left(\stackrel{\circ}{\nabla}^{C} \stackrel{\circ}{\nabla}^{D} h\right)\right]+2(h-\tau) \triangle_{\mathfrak{q}} h+(h-\tau)^{2}}{\left[(h-\tau)^{2}+(h-\tau) \triangle_{\dot{q}} h+\frac{1}{2}\left[\left(\triangle_{\dot{q}} h\right)^{2}-\left(\stackrel{\circ}{\nabla}_{C} \stackrel{\circ}{\nabla}_{D} h\right)\left(\stackrel{\circ}{\nabla}^{C} \stackrel{\circ}{\nabla}^{D} h\right)\right]\right]^{2}},
\end{aligned}
$$

and

$$
\begin{aligned}
W_{2}:=W_{2}\left(A_{0}, f\right) & =\frac{\operatorname{tr}\left(\boldsymbol{A}_{0}\right)+2 f}{\left[\operatorname{det}\left(\boldsymbol{A}_{0}+f \boldsymbol{I} \boldsymbol{d}\right)\right]^{2}} \\
& =\frac{\triangle_{\mathfrak{q}} h+2(h-\tau)}{\left[(h-\tau)^{2}+(h-\tau) \triangle_{q} h+\frac{1}{2}\left[\left(\triangle_{\dot{q}} h\right)^{2}-\left(\stackrel{\circ}{\nabla}_{C} \stackrel{\circ}{\nabla}_{D} h\right)\left(\stackrel{\circ}{\nabla}^{C} \stackrel{\circ}{\nabla}^{D} h\right)\right]\right]^{2}},
\end{aligned}
$$

in terms of which we can write

$$
(\boldsymbol{B}-\tau \boldsymbol{l} \boldsymbol{d})^{-2}=W_{1} \boldsymbol{l d}-W_{2} \boldsymbol{A}_{0} .
$$

Substituting (4.39), (4.40) and (4.41) into the left-hand side of inequality (4.18) gives the left-hand side of (4.36). In particular, we have obtained an explicit formula for the integral of $\theta_{\ell}$ on $S$, namely

$$
\int_{S} \theta_{\ell} \boldsymbol{\eta}_{S}=\int_{\mathbb{S}^{2}}\left(1+W_{1}|\stackrel{\circ}{\nabla} \tau|_{\grave{q}}^{2}-W_{2}\left(\stackrel{\circ}{\nabla}^{A} \stackrel{\circ}{\nabla}^{B} h\right) \stackrel{\circ}{\nabla}_{A} \tau \stackrel{\circ}{\nabla}_{B} \tau\right)\left(\triangle_{\dot{q}} h+2(h-\tau)\right) \boldsymbol{\eta}_{\dot{q}}
$$


For the right-hand side of (4.36) we need to calculate $|S|=\int_{\mathbb{S}^{2}} \operatorname{det}(\mathbf{B}-\tau \boldsymbol{l} \boldsymbol{d}) \boldsymbol{\eta}_{\mathfrak{q}}$. In particular, we need to integrate $\left(\triangle_{\mathfrak{q}} h\right)^{2}-\left(\stackrel{\circ}{\nabla}_{C} \dot{\circ}_{D} h\right)\left(\stackrel{\circ}{\nabla}^{C} \stackrel{\circ}{\nabla}^{D} h\right)$ on the sphere. We note the following identity:

$$
\begin{aligned}
& \stackrel{\circ}{\nabla}_{C}\left[\left(\stackrel{\circ}{\nabla}^{C} h\right)\left(\stackrel{\circ}{\nabla}_{D} \stackrel{\circ}{\nabla}^{D} h\right)\right]-\stackrel{\circ}{\nabla}_{C}\left[\left(\stackrel{\circ}{\nabla}^{D} h\right)\left(\stackrel{\circ}{\nabla}^{C} \stackrel{\circ}{\nabla}_{D} h\right)\right]= \\
& =\left(\triangle_{\dot{q}} h\right)^{2}-\left(\stackrel{\circ}{\nabla}_{C} \stackrel{\circ}{\nabla}_{D} h\right)\left(\stackrel{\circ}{\nabla}^{C} \stackrel{\circ}{\nabla}^{D} h\right)+\left(\stackrel{\circ}{\nabla}^{C} h\right)\left[\stackrel{\circ}{\nabla}_{C} \stackrel{\circ}{\nabla}_{D} \stackrel{\circ}{\nabla}^{D} h-\stackrel{\circ}{\nabla}_{D} \stackrel{\circ}{\nabla}^{D} \stackrel{\circ}{\nabla}_{C} h\right]= \\
& =\left(\triangle_{\dot{q}} h\right)^{2}-\left(\stackrel{\circ}{\nabla}_{C} \dot{\circ}_{D} h\right)\left(\stackrel{\circ}{\nabla}^{C} \stackrel{\circ}{\nabla}^{D} h\right)+\left(\stackrel{\circ}{\nabla}^{C} h\right)\left[\stackrel{\circ}{\nabla}_{C} \stackrel{\circ}{\nabla}_{D} \stackrel{\circ}{\nabla}^{D} h-\stackrel{\circ}{\nabla}_{D} \stackrel{\circ}{\nabla}_{C} \stackrel{\circ}{\nabla}^{D} h\right]= \\
& =\left(\triangle_{\dot{q}} h\right)^{2}-\left(\stackrel{\circ}{\nabla}_{C} \stackrel{\circ}{\nabla}_{D} h\right)\left(\stackrel{\circ}{\nabla}^{C} \stackrel{\circ}{\nabla}^{D} h\right)+\left(\stackrel{\circ}{\nabla}^{C} h\right)\left(-\stackrel{\circ}{L} L C^{\circ} \stackrel{\circ}{\nabla}^{L} h\right)= \\
& =\left(\triangle_{\dot{q}} h\right)^{2}-\left(\stackrel{\circ}{\nabla}_{C} \stackrel{\circ}{\nabla}_{D} h\right)\left(\stackrel{\circ}{\nabla}^{C} \stackrel{\circ}{\nabla}^{D} h\right)-|\stackrel{\circ}{\nabla} h|_{\stackrel{q}{q}}^{2}
\end{aligned}
$$

where in the third equality we have used the definition of the Riemann tensor and the fact that the sphere has constant curvature equal to one. Integrating (4.43) and using the fact that the left-hand side of this expression is a divergence, it follows

$$
\int_{\mathbb{S}^{2}}\left(\left(\triangle_{\dot{q}} h\right)^{2}-\left(\stackrel{\circ}{\nabla}_{C} \stackrel{\circ}{\nabla}_{D} h\right)\left(\stackrel{\circ}{\nabla}^{C} \stackrel{\circ}{\nabla}^{D} h\right)\right) \boldsymbol{\eta}_{\grave{q}}=\int_{\mathbb{S}^{2}}|\stackrel{\circ}{\nabla} h|_{\dot{q}}^{2} \boldsymbol{\eta}_{\grave{q}}=\int_{\mathbb{S}^{2}}-\left(h \triangle_{\dot{q}} h\right) \boldsymbol{\eta}_{\dot{q}}
$$

where in the last step we have integrated by parts. Summing up,

$$
|S|=\int_{\mathbb{S}^{2}}\left((h-\tau)^{2}+\left(\triangle_{\mathfrak{q}} h\right)(h-\tau)-\frac{1}{2} h \triangle_{\tilde{q}} h\right) \boldsymbol{\eta}_{\tilde{q}}
$$

which inserted into the right-hand side of (4.18) gives the right-hand side of (4.36) (recall that $\omega_{2}=4 \pi$ ).

It is well-known that when the surface $S$ lies in a hyperplane of the Minkowski spacetime, the Penrose inequality (1.4) becomes the classic Minkowski inequality for the total mean curvature $H$ of a surface in Euclidean space (this is a consequence of formula (3.53) of the previous chapter, by setting $\tau=0$ ). In the case of $3+1$ dimensions, the Minkowski inequality reads (see Figure 4.3)

$$
\int_{S} H \eta_{S} \geq \sqrt{16 \pi|S|}
$$

Using the theorem above we can obtain the explicit form of the Minkowski inequality in terms of the support function.

Corollary 4.4.7 (Minkowski inequality in $\left(\mathbb{R}^{3}, g_{E}\right)$ in terms of the support function). Let $S$ be a spacetime strictly convex surface embedded in a constant time hyperplane of the Minkowski spacetime $\left(\mathcal{M}^{1,3}, \eta\right)$. Then, the Minkowski inequality (4.45) in terms of the support function $h$ of $S$ takes the form

$$
\left(\int_{\mathbb{S}^{2}} h \boldsymbol{\eta}_{\dot{q}}\right) \geqslant \sqrt{4 \pi \int_{\mathbb{S}^{2}}\left(h^{2}+\frac{1}{2} h \triangle_{\dot{q}} h\right) \boldsymbol{\eta}_{\dot{q}}}
$$




\section{Projection along the past null cone $\Omega$ in Minkowski}

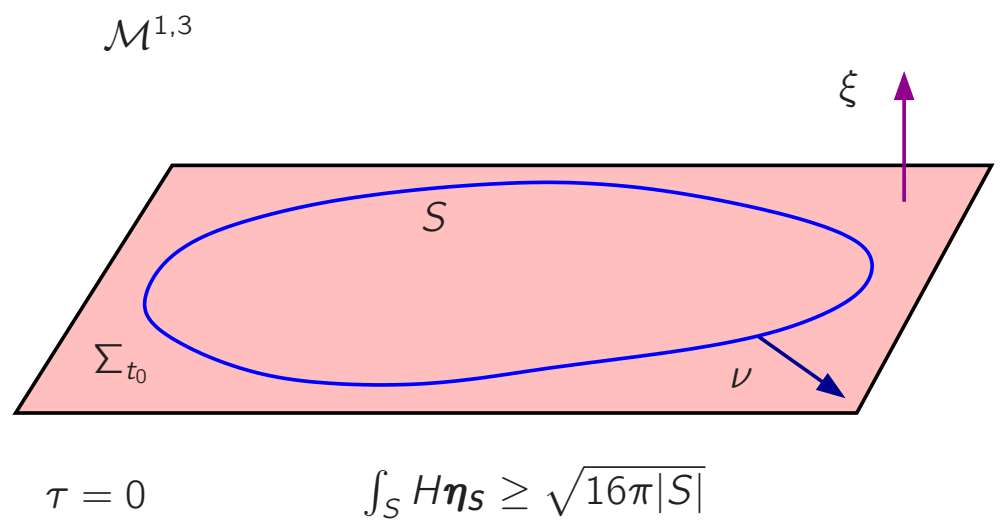

Figure 4.3: When the spacetime convex surface $S$ lies in a constant time hyperplane $\Sigma_{t_{0}}$, the surface $S$ is by definition convex. This is a particular case of the projection in Figure 3.3. where the two Killings $\xi$ and $\xi^{\prime}$ are the same and $\Sigma_{t_{0}}$ and $\Sigma_{t_{0}^{\prime}}^{\prime}$ coincide. In this situation $f=1$ and the shell Penrose inequality with respect to $\xi$ transforms into a Minkowski inequality for euclidean surfaces $\int_{S} H \eta_{S} \geq \sqrt{16 \pi|S|}$, which is known to hold in full generality (Minkowski theorem).

Proof. Without loss of generality choose $t_{0}$ as the value of $t$ on the hyperplane where $\widehat{S}_{0}$ lies. This choice implies $\tau=0$ and that $h$ is the support function of $\widehat{S}_{0}$. Since $\stackrel{\circ}{\nabla}_{A} \tau=0$, inequality (4.36) reduces to (4.46).

Inequality (4.36) in terms of the support function is still formidable. However, it is completely explicit in terms of two functions on the sphere. In the next section we prove its validity for a subset of admissible functions $\{h, \tau\}$. This subset has not-empty interior (in any reasonable topology) so the class of surfaces where the inequality is proved is rather large. The proof is inspired in the flow of surfaces put forward by Ludvigsen \& Vickers [67] in their attempt to prove the general Penrose inequality in terms of the Bondi mass. However, Bergqvist [8] found a gap in the argument and showed that the method provides a proof only under additional circumstances which are, in principle, not straightforward to control directly in terms of the initial surface. In our situation we have very explicit control of the whole flow of surfaces. This allows us, on the one hand, to find sufficient conditions for the validity of the Penrose inequality directly in terms of the geometry of the initial surface and, on the other, to prove the inequality for a much larger class than the one covered by Bergqvist's argument. In Chapter 7 we study in detail the relationship between the argument here and the proof in [8] and we conclude that the argument in this chapter admits a generalization to general spacetimes with complete past null infinity. 


\subsection{Dragging the surface along its past null cone}

\subsection{Dragging the surface along its past null cone}

In their approach to the Penrose inequality, both Ludvigsen \& Vickers [67] and Bergqvist [8] used flows of surfaces along null hypersurfaces embedded in spacetimes satisfying the dominant energy condition. These flows consist in dragging the initial surface $S$ along its outer directed past null cone along affinely parametrized null geodesics. The key property that makes these flows useful is the existence of a monotonic quantity, often called Bergqvist mass. In this section we use foliations moving along null hypersurfaces at a suitable velocity in the Minkowski spacetime, and we exploit the monotonic properties of the Bergqvist mass along such flows to address the shell Penrose inequality in Minkowski. This idea will be applied in Chapter 7 to more general spacetimes to tackle the general version of the null Penrose inequality. We start by introducing the flow and defining the Bergqvist mass in our context.

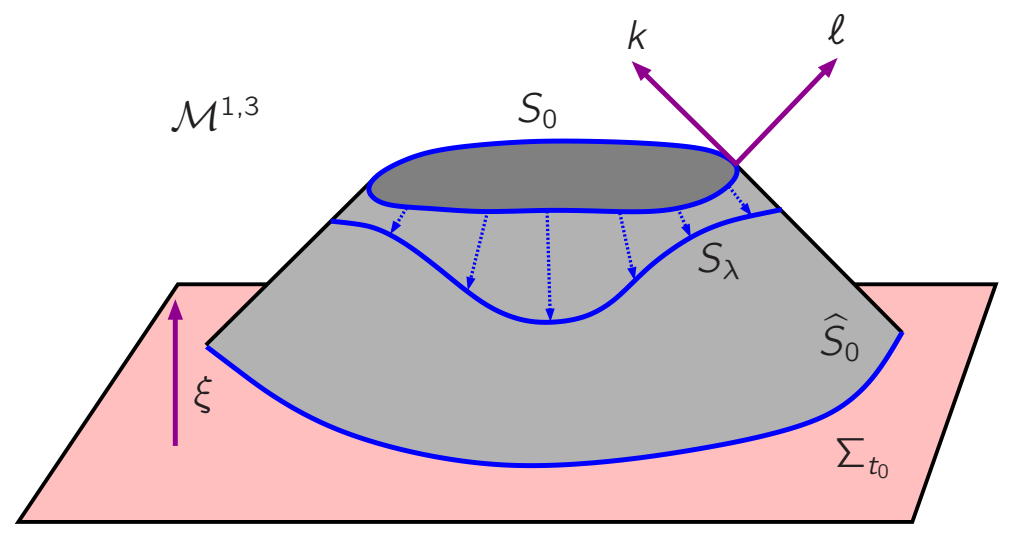

Figure 4.4: The flow of surfaces $\left\{S_{\lambda}\right\}$ moves along the null hypersurface $\Omega$ embedded in $\mathcal{M}^{1,3}$ as $\lambda$ increases. The collection $\left\{S_{\lambda}\right\}$ is generated as the level sets of the function $\lambda: \Omega \rightarrow \mathbb{R}$, which solves $k(\lambda)=-1$. The starting surface of the flow is $S=S_{0}, k$ is normalized so that $\langle k, \xi\rangle=-1$, and $\ell$ satisfies $\langle k, \ell\rangle=-2$.

We put ourselves in the setting where $S$ is a spacetime strictly convex surface in the four-dimensional Minkowski spacetime $\left(\mathcal{M}^{1,3}, \eta\right), \Omega$ is the spacetime convex null hypersurface where it sits and $\widehat{S}_{0}=\Omega \cap \Sigma_{t_{0}}$ is closed. We have introduced in Section 4.3 a smooth function $\sigma: \Omega \rightarrow \mathbb{R}$ which assigns to every point $p \in \Omega$, the affine parameter at $p$ of the null geodesic tangent to the null vector $k$ starting on $S$. By construction, $\sigma$ vanishes on $\widehat{S}_{0}$ and takes the values $\left.\sigma\right|_{S}=\tau$. Let us extend $\tau$ to a function $\tau: \Omega \rightarrow \mathbb{R}$ by imposing $k(\tau)=0$ and introduce a new smooth function $\lambda: \Omega \rightarrow \mathbb{R}$ by $\lambda=\tau-\sigma$. Geometrically, $\lambda$ is just an affine parameter along the null geodesics ruling $\Omega$ (with this parameter the tangent vector is $-k$ and the geodesics start on $S$ ). It is immediate to see that the level sets $S_{\lambda_{0}}=\left\{\lambda^{-1}\left(\lambda_{0}\right), \lambda_{0} \geq 0\right\}$ of this function define spacetime convex surfaces embedded in $\Omega$ (see Figure 4.4). The collection of $\left\{S_{\lambda}\right\}, \lambda \in[0, \infty$ ) defines a flow starting at $S=S_{0}$. Let us denote by $\gamma_{\lambda}$ and $\boldsymbol{\eta}_{S_{\lambda}}$ the induced metric and 


\section{Projection along the past null cone $\Omega$ in Minkowski}

volume form of $S_{\lambda}$ and by $\theta_{\ell}(\lambda)$ the outer null expansion of $S_{\lambda}$ (with the normalization $\langle\ell, k\rangle=-2$, as before). Then, the Bergqvist mass is defined by

$$
M_{b}(\lambda):=\frac{\lambda}{2}-\frac{1}{16 \pi} \int_{S_{\lambda}} \theta_{\ell}(\lambda) \eta_{S_{\lambda}}
$$

Bergqvist introduced this functional in [8] and proved its monotonicity by differentiating $M_{b}$ with respect to $\lambda$. We use this functional in this chapter in the Minkowski spacetime and in Chapter 7 we exploit its properties in more general backgrounds. In this chapter we give a different prove of the monotonicity of $M_{b}$ tailored to the Minkowski setting. We first need the following lemma:

Lemma 4.5.1. Let $\left(\mathcal{M}^{1,3}, \eta\right)$ be the four dimensional Minkowski spacetime. Let $S$ be a two-dimensional spacelike surface embedded in it. Consider the time translation $\boldsymbol{\xi}=-d t$, where $t$ is a Minkowskian time, and the null normals $\{k, \ell\}$ normalized so that $\langle k, \xi\rangle=-1$ and $\langle k, \ell\rangle=-2$. Then

$$
\left(s_{\ell}\right)_{A}=\tau^{, B} K_{B A}^{k},
$$

where $s_{\ell}(X)=\frac{1}{2}\left\langle\nabla_{X} k, \ell\right\rangle$, and $\tau=\left.t\right|_{s}$. In addition the following identity holds:

$$
\begin{aligned}
0= & \mathrm{Scal}^{S}+\left(K_{A B}^{k} K^{k^{A B}}-\theta_{k}^{2}\right)\left(1+|D \tau|_{\gamma}^{2}\right)+2\left(\theta_{k}\left(s_{\ell}\right)_{A} \tau^{, A}-\left(s_{\ell}\right)_{A}\left(s_{\ell}\right)^{A}\right) \\
& +2 D_{A}\left(\theta_{k} \tau^{, A}-\tau_{, B} K^{k^{B A}}\right) .
\end{aligned}
$$

Proof. To find the expression that the one-form $\left(s_{\ell}\right)_{A}$ has in Minkowski, we decompose the Killing vector $\xi$ into normal and tangential components to $S$ as in (3.26). Solving for $\ell$ and inserting into the definition of $s_{\ell}$ we have

$$
s_{\ell A}=\frac{1}{2}\left\langle\nabla_{X_{A}} k, \ell\right\rangle=\left\langle\nabla_{X_{A}} k, \xi-\frac{\left(1+|D \tau|_{\gamma}^{2}\right)}{2} k+\operatorname{grad} \tau\right\rangle=\left\langle\nabla_{X_{A}} k, \xi\right\rangle+\tau^{, B} K_{A B}^{k}
$$

Now, from $\langle k, \xi\rangle=-1$ we have $\left\langle\nabla_{X_{A}} k, \xi\right\rangle=-\left\langle k, \nabla_{X_{A}} \xi\right\rangle=0$ because $\xi$ is covariantly constant and (4.48) follows.

For the second identity, we use the Gauss identity (equation (A.30) in Appendix A ). In the Minkowski spacetime, it reads

$$
\mathrm{Scal}^{S}=-\theta_{k} \theta_{\ell}+K_{A B}^{k} K^{\ell^{A B}} .
$$

This relation can be rewritten fully in terms of the geometry of $K^{k}$ by exploiting the curvature equation (3.25), that in the present case reads

$$
K_{A B}^{\ell}+\left(1+|D \tau|_{\gamma_{\lambda}}^{2}\right) K_{A B}^{k}-2 D_{A} D_{B} \tau=0 .
$$

The result is that (4.50) becomes

$$
0=\mathrm{Scal}^{S}+\left(K_{A B}^{k} K^{k^{A B}}-\theta_{k}^{2}\right)\left(1+|D \tau|_{\gamma}^{2}\right)+2\left(\left(\triangle_{\gamma} \tau\right) \theta_{k}-\left(D_{A} D_{B} \tau\right) K^{k A B}\right) .
$$




\subsection{Dragging the surface along its past null cone}

We want to convert all second derivatives into a divergence. Specifically we use $\left(\triangle_{\gamma} \tau\right) \theta_{k}=D_{A}\left(\tau^{, A} \theta_{k}\right)-\left(\tau^{, A}\right) D_{A} \theta_{k}$ and $\left(D_{A} D_{B} \tau\right) K^{k A B}=D_{A}\left(\tau_{, B} K^{k A B}\right)-\tau_{{ }_{B}} D_{A} K^{k A B}$ to write

$$
\left(\triangle_{\gamma} \tau\right) \theta_{k}-\left(D_{A} D_{B} \tau\right) K^{k A B}=\tau_{, B} D_{A} K^{k A B}-\tau^{, A} D_{A} \theta_{k}+D_{A}\left(\tau^{, A} \theta_{k}-\tau_{, B} K^{k B A}\right) .
$$

The trace of the Codazzi identity (see equation (B.38) in Appendix $(\mathrm{B})$ ), simplifies in the Minkowski spacetime to

$$
D_{L}\left(K^{k}\right)_{A}^{L}-D_{A} \theta_{k}+\left(s_{\ell}\right)^{L} K_{L A}^{k}-\theta_{k}\left(s_{\ell}\right)_{A}=0 .
$$

Solving for $D_{A} \theta_{k}$ and substituting in (4.52) one finds

$$
\left(\triangle_{\gamma} \tau\right) \theta_{k}-\left(D_{A} D_{B} \tau\right) K^{k A B}=-\left(s_{\ell}\right)_{A}\left(s_{\ell}\right)^{A}+\theta_{k}\left(s_{\ell}\right)_{A} \tau^{, A}+D_{A}\left(\tau^{, A} \theta_{k}-\tau_{, B} K^{k B A}\right),
$$

where we have used the relation between $\boldsymbol{s}_{\boldsymbol{\ell}}$ and $K^{k}$ in (4.48). Inserting (4.53) into (4.51) yields the result.

We can now establish the following result that proves the monotonicity of the Bergqvist mass along the flow:

Theorem 4.5.2 (Bergqvist [8]). With the definitions above we have

$$
\frac{d M_{b}(\lambda)}{d \lambda}=\frac{1}{8 \pi} \int_{S_{\lambda}}\left\langle s_{\lambda}, s_{\lambda}\right\rangle_{\gamma_{\lambda}} \boldsymbol{\eta}_{S_{\lambda}} \geq 0,
$$

where $s_{\lambda}$ is the connection one-form of $S_{\lambda}$, defined as $s_{\lambda}(X):=\frac{1}{2}\left\langle\nabla_{X} k, \ell\right\rangle_{\gamma_{\lambda}}$ for any vector field $X$ tangent to $S_{\lambda}$.

Proof. We first rewrite the Bergqvist mass in terms of the null expansion $\theta_{k}$. Integrating (4.3) and using the Gauss theorem, the Bergqvist mass becomes

$$
M_{b}(\lambda):=\frac{\lambda}{2}+\frac{1}{16 \pi} \int_{S_{\lambda}} \theta_{k}(\lambda)\left(1+|D \tau|_{\gamma_{\lambda}}^{2}\right) \boldsymbol{\eta}_{S_{\lambda}}
$$

To compute the derivation of $M_{b}$ we need the evolution equations for $\theta_{k}, \gamma_{\lambda}$ and $\boldsymbol{\eta}_{S_{\lambda}}$. They are obtained in Appendix $B$ (see formulas (B.14), (B.17) and (B.41) ) and read

$$
\frac{d}{d \lambda} \theta_{k}(\lambda)=K_{A B}^{k} K^{k A B}, \quad \frac{d}{d \lambda} \gamma(\lambda)^{A B}=2\left(K^{k}\right)^{A B}, \quad £_{-k} \boldsymbol{\eta}_{S_{\lambda}}=-\theta_{k}(\lambda) \boldsymbol{\eta}_{S_{\lambda}} .
$$

As a consequence $\frac{d M_{b}}{d \lambda}$ takes the form

$$
\frac{d M_{b}}{d \lambda}=\frac{1}{2}+\frac{1}{16 \pi} \int_{S_{\lambda}}\left(\left(K_{A B}^{k} K^{k A B}-\theta_{k}^{2}\right)\left(1+|D \tau|_{\gamma_{\lambda}}^{2}\right)+2 \theta_{k}\left(s_{\ell}\right)_{A} \mathcal{T}^{, A}\right) \boldsymbol{\eta}_{S_{\lambda}},
$$




\section{Projection along the past null cone $\Omega$ in Minkowski}

where we have used relation (4.48).

Note the similarity between the integrand in this expression and identity (4.49). Integrating this identity the divergence term cancels out and the curvature scalar term gives $\int_{S_{\lambda}}$ Scal $^{S_{\lambda}} \boldsymbol{\eta}_{S_{\lambda}}=8 \pi$ by the Gauss-Bonnet theorem and we find $\frac{d M_{b}(\lambda)}{d \lambda}=$ $\frac{1}{8 \pi} \int_{S_{\lambda}}\left\langle\boldsymbol{s}_{\boldsymbol{\lambda}}, \boldsymbol{s}_{\boldsymbol{\lambda}}\right\rangle_{\gamma_{\lambda}} \boldsymbol{\eta}_{\boldsymbol{S}_{\boldsymbol{\lambda}}}$ as stated in the theorem.

The following result is the main one in this chapter. Using the monotonicity of the Bergqvist mass, we obtain a class of surfaces for which the shell Penrose inequality in Minkowski holds. This family of surfaces are determined in terms of the support function $h$ and the time-height function $\tau$.

Theorem 4.5.3 (Class of surfaces where the shell Penrose inequality in $\mathcal{M}^{1,3}$ holds). Let $(S, \gamma)$ be a spacetime strictly convex surface in $\left(\mathcal{M}^{1,3}, \eta\right)$. With the same assumptions and notation as in Theorem 4.4.6, let $h$ be the support function of $\widehat{S}_{0}$ as a hypersurface of Euclidean space and $\tau=\left.t\right|_{S}-t_{0}$. If these two functions satisfy the inequality

$$
4 \pi \int_{\mathbb{S}^{2}}\left(\left(\triangle_{\dot{q}} h\right)^{2}+2 h \triangle_{\dot{q}} h\right) \boldsymbol{\eta}_{\dot{q}} \geqslant 4 \pi \int_{\mathbb{S}^{2}} u^{2} \boldsymbol{\eta}_{\dot{q}}-\left(\int_{\mathbb{S}^{2}} u \boldsymbol{\eta}_{\dot{q}}\right)^{2},
$$

where $u:=\triangle_{q} h+2(h-\tau)$, then the shell Penrose inequality (2.34) holds for $S$.

Proof. In analogy with the definition of $M_{b}(\lambda)$ (4.47), we define a function $D(\lambda)$ by

$$
D(\lambda):=\sqrt{\frac{\left|S_{\lambda}\right|}{16 \pi}}-\frac{\lambda}{2} .
$$

With this definition, the shell Penrose inequality (1.4) for $S_{\lambda}$ can be written in the form

$$
M_{b}(\lambda)+D(\lambda) \leq 0
$$

Our aim is to prove $M_{b}(\lambda=0)+D(\lambda=0) \leq 0$. Since Bergqvist's Theorem 4.5 .2 ensures that $M_{b}(\lambda)$ is monotonically non-decreasing in $\lambda$, the idea of the proof is to study the monotonicity properties of $D(\lambda)$ together with the limiting behaviour of both functions when $\lambda \rightarrow \infty$ in order to see if sufficient conditions can be obtained so that $M_{b}(\lambda=0)+D(\lambda=0) \leq 0$ holds.

Let us start with the limit of $M_{b}(\lambda)$ at infinity. We want to exploit the fact that we obtained in (4.42) a general expression for the total integral of the outer null expansion

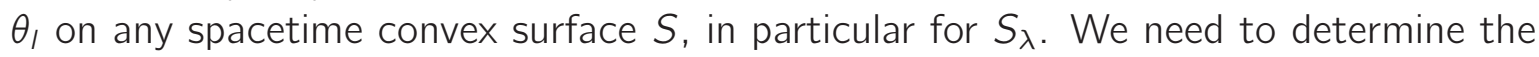
support and time height function of $S_{\lambda}$. Although it is not the only natural possibility, a convenient choice is to fix one hyperplane $\Sigma_{t_{0}}$ and project all surfaces $S_{\lambda}$ along $\Omega$ onto $\Sigma_{t_{0}}$. This procedure has the advantage that $\widehat{S}_{0}$ is the same surface for all $S_{\lambda}$ 


\subsection{Dragging the surface along its past null cone}

and hence that the support function $h$ is independent of $\lambda$. With this choice, the time height function $\tau_{\lambda}$ of $S_{\lambda}$ is

$$
\tau_{\lambda}=\left.t\right|_{S_{\lambda}}-t_{0}=\left.\sigma\right|_{S_{\lambda}}=\tau-\lambda .
$$

Inserting these functions in (4.42) we find

$$
\begin{aligned}
& M_{b}(\lambda)=\frac{-1}{16 \pi} \int_{\mathbb{S}^{2}}\left[\triangle_{\dot{q}} h+2(h-\tau)\right. \\
& \left.+\left(\triangle_{\dot{q}} h+2(h-\tau+\lambda)\right)\left(W_{1}(\lambda)|\stackrel{\circ}{\nabla}|_{\stackrel{q}{q}}^{2}-W_{2}(\lambda)\left(\stackrel{\circ}{\nabla}^{A} \stackrel{\circ}{\nabla}^{B} h\right) \stackrel{\circ}{\nabla}_{A} \tau \stackrel{\circ}{\nabla}_{B} \tau\right)\right] \boldsymbol{\eta}_{\dot{q}},
\end{aligned}
$$

where $W_{1}(\lambda)$ and $W_{2}(\lambda)$ are obtained from (4.37)-(4.38) after substituting $\tau \rightarrow \tau-\lambda$. Since $W_{1}(\lambda)$ and $W_{2}(\lambda)$ vanish as $\lambda^{-2}$ when $\lambda \rightarrow \infty$ the limit of $M_{b}(\lambda)$ is simply

$$
\lim _{\lambda \rightarrow \infty} M_{b}(\lambda)=\frac{-1}{16 \pi} \int_{\mathbb{S}^{2}}\left(\triangle_{\dot{q}} h+2(h-\tau)\right) \boldsymbol{\eta}_{\dot{q}}
$$

Regarding $D(\lambda)$, we substitute $\tau \rightarrow \tau-\lambda$ in (4.44) to obtain

$$
\left|S_{\lambda}\right|=\int_{\mathbb{S}^{2}}\left((h-\tau+\lambda)^{2}+\triangle_{\mathfrak{q}} h\left(\frac{h}{2}-\tau+\lambda\right)\right) \boldsymbol{\eta}_{\tilde{q}},
$$

so that

$$
D(\lambda)=\sqrt{\frac{1}{16 \pi} \int_{\mathbb{S}^{2}}\left((h-\tau+\lambda)^{2}+\triangle_{\dot{q}} h\left(\frac{h}{2}-\tau+\lambda\right)\right) \boldsymbol{\eta}_{\mathfrak{q}}}-\frac{\lambda}{2} .
$$

It is straightforward to check that the limit of this expression at infinity is

$$
\lim _{\lambda \rightarrow \infty} D(\lambda)=\frac{1}{16 \pi} \int_{\mathbb{S}^{2}}\left(\triangle_{\dot{q}} h+2(h-\tau)\right) \boldsymbol{\eta}_{\tilde{q}}
$$

which together with (4.57) implies $\lim _{\lambda \rightarrow \infty}\left(M_{b}(\lambda)+D(\lambda)\right)=0$. Since $M_{b}(\lambda)$ is monotonically non-decreasing, and $M_{b}(\lambda)+D(\lambda)$ tends to zero at infinity, a sufficient condition for the validity of $M_{b}(\lambda=0)+D(\lambda=0) \leq 0$ is that $D(\lambda)$ is monotonically increasing. From the definition (4.55) it follows

$$
\frac{d D(\lambda)}{d \lambda}=\sqrt{\frac{1}{64 \pi\left|S_{\lambda}\right|}}\left(\frac{d\left|S_{\lambda}\right|}{d \lambda}-\sqrt{16 \pi\left|S_{\lambda}\right|}\right) .
$$

It only remains to find under which conditions the right-hand side of (4.59) is nonnegative. Since $\frac{d\left|S_{\lambda}\right|}{d \lambda} \geq 0$ (because $\theta_{k} \leq 0$ on $\Omega$ and $\frac{d \boldsymbol{\eta}_{S_{\lambda}}}{d \lambda}=-\theta_{k}(\lambda) \boldsymbol{\eta}_{S_{\lambda}}$ ), this is equivalent to

$$
\left(\frac{d\left|S_{\lambda}\right|}{d \lambda}\right)^{2}-16 \pi\left|S_{\lambda}\right| \geq 0
$$




\section{Projection along the past null cone $\Omega$ in Minkowski}

Recalling expression (4.58) for the area of $S_{\lambda}$, it follows

$$
\frac{d\left|S_{\lambda}\right|}{d \lambda}=\int_{\mathbb{S}^{2}}\left(2(h-\tau+\lambda)+\triangle_{\dot{q}} h\right) \boldsymbol{\eta}_{\tilde{q}}=8 \pi \lambda+\int_{\mathbb{S}^{2}} u \boldsymbol{\eta}_{\tilde{q}}
$$

On the other hand, using again expression (4.58) we have

$$
\begin{aligned}
16 \pi\left|S_{\lambda}\right| & =16 \pi \int_{\mathbb{S}^{2}}\left((h-\tau)^{2}+\lambda^{2}+2 \lambda(h-\tau)+\triangle_{\dot{q}} h\left(\frac{h}{2}-\tau\right)+\lambda \triangle_{\dot{q}} h\right) \boldsymbol{\eta}_{\dot{q}} \\
& =64 \pi^{2} \lambda^{2}+16 \pi\left(\int_{\mathbb{S}^{2}} u \boldsymbol{\eta}_{\dot{q}}\right) \lambda+16 \pi \int_{\mathbb{S}^{2}}\left((h-\tau)^{2}+\triangle_{\dot{q}} h\left(\frac{h}{2}-\tau\right)\right) .
\end{aligned}
$$

Combining (4.61) and (4.62), it is clear that (4.60) is equivalent to

$$
\left(\int_{\mathbb{S}^{2}} u \boldsymbol{\eta}_{\dot{q}}\right)^{2} \geq 16 \pi \int_{\mathbb{S}^{2}}\left((h-\tau)^{2}+\triangle_{\dot{q}} h\left(\frac{h}{2}-\tau\right)\right) \boldsymbol{\eta}_{\dot{q}}
$$

Since $u=2(h-\tau)+\triangle_{\dot{q}} h$, squaring yields

$$
(h-\tau)^{2}=\frac{u^{2}}{4}-\frac{\left(\triangle_{q} h\right)^{2}}{4}-\triangle_{q} h(h-\tau),
$$

so that the right hand-side of the inequality (4.63) becomes

$$
\left(\int_{\mathbb{S}^{2}} u \boldsymbol{\eta}_{\dot{q}}\right)^{2} \geq 16 \pi \int_{\mathbb{S}^{2}}\left(\frac{u^{2}}{4}-\frac{\left(\triangle_{\dot{q}} h\right)^{2}}{4}-\triangle_{\dot{q}} h(h-\tau)+\triangle_{\dot{q}} h\left(\frac{h}{2}-\tau\right)\right) \boldsymbol{\eta}_{\dot{q}}
$$

After simplification we obtain

$$
\left(\int_{\mathbb{S}^{2}} u \boldsymbol{\eta}_{\tilde{q}}\right)^{2} \geq 4 \pi \int_{\mathbb{S}^{2}}\left(u^{2}-\left(\triangle_{\dot{q}} h\right)^{2}-2 h \triangle_{\dot{q}} h\right) \boldsymbol{\eta}_{\tilde{q}}
$$

which is precisely (4.54).

Theorem 4.5 .3 gives a class of spacetime strictly convex surfaces in the Minkowski spacetime for which the shell Penrose inequality holds. An important question regarding this result is how large is the class of surfaces covered by the theorem. Since inequality (4.54) is quadratic in $h, u$ and its derivatives, a natural strategy is to expand these functions in terms of spherical harmonics and to rewrite (4.54) as an inequality for the coefficients of these expansions.

Let $r \in \mathbb{N} \cup 0$ and $\widehat{Y}_{m}^{r}(m=-r, \cdots r)$ be $2 r+1$ linearly independent eigenfunctions of the spherical Laplacian with eigenvalue $-r(r+1)$, i.e. $\triangle_{\mathfrak{q}} \widehat{Y}_{m}^{r}=-r(r+1) \widehat{Y}_{m}^{r}$. Without loss of generality we assume that they form an orthonormal basis of $L^{2}\left(\mathbb{S}^{2}\right)$, 


\subsection{Dragging the surface along its past null cone}

i.e. $\int_{\mathbb{S}^{2}} \widehat{Y}_{i}^{r} \widehat{Y}_{j}^{s} \boldsymbol{\eta}_{\tilde{q}}=\delta^{r s} \delta_{i j}$. Any smooth function $f$ on the sphere can be decomposed in this basis as

$$
f=\sum_{r=0}^{\infty} a_{r} \cdot \widehat{Y}^{r}
$$

where here and in the following we use the notation $a_{r} \cdot \widehat{Y}^{r}:=\sum_{m=-r}^{r} a_{r}^{m} \widehat{Y}_{m}^{r}$. Similarly we write $a_{r}^{2}:=\sum_{m=-r}^{r}\left(a_{r}^{m}\right)^{2}$. The following corollary identifies the class of surfaces covered in Theorem 4.5 .3 in terms of the spherical harmonic decompositions of $h$ and $u$.

Corollary 4.5.4. With the notation of Theorem 4.5.3, let us expand the functions $h$ and $u$ in terms of spherical harmonics as

$$
h=\sum_{r=0}^{\infty} a_{r} \cdot \widehat{Y}^{r}, \quad u=\sum_{r=0}^{\infty} b_{r} \cdot \widehat{Y}^{r} .
$$

If the coefficients satisfy the inequality

$$
\sum_{r=2}^{\infty} a_{r}^{2} r(r+1)(r-1)(r+2) \geqslant \sum_{r=1}^{\infty} b_{r}^{2},
$$

then the shell Penrose inequality holds for the spacetime convex surface $S$ defined by the support function $h$ and the time height function $\tau=h-\frac{1}{2} \triangle_{\mathfrak{q}} h-\frac{u}{2}$.

Proof. The orthogonality relations of the spherical harmonics imply

$$
\int_{\mathbb{S}^{2}}\left(\triangle_{\dot{q}} h\right)^{2} \boldsymbol{\eta}_{\dot{q}}=\sum_{r=0}^{\infty} a_{r}^{2} r^{2}(r+1)^{2}, \quad \int_{\mathbb{S}^{2}} h \triangle_{\dot{q}} h \boldsymbol{\eta}_{\tilde{q}}=-\sum_{r=0}^{\infty} r(r+1) a_{r}^{2},
$$

so that the left-hand side of (4.54) reads

$$
4 \pi \int_{\mathbb{S}^{2}}\left(\left(\triangle_{\tilde{q}} h\right)^{2}+2 h \triangle_{\tilde{q}} h\right) \boldsymbol{\eta}_{\tilde{q}}=4 \pi \sum_{r=2}^{\infty} a_{r}^{2} r(r-1)(r+1)(r+2) .
$$

On the other hand, the spherical harmonic decomposition of $u$ implies $\int_{\mathbb{S}^{2}} u \boldsymbol{\eta}_{\tilde{q}}=\sqrt{4 \pi} b_{0}^{0}$ and

$$
4 \pi \int_{\mathbb{S}^{2}} u^{2} \boldsymbol{\eta}_{\grave{q}}-\left(\int_{\mathbb{S}^{2}} u \boldsymbol{\eta}_{\grave{q}}\right)^{2}=4 \pi \sum_{r=0}^{\infty} b_{r}^{2}-\left(\sqrt{4 \pi} b_{0}^{0}\right)^{2}=4 \pi \sum_{r=1}^{\infty} b_{r}^{2} .
$$

Using (4.66) and (4.67), we obtain (4.65), as claimed (we note that (4.66) and (4.67) imply that both sides in inequality (4.54) are non-negative, which will be used in the following remark). 


\section{Projection along the past null cone $\Omega$ in Minkowski}

Remark 4.5.5. In Theorem 4.5.3 we have shown that the Penrose inequality in the spherical case holds as a consequence of the Beckner inequality (or as a consequence of the Sobolev inequality in $\mathbb{R}^{m}$ in the case of four spacetime dimensions [101]). It is interesting to see how does the spherical case fit into the class of functions covered in Theorem 4.5.3. As we checked in Appendix C the support function of a sphere is either a constant (if the origin of Euclidean space coincides with the center of the sphere) or a linear combination of $r=0,1$ spherical harmonics (when the sphere is displaced from the origin). In either case, the left-hand side of (4.65) is identically vanishing, so that the inequality can only hold if the right-hand side also vanishes (because the righthand side is in any case non-negative). This forces $u=$ const. and hence $\tau=$ const. too. We see that that the only "spherical case" included in Theorem 4.5.3 is when the surface $S$ itself is spherically symmetric, which is a trivial case. Thus, in some sense, the cases covered by Beckner's inequality (which is essentially analytic in nature) and the cases covered by the geometric flow used in Theorem 4.5.3 are mutually exclusive. This seems to indicate that any attempt of proving the shell Penrose inequality for spacetime convex surfaces in the general case most likely needs some sort of combination of both ingredients and almost surely a combination of analytic and geometric arguments.

Remark 4.5.6. The other case where the Penrose inequality in Minkowski was known to hold involves surfaces lying in a constant time hyperplane. It is also natural to see how this case fits into the class of surfaces covered by Theorem 4.5.3. In this situation we have $\tau=0$ and hence $u=\triangle_{q} h+2 h$. Inserting this function in (4.54), this inequality becomes

$$
\left(\int_{\mathbb{S}^{2}} h \boldsymbol{\eta}_{\dot{q}}\right)^{2} \geqslant 4 \pi \int_{\mathbb{S}^{2}}\left(h^{2}+\frac{1}{2} h \triangle_{\dot{q}} h\right) \boldsymbol{\eta}_{\dot{q}},
$$

which is exactly the Minkowski inequality for 2-dimensional euclidean surfaces in terms of the support function (see formula (4.46)). Since the Minkowski inequality is true, it follows that the class of surfaces covered by Theorem 4.5.3 includes the case of convex surfaces lying on constant time hyperplanes (incidentally, it is immediate to prove directly the validity of (4.68) by using the spherical harmonic decomposition $\left.h=\sum_{r=0}^{\infty} a_{r} \cdot \widehat{Y}^{r}\right)$.

We finish this section, and the chapter, with a particular case of Theorem 4.5.3 where the inequality (4.54) can be interpreted nicely in terms of the geometry of the projected surface $\widehat{S}_{0}$ and of the height function $\tau$ of $S$. In Chapter 7 we will see that the set of solutions covered by the following Corollary includes as a particular one the situation covered by the original argument of Ludvigsen \& Vickers and Bergqvist when applied to the Minkowski spacetime. Since the corollary below restricts $\tau$ to depend on constants (when $h$ is already fixed) we conclude that the original method by Ludvigsen \& Vickers 


\subsection{Dragging the surface along its past null cone}

and Bergqvist is very restrictive. This is to be compared with our Theorem 4.5.3 above which includes the function $\tau$ (when $h$ is already fixed).

Corollary 4.5.7. Let $\widehat{S}_{0}$ be a strictly convex surface embedded in a hyperplane $\Sigma_{t_{0}}$ and let $\Omega$ the spacetime convex null hypersurface containing $\widehat{S}_{0}$. The shell Penrose inequality holds for any surface $S$ embedded in $\Omega$ and defined by a function $\tau=\left.t\right|_{S}-t_{0}$ of the form

$$
\tau=2 \alpha \frac{H\left(\widehat{S}_{0}\right)}{\operatorname{Scal}\left(\widehat{S}_{0}\right)}-\beta
$$

where $\alpha \in[0,1], \beta>2 \frac{H\left(\widehat{S}_{0}\right)}{\operatorname{Scal}\left(\widehat{S}_{0}\right)}-\frac{1}{\max _{1 \leq A \leq n}\left\{\kappa_{A}\right\}}$ and $H\left(\widehat{S}_{0}\right)$, Scal $\left(\widehat{S}_{0}\right)$ are, respectively, the mean curvature and scalar curvature of $\widehat{S}_{0}$ as a hypersurface of Euclidean space.

Proof. First of all observe that if $\tau$ has the form (4.69) and satisfies the bound (4.16), $\beta$ must satisfy

$$
\tau=2 \alpha \frac{H\left(\widehat{S}_{0}\right)}{\operatorname{Scal}\left(\widehat{S}_{0}\right)}-\beta<\frac{1}{\max _{1 \leq A \leq n}\left\{\kappa_{A}\right\}} \Longleftrightarrow 2 \alpha \frac{H\left(\widehat{S}_{0}\right)}{\operatorname{Scal}\left(\widehat{S}_{0}\right)}-\frac{1}{\max _{1 \leq A \leq n}\left\{\kappa_{A}\right\}}<\beta \quad \forall \alpha \in[0,1],
$$

which given the positivity of $H\left(\widehat{S}_{0}\right)$ and Scal $\left(\widehat{S}_{0}\right)$ is equivalent to $\beta>2 \frac{H\left(\widehat{S}_{0}\right)}{\operatorname{Scal}\left(\widehat{S}_{0}\right)}-\frac{1}{\max _{1 \leq A \leq n}\left\{\kappa_{A}\right\}}$. For surfaces in $\mathbb{R}^{3}$, the scalar curvature can be written as $\operatorname{Scal}\left(\widehat{S}_{0}\right)=2 \kappa_{1} \kappa_{2}$ where $\kappa_{1}$ and $\kappa_{2}$ are the principal curvatures of $\widehat{S}_{0}$ (hence positive everywhere since $\widehat{S}_{0}$ is strictly convex). Since $H=\kappa_{1}+\kappa_{2}$ it follows

$$
\begin{aligned}
\tau=2 \alpha \frac{H\left(\widehat{S}_{0}\right)}{\operatorname{Scal}\left(\widehat{S}_{0}\right)}-\beta=\alpha\left(\frac{1}{\kappa_{1}}+\frac{1}{\kappa_{2}}\right)-\beta=\alpha \operatorname{tr}\left(\mathbf{K}_{0}^{-1}\right)-\beta & =\alpha \operatorname{tr}(\mathbf{B})-\beta \\
& =\alpha\left(\triangle_{q} h+2 h\right)-\beta .
\end{aligned}
$$

As a consequence, the function $u=\triangle_{q} h+2(h-\tau)$ takes the form

$$
u=(1-2 \alpha)\left(\triangle_{\mathfrak{q}} h+2 h\right)+2 \beta \text {, }
$$

which in terms of the coefficients in the expansion (4.64) implies

$$
b_{r}=-(1-2 \alpha)(r+2)(r-1) a_{r} \quad r \geqslant 1 \text {. }
$$

Inserting this into (4.65) we find that this inequality becomes

$$
\sum_{r=2}^{\infty} a_{r}^{2}(r+2)(r-1)\left[r(r+1)-(1-2 \alpha)^{2}(r+2)(r-1)\right] \geqslant 0 .
$$


Since $h$ is basically arbitrary (it is only restricted by the condition that it defines a strictly convex surface) we need to impose that each term of the sum is non-negative. This is achieved only if

$$
(1-2 \alpha)^{2} \leqslant \frac{r(r+1)}{(r+2)(r-1)}:=Z(r), \quad \forall r \geq 2 .
$$

Since the sequence $Z(r)$ is decreasing and its limit is 1 we see that this inequality holds if $(1-2 \alpha)^{2} \leqslant 1$, which is equivalent to $\alpha \in[0,1]$. But $\alpha$ is restricted to this range by hypothesis, so (4.70) holds true and the corollary follows. 



\section{Normal graphs in Euclidean space. Applications to the shell Penrose inequality}

\subsection{Introduction}

In the previous two chapters we performed two natural projections of spacelike surfaces in the Minkowski spacetime. In Chapter 3 we obtained explicit expressions for the geometry of the initial surface $S$ in terms of its projection along the Killing direction. In Chapter 4 , the solution to the Ricatti and the metric equation along the past null cone of $S$ was used to find the relation between the geometry of $S$ and the geometry of the convex surface corresponding to the intersection of the null cone with a constant time hyperplane. The next natural step is the relate the geometries of the two projected surfaces. This is the purpose of this chapter.

Let $\bar{S}$ be the vertical projection of the initial surface $S$ onto the constant time hyperplane $\left\{t=t_{0}\right\}$, and $\widehat{S}_{0}$ the projection of $S$ along its past null cone $\Omega$ onto the same euclidean hyperplane. It is intuitively clear, and will be proven later, that $\bar{S}$ is uniquely determined once we know $\widehat{S}_{0}=\Omega \cap\left\{t=t_{0}\right\}$ and the time height function $\tau=\left.t\right|_{S}-t_{0}$, which describes how far $S$ lies from $\left\{t=t_{0}\right\}$. In this construction, $\bar{S}$ turns out to be a graph over $\widehat{S}_{0}$ within the euclidean space $\left\{t=t_{0}\right\}$. For this reason, we devote the first part of the chapter to compute the expressions that relate the geometry of a given orientable hypersurface embedded in the arbitrary dimensional Euclidean space with another hypersurface which is a graph over the former one. For the sake of completeness, we also give the analogous relations when the manifolds are embedded in the arbitrary Minkowski spacetime, and are strictly spacelike or timelike. These computations were published in [75] and, when applied to the context above, provide the first and the second fundamental forms of the vertical projection $\bar{S}$ in terms of the first and second fundamental forms of $\widehat{S}_{0}$ and the function $\tau$. 


\subsection{Geometry of normal graphs on hypersurfaces in the Euclidean space}

Recall that Brendle and Wang (Chapter 3, Theorem 3.3.7) found that the convexity of the surface $\bar{S}$ was a sufficient condition for the validity of the shell Penrose inequality in Minkowski. As a consequence of our results, we are able to rewrite this condition explicitly as an inequality involving a tensor $\mathcal{T}_{A B}$ defined on $\widehat{S}_{0}$, and which involves the geometry of $\widehat{S}_{0}$ and the time-height function $\tau$ of $S$.

To conclude the chapter, we present two simple examples. In the first example $\bar{S}$ is a graph over a cylinder (suitably truncated and closed) and in the second example $\bar{S}$ is a graph over a sphere. In both cases the tensor $\mathcal{T}_{A B}$ that codifies the convexity conditions for the respective graphs is computed explicitly.

\subsection{Geometry of normal graphs on hypersurfaces in the Euclidean space}

In this section, the ambient manifold is the $(n+1)$-Euclidean space $\left(\mathbb{R}^{n+1}, g_{E}\right), n \geq 2$. The flat connection is denoted by $\nabla$ and the corresponding (global) parallel transport by $\mathcal{T}_{p_{1} \rightarrow p_{2}}: T_{p_{1}} \mathbb{R}^{n+1} \longrightarrow T_{p_{2}} \mathbb{R}^{n+1}$, for any $p_{1}, p_{2} \in \mathbb{R}^{n+1}$. Obviously, in Cartesian coordinates $\left\{x^{\alpha}\right\},(\alpha=1, \ldots, n+1)$ this map simply preserves the coefficients of any vector $V \in T_{p_{1}} \mathbb{R}^{n+1}$ in the basis $\left\{\partial_{x^{\alpha}}\right\} .\left\{x^{\alpha}\right\}$ will always refer to a Cartesian coordinate system.

Consider two embedded submanifolds $\widehat{S}_{0}$ and $\bar{S}$ in $\mathbb{R}^{n+1}$ and assume there is diffeomorphism $\psi: \widehat{S}_{0} \longrightarrow \bar{S}$. The following result relates the tangential covariant derivative of vector fields along $\widehat{S}_{0}$ (not necessarily tangent to $\widehat{S}_{0}$ ) with the corresponding parallely transported vector field on $\bar{S}$ (see Figure 5.1). This result will play an important role below.

Lemma 5.2.1. Let $\widehat{S}_{0}, \bar{S}$ and $\psi$ as above. Let $Z$ be a vector field along $\widehat{S}_{0}$. Consider $X$ a vector field tangent to $\widehat{S}_{0}$ and define $\left.\mathcal{T} Z\right|_{\psi(p)}:=\mathcal{T}_{p \rightarrow \psi(p)} Z_{p} \forall p \in \widehat{S}_{0}$. Then

$$
\mathcal{T}_{p \rightarrow \psi(p)}\left(\left.\nabla_{X} Z\right|_{p}\right)=\left.\left(\nabla_{d \psi(X)} \mathcal{T} Z\right)\right|_{\psi(p)}
$$

Proof. The left-hand side of (5.1) is

$$
\mathcal{T}_{p \rightarrow \psi(p)}\left(\left.\nabla_{X} Z\right|_{p}\right)=\mathcal{T}_{p \rightarrow \psi(p)}\left(\left.X\left(Z^{\alpha}\right) \partial_{X^{\alpha}}\right|_{p}\right)=\left.\left.X\left(Z^{\alpha}\right)\right|_{p} \partial_{X^{\alpha}}\right|_{\psi(p)} .
$$

On the other hand, on $\bar{S}$ we have $\mathcal{T} Z=Z^{* \alpha} \partial_{x^{\alpha}}$, where $Z^{* \alpha}=Z^{\alpha} \circ \psi^{-1}$. Viewing $Z^{\alpha}$ as scalar functions we can also write $Z^{* \alpha}=\left(\psi^{-1}\right)^{\star}\left(Z^{\alpha}\right)$. Its covariant derivative along $\left.d \psi\right|_{p}(X)$ is

$$
\begin{aligned}
\left.\left(\nabla_{d \psi(X)} \mathcal{T} Z\right)\right|_{\psi(p)} & =\left.\nabla_{d \psi(X)}\left(Z^{* \alpha} \partial_{X^{\alpha}}\right)\right|_{\psi(p)}= \\
& =\left.d \psi(X)\left(\left(\psi^{-1}\right)^{\star}\left(Z^{\alpha}\right)\right) \partial_{X^{\alpha}}\right|_{\psi(p)}= \\
& =\left.\left.X\left(Z^{\alpha}\right)\right|_{p} \partial_{X^{\alpha}}\right|_{\psi(p)},
\end{aligned}
$$




\section{Applications of Euclidean normal graphs to the shell Penrose inequality}

which is the same as (5.2).

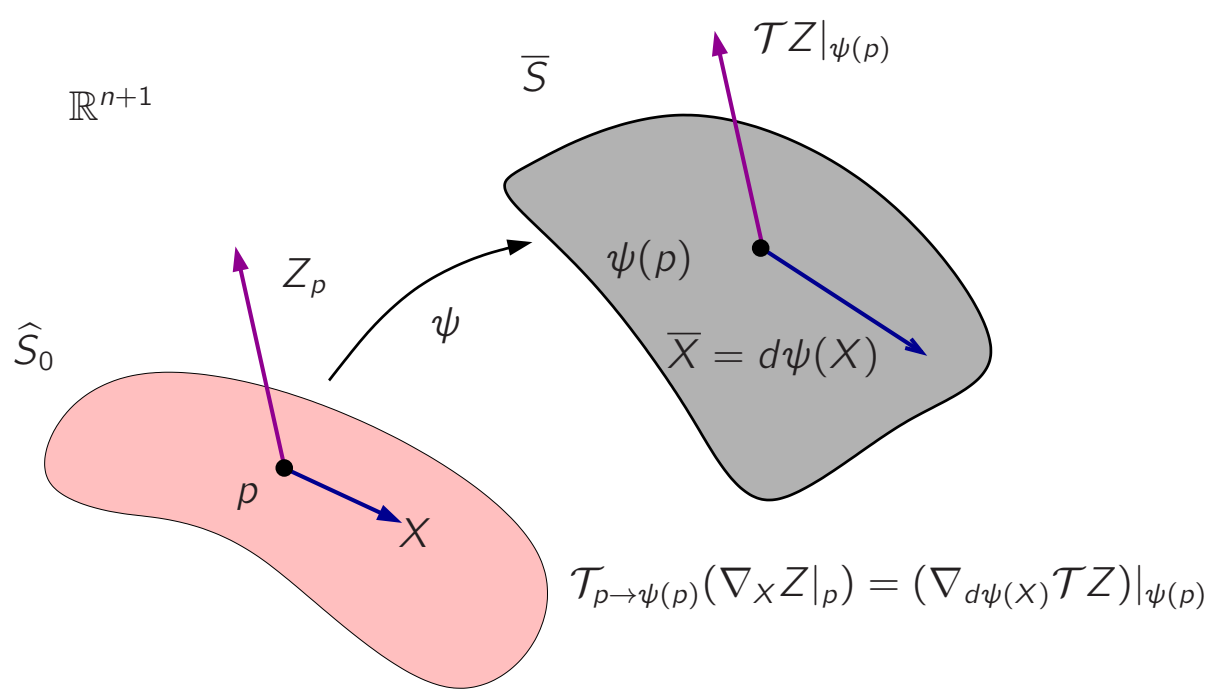

Figure 5.1: Relation between tangential covariant derivatives of vector fields along $\widehat{S}_{0}$ with the corresponding parallely transported vector field on $\bar{S}$. Here $\widehat{S}_{0}$ and $\bar{S}$ are two embedded manifolds in $\mathbb{R}^{n+1}$ diffeomorphically related by $\psi . Z$ is the vector field defined in a neighbourhood of $p \in \widehat{S}_{0}$, and $\mathcal{T} Z$ the corresponding parallely transported vector field defined near $\psi(p) \in \bar{S}$. $X_{p}$ is any tangent vector to $\widehat{S}_{0}$ at $p$, and $\bar{X}_{\psi(p)}$ the pushforward of $X_{p}$ by $\psi$. The covariant derivative of $Z$ along $X_{p}$ coincides with the covariant derivative of $\mathcal{T} Z$ along $\bar{X}_{\psi(p)}$ up to a translation.

Assume now that $\widehat{S}_{0}$ is an orientable hypersurface and select a unit normal vector field $\nu$. Choose a smooth function $\sigma: \widehat{S}_{0} \longrightarrow \mathbb{R}$ and consider the set of points at signed distance $\sigma$ from each $p \in \widehat{S}_{0} \subset \mathbb{R}^{n+1}$ along the normal $\nu(p)$. The congruence of normal geodesics to $\widehat{S}_{0}$ meets no focal points for distances $\sigma$ satisfying the bounds

$$
1+\sigma \kappa_{A}>0 \quad A=1, \cdots, n
$$

where $\left\{\kappa_{A}\right\}$ are the principal curvatures of $\widehat{S}_{0}$. Assuming this bound from now on, we have that the map $\psi^{\prime}: \widehat{S}_{0} \rightarrow \mathbb{R}^{n+1}$ defined by

$$
\psi^{\prime}(p)=p+\sigma(p) \nu(p)
$$

(where we are obviously using the affine structure of $\mathbb{R}^{n+1}$ ) is such that $\bar{S}:=\psi^{\prime}\left(\widehat{S}_{0}\right)$ is an embedded hypersurface of Euclidean space, and, in fact, a graph over $\widehat{S}_{0}$ (see Figure 5.2). Our aim is to relate the induced metrics and second fundamental forms of $\widehat{S}_{0}$ and $\bar{S}$.

It is clear that the restriction of $\psi^{\prime}$ onto its image is a diffeomorphism between $\widehat{S}_{0}$ and $\bar{S}$, which will be denoted by $\psi$. Let $X \in \mathfrak{X}\left(\widehat{S}_{0}\right)$ be a vector field tangent to $\widehat{S}_{0}$ and define $\bar{X}:=d \psi(X)$, which is obviously tangent to $\bar{S}$. 


\subsection{Geometry of normal graphs on hypersurfaces in the Euclidean space}

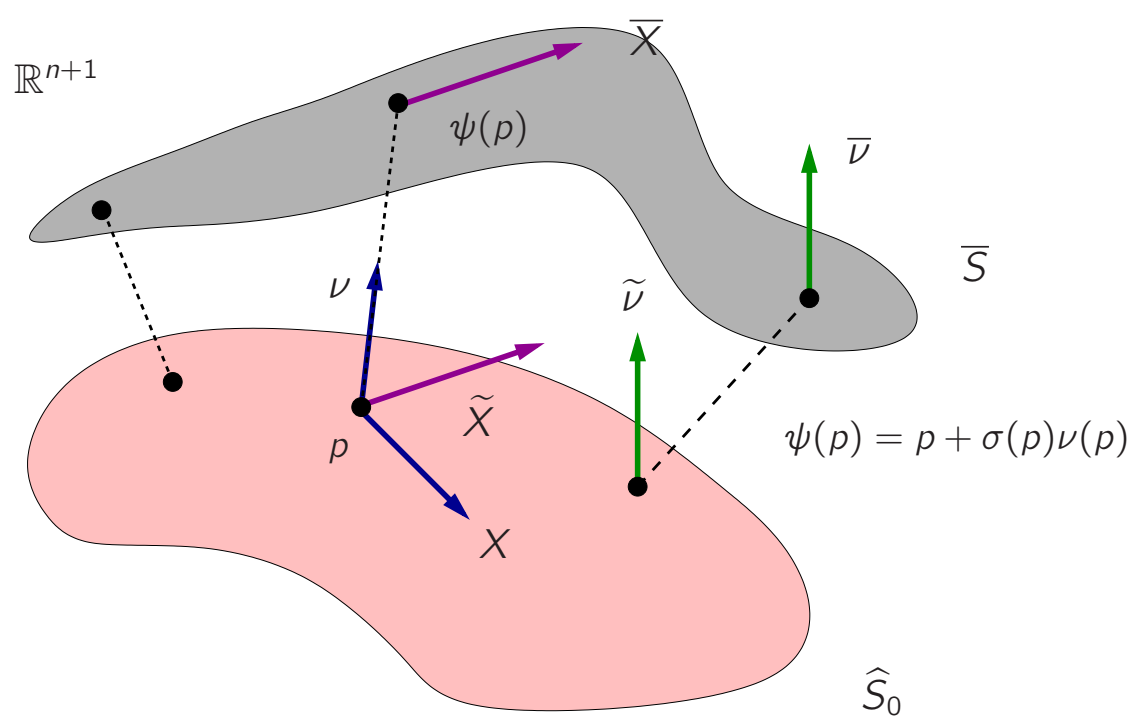

Figure 5.2: Particular case of diffeomorphism relating the surfaces $\widehat{S}_{0}$ and $\bar{S}$ in Figure 5.1. In this case the set of points at signed distance $\sigma(p)$ from $p \in \widehat{S}_{0} \subset \mathbb{R}^{n+1}$ along the normal $\nu(p)$ forms the embedded hypersurface $\bar{S}$, which is in fact a graph over $\widehat{S}_{0}$. Any tangent vector field $\bar{X}$ to $\bar{S}$ and the normal $\bar{\nu}$ can be parallely transported to $\widehat{S}_{0}$ providing the vector fields $\widetilde{X}$ and $\widetilde{\nu}$ respectively. This parallel transport allows one to perform all computations in a single manifold.

For the purposes of this section, it is convenient to transport parallely $\bar{X}$ from $\psi(p)$ to $p$ because this will allow us to perform all calculations in a single manifold. Thus, let us define the vector field $\widetilde{X} \in \mathfrak{X}\left(\widehat{S}_{0}\right)$ as $\left.\widetilde{X}\right|_{p}:=\mathcal{T}_{\psi(p) \rightarrow p}\left(\left.\bar{X}\right|_{\psi(p)}\right)$. The first aim is to relate $\widetilde{X}$ with $X$. Consider any curve $c(s)$ in $\widehat{S}_{0}$ passing through $p \in \widehat{S}_{0}$ with tangent vector $\left.X\right|_{p}$. From the definition of $\psi$, the curve $\bar{c}:=\psi \circ c$ has tangent vector $\left.\bar{X}\right|_{\psi(p)}$ at $\psi(p)$. The curves expressed in Cartesian coordinates are related by

$$
\bar{c}^{\alpha}=c^{\alpha}+\sigma \nu^{\alpha}
$$

Differentiating this relation with respect to the parameter $s$ of both curves, we have

$$
\frac{d \bar{c}^{\alpha}}{d s}=\frac{d c^{\alpha}}{d s}+\frac{d \sigma}{d s} \nu^{\alpha}+\sigma \frac{d \nu^{\alpha}}{d s}
$$

In Cartesian coordinates,

$$
\frac{d \bar{c}^{\alpha}}{d s}=\left(\bar{X}^{\alpha} \circ \psi\right)(p), \quad \frac{d c^{\alpha}}{d s}=X^{\alpha}(p), \quad \frac{d \sigma}{d s}=X(\sigma),\left.\quad\left(\nabla_{X} \nu\right)^{\alpha}\right|_{p}=\frac{d \nu^{\alpha}}{d s} .
$$

Hence, relation (5.4) can be rewritten in a tensorial way as

$$
\mathcal{T}_{\psi(p) \rightarrow p}\left(\left.\bar{X}\right|_{\psi(p)}\right)=\left.\left(X+X(\sigma) \nu+\sigma \nabla_{X} \nu\right)\right|_{p}
$$




\section{Applications of Euclidean normal graphs to the shell Penrose inequality}

Recalling that the Weingarten map $K_{0}: T_{p} \widehat{S}_{0} \longrightarrow T_{p} \widehat{S}_{0}$ is defined by $K_{0}(X):=\nabla_{X} \nu$ we conclude

$$
\left.\left.\widetilde{X}\right|_{p}=\left.\left(X+\sigma \nabla_{X} \nu+X(\sigma) \nu\right)\right|_{p}=\left(\boldsymbol{I d}+\sigma \boldsymbol{K}_{0}+d \sigma \otimes \nu\right)(X)\right)\left.\right|_{p}
$$

From the geometric construction of $\bar{S}$ it is intuitively clear that the normal vector $\bar{\nu}$ orthogonal to $\bar{S}$ must satisfy $g_{E}\left(\left.\widetilde{\nu}\right|_{p},\left.\nu\right|_{p}\right) \neq 0$ for all $p \in \widehat{S}_{0}$, where $\left.\widetilde{\nu}\right|_{p}:=\mathcal{T}_{\psi(p) \rightarrow p}\left(\left.\bar{\nu}\right|_{\psi(p)}\right)$. For a rigorous proof we use (5.5) as follows. Given that $\mathcal{T}_{p \rightarrow \psi(p)}$ is an isometry, (5.5) implies the following identity, valid for any $X \in T_{p} \widehat{S}_{0}$ :

$$
\begin{aligned}
0 & =\left.g_{E}(\bar{\nu}, \bar{X})\right|_{\psi(p)}=\left.g_{E}\left(\mathcal{T}_{p \rightarrow \psi(p)}(\widetilde{\nu}), \mathcal{T}_{p \rightarrow \psi(p)}(\widetilde{X})\right)\right|_{\psi(p)}=\left.g_{E}(\widetilde{\nu}, \widetilde{X})\right|_{p} \\
& =\left.g_{E}\left(\widetilde{\nu},\left(\boldsymbol{I} \boldsymbol{d}+\sigma \boldsymbol{K}_{0}\right)(X)\right)\right|_{p}+\left.d \sigma(X) g_{E}(\widetilde{\nu}, \nu)\right|_{p}
\end{aligned}
$$

Assume there is $q \in \widehat{S}_{0}$ such that $\left.\widetilde{\nu}\right|_{q} \in T_{q} \widehat{S}_{0}$ (i.e. $\left.g_{E}(\widetilde{\nu}, \nu)=0\right)$. Then $g_{E}(\widetilde{\nu},(\boldsymbol{I} \boldsymbol{d}+$ $\left.\left.\sigma K_{0}\right)(X)\right)\left.\right|_{q}=0$ for any $X \in T_{q} \widehat{S}_{0}$, which is a contradiction with the fact that the bound (5.3) implies that the endomorphism $\boldsymbol{I} \boldsymbol{d}+\sigma \boldsymbol{K}_{0}$ is invertible.

Let us choose the orientation of $\bar{\nu}$ so that $W:=g_{E}(\widetilde{\nu}, \nu)>0$ on $\widehat{S}_{0}$. Thus, we can decompose $\widetilde{\nu}=W(\nu-T)$ on $\widehat{S}_{0}$, where $T \in \mathfrak{X}\left(\widehat{S}_{0}\right)$ is a tangent vector field. From equation (5.6) we have

$$
0=\left.g_{E}\left(W(\nu-T),\left(\boldsymbol{I}+\sigma \boldsymbol{K}_{0}\right)(X)\right)\right|_{p}+\left.W \nabla_{X} \sigma\right|_{p}
$$

and consequently

$$
\left.g_{E}\left(T,\left(\boldsymbol{I d}+\sigma \boldsymbol{K}_{0}\right)(X)\right)\right|_{p}=\left.\nabla_{X} \sigma\right|_{p} .
$$

Using that the endomorphism $\boldsymbol{I} \boldsymbol{d}+\sigma \boldsymbol{K}_{0}$ is self-adjoint, the vector

$$
T=\left(\boldsymbol{I d}+\sigma \boldsymbol{K}_{0}\right)^{-1}\left(\operatorname{grad}_{\gamma_{0}}(\sigma)\right)
$$

solves relation (5.7), where $\operatorname{grad}_{\gamma_{0}}(\sigma)$ is the gradient of $\sigma$ with respect to the induced metric $\gamma_{0}$. For notational simplicity, define the invertible endomorphism $\boldsymbol{C}:=(\boldsymbol{I} \boldsymbol{d}+$ $\left.\sigma K_{0}\right)$ so that $T=C^{-1}\left(\operatorname{grad}_{\gamma_{0}}(\sigma)\right)$. The condition of $\bar{\nu}$ being unit fixes $W$ to satisfy $W^{2}\left(1+\gamma_{0}(T, T)\right)=1$, which, given our choice of normal in $\bar{S}$, implies

$$
W=\frac{1}{\sqrt{1+\gamma_{0}(T, T)}} .
$$

We are ready to prove our main result of this section, which relates the geometry of the graph $\bar{S}$ with the geometry of its base $\widehat{S}_{0}$.

Theorem 5.2.2. Consider the hypersurfaces $\widehat{S}_{0}, \bar{S}$ of Euclidean space $\left(\mathbb{R}^{n+1}, g_{E}\right)$ with signed distance function $\sigma$ and diffeomorphism $\psi$, as above. The respective induced 


\subsection{Geometry of normal graphs on hypersurfaces in the Euclidean space}

metrics $\gamma_{0}$ and $\bar{\gamma}$ and second fundamental forms $K_{0}$ and $\bar{K}$ with respect to the normals $\nu$ and $\bar{\nu}$ are related by

$$
\begin{aligned}
\psi^{\star}(\bar{\gamma})= & \gamma_{0}+2 \sigma K_{0}+\sigma^{2} K_{0} \circ K_{0}+d \sigma \otimes d \sigma \\
\frac{1}{W} \psi^{\star}(\bar{K})= & K_{0}+\sigma K_{0} \circ K_{0}+\sigma D K_{0}(\cdot, T, \cdot)+d \sigma \otimes K_{0}(T, \cdot)+ \\
& +K_{0}(T, \cdot) \otimes d \sigma-\operatorname{Hess}_{\gamma_{0}}(\sigma),
\end{aligned}
$$

where $T$ and $W$ are defined in (5.8)-(5.9), $K_{0} \circ K_{0}$ is the trace of $K_{0} \otimes K_{0}$ in the second and third indices, $D$ is the Levi-Civita derivative of $\gamma_{0}$ and $\operatorname{Hess}_{\gamma_{0}}(\sigma)$ is the Hessian of $\sigma$ in this metric.

Remark 5.2.3. These expressions reduce to well-known results when either $\sigma$ is constant or when the base surface is a hyperplane.

Remark 5.2.4. It is interesting that in (5.11) the symmetry of $\bar{K}$ for any $\sigma$ is equivalent to the Codazzi identity $D K_{0}\left(X_{1}, \cdot, X_{3}\right)=D K_{0}\left(X_{3}, \cdot, X_{1}\right)$ for $\widehat{S}_{0}$. So, properties of normal graphs can be used to derive curvature identities on the base hypersurface, which usually would require different methods.

Proof. Let $X, Y \in \mathfrak{X}\left(\widehat{S}_{0}\right)$ be arbitrary tangent vector fields. We start with (15.10). With the notation above, and using that the parallel transport is an isometry:

$$
\begin{aligned}
\left.\psi^{\star}(\bar{\gamma})(X, Y)\right|_{\psi(p)} & =\bar{\gamma}\left(\left.d \psi\right|_{p}(X),\left.d \psi\right|_{p}(Y)\right)=\left.g_{E}(\bar{X}, \bar{Y})\right|_{\psi(p)}=\left.g_{E}(\tilde{X}, \tilde{Y})\right|_{p} \\
& =\left.g_{E}(\boldsymbol{C}(X), \boldsymbol{C}(Y))\right|_{p}+\left.d \sigma \otimes d \sigma\right|_{p}(X, Y) \\
& =\left.\gamma_{0}\left(\left(\boldsymbol{I} \boldsymbol{d}+\sigma \boldsymbol{K}_{0}\right)(X),\left(\boldsymbol{I} \boldsymbol{d}+\sigma \boldsymbol{K}_{0}\right)(Y)\right)\right|_{p}+\left.d \sigma \otimes d \sigma\right|_{p}(X, Y)
\end{aligned}
$$

where in the fourth equality we used (5.5). This establishes (5.10). To prove (5.11) we first apply Lemma 5.2.1 to find the identity

$$
\begin{aligned}
\left.g_{E}\left(\nabla_{X} \widetilde{\nu}, \widetilde{Y}\right)\right|_{p} & =\left.g_{E}\left(\mathcal{T}_{p \rightarrow \psi(p)}\left(\nabla_{X} \widetilde{\nu}\right), \mathcal{T}_{p \rightarrow \psi(p)}(\widetilde{Y})\right)\right|_{\psi(p)} \\
& =\left.g_{E}\left(\nabla_{\bar{X}} \bar{\nu}, \bar{Y}\right)\right|_{\psi(p)}=\left.\bar{K}(\bar{X}, \bar{Y})\right|_{\psi(p)}=\left.\psi^{\star}(\bar{K})(X, Y)\right|_{p}
\end{aligned}
$$

To evaluate the left-hand side we recall the fundamental identity, $\nabla_{X} Y=D_{X} Y-$ $K_{0}(X, Y) \nu$ (cf. formula (A.12) in Appendix $\mathrm{A}$ ), valid for any pair of tangential vector fields. Given that $g_{E}(\widetilde{\nu}, \widetilde{Y})=0$, the left-hand side of (5.12) becomes

$$
\begin{aligned}
g_{E}\left(\nabla_{X} \widetilde{\nu}, \widetilde{Y}\right) & =\frac{X(W)}{W} g_{E}(\widetilde{\nu}, \widetilde{Y})+W g_{E}\left(\nabla_{X}(\nu-T), d \sigma(Y) \nu+C(Y)\right) \\
& =W \gamma_{0}\left(K_{0}(X)-D_{X} T, C(Y)\right)+W K_{0}(X, T) d \sigma(Y) .
\end{aligned}
$$

The first term is immediately $W \gamma_{0}\left(K_{0}(X), C(Y)\right)=W\left(K_{0}+\sigma K_{0} \circ K_{0}\right)(X, Y)$. To elaborate the second term, we use that the endomorphism $C$ is symmetric with respect 


\section{Applications of Euclidean normal graphs to the shell Penrose inequality}

to $\gamma_{0}$, i.e. $\gamma_{0}\left(X_{1}, \boldsymbol{C}\left(X_{2}\right)\right)=\gamma_{0}\left(\boldsymbol{C}\left(X_{1}\right), X_{2}\right)$. Thus,

$$
\begin{aligned}
-\gamma_{0}\left(D_{X} T, C(Y)\right) & =-\gamma_{0}\left(\left(C \circ D_{X} C^{-1}\right)\left(\operatorname{grad}_{\gamma_{0}}(\sigma)\right), Y\right)-\gamma_{0}\left(D_{X} \operatorname{grad}_{\gamma_{0}}(\sigma), Y\right) \\
& =\gamma_{0}\left(\left(D_{X} C\right)(T), Y\right)-\operatorname{Hess}_{\gamma_{0}}(\sigma)(X, Y) \\
& =d \sigma(X) K_{0}(T, Y)+\sigma D K_{0}(X, T, Y)-\operatorname{Hess}_{\gamma_{0}}(\sigma)(X, Y),
\end{aligned}
$$

where in the first equality we used (5.8) and in the second equality $-C \circ\left(D_{X} C^{-1}\right)=$ $\left(D_{X} C\right) \circ C^{-1}$. Inserting this into (5.13) yields the result.

Remark 5.2.5. The Riemannian character of the ambient Euclidean space has only been used when evaluating $g_{E}(\nu, \nu)$ and $g_{E}(\bar{\nu}, \bar{\nu})$. With the same arguments as before, let $\widehat{S}_{0}$ be an embedded submanifold of the Minkowski spacetime $\left(\mathcal{M}^{1, n+1}, \eta\right)$ with nondegenerate induced metric $\gamma_{0}$ and unit normal $\nu$ satisfying $\langle\nu, \nu\rangle_{\eta}=\epsilon$ with $\epsilon= \pm 1$. $\bar{S}$ is constructed as before, where the orientation of the unit normal $\bar{\nu}$ is selected so that it satisfies $\langle\widetilde{\nu}, \nu\rangle_{\eta}=\epsilon W$, with $W>0$. Under these conditions:

$$
\begin{aligned}
\psi^{\star}(\bar{\gamma}) & =\gamma_{0}+2 \sigma K_{0}+\sigma^{2} K_{0} \circ K_{0}+\epsilon d \sigma \otimes d \sigma \\
\frac{1}{W} \psi^{\star}(\bar{K}) & =K_{0}+\sigma K_{0} \circ K_{0}+\sigma D K_{0}(\cdot, T, \cdot)+d \sigma \otimes K_{0}(T, \cdot)+ \\
& +K_{0}(T, \cdot) \otimes d \sigma-\epsilon \operatorname{Hess}_{\gamma_{0}}(\sigma),
\end{aligned}
$$

where all definitions are as before and the decomposition $\widetilde{\nu}=W(\nu-T)$ still holds, but this time $T$ reads $T=\epsilon\left(\boldsymbol{I} \boldsymbol{d}+\sigma \boldsymbol{K}_{0}\right)^{-1}\left(\operatorname{grad}_{\gamma_{0}}(\sigma)\right)$ and $W$ is

$$
W=\frac{1}{\sqrt{1+\epsilon \gamma_{0}(T, T)}} .
$$

The condition $1+\epsilon \gamma_{0}(T, T)>0$ is necessary for $\bar{S}$ to be of the same causal character as $\widehat{S}_{0}$.

\subsection{Matching the two different projections}

Let $\left(\mathcal{M}^{1, n+1}, \eta\right)$ be the $(n+2)$-dimensional Minkowski spacetime $(n \geq 2)$, and consider the same setting as in Section 4.3 of Chapter 4 . Specifically, consider three different surfaces: the initial spacetime convex surface $S$ embedded in the null hypersurface $\Omega$, the euclidean surface $\widehat{S}_{0}=\Omega \cap \Sigma_{t_{0}}$, and the euclidean surface $\bar{S}$, which is the projection of $S$ along the Killing direction $\xi$ onto the constant time hyperplane $\Sigma_{t_{0}}$.

For any closed spacetime convex surface $S, \bar{S}$ must be embedded in $\Sigma_{t_{0}}$ (otherwise two different points of $S$ with different time heights would project the same point onto $\Sigma_{t_{0}}$ which is impossible given that they lie on a smooth null hypersurface). We can apply Theorem 5.2.2 to relate the geometry of $\widehat{S}_{0}$ and $\bar{S}$ as follows: Theorem 3.3 .7 


\subsection{Matching the two different projections}

required the convexity of the projected surface $\bar{S}$ as the main condition for the validity of the shell Penrose inequality for $S$. In the construction above and using the results of Theorem 5.2.2, we can rewrite the second fundamental form of $\bar{S}$ in terms of the second fundamental form of $\widehat{S}_{0}$ and the signed function between the two euclidean surfaces, which as we will see coincides with time height function $\tau$ of $S$ up to a sign. This way the convexity condition for $\bar{S}$ can be described by suitable conditions on the geometry of the surface $\widehat{S}_{0}$ and the time height that separates the surface $S$ from the constant time hyperplane $\Sigma_{t_{0}}$. This underlying convexity condition of $\bar{S}$ can be translated to a positive definite condition of a two-covariant tensor defined on the surface $\widehat{S}_{0}$, as we show next:

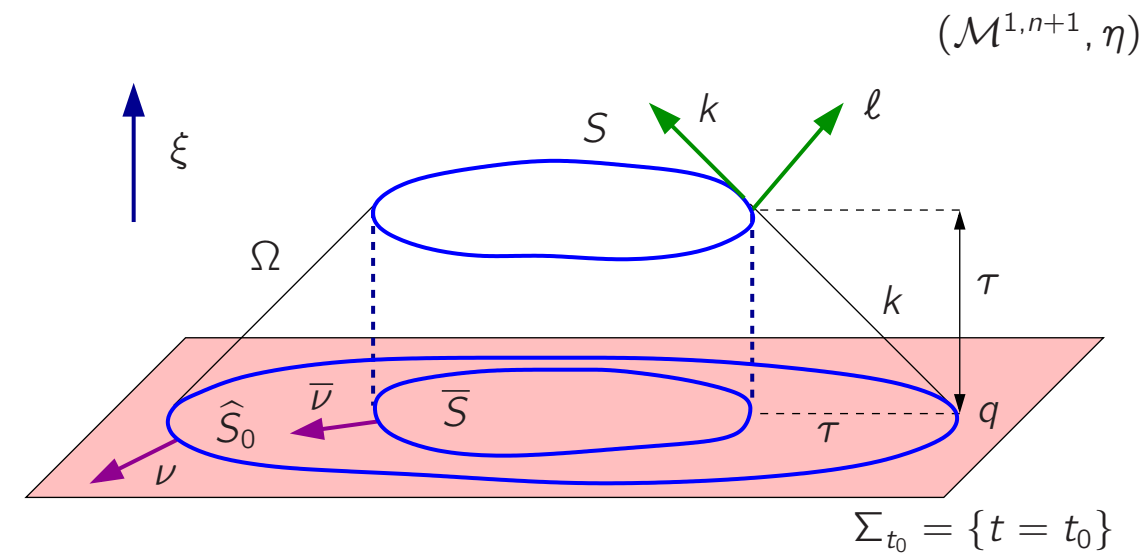

Figure 5.3: Schematic figure combining both projections: the spacetime convex surface $S$ is projected along $\Omega$ onto $\Sigma_{t_{0}}$, with $\widehat{S}_{0}=\Omega \cap \Sigma_{t_{0}}$. $\bar{S}$ is obtained by projecting $S$ along the Killing $\xi$. $\{k, \ell\}$ are normalized so that $\langle k, \ell\rangle_{\eta}=-2$.

Theorem 5.3.1 (Sufficient condition for the Penrose inequality in Minkowski in terms of spacetime convex geometry). Let $\left(\mathcal{M}^{1, n+1}, \eta\right)$ be the $(n+2)$-dimensional Minkowski spacetime with $t$ a Minkowskian time defining a unit Killing $\boldsymbol{\xi}=-d t$. Let $S$ be a closed, connected, orientable and spacetime convex surface in $\left(\mathcal{M}^{1, n+1}, \eta\right)$ and $\Omega$ the convex null hypersurface containing $S$. Consider $\widehat{S}_{0}:=\Omega \cap \Sigma_{t_{0}}$ and let $K_{0}$ be its second fundamental form as an euclidean surface of $\Sigma_{t_{0}}$ with respect to its outer unit normal $\nu$ (see Figure [5.3), $D$ the Levi-Civita connection of the metric $\gamma_{0}$ of $\widehat{S}_{0}$, and $\operatorname{grad}_{\gamma_{0}}(\tau)$ and $\operatorname{Hess}_{\gamma_{0}}(\tau)$ the gradient and Hessian of $\tau$ in the metric $\gamma_{0}$ respectively, where $\tau:=\left.t\right|_{s}-t_{0}$. If the tensor

$$
\mathcal{T}=K_{0}-\tau K_{0} \circ K_{0}-\tau D K_{0}(\cdot, T, \cdot)-d \tau \otimes K_{0}(T, \cdot)-K_{0}(T, \cdot) \otimes d \tau+\operatorname{HesS}_{\gamma_{0}}(\tau)
$$

is positive semidefinite, where $T=-\left(\boldsymbol{I} \boldsymbol{d}-\tau \boldsymbol{K}_{0}\right)^{-1}\left(\operatorname{grad}_{\gamma_{0}}(\tau)\right)$, then the Penrose inequality with respect to $\xi$ holds for $S$.

Proof. Observe that in the euclidean hyperplane $\Sigma_{t_{0}}$ we can obtain $\bar{S}$ as a graph over $\widehat{S}_{0}$ moving inwards along the inner normal to $\widehat{S}_{0}$. Indeed, let $\nu$ and $\bar{\nu}$ be the outer unit 


\section{Applications of Euclidean normal graphs to the shell Penrose inequality}

normals of $\widehat{S}_{0}$ and $\bar{S}$. Moving along geodesics tangent to $k$ in the past null cone $\Omega$ a time height $\tau$ with respect to $\Sigma_{t_{0}}$ is equivalent to the projected trajectory moving inwards the same signed distance $\tau$ (see Figure 5.3). Thus, we can apply Theorem 5.2 .2 with $\sigma=-\mathcal{\tau}$ and conclude that $\mathcal{T}=\frac{1}{W} \psi^{*}(\bar{K})$ with $W>0$. The validity of the Penrose inequality for $S$ is then a consequence of Theorem 3.3.7.

Remark 5.3.2. The expression of $\mathcal{T}$ with an index notation is

$$
\mathcal{T}_{A B}=K_{0 A B}-\tau K_{0 A L} K_{0}{ }_{B}-\tau T^{L} D_{A} K_{0 B L}-T^{L}\left(\tau_{, A} K_{0 B L}+\tau_{{ }_{B}} K_{0 A L}\right)+D_{A} D_{B} \tau
$$

where $T^{L}=-\tau_{M}\left[\left(\boldsymbol{I d}-\tau \boldsymbol{K}_{0}\right)^{-1}\right]^{M L}$.

To get a flavour of the range of applicability of this result, let us consider a few examples. Consider a closed, axially symmetric convex surface $\widehat{S}_{0}$ in a spacelike hyperplane $\Sigma_{t_{0}}$ of four-dimensional Minkowski spacetime $\mathcal{M}^{1,3}$, and assume that this surface is a cylinder between two parallel planes $z=z_{0}$ and $z=z_{1}$ orthogonal to the axis of symmetry. Let $\rho_{0}$ be the radius of the cylinder (see Figure 5.4).

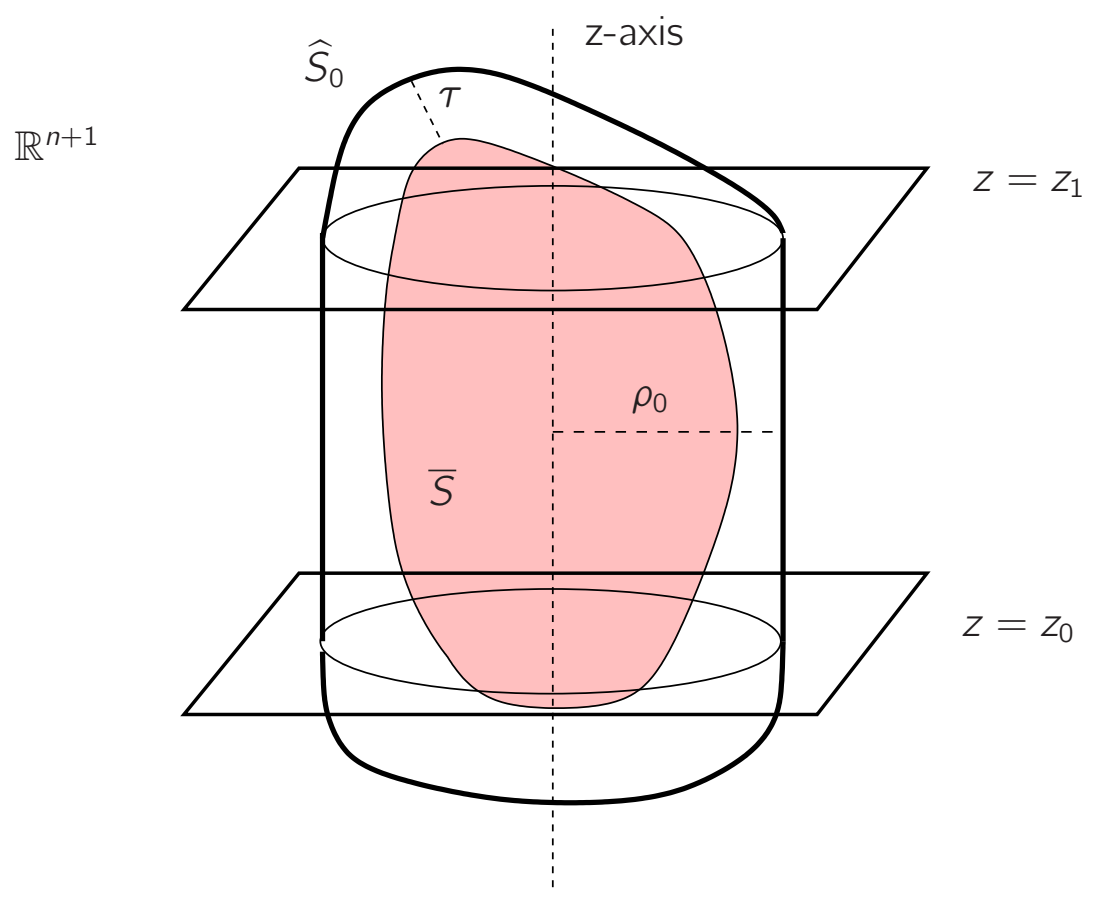

Figure 5.4: Particular case of the construction in Figure 5.3, where the projection $\widehat{S}_{0}$ onto a constant time hyperplane $\Sigma_{t_{0}}$ of the spacetime convex surface $S$ embedded in $\mathcal{M}^{1,3}$ along its past null cone $\Omega$ is a cylinder of radius $\rho_{0}$ between the two parallel planes $z=z_{0}$ and $z=z_{1}$ orthogonal to the axis of symmetry. For all those $\tau$ for which $\mathcal{T}$ is positive semidefinite, i.e. those ones that make $\bar{S} \subset \mathbb{R}^{n+1}$ convex, the shell Penrose inequality for $S$ holds. 


\subsection{Matching the two different projections}

In cylindrical coordinates $\{\varphi, z\}$, the first and second fundamental form of the cylinder read

$$
\gamma_{0}=\rho_{0}^{2} d \varphi^{2}+d z^{2}, \quad K_{0}=\rho_{0} d \varphi^{2} .
$$

It is immediate to see that

$$
K_{0 A L} K_{0}{ }_{B}=\delta_{A}^{\varphi} \delta_{B}^{\varphi}
$$

with $\delta$ the Kronecker delta. In addition, the coefficients of the metric tensor $\gamma_{0}$ are constant. This implies that the Christoffel symbols of the metric are zero, and consequently $D_{A} K_{O B C}=0$, because the coefficients of $K_{0}$ are also constant. Inverting $\left(\boldsymbol{I} \boldsymbol{d}-\tau \boldsymbol{K}_{0}\right)_{B}^{A}=\frac{\rho_{0}-\tau}{\rho_{0}} \boldsymbol{\delta}_{\varphi}^{A} \boldsymbol{\delta}_{B}^{\varphi}+\boldsymbol{\delta}_{z}^{A} \boldsymbol{\delta}_{B}^{Z}$, we find $\left[\left(\boldsymbol{I} \boldsymbol{d}-\tau \boldsymbol{K}_{0}\right)^{-1}\right]_{B}^{A}=\frac{\rho_{0}}{\rho_{0}-\tau} \boldsymbol{\delta}_{\varphi}^{A} \boldsymbol{\delta}_{B}^{\varphi}+\boldsymbol{\delta}_{z}^{A} \boldsymbol{\delta}_{B}^{Z}$, and the following expression for the vector $T$ is obtained:

$$
T^{L}=-\tau_{M}\left[\left(\boldsymbol{I d}-\tau \boldsymbol{K}_{0}\right)^{-1}\right]^{M L}=\frac{-\tau, \varphi}{\rho_{0}\left(\rho_{0}-\tau\right)} \boldsymbol{\delta}_{\varphi}^{L} .
$$

Thus we obtain $T^{L} K_{0 A L}=\frac{-\tau, \varphi}{\rho_{0}-\tau} \delta_{A}^{\varphi}$, and it follows

$$
-T^{L}\left(\tau_{, A} K_{0 B L}+\tau_{, B} K_{0 A L}\right)=\frac{\tau, \varphi}{\rho_{0}-\tau}\left(\tau_{, A} \delta_{B}^{\varphi}+\tau_{, B} \delta_{A}^{\varphi}\right) .
$$

Using all this information, in cylindrical coordinates $\{\varphi, z\}$, (5.16) becomes, in the region $z_{0} \leq z \leq z_{1}$,

$$
\mathcal{T}_{A B}=\left(\rho_{0}-\tau\right) \boldsymbol{\delta}_{A}^{\varphi} \boldsymbol{\delta}_{B}^{\varphi}+\tau_{, A B}+\frac{\tau_{, \varphi}}{\rho_{0}-\tau}\left(\tau_{, A} \boldsymbol{\delta}_{B}^{\varphi}+\tau_{, B} \boldsymbol{\delta}_{A}^{\varphi}\right)
$$

Assuming $\tau$ also axially symmetric, then $\mathcal{T}$ is positive semidefinite if and only if $\tau_{, z z} \geq 0$. So, any smooth axially symmetric surface $S$ projecting to $\widehat{S}_{0}$ along the past null cone and for which $\tau$ fulfills $\tau_{, z z} \geq 0$ on $z \in\left[z_{0}, z_{1}\right]$, and is a constant $\tau_{1}$ on $z \geq z_{1}$ and a constant $\tau_{0}$ on $z \leq z_{0}$ (although it is not the only possible way to define it, we consider such a $\tau$ in order to obtain a compact projected surface $\bar{S}$ ), satisfies the Penrose inequality (with respect to the time translation orthogonal to the hyperplane $\Sigma_{t_{0}}$ ).

Another simple example is obtained when $\widehat{S}_{0}$ is a sphere of radius $r_{0}$ in $\Sigma_{t_{0}}$ (see Figure 5.5). In this case $K_{0}=\frac{1}{r_{0}} \gamma_{0}$, where $\gamma_{0}$ is the metric of the two-sphere of radius $r_{0}$. Clearly

$$
K_{0 A L} K_{0}{ }_{B}=\frac{1}{r_{0}^{2}} \gamma_{0}
$$

and we also have $D_{A} K_{0 B C}=0$. Inverting $\left(\boldsymbol{I} \boldsymbol{d}-\tau \boldsymbol{K}_{0}\right)^{A}{ }_{B}=\frac{r_{0}-\tau}{r_{0}} \boldsymbol{\delta}_{B}^{A}$, is now immediate $\left[\left(\boldsymbol{I} \boldsymbol{d}-\tau \boldsymbol{K}_{0}\right)^{-1}\right]_{B}^{A}=\frac{r_{0}}{r_{0}-\tau} \boldsymbol{\delta}_{B}^{A}$, and the expression for $T$ is

$$
T^{L}=-\tau_{M}\left[\left(\boldsymbol{I d}-\tau \boldsymbol{K}_{0}\right)^{-1}\right]^{M L}=\frac{-r_{0}}{r_{0}-\tau} \tau^{, L} .
$$

The contraction $T^{L} K_{0 A L}=\frac{-1}{r_{0}-\tau} \tau_{, A}$ yields

$$
-T^{L}\left(\tau_{, A} K_{0 B L}+\tau_{{ }_{B}} K_{0 A L}\right)=\frac{2 \tau_{, A} \tau_{, B}}{r_{0}-\tau} .
$$




\section{Applications of Euclidean normal graphs to the shell Penrose inequality}

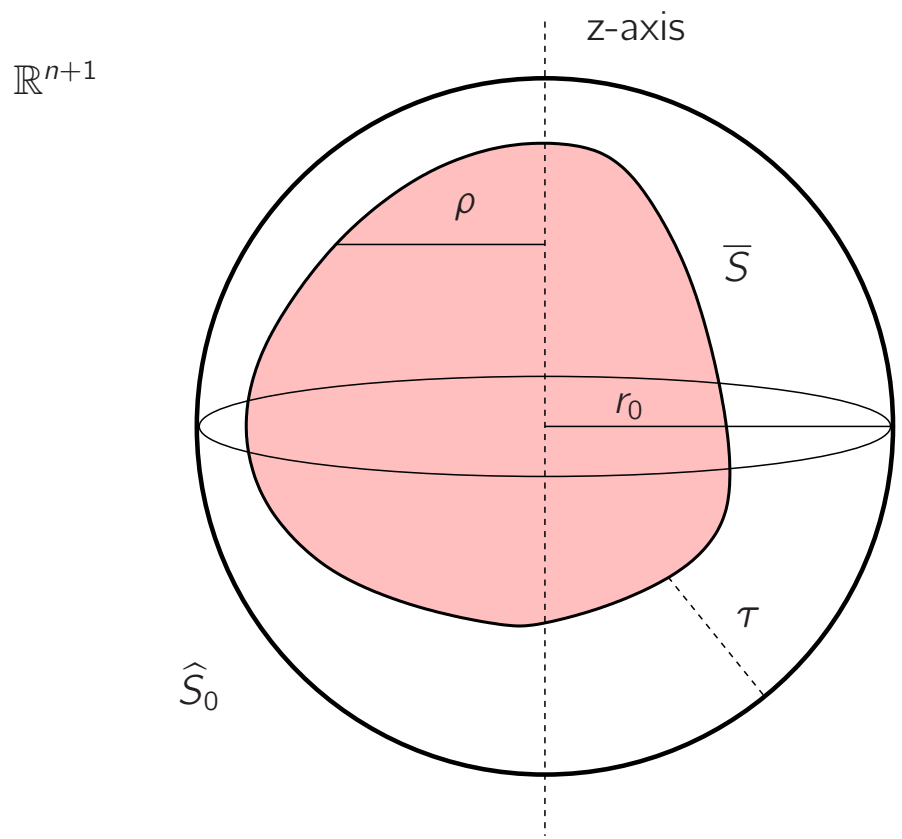

Figure 5.5: Particular case of construction in Figure 5.3, where the projection $\widehat{S}_{0}$ onto a constant time hyperplane $\Sigma_{t_{0}}$ of the spacetime convex surface $S$ embedded in $\mathcal{M}^{1,3}$ along its past null cone $\Omega$ is a sphere of radius $r_{0}$. As in Figure 5.4, for all those $\tau$ for which $\mathcal{T}$ is positive semidefinite, i.e. those ones that make $\bar{S} \subset \mathbb{R}^{n+1}$ convex, the shell Penrose inequality for $S$ holds.

Using all the above information, in spherical coordinates $\{\theta, \varphi\}$ non-negativity of the tensor $\mathcal{T}$ reads

$$
\mathcal{T}_{A B}=\left(\frac{r_{0}-\tau}{r_{0}^{2}}\right) \gamma_{0 A B}+D_{A} D_{B} \tau+\frac{2}{r_{0}-\tau} \tau_{, A} \tau_{, B} \geq 0 .
$$

A direct computation gives that the Hessian of $\tau$ is $D_{A} D_{B} \tau=\tau_{, A B}+\sin \theta \cos \theta \tau_{, \theta} \boldsymbol{\delta}_{A}^{\varphi} \boldsymbol{\delta}_{B}^{\varphi}-$ $\frac{\cos \theta}{\sin \theta} \tau_{, \varphi}\left(\boldsymbol{\delta}_{A}^{\theta} \boldsymbol{\delta}_{B}^{\varphi}+\boldsymbol{\delta}_{A}^{\varphi} \boldsymbol{\delta}_{B}^{\theta}\right)$. In the case that $S$ is axially symmetric, the conditions $\mathcal{T}_{\theta \theta} \geq 0$ and $\mathcal{T}_{\varphi \varphi} \geq 0$ are equivalent to (in spherical coordinates where $\tau(\theta)$ )

$$
\left(r_{0}-\tau\right)^{2}+\left(r_{0}-\tau\right) \tau_{, \theta \theta}+2\left(\tau_{, \theta}\right)^{2} \geq 0, \quad\left(r_{0}-\tau\right) \sin \theta+\cos \theta \tau_{, \theta} \geq 0
$$

Let us solve these inequalities in the strictly convex case (i.e. with strict inequalities in (5.17) $)$. With the definition $z(\theta):=\left(r_{0}-\tau(\theta)\right) \cos \theta$, the second inequality becomes immediately $z_{, \theta}<0$, which can be inverted to define $\theta(z)$. With the definition $\rho(\theta(z)):=\left.\left(r_{0}-\tau(\theta)\right) \sin \theta\right|_{\theta(z)}$, the first inequality becomes, $\rho_{, z z}<0$ as we show next. Using the chain rule we have

$$
\rho_{, z z}=\frac{d^{2} \rho}{d \theta^{2}}\left(\frac{d \theta}{d z}\right)^{2}+\frac{d \rho}{d \theta} \frac{d^{2} \theta}{d z^{2}} .
$$




\subsection{Matching the two different projections}

From $\frac{d \theta}{d z}=\frac{1}{z_{, \theta}}$, we have $\frac{d^{2} \theta}{d z^{2}}=\frac{-z_{, \theta \theta}}{\left(z_{\theta}\right)^{3}}$, which inserted into (5.18), transforms this expression into

$$
\rho_{, z z}=\frac{\rho_{, \theta \theta} Z_{, \theta}-\rho_{, \theta} z_{\theta \theta}}{\left(z_{, \theta}\right)^{3}}
$$

Hence the condition $\rho_{, z z}<0$ is equivalent to $\rho_{\theta \theta} z_{\theta}-\rho_{\theta} z_{, \theta \theta}>0$ after taking into account that $z_{, \theta}<0$. On the other hand, the definitions of $\rho$ and $z$ imply

$$
\begin{aligned}
\rho_{, \theta} & =-\tau_{, \theta} \sin \theta+\left(r_{0}-\tau\right) \cos \theta, \\
\rho_{, \theta \theta} & =-\left(\tau_{, \theta \theta}+r_{0}-\tau\right) \sin \theta-2 \tau_{, \theta} \cos \theta,
\end{aligned}
$$

and

$$
\begin{aligned}
z_{, \theta} & =-\tau_{, \theta} \cos \theta-\left(r_{0}-\tau\right) \sin \theta, \\
z_{, \theta \theta} & =-\left(\tau_{, \theta \theta}+r_{0}-\tau\right) \cos \theta+2 \tau_{, \theta} \sin \theta .
\end{aligned}
$$

The combination $\rho_{, \theta \theta} Z_{, \theta}-\rho_{, \theta} z_{, \theta \theta}$ is

$$
\rho_{, \theta \theta} Z_{, \theta}-\rho_{, \theta} Z_{, \theta \theta}=\left(r_{0}-\tau\right)^{2}+\left(r_{0}-\tau\right) \tau_{, \theta \theta}+2\left(\tau_{, \theta}\right)^{2} \geq 0,
$$

which implies that the first inequality in (5.17) is equivalent to $\rho_{, z z} \leq 0$ as claimed. Note also that

$$
\rho-z \rho_{, z}=\rho-\rho_{, \theta} \frac{z}{z_{, \theta}}=-\frac{\left(r_{0}-\tau\right)^{2}}{z_{, \theta}}>0
$$

as a consequence of their definitions. Conversely, let $\rho(z)$ satisfy $\rho_{, z z}<0$ and $\rho-z \rho_{, z}>$ 0 . We want to define the function $z(\theta)$ by using the implicit function theorem in the relation

$$
\cos \theta=\left.z\left(\sqrt{z^{2}+\rho(z)^{2}}\right)^{-1}\right|_{z=z(\theta)} .
$$

This can be achieved by considering the function $F(\theta, z)=z\left(\sqrt{z^{2}+\rho(z)^{2}}\right)^{-1}-\cos \theta$ (note that (5.19) ) is equivalent to $F(\theta, z)=0$ ). It holds $\frac{\partial F}{\partial z}=\frac{\rho\left(\rho-z \rho_{. z}\right)}{\left(z^{2}+\rho^{2}\right) \sqrt{z^{2}+\rho^{2}}}>0$ from the conditions $\rho>0$ and $\rho-z \rho_{, z}>0$ and the implicit function theorem can be used to define $z(\theta)$. We can now construct a function $\tau(\theta)$ be means of

$$
\tau=r_{0}-\left.\sqrt{z^{2}+\rho(z)^{2}}\right|_{z=z(\theta)} .
$$

Then the surface $S$ defined by this time height over the sphere $\widehat{S}_{0}$ projects onto a convex euclidean surface $\bar{S}$ lying in the constant time hyperplane $\Sigma_{t_{0}}$, and therefore using Brendle and Wang's Theorem 3.3.7, we prove that the constructed surface $S$ satisfies the shell Penrose inequality.

We note that the Penrose inequality for surfaces $S$ lying in the past null cone of a point in the Minkowski spacetime has been established in full generality in [101] (for dimension 4) and in Chapter 4 of this thesis for any dimension. So, the second example above does not extend in any way the class of surfaces for which the inequality holds. 


\section{Applications of Euclidean normal graphs to the shell Penrose inequality}

However, besides giving us an idea of the proportion of surfaces in the null cone case covered by Theorem 3.3.7 in Chapter 3, it also provides a method to construct a wide family of axially symmetric surfaces $S$ for which the Penrose inequality holds. Indeed, assume now that $\widehat{S}_{0}$ is axially symmetric and consider axially symmetric functions $\tau$ on $\widehat{S}_{0}$ so that $\bar{S}$ is strictly convex. Let $e_{z}$ be the unit field tangent to the axis of symmetry and $e_{\rho}$ the unit field radially outward from the axis of symmetry. Define the two functions on $\widehat{S}_{0}$

$$
z(p):=\left.g_{E}\left(x-\tau \nu, e_{z}\right)\right|_{p}, \quad \rho(p):=\left.g_{E}\left(x-\tau \nu, e_{\rho}\right)\right|_{p},
$$

where $x$ is the position vector of a point $p$ on $\widehat{S}_{0}$ with respect to an origin on the axis of symmetry and $\nu$ the outward normal at $p$. The strict inequality $\mathcal{T}>0$ is equivalent to (i) $z$ being a coordinate on $\widehat{S}_{0}$ away from points on the axis of symmetry and (ii) $\rho(z)$ satisfying $\rho_{, z z}<0$. Conversely, given any function $\rho(z)$ satisfying $\rho_{, z z}<0$, if there are two maps $z, \tau: S \rightarrow \mathbb{R}$ solving the algebraic equations (5.20) with $\rho(p):=\rho(z(p)$ ), then the spacetime surface $S$ defined by this time height function over $\widehat{S}_{0}$ satisfies the Penrose inequality. The algebraic equations will be solvable provided the parametric surface $\{\rho(z), z, \varphi\}$ in cylindrical coordinates is a normal graph over $\widehat{S}_{0}$. It is obvious that this is not always the case, so restrictions are necessary. In the spherical case above, this restriction is precisely $\rho-z \rho_{z}>0$. 



\section{The Hawking energy along null AF hypersurfaces}

\subsection{Introduction}

From this chapter onwards we move on from the Minkowski case and start analyzing the null Penrose inequality in a general context. The main strategy we shall follow is a generalization of the ideas in Chapter 4 where the surface was dragged along the past null cone of the surface. As it will become clear in Chapter 7 , the method requires understanding in detail the limit of the Hawking energy along general flows by cross sections of $\Omega$.

Recall that the Hawking energy is a functional on surfaces which is known to approach the Bondi energy along flows approaching large spheres. The main purpose of this chapter is the study of the limit of the Hawking mass when this condition is relaxed. To do that we study the geometric elements (metric, extrinsic curvatures and connection one-form) on the leaves of general flows in terms of the geometric elements of a special reference flow which we call background foliation. This allows us to analyze their asymptotic behaviour at past null infinity. As a consequence, we obtain in Theorem 6.6.2 an expression for the limit of the Hawking mass along these general flows in terms of the geometry of the background foliation. These results were published in [76].

In the final part of the chapter, we particularize to those flows along $\Omega$ which tend to large spheres, and consider the one-to-one correspondence between their asymptotic limit at past null infinity and the solutions of the large sphere equation. The solutions of this equation are determined by three real parameters, which also determine a unique four-vector observer $u$ in an abstract Minkowski spacetime that is assigned to the flow. It is well known that the limit of the Hawking energy along such flow is the Bondi energy $E_{B}^{u}$ associated to the observer $u$, i.e. the component along $u$ of an energy-momentum four vector $P_{B}$. Our results allow us to give (Corollary 6.7.3) an explicit expression for $P_{B}$ in terms of the background foliation that appears to be new. 


\subsection{Preliminaries}

In the previous chapter we were mainly working in the Minkowski spacetime. Let us go back to a more general setting dealing with a time-oriented spacetime $(M, g)$ of dimension $n+2, n \geq 2$, admitting an embedded smooth, connected, null hypersurface $\Omega$. Let $k$ be a smooth, nowhere zero, future directed null vector field tangent to $\Omega$ (i.e. a null generator). Since the integral curves of $k$ are geodesics, there exists $Q_{k} \in \mathcal{F}(\Omega)$ such that $\nabla_{k} k=Q_{k} k$. We make the assumption that there is an embedded spacelike connected hypersurface $S_{0}$ in $\Omega$ (with embedding $\Phi_{0}$ ) such that any integral curve of $-k$ intersects $S_{0}$ precisely once. This implies the existence of a smooth map $\pi: \Omega \longrightarrow S_{0}$ (we identify $S_{0}$ with its image, the meaning being clear from the context) which sends $p \in \Omega$ to the intersection of the integral curve $\alpha_{p}^{k}$ of $-k$ passing through $p$ with $S_{0}$. The map $\pi$ is clearly a submersion. We choose the parameter $\lambda$ of the curve $\alpha_{p}^{k}$ so that $\alpha_{p}^{k}(0)=p$.

Given $k$ and $S_{0}$, a scalar function $\lambda \in \mathcal{F}(\Omega)$ is defined by $k(\lambda)=-1$ and $\lambda(p)=0$ for all $p \in S_{0}$. Let $\left(\lambda_{-}(p), \lambda_{+}(p)\right)$ be the range of the function $\lambda$ restricted to the curve $\alpha_{p}^{k}$. We also assume that the open interval $\left(\Lambda_{-}:=\sup _{S_{0}} \lambda_{-}, \Lambda_{+}:=\inf _{S_{0}} \lambda_{+}\right)$is non-empty. The function $\lambda$ having nowhere zero gradient, the level sets $S_{\lambda_{1}}=\left\{\lambda=\lambda_{1}\right\}$ are either empty or smooth embedded (not necessarily connected) hypersurfaces. The collection $\left\{S_{\lambda}\right\}$ is a foliation of $\Omega$. For $\lambda_{1} \in\left(\Lambda_{-}, \Lambda_{+}\right)$the hypersurfaces $S_{\lambda_{1}}$ are in fact connected and diffeomorphic to $S_{0}$.

At any $p \in \Omega$ let $\left.\ell\right|_{p} \in T_{p} M$ be the unique null vector field satisfying $\left.\langle k, \ell\rangle\right|_{p}=-2$ and $\left.\langle\ell, X\rangle\right|_{p}=0$ for any $X \in T_{p} S_{\lambda(p)} . S_{\lambda}$ is endowed with an induced metric $\gamma_{S_{\lambda}}$, with two null second fundamental forms $K^{k}, K^{\ell}$ and with a normal bundle connection one-form $s_{\ell}(X):=\frac{1}{2}\left\langle\nabla_{X} k, \ell\right\rangle, X \in \mathfrak{X}\left(S_{\lambda}\right)$.

In order to obtain the limit of the Hawking energy as described in Section 6.1, we need to relate the geometry of different spacelike surfaces embedded in $\Omega$. Consider a spacelike embedded hypersurface $S$ in $\Omega$ with embedding $\Phi: S \longrightarrow \Omega$ and let $p \in S$. This hypersurface is uniquely defined by a diffeomorphism $\psi: S \longrightarrow \psi(S) \subset S_{0}$ and a function $F \in \mathcal{F}(S)$ as follows. For all $p \in S$ define $F(p)=\lambda(\Phi(p))$ and $\Psi(p)=(\pi \circ \Phi)(p)$. Conversely, a function $F \in \mathcal{F}(S)$ with image in $\left(\lambda_{-}(p), \lambda_{+}(p)\right)$ and a diffeomorphism $\psi$ as above defines an embedding (see Figure 6.1)

$$
\begin{aligned}
\Phi: S & \longrightarrow \Omega \\
& p \longrightarrow \alpha_{\psi(p)}^{k}(\lambda=F(p)) .
\end{aligned}
$$

We want to relate the intrinsic and extrinsic geometry of $S$ at $p$ with the geometry of the surface $S_{\lambda=F(p)}$. Since this is all local we can assume $\Psi(S)=S_{0}$, which makes the presentation simpler. We extend $F$ to a function on $\Omega$ defined by $F(q)=F\left(\left(\Psi^{-1}\right.\right.$ o $\pi)(q))$. We keep the same symbol for the extension. It is clear that $k(F)=0$. For 


\section{The Hawking energy along null AF hypersurfaces}

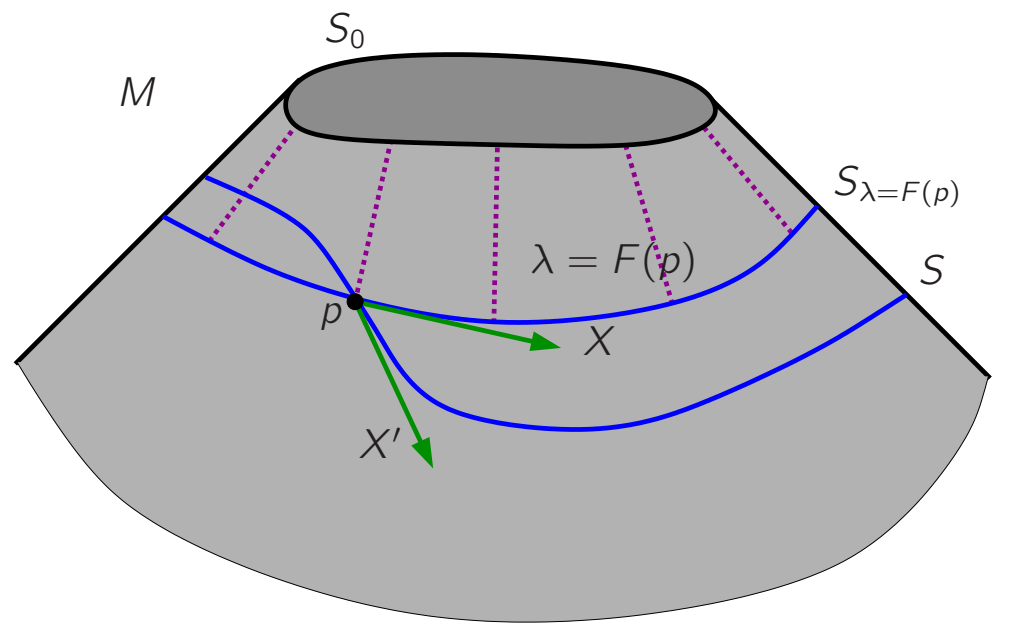

Figure 6.1: Embedding of the surface $S$ in the past null cone $\Omega$ of $S_{0}$. The flow $\left\{S_{\lambda}\right\}$ of surfaces along $\Omega$ defined as the level sets of the function $\lambda: \Omega \rightarrow \mathbb{R}$ satisfying $k(\lambda)=-1$, and $\left.\lambda\right|_{S_{0}}=0$. The "distance" along $\Omega$ from $S_{0}$ to $S$ is given by the graph function $F: S \rightarrow \mathbb{R}$. For a point $p \in S$, and the surface $S_{\lambda=F(p)}$ of the flow, we can rewrite the tangent vectors, metric, second fundamental forms along $k$ and $\ell_{S}$, and the connection one form $s_{\ell_{S}}$ of $S$ in terms of the geometry of the leaf of the flow $S_{\lambda=F(p)}$.

the extrinsic geometry of $S$ (we again identify $S$ with its image) we define at $p \in S$, the null normal $\left.\ell_{S}\right|_{p}$ by the conditions $\left.\left\langle\ell_{S}, k\right\rangle\right|_{p}=-2$ and $\left\langle\ell_{S}, X\right\rangle=0$ for all $X \in T_{p} S$. The induced metric $\gamma_{S}$, the null second fundamental forms $K^{\ell_{s}}, K^{k}$ and the normal connection one-form $s_{\ell_{S}}$ are defined similarly as before. The following proposition is known (see e.g [93]) when the background foliation $\left\{S_{\lambda}\right\}$ is affine (i.e. $Q^{k}=0$ ). Although this is the situation we will require later, we include for the sake of generality the non-affine case as well. Our proof also follows a somewhat different approach.

Proposition 6.2.1. Let $p \in S$, then the map

$$
\begin{aligned}
T_{F}: T_{p} S_{\lambda=F(p)} & \longrightarrow T_{p} S \\
X & \longrightarrow X^{\prime}:=X-X(F) k
\end{aligned}
$$

is a well-defined isomorphism. The induced metric $\gamma_{S}$, null second fundamental forms $K^{\ell_{S}}, K^{k}$ and normal bundle connection $s_{\ell_{S}}$ of $S$ are given by

$$
\begin{aligned}
\left.\gamma_{S}\right|_{p}\left(X^{\prime}, Y^{\prime}\right)= & \gamma(X, Y) \\
\left.K^{k}\left(X^{\prime}, Y^{\prime}\right)\right|_{p}= & K^{k}(X, Y), \\
\left.s_{\ell_{S}}\left(X^{\prime}\right)\right|_{p}= & s_{\boldsymbol{\ell}}(X)-K^{k}(X, \operatorname{grad} F)+X(F) Q_{k}, \\
\left.K^{\ell_{S}}\left(X^{\prime}, Y^{\prime}\right)\right|_{p}= & K^{\ell}(X, Y)+|D F|^{2} K^{k}(X, Y)+2 X(F) s_{\boldsymbol{\ell}}(Y)+2 Y(F) s_{\boldsymbol{\ell}}(X) \\
& -2 X(F) K^{k}(Y, \operatorname{grad} F)-2 Y(F) K^{k}(X, \operatorname{grad} F)-2 \operatorname{Hess} F(X, Y) \\
& +2 Q_{k} X(F) Y(F),
\end{aligned}
$$


where $\gamma, K^{k}, K^{\ell}, s_{\ell}$, grad, Hess and $|D F|^{2}=\langle\operatorname{grad} F, \operatorname{grad} F\rangle$ refer to the surface $S_{\lambda=F(p)}$ and are evaluated at $p$.

The proof is based on the following simple identity that may be useful in other contexts.

Lemma 6.2.2. Let $S$ be an embedded spacelike surface with embedding $\Psi: S \longrightarrow M$. Select a pair of null normal vector fields $\{k, \ell\}$ along $S$ satisfying $\langle k, \ell\rangle=-2$. For any vector field $\xi$ on a spacetime neighbourhood of $S$ write its deformation tensor as $£_{\xi} g:=a_{\xi}$. Then

$$
a_{\xi}(X, Y)=-\langle\xi, \ell\rangle K^{k}(X, Y)-\langle\xi, k\rangle K^{\ell}(X, Y)+\left(D_{X} \boldsymbol{\xi}^{\|}\right)(Y)+\left(D_{Y} \boldsymbol{\xi}^{\|}\right)(X),
$$

$X, Y \in \mathfrak{X}(S)$, where $D$ is the Levi-Civita covariant derivative of $S, \boldsymbol{\xi}^{\|}:=\Psi^{\star}(\boldsymbol{\xi})$ and $\boldsymbol{\xi}:=g(\xi, \cdot)$.

Remark 6.2.3. Given $\xi$ merely along $S$, this result can be applied to any extension of $\xi$ to a neighbourhood of $S$, the result being independent of the extension.

Proof. Decompose $\xi$ in tangential and normal parts $\xi=\xi^{\perp}+\xi^{\|}$and $\xi^{\perp}$ in the null basis $\{k, \ell\}$ as in (3.26), i.e., $\xi=-\frac{1}{2}\langle\xi, \ell\rangle k-\frac{1}{2}\langle\xi, k\rangle \ell+\xi^{\|}$so that

$$
\begin{aligned}
a_{\xi}(X, Y) & =\left\langle\nabla_{X} \xi, Y\right\rangle+\left\langle\nabla_{Y} \xi, X\right\rangle \\
& =-\langle\xi, \ell\rangle K^{k}(X, Y)-\langle\xi, k\rangle K^{\ell}(X, Y)+\left\langle\nabla_{X} \xi^{\|}, Y\right\rangle+\left\langle\nabla_{Y} \xi^{\|}, X\right\rangle \\
& =-\langle\xi, \ell\rangle K^{k}(X, Y)-\langle\xi, k\rangle K^{\ell}(X, Y)+\left\langle D_{X} \xi^{\|}, Y\right\rangle+\left\langle D_{Y} \xi^{\|}, X\right\rangle \\
& =-\langle\xi, \ell\rangle K^{k}(X, Y)-\langle\xi, k\rangle K^{\ell}(X, Y)+\left(D_{X} \boldsymbol{\xi}^{\|}\right)(Y)+\left(D_{Y} \boldsymbol{\xi}^{\|}\right)(X) .
\end{aligned}
$$

Proof of Proposition 6.2.1. $T_{F}$ is well-defined provided $X-X(F) k$ is tangent to $S$. This follows because $S$ is defined by $\lambda-F=0$ (note that $d(\lambda-F) \neq 0$ everywhere) and $\mathcal{L}_{X-X(F) k}(\lambda-F)=-X(F)-X(F) k(\lambda)=0$. $T_{F}$ is obviously injective, hence an isomorphism. Properties (6.1) and (6.2) are well-known. Anyhow we also prove them for completeness. First of all,

$$
\gamma_{S}\left(X^{\prime}, Y^{\prime}\right)=\langle X-X(F) k, Y-Y(F) k\rangle=\langle X, Y\rangle=\gamma(X, Y)
$$

and also

$$
\begin{aligned}
K^{k}\left(X^{\prime}, Y^{\prime}\right) & =\left\langle\nabla_{X^{\prime}} k, Y^{\prime}\right\rangle=\left\langle\nabla_{X-X(F) k} k, Y-Y(F) k\right\rangle \\
& =\left\langle\nabla_{X} k-X(F) Q_{k} k, Y-Y(F) k\right\rangle=\left\langle\nabla_{X} k, Y\right\rangle=K^{k}(X, Y) .
\end{aligned}
$$

These two identities recover the fact that the objects $\gamma$ and $K^{k}$ are intrinsic to $\Omega$ at $p$ and independent of the details of how $S$ containing $p$ is embedded in $\Omega$. 


\section{The Hawking energy along null AF hypersurfaces}

For the remaining parts of the proposition we note the decomposition

$$
\left.\ell_{S}\right|_{p}=\ell+|D F|^{2} k-\left.2 \operatorname{grad} F\right|_{p}, \quad p \in S,
$$

which holds because the right-hand side is null:

$$
\begin{aligned}
\left\langle\ell_{s}, \ell_{S}\right\rangle & =\left\langle\ell+|D F|^{2} k-2 \operatorname{grad} F, \ell+|D F|^{2} k-2 \operatorname{grad} F\right\rangle \\
& =-4|D F|^{2}-4 \ell(F)+4|D F|^{2}=0
\end{aligned}
$$

is orthogonal to $X^{\prime}=T_{F}(X)$, for all $X \in T_{p} S$ :

$\left\langle\ell_{S}, X^{\prime}\right\rangle=\left\langle\ell+|D F|^{2} k-2 \operatorname{grad} F, X-X(F) k\right\rangle=2 X(F)-2 X(F)+2 X(F) k(F)=0 ;$

and clearly satisfies $\left\langle\ell_{s}, k\right\rangle=-2$.

To show (6.3) we compute

$$
\begin{aligned}
s_{\ell_{S}}\left(X^{\prime}\right) & =-\frac{1}{2}\left\langle\nabla_{X^{\prime} \ell_{S}}, k\right\rangle=\frac{1}{2}\left\langle\nabla_{X^{\prime}} k, \ell_{S}\right\rangle=\frac{1}{2}\left\langle\nabla_{X} k-X(F) \nabla_{k} k, \ell_{S}\right\rangle \\
& =s_{\ell}(X)-K^{k}(X, \operatorname{grad} F)+X(F) Q_{k} .
\end{aligned}
$$

For the null extrinsic curvature $K^{\ell_{S}}$ we use Lemma 6.2.2. First observe that the righthand side of (6.2) makes sense for all $p \in \Omega$, so it defines an extension of $\ell_{S}$ which remains null and satisfying $\left\langle\ell_{S}, k\right\rangle=-2$. Extend also $Y \in T_{p} S_{\lambda=F(p)}$ to a neighbourhood under the condition that remains tangent to the foliation $\left\{S_{\lambda}\right\}$. This induces an extension of $Y^{\prime}$ which remains orthogonal to $\ell_{S}$. Note

$$
\left[k, Y^{\prime}\right]=[k, Y]-k(Y(F)) k=[k, Y]-[k, Y](F)=([k, Y])^{\prime},
$$

which shows that $\left[k, Y^{\prime}\right]$ is tangent to $S$ at $p$ (and we used that $\left.[k, Y]\right|_{p}$ is tangent to $S_{\lambda=F(p)}$ ). We apply Lemma 6.2.2 on the surface $S_{\lambda=F(p)}$ and to the vector field $\ell_{S}$. Concerning the deformation tensor

$$
\begin{aligned}
& \left\langle\nabla_{X} \ell_{S}, Y\right\rangle=\left\langle\nabla_{X^{\prime}+X(F) k} \ell_{S}, Y^{\prime}+Y(F) k\right\rangle \\
& =\left\langle\nabla_{X^{\prime}} \ell_{S}, Y^{\prime}\right\rangle+X(F)\left\langle\nabla_{k} \ell_{S}, Y^{\prime}\right\rangle+Y(F)\left\langle\nabla_{X^{\prime}} \ell_{S}, k\right\rangle+X(F) Y(F)\left\langle\nabla_{k} \ell_{S}, k\right\rangle \\
& =K^{\ell_{S}}\left(X^{\prime}, Y^{\prime}\right)-X(F)\left\langle\nabla_{k} Y^{\prime}, \ell_{S}\right\rangle-2 Y(F) s_{\ell_{S}}\left(X^{\prime}\right)-X(F) Y(F)\left\langle\nabla_{k} k, \ell_{S}\right\rangle \\
& =K^{\ell_{S}}\left(X^{\prime}, Y^{\prime}\right)-X(F)\left\langle\left[k, Y^{\prime}\right]+\nabla_{Y^{\prime}} k, \ell_{S}\right\rangle-2 Y(F) s_{\ell_{S}}\left(X^{\prime}\right) \\
& +2 Q_{k} X(F) Y(F) \\
& =K^{\ell_{S}}\left(X^{\prime}, Y^{\prime}\right)-2 X(F) s_{\ell_{S}}\left(Y^{\prime}\right)-2 Y(F) s_{\ell_{S}}\left(X^{\prime}\right)+2 Q_{k} X(F) Y(F) \text {, }
\end{aligned}
$$

where in the last equality we used that $\left.\left[k, Y^{\prime}\right]\right|_{p}$ is tangent to $S$. Hence

$$
\begin{aligned}
a_{\ell_{S}}(X, Y)= & 2 K^{\ell_{S}}\left(X^{\prime}, Y^{\prime}\right)-4 X(F) s_{\ell_{S}}\left(Y^{\prime}\right)-4 Y(F) s_{\ell_{S}}\left(X^{\prime}\right)+4 Q_{k} X(F) Y(F) \\
= & 2 K^{\ell_{S}}\left(X^{\prime}, Y^{\prime}\right)-4 X(F) s_{\ell}(Y)-4 Y(F) s_{\ell}(X)+4 X(F) K^{k}(Y, \operatorname{grad} F) \\
& +4 Y(F) K^{k}(X, \operatorname{grad} F)-4 Q_{k} X(F) Y(F)
\end{aligned}
$$


after using (6.3) in the second equality. Now Lemma 6.2 .2 gives

$$
a_{\ell_{S}}(X, Y)=2|D F|^{2} K^{k}(X, Y)+2 K^{\ell}(X, Y)-4 \operatorname{Hess} F(X, Y) \text {. }
$$

Solving for $K^{\ell_{S}}\left(X^{\prime}, Y^{\prime}\right)$ in (6.5) and (6.6) yields the result.

The following corollary is a consequence of how the null second fundamental forms and the normal bundle connection transforms under a boost in $\left\{\ell_{S}, k\right\}$.

Corollary 6.2.4. Let $S$ as before and for all $p \in S$ let $\left.k^{\prime}\right|_{p}=\left.\alpha(p) k\right|_{p}$ and $\left.\ell_{S}^{\prime}\right|_{p}=\frac{1}{\alpha(p)} \ell_{S}$, where $\alpha: S \mapsto \mathbb{R}$ is a smooth positive function extended to $\Omega$ by $k(\alpha)=0$. Then

$$
\begin{aligned}
s_{\ell_{S}^{\prime}}\left(X^{\prime}\right),= & s_{\boldsymbol{\ell}}(X)-K^{k}(X, \operatorname{grad} F)+X(F) Q_{k}-\frac{1}{\alpha} X(\alpha), \\
K^{k^{\prime}}\left(X^{\prime}, Y^{\prime}\right)= & \alpha K^{k}(X, Y), \\
\left.K^{\ell^{\prime}}\left(X^{\prime}, Y^{\prime}\right)\right|_{p}=\frac{1}{\alpha} & \left(K^{\ell}(X, Y)+|D F|^{2} K^{k}(X, Y)+2 X(F) s_{\ell}(Y)+2 Y(F) s_{\ell}(X)\right. \\
& -2 H \operatorname{Hess} F(X, Y)-2 X(F) K^{k}(Y, \operatorname{grad} F)-2 Y(F) K^{k}(X, \operatorname{grad} F) \\
& \left.+2 Q_{k} X(F) Y(F)\right) .
\end{aligned}
$$

Proof. We first compute how $\boldsymbol{s}_{\boldsymbol{\ell}}$ transforms under change of $\ell$ :

$$
\begin{aligned}
s_{\ell_{S}^{\prime}}\left(X^{\prime}\right) & =\frac{1}{2}\left\langle\nabla_{X^{\prime}} k^{\prime}, \ell_{S}^{\prime}\right\rangle=\frac{1}{2}\left\langle\nabla_{X^{\prime}}(\alpha k), \frac{1}{\alpha} \ell_{S}\right\rangle=\frac{1}{2}\left\langle X^{\prime}(\alpha) k+\alpha \nabla_{X^{\prime}} k, \frac{1}{\alpha} \ell_{S}\right\rangle \\
& =\frac{-X^{\prime}(\alpha)}{\alpha}+s_{\ell_{S}}\left(X^{\prime}\right) .
\end{aligned}
$$

Using relation (6.3) and $X^{\prime}(\alpha)=X(\alpha)-X(F) k(\alpha)=X(\alpha)$ yields (6.7). The change of $K^{k^{\prime}}$ under rescaling is very simple:

$$
K^{k^{\prime}}\left(X^{\prime}, Y^{\prime}\right)=\left\langle\nabla_{X^{\prime}}(\alpha k), Y^{\prime}\right\rangle=\left\langle X^{\prime}(\alpha) k+\alpha \nabla_{X^{\prime}} k, Y^{\prime}\right\rangle=\alpha K^{k}\left(X^{\prime}, Y^{\prime}\right),
$$

which in combination with (6.2) gives (6.8).

An analogous reasoning gives $K^{\ell_{S}^{\prime}}\left(X^{\prime}, Y^{\prime}\right)=\frac{1}{\alpha} K^{\ell_{S}}\left(X^{\prime}, Y^{\prime}\right)$, and (6.9) follows from (6.4).

The trace of $K^{k}$ and $K^{\ell}$ on $S_{\lambda}$ with the induced metric define the null expansions of $S_{\lambda}$ and are denoted respectively as $\theta_{k}$ and $\theta_{\ell}$. The relationship between the null expansions $\theta_{k}, \theta_{\ell_{S}}$ of a graph $S$ with the corresponding ones at the level set $S_{\lambda=F(p)}$ follow from Proposition 6.2.1. 


\section{The Hawking energy along null AF hypersurfaces}

Corollary 6.2.5. Let $S, k^{\prime}$ and $\ell_{S}^{\prime}$ as in Corollary 6.2.4. The null expansions $\theta_{k^{\prime}}$ and $\theta_{\ell^{\prime}}$ at $p \in S$ and the null expansions $\theta_{k}, \theta_{\ell}$ of $S_{\lambda=F(p)}$ at $p$ are related by

$$
\begin{aligned}
& \theta_{k^{\prime}}=\alpha \theta_{k}, \\
& \theta_{\ell_{s}^{\prime}}=\frac{1}{\alpha}\left(\theta_{\ell}+|D F|^{2} \theta_{k}+4 s_{\ell}(\operatorname{grad} F)-4 K^{k}(\operatorname{grad} F, \operatorname{grad} F)-2 \Delta F+2 Q_{k}|D F|^{2}\right),
\end{aligned}
$$

where $\Delta F$ is the Laplacian of $S_{\lambda}$ with the induced metric.

Another useful identity that will play a role later is the evolution equation for the connection of the normal bundle. The proof of the following result is given in Appendix B, formula (B.39):

Proposition 6.2.6. With the same notation as above, let $X \in \mathfrak{X}(\Omega)$ be a vector field satisfying $[k, X]=0$ and tangent to $S$. Then

$$
k\left(s_{\ell}(X)\right)=-X\left(Q_{k}\right)-s_{\ell}(X) \theta_{k}+\left(\operatorname{div}_{S_{r}} K^{k}\right)(X)-D_{X} \theta_{k}-\operatorname{Ein}^{g}(k, X),
$$

where $\operatorname{Ein}^{g}$ is the Einstein tensor of $(M, g)$.

\subsection{Null asymptotic flatness of $\Omega$ and asymptotic be- haviour}

The previous section involved general properties of $\Omega$ of local nature and valid in any spacetime dimension. We now impose global conditions and restrict to dimension four. First of all we assume that $\Omega$ admits a global cross section $S_{0}$ (i.e. a smooth embedded spacelike surface intersected precisely once by every inextendible curve along the null generators) of spherical topology $S_{0}$. We also assume that for one (and hence any) choice of affine null generator $k$ (i.e. satisfying $\nabla_{k} k=0$ ) the corresponding integral curve starting at $p \in S_{0}$ has maximal domain $\left(-\infty, \lambda_{+}(p)\right)$, i.e. the null generators are past complete. After possibly removing portions of $\Omega$ lying to the future of $S_{0}$ we can assume that $\Omega$ is foliated by the level sets $\left\{S_{\lambda}\right\}$ of the function $\lambda \in \mathcal{F}(\Omega)$ defined by $k(\lambda)=-1,\left.\lambda\right|_{S_{0}}=0$ and that all these level sets are diffeomorphic to $S_{0}$ (so that in particular $\Omega=S_{0} \times(\rho, \infty)$ ). The function $\lambda$ is called level set function of $k$. If we change the selection of null affine generator $k$, the set of points to be removed is different, but since we are only interested in the past of $S_{0}$ this is irrelevant, and we keep the same name $\Omega$. A null hypersurface $\Omega$ satisfying these properties is called extending to past null infinity, and as mentioned in Section 6.1, it generalizes the concept of spacetime convex null hypersurface defined in Chapter 4 for hypersurfaces in the Minkowski spacetime that also extend to past null infinity. 


\subsection{Null asymptotic flatness of $\Omega$ and asymptotic behaviour}

In order to define asymptotic flatness along $\Omega$ we need to impose decay of various objects at infinity. First note the existence of covariant tensor fields $T$ on $\Omega$ completely orthogonal to $k$ (i.e. satisfying $T(k, \cdots)=T(\ldots, k)=0)$. We call such tensors transversal. An immediate example is the first fundamental form $\gamma$. The collection of second fundamental forms $K_{S_{\lambda}}^{k}$ defines a transversal tensor denoted simply by $K^{k}$ (this is compatible with the notation already used in the previous section). A tensor field $T$ (not necessarily transversal) is called Lie constant if and only if $£_{k} T=0$.

A local basis $\left\{X_{A}\right\}$ for any cross section of $\mathfrak{X}(\Omega)$ extended by $\left[k, X_{A}\right]=0$ implies

$$
\left(£_{k} T\right)\left(X_{A_{1}}, \cdots, X_{A_{q}}\right)=k\left(T\left(X_{A_{1}}, \cdots, X_{A_{q}}\right)\right) .
$$

The following result shows the relation of transversal and Lie constant tensors with collections of tensors just defined on the leaves of the foliation $\left\{S_{\lambda}\right\}$ along $\Omega$ :

Lemma 6.3.1. A transversal tensor $T$ on $\Omega$ is in one-to-one correspondence with a smooth collection of covariant tensor fields $\{T(\lambda)\}$ with each $T(\lambda)$ defined on each level set $S_{\lambda}$. If in addition $T$ is Lie constant, then $T$ is in one-to-one correspondence with covariant tensors $\hat{T}$ on a fixed leaf $S_{\lambda}$. Moreover, $T(\lambda)$ are all diffeomorphically related to each other and to $\hat{T}$.

Proof. Assume first that $T\left(X_{A_{1}}, \cdots, X_{A_{q}}\right)$ defined on $\Omega$ is transversal. Then for each $\lambda$, and $\left\{X_{A_{1}}^{\lambda}, \cdots, X_{A_{q}}^{\lambda}\right\}$ tangent to $\mathfrak{X}\left(S_{\lambda}\right)$, we define the family $\{T(\lambda)\}$ as

$$
T(\lambda)\left(X_{A_{1}}^{\lambda}, \cdots, X_{A_{q}}^{\lambda}\right):=\left.T\right|_{S_{\lambda}}\left(X_{A_{1}}^{\lambda}, \cdots, X_{A_{q}}^{\lambda}\right)=T\left(X_{A_{1}}^{\lambda}, \cdots, X_{A_{q}}^{\lambda}\right) .
$$

Conversely, given a family $\{T(\lambda)\}$, with $T(\lambda)$ defined on $S_{\lambda}$, we define the following tensor $T$ on $\Omega$. For any $p \in \Omega$, there is a unique $\lambda^{\star}$ so that $p \in S_{\lambda^{\star}}$. Each $X_{A_{i}} \in T_{p} \Omega$ can be decomposed as

$$
X_{A_{i}}=X_{A_{i}}^{\lambda^{\star}}+\alpha k
$$

where $X_{A_{i}}^{\lambda^{\star}} \in \mathfrak{X}\left(S_{\lambda^{\star}}\right)$. With the definition

$$
T_{p}\left(X_{A_{1}}, \cdots, X_{A_{q}}\right):=T_{p}\left(\lambda^{\star}\right)\left(X_{A_{1}}^{\lambda^{\star}}, \cdots, X_{A_{q}}^{\lambda^{\star}}\right),
$$

$T$ is transversal, and the first statement of the Lemma is proved. If in addition a transversal $T$ is also Lie constant, we have that

$$
0=\left(£_{k} T\right)\left(X_{A_{1}}, \cdots, X_{A_{q}}\right)=k\left(T\left(X_{A_{1}}, \cdots, X_{A_{q}}\right)\right) \text {, }
$$

provided $X_{A_{i}}$ satisfy $\left[k, X_{A_{i}}\right]=0$. Thus $T\left(X_{A_{1}}, \cdots, X_{A_{q}}\right)$ is constant along $k$. For any a priori chosen $\hat{\lambda}$, let us define $\hat{T}:=T(\hat{\lambda})$. This defines $\hat{T}$ on $S_{\hat{\lambda}}$ uniquely.

Conversely given a fixed $\hat{\lambda}$ and a covariant tensor $\hat{T}$ on $S_{\hat{\lambda}}$, we can build a tensor $T$ on $\Omega$ transverse and Lie constant. Given any $p \in \Omega$, let $\hat{\pi}(p)$ be the unique point of $S_{\hat{\lambda}}$ 


\section{The Hawking energy along null AF hypersurfaces}

lying in the geodesic that contains $p$ and is tangent to $k$. Let $X_{A_{i}}$ be vectors in $T_{p} \Omega$ and extend them as usual by $\left[X_{A_{i}}, k\right]=0$ along $k$. At $\hat{\pi}(p)$ we decompose them as before, i.e.

$$
X_{A_{i}}=X_{A_{i}}^{\hat{\lambda}}+\alpha k,
$$

with $X_{A_{i}}^{\hat{\lambda}}$ tangent to $S_{\hat{\lambda}}$. The tensor field

$$
T_{p}\left(X_{A_{1}}, \cdots, X_{A_{q}}\right):=\hat{T}_{\hat{\pi}(p)}\left(X_{A_{1}}^{\hat{\lambda}}, \cdots, X_{A_{q}}^{\hat{\lambda}}\right)
$$

is transverse and Lie constant, as claimed. Observe that given an initial transversal tensor $T$ on $\Omega$ (not necessarily Lie constant), we can obtain an associated $\hat{T}$, and using (6.15) we define another tensor that in general is not the same as the initial $T$. If $T$ is Lie constant, both tensors coincide due to property (6.14).

We conclude by showing that in the Lie constant case the tensors $T(\lambda)$ are diffeomorphic to each other. Indeed take two different values $\lambda_{1}, \lambda_{2}$, and two different points $p_{1} \in S_{\lambda_{1}}$ and $p_{2} \in S_{\lambda_{2}}$ so that they lie in the same geodesic tangent to $k_{p_{1}}$. From (6.15),

$$
\begin{aligned}
T_{p_{1}}\left(\lambda_{1}\right)\left(X_{A_{1}}^{\lambda_{1}}, \cdots, X_{A_{q}}^{\lambda_{1}}\right) & =T_{p_{1}}\left(X_{A_{1}}^{\lambda_{1}}, \cdots, X_{A_{q}}^{\lambda_{1}}\right)=\hat{T}_{\hat{\pi}\left(p_{1}\right)}\left(X_{A_{1}}^{\hat{\lambda}}, \cdots, X_{A_{q}}^{\hat{\lambda}}\right) \\
& =\hat{T}_{\hat{\pi}\left(p_{2}\right)=\hat{\pi}\left(p_{1}\right)}\left(X_{A_{1}}^{\hat{\lambda}}, \cdots, X_{A_{q}}^{\hat{\lambda}}\right)=T_{p_{2}}\left(\lambda_{2}\right)\left(X_{A_{1}}^{\lambda_{2}}, \cdots, X_{A_{q}}^{\lambda_{2}}\right),
\end{aligned}
$$

and it is precisely in this sense that we say that $T\left(\lambda_{1}\right)$ and $T\left(\lambda_{2}\right)$ are diffeomorphically related to each other.

Remark 6.3.2. Using the above lemma, with the collections $K_{S_{\lambda}}^{\ell}$ and $\boldsymbol{s}_{\ell}\left(S_{\lambda}\right)$ we can define the corresponding transversal tensors $K^{\ell}$ and $s_{\ell}$.

Definition 6.3.3. A transversal tensor field $T$ is positive definite if and only if the corresponding collection of tensors $T(\lambda)$ is positive definite for all $\lambda$.

In the proof of Lemma 6.3.1 we have used vectors $X_{A}$ tangent to $\Omega$ (and not necessarily tangent to the leaves $S_{\lambda}$ of the foliation). For the sake of simplicity and in order to avoid using the decomposition (6.13), we consider a local basis $\left\{X_{A}\right\}$ on $S_{0}$ extended uniquely to $\mathfrak{X}(\Omega)$ by $\left[k, X_{A}\right]=0$. With this extension $\left\{X_{A}\right\}$ defines a basis of each level set $S_{\lambda}$. From now on $\left\{X_{A}\right\}$ will denote one basis constructed this way.

We shall say that a transversal tensor field $T$ on $\Omega$ is $T=O(1)$ if and only if $T_{A_{1} \cdots A_{q}}:=$ $T\left(X_{A_{1}}, \cdots, X_{A_{q}}\right)$ is uniformly bounded. We write $T=O_{n}\left(\lambda^{-q}\right), q \in \mathbb{R}, n \in \mathbb{N}$ if and only if

$$
\lambda^{q} T=O(1), \quad \lambda^{q+1} £_{k} T=O(1), \quad \cdots \quad, \lambda^{q+n} \underbrace{£_{k} \cdots £_{k} T}_{n}=O(1) .
$$




\subsection{Null asymptotic flatness of $\Omega$ and asymptotic behaviour}

We also write $T=O\left(\lambda^{-q}\right)$ if and only if $\lim _{\lambda \rightarrow \infty} \lambda^{q} T(\lambda)_{A_{1} \cdots A_{q}}=0$ and $T=O_{n}\left(\lambda^{-q}\right)$ if and only if $\lambda^{i+q}\left(£_{k}\right)^{i} T=o(1)$ for all $i=0,1, \cdots, n$. Given a transversal tensor (field) $T$ the tensor $£_{X_{A}} T$ is also transversal. We write $T=o_{n}^{X}\left(\lambda^{-q}\right)$ if and only if

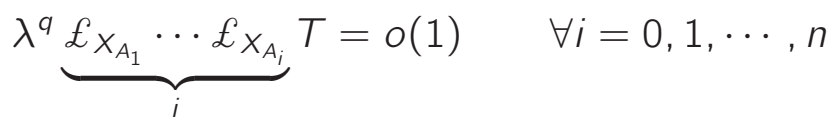

It is clear that all these definitions are independent of the choice of $\left\{X_{A}\right\}$.

Definition 6.3.4. Let $(M, g)$ be a four-dimensional spacetime. A null hypersurface $\Omega$ is past asymptotically flat if it extends to past null infinity and there exists a choice of cross section $S_{0}$ and null affine generator $k$ with corresponding level set function $\lambda$ with the following properties:

(i) There exist two symmetric 2-covariant transversal and Lie constant tensor fields $\hat{q}$ and $h$ such that $\tilde{\gamma}:=\gamma-\lambda^{2} \hat{q}-\lambda h$ is $\tilde{\gamma}=o_{1}(\lambda) \cap o_{2}^{X}(\lambda)$.

(ii) There exists a transversal, Lie constant one-form $\boldsymbol{s}_{\boldsymbol{\ell}}^{(1)}$ such that $\tilde{s}_{\boldsymbol{\ell}}:=\boldsymbol{s}_{\boldsymbol{\ell}}-\frac{\boldsymbol{s}_{\boldsymbol{\ell}}^{(1)}}{\lambda}$ is $\tilde{s}_{\ell}=o_{1}\left(\lambda^{-1}\right)$.

(iii) There exist Lie constant functions $\theta_{\ell}^{(0)}$ and $\theta_{\ell}^{(1)}$ such that $\tilde{\theta}_{\ell}:=\theta_{\ell}-\frac{\theta_{\ell}^{(0)}}{\lambda}-\frac{\theta_{\ell}^{(1)}}{\lambda^{2}}$ is $\tilde{\theta}_{\ell}=o\left(\lambda^{-2}\right)$.

(iv) The scalar $\operatorname{Riem}^{g}\left(X_{A}, X_{B}, X_{C}, X_{D}\right)$ along $\Omega$ is such that

$$
\lim _{\lambda \rightarrow \infty} \frac{1}{\lambda^{2}} \operatorname{Riem}^{g}\left(X_{A}, X_{B}, X_{C}, X_{D}\right)<\infty,
$$

and its double trace satisfies $2 \operatorname{Ein}^{g}(k, \ell)-\operatorname{Scal}^{g}-\frac{1}{2} \operatorname{Riem}^{g}(\ell, k, \ell, k)=o\left(\lambda^{-2}\right)$.

We remark that several parts of this definition involve foliation dependent quantities. However, the definition is intrinsic to the null hypersurface $\Omega$ because the definition requires the existence of a foliation with the desired properties.

This definition of asymptotic flatness is weaker in many aspects than most existing definitions. Compared, for instance, to the definition used in [23] we demand $\tilde{\gamma}=$ $o_{1}(\lambda) \cap o_{2}^{X}(\lambda)$ instead of the stronger condition $\tilde{\gamma}=O(1) \cap O_{2}^{X}(1)$ and $£_{k} \tilde{\gamma}=O_{1}\left(\lambda^{-2}\right)$ assumed there. We also emphasize that this definition is different to the global definition of asymptotic flatness discussed in Chapter 2 in connection with the heuristics of the Penrose inequality. The present definition only involves properties along $\Omega$ and makes no assumptions on the future evolution of the spacetime where $\Omega$ is embedded. This is in accordance with the spirit of the Penrose inequality where global assumptions are only allowed at one instant of "time".

Sometimes it will be convenient to supplement this definition with a stronger notion where additional decay for some components of the Einstein tensor and for the remainder 


\section{The Hawking energy along null AF hypersurfaces}

tensor $\tilde{\gamma}$ is assumed. Specifically, we say that an asymptotically flat null hypersurface $\Omega$ satisfies the energy flux decay condition if

$$
\left.\operatorname{Ein}^{g}\left(k, X_{A}\right)\right|_{\Omega}=o\left(\lambda^{-2}\right), \quad £_{k} \tilde{\gamma}=o_{1}^{X}(1) .
$$

The name is motivated by the analogous role of density flux that the Einstein tensor component $\operatorname{Ein}^{g}\left(k, X_{A}\right)$ plays in the constraint equations for null hypersurfaces (see e.g. [71).

Let us consider a past asymptotically flat null hypersurface $\Omega$ with a choice of $k$ and level set function $\lambda$. The following proposition determines the asymptotic expansion of $K^{k}$ and provides an explicit expression for $\theta_{\ell}^{(0)}$.

Proposition 6.3.5. Let $\Omega$ be a past asymptotically flat null hypersurface with a choice of affinely parametrized null generator $k$ and corresponding level set function $\lambda$. Let $\gamma(\lambda)^{A B}$ be the inverse of $\gamma(\lambda)$. Then

$$
\begin{aligned}
\gamma(\lambda)^{A B} & =\frac{1}{\lambda^{2}} \hat{q}^{A B}-\frac{1}{\lambda^{3}} \hat{h}^{A B}+o\left(\lambda^{-3}\right), \\
K_{A B}^{k} & =-\hat{q}_{A B} \lambda-\frac{1}{2} h_{A B}+o(1), \\
\theta_{\ell} & =\frac{2 \mathcal{K}_{\hat{q}}}{\lambda}+\frac{\theta_{\ell}^{(1)}}{\lambda^{2}}+o\left(\lambda^{-2}\right) .
\end{aligned}
$$

$\hat{q}^{A B}$ is the inverse of $\hat{q}_{A B}$, indices in hatted tensors are raised and lowered with these metrics and $\mathcal{K}_{\hat{q}}$ is the Gauss curvature of $\hat{q}_{A B}$.

Proof. Expression (6.16) is an immediate consequence of item (i) in the definition of asymptotic flatness, namely

$$
\gamma_{A B}=\hat{q}_{A B} \lambda^{2}+h_{A B} \lambda+o_{1}(\lambda) \cap o_{2}^{X}(\lambda) .
$$

For expression (6.17) we use the evolution equation (B.12) in Appendix B. Inserting (6.19) yields (6.17) immediately. Concerning the expansion for $\theta_{\ell}$ we invoke the Gauss identity for $\left(S_{\lambda}, \gamma(\lambda)\right)((\mathbb{A} .27)$ in Appendix $₫$ with $\varphi=2)$, which says

$$
\operatorname{Riem}_{A B C D}^{g}=\operatorname{Riem}_{A B C D}^{\gamma(\lambda)}-\frac{1}{2}\left(K_{B C}^{k} K_{A D}^{\ell}+K_{A D}^{k} K_{B C}^{\ell}\right)+\frac{1}{2}\left(K_{A C}^{k} K_{B D}^{\ell}+K_{B D}^{k} K_{A C}^{\ell}\right) .
$$

The decomposition (6.19) implies that $\operatorname{Riem}_{A B C D}^{\gamma(\lambda)}=\lambda^{2} \operatorname{Riem}_{A B C D}^{\hat{q}}+O(\lambda)$ and given that $K_{A B}^{k}=-\hat{q}_{A B} \lambda+O(1)$, it follows from item (iv) in Definition 6.3.4 that $K_{A B}^{\ell}$ is of the form

$$
K_{A B}^{\ell}=\lambda K_{(0) A B}^{\ell}+O(\lambda)
$$




\subsection{Null asymptotic flatness of $\Omega$ and asymptotic behaviour}

with $K_{(0)}^{\ell}$ a transverse Lie constant symmetric tensor on $\Omega$. The trace of the Gauss identity (equation (A.30) in Appendix A with $\varphi=2$ ) is

$$
2 \operatorname{Ein}^{g}(k, \ell)-\operatorname{Scal}^{g}-\frac{1}{2} \operatorname{Riem}^{g}(\ell, k, \ell, k)=2 \mathcal{K}_{\gamma(\lambda)}+\theta_{\ell} \theta_{k}-K_{A B}^{k} K^{\ell A B} .
$$

Now, $\mathcal{K}_{\gamma(\lambda)}=\frac{1}{\lambda^{2}} \mathcal{K}_{\hat{q}}+o\left(\lambda^{-2}\right)$ and the decompositions (6.16)-(6.17), (6.21) and the trace condition in item (iv) of Definition 6.3.4 imply

$$
0=\frac{1}{\lambda^{2}}\left(2 \mathcal{K}_{\hat{q}}-\theta_{\ell}^{(0)}\right)+o\left(\lambda^{-2}\right),
$$

which, together with item (iii) in Definition 6.3.4 proves (6.18).

Remark 6.3.6. Note that the expansion for $K^{k}$ only depends on item (i) in the definition of asymptotic flatness. The expression for $\theta_{\ell}^{(0)}$ depends on items (i), (iii) and (iv).

Remark 6.3.7. We can raise the index to the tensor $K^{k}(\lambda)$ with the contravariant metric $\gamma^{A B}$. Combining the asymptotic expansions (6.16) and (6.17) yields

$$
K^{k}(\lambda)_{B}^{A}=-\frac{1}{\lambda} \delta_{B}^{A}+\frac{1}{2} \hat{h}_{B}^{A} \frac{1}{\lambda^{2}}+o\left(\lambda^{-2}\right),
$$

and taking trace

$$
\theta_{k}=-\frac{2}{\lambda}+\frac{1}{2}\left(\operatorname{tr}_{\hat{q}} \hat{h}\right) \frac{1}{\lambda^{2}}+o\left(\lambda^{-2}\right):=-\frac{2}{\lambda}+\theta_{k}^{(1)} \frac{1}{\lambda^{2}}+o\left(\lambda^{-2}\right) .
$$

It will be convenient to endow each level set $\left\{S_{\lambda}\right\}$ with a covariant derivative independent of $\lambda$. The natural choice is $\hat{q}$ which is Lie constant, a metric on each $S_{\lambda}$ and gives the leading term of the asymptotic expansion of $\gamma(\lambda)$. Denote by $\hat{D}$ the covariant derivative of $\hat{q}$. In the following lemma we find the asymptotic expansion of the difference tensor

$$
D_{X} Y-\hat{D}_{X} Y=Z(X, Y)
$$

and apply it to relate the Laplacians on functions with respect to the metrics $\gamma(\lambda)$ and $\hat{q}$. This will be needed later when relating two different foliations on $\Omega$.

Lemma 6.3.8. The difference tensor $Z$ admits the decomposition

$$
Z_{A B}^{C}=\frac{1}{2}\left(\hat{D}_{A} \hat{h}_{B}^{C}+\hat{D}_{B} \hat{h}_{A}^{C}-\hat{D}^{C} h_{A B}\right) \frac{1}{\lambda}+O\left(\lambda^{-2}\right) .
$$

Moreover, if $F$ is a Lie constant function on $\Omega$ then

$$
\triangle_{\gamma} F=\triangle_{\hat{q}} F \frac{1}{\lambda^{2}}+\left(-\hat{h}^{A B} \hat{D}_{A} \hat{D}_{B} F-\left(\hat{D}_{A} \hat{h}^{C A}\right) F_{, C}+\left(\hat{D}^{C} \theta_{k}^{(1)}\right) F_{, C}\right) \frac{1}{\lambda^{3}}+o\left(\lambda^{-3}\right) .
$$




\section{The Hawking energy along null AF hypersurfaces}

Proof. We use the general formula for the difference tensor of Levi-Civita covariant derivatives, see e.g. [106],

$$
Z_{A B}^{C}=\frac{1}{2} \gamma^{C D}\left(\hat{D}_{A} \gamma_{D B}+\hat{D}_{B} \gamma_{D A}-\hat{D}_{D} \gamma_{A B}\right)
$$

Given that $\hat{D}_{A} \gamma_{D B}=\left(\hat{D}_{A} h_{D B}\right) \lambda+O(1)$ and $\gamma^{C D}=\frac{1}{\lambda^{2}} \hat{q}^{C D}+O\left(\lambda^{-3}\right)$, expression (6.24) follows. For the Laplacian we use

$$
\begin{aligned}
\triangle_{\gamma} F= & \gamma^{A B} D_{A} D_{B} F=\gamma^{A B}\left(\hat{D}_{A} \hat{D}_{B} F-Z_{A B}^{C} \hat{D}_{C} F\right) \\
= & \left(\frac{1}{\lambda^{2}} \hat{q}^{A B}-\frac{1}{\lambda^{3}} \hat{h}^{A B}+o\left(\lambda^{-3}\right)\right) \times \\
& \left(\hat{D}_{A} \hat{D}_{B} F-\frac{1}{2}\left(\hat{D}_{A} \hat{h}_{B}^{C}+\hat{D}_{B} \hat{h}^{C}{ }_{A}-\hat{D}^{C} h_{A B}\right) F_{, C} \frac{1}{\lambda}+O\left(\lambda^{-2}\right)\right) \\
= & \frac{1}{\lambda^{2}} \triangle_{\hat{q}} F+\left(-\hat{h}^{A B} \hat{D}_{A} \hat{D}_{B} F-\left(\hat{D}_{A} \hat{h}^{C A}\right) F_{, C}+\frac{1}{2} \hat{D}^{C}\left(\hat{q}^{A B} h_{A B}\right) F_{, C}\right) \frac{1}{\lambda^{3}}+o\left(\lambda^{-3}\right),
\end{aligned}
$$

which is (6.25) after recalling that $\operatorname{tr}_{\hat{q}} \hat{h}=2 \theta_{k}^{(1)}$.

We conclude this section by showing that the leading term $s_{\ell}^{(1)}$ of $s_{\ell}$ is fully determined in terms of the rest of objects whenever the energy flux decay condition is assumed.

Proposition 6.3.9. Let $\Omega$ be a past asymptotically flat null hypersurface with a choice of affinely parametrized null generator $k$ and corresponding level set function $\lambda$. Assume that the energy flux decay condition holds. Then

$$
s_{\ell}^{(1)}{ }_{A}=\hat{D}_{A} \theta_{k}^{(1)}-\frac{1}{2} \hat{D}_{B} \hat{h}_{A}^{B} .
$$

Proof. From item (ii) in Definition 6.3.4 and the decomposition (6.23) we have $£_{k} s_{A}=\frac{s_{l}^{(1)} A}{\lambda^{2}}+o\left(\lambda^{-2}\right), \quad \theta_{k} s_{\ell A}=-\frac{2 s_{l}^{(1)} A}{\lambda^{2}}+o\left(\lambda^{-2}\right) \quad$ and $\quad \hat{D}_{A} \theta_{k}=\frac{\hat{D}_{A} \theta_{k}^{(1)}}{\lambda^{2}}+o\left(\lambda^{-2}\right)$.

Concerning the divergence of $K_{B}^{k A}$ in the metric $\gamma(\lambda)$ we use that the leading term in (6.22) is covariantly constant and then replace the $D$-covariant derivative by the $\hat{D}$-derivative and use $Z_{A B}^{A}=O\left(\lambda^{-1}\right)$ to obtain

$$
D_{B} K^{k B}{ }_{C}=\frac{1}{2 \lambda^{2}} D_{B} \hat{h}^{B}{ }_{C}+o\left(\lambda^{-2}\right)=\frac{1}{2 \lambda^{2}} \hat{D}_{B} \hat{h}^{B}{ }_{C}+o\left(\lambda^{-2}\right) .
$$

Thus, identity (6.12) (with $Q_{k}=0$ ) becomes

$$
\frac{1}{\lambda^{2}}\left(s_{\ell}^{(1)} A+\frac{1}{2} \hat{D}_{B} \hat{h}_{A}^{B}-\hat{D}_{A} \theta_{k}^{(1)}\right)+o\left(\lambda^{-2}\right)=0,
$$

and the result follows. 


\subsection{Background foliation approaching large spheres}

\subsection{Background foliation approaching large spheres}

As discussed in Section 6.1, the Hawking energy has the interesting and well-known property of approaching the Bondi energy when the surfaces approach large spheres in a suitable sense. Our general limiting expressions for the Hawking energy will of course have to recover this fact. To that aim, it is useful to restrict the choice of affinely parametrized null generator $k$ and corresponding level set function $\lambda$ so that the geometry of the level sets $S_{\lambda}$ approaches, after rescaling, the standard metric of unit radius on the sphere, denoted by $\stackrel{q}{q}$.

Definition 6.4.1. Let $\Omega$ be null and past asymptotically flat with a choice of affine null generator $k$ and level set function $\lambda$. The foliation $\left\{S_{\lambda}\right\}$ is said to approach large spheres if and only if the leading term $\hat{q}$ in the expansion (6.19) of $\gamma$ is the standard metric of a unit two-sphere.

Our definition of approaching large spheres is equivalent to demanding that the rescaled metric $\frac{1}{\lambda^{2}} \gamma(\lambda)$, has a limit $\stackrel{q}{ }$ when $\lambda \rightarrow \infty$. In [93] the definition of approaching large spheres is defined more generally for exhaustions $\left\{S_{s}\right\}$ of $\Omega$ with all elements diffeomorphic to each other by demanding that the rescaled metric $\frac{4 \pi}{\left|S_{s}\right|} \gamma_{s}$ has a limit when $s \rightarrow \infty$ and defines a metric $\stackrel{q}{q}$ of constant unit curvature. It is clear that both definitions agree for the affine foliations $\left\{S_{\lambda}\right\}$ that we are using in this chapter.

Our aim is to consider very general exhaustions $\left\{S_{\lambda^{\prime}}\right\}$ on $\Omega$ and obtain the limit of the Hawking energy along them by referring all objects to an affine background foliation approaching large spheres. It is important to note that, since all Riemannian metrics on a manifold $\simeq \mathbb{S}^{2}$ are conformal to the standard metric $\stackrel{q}{q}$, there always exists a (nonunique) choice of affine null generator $k$ in an asymptotically flat $\Omega$ with corresponding background foliation $\left\{S_{\lambda}\right\}$ approaching large spheres (cf. Remark 6.5.2 below). Tensors raised and lowered with the metric $\stackrel{\circ}{q}_{A B}$ and its inverse $\stackrel{\circ}{q}^{A B}$ will have a circle on top, so that for instance (6.26) reads

$$
\gamma(\lambda)^{A B}=\frac{1}{\lambda^{2}} \stackrel{q}{q}^{A B}-\frac{1}{\lambda^{3}} \stackrel{h}{h}^{A B}+o\left(\lambda^{-3}\right) .
$$

Note also that since $q$ has constant unit curvature, the null expansion $\theta_{\ell}$ has asymptotic behaviour

$$
\theta_{\ell}=\frac{2}{\lambda}+\frac{\theta_{\ell}^{(1)}}{\lambda^{2}}+o\left(\lambda^{-2}\right)
$$

We will consider three types of foliations $\left\{S_{\lambda^{\prime}}\right\}$ and then combine them to obtain the general case treated in Theorem 6.6.2. Given a null basis $\left\{k^{\prime}, \ell^{\prime}\right\}$ orthogonal to a section $S$ in $\Omega$ and satisfying $\left\langle k^{\prime}, \ell^{\prime}\right\rangle=-2$, the mean curvature $\vec{H}$ of $S$ decomposes as $\vec{H}=-\frac{1}{2}\left(\theta_{k^{\prime}} \ell^{\prime}+\theta_{\ell^{\prime}} k^{\prime}\right)$ and the Hawking energy is

$$
m_{H}(S)=\sqrt{\frac{|S|}{16 \pi}}\left(1+\frac{1}{16 \pi} \int_{S} \theta_{k^{\prime}} \theta_{\ell^{\prime}} \eta_{S}\right),
$$




\section{The Hawking energy along null AF hypersurfaces}

so our aim will be to compute the limit of the areas $\left|S_{\lambda^{\prime}}\right|$ and of $\theta_{k^{\prime}} \theta_{\ell^{\prime}} \eta_{S_{\lambda^{\prime}}}$ of the new foliation $\left\{S_{\lambda^{\prime}}\right\}$ in terms of the background foliation geometry. The following section deals with the case when $\left\{S_{\lambda^{\prime}}\right\}$ is any affine foliation (not necessarily approaching large spheres).

\subsection{Limit of the Hawking energy for affine foliations}

In this section we assume that $\Omega$ is null and past asymptotically flat and endowed with a foliation $\left\{S_{\lambda}\right\}$ associated to an affinely parametrized null generator $k$ and approaching large spheres. By definition, an affine foliation in $\Omega$ is a foliation $\left\{S_{\lambda^{\prime}}\right\}$ by cross sections defined by the function $\lambda^{\prime} \in \mathcal{F}(\Omega)$ such that the (unique) null generator $k^{\prime}$ satisfying $k^{\prime}\left(\lambda^{\prime}\right)=-1$ is affinely parametrized. Thus, there exists a positive function $\phi \in \mathcal{F}(\Omega)$, Lie constant along $k$ such that $k^{\prime}=\phi k$. Consequently, the level set functions $\lambda$ and $\lambda^{\prime}$ are necessarily related by $\lambda=\tau+\phi \lambda^{\prime}$ where $\tau \in \mathcal{F}(\Omega)$ is a Lie constant function. We first consider the case when the two foliations $\left\{S_{\lambda}\right\}$ and $\left\{S_{\lambda^{\prime}}\right\}$ have the same starting surface, i.e. that $S_{\lambda^{\prime}=0}=S_{\lambda=0}$, which fixes $\tau=0$. The leaves of the new foliation are defined as (see Figure 6.2)

$$
S_{\lambda^{\prime}}=\left\{p \in \Omega: \lambda(p)=\phi(p) \lambda^{\prime}\right\}
$$

Thus, each of the surfaces $\left\{S_{\lambda^{\prime}}\right\}$ can be described by a graph function

$$
\left.\lambda\right|_{S_{\lambda^{\prime}}}=F_{\lambda^{\prime}}=\left.\phi\right|_{S_{\lambda^{\prime}}} \lambda^{\prime}
$$

where here $F_{\lambda^{\prime}}$ is a function on $S_{\lambda^{\prime}}$, not its extension to $\Omega$ satisfying $k\left(F_{\lambda^{\prime}}\right)=0$.

Our strategy is to use the general expressions in Section 6.2 for the geometry of a graph $\lambda=F$ in a background foliation, insert the resulting expressions in (6.27) and take the limit when $\lambda^{\prime} \rightarrow \infty$. We start with the following Proposition.

Proposition 6.5.1. Let $\Omega$ be a past asymptotically flat null hypersurface endowed with an affinely parametrized background foliation $\left\{S_{\lambda}\right\}$ with generator $k$ and approaching large spheres. Let $\phi>0$ be a Lie constant function and define the affine foliation $\left\{S_{\lambda^{\prime}}\right\}$ as $S_{\lambda^{\prime}}=\left\{p \in \Omega: \lambda(p)=\phi(p) \lambda^{\prime}\right\}$, where the graph functions read

$$
\left.\lambda\right|_{s_{\lambda^{\prime}}}=F_{\lambda^{\prime}}:=\left.\phi\right|_{s_{\lambda^{\prime}}} \lambda^{\prime}
$$

Let $k^{\prime}=\phi k$ and $\ell^{\prime}$ be the null normal orthogonal to $\left\{S_{\lambda^{\prime}}\right\}$ satisfying $\left\langle k^{\prime}, \ell^{\prime}\right\rangle=-2$. Then the induced metric $\gamma\left(\lambda^{\prime}\right)$, volume form $\eta_{S_{\lambda^{\prime}}}$, null expansions $\theta_{k^{\prime}}, \theta_{\ell^{\prime}}$ and the connection 


\subsection{Limit of the Hawking energy for affine foliations}

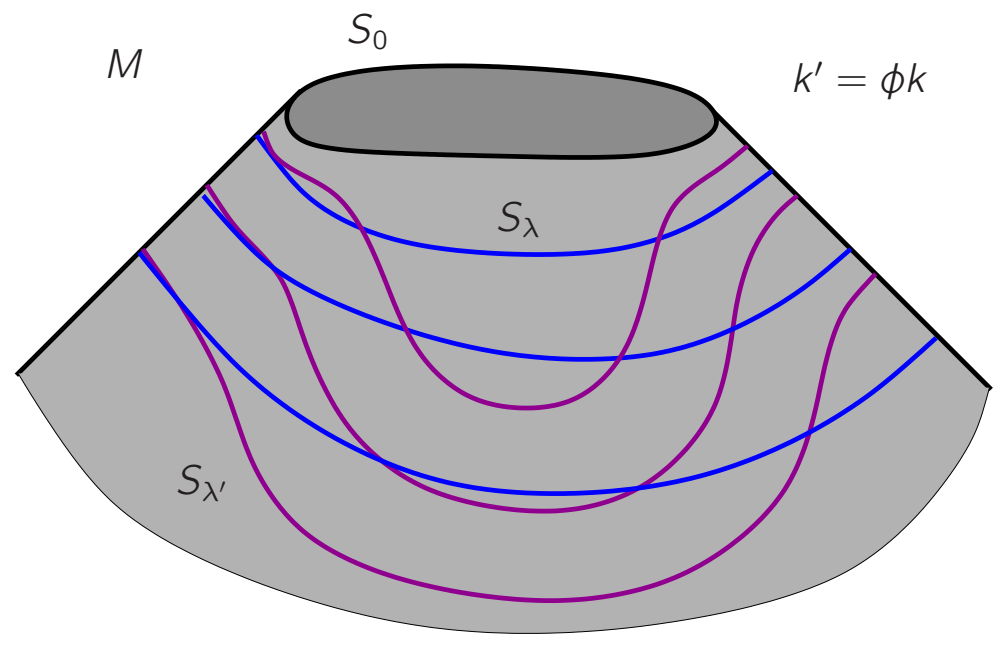

Figure 6.2: Representation of two different flows $\left\{S_{\lambda}\right\}$ and $\left\{S_{\lambda^{\prime}}\right\}$ associated to different parameters $\lambda$ and $\lambda^{\prime}$. Both flows have the same starting surface $S_{0}$, and their velocities are related by a Lie constant function $\phi$ as $k^{\prime}=\phi k$.

of the normal bundle $\boldsymbol{s}_{\boldsymbol{\ell}^{\prime}}$ can be expressed in terms of the background geometry as

$$
\begin{aligned}
& \gamma_{A B}^{\prime}:=\gamma^{\prime}\left(X_{A}^{\prime}, X_{B}^{\prime}\right)=\phi^{2} \stackrel{\circ}{q}_{A B} \lambda^{\prime 2}+\phi h_{A B} \lambda^{\prime}+o\left(\lambda^{\prime}\right), \\
& \boldsymbol{\eta}_{S_{\lambda^{\prime}}}=\left(\phi^{2} \lambda^{\prime 2}+\phi \theta_{k}^{(1)} \lambda^{\prime}+o\left(\lambda^{\prime}\right)\right) \boldsymbol{\eta}_{\tilde{q}} \text {, } \\
& \theta_{k^{\prime}}=-\frac{2}{\lambda^{\prime}}+\frac{\theta_{k}^{(1)}}{\phi} \frac{1}{\lambda^{\prime 2}}+o\left(\lambda^{\prime-2}\right)
\end{aligned}
$$

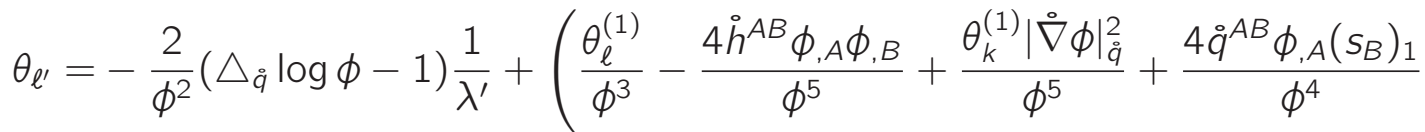

$$
\begin{aligned}
& \left.+\frac{2 \stackrel{\circ}{h}^{A B} \stackrel{\circ}{\nabla}_{A} \stackrel{\circ}{\nabla}_{B} \phi}{\phi^{4}}+\frac{2 \stackrel{\circ}{\nabla}_{A} \stackrel{\circ}{h}^{A C} \phi, C}{\phi^{4}}-\frac{2 \stackrel{\circ}{\nabla}^{C} \theta_{k}^{(1)} \phi, C}{\phi^{4}}\right) \frac{1}{\lambda^{\prime 2}}+o\left(\lambda^{\prime-2}\right), \\
& s_{\ell^{\prime} A}=\left(\frac{s_{\ell}^{(1)} A}{\phi}-\frac{\phi_{, L} \stackrel{\circ}{L}_{A}}{2 \phi^{2}}\right) \frac{1}{\lambda^{\prime}}+o\left(\lambda^{\prime-1}\right),
\end{aligned}
$$

where $X_{A}^{\prime}:=X_{A}-X_{A}\left(F_{\lambda^{\prime}}\right) k$.

Proof. As already mentioned $k^{\prime}$ is the generator of the foliation $\left\{S_{\lambda^{\prime}}\right\}$. For any point $p \in S_{\lambda^{\prime}}$, the level set passing through $p$ has $\lambda=F_{\lambda^{\prime}}(p)=\phi(p) \lambda^{\prime}$. Thus (6.1) and the background expansion (6.19) with $\hat{q} \rightarrow \stackrel{q}{q}$ gives

$$
\left.\gamma_{A B}^{\prime}\right|_{p}=\left.\gamma_{A B}\right|_{S_{\lambda=\phi(p) \lambda^{\prime}}}=\stackrel{\circ}{q B}_{A B} \lambda^{2}+h_{A B} \lambda+\left.\tilde{\gamma}\right|_{\lambda=\phi \lambda^{\prime}},
$$

which is (6.28). (6.29) follows by taking determinants and using the standard identity

$$
\operatorname{det}(M+s B)=(\operatorname{det} M)\left(1+s \operatorname{tr}\left(M^{-1} B\right)+O\left(s^{2}\right)\right)
$$




\section{The Hawking energy along null AF hypersurfaces}

valid for any invertible matrix $M$. For $\theta_{k^{\prime}}$ we use the fact that $\theta_{k}$ is a property of $\Omega$ and not of the surface embedded in $\Omega$ passing through that point, cf. Corollary 6.2.5. Thus

$$
\theta_{k^{\prime}}=\left.\phi \theta_{k}\right|_{\lambda=\phi \lambda^{\prime}}=-\frac{2}{\lambda^{\prime}}+\frac{\theta_{k}^{(1)}}{\phi} \frac{1}{\lambda^{\prime 2}}+o\left(\lambda^{\prime-2}\right)
$$

as claimed in (6.30). To compute $\theta_{\ell^{\prime}}$ we use expression (6.11) with $Q_{k}=0, \alpha=\phi$ and graph function $F=F_{\lambda^{\prime}}=\phi \lambda^{\prime}$. As before, the right-hand side has to be evaluated at $\lambda=\phi \lambda^{\prime}$. We work out each term separately:

$$
\begin{aligned}
\theta_{\ell} & =\frac{2}{\lambda}+\frac{\theta_{\ell}^{(1)}}{\lambda^{2}}+o\left(\lambda^{-2}\right)=\frac{2}{\phi} \frac{1}{\lambda^{\prime}}+\frac{\theta_{\ell}^{(1)}}{\phi^{2}} \frac{1}{\lambda^{\prime 2}}+o\left(\lambda^{\prime-2}\right) \\
\theta_{k}\left|D F_{\lambda^{\prime}}\right|^{2} & =\theta_{k} \gamma^{A B} \nabla_{A} F_{\lambda^{\prime}} \nabla_{B} F_{\lambda^{\prime}} \\
& =\left(-\frac{2}{\phi} \frac{1}{\lambda^{\prime}}+\frac{\theta_{k}^{(1)}}{\phi^{2}} \frac{1}{\lambda^{\prime 2}}+o\left(\lambda^{\prime-2}\right)\right)\left(\frac{\stackrel{\circ}{q}^{A B}}{\phi^{2}} \frac{1}{\lambda^{\prime 2}}-\frac{\stackrel{h}{h}^{A B}}{\phi^{3}} \frac{1}{\lambda^{\prime 3}}+O\left(\lambda^{\prime-4}\right)\right) \phi_{, A} \phi_{, B} \lambda^{\prime 2} \\
& =-\frac{2|\stackrel{\circ}{\nabla} \phi|_{\stackrel{q}{q}}^{2}}{\phi^{3}} \frac{1}{\lambda^{\prime}}+\left(\frac{2 \stackrel{\circ}{h}^{A B} \phi_{, A} \phi_{, B}}{\phi^{4}}+\frac{\theta_{k}^{(1)}|\stackrel{\circ}{\nabla} \phi|_{\stackrel{q}{q}}^{2}}{\phi^{4}}\right) \frac{1}{\lambda^{\prime 2}}+o\left(\lambda^{\prime-2}\right) .
\end{aligned}
$$

For the Laplacian of $F_{\lambda^{\prime}}$ we use (6.25) which gives, using $\nabla F_{\lambda^{\prime}}=(\nabla \phi) \lambda^{\prime}$, Hess ${ }_{\dot{q}} F_{\lambda^{\prime}}=$ $\left(\operatorname{Hess}_{\tilde{q}} \phi\right) \lambda^{\prime}$ and $\triangle_{\dot{q}} F_{\lambda^{\prime}}=\left(\triangle_{\dot{q}} \phi\right) \lambda^{\prime}$,

$$
\triangle_{\gamma} F_{\lambda^{\prime}}=\frac{\triangle_{q} \phi}{\phi^{2}} \frac{1}{\lambda^{\prime}}+\left(-\frac{\stackrel{\circ}{h}^{A B} \stackrel{\circ}{\nabla}_{A} \stackrel{\circ}{\nabla}_{B} \phi}{\phi^{3}}-\frac{\stackrel{\circ}{\nabla}_{A} \stackrel{\circ}{h}^{C A} \phi, C}{\phi^{3}}+\frac{\left(\stackrel{\circ}{\nabla}^{C} \theta_{k}^{(1)}\right) \phi, C}{\phi^{3}}\right) \frac{1}{\lambda^{\prime 2}}+o\left(\lambda^{\prime-2}\right) .
$$

For the term $s_{\ell}\left(\operatorname{grad} F_{\lambda^{\prime}}\right)$

$$
\begin{aligned}
s_{\ell}\left(\operatorname{grad} F_{\lambda^{\prime}}\right) & =\gamma^{A B} s_{\ell A} \nabla_{B} F_{\lambda^{\prime}}=\left(\frac{\dot{q}^{A B}}{\phi^{2}} \frac{1}{\lambda^{\prime 2}}+o\left(\lambda^{\prime-2}\right)\right)\left(\frac{s_{\ell}^{(1)} A}{\phi} \frac{1}{\lambda^{\prime}}+o\left(\lambda^{\prime-1}\right)\right) \phi_{, B} \lambda^{\prime} \\
& =\frac{\dot{q}^{A B} s_{\ell}^{(1)} A \phi_{, B}}{\phi^{3}} \frac{1}{\lambda^{\prime 2}}+o\left(\lambda^{\prime-2}\right)
\end{aligned}
$$

and finally $K^{k}\left(\operatorname{grad} F_{\lambda^{\prime}}, \operatorname{grad} F_{\lambda^{\prime}}\right)$ is, inserting (6.16) and (6.17),

$$
\begin{aligned}
& K^{k}\left(\operatorname{grad} F_{\lambda^{\prime}}, \operatorname{grad} F_{\lambda^{\prime}}\right)=\gamma^{A B} \gamma^{C D} K_{B D}^{k} \phi_{, A} \phi_{, B} \lambda^{\prime 2}=\frac{\phi_{, A} \phi_{, B}}{\phi^{4} \lambda^{\prime 2}}\left(\stackrel{\circ}{q} A B-\frac{1}{\phi \lambda^{\prime}} \stackrel{\circ}{A}^{A B}+o\left(\lambda^{\prime-1}\right)\right) \times \\
& \left(\stackrel{\circ}{q}^{C D}-\frac{1}{\phi \lambda^{\prime}} \stackrel{\circ}{C D}^{C D} \circ\left(\lambda^{\prime-1}\right)\right)\left(-\stackrel{\circ}{q}_{B D} \phi \lambda^{\prime}-\frac{1}{2} h_{B D}+o(1)\right) \\
& =\frac{-|\stackrel{\circ}{\nabla} \phi|_{\stackrel{9}{q}}^{2}}{\phi^{3}} \frac{1}{\lambda^{\prime}}+\frac{3 \stackrel{h}{L}^{L A} \phi, A}{\phi_{, L}} \frac{1}{2 \phi^{4}}+o\left(\lambda^{\prime-2}\right) \text {. }
\end{aligned}
$$

Putting things together leads to (6.31) after using $\frac{{\triangle_{\tilde{q}} \phi}_{\phi}}{\phi}-\frac{\left|\nabla^{\circ} \phi\right|_{\hat{q}}^{2}}{\phi^{2}}=\triangle_{\mathfrak{q}} \log \phi$. 


\subsection{Limit of the Hawking energy for affine foliations}

Finally, if we substitute in expression (6.7) the asymptotic expansion of item (ii) in Definition 6.3.4, and the expansion of $K^{k}$ of (6.22), we have $s_{\ell^{\prime} A}=-\frac{\phi, A}{\phi}+\frac{s_{l}^{(1)} A}{\phi} \frac{1}{\lambda^{\prime}}+\frac{\phi_{, A}}{\phi}-\frac{\phi_{, L} h^{L}{ }_{A}}{2 \phi^{2}} \frac{1}{\lambda^{\prime}}+o\left(\lambda^{\prime-1}\right)=\left(\frac{s_{l}^{(1)} A}{\phi}-\frac{\phi_{, L} \stackrel{\circ}{L}_{A}}{2 \phi^{2}}\right) \frac{1}{\lambda^{\prime}}+o\left(\lambda^{\prime-1}\right)$, where the term in $\frac{1}{\lambda^{\prime}}$ is $S_{\ell^{\prime}}^{(1)} A$.

Remark 6.5.2. The foliation $\left\{S_{\lambda^{\prime}}\right\}$ has limit metric $\lim _{\lambda^{\prime} \rightarrow \infty} \frac{\gamma^{\prime}}{\lambda^{\prime 2}}=\phi^{2} \stackrel{q}{q}:=\hat{q}$. Using the formula for the scalar curvature of a conformal metric it follows

$$
\mathcal{K}_{\hat{q}}=\frac{1-\triangle_{\mathfrak{q}} \log \phi}{\phi^{2}}
$$

so that the expansion of $\theta_{\ell^{\prime}}$ is $\theta_{\ell^{\prime}}=\frac{2 \mathcal{K}_{\hat{q}}}{\lambda^{\prime}}+o\left(\lambda^{\prime-1}\right)$ in agreement with Proposition 6.3.5. Note that the transformation law $\hat{q} \rightarrow \phi^{2} \hat{q}^{\prime}$ for the leading term $\hat{q}$ in the metric $\gamma(\lambda)$ under change of foliation $k^{\prime}=\phi k$ holds irrespectively of whether the background foliation approaches large spheres or not. Since as mentioned above, any metric on $\mathbb{S}^{2}$ is conformal to $\stackrel{q}{q}$, it follows that any asymptotically flat $\Omega$ admits a background foliation approaching large spheres.

We can now evaluate the limit of the Hawking energy for the foliation $\left\{S_{\lambda^{\prime}}\right\}$ (we write $\phi=\frac{1}{\psi}$ for later convenience):

Theorem 6.5.3. Let $\Omega$ be a past asymptotically flat null hypersurface endowed with an affinely parametrized background foliation $\left\{S_{\lambda}\right\}$ with generator $k$ and approaching large spheres. Let $\Psi>0$ be a Lie constant function and define the affine foliation $\left\{S_{\lambda^{\prime}}\right\}$ as $S_{\lambda^{\prime}}=\left\{p \in \Omega: \lambda(p)=\frac{1}{\Psi(p)} \lambda^{\prime}\right\}$, where the graph functions read

$$
\left.\lambda\right|_{S_{\lambda^{\prime}}}=F_{\lambda^{\prime}}:=\left.\frac{1}{\psi}\right|_{S_{\lambda^{\prime}}} \lambda^{\prime}
$$

The limit of the Hawking energy along the foliation $\left\{S_{\lambda^{\prime}}\right\}$ is, in terms of the background geometry,

$\lim _{\lambda^{\prime} \rightarrow \infty} m_{H}\left(S_{\lambda^{\prime}}\right)=\frac{1}{8 \pi \sqrt{16 \pi}}\left(\sqrt{\int_{\mathbb{S}^{2}} \frac{1}{\Psi^{2}} \boldsymbol{\eta}_{\dot{q}}}\right) \int_{\mathbb{S}^{2}}\left(\triangle_{\dot{q}} \theta_{k}^{(1)}-\left(\theta_{k}^{(1)}+\theta_{\ell}^{(1)}\right)-4 \operatorname{div}_{\dot{q}}\left(s_{\ell}^{(1)}\right)\right) \psi \boldsymbol{\eta}_{\dot{q}}$.

Proof. Set $\phi=\Psi^{-1}$ so that we can use the expressions in Proposition 6.5.1. We need to compute $\theta_{k^{\prime}} \theta_{\ell^{\prime}} \boldsymbol{\eta}_{S_{\lambda^{\prime}}}$. Denoting by $\theta_{\ell^{\prime}}^{(1)}$ the coefficient of the term $\frac{1}{\lambda^{\prime 2}}$ in $\theta_{\ell^{\prime}}$ we immediately find, from (6.29)-(6.31),

$$
\theta_{k^{\prime}} \theta_{\ell^{\prime}} \boldsymbol{\eta}_{S_{\lambda^{\prime}}}=\left(4\left(\triangle_{\dot{q}} \log \phi-1\right)+\left(-2 \mathcal{K}_{\hat{q}} \theta_{k}^{(1)} \phi-2 \theta_{\ell^{\prime}}^{(1)} \phi^{2}\right) \frac{1}{\lambda^{\prime}}+o\left(\lambda^{\prime-1}\right)\right) \boldsymbol{\eta}_{\dot{q}}
$$




\section{The Hawking energy along null AF hypersurfaces}

and hence

$$
\begin{aligned}
1+\frac{1}{16 \pi} \int_{S_{\lambda^{\prime}}} \theta_{k^{\prime}} \theta_{\ell^{\prime}} \boldsymbol{\eta}_{S_{\lambda^{\prime}}} & =1+\frac{1}{16 \pi} \int_{\mathbb{S}^{2}}\left(4\left(\triangle_{\dot{q}} \log \phi-1\right)+\left(-2 \mathcal{K}_{\hat{q}} \theta_{k}^{(1)} \phi-2 \theta_{\ell^{\prime}}^{(1)} \phi^{2}\right) \frac{1}{\lambda^{\prime}}\right. \\
& \left.+o\left(\lambda^{\prime-1}\right)\right) \boldsymbol{\eta}_{\mathfrak{q}}=\frac{1}{16 \pi \lambda^{\prime}} \int_{\mathbb{S}^{2}} \underbrace{\left(-2 \mathcal{K}_{\hat{q}} \theta_{k}^{(1)} \phi-2 \theta_{\ell^{\prime}}^{(1)} \phi^{2}\right)}_{\mathcal{I}} \boldsymbol{\eta}_{\mathfrak{q}}+o\left(\lambda^{\prime-1}\right) .
\end{aligned}
$$

Concerning the area term

$$
\left|S_{\lambda^{\prime}}\right|=\int_{S_{\lambda^{\prime}}} \boldsymbol{\eta}_{S_{\lambda^{\prime}}}=\left(\int_{\mathbb{S}^{2}} \phi^{2} \boldsymbol{\eta}_{\dot{q}}\right) \lambda^{\prime 2}+O\left(\lambda^{\prime}\right) \Longrightarrow \sqrt{\left|S_{\lambda^{\prime}}\right|}=\lambda^{\prime} \sqrt{\int_{\mathbb{S}^{2}} \phi^{2} \boldsymbol{\eta}_{\dot{q}}}+O(1),
$$

so that the limit of the Hawking energy is

$$
\lim _{\lambda^{\prime} \rightarrow \infty} m_{H}\left(S_{\lambda^{\prime}}\right)=\frac{1}{16 \pi \sqrt{16 \pi}}\left(\sqrt{\int_{\mathbb{S}^{2}} \phi^{2} \boldsymbol{\eta}_{\dot{q}}}\right) \int_{\mathbb{S}^{2}} \mathcal{I} \boldsymbol{\eta}_{\dot{q}} .
$$

We now compute $\mathcal{I}$. Using the explicit form of $\mathcal{K}_{\hat{q}}$ and $\theta_{\ell^{\prime}}^{(1)}$ it follows

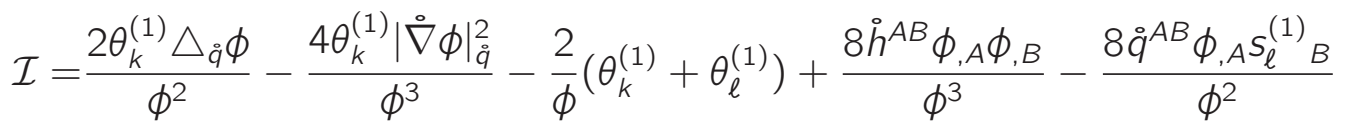

$$
\begin{aligned}
& -\frac{4 \stackrel{\circ}{h}^{A B} \stackrel{\circ}{\nabla}_{A} \stackrel{\circ}{\nabla}_{B} \phi}{\phi^{2}}-\frac{4 \stackrel{\circ}{\nabla}_{A} \stackrel{\circ}{h}^{A B} \phi_{, B}}{\phi^{2}}+\frac{4 \stackrel{\circ}{\nabla}^{B} \theta_{k}^{(1)} \phi_{, B}}{\phi^{2}} \\
& =\stackrel{\circ}{\nabla}_{A}\left(-\frac{4}{\phi^{2}} \stackrel{\circ}{A B}^{A B} \phi_{, B}+\frac{2 \theta_{k}^{(1)} \stackrel{\circ}{\nabla}^{A} \phi}{\phi^{2}}+\frac{8 s_{\ell}^{(1) A}}{\phi}\right)+\frac{2 \stackrel{\circ}{\nabla}^{A} \theta_{k}^{(1)} \phi_{, A}}{\phi^{2}}-\frac{2}{\phi}\left(\theta_{k}^{(1)}+\theta_{\ell}^{(1)}\right) \\
& -\frac{8}{\phi} \operatorname{div}_{\mathfrak{q}}\left(s_{\ell}^{(1)}\right)= \\
& =\stackrel{\circ}{\nabla}_{A}\left(-\frac{4}{\phi^{2}} \stackrel{h}{A B}^{A B}, \frac{2 \theta_{k}^{(1)} \stackrel{\circ}{\nabla}^{A} \phi}{\phi^{2}}+\frac{8 s_{\ell}^{(1) A}}{\phi}-\frac{2}{\phi} \stackrel{\circ}{\nabla}^{A} \theta_{k}^{(1)}\right) \\
& +\frac{2}{\phi} \triangle_{\dot{q}} \theta_{k}^{(1)}-\frac{2}{\phi}\left(\theta_{k}^{(1)}+\theta_{\ell}^{(1)}\right)-\frac{8}{\phi} \operatorname{div}_{\tilde{q}}\left(s_{\ell}^{(1)}\right) \text {, }
\end{aligned}
$$

and (6.35) becomes (6.34) after using the Gauss identity and $\phi=\psi^{-1}$.

Remark 6.5.4. It is interesting that all terms involving derivatives of $\phi$ (or $\Psi$ ) combine themselves into a divergence and drop out after integration. The behaviour of the limit of the Hawking energy under change of affine foliation is hence much simpler than one might have expected a priori.

Given a past asymptotically flat null hypersurface, there are many possible choices of affine background foliations approaching large spheres. Any two such foliations are related by $\lambda=\phi \lambda^{\prime}$ with $\phi$ satisfying

$$
\triangle_{q} \log \phi+\phi^{2}=1
$$




\subsection{Limit of the Hawking energy for affine foliations}

so that the Gauss curvature (6.33) of $\hat{q}$ is also one. In this case the limit of the Hawking energy of the foliation $\left\{S_{\lambda^{\prime}}\right\}$ can be computed in two different ways, namely referring $\left\{S_{\lambda^{\prime}}\right\}$ to the background foliation $\left\{S_{\lambda}\right\}$ and using Theorem 6.5.3 or considering $\left\{S_{\lambda^{\prime}}\right\}$ itself as a background foliation (so that the result would be (6.34) with $\psi=1$ and $\theta_{k}^{(1)}$, $\theta_{\ell}^{(1)}, s_{\ell}^{(1)}$ all referred to the foliation $\left.\left\{S_{\lambda^{\prime}}\right\}\right)$. It is clear that both results must agree. This requires a kind of covariance property of the integral in (6.34). Remarkably, this covariance occurs already at the level of the integrand, as we show next. All geometric objects referred to the affine foliation $\left\{S_{\lambda^{\prime}}\right\}$ will carry a prime.

Theorem 6.5.5. Let $\Omega$ be a past asymptotically flat null hypersurface endowed with an affinely parametrized background foliation $\left\{S_{\lambda}\right\}$ with generator $k$ and approaching large spheres. Let $\phi>0$ be a Lie constant function and define the affine foliation $\left\{S_{\lambda^{\prime}}\right\}$ as $S_{\lambda^{\prime}}=\left\{p \in \Omega: \lambda(p)=\phi(p) \lambda^{\prime}\right\}$, where the graph functions read

$$
\left.\lambda\right|_{S_{\lambda^{\prime}}}=F_{\lambda^{\prime}}:=\left.\phi\right|_{S_{\lambda^{\prime}}} \lambda^{\prime},
$$

with $\phi$ satisfying (6.36). Then

$$
\left(\triangle_{\tilde{q}} \theta_{k}^{(1)}-\left(\theta_{k}^{(1)}+\theta_{\ell}^{(1)}\right)-4 \operatorname{div}_{\tilde{q}} s_{\ell}^{(1)}\right) \frac{1}{\phi} \boldsymbol{\eta}_{\grave{q}}=\left(\triangle_{\tilde{q}^{\prime}} \theta_{k^{\prime}}^{(1)}-\left(\theta_{k^{\prime}}^{(1)}+\theta_{\ell^{\prime}}^{(1)}\right)-4 \operatorname{div}_{\tilde{q}^{\prime}} \boldsymbol{s}_{\ell^{\prime}}^{(1)}\right) \boldsymbol{\eta}_{\dot{q}^{\prime}}
$$

As a consequence we have the necessary invariance of the limit of the Hawking energy

$$
\begin{aligned}
\lim _{\lambda^{\prime} \rightarrow \infty} m_{H}\left(S_{\lambda^{\prime}}\right) & =\frac{-1}{16 \pi} \int_{\mathbb{S}^{2}}\left(\theta_{k^{\prime}}^{(1)}+\theta_{\ell^{\prime}}^{(1)}\right) \boldsymbol{\eta}_{\dot{q}^{\prime}} \\
& =\frac{1}{16 \pi} \int_{\mathbb{S}^{2}}\left(\triangle_{\dot{q}} \theta_{k}^{(1)}-\left(\theta_{k}^{(1)}+\theta_{\ell}^{(1)}\right)-4 \operatorname{div}_{\mathfrak{q}} \boldsymbol{s}_{\ell}^{(1)}\right) \frac{1}{\phi} \boldsymbol{\eta}_{\grave{q}} .
\end{aligned}
$$

Proof. The general expressions (6.28)-(6.31) give the explicit form of the geometric objects of $\left\{S_{\lambda^{\prime}}\right\}$ in terms of the background foliation $\left\{S_{\lambda}\right\}$, namely

$$
\begin{aligned}
& \stackrel{q}{q}_{A B}^{\prime}=\phi^{2} \stackrel{q}{q}_{A B}, \quad \theta_{k^{\prime}}^{(1)}=\frac{\theta_{k}^{(1)}}{\phi}, \quad \theta_{\ell^{\prime}}^{(1)}=\frac{\theta_{\ell}^{(1)}}{\phi^{3}}-\frac{4 \stackrel{h}{h}^{A B} \phi_{, A} \phi_{, B}}{\phi^{5}}+\frac{\theta_{k}^{(1)}|\stackrel{\circ}{\nabla} \phi|_{\grave{q}}^{2}}{\phi^{5}}+\frac{4 \dot{q}^{A B} \phi_{, A}\left(s_{B}\right)_{1}}{\phi^{4}} \\
& +\frac{2 \stackrel{\circ}{h}^{A B} \stackrel{\circ}{\nabla}_{A} \stackrel{\circ}{\nabla}_{B} \phi}{\phi^{4}}+\frac{2 \stackrel{\circ}{\nabla}_{A} \stackrel{\circ}{A}^{A C} \phi, C}{\phi^{4}}-\frac{2 \stackrel{\nabla}{\nabla}^{C} \theta_{k}^{(1)} \phi, C}{\phi^{4}}, \quad s_{\ell^{\prime}}^{(1)} A=\frac{s_{\ell}^{(1)} A}{\phi}-\frac{\phi_{, L} \stackrel{h}{L}_{A}}{2 \phi^{2}} \text {. }
\end{aligned}
$$

The Laplacian in two-dimensions is conformally covariant so that $\triangle_{\tilde{q}^{\prime}} \theta_{k^{\prime}}^{(1)}=\frac{1}{\phi^{2}} \triangle_{q_{q}} \theta_{k^{\prime}}^{(1)}$, and hence

$$
\triangle_{q^{\prime}} \theta_{k^{\prime}}^{(1)}=\frac{1}{\phi^{2}} \triangle_{\dot{q}} \theta_{k^{\prime}}^{(1)}=\frac{\triangle_{\dot{q}} \theta_{k}^{(1)}}{\phi^{3}}-\frac{\theta_{k}^{(1)} \triangle_{\dot{q}} \phi}{\phi^{4}}-\frac{2 \nabla^{A} \theta_{k}^{(1)} \phi, C}{\phi^{4}}+\frac{2 \theta_{k}^{(1)}\left|\dot{\nabla}^{\circ} \phi\right|_{\stackrel{q}{q}}^{2}}{\phi^{5}} .
$$

The divergence of a one-form in two-dimensions is also conformally covariant $\operatorname{div}_{\tilde{q}^{\prime}} \boldsymbol{s}_{\ell^{\prime}}^{(1)}=$ $\frac{1}{\phi^{2}} \operatorname{div}_{\tilde{q}} s_{\ell^{\prime}}^{(1)}$ and

$$
\operatorname{div}_{\tilde{q}^{\prime}} S_{\ell^{\prime}}^{(1)}=\frac{\stackrel{\circ}{\nabla}^{A} S_{\ell}^{(1)} A}{\phi^{3}}-\frac{s_{l}^{(1)}{ }_{A} \stackrel{\circ}{\nabla}^{A} \phi}{\phi^{4}}+\frac{1}{\phi^{5}} \phi_{, A} \phi, L \stackrel{\circ}{h}^{A L}-\frac{1}{2 \phi^{4}} \stackrel{\circ}{h}^{A L} \stackrel{\circ}{\nabla}_{A} \stackrel{\circ}{\nabla}_{L} \phi-\frac{1}{2 \phi^{4}} \phi_{L} \stackrel{\circ}{\nabla}_{A} \stackrel{\circ}{h}^{L A} .
$$




\section{The Hawking energy along null AF hypersurfaces}

Putting things together many terms cancel out and we find

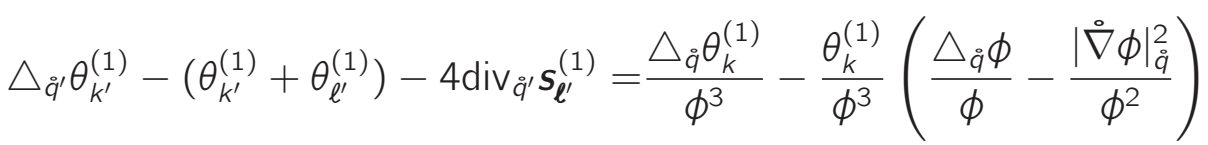

$$
\begin{aligned}
& -\frac{\theta_{k}^{(1)}}{\phi}-\frac{\theta_{\ell}^{(1)}}{\phi^{3}}-\frac{4}{\phi^{2}} \operatorname{div}_{\tilde{q}} s_{\ell}^{(1)} \text {. }
\end{aligned}
$$

Using the large sphere equation (6.36) $\frac{\triangle_{\tilde{q}} \phi}{\phi}-\frac{\left.||^{\nabla} \phi\right|_{\hat{q}} ^{2}}{\phi^{2}}=\triangle_{\tilde{q}} \log \phi=1-\phi^{2}$ we find

$$
\triangle_{\mathfrak{q}^{\prime}} \theta_{k^{\prime}}^{(1)}-\left(\theta_{k^{\prime}}^{(1)}+\theta_{\ell^{\prime}}^{(1)}\right)-4 \operatorname{div}_{\tilde{q}^{\prime}} s_{\ell^{\prime}}^{(1)}=\left(\triangle_{s} \theta_{k}^{(1)}-\left(\theta_{k}^{(1)}+\theta_{\ell}^{(1)}\right)-4 \operatorname{div}_{\mathfrak{q}} s_{\ell}^{(1)}\right) \frac{1}{\phi^{3}} .
$$

Since the volume forms are related by $\boldsymbol{\eta}_{\tilde{\boldsymbol{q}}^{\prime}}=\phi^{2} \boldsymbol{\eta}_{\boldsymbol{q}}$, the result follows.

We have considered so far the limit of the Hawking energy for affine foliations with fixed initial surface $S_{0}$. The second step is to consider affine foliations with a different initial surface. Let us fix an affine background foliation $\left\{S_{\lambda}\right\}$ approaching large spheres and, as usual, let $k$ be the associated null generator $k$ satisfying $k(\lambda)=-1$. Any affine foliation with the same null generator is defined by the equation $\lambda^{\prime}=$ const, where $\lambda^{\prime}$ is any solution of $k\left(\lambda^{\prime}\right)=-1$. Hence $k\left(\lambda-\lambda^{\prime}\right)=0$ and the function $\tau:=\lambda-\lambda^{\prime}$ is Lie constant. This function can be interpreted as the graph function of the initial surface $S_{\lambda^{\prime}=0}$ in the original foliation $\left\{S_{\lambda^{\prime}}\right\}$ (see Figure [6.3). If $\left\{S_{\lambda^{\prime}}\right\}$ starts at a larger initial value $\lambda_{0}^{\prime}>0$, the graph function of $S_{\lambda_{0}^{\prime}}$ is given by an appropriate constant shift of $\tau$, namely $\tau+\lambda_{0}^{\prime}$. The following theorem gives the limit of the Hawking energy for the foliation $\left\{S_{\lambda^{\prime}}\right\}$ and shows that the integrand is also covariant (in fact invariant) for this change of foliation.

Theorem 6.5.6. Let $\Omega$ be a past asymptotically flat null hypersurface endowed with an affinely parametrized background foliation $\left\{S_{\lambda}\right\}$ with generator $k$ and approaching large spheres. Let $\tau$ be a Lie constant function and define the affine foliation $\left\{S_{\lambda^{\prime}}\right\}$ as $S_{\lambda^{\prime}}=\left\{p \in \Omega: \lambda(p)=\tau(p)+\lambda^{\prime}\right\}$, where the graph functions read

$$
\left.\lambda\right|_{s_{\lambda^{\prime}}}=F_{\lambda^{\prime}}:=\left.\tau\right|_{s_{\lambda^{\prime}}}+\lambda^{\prime} .
$$

Then we have $\dot{q}^{\prime}=\stackrel{q}{q}$,

$$
\begin{aligned}
\theta_{k^{\prime}} & =\frac{-2}{\lambda^{\prime}}+\frac{\theta_{k}^{(1)}+2 \tau}{\lambda^{\prime 2}}+o\left(\lambda^{\prime-2}\right), \quad \theta_{\ell^{\prime}}=\frac{2}{\lambda^{\prime}}+\frac{\theta_{\ell}^{(1)}-2 \tau-2 \triangle_{\tilde{q}} \tau}{\lambda^{\prime 2}}+o\left(\lambda^{\prime-2}\right), \\
s_{\ell^{\prime} A} & =\frac{s_{\ell}^{(1)} A+\tau_{, A}}{\lambda^{\prime}}+o\left(\lambda^{\prime-1}\right),
\end{aligned}
$$

and

$$
\left(\triangle_{\tilde{q}} \theta_{k}^{(1)}-\left(\theta_{k}^{(1)}+\theta_{\ell}^{(1)}\right)-4 \operatorname{div}_{\tilde{q}} s_{\ell}^{(1)}\right) \boldsymbol{\eta}_{\tilde{q}}=\left(\triangle_{\tilde{q}^{\prime}} \theta_{k^{\prime}}^{(1)}-\left(\theta_{k^{\prime}}^{(1)}+\theta_{\ell^{\prime}}^{(1)}\right)-4 \operatorname{div}_{\tilde{q}^{\prime}} s_{\ell^{\prime}}^{(1)}\right) \boldsymbol{\eta}_{\tilde{q}^{\prime}} .
$$




\subsection{Limit of the Hawking energy for affine foliations}

Moreover, the limits of the Hawking energies along the two foliations coincide and read

$$
\lim _{\lambda \rightarrow \infty} m_{H}\left(S_{\lambda}\right)=\lim _{\lambda^{\prime} \rightarrow \infty} m_{H}\left(S_{\lambda^{\prime}}\right)=\frac{-1}{16 \pi} \int_{\mathbb{S}^{2}}\left(\theta_{k}^{(1)}+\theta_{\ell}^{(1)}\right) \boldsymbol{\eta}_{\dot{q}}=\frac{-1}{16 \pi} \int_{\mathbb{S}^{2}}\left(\theta_{k^{\prime}}^{(1)}+\theta_{\ell^{\prime}}^{(1)}\right) \boldsymbol{\eta}_{\dot{q}^{\prime}} .
$$

Proof. Changing parameters in the first fundamental form

$$
\gamma_{A B}=\stackrel{\circ}{q}_{A B} \lambda^{2}+h_{A B} \lambda+\Psi_{A B}=\stackrel{\circ}{q}_{A B}\left(\tau+\lambda^{\prime}\right)^{2}+O\left(\lambda^{\prime}\right)=\stackrel{\circ}{q}_{A B} \lambda^{\prime 2}+O\left(\lambda^{\prime}\right),
$$

it follows $\stackrel{q}{q}^{\prime}=\stackrel{\circ}{q}$. Similarly, (6.23) gives

$\theta_{k}=\frac{-2}{\lambda}+\frac{\theta_{k}^{(1)}}{\lambda^{2}}+o\left(\lambda^{-2}\right)=\frac{-2}{\tau+\lambda^{\prime}}+\frac{\theta_{k}^{(1)}}{\left(\tau+\lambda^{\prime}\right)^{2}}+o\left(\lambda^{\prime-2}\right)=\frac{-2}{\lambda^{\prime}}+\frac{\theta_{k}^{(1)}+2 \tau}{\lambda^{\prime 2}}+o\left(\lambda^{\prime-2}\right)$,

so that $\theta_{k^{\prime}}^{(1)}=\theta_{k}^{(1)}+2 \tau$. For $\theta_{\ell^{\prime}}$ we use (6.11) with $\alpha=1, Q_{k}=0$ and $F=F_{\lambda^{\prime}}$. First we change parameter in the expansion of the first terms in the right-hand side

$$
\theta_{\ell}=\frac{2}{\lambda}+\frac{\theta_{\ell}^{(1)}}{\lambda^{2}}+o\left(\lambda^{-2}\right)=\frac{2}{\tau+\lambda^{\prime}}+\frac{\theta_{\ell}^{(1)}}{\left(\tau+\lambda^{\prime}\right)^{2}}+o\left(\lambda^{\prime-2}\right)=\frac{2}{\lambda^{\prime}}+\frac{\theta_{\ell}^{(1)}-2 \tau}{\lambda^{\prime 2}}+o\left(\lambda^{\prime-2}\right)
$$

Since $D F_{\lambda^{\prime}}=D \tau$ independent of $\lambda$ and $\gamma^{A B}=O\left(\lambda^{\prime-2}\right)$, cf. (6.16), we have $\left|D F_{\lambda^{\prime}}\right|_{\gamma}^{2}=$ $O\left(\lambda^{\prime-2}\right)$ and the term $\theta_{k}\left|D F_{\lambda^{\prime}}\right|_{\gamma}^{2}=O\left(\lambda^{\prime-3}\right)$. The same argument shows that $s_{\boldsymbol{\ell}}(\operatorname{grad} F)=$ $O\left(\lambda^{\prime-3}\right)$ and $K^{k}(\operatorname{grad} F, \operatorname{grad} F)=O\left(\lambda^{\prime-3}\right)$. The Laplacian term can be computed, using (6.25), as

$$
\triangle_{\gamma} F_{\lambda^{\prime}}=\frac{\triangle_{\mathfrak{q}} \tau}{\lambda^{2}}+o\left(\lambda^{-2}\right)=\frac{\triangle_{\mathfrak{q}} \tau}{\lambda^{\prime 2}}+o\left(\lambda^{\prime-2}\right)
$$

Inserting also these into expression (6.11) yields

$$
\theta_{\ell^{\prime}}=\frac{2}{\lambda^{\prime}}+\frac{\theta_{\ell}^{(1)}-2 \tau}{\lambda^{\prime 2}}-\frac{2 \triangle_{\mathfrak{q}} \tau}{\lambda^{\prime 2}}+o\left(\lambda^{\prime-2}\right)=\frac{2}{\lambda^{\prime}}+\frac{\theta_{\ell}^{(1)}-2 \tau-2 \triangle_{\mathfrak{q}} \tau}{\lambda^{\prime 2}}+o\left(\lambda^{\prime-2}\right)
$$

as claimed. Note in particular that $\theta_{\ell^{\prime}}^{(1)}=\theta_{\ell}^{(1)}-2 \tau-2 \triangle_{\mathfrak{q}} \tau$. The expansion of the connection one-form $s_{\ell^{\prime}}^{(1)}$ is obtained from (6.7) with $\alpha=1$ and $Q_{k}=0$. The term $-K^{k}\left(X_{A}, \operatorname{grad} F_{\lambda^{\prime}}\right)$ is $-K^{k}\left(X_{A}, \operatorname{grad} F_{\lambda^{\prime}}\right)=-K^{k}\left(X_{A}, \operatorname{grad} \tau\right)=\frac{1}{\lambda} \tau_{A}+O\left(\lambda^{-2}\right)$ after using (6.22). Given the expansion of $s_{\boldsymbol{\ell}}$ in item (ii) of Definition 6.3.4 we conclude

$$
\begin{gathered}
s_{\ell^{\prime} A}=\frac{s_{\ell}^{(1)} A}{\lambda}+\frac{\tau_{, A}}{\lambda}+o\left(\frac{1}{\lambda}\right)=\frac{s_{\ell}^{(1)} A+\tau_{, A}}{\lambda}+o\left(\frac{1}{\lambda}\right)=\frac{s_{\ell}^{(1)} A+\tau_{, A}}{\lambda^{\prime}}+o\left(\frac{1}{\lambda^{\prime}}\right) \Longrightarrow \\
s_{\ell^{\prime}}^{(1)} A=s_{\ell}^{(1)} A+\tau_{, A} .
\end{gathered}
$$

Inserting $\stackrel{q}{q}^{\prime}, \theta_{k^{\prime}}^{(1)}, \theta_{\ell^{\prime}}^{(1)}$ and $\boldsymbol{s}_{\boldsymbol{\ell}^{\prime}}^{(1)}$ in $\left(\triangle_{\dot{q}^{\prime}} \theta_{k^{\prime}}^{(1)}-\left(\theta_{k^{\prime}}^{(1)}+\theta_{\ell^{\prime}}^{(1)}\right)-4 \operatorname{div}_{q^{\prime}} s_{\ell^{\prime}}^{(1)}\right) \boldsymbol{\eta}_{\dot{q}^{\prime}}$ all terms in $\tau$ cancel out and the invariance (6.37) is established. For the last statement we use the fact that both foliations $\left\{S_{\lambda}\right\}$ and $\left\{S_{\lambda^{\prime}}\right\}$ are affine foliations approaching large spheres. 


\section{The Hawking energy along null AF hypersurfaces}

So, both can be taken as background foliations and in each case we can apply Theorem 6.5 .3 with $\psi=1$. Using the Gauss identity, the equalities

$$
\lim _{\lambda \rightarrow \infty} m_{H}\left(S_{\lambda}\right)=\frac{-1}{16 \pi} \int_{\mathbb{S}^{2}}\left(\theta_{k}^{(1)}+\theta_{\ell}^{(1)}\right) \boldsymbol{\eta}_{\grave{q}} \quad \text { and } \quad \lim _{\lambda^{\prime} \rightarrow \infty} m_{H}\left(S_{\lambda^{\prime}}\right)=\frac{-1}{16 \pi} \int_{\mathbb{S}^{2}}\left(\theta_{k^{\prime}}^{(1)}+\theta_{\ell^{\prime}}^{(1)}\right) \boldsymbol{\eta}_{\tilde{q}^{\prime}}
$$

hold. Invoking the invariance (6.37) the remaining equality $\lim _{\lambda \rightarrow \infty} m_{H}\left(S_{\lambda}\right)=\lim _{\lambda^{\prime} \rightarrow \infty} m_{H}\left(S_{\lambda^{\prime}}\right)$ follows.

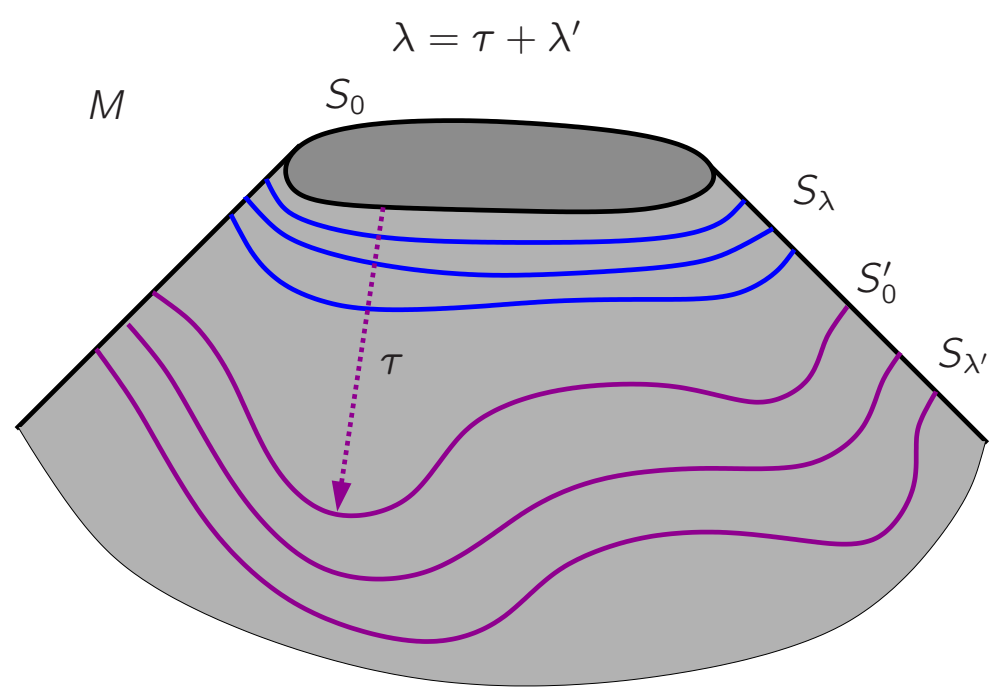

Figure 6.3: Representation of two different flows $\left\{S_{\lambda}\right\}$ and $\left\{S_{\lambda^{\prime}}\right\}$ associated to different parameters $\lambda$ and $\lambda^{\prime}$. Both flows have the same velocity, but start on different surfaces separated by a "distance" $\tau$. More precisely, the starting surface $S_{0}^{\prime}$ of $\left\{S_{\lambda^{\prime}}\right\}$ corresponds to the graph surface $\lambda=\tau$. Extending $\tau$ to a Lie constant function on $\Omega$ one has $\lambda=\tau+\lambda^{\prime}$ everywhere.

\subsection{Limit of the Hawking energy for non-affine folia- tions}

Our aim in this chapter is to obtain the limit of the Hawking energy along very general foliations $\left\{S_{\lambda^{*}}\right\}$. In the previous section we dealt with the general case when the foliations are affine. In order to go into more general settings we need to consider vector fields $k^{\prime}$ not affinely parametrized. We will however assume that $k^{\prime}$ is nowhere zero even at the limit at infinity. More specifically, we assume that there exists a function $\lambda^{\prime} \in \mathcal{F}(\Omega)$ satisfying $k^{\prime}\left(\lambda^{\prime}\right)=-1$ and an affine background foliation (not necessarily approaching large spheres) defined as the level sets of a function $\lambda \in \mathcal{F}(\Omega)$ such that 


\subsection{Limit of the Hawking energy for non-affine foliations}

$\xi:=\lambda-\lambda^{\prime}$ decays at infinity in an appropriate way. In other words, the foliation $\left\{S_{\lambda^{\prime}}\right\}$ is assumed to approach at infinity an affine foliation $\left\{S_{\lambda}\right\}$ at an appropriate rate. Conversely, given an affine background foliation and a function $\xi \in \mathcal{F}(\Omega)$ satisfying $\xi=o_{1}(1)$ we can define a function $\lambda^{\prime}:=\lambda-\xi \in \mathcal{F}(\Omega)$. The level sets of this function are smooth surfaces at least for points at large enough $\lambda$. This is because $d \lambda^{\prime}(k)=k(\lambda-\xi)=-1-k(\xi) \neq 0$ given that $k(\xi)$ decays at infinity. Thus, for $\lambda$ bigger than some (possible large) value $\rho_{1}$, the level sets $\lambda^{\prime}=$ const define a foliation $\left\{S_{\lambda^{\prime}}\right\}$. Each surface on this foliation is transverse to $k$ and hence spacelike. The null generator $k^{\prime}$ satisfying $k^{\prime}\left(\lambda^{\prime}\right)=-1$ is given by $k^{\prime}=\frac{1}{1+k(\xi)} k$ because

$$
k^{\prime}\left(\lambda^{\prime}\right)=\frac{1}{1+k(\xi)} k\left(\lambda^{\prime}\right)=\frac{1}{1+k(\xi)} k(\lambda-\xi)=-1 .
$$

It is clear that the foliation $\left\{S_{\lambda^{\prime}}\right\}$ is not affine in general. Given a value $\lambda^{\prime}$ large enough, the surface $S_{\lambda^{\prime}}$ is a graph on the background foliation $\left\{S_{\lambda}\right\}$, and in particular on $S_{0}$. The graph function $\left.\lambda\right|_{S_{\lambda^{\prime}}}=F_{\lambda^{\prime}}$ is given by $F_{\lambda^{\prime}}(p)=\lambda^{\prime}+\xi(p)$ for all $p \in S_{\lambda^{\prime}}$. As usual, we extend the graph function to $\Omega$ by Lie dragging along $k$. Note that $F_{\lambda^{\prime}}$ extended this way is not $\lambda^{\prime}+\xi$, but both agree on $S_{\lambda^{\prime}}$. Thus we can safely abuse notation and write the graph simply as $F_{\lambda^{\prime}}=\lambda^{\prime}+\left.\xi\right|_{s_{\lambda^{\prime}}}$. The following theorem gives the limit of the Hawking energy for the foliation $\left\{S_{\lambda^{\prime}}\right\}$.

Theorem 6.6.1. Let $\Omega$ be a past asymptotically flat null hypersurface endowed with an affinely parametrized background foliation $\left\{S_{\lambda}\right\}$ with generator $k$. Let $\xi \in \mathcal{F}(\Omega)$ satisfy $\xi=o_{1}(1) \cap o_{2}^{X}(1)$, and $k(\xi)=o_{1}^{X}\left(\lambda^{-1}\right)$. Define the foliation $\left\{S_{\lambda^{\prime}}\right\}$ as $S_{\lambda^{\prime}}=\{p \in \Omega$ : $\left.\lambda(p)=\lambda^{\prime}+\xi(p)\right\}$, where the graph functions read

$$
\left.\lambda\right|_{S_{\lambda^{\prime}}}=F_{\lambda^{\prime}}:=\lambda^{\prime}+\left.\xi\right|_{S_{\lambda^{\prime}}} .
$$

Then the null expansions and the connection one-form of $\left\{S_{\lambda^{\prime}}\right\}$ have, to leading orders, the same form as for the background foliation, i.e.

$$
\theta_{k^{\prime}}=\frac{-2}{\lambda^{\prime}}+\frac{\theta_{k}^{(1)}}{\lambda^{\prime 2}}+o\left(\lambda^{\prime-2}\right), \quad \theta_{\ell^{\prime}}=\frac{2 \mathcal{K}_{\hat{q}}}{\lambda^{\prime}}+\frac{\theta_{\ell}^{(1)}}{\lambda^{\prime 2}}+o\left(\lambda^{\prime-2}\right), \quad s_{\ell^{\prime} A}=\frac{\left(s_{\ell}^{(1)}\right)_{A}}{\lambda^{\prime}}+o\left(\lambda^{\prime-1}\right),
$$

where all objects in the right-hand side refer to the background foliation. The limit of the Hawking energy along $\left\{S_{\lambda^{\prime}}\right\}$ is the same as the limit along $\left\{S_{\lambda}\right\}$ and reads

$$
\lim _{\lambda \rightarrow \infty} m_{H}\left(S_{\lambda}\right)=\lim _{\lambda^{\prime} \rightarrow \infty} m_{H}\left(S_{\lambda^{\prime}}\right)=\frac{-1}{8 \pi \sqrt{16 \pi}} \sqrt{|\hat{S}|} \int_{\hat{S}}\left(\mathcal{K}_{\hat{q}} \theta_{k}^{(1)}+\theta_{\ell}^{(1)}\right) \boldsymbol{\eta}_{\hat{q}}
$$

where $|\hat{S}|$ is the area of $\left(S_{0}, \hat{q}\right)$ and $\boldsymbol{\eta}_{\hat{q}}$ the corresponding volume form.

Proof. The asymptotic expansion for $\theta_{k}$ is obtained by simply changing the parameter of the foliation $\lambda=\lambda^{\prime}+\xi$ :

$\theta_{k}=\frac{-2}{\lambda}+\frac{\theta_{k}^{(1)}}{\lambda^{2}}+o\left(\lambda^{-2}\right)=\frac{-2}{\lambda^{\prime}+\xi}+\frac{\theta_{k}^{(1)}}{\left(\lambda^{\prime}+\xi\right)^{2}}+o\left(\lambda^{\prime-2}\right)=\frac{-2}{\lambda^{\prime}}+\frac{\theta_{k}^{(1)}}{\lambda^{\prime 2}}+o\left(\lambda^{\prime-2}\right)$, 


\section{The Hawking energy along null AF hypersurfaces}

where $\xi=o_{1}(1)$ has been used in the last equality. Given that $k^{\prime}=\left(\frac{1}{1+k(\xi)}\right) k$ and $k(\xi)=o\left(\lambda^{-1}\right)$, the null expansion along $k^{\prime}$ is

$$
\theta_{k^{\prime}}=\left(\frac{1}{1+k(\xi)}\right) \theta_{k}=\frac{-2}{\lambda^{\prime}}+\frac{\theta_{k}^{(1)}}{\lambda^{\prime 2}}+o\left(\lambda^{\prime-2}\right)
$$

which is the first expression in (6.39). We next compute $\theta_{\ell^{\prime}}$ from (6.11) with $\alpha=\frac{1}{1+k(\xi)}$. Changing parameters in the first term of the right hand side,

$\theta_{\ell}=\frac{2 \mathcal{K}_{\hat{q}}}{\lambda^{\prime}+\xi}+\frac{\theta_{\ell}^{(1)}}{\left(\lambda^{\prime}+\xi\right)^{2}}+o\left(\lambda^{\prime-2}\right)=\frac{2 \mathcal{K}_{\hat{q}}}{\lambda^{\prime}}+\frac{\theta_{\ell}^{(1)}-2 \xi}{\lambda^{\prime 2}}+o\left(\lambda^{\prime-2}\right)=\frac{2 \mathcal{K}_{\hat{q}}}{\lambda^{\prime}}+\frac{\theta_{\ell}^{(1)}}{\lambda^{\prime 2}}+o\left(\lambda^{\prime-2}\right)$.

For the terms involving the graph function, it is immediate to check that $\left|D F_{\lambda^{\prime}}\right|_{\gamma}^{2} \theta_{k}=$ $o\left(\lambda^{\prime-3}\right), s_{\ell}\left(\operatorname{grad} F_{\lambda^{\prime}}\right)=o\left(\lambda^{\prime-3}\right), K^{k}\left(\operatorname{grad} F_{\lambda^{\prime}}, \operatorname{grad} F_{\lambda^{\prime}}\right)=o\left(\lambda^{\prime-3}\right)$. For the Laplacian term (6.25) gives

$$
\triangle_{\gamma} F_{\lambda^{\prime}}=\frac{\triangle_{\hat{q}} F_{\lambda^{\prime}}}{\lambda^{2}}+o\left(\lambda^{-2}\right)=\frac{\triangle_{\hat{q}} F_{\lambda^{\prime}}}{\lambda^{\prime 2}}+o\left(\lambda^{\prime-2}\right)=\frac{\triangle_{\hat{q}} \xi}{\lambda^{\prime 2}}+o\left(\lambda^{\prime-2}\right)=o\left(\lambda^{\prime-2}\right),
$$

because $\xi=o_{2}^{X}(1)$. Finally, $k(\xi)=o\left(\lambda^{\prime-1}\right)$ implies $\frac{1}{\alpha}=1+k(\xi)=1+o\left(\lambda^{\prime-1}\right)$ and (6.11) is simply

$$
\theta_{\ell^{\prime}}=\left(1+o\left(\lambda^{\prime-1}\right)\right)\left(\frac{2 \mathcal{K}_{\hat{q}}}{\lambda^{\prime}}+\frac{\theta_{\ell}^{(1)}}{\lambda^{\prime 2}}+o\left(\lambda^{\prime-2}\right)\right)=\frac{2 \mathcal{K}_{\hat{q}}}{\lambda^{\prime}}+\frac{\theta_{\ell}^{(1)}}{\lambda^{\prime 2}}+o\left(\lambda^{\prime-2}\right),
$$

as stated in the theorem. The connection one-form $s_{\ell^{\prime}}$ is obtained from (66.7) with $Q_{k}=0$ and $\alpha=\frac{1}{1+k(\xi)}$. Given that $\alpha_{, A}=o\left(\lambda^{\prime-1}\right)$ (because $k(\xi)=o_{1}^{X}\left(\lambda^{\prime-1}\right)$ ) and $s_{\ell}\left(\operatorname{grad} F_{\lambda^{\prime}}\right)=o\left(\lambda^{\prime-2}\right)$, we conclude

$$
s_{\ell^{\prime} A}=\frac{\left(s_{\ell}^{(1)}\right)_{A}}{\lambda^{\prime}}+o\left(\lambda^{\prime-1}\right) .
$$

We next compute the limit of the Hawking energy along $\left\{S_{\lambda^{\prime}}\right\}$. The metric in $S_{\lambda^{\prime}}$ is

$$
\gamma\left(\lambda^{\prime}\right)_{A B}=\hat{q}_{A B} \lambda^{2}+o\left(\lambda^{2}\right)=\hat{q}_{A B}\left(\lambda^{\prime}+\xi\right)^{2}+o\left(\lambda^{\prime 2}\right)=\hat{q}_{A B} \lambda^{\prime 2}+o\left(\lambda^{\prime 2}\right),
$$

so that in particular the rescaled limit metric and corresponding volume forms remain unchanged, $\hat{q}^{\prime}=\hat{q}$ and $\boldsymbol{\eta}_{\hat{q}^{\prime}}=\boldsymbol{\eta}_{\hat{q}}$. This, together with the expansions (6.39), already implies that the limit of the Hawking energy along $\left\{S_{\lambda^{\prime}}\right\}$ and along $\left\{S_{\lambda}\right\}$ are the same. To obtain expression (6.40) we need the volume form of $S_{\lambda^{\prime}}$. As with the metric $\gamma(\lambda)$ or with the null expansion $\theta_{k}$ it suffices to change parameter in the volume form $\boldsymbol{\eta}_{\boldsymbol{s}_{\lambda}}$ which is given by (6.29) with $\phi=1$

$$
\boldsymbol{\eta}_{\boldsymbol{S}_{\lambda^{\prime}}}=\left(\left(\lambda^{\prime}+\xi\right)^{2}+\theta_{k}^{(1)}\left(\lambda^{\prime}+\xi\right)+o\left(\lambda^{\prime}\right)\right) \boldsymbol{\eta}_{\hat{\boldsymbol{q}}}=\left(\lambda^{\prime 2}+\theta_{k}^{(1)} \lambda^{\prime}+o\left(\lambda^{\prime}\right)\right) \boldsymbol{\eta}_{\hat{\boldsymbol{q}}} .
$$




\subsection{Limit of the Hawking energy for non-affine foliations}

A straightforward computation shows that the product $\theta_{k^{\prime}} \theta_{\ell^{\prime}} \boldsymbol{\eta}_{S_{\lambda^{\prime}}}$ is

$$
\theta_{k^{\prime}} \theta_{\ell^{\prime}} \boldsymbol{\eta}_{S_{\lambda^{\prime}}}=\left(-4 \mathcal{K}_{\hat{q}}+\left(-2 \mathcal{K}_{\hat{q}} \theta_{k}^{(1)}-2 \theta_{\ell}^{(1)}\right) \frac{1}{\lambda^{\prime}}+o\left(\lambda^{\prime-1}\right)\right) \boldsymbol{\eta}_{\hat{q}}
$$

so that, using Gauss-Bonnet $\int_{\hat{S}} \mathcal{K}_{\hat{q}} \boldsymbol{\eta}_{\hat{q}}=4 \pi$,

$$
1+\frac{1}{16 \pi} \int_{S_{\lambda^{\prime}}} \theta_{k^{\prime}} \theta_{\ell^{\prime}} \boldsymbol{\eta}_{S_{\lambda^{\prime}}}=\frac{1}{16 \pi} \int_{\hat{S}}\left(-2 \mathcal{K}_{\hat{q}} \theta_{k}^{(1)}-2 \theta_{\ell}^{(1)}\right) \boldsymbol{\eta}_{\hat{q}} \frac{1}{\lambda^{\prime}}+o\left(\lambda^{\prime-1}\right) .
$$

On the other hand $\left|S_{\lambda^{\prime}}\right|=\int_{\hat{S}}\left(\lambda^{\prime 2}+\theta_{k}^{(1)} \lambda^{\prime}+o\left(\lambda^{\prime}\right)\right) \boldsymbol{\eta}_{\hat{q}}=|\hat{S}| \lambda^{\prime 2}+o\left(\lambda^{\prime 2}\right) \Rightarrow \sqrt{\left|S_{\lambda^{\prime}}\right|}=$ $\sqrt{|\hat{S}|} \lambda^{\prime}+o\left(\lambda^{\prime}\right)$ and

$$
\begin{aligned}
m_{H}\left(S_{\lambda^{\prime}}\right) & =\frac{1}{\sqrt{16 \pi}}\left(\sqrt{|\hat{S}|} \lambda^{\prime}+o\left(\lambda^{\prime}\right)\right)\left(\frac{1}{16 \pi} \int_{\hat{S}}\left(-2 \mathcal{K}_{\hat{q}} \theta_{k}^{(1)}-2 \theta_{\ell}^{(1)}\right) \boldsymbol{\eta}_{\hat{q}} \frac{1}{\lambda^{\prime}}+o\left(\lambda^{\prime-1}\right)\right) \\
& =\frac{-1}{8 \pi \sqrt{16 \pi}} \sqrt{|\hat{S}|} \int_{\hat{S}}\left(\mathcal{K}_{\hat{q}} \theta_{k}^{(1)}+\theta_{\ell}^{(1)}\right) \boldsymbol{\eta}_{\hat{q}}+o(1) .
\end{aligned}
$$

We are ready to obtain our main Theorem 6.6 .2 by simply combining the previous results. In fact, we state and prove a slightly more complete theorem that provides two different expressions for the limit.

Theorem 6.6.2 (General Hawking energy limit). Let $\Omega$ be a past asymptotically flat null hypersurface endowed with an affinely parametrized background foliation $\left\{S_{\lambda}\right\}$ with generator $k$ that tends to large spheres. Define the foliation $\left\{S_{\lambda^{*}}\right\}$ as $S_{\lambda^{*}}=\{p \in \Omega$ : $\left.\lambda(p)=\frac{1}{\Psi(p)} \lambda^{\star}+\tau(p)+\xi(p)\right\}$, where the graph functions read

$$
\left.\lambda\right|_{S_{\lambda^{\star}}}=F_{\lambda^{\star}}:=\left.\frac{1}{\psi}\right|_{S_{\lambda^{\star}}} \lambda^{\star}+\left.\tau\right|_{S_{\lambda^{\star}}}+\left.\xi\right|_{S_{\lambda^{\star}}},
$$

with $\Psi>0$, and $\tau$ being Lie constant functions on $\Omega$ and $\xi=o_{1}(1) \cap o_{2}^{X}(1)$ with $k(\xi)=o_{1}^{X}\left(\lambda^{-1}\right)$. The limit of the Hawking energy along $\left\{S_{\lambda^{\star}}\right\}$ is

$$
\begin{aligned}
\lim _{\lambda^{\star} \rightarrow \infty} m_{H}\left(S_{\lambda^{\star}}\right)= & \frac{-1}{8 \pi \sqrt{16 \pi}}\left(\sqrt{\int_{\mathbb{S}^{2}} \boldsymbol{\eta}_{\hat{q}}}\right) \int_{\mathbb{S}^{2}}\left(\mathcal{K}_{\hat{q}} \theta_{k^{\star}}^{(1)}+\theta_{\ell^{\star}}^{(1)}\right) \boldsymbol{\eta}_{\hat{q}} \\
= & \frac{1}{8 \pi \sqrt{16 \pi}}\left(\sqrt{\int_{\mathbb{S}^{2}} \frac{1}{\Psi^{2}} \boldsymbol{\eta}_{\grave{q}}}\right) \\
& \times \int_{\mathbb{S}^{2}}\left(\triangle_{\dot{q}} \theta_{k}^{(1)}-\left(\theta_{k}^{(1)}+\theta_{\ell}^{(1)}\right)-4 \operatorname{div}_{\mathfrak{q}}\left(\boldsymbol{s}_{\ell}^{(1)}\right)\right) \Psi \boldsymbol{\eta}_{\dot{q}},
\end{aligned}
$$

where $\hat{q}, \theta_{k^{\star}}^{(1)}$ and $\theta_{\ell^{\star}}^{(1)}$ refer either to the foliation $\left\{S_{\lambda^{\star}}\right\}$ or to the affine foliation $\left\{S_{\lambda^{\prime \prime}}\right\}$ defined as $S_{\lambda^{\prime \prime}}=\left\{p \in \Omega: \lambda(p)=\frac{1}{\Psi(p)} \lambda^{\prime \prime}+\tau(p)\right\}$ and whose respective graph functions 


\section{The Hawking energy along null AF hypersurfaces}

read $\left.\lambda\right|_{S_{\lambda^{\prime \prime}}}=F_{\lambda^{\prime \prime}}:=\left.\frac{1}{\psi}\right|_{S_{\lambda^{\prime \prime}}} \lambda^{\prime \prime}+\left.\tau\right|_{S_{\lambda^{\prime \prime}}}$, and $\stackrel{\circ}{q}, \theta_{k}^{(1)}, \theta_{\ell}^{(1)}$ and $s_{\ell}^{(1)}$ refer to the background foliation $\left\{S_{\lambda}\right\}$.

Proof. The strategy is to pass from the background foliation to $\left\{S_{\lambda^{*}}\right\}$ in three steps. The geometric elements of each foliation use the same symbol as the foliation, so the meaning of each quantity should be clear. Consider first a foliation defined by the level sets of $\lambda^{\prime}:=\lambda-\tau$. Theorem 6.5 .6 gives

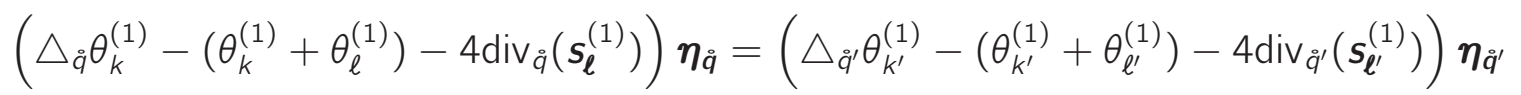

Consider next the foliation defined by the level sets of $\lambda^{\prime \prime}$, where $\lambda^{\prime}=\psi^{-1} \lambda^{\prime \prime}$. Since $\left\{S_{\lambda^{\prime}}\right\}$ is an affine foliation approaching large spheres, Theorem 6.5.3 implies the limit of the Hawking energy is

$$
\begin{aligned}
\lim _{\lambda^{\prime \prime} \rightarrow \infty} m_{H}\left(S_{\lambda^{\prime \prime}}\right)= & \frac{1}{8 \pi \sqrt{16 \pi}}\left(\sqrt{\int_{\mathbb{S}^{2}} \frac{1}{\Psi^{2}} \boldsymbol{\eta}_{\dot{q}^{\prime}}}\right) \\
& \times \int_{\mathbb{S}^{2}}\left(\triangle_{\tilde{q}^{\prime}} \theta_{k^{\prime}}^{(1)}-\left(\theta_{k^{\prime}}^{(1)}+\theta_{\ell^{\prime}}^{(1)}\right)-4 \operatorname{div}_{\tilde{q}^{\prime}}\left(\boldsymbol{s}_{\ell^{\prime}}^{(1)}\right)\right) \Psi \boldsymbol{\eta}_{\dot{q}^{\prime}}
\end{aligned}
$$

which, upon using (6.6) and $\boldsymbol{\eta}_{\grave{q}}=\boldsymbol{\eta}_{\dot{q}^{\prime}}$, implies

$$
\begin{aligned}
\lim _{\lambda^{\prime \prime} \rightarrow \infty} m_{H}\left(S_{\lambda^{\prime \prime}}\right)= & \frac{1}{8 \pi \sqrt{16 \pi}}\left(\sqrt{\int_{\mathbb{S}^{2}} \frac{1}{\Psi^{2}} \boldsymbol{\eta}_{\dot{q}}}\right) \\
& \times \int_{\mathbb{S}^{2}}\left(\triangle_{\dot{q}} \theta_{k}^{(1)}-\left(\theta_{k}^{(1)}+\theta_{\ell}^{(1)}\right)-4 \operatorname{div}_{\dot{q}}\left(s_{\ell}^{(1)}\right)\right) \Psi \boldsymbol{\eta}_{\dot{q}} .
\end{aligned}
$$

Now, $\left\{S_{\lambda^{\prime \prime}}\right\}$ is affine but does not necessarily tend to large spheres. The final foliation $\left\{S_{\lambda^{*}}\right\}$ is related to $\left\{S_{\lambda^{\prime \prime}}\right\}$ by $\lambda^{\prime \prime}=\lambda^{\star}+\psi \xi$. Since $\psi \xi$ satisfies the hypotheses of Theorem 6.6.1 we conclude that the Hawking energy has the same limit along $\left\{S_{\lambda^{\prime \prime}}\right\}$ and along $\left\{S_{\lambda^{*}}\right\}$. In combination with (6.42) this proves the second equality in (6.41). For the first equality we simply note that the rescaled limit metric of $\left\{S_{\lambda^{\prime \prime}}\right\}$ is $\hat{q}=\Psi^{-2} \dot{q}$ and apply again Theorem 6.6.1.

Remark 6.6.3. In this chapter we have considered null hypersurfaces extending to past null infinity. Obviously similar results apply for asymptotically flat null hypersurfaces extending to future null infinity. By repeating the arguments before, the following result is obtained: consider a future directed affine null vector $\bar{k}$ tangent to $\Omega$ and define the function $\lambda \in \mathcal{F}(\Omega)$ by $\bar{k}(\lambda)=1$ with $\lambda=0$ on some initial cross section. The level sets $\left\{S_{\lambda}\right\}$ define a foliation which allows to construct a transversal future directed null normal $\bar{\ell}$ satisfying $\langle\bar{k}, \bar{\ell}\rangle=-2$. If the rescaled asymptotic metric of the foliation 


\subsection{The large sphere equation and the Bondi energy-momentum}

is spherical $\stackrel{q}{q}$, the expansions of the null second fundamental forms and connection one-form take the form (note the change of signs with respect to the past null case)

$$
\theta_{\bar{k}}=\frac{2}{\lambda}+\frac{\theta_{\bar{k}}^{(1)}}{\lambda^{2}}+o\left(\lambda^{-2}\right), \quad \theta_{\bar{\ell}}=\frac{-2}{\lambda}+\frac{\theta_{\bar{\ell}}^{(1)}}{\lambda^{2}}+o\left(\lambda^{-2}\right) \quad s_{\bar{\ell} A}=\frac{s_{\bar{\ell} A}^{(1)}}{\lambda}+o\left(\lambda^{-1}\right)
$$

and the limit of the Hawking energy along $\left\{S_{\lambda^{*}}\right\}$ (with the same definition as in Theorem 6.6.2) is

$$
\begin{aligned}
& \lim _{\lambda^{*} \rightarrow \infty} m_{H}\left(S_{\lambda^{*}}\right)= \\
& =\frac{1}{8 \pi \sqrt{16 \pi}}\left(\sqrt{\int_{\mathbb{S}^{2}} \frac{1}{\Psi^{2}} \boldsymbol{\eta}_{\tilde{q}}}\right) \int_{\mathbb{S}^{2}}\left(-\triangle_{\tilde{q}} \theta_{\bar{k}}^{(1)}+\left(\theta_{\bar{k}}^{(1)}+\theta_{\bar{\ell}}^{(1)}\right)-4 \operatorname{div}_{\tilde{q}}\left(s_{\bar{\ell}}^{(1)}\right)\right) \psi \boldsymbol{\eta}_{\tilde{q}} .
\end{aligned}
$$

\subsection{The large sphere equation and the Bondi energy- momentum}

As mentioned in Section 6.1, the limit of the Hawking energy when the foliation approaches large spheres is the Bondi energy. In this section we want to recover this fact from our general expressions. Recall first that the conformal group of the two-sphere

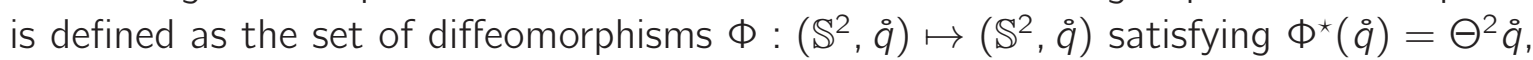
$\Theta \in \mathcal{F}\left(\mathbb{S}^{2}, \mathbb{R}^{+}\right)$(i.e. the set of conformal diffeomorphisms). We restrict ourselves to the connected component of the identity of this group. It is well-known (see e.g. [87]) that this group is isomorphic to the connected component of the identity of Lorentz group of Minkowski space $\mathcal{M}^{1,3}$, and also isomorphic to the Möbius group of the Riemann sphere

$$
\begin{aligned}
F: \mathbb{S}^{2} & \mapsto \mathbb{S}^{2} \\
z & \mapsto F(z)=\frac{\alpha z+\beta}{\gamma z+\delta} \quad\left(\begin{array}{ll}
\alpha & \beta \\
\gamma & \delta
\end{array}\right) \in S L(2, \mathbb{C}),
\end{aligned}
$$

where $z \in \mathbb{C} \cup\{\infty\} \simeq \mathbb{S}^{2}$. In these coordinates, the standard metric on the sphere is $\stackrel{q}{q}=\frac{4}{(1+z \bar{z})^{2}} d z d \bar{z}$ and the $I=1$ spherical harmonics read

$$
Y_{1}^{1}=\frac{z+\bar{z}}{1+z \bar{z}}, \quad Y_{2}^{1}=\frac{z-\bar{z}}{i(1+z \bar{z})}, \quad Y_{3}^{1}=\frac{z \bar{z}-1}{1+z \bar{z}} .
$$

Remark 6.7.1. The notation used in this section for the spherical harmonics differs from the one used in Section 4.5 in Chapter 4 because we do not consider here the normalized version of such functions. 


\section{The Hawking energy along null AF hypersurfaces}

For a vector $a \in \mathbb{R}^{3}$ we write $a \cdot Y^{1}:=\sum_{i=1}^{3} a^{i} Y_{i}^{1}$. These properties allow us to obtain easily the general solution to the large sphere equation (6.36).

Proposition 6.7.2 (Solution of the large sphere equation). A smooth function $\phi$ : $\mathbb{S}^{2} \mapsto \mathbb{R}^{+}$solves equation (6.36) if and only if there exists $a=\left(a^{1}, a^{2}, a^{3}\right) \in \mathbb{R}^{3}$ such that

$$
\Psi:=\frac{1}{\phi}=\sqrt{1+|a|^{2}}+a \cdot Y^{1} .
$$

Proof. In terms of $\psi:=\frac{1}{\phi}$, equation (6.36) becomes

$$
\psi^{2}+\left(\triangle_{\tilde{q}} \psi\right) \psi-|\circ \nabla \psi|_{\grave{q}}^{2}=1 .
$$

We first show that (6.45) solves this equation. Applying a rotation to $\mathbb{S}^{2}$ we can assume without loss of generality that $a=(0,0, c)$ and hence $\Psi=\sqrt{1+c^{2}}+c Y_{3}^{1}$. Thus $\triangle_{\tilde{q}} \Psi=c \triangle_{\hat{q}} Y_{3}^{1}=-2 c Y_{3}^{1}=-2 \psi+2 \sqrt{1+c^{2}}$, and $\left|\nabla^{\circ} \psi\right|_{\tilde{q}}^{2}=(1+z \bar{z})^{2} \partial_{z} \psi \partial_{\bar{z}} \psi=$ $\frac{4 c^{2} z \bar{z}}{(1+z \bar{z})^{2}}=-\left(\Psi^{2}+1-2 \sqrt{1+c^{2}} \Psi\right)$, and (6.46) holds after immediate cancellations.

To show the converse we recall that equation (6.36) is the statement that the Gauss curvature $\mathcal{K}_{\phi^{2} \grave{q}}=1$. This means that there exist coordinates $z^{\prime} \in \mathbb{C} \cup\{\infty\}$ where $\phi^{2} \stackrel{\circ}{q}=\frac{4 d z^{\prime} d z^{\prime}}{\left(1+z^{\prime} z^{\prime}\right)^{2}}$. We can assume without loss of generality that the map $F(z)=z^{\prime}$ is orientation preserving. Since it is also an element of the conformal group, it must be an element of the Möbius group (6.44). Performing the pull-back of $\stackrel{q}{q}$

$$
\phi^{2} \stackrel{\circ}{q}=\frac{4\left|\frac{\partial F}{\partial z}\right|^{2}}{\left(1+|F|^{2}\right)^{2}} d z d \bar{z} .
$$

Thus $\phi=\frac{1+|z|^{2}}{1+|F|^{2}}\left|\frac{\partial F}{\partial z}\right|$. Since $\frac{\partial F}{\partial z}=\frac{1}{(\gamma z+\delta)^{2}}$ if follows

$$
\Psi=\frac{1}{\phi}=\frac{|\alpha z+\beta|^{2}+|\gamma z+\delta|^{2}}{\left(1+|z|^{2}\right)} .
$$

This function can be expanded in terms of $I=1$ spherical harmonics as

$$
\begin{aligned}
\Psi= & \frac{|\alpha|^{2}+|\beta|^{2}+|\gamma|^{2}+|\delta|^{2}}{2}+\operatorname{Re}(\bar{\alpha} \beta+\bar{\gamma} \delta) Y_{1}^{1}+\operatorname{Im}(\bar{\alpha} \beta+\bar{\gamma} \delta) Y_{2}^{1} \\
& +\frac{|\alpha|^{2}-|\beta|^{2}+|\gamma|^{2}-|\delta|^{2}}{2} Y_{3}^{1} .
\end{aligned}
$$

It is straightforward to check that this expression is of the form $\Psi=\sqrt{1+|a|^{2}}+a \cdot Y^{1}$ with $a \in \mathbb{R}^{3}$. Indeed the vector $a$ is

$$
a=\left(a^{1}, a^{2}, a^{3}\right)=\left(\operatorname{Re}(\bar{\alpha} \beta+\bar{\gamma} \delta), \operatorname{Im}(\bar{\alpha} \beta+\bar{\gamma} \delta), \frac{|\alpha|^{2}-|\beta|^{2}+|\gamma|^{2}-|\delta|^{2}}{2}\right),
$$




\subsection{The large sphere equation and the Bondi energy-momentum}

and we need to prove

$$
1+|a|^{2}=\frac{\left(|\alpha|^{2}+|\beta|^{2}+|\gamma|^{2}+|\delta|^{2}\right)^{2}}{4}
$$

The associated complex matrix that determines $F$ belongs to $S L(2, \mathbb{C})$. Therefore, $\alpha \delta-\beta \gamma=1$ and in particular $|\alpha \delta-\beta \gamma|^{2}=1$ which, when expanded becomes

$$
2 \operatorname{Re}(\alpha \bar{\beta} \bar{\gamma} \delta)=|\alpha|^{2}|\delta|^{2}+|\beta|^{2}|\gamma|^{2}-1
$$

Now

$$
\begin{aligned}
\left(a^{1}\right)^{2}+\left(a^{2}\right)^{2} & =(\operatorname{Re}(\bar{\alpha} \beta+\bar{\gamma} \delta))^{2}+(\operatorname{Im}(\bar{\alpha} \beta+\bar{\gamma} \delta))^{2}=|\bar{\alpha} \beta+\bar{\gamma} \delta|^{2} \\
& =|\alpha|^{2}|\beta|^{2}+2 \operatorname{Re}(\alpha \bar{\beta} \bar{\gamma} \delta)+|\gamma|^{2}|\delta|^{2} \\
& =|\alpha|^{2}|\beta|^{2}+|\gamma|^{2}|\delta|^{2}+|\alpha|^{2}|\delta|^{2}+|\beta|^{2}|\gamma|^{2}-1 \\
& =\left(|\alpha|^{2}+|\gamma|^{2}\right)\left(|\beta|^{2}+|\delta|^{2}\right)-1,
\end{aligned}
$$

after using (6.48) in the last but one equality. Thus

$$
\begin{aligned}
1+|a|^{2} & =\left(|\alpha|^{2}+|\gamma|^{2}\right)\left(|\beta|^{2}+|\delta|^{2}\right)+\frac{\left(|\alpha|^{2}-|\beta|^{2}+|\gamma|^{2}-|\delta|^{2}\right)^{2}}{4} \\
& =\frac{\left(|\alpha|^{2}+|\beta|^{2}+|\gamma|^{2}+|\delta|^{2}\right)^{2}}{4},
\end{aligned}
$$

as a consequence of the trivial identity $A B+\frac{1}{4}(A-B)^{2}=\frac{1}{4}(A+B)^{2}$.

The Bondi energy-momentum is a vector in an abstract Minkowski space. Let us recall the construction for the sake of completeness and because of a subtlety that arises in the case of past null hypersurfaces. In [87] $t$ is shown that every Möbius transformation corresponds to a unique restricted Lorentz transformation; conversely every restricted Lorentz transformation corresponds to precisely two Möbius transformations, one being the negative of the other. The Lorentz transformation $x^{\prime \mu}=\Lambda(F)^{\mu}{ }_{\nu} x^{\nu}$ associated to the Möbius transformation $F$ has the form (see page 17 in [87])

$$
\frac{1}{2}\left(\begin{array}{cccc}
\alpha \bar{\alpha}+\beta \bar{\beta}+\gamma \bar{\gamma}+\delta \bar{\delta} & \alpha \bar{\beta}+\beta \bar{\alpha}+\gamma \bar{\delta}+\delta \bar{\gamma} & i(\alpha \bar{\beta}-\beta \bar{\alpha}+\gamma \bar{\delta}-\delta \bar{\gamma}) & \alpha \bar{\alpha}-\beta \bar{\beta}+\gamma \bar{\gamma}-\delta \bar{\delta} \\
\alpha \bar{\gamma}+\gamma \bar{\alpha}+\beta \bar{\delta}+\delta \bar{\beta} & \alpha \bar{\delta}+\delta \bar{\alpha}+\beta \bar{\gamma}+\gamma \bar{\beta} & i(\alpha \bar{\delta}-\delta \bar{\alpha}+\gamma \bar{\beta}-\beta \bar{\gamma}) & \alpha \bar{\gamma}+\gamma \bar{\alpha}-\beta \bar{\delta}-\delta \bar{\beta} \\
i(\gamma \bar{\alpha}-\alpha \bar{\gamma}+\delta \bar{\beta}-\beta \bar{\delta}) & i(\delta \bar{\alpha}-\alpha \bar{\delta}+\gamma \bar{\beta}-\beta \bar{\gamma}) & \alpha \bar{\delta}+\delta \bar{\alpha}-\beta \bar{\gamma}-\gamma \bar{\beta} & i(\gamma \bar{\alpha}-\alpha \bar{\gamma}+\beta \bar{\delta}-\delta \bar{\beta}) \\
\alpha \bar{\alpha}+\beta \bar{\beta}-\gamma \bar{\gamma}-\delta \bar{\delta} & \alpha \bar{\beta}+\beta \bar{\alpha}-\gamma \bar{\delta}-\delta \bar{\gamma} & i(\alpha \bar{\beta}-\beta \bar{\alpha}+\delta \bar{\gamma}-\gamma \bar{\delta}) & \alpha \bar{\alpha}-\beta \bar{\beta}-\gamma \bar{\gamma}+\delta \bar{\delta}
\end{array}\right)
$$

To a background foliation $\left\{S_{\lambda}\right\}$ of $\Omega$ approaching large spheres with asymptotic rescaled metric $\stackrel{q}{q}$ one can assign an asymptotic inertial reference frame $\left\{t, x^{i}\right\}$ in an (abstract) Minkowski spacetime. This is possible because given another such foliation $\left\{S_{\lambda^{\prime}}\right\}$ with asymptotic rescaled metric $\phi^{2} \stackrel{\circ}{q}$, (with $\phi$ a solution of the large sphere equation) one associates a Möbius transformation $F$ (determined by the function $\phi$ ), 


\section{The Hawking energy along null AF hypersurfaces}

and to $F$ one can associate a unique restricted Lorentz transformation $\Lambda(F)$. In turn $\Lambda(F)$ defines a new asymptotic inertial reference frame $\left\{t^{\prime}, x^{\prime \prime}\right\}$. The inertial observer associated to $\Lambda(F)$ is defined by the vector

$$
u=\partial_{t^{\prime}}
$$

The transition from $\phi$ to $F$ is not unique because given $a^{1}, a^{2}, a^{3}$ there are many $F(z)=\frac{\alpha z+\beta}{\gamma z+\delta}$ that give $a^{i}$ as in (6.47). However this freedom corresponds explicitly to rotations in the spacial axes and hence do not change the new time direction $\partial_{t^{\prime}}$. All this construction makes sense because the Bondi energy $E_{B}^{u}$ associated to a round asymptotic metric $q$ व corresponding to an asymptotic inertial observer $u$ can be written in the form ( $\eta$ is the Minkowski metric)

$$
E_{B}^{u}=-\eta\left(P_{B}, u\right),
$$

where $P_{B}$ is independent of $u . P_{B}$ is the Bondi four-momentum. In Corollary 6.7.3 below we recover all these facts from our results on the limit of the Hawking energy. To do that we need to identify $\partial_{t^{\prime}}$ in terms of $a^{i}$. Using that $x^{\mu}=\Lambda^{-1}(F)^{\mu}{ }_{\nu} x^{\prime \nu}$, where $\Lambda^{-1}(F)=\eta \Lambda^{t}(F) \eta$ and $\eta$ the matrix associated to the Minkowski metric, we have

$$
u:=\partial_{t^{\prime}}=\frac{\partial t}{\partial t^{\prime}} \partial_{t}+\sum_{i=1}^{3} \frac{\partial x^{i}}{\partial t^{\prime}} \partial_{x^{i}}=\Lambda(F)_{0}^{0} \partial_{t}-\sum_{i=1}^{3} \Lambda(F)^{0}{ }_{i} \partial_{x^{i}} .
$$

Comparing $\Lambda^{0}{ }_{i}(F)$ in the above Lorentz transformation with the expression for $a^{i}$ in (6.47) it follows that in the case of future directed null hypersurfaces extending to future null infinity,

$$
u=\sqrt{1+|a|^{2}} \partial_{t}-a^{i} \partial_{x^{i}} .
$$

The construction of $\Lambda(F)$ in [87] is performed with the unit sphere lying at the intersection of the hyperplane $t=1$ and the future null cone of the origin (see Figure 6.4). It is hence adapted to future directed null hypersurfaces extending to future null infinity. In this chapter we have considered null hypersurfaces extending to past null infinity. This case is obtained from the previous one by a time inversion, which has the effect that the observer $u$ is expressed for past directed null hypersurfaces extending to past null infinity as

$$
u=\sqrt{1+|a|^{2}} \partial_{t}+a^{i} \partial_{x^{i}}
$$

in terms of the coefficients $a^{i}$ in the conformal factor $\phi$.

We can now recover the result that the Hawking energy approaches the Bondi energy for spherical foliations. 


\subsection{The large sphere equation and the Bondi energy-momentum}

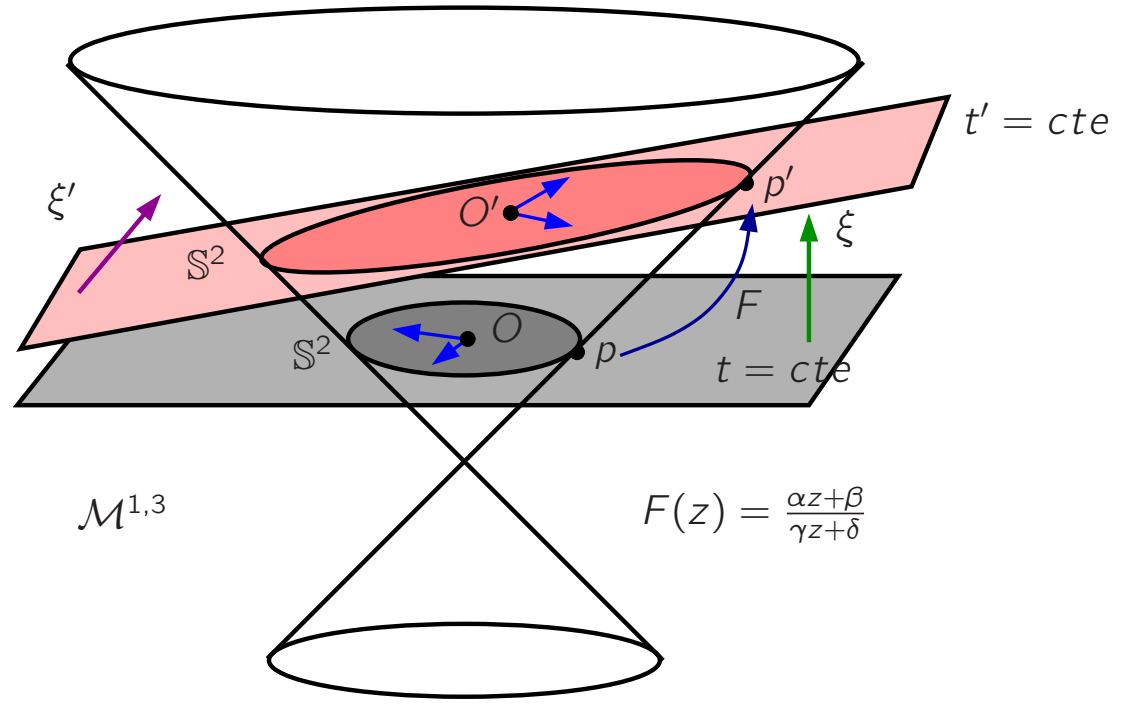

Figure 6.4: The intersection of the light cone of a point with two hyperplanes in $\mathcal{M}^{1,3}$ define two two-spheres $\mathbb{S}^{2}$. In the Figure, two orthonormal basis associated to the centers $O$ and $O^{\prime}$ of the spheres are represented. The null generators of the light cone generate a conformal diffeomorphism $F: \mathbb{S}^{2} \rightarrow \mathbb{S}^{2}$ which induces a Lorentz transformation $\Lambda(F)$ in $\mathcal{M}^{1,3}$. The function $\phi$ defined by the pullback of the spherical metric $F^{*}(\stackrel{\circ}{q})=\phi^{2} \stackrel{q}{q}$ satisfies the large sphere equation, and is determined by three real numbers, which combined with the rotation parameters between the orthonormal frames in $O$ and $O^{\prime}$ determine the complex numbers $\alpha, \beta, \gamma$ and $\delta$ (with $\alpha \delta-\beta \gamma=1$ ) that define the Möbius transformation $F$. The new asymptotic inertial frame $\left\{t^{\prime}, x^{\prime \prime}\right\}$ defines with respect to the initial inertial frame $\left\{t, x^{i}\right\}$ a unique Lorentz transformation $\Lambda(F)$.

Corollary 6.7.3. Let $\Omega$ be a past asymptotically flat null hypersurface endowed with an affinely parametrized background foliation $\left\{S_{\lambda}\right\}$ with generator $k$ that tends to large spheres. Consider another foliation associated to the parameter $\lambda^{*}$ so that $\lambda=$ $\phi \lambda^{*}+\tau+\xi$, as in Theorem [6.6.2, where $\phi>0$ satisfies the large sphere equation (6.36). Let $u \in \mathcal{M}^{1,3}$ be the asymptotic inertial observer associated to this foliation. Then

$$
\lim _{\lambda^{*} \rightarrow \infty} m_{H}\left(S_{\lambda^{*}}\right)=-\eta\left(P_{B}, u\right):=E_{B}^{u},
$$

where $\eta$ is the Minkowski metric and the Bondi four-momentum vector $P_{B}$ reads

$$
\begin{aligned}
E_{B} & :=P_{B}^{0}:=\frac{-1}{16 \pi} \int_{\mathbb{S}^{2}}\left(\theta_{k}^{(1)}+\theta_{\ell}^{(1)}\right) \boldsymbol{\eta}_{\dot{q}}, \\
P_{B}^{i}: & :=\frac{1}{16 \pi} \int_{\mathbb{S}^{2}}\left(-\triangle_{\tilde{q}} \theta_{k}^{(1)}+\left(\theta_{k}^{(1)}+\theta_{\ell}^{(1)}\right)+4 \operatorname{div}_{\tilde{q}} s_{\ell}^{(1)}\right) Y_{i}^{1} \boldsymbol{\eta}_{\mathfrak{q}}, \quad i \in\{1,2,3\} .
\end{aligned}
$$

If, in addition, the energy flux decay condition of Proposition 6.3.9 is satisfied, then the 


\section{The Hawking energy along null AF hypersurfaces}

Bondi three-momentum simplifies to

$$
P_{B}^{i}=\frac{1}{16 \pi} \int_{\mathbb{S}^{2}}\left(\triangle_{\dot{q}} \theta_{k}^{(1)}+\left(\theta_{k}^{(1)}+\theta_{\ell}^{(1)}\right)\right) Y_{i}^{1} \boldsymbol{\eta}_{\dot{q}}, \quad i \in\{1,2,3\}
$$

Proof. We can use expression (6.41) with $\psi$ as in (6.45) so that

$$
\begin{aligned}
\lim _{\lambda^{*} \rightarrow \infty} m_{H}\left(S_{\lambda^{*}}\right)= & \frac{1}{16 \pi} \int_{\mathbb{S}^{2}}\left(\triangle_{\dot{q}} \theta_{k}^{(1)}-\left(\theta_{k}^{(1)}+\theta_{\ell}^{(1)}\right)-4 \operatorname{div}_{q} s_{\ell}^{(1)}\right) \\
& \times\left(\sqrt{1+|a|^{2}}+\sum_{i=1}^{3} a^{i} Y_{i}^{1}\right) \boldsymbol{\eta}_{\dot{q}} \\
= & \left(\frac{-1}{16 \pi} \int_{\mathbb{S}^{2}}\left(\theta_{k}^{(1)}+\theta_{\ell}^{(1)}\right) \boldsymbol{\eta}_{\dot{q}}\right) \sqrt{1+|a|^{2}}+ \\
& +\sum_{i=1}^{3}\left(\frac{1}{16 \pi} \int_{\mathbb{S}^{2}}\left(\triangle_{\dot{q}} \theta_{k}^{(1)}-\left(\theta_{k}^{(1)}+\theta_{\ell}^{(1)}\right)-4 \operatorname{div}_{\dot{q}} s_{\ell}^{(1)}\right) Y_{i}^{1} \boldsymbol{\eta}_{\dot{q}}\right) a^{i} \\
= & -\eta\left(u, P_{B}\right),
\end{aligned}
$$

with $u=\left(\sqrt{1+|a|^{2}}, a^{1}, a^{2}, a^{3}\right)$ and $P_{B}^{i}$ as given in the statement of the corollary.

When the energy flux decay condition holds, we have from Proposition 6.3.9 $s_{\ell}^{(1)}{ }_{A}=$ $\stackrel{\circ}{\nabla}_{A} \theta_{k}^{(1)}-\frac{1}{2} \stackrel{\circ}{\nabla}_{B} \stackrel{\circ}{h}_{A}$, and the integral (6.52) becomes

$$
\int_{\mathbb{S}^{2}}\left(-3 \triangle_{\dot{q}} \theta_{k}^{(1)}-\left(\theta_{k}^{(1)}+\theta_{\ell}^{(1)}\right)+2 \stackrel{\circ}{\nabla}_{A} \circ_{B} \stackrel{\circ}{A}^{A B}\right) \Psi_{a} \boldsymbol{\eta}_{\dot{q}}
$$

where $\psi_{a}:=\sqrt{1+|a|^{2}}+a \cdot Y^{1}$. Integrating by parts the last term and using that

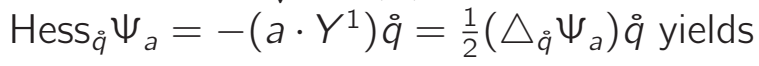

$$
\int_{\mathbb{S}^{2}} 2 \stackrel{\circ}{\nabla}_{A} \stackrel{\circ}{\nabla}_{B} \stackrel{h}{A}^{A B} \Psi \boldsymbol{\eta}_{\dot{q}}=\int_{\mathbb{S}^{2}} 2 \theta_{k}^{(1)} \triangle_{\dot{q}} \Psi_{a} \boldsymbol{\eta}_{\dot{q}}=\int_{\mathbb{S}^{2}} 2\left(\triangle_{\dot{q}} \theta_{k}^{(1)}\right) \Psi_{a} \boldsymbol{\eta}_{\dot{q}}
$$

where in the first equality we used $\operatorname{tr}_{\mathscr{q}} h=2 \theta_{k}^{(1)}$ and in the second we performed another integration by parts. Arguing as before, the expression (6.51) for $P_{B}^{i}$ follows.

Remark 6.7.4. An analogous result can be obtained for the case of asymptotically flat null hypersurfaces $\Omega$ approaching future null infinity. Using the general expression in Remark 6.6.3 for the limit of the Hawking energy in this case and using the fact that $\Psi_{a}=\sqrt{1+|a|^{2}}+a \cdot Y$ corresponds now to the asymptotic observer with four velocity $u=\left(\sqrt{1+|a|^{2}},-a^{1},-a^{2},-a^{3}\right)$, the Bondi energy-momentum vector $P_{B}$ satisfying $\lim _{\lambda^{\star} \rightarrow \infty} M_{H}\left(S_{\lambda^{\star}}\right)=-\eta\left(u, P_{B}\right):=E_{B}^{u}$ is

$$
\begin{aligned}
E_{B} & :=P_{B}^{0}:=\frac{1}{16 \pi} \int_{\mathbb{S}^{2}}\left(\theta_{\bar{k}}^{(1)}+\theta_{\bar{\ell}}^{(1)}\right) \boldsymbol{\eta}_{\tilde{q}} \\
P_{B}^{i} & :=\frac{1}{16 \pi} \int_{\mathbb{S}^{2}}\left(-\triangle_{\dot{q}} \theta_{\bar{k}}^{(1)}+\left(\theta_{\bar{k}}^{(1)}+\theta_{\bar{\ell}}^{(1)}\right)-4 \operatorname{div}_{\grave{q}} s_{\bar{\ell}}^{(1)}\right) Y_{i}^{1} \boldsymbol{\eta}_{\dot{q}} .
\end{aligned}
$$




\subsection{The large sphere equation and the Bondi energy-momentum}

The energy flux decay condition in this case implies (i.e. the analogous on Proposition 6.3.9)

$$
s_{\bar{\ell}}^{(1)}{ }_{A}=-\hat{D}_{A} \theta_{\bar{k}}^{(1)}-\frac{1}{2} \hat{D}_{B} \hat{h}_{A}^{B},
$$

and the Bondi momentum simplifies to

$$
P_{B}^{i}=\frac{1}{16 \pi} \int_{\mathbb{S}^{2}}\left(\triangle_{\dot{q}} \theta_{\bar{k}}^{(1)}+\left(\theta_{\bar{k}}^{(1)}+\theta_{\bar{\ell}}^{(1)}\right)\right) Y_{i}^{1} \boldsymbol{\eta}_{\dot{q}}, \quad i \in\{1,2,3\} .
$$

Note that in this case

$$
E_{B}^{u}:=-\eta\left(u, P_{B}\right)=\frac{1}{16 \pi} \int_{\mathbb{S}^{2}}\left(\triangle_{\dot{q}} \theta_{\bar{k}}^{(1)}+\left(\theta_{\bar{k}}^{(1)}+\theta_{\bar{\ell}}^{(1)}\right)\right)\left(u^{0}-u^{i} Y_{i}^{1}\right) \boldsymbol{\eta}_{\dot{q}}
$$

Remark 6.7.5. The relationship between the limit of the Hawking energy and the Bondi four-momentum for foliations approaching large spheres has been investigated in [87] and [6] (see also Definition 4.2 in [93]). As a useful check, it is convenient to see how the results in this chapter fit with the results in [6]. The setup there involves so-called null quasi-spherical coordinates which are adapted to a foliation by future outgoing null hypersurfaces $\left\{\mathcal{N}_{z}\right\}$, each of them foliated by codimension-two spacelike surfaces $S_{z, r_{B}}$, where the parameter $r_{B}$ takes a value $r_{0}$ at the initial surface. An explicit computation shows that the parameter $r_{B}$ is not affine in general.

Each $S_{z, r_{B}}$ has induced metric isometric to the standard sphere of radius $r_{B}$. In fact, the null quasi-spherical coordinates $\left\{z, r_{B}, \theta, \phi\right\}$ are such that the surface $\left\{z=\right.$ const, $r_{B}=$ const $\}$ has induced metric $r_{B}^{2}\left(d \theta^{2}+\sin ^{2} \theta d \phi\right):=r_{B}^{2} \stackrel{\circ}{q}$, which in particular selects the diffeomorphism of $S_{r_{B}, z}$ with the standard unit sphere $\left(\mathbb{S}^{2}, \stackrel{q}{q}\right)$. Under asymptotic conditions along the null hypersurface involving the shear and its angular derivative, Bartnik shows, among various other things, that the Bondi energy-momentum is well-defined and agrees with the limit of the Hawking energy along the quasi-spherical foliation $S_{z, r_{B}}$. More precisely, defining the mass aspect function $m=\frac{1}{2} r_{B}\left(1-\frac{1}{4} \vec{H}^{2} r_{B}^{2}\right)$ of the sphere $S_{z, r_{B}}$ so that $m_{H}\left(z, r_{B}\right)=\frac{1}{4 \pi} \int_{S_{z, r_{B}}^{2}} m \boldsymbol{\eta}_{\mathfrak{q}}$ (recall that $\vec{H}$ is the mean curvature vector of the surface), Bartnik shows that $\lim _{r_{B} \rightarrow \infty} m=m_{0}$ with $m_{0} \in C^{\infty}\left(\mathbb{S}^{2}\right)$ and that, under sufficient decay of suitable components of the Einstein tensor which include the energy flux decay condition of this chapter,

$$
\begin{aligned}
& E_{B}=\lim _{r_{B} \rightarrow \infty} \frac{1}{4 \pi} \int_{\mathbb{S}^{2}} m \boldsymbol{\eta}_{\dot{q}}=\frac{1}{4 \pi} \int_{\mathbb{S}^{2}} m_{0} \boldsymbol{\eta}_{\dot{q}}, \\
& P_{B}^{i}=\lim _{r_{B} \rightarrow \infty} \frac{1}{4 \pi} \int_{\mathbb{S}^{2}} m Y_{i}^{1} \boldsymbol{\eta}_{\mathfrak{q}}=\frac{1}{4 \pi} \int_{\mathbb{S}^{2}} m_{0} Y_{i}^{1} \boldsymbol{\eta}_{\grave{q}} .
\end{aligned}
$$

We have also found in Theorem 6.6.1 an expression for the limit of the Hawking energy for non-affine foliations in terms of the geometry of an (affine) background foliation 


\section{The Hawking energy along null AF hypersurfaces}

$\left\{S_{\lambda}\right\}$. Define a new affine parameter $r:=r_{0}+\lambda$, such that it also becomes $r_{0}$ when evaluated at the initial surface. Let us define $\xi$ as the difference between the parameter $r$ (that generates the affine foliation $\left\{S_{r}\right\}$ ) and Bartnik's parameter $r_{B}$, namely

$$
r-r_{B}=\xi
$$

Assume that $\xi$ satisfies the decay conditions of Theorem [6.6.1. Then we recover Bartnik's Bondi energy formula (6.54) from our expression (6.53), as we show next.

Recall that in this case the null hypersurface extends to future null infinity, and Bartnik's future null basis $\left\{\bar{k}_{B}, \bar{\ell}_{B}\right\}$ is normalized so that $\left\langle\bar{k}_{B}, \bar{\ell}_{B}\right\rangle=-2$, being $\bar{k}_{B}$ the future null normal tangent to the hypersurface. Let $\{\bar{k}, \bar{\ell}\}$ the null basis associated to the background foliation $\left\{S_{r}\right\}$. The expansions associated to the background foliation read

$$
\theta_{\bar{k}}=\frac{2}{r-r_{0}}+\frac{\theta_{\bar{k}}^{(1)}}{\left(r-r_{0}\right)^{2}}+o\left(\left(r-r_{0}\right)^{-2}\right), \quad \theta_{\bar{\ell}}=\frac{-2}{r-r_{0}}+\frac{\theta_{\bar{\ell}}^{(1)}}{\left(r-r_{0}\right)^{2}}+o\left(\left(r-r_{0}\right)^{-2}\right) .
$$

We showed in Theorem 6.6.1 that under the change of parameter (6.55), the expansions associated to the Bartnik foliation preserve the form up to leading orders, that is to say

$\theta_{\bar{k}_{B}}=\frac{2}{r_{B}-r_{0}}+\frac{\theta_{\bar{k}}^{(1)}}{\left(r_{B}-r_{0}\right)^{2}}+o\left(\left(r_{B}-r_{0}\right)^{-2}\right), \quad \theta_{\bar{\ell}_{B}}=\frac{-2}{r_{B}-r_{0}}+\frac{\theta_{\bar{\ell}}^{(1)}}{\left(r_{B}-r_{0}\right)^{2}}+o\left(\left(r_{B}-r_{0}\right)^{-2}\right)$,

or equivalently

$$
\theta_{\bar{k}_{B}}=\frac{2}{r_{B}}+\frac{\theta_{\bar{k}}^{(1)}+2 r_{0}}{r_{B}^{2}}+o\left(r_{B}^{-2}\right), \quad \theta_{\bar{\ell}_{B}}=\frac{-2}{r_{B}}+\frac{\theta_{\bar{l}}^{(1)}-2 r_{0}}{r_{B}^{2}}+o\left(r_{B}^{-2}\right) .
$$

The null quasi-spherical gauge has the key property that $\theta_{\bar{k}_{B}}-\frac{2}{r_{B}}$ is automatically a divergence on each $S_{r_{B}}$. Hence Bartnik finds that the null expansions have the form

$$
\theta_{\bar{k}_{B}}=\frac{2}{r_{B}}-\frac{\operatorname{div} b_{0}}{r_{B}^{2}}+o\left(r_{B}^{-2}\right) \quad \theta_{\bar{\ell}_{B}}=\frac{-2}{r_{B}}+\frac{a}{r_{B}^{2}}+o\left(r_{B}^{-2}\right),
$$

and proves after suitable computations that

$$
m_{0}=\frac{1}{4}\left(\operatorname{div} b_{0}+a\right) .
$$

In terms of our notation one finds

$$
\begin{aligned}
-\operatorname{div} b_{0} & =\theta_{\bar{k}}^{(1)}+2 r_{0}, \\
a & =\theta_{\bar{l}}^{(1)}-2 r_{0},
\end{aligned}
$$




\subsection{The large sphere equation and the Bondi energy-momentum}

and hence $m_{0}=\frac{1}{4}\left(\theta_{\bar{\ell}}^{(1)}-\theta_{\bar{k}}^{(1)}-4 r_{0}\right)$. We can now recover Bartnik's result from (6.53) because, with the shorthand $\Psi_{u}:=u_{0}-u^{i} Y_{i}^{1}$,

$$
\begin{aligned}
E_{B}^{u} & =\frac{1}{16 \pi} \int_{\mathbb{S}^{2}}\left(\triangle_{\dot{q}} \theta_{\bar{k}}^{(1)}+\left(\theta_{\bar{k}}^{(1)}+\theta_{\bar{\ell}}^{(1)}\right)\right) \Psi_{u} \boldsymbol{\eta}_{\dot{q}} \\
& =\frac{1}{16 \pi} \int_{\mathbb{S}^{2}}\left(\triangle_{\dot{q}} \theta_{\bar{k}}^{(1)}+\left(\theta_{\bar{k}}^{(1)}+\theta_{\bar{\ell}}^{(1)}\right)-\left(\triangle_{\dot{q}}+2\right)\left(\theta_{\bar{k}}^{(1)}+2 r_{0}\right)\right) \Psi_{u} \boldsymbol{\eta}_{\dot{q}} \\
& =\frac{1}{16 \pi} \int_{\mathbb{S}^{2}}\left(\theta_{\bar{\ell}}^{(1)}-\theta_{\bar{k}}^{(1)}-4 r_{0}\right) \Psi_{u} \boldsymbol{\eta}_{\dot{q}}=\frac{1}{4 \pi} \int_{\mathbb{S}^{2}} m_{0} \Psi_{u} \boldsymbol{\eta}_{\dot{q}},
\end{aligned}
$$

where in the second expression we added zero in the form

$$
0=\int_{\mathbb{S}^{2}}\left(-\left(\triangle_{\mathfrak{q}}+2\right)\left(\theta_{\bar{k}}^{(1)}+2 r_{0}\right)\right) \Psi_{u} \boldsymbol{\eta}_{\dot{q}}
$$

Note that this integral vanishes because $\theta_{\bar{k}}^{(1)}+2 r_{0}$ is a divergence. So, the form $E_{B}^{u}=$ $\frac{1}{4 \pi} \int_{\mathbb{S}^{2}} m_{0} \boldsymbol{\eta}_{\dot{q}}$ obtained by Bartnik depends crucially on the assumption that the foliation is quasi-spherical. For general foliations, the right expression for $E_{B}^{u}$ is given by (6.53). 


\section{On the Penrose inequality along null hypersurfaces}

\subsection{Introduction}

We devote the last chapter of this thesis to study the main properties of general flows along null hypersurfaces in a AF spacetimes satisfying the dominant energy condition with the purpose of addressing the null version of the Penrose inequality. We prove this inequality for a large class of surfaces. Moreover, a different geometric inequality in the same spirit as the null Penrose inequality is proven in full generality. The results of this chapter were published in [77].

The key tool that we use in this chapter is a functional on surfaces which bounds from above the square root of the area of any weakly outer trapped surface. If the foliation tends to large spheres, the limit of such functional is the Bondi energy measured by the observer determined by the flow. The functional is not monotonic in general, but it can be split into a monotonic part, which we defined as Bergqvist mass in Chapter 4 , and a term $D$ which is a renormalization of the area of each leaf. The latter will play an important role to find sufficient conditions for the validity of the null Penrose inequality.

Two main foliations are essential for the obtention of our results. We call the first Geodesic Asymptotically Bondi (GAB) foliation. A GAB foliation is defined by the condition that the first non-trivial coefficient of the asymptotic expansion of $\theta_{k}$ is constant. We show that given any initial cross section $S$ there exists a unique (up to trivial reparametrization) GAB foliation starting at $S$. However, this foliation needs not approach large spheres at infinity. Using GAB foliations we prove in full generality (Theorem 7.3 .8 ) that the square root of the area (with the usual factor) of a WOTS $S$ is bounded above by the limit of the Hawking energy along the GAB foliation associated to $S$. This theorem recovers Ludvigsen \& Vicker's [67] and Bergqvist's [8] results in case that the GAB foliation tends to large spheres. Applications of the GAB foliations to the Minkowski shell case are discussed in Section 7.8. 
The second family of foliations that we consider are geodesic foliations approaching large spheres. The idea is to generalize the methods used in the Minkowski spacetime in previous chapters to the general case. The functional $D$ was introduced in Chapter 4 in the Minkowski case and we showed that monotonicity of $D$ implied the shell Penrose inequality. Here we show that the crucial property that $D$ must satisfy for the general null Penrose inequality to hold is that it is bounded above by its limit at infinity. One way of achieving this is imposing monotonicity on $D$. We refer to this method as Renormalized Area Method. In this chapter we find two sufficient conditions in Theorem 7.5.5 under which the Renormalized Area Method applies. Sections 7.7 and 7.8 are devoted to the study of applicability of the method to the case where the null hypersurfaces $\Omega$ is shearfree, and to the four dimensional Minkowski spacetime respectively. In the Minkowski case both conditions determine the same class of surfaces already obtained in Chapter 4.

The chapter is concluded by showing yet another general inequality bounding the area of a closed spacelike surface embedded in a past AF null hypersurface $\Omega$ in terms of an asymptotic quantity intrinsic to $\Omega$.

\subsection{A functional on two-surfaces}

In Chapter 4 the shell Penrose inequality was approached by using two functionals on an affine foliation $\left\{S_{\lambda}\right\}$ that tends to large spheres along a past null hypersurface $\Omega$, namely

$$
M_{b}(\lambda):=\frac{\lambda}{2}-\frac{1}{16 \pi} \int_{S_{\lambda}} \theta_{\ell}(\lambda) \boldsymbol{\eta}_{S_{\lambda}},
$$

that was defined as the Bergqvist mass and was monotonic (see Theorem 4.5.2 in Chapter 4), and

$$
D(\lambda):=\sqrt{\frac{\left|S_{\lambda}\right|}{16 \pi}}-\frac{\lambda}{2}
$$

that was defined in analogy with the definition of $M_{b}$. The shell Penrose inequality was equivalent to prove (see formula (4.56))

$$
M_{b}(\lambda=0)+D(\lambda=0) \leq 0 .
$$

This expression suggests that a new functional defined by $M_{b}(\lambda)+D(\lambda)$ may be of interest on its own. In the sum, the terms in $\lambda$ cancel out and we find an expression that depends only on the surface $S$ and on the choice of $\ell$ (because of the term $\theta_{\ell}$ in (7.1) ). In this chapter a critical object is the functional

$$
M(S, \ell):=\sqrt{\frac{|S|}{16 \pi}}-\frac{1}{16 \pi} \int_{S} \theta_{\ell} \boldsymbol{\eta}_{S}
$$




\section{On the Penrose inequality along null hypersurfaces}

We will analyze its properties and study whether it is a useful tool to approach the Penrose inequality.

This quantity has geometric units of length so one may be tempted to assign to it a physical interpretation of quasi-local mass of $S$. However, $M(S, \ell)$ is not truly a quasi-local quantity on the surface because it depends on the choice of null normal $\ell$, which cannot be uniquely fixed a priori in the absence of additional geometric structure. Note, however, that a weakly outer trapped surface $S$ satisfies, by definition, $\theta_{\ell} \leq 0$ irrespectively of the scaling of $\ell$, and hence

$$
\sqrt{\frac{|S|}{16 \pi}} \leq M(S, \ell) .
$$

So, if $M(S, \ell)$ enjoyed good monotonicity properties under suitable flows and its value on very large surfaces in an asymptotically flat context could be related to the total mass of the spacetime, this object would be potentially useful to address the Penrose inequality and play perhaps a similar role as the Hawking energy does in the timesymmetric context. In this chapter we restrict ourselves to null flows because in this case $M(S, \ell)$ does satisfy an interesting evolution equation. However, there are many other possibilities that may well be worth exploring.

Since we deal with null flows we shall work on a null hypersurface $\Omega$. Thus, let $\Omega$ be a smooth, connected null hypersurface embedded in $(M, g)$ and admitting a global cross section $S_{0}$ (i.e. a smooth embedded spacelike surface intersected precisely once by every inextendible curve along the null generators of $\Omega$ ). In this section we use the same setting as in Section 6.2 of Chapter 6 , and we consider foliations $\left\{S_{\lambda}\right\}$ along $\Omega$. We want to investigate the derivative of $M\left(S_{\lambda}, \ell\right)$ with respect to $\lambda$. In order to maintain the generality, we do not make any assumption on the null vector field $\ell$ orthogonal to $S_{\lambda}$ (other than being future directed and transverse to $\Omega$ ). The null generators of $\Omega$ do not have to be necessarily affinely parametrized, that is to say, it holds

$$
\nabla_{k} k=Q_{k} k
$$

with $Q_{k}$ not necessarily zero. Given a smooth positive function $\varphi: \Omega \mapsto \mathbb{R}^{+}$, there is a unique choice of null normal $\ell$ to $S_{\lambda}$ (denoted by $\ell^{\varphi}$ ) satisfying

$$
\left\langle k, \ell^{\varphi}\right\rangle=-\varphi .
$$

Obviously $\ell^{\varphi}$ depends on the foliation $\left\{S_{\lambda}\right\}$, so it is not an intrinsic property of $\Omega$. The choice $\varphi=2$ will be relevant later and we will denote $\ell^{\varphi=2}$ simply by $\ell$ from now on. As before, $s_{\ell^{\varphi}}$ is also a foliation dependent quantity. The evolution of $M\left(S_{\lambda}, \ell^{\varphi}\right)$ in this general setting is given in the following lemma.

Lemma 7.2.1. Let $\Omega$ be a null hypersurface embedded in a spacetime $\left(M^{4}, g\right)$. Assume that $\Omega$ has topology $S \times \mathbb{R}$ with the null generator tangent to the $\mathbb{R}$ factor. Consider 
a foliation $\left\{S_{\lambda}\right\}$ of $\Omega$ by spacelike hypersurfaces, all diffeomorphic to $S$. Let $k$ be the future null generator satisfying $k(\lambda)=-1$ and $\ell^{\varphi}$ the null normal to $S_{\lambda}$ satisfying $\left\langle k, \ell^{\varphi}\right\rangle=-\varphi$. Then

$$
\begin{aligned}
\frac{d M\left(S_{\lambda}, \ell^{\varphi}\right)}{d \lambda}= & \frac{1}{\sqrt{64 \pi\left|S_{\lambda}\right|}} \int_{S_{\lambda}}\left(-\theta_{k}\right) \boldsymbol{\eta}_{S_{\lambda}}+\frac{1}{16 \pi} \int_{S_{\lambda}}\left[\operatorname{Ein}^{g}(\ell, k)-\frac{\varphi}{2} S_{c a l} S_{\lambda}\right. \\
& \left.+\varphi\left(-\operatorname{div}_{S_{\lambda}} S_{\ell^{\varphi}}+\left|S_{\ell^{\varphi}}\right|_{\gamma_{S_{\lambda}}}^{2}\right)+\left(\frac{1}{\varphi} k(\varphi)-Q_{k}\right) \theta_{\ell^{\varphi}}\right] \boldsymbol{\eta}_{S_{\lambda}}
\end{aligned}
$$

where $Q_{k}$ is defined by $\nabla_{k} k=Q_{k} k$, Ein ${ }^{g}$ is the Einstein tensor of $\left(M^{4}, g\right)$, Scal ${ }^{S_{\lambda}}$ the curvature scalar of $\left(S_{\lambda}, \gamma_{S_{\lambda}}\right)$, and $s_{\ell^{\varphi}}$ is the connection of the normal bundle of $S_{\lambda}$. If, moreover, $\varphi$ is constant and $k$ is affine $\left(Q_{k}=0\right)$ then

$$
\begin{array}{r}
\frac{d M\left(S_{\lambda}, \ell^{\varphi}\right)}{d \lambda}=\frac{1}{\sqrt{64 \pi\left|S_{\lambda}\right|}} \int_{S_{\lambda}}\left(-\theta_{k}\right) \eta_{S_{\lambda}}-\frac{\varphi \chi\left(S_{\lambda}\right)}{8}+\frac{1}{16 \pi} \int_{S_{\lambda}}\left(\operatorname{Ein}^{g}\left(\ell^{\varphi}, k\right)\right. \\
\left.+\varphi\left|S_{\ell^{\varphi}}\right|_{\gamma_{S_{\lambda}}}^{2}\right) \eta_{S_{\lambda}}
\end{array}
$$

where $\chi\left(S_{\lambda}\right)$ is the Euler characteristic of $S_{\lambda}$.

Proof. We drop all reference to $\lambda$ for simplicity. The volume form satisfies relation (B.41) in Appendix B, so the derivative along $-k$ of $M\left(S, \ell^{\varphi}\right)$ is, using formula (B.29) in Appendix B,

$$
\begin{aligned}
\frac{d M\left(S, \ell^{\varphi}\right)}{d \lambda}= & \frac{1}{\sqrt{64 \pi|S|}} \int_{S}\left(-\theta_{k}\right) \boldsymbol{\eta}_{S}+\frac{1}{16 \pi} \int_{S}\left[\operatorname{Ein}^{g}\left(\ell^{\varphi}, k\right)-\frac{\varphi}{2} S_{c a l}{ }^{\gamma_{S}}\right. \\
& \left.+\varphi\left(-\operatorname{div}_{S} S_{\ell^{\varphi}}+\left|s_{\ell^{\varphi}}\right|_{\gamma_{S}}^{2}\right)+\left(\frac{1}{\varphi} k(\varphi)-Q_{k}\right) \theta_{\ell^{\varphi}}\right] \boldsymbol{\eta}_{S} .
\end{aligned}
$$

This is precisely (7.3). When $\varphi=$ const and $Q_{k}=0$, (7.4) follows directly from (7.3) as a consequence of the Gauss-Bonnet theorem $\int_{S} S_{\text {cal }}{ }^{\gamma_{S}} \boldsymbol{\eta}_{S}=4 \pi \chi(S)$.

Our purpose in deriving the general variation formula (7.3) is to show that indeed $\varphi=$ const and $Q_{k}=0$ are the only clear situations leading to a (nearly) monotonic behaviour. Indeed, the divergence term $\operatorname{div}_{S} \boldsymbol{S}_{\ell^{\varphi}}$ has no sign (unless identically zero) which strongly suggests the choice $\varphi=$ const. The term in $\theta_{\ell^{\varphi}}$, which again has no sign a priori, suggest making the choice $Q_{k}=0$ (the seemingly more general condition of making $\varphi$ constant only within the leaves and $Q_{k}=\varphi^{-1} k(\varphi)$ is simply a reparametrization of the previous one).

Under the dominant energy condition (DEC) on $\left(M^{4}, g\right)$, this lemma implies that if $S$ is connected and non-spherical, then $M\left(S_{\lambda}, \ell^{\varphi}\right)$ is monotonically increasing along any affine flow for any past expanding (i.e. with $\theta_{-k} \geq 0$ ) null hypersurface. 


\section{On the Penrose inequality along null hypersurfaces}

Let us assume that $\lambda$ is from now on an affine parameter. For the Penrose inequality in an asymptotically flat context, the spherical topology is the relevant one. In this setting, $M\left(S_{\lambda}, \ell\right)$ is not always monotonic. However, under certain circumstances one can relate its value on the initial surface and its asymptotic value at infinity. In fact, obtaining such relations will be the main theme of this chapter. We first need to specify our asymptotic conditions. We adopt here the same setting and definitions as in Section 6.3 in Chapter 6, where a detailed analysis of the limit of the Hawking energy along null flows was obtained. We make the global assumption that $\Omega=\mathbb{S}^{2} \times \mathbb{R}$ with the affine null generator $k$ tangent along the $\mathbb{R}$-factor. Throughout this chapter we use the notions transversal and Lie constant tensor, and asymptotic flatness, introduced in Chapter 6 .

Our next aim is to analyze the limit of $M\left(S, \ell^{\varphi}\right)$ at infinity. From item (i) in Definition 6.3.4 in Chapter [6, it follows that the volume form $\eta_{S_{\lambda}}$ of each $S_{\lambda}$ satisfies

$$
\boldsymbol{\eta}_{S_{\lambda}}=\left(\lambda^{2}+\theta_{k}^{(1)} \lambda+o(\lambda)\right) \boldsymbol{\eta}_{\hat{\boldsymbol{q}}}
$$

where the Lie constant function $\theta_{k}^{(1)}$ is defined by the expansion

$$
\theta_{k}=\frac{-2}{\lambda}+\frac{\theta_{k}^{(1)}}{\lambda^{2}}+o\left(\lambda^{-2}\right)
$$

The expressions become simpler if we introduce the area radius at infinity as

$$
R_{\hat{q}}^{2}:=\frac{1}{4 \pi} \int_{\hat{S}} \boldsymbol{\eta}_{\hat{q}}
$$

where $\hat{S}$ represents the surface $S$ endowed with the asymptotic metric $\hat{q}$ along the foliation $\left\{S_{\lambda}\right\}$. At this point we do not assume that the foliation approaches large spheres. The area $S_{\lambda}$ has the expansion

$$
\left|S_{\lambda}\right|=\int_{S_{\lambda}} \boldsymbol{\eta}_{S_{\lambda}}=\int_{\hat{S}}\left(\lambda^{2}+\theta_{k}^{(1)} \lambda+o(\lambda)\right) \boldsymbol{\eta}_{\hat{q}}=4 \pi R_{\hat{q}}^{2} \lambda^{2}+\left(\int_{\hat{S}} \theta_{k}^{(1)} \boldsymbol{\eta}_{\hat{q}}\right) \lambda+o(\lambda),
$$

and therefore

$$
\sqrt{\left|S_{\lambda}\right|}=\sqrt{4 \pi R_{\hat{q}}^{2}} \lambda+\frac{\int_{\hat{S}} \theta_{k}^{(1)} \boldsymbol{\eta}_{\hat{q}}}{2 \sqrt{4 \pi R_{\hat{q}}^{2}}}+o(1)
$$

We next compute the asymptotic behaviour of the second term in $M\left(S, \ell^{\varphi}\right)$. Using item (iii) in Definition 6.3.4 and noticing that $\theta_{\ell^{\varphi}}=\frac{\varphi}{2} \theta_{\ell}$ (because of the scaling relation $\ell^{\varphi}=\frac{\varphi}{2} \ell$ ), it follows (we are assuming $\varphi$ constant here and in what follows)

$$
\begin{aligned}
\int_{S_{\lambda}} \theta_{\ell^{\varphi}}(\lambda) \boldsymbol{\eta}_{S_{\lambda}} & =\int_{\hat{s}}\left(\frac{\varphi \mathcal{K}_{\hat{q}}}{\lambda}+\frac{\varphi \theta_{\ell}^{(1)}}{2 \lambda^{2}}+o\left(\lambda^{2}\right)\right)\left(\lambda^{2}+\theta_{k}^{(1)} \lambda+o(\lambda)\right) \boldsymbol{\eta}_{\hat{q}} \\
& =4 \pi \varphi \lambda+\int_{\hat{s}}\left(\varphi \mathcal{K}_{\hat{q}} \theta_{k}^{(1)}+\frac{\varphi}{2} \theta_{\ell}^{(1)}\right) \boldsymbol{\eta}_{\hat{q}}+o(1) .
\end{aligned}
$$


Combining (7.7) and (7.8) into (7.2) gives

$$
M\left(S, \ell^{\varphi}\right)=\left(\frac{R_{\hat{q}}}{2}-\frac{\varphi}{4}\right) \lambda+\frac{1}{16 \pi} \int_{\hat{S}}\left(\theta_{k}^{(1)}\left(\frac{1}{R_{\hat{q}}}-\varphi \mathcal{K}_{\hat{q}}\right)-\frac{\varphi}{2} \theta_{\ell}^{(1)}\right) \eta_{\hat{q}}+o(1) .
$$

This expression has a finite limit at infinity if and only if the scaling of $\ell^{\varphi}$ is chosen so that $\varphi=2 R_{\hat{q}}$. This leads to the following definition:

Definition 7.2.2. Let $\Omega$ be a past asymptotically flat null hypersurface and $\left\{S_{\lambda}\right\}$ the foliation whose existence is assumed in Definition 6.3.4 in Chapter 6. The vector field $\ell^{\star}$ is defined to be null, orthogonal to each leaf $S_{\lambda}$ and normalized by $\left\langle k, \ell^{\star}\right\rangle=-2 R_{\hat{q}}$.

Note that $\ell^{\star}$ is defined by $\varphi=2 R_{\hat{q}}$ so that its relation to the canonical $\ell$ is $\ell^{\star}=R_{\hat{q}} \ell$. With this choice,

$$
\lim _{\lambda \rightarrow \infty} M\left(S_{\lambda}, \ell^{\star}\right)=\frac{1}{16 \pi} \int_{\hat{S}}\left(\theta_{k}^{(1)}\left(\frac{1}{R_{\hat{q}}}-2 R_{\hat{q}} \mathcal{K}_{\hat{q}}\right)-R_{\hat{q}} \theta_{\ell}^{(1)}\right) \boldsymbol{\eta}_{\hat{q}} .
$$

It is useful to relate this limit to the corresponding limit of the Hawking energy along $\left\{S_{\lambda}\right\}$. The limit of $m_{H}\left(S_{\lambda}\right)$ was investigated in detail in Chapter 6. In particular, Theorem 6.6.1 in this chapter gives

$$
\lim _{\lambda \rightarrow \infty} m_{H}\left(S_{\lambda}\right)=\frac{-R_{\hat{q}}}{16 \pi} \int_{\hat{S}}\left(\mathcal{K}_{\hat{q}} \theta_{k}^{(1)}+\theta_{\ell}^{(1)}\right) \boldsymbol{\eta}_{\hat{q}} .
$$

Combining (7.9) and (7.10), the following proposition is proved:

Proposition 7.2.3. With the choice $\ell^{\star}=R_{\hat{q}} \ell$, the limits of $M\left(S_{\lambda}, \ell^{\star}\right)$ and $m_{H}\left(S_{\lambda}\right)$ are related by

$$
\lim _{\lambda \rightarrow \infty} M\left(S_{\lambda}, \ell^{\star}\right)=\lim _{\lambda \rightarrow \infty} m_{H}\left(S_{\lambda}\right)+\frac{1}{16 \pi} \int_{\hat{S}} \theta_{k}^{(1)}\left(\frac{1}{R_{\hat{q}}}-R_{\hat{q}} \mathcal{K}_{\hat{q}}\right) \boldsymbol{\eta}_{\hat{q}} .
$$

Remark 7.2.4. There are two interesting cases where the limit of $M\left(S_{\lambda}, \ell^{\star}\right)$ agrees with the limit of the Hawking energy along the foliation. The first one occurs when $\hat{q}$ has positive constant curvature, in which case the area radius $R_{\hat{q}}$ and the Gauss curvature are related by $\mathcal{K}_{\hat{q}}=\frac{1}{R_{\hat{q}}^{2}}$ and the integrand in the second term of (7.11) vanishes. Recall that such foliations are called "approaching to large spheres" because the geometry of the leaves tends, after a suitable rescaling, to the round spherical metric. This situation is particularly relevant because as already discussed in the previous chapter, then the limit of the Hawking energy is the Bondi energy measured by the observer defined by the foliation $\left\{S_{\lambda}\right\}$.

The other case corresponds to those foliations satisfying $\theta_{k}^{(1)}=$ constant. In this case we have

$$
\int_{\hat{S}} \theta_{k}^{(1)}\left(\frac{1}{R_{\hat{q}}}-R_{\hat{q}} \mathcal{K}_{\hat{q}}\right) \boldsymbol{\eta}_{\hat{q}}=\theta_{k}^{(1)} \int_{\hat{S}}\left(\frac{1}{R_{\hat{q}}}-R_{\hat{q}} \mathcal{K}_{\hat{q}}\right) \boldsymbol{\eta}_{\hat{q}}=\theta_{k}^{(1)}\left(4 \pi R_{\hat{q}}-4 \pi R_{\hat{q}}\right)=0,
$$

where in the second equality we have used the Gauss-Bonnet theorem. We devote the next section to study in detail geodesic foliations with constant $\theta_{k}^{(1)}$. 


\subsection{GAB foliations and a Penrose type inequality}

As discussed in Section 7.1, Ludvigsen \& Vickers [67] and Bergqvist [8] considered the Penrose inequality for null hypersurfaces. A fundamental ingredient of their work involved affine foliations for which $\theta_{k}^{(1)}$ vanishes identically. As we will see below, such foliations are closely related to affine foliations with $\theta_{k}^{(1)}$ constant. We devote this section to study such foliations. Our main result is a Penrose-type inequality valid in full generality and which reduces to the Penrose inequality when the foliation approaches large spheres. Besides its intrinsic interest, the general Penrose-type inequality helps also putting the result of Ludvigsen \& Vickers and Bergqvist into a broader perspective and clarifies both its scope and its range of validity.

We first need a lemma showing that, no matter which affine foliation is taken, the leading term $\theta_{k}^{(1)}$ is always strictly positive. This may seem to contradict the original Ludvigsen \& Vickers assumption (1.5), but this is not the case because $\lambda=0$ corresponds to a cross section on $\Omega$, while the corresponding condition for $r$ was not assumed (and in fact does not hold) in (1.5).

Lemma 7.3.1. Let $\Omega$ be a past asymptotically flat null hypersurface with a choice of affinely parametrized null generator $k$ and corresponding level set function $\lambda$. Assume that the spacetime satisfies the dominant energy condition, then $\theta_{k}^{(1)}>0$.

Proof. Let $\left\{S_{\lambda}\right\}$ the affine foliation defined by $\lambda$ and consider the function $\rho(\lambda)=$ $\theta_{k} \mid s_{\lambda} \lambda^{2}+2 \lambda$. Using the Raychaudhuri equation (B.18), which can be written as $\frac{d \theta_{k}(\lambda)}{d \lambda}=$ $\frac{\theta_{k}^{2}}{2}+W$ with $W \geq 0$ under DEC, the derivative of $\rho$ satisfies

$$
\rho^{\prime}(\lambda)=2 \lambda \theta_{k}+\lambda^{2}\left(\frac{1}{2} \theta_{k}^{2}+W\right)+2 \geq \frac{\left(\lambda \theta_{k}+2\right)^{2}}{2} \geq 0 .
$$

Since $\rho$ vanishes at $\lambda=0$, it follows that $0 \leq \rho(\lambda) \leq \lim _{\lambda \rightarrow \infty} \rho(\lambda)=\theta_{k}^{(1)}$ where the last equality follows from the expansion (7.6). To show the strict inequality $\theta_{k}^{(1)}>0$ we argue by contradiction. Assume that there is some null geodesic $\alpha_{p}$ in $\Omega$ where $\theta_{k}^{(1)}=0$. Then $\rho(\lambda)$ necessarily vanishes on this curve and

$$
\left.\theta_{k}\right|_{\alpha_{p}(\lambda)} \lambda^{2}+2 \lambda=\left.0 \quad \Longrightarrow \quad \theta_{k}\right|_{\alpha_{p}(\lambda)}=\left.\frac{-2}{\lambda} \quad \Longrightarrow \quad \lim _{\lambda \rightarrow 0^{+}} \theta_{k}\right|_{\alpha_{p}(\lambda)}=-\infty,
$$

which is a contradiction to the smoothness of $\Omega$ at $S_{0}$.

The following result deals with the existence of foliations with constant $\theta_{k}^{(1)}$.

Lemma 7.3.2. Let $\Omega$ be a past asymptotically flat null hypersurface with a choice of affinely parametrized null generator $k$ and corresponding level set function $\lambda$. There exists a Lie constant positive function $f \in \mathcal{F}(\Omega)$ and a rescaling $k^{\prime}=f k$ such that the term $\theta_{k^{\prime}}^{(1)}$ in the asymptotic expansion of $\theta_{k^{\prime}}$ is constant. 


\subsection{GAB foliations and a Penrose type inequality}

Proof. Let $\left\{S_{\lambda^{\prime}}\right\}$ be the foliation associated to $k^{\prime}$. Thus $\lambda=f \lambda^{\prime}$ because $k^{\prime}\left(\lambda^{\prime}\right)=-1$ and $\left.\lambda^{\prime}\right|_{S_{0}}=0$. We use again that the null expansion $\left.\theta_{k}\right|_{p}$ is a property of $\Omega$ at $p \in \Omega$, independent of the cross section passing through $p$. Thus, as in the previous chapter, we can transform the expansion (7.6) under the change of foliation $\lambda=f \lambda^{\prime}$ simply as

$$
\theta_{k}=\frac{-2}{f} \frac{1}{\lambda^{\prime}}+\frac{\theta_{k}^{(1)}}{f^{2}} \frac{1}{\lambda^{\prime 2}}+o\left(\lambda^{\prime-2}\right) .
$$

Using now $k^{\prime}=f k$ and the fact that $\theta_{k}$ scales as $k$,

$$
\theta_{k^{\prime}}=\frac{-2}{\lambda^{\prime}}+\frac{\theta_{k}^{(1)}}{f} \frac{1}{\lambda^{\prime 2}}+o\left(\lambda^{\prime-2}\right)
$$

Since $\theta_{k}^{(1)}>0$, we can choose $f=\frac{\theta_{k}^{(1)}}{c}$ for any given constant $c>0$. The foliation $\left\{S_{\lambda^{\prime}}\right\}$ has $\theta_{k^{\prime}}^{(1)}=c$, as claimed.

Note that, by construction, the foliation $\left\{S_{\lambda^{\prime}}\right\}$ in this lemma is also an affine foliation. Once $\theta_{k}^{(1)}$ is constant, it can be made zero by a constant shift of $\lambda$. Indeed, let $\lambda$ be an affine parameter and define $\lambda=\lambda^{\prime}+\lambda_{0}$ with $\lambda_{0}$ constant. The null generator $k$ now remains unchanged and

$$
\theta_{k}=\frac{-2}{\lambda}+\frac{\theta_{k}^{(1)}}{\lambda^{2}}+o\left(\lambda^{-2}\right)=\frac{-2}{\lambda^{\prime}}+\frac{\theta_{k}^{(1)}+2 \lambda_{0}}{\lambda^{\prime 2}}++o\left(\lambda^{\prime-2}\right) .
$$

Thus, the coefficient $\theta_{k}^{(1)}$ along an affine foliation can be made zero by a change of origin if and only if it is constant. As mentioned above, Ludvigsen \& Vickers and Bergqvist considered foliations with vanishing $\theta_{k}^{(1)}$. Such foliations arise naturally in the context of conformal compactifications of null infinity and are related to the Bondi coordinates near null infinity. This motivates the following definition.

Definition 7.3.3 (Geodesic asymptotically Bondi foliation associated to $S_{0}$ ). Consider a past asymptotically flat null hypersurface $\Omega$ with a choice of cross section $S_{0}$. A geodesic-affine foliation $\left\{S_{\lambda}\right\}$ is called geodesic asymptotically Bondi (GAB) and associated to $S_{0}$ if and only if

(i) $S_{\lambda=0}=S_{0}$,

(ii) $\theta_{k}^{(1)}$ is constant.

In the following lemma we show that two GAB foliations associated to $S_{0}$ are necessarily related by a constant rescaling of parameter, $\lambda=a \lambda^{\prime}$ with $a \in \mathbb{R}^{+}$. Thus, the collection of surfaces $\left\{S_{\lambda}\right\}$ remain unchanged, and GAB foliations associated to a given $S_{0}$ are geometrically unique. Obviously, when $S_{0}$ changes, the corresponding unique GAB foliation (which exists by Lemma 7.3.2) also changes. 
Lemma 7.3.4 (Uniqueness of GABs). Let $\Omega$ be a past asymptotically flat null hypersurface and $S_{0}$ a cross section. Two GAB foliations $\left\{S_{\lambda}\right\}$ and $\left\{S_{\lambda^{\prime}}\right\}$ associated to $S_{0}$ are related by $\lambda=a \lambda^{\prime}$ for some positive constant $a$.

Proof. Let $k$ and $k^{\prime}$ be the null generators of $\left\{S_{\lambda}\right\}$ and $\left\{S_{\lambda^{\prime}}\right\}$. Since both are affine, there exists a Lie constant positive function $f$ such that $k^{\prime}=f k$. We have shown in (7.12) that

$$
\theta_{k^{\prime}}=\frac{-2}{\lambda^{\prime}}+\frac{\theta_{k}^{(1)}}{f} \frac{1}{\lambda^{\prime 2}}+o\left(\lambda^{\prime-2}\right)=\frac{-2}{\lambda^{\prime}}+\frac{\theta_{k}^{(1)^{\prime}}}{\lambda^{\prime 2}}+o\left(\lambda^{\prime-2}\right)
$$

By definition of GAB foliation, both $\theta_{k}^{(1)}$ and $\theta_{k}^{(1)^{\prime}}$ are constant. Thus $f$ is a positive constant (say a) and the affine parameters are related by $\lambda=f \lambda^{\prime}=a \lambda^{\prime}$.

The main result in the work by Ludvigsen \& Vickers and Bergqvist can be formulated in terms of GABs as follows.

Theorem 7.3.5 (Ludvigsen \& Vickers [67], Bergqvist [8]). Let $\Omega$ be a past asymptotically flat null hypersurface $\Omega$ in a spacetime satisfying the DEC. Assume that $\Omega$ admits a weakly outer trapped cross section $S_{0}$. If $\Omega$ admits a GAB foliation $\left\{S_{\lambda}\right\}$ associated to $S_{0}$ and approaching large spheres, then the Penrose inequality

$$
E_{B} \geq \sqrt{\frac{\left|S_{0}\right|}{16 \pi}}
$$

holds, where $E_{B}$ is the Bondi energy associated to the observer at infinity defined by the foliation $\left\{S_{\lambda}\right\}$.

As mentioned in Section 7.1, the possibility that the foliation can be chosen to approach large spheres was assumed implicitly in the work by Ludvigsen \& Vickers. The necessity to add this restriction explicitly was noticed by Bergqvist. Since GAB foliations associated to a given $S_{0}$ are unique, the condition of approaching large spheres is indeed a strong additional assumption, that will only be satisfied in very special circumstances. It makes sense to study GAB foliations in detail dropping the assumption of approaching large spheres. By doing this we will be able to obtain an interesting Penrose-type inequality relating the area of $S_{0}$, not to the Bondi energy, but to the limit of the Hawking energy along the foliation. Since the Hawking energy approaches the Bondi energy for asymptotically spherical foliations, our result will automatically include Theorem 7.3.5 as a corollary. In particular, this will help to clarify the role played by the asymptotically spherical condition in Theorem 7.3.5.

We have shown in Proposition 7.2.3 (cf. Remark 7.2.4) that for GAB foliations, the limit of the functional $M\left(S, \ell^{\star}\right)$ is the same as the limit of the Hawking energy at infinity. To obtain a Penrose-type inequality we need to relate the value of $M\left(S, \ell^{\star}\right)$ at the initial 


\subsection{GAB foliations and a Penrose type inequality}

surface with its asymptotic value. The functional $M\left(S_{\lambda}, \ell^{\star}\right)$ is not monotonic, so this cannot be done straight away. However, as we saw in Section $7.2, M\left(S_{\lambda}, \ell^{\varphi}\right)$ can be split in two terms one of which is automatically monotonic, so that we concentrate in studying the non-monotonic term. In fact this splitting corresponds to the two functionals $M_{b}(\lambda)$ and $D(\lambda)$ used in Section 7.1 .

The only difference is that now $\ell^{\varphi}$ is kept free. Thus, we introduce the following functionals, which in the case $\varphi=2$ reduce to $M_{b}(\lambda)$ and $D(\lambda)$ defined in Minkowski in Section 7.2 .

$$
\begin{aligned}
D\left(S, \ell^{\varphi}\right) & :=\sqrt{\frac{|S|}{16 \pi}}-\frac{\varphi}{4} \lambda, \\
M_{b}\left(S, \ell^{\varphi}\right) & :=\frac{\varphi}{4} \lambda-\frac{1}{16 \pi} \int_{S} \theta_{\ell^{\varphi}} \boldsymbol{\eta}_{S} .
\end{aligned}
$$

We obviously have $M\left(S, \ell^{\varphi}\right)=D\left(S, \ell^{\varphi}\right)+M_{b}\left(S, \ell^{\varphi}\right)$. In Chapter 4 we studied conditions under which $D(\lambda)$ is monotonic. In this chapter we intend to investigate in detail the properties of the functional $D\left(S, \ell^{\varphi}\right)$. The computation in Lemma 7.2 .1 implies that for affine flows and $\varphi=$ const (recall that the cross sections of $\Omega$ are topological spheres, so that $\chi(S)=2)$

$$
\begin{aligned}
\frac{d D\left(S_{\lambda}, \ell^{\varphi}\right)}{d \lambda} & =\frac{1}{\sqrt{64 \pi\left|S_{\lambda}\right|}} \int_{S_{\lambda}}\left(-\theta_{k}\right) \eta_{S_{\lambda}}-\frac{\varphi}{4} \\
\frac{d M_{b}\left(S_{\lambda}, \ell^{\varphi}\right)}{d \lambda} & =\frac{1}{16 \pi} \int_{S_{\lambda}}\left(\operatorname{Ein}^{g}\left(\ell^{\varphi}, k\right)+\varphi\left|s_{\ell^{\varphi}}\right|_{\gamma_{S_{\lambda}}}^{2}\right) \eta_{S_{\lambda}} \quad(\geq 0 \quad \text { under DEC }) .
\end{aligned}
$$

Recall that the monotonicity of $M_{b}(\lambda)$ in the Minkowski setting required using specific properties of flat space. This general expression for $M_{b}\left(S_{\lambda}, \ell^{\varphi}\right)$ shows that the monotonicity is in fact a general property of spacetimes satisfying the DEC. A direct consequence of $M_{b}\left(S, \ell^{\varphi}\right)$ being monotonically increasing is that its initial value is bounded above by its value at infinity. From (17.8), this limit is given by

$$
\lim _{\lambda \rightarrow \infty} M_{b}\left(S_{\lambda}, \ell^{\varphi}\right)=-\frac{1}{16 \pi} \int_{\hat{S}} \varphi\left(\mathcal{K}_{\hat{q}} \theta_{k}^{(1)}+\frac{1}{2} \theta_{\ell}^{(1)}\right) \boldsymbol{\eta}_{\hat{q}}
$$

which is finite irrespectively of the choice of $\ell^{\varphi}$. On the other hand, $D\left(S_{\lambda}, \ell^{\varphi}\right)$ is not necessarily monotonic and its limit at infinity is finite only for the choice $\ell^{\star}=R_{\hat{q}} \ell$ and given by (see (7.7) )

$$
\lim _{\lambda \rightarrow \infty} D\left(S_{\lambda}, \ell^{\star}\right)=\frac{\int_{\hat{S}} \theta_{k}^{(1)} \boldsymbol{\eta}_{\hat{q}}}{16 \pi R_{\hat{q}}} .
$$

To bound $M\left(S_{\lambda}, \ell^{\star}\right)$ from above we need to find an upper bound for $D\left(S_{\lambda}, \ell^{\star}\right)$. In fact, we shall prove $D\left(S_{\lambda}, \ell^{\star}\right) \leq \lim _{\lambda \rightarrow \infty} D\left(S_{\lambda}, \ell^{\star}\right)$ provided the foliation $\left\{S_{\lambda}\right\}$ is GAB. In the following lemma we introduce a functional that turns out to be monotonic for $G A B$ foliations. 


\section{On the Penrose inequality along null hypersurfaces}

Lemma 7.3.6. Let $\Omega$ be a past asymptotically flat null hypersurface with a choice of affinely parametrized null generator $k$ and corresponding level set function $\lambda$. Assume that the spacetime satisfies the dominant energy condition. Consider the functional

$$
F\left(S_{\lambda}\right)=\frac{\left|S_{\lambda}\right|}{\left(8 \pi R_{\hat{q}}^{2} \lambda+\int_{\hat{S}} \theta_{k}^{(1)} \boldsymbol{\eta}_{\hat{q}}\right)^{2}} .
$$

If $\left\{S_{\lambda}\right\}$ is the GAB foliation associated to $S_{0}$, then $F\left(S_{\lambda}\right)$ is monotonically increasing. Proof. Writing $F\left(S_{\lambda}\right)$ as

$$
F\left(S_{\lambda}\right)=\int_{S_{\lambda}} \frac{\boldsymbol{\eta}_{S_{\lambda}}}{\left(8 \pi R_{\hat{q}}^{2} \lambda+\int_{\hat{S}} \theta_{k}^{(1)} \boldsymbol{\eta}_{\hat{q}}\right)^{2}}
$$

and using $£_{-k} \boldsymbol{\eta}_{S_{\lambda}}=-\theta_{k} \boldsymbol{\eta}_{S_{\lambda}}$, the derivative of $F\left(S_{\lambda}\right)$ is

$$
\begin{aligned}
\frac{d}{d \lambda} F\left(S_{\lambda}\right) & =\int_{S_{\lambda}}\left(\frac{-16 \pi R_{\hat{q}}^{2}}{\left(8 \pi R_{\hat{q}}^{2} \lambda+\int_{\hat{S}} \theta_{k}^{(1)} \boldsymbol{\eta}_{\hat{q}}\right)^{3}}+\frac{-\theta_{k}}{\left(8 \pi R_{\hat{q}}^{2} \lambda+\int_{\hat{S}} \theta_{k}^{(1)} \boldsymbol{\eta}_{\hat{q}}\right)^{2}}\right) \boldsymbol{\eta}_{S_{\lambda}} \\
& =\int_{S_{\lambda}}\left(\frac{-16 \pi R_{\hat{q}}^{2}+\left(-\theta_{k}\right)\left(8 \pi R_{\hat{q}}^{2} \lambda+\int_{\hat{S}} \theta_{k}^{(1)} \boldsymbol{\eta}_{\hat{q}}\right)}{\left(8 \pi R_{\hat{q}}^{2} \lambda+\int_{\hat{S}} \theta_{k}^{(1)} \boldsymbol{\eta}_{\hat{q}}\right)^{3}}\right) \boldsymbol{\eta}_{S_{\lambda} .}
\end{aligned}
$$

This derivative is non-negative provided

$$
\left(-\theta_{k}\right)\left(8 \pi R_{\hat{q}}^{2} \lambda+\int_{\hat{S}} \theta_{k}^{(1)} \boldsymbol{\eta}_{\hat{q}}\right) \geq 16 \pi R_{\hat{q}}^{2} \Longleftrightarrow \frac{1}{-\theta_{k}}-\frac{\lambda}{2} \leq \frac{1}{16 \pi R_{\hat{q}}^{2}} \int_{\hat{S}} \theta_{k}^{(1)} \boldsymbol{\eta}_{\hat{q}} .
$$

The Raychaudhuri equation (B.18) implies that the function $\frac{1}{-\theta_{k}}-\frac{\lambda}{2}$ has non-negative derivative (under DEC). Since its limit at infinity is $\frac{\theta_{k}^{(1)}}{4}$ it follows

$$
\frac{1}{-\theta_{k}}-\frac{\lambda}{2} \leq \frac{\theta_{k}^{(1)}}{4}
$$

which holds true for any affine foliation. For GAB foliations we have, using $\int_{\hat{S}} \boldsymbol{\eta}_{\hat{q}}=$ $4 \pi R_{\hat{q}}^{2}$,

$$
\frac{\theta_{k}^{(1)}}{4}=\frac{1}{16 \pi R_{\hat{q}}^{2}} \int_{\hat{S}} \theta_{k}^{(1)} \boldsymbol{\eta}_{\hat{\boldsymbol{q}}},
$$

and (7.16) is exactly (7.15). 


\subsection{GAB foliations and a Penrose type inequality}

The monotonicity of the functional $F\left(S_{\lambda}\right)$ is useful to establish an upper bound for $D\left(S_{\lambda}, \ell^{\star}\right)$, irrespectively of whether the foliation is GAB or not.

Lemma 7.3.7. Let $\left\{S_{\lambda}\right\}$ be an affine foliation with leading term metric $\hat{q}$. If the functional $F\left(S_{\lambda}\right)$ is monotonically increasing, then

$$
D\left(S_{\lambda}, \ell^{\star}\right) \leq \lim _{\lambda \rightarrow \infty} D\left(S_{\lambda}, \ell^{\star}\right) .
$$

Proof. The monotonicity of the functional $F\left(S_{\lambda}\right)$ along $\left\{S_{\lambda}\right\}$ implies

$$
F\left(S_{\lambda}\right) \leq \lim _{\lambda \rightarrow \infty} F\left(S_{\lambda}\right) .
$$

To compute this limit we use

$$
\left|S_{\lambda}\right|=\int_{S_{\lambda}} \boldsymbol{\eta}_{S_{\lambda}}=\int_{\hat{S}}\left(\lambda^{2}+\theta_{k}^{(1)} \lambda+o(\lambda)\right) \boldsymbol{\eta}_{\hat{q}}=4 \pi R_{\hat{q}}^{2} \lambda^{2}+o(\lambda),
$$

which follows from (7.5) and $\int_{\hat{S}} \boldsymbol{\eta}_{\hat{q}}=4 \pi R_{\hat{q}}^{2}$. Hence

$$
\lim _{\lambda \rightarrow \infty} F\left(S_{\lambda}\right)=\lim _{\lambda \rightarrow \infty} \frac{\left|S_{\lambda}\right|}{\left(8 \pi R_{\hat{q}}^{2} \lambda+\int_{\hat{S}} \theta_{k}^{(1)} \eta_{\hat{q}}\right)^{2}}=\frac{1}{16 \pi R_{\hat{q}}^{2}}
$$

and (7.18) yields

$$
\frac{\left|S_{\lambda}\right|}{\left(8 \pi R_{\hat{q}}^{2} \lambda+\int_{\hat{S}} \theta_{k}^{(1)} \eta_{\hat{q}}\right)^{2}} \leq \frac{1}{16 \pi R_{\hat{q}}^{2}} \Longleftrightarrow \frac{\left|S_{\lambda}\right|}{16 \pi} \leq\left(\frac{R_{\hat{a}}}{2} \lambda+\frac{1}{16 \pi R_{\hat{q}}} \int_{\hat{S}} \theta_{k}^{(1)} \boldsymbol{\eta}_{\hat{q}}\right)^{2} .
$$

From the definition of $D\left(S_{\lambda}, \ell^{\star}\right)$ and using $\varphi=2 R_{\hat{q}}$ we have

$$
D\left(S_{\lambda}, \ell^{\star}\right)=\sqrt{\frac{\left|S_{\lambda}\right|}{16 \pi}}-\frac{R_{\hat{q}}}{2} \lambda \leq \frac{\int_{\hat{S}} \theta_{k}^{(1)} \eta_{\hat{q}}}{16 \pi R_{\hat{q}}}
$$

after using (7.19). Since the right-hand side is the limit of $D\left(S_{\lambda}, \ell^{\star}\right)$ at infinity (7.13), we conclude (7.17).

We can now establish our main result concerning GAB foliations.

Theorem 7.3.8 (A Penrose type inequality for GAB foliations). Let $\Omega$ be a past asymptotically flat null hypersurface and $S_{0}$ a cross section. Assume that the spacetime satisfies the dominant energy condition. Then, the area $\left|S_{0}\right|$ satisfies the bound

$$
\sqrt{\frac{\left|S_{0}\right|}{16 \pi}}-\frac{1}{16 \pi} \int_{S_{0}} \theta_{\ell^{\star}} \boldsymbol{\eta}_{S_{0}} \leq \lim _{\lambda \rightarrow \infty} m_{H}\left(S_{\lambda}\right),
$$

where the limit is taken along the GAB foliation $\left\{S_{\lambda}\right\}$ associated to $S_{0}$. In particular, if $S_{0}$ is a weakly outer trapped cross section, then

$$
\sqrt{\frac{\left|S_{0}\right|}{16 \pi}} \leq \lim _{\lambda \rightarrow \infty} m_{H}\left(S_{\lambda}\right)
$$




\section{On the Penrose inequality along null hypersurfaces}

Proof. From Lemmas 7.3.6 and 7.3.7, $D\left(S_{\lambda}, \ell^{\star}\right)$ is bounded above by its limit at infinity. The monotonicity of $M_{b}\left(S_{\lambda}, \ell^{\star}\right)$ then implies

$$
M\left(S_{\lambda}, \ell^{\star}\right) \leq \lim _{\lambda \rightarrow \infty} M\left(S_{\lambda}, \ell^{\star}\right)=\lim _{\lambda \rightarrow \infty} m_{H}\left(S_{\lambda}\right),
$$

where the last equality follows from Proposition 7.2.3, since $\left\{S_{\lambda}\right\}$ is GAB. In particular, for $\lambda=0$ we have (7.20). For the last statement we simply use that $\theta_{\ell^{\star}} \leq 0$ for weakly outer trapped surfaces.

Inequality (7.21) gives a completely general upper bound for the area of weakly outer trapper surfaces $S_{0}$ in terms of an energy-type quantity evaluated at infinity along the outward past null hypersurface generated by $S_{0}$, provided the latter stays regular all the way to infinity. In combination to the general analysis of the limit of the Hawking energy at infinity carried out in Chapter 6, this provides a Penrose-type inequality with potentially interesting consequences. Obviously, this inequality will only correspond to the Penrose inequality whenever the limit of the Hawking energy agrees with the Bondi energy of the cut at infinity defined by $\Omega$. As already mentioned, this is known to occur for foliations approaching large spheres. When $\Omega$ admits a GAB foliation approaching large spheres, then the limit of the Hawking energy along this foliation is the Bondi energy $E_{B}$ associated to this observer at infinity, and the Penrose-type inequality in Theorem 7.3 .8 becomes the standard Penrose inequality, thus recovering the original result by Ludvigsen \& Vickers and Bergqvist quoted as Theorem 7.3.5.

\subsection{On the inequality $D\left(S_{\lambda}, \ell\right) \leq \lim _{\lambda \rightarrow \infty} D\left(S_{\lambda}, \ell\right)$}

The key ingredient that allowed us to prove the Penrose-type inequality (7.21) is $D\left(S_{\lambda}, \ell^{\star}\right) \leq \lim _{\lambda \rightarrow \infty} D\left(S_{\lambda}, \ell^{\star}\right)$. In fact, the argument in the proof of Theorem 7.3 .8 combined with Proposition 7.2 .3 shows that any surface $S_{0}$ satisfying the inequality

$$
D\left(S_{0}, \ell^{\star}\right) \leq \lim _{\lambda \rightarrow \infty} D\left(S_{\lambda}, \ell^{\star}\right)=\frac{1}{16 \pi R_{\hat{q}}} \int_{\hat{S}} \theta_{k}^{(1)} \boldsymbol{\eta}_{\hat{q}}
$$

along an affine foliation $\left\{S_{\lambda}\right\}$ starting at $S_{0}$, in a spacetime satisfying the dominant energy condition, automatically satisfies the inequality

$$
\sqrt{\frac{\left|S_{0}\right|}{16 \pi}}-\frac{1}{16 \pi} \int_{S_{0}} \theta_{\ell^{\star}} \boldsymbol{\eta}_{S_{0}} \leq \lim _{\lambda \rightarrow \infty} m_{H}\left(S_{\lambda}\right)+\frac{1}{16 \pi} \int_{\hat{S}} \theta_{k}^{(1)}\left(\frac{1}{R_{\hat{q}}}-R_{\hat{q}} \mathcal{K}_{\hat{q}}\right) \boldsymbol{\eta}_{\hat{\boldsymbol{q}}} .
$$

This is the Penrose inequality provided $S_{0}$ is a weakly outer trapped surface and the right hand is the Bondi energy $E_{B}$ along $\left\{S_{\lambda}\right\}$. For this it is sufficient that $\left\{S_{\lambda}\right\}$ approaches 


\subsection{On the inequality $D\left(S_{\lambda}, \ell\right) \leq \lim _{\lambda \rightarrow \infty} D\left(S_{\lambda}, \ell\right)$}

large spheres and this will be the case we will be interested from now on. However, we postpone making the assumption that $\hat{q}$ is the round metric until subsection 7.5 because Proposition 7.4 .1 below (which holds for arbitrary affine foliations) may be of independent interest.

In the previous section the validity of (7.22) followed from the monotonicity of $F\left(S_{\lambda}\right)$ along GAB foliations. As shown in Lemma 7.3.7 monotonicity of $F\left(S_{\lambda}\right)$ is sufficient to establish (7.22) for arbitrary affine foliations. Since the derivative of (7.14) is

$$
\frac{d}{d \lambda} F\left(S_{\lambda}\right)=\frac{1}{\left(8 \pi R_{\hat{q}}^{2} \lambda+\int_{\hat{S}} \theta_{k}^{(1)} \eta_{\hat{q}}\right)^{2}}\left(\frac{d}{d \lambda}\left|S_{\lambda}\right|-\frac{16 \pi R_{\hat{q}}^{2}\left|S_{\lambda}\right|}{8 \pi R_{\hat{q}}^{2} \lambda+\int_{\hat{S}} \theta_{k}^{(1)} \boldsymbol{\eta}_{\hat{q}}}\right),
$$

we have established:

Proposition 7.4.1. Let $\Omega$ be a past asymptotically flat null hypersurface in a spacetime satisfying the dominant energy condition and $\left\{S_{\lambda}\right\}$ an affine foliation. If

$$
\frac{d}{d \lambda}\left|S_{\lambda}\right| \geq \frac{16 \pi R_{\hat{q}}^{2}\left|S_{\lambda}\right|}{8 \pi R_{\hat{q}}^{2} \lambda+\int_{\hat{S}} \theta_{k}^{(1)} \eta_{\hat{q}}}
$$

holds for all $\lambda \geq 0$ then the inequality (7.23) holds. In particular if $\left\{S_{\lambda}\right\}$ approaches large spheres and $(7.24)$ is satisfied, then the Penrose inequality $E_{B} \geq \sqrt{\frac{\left|S_{0}\right|}{16 \pi}}$ holds, where $E_{B}$ is the Bondi energy associated to the observer defined by $\left\{S_{\lambda}\right\}$.

Remark 7.4.2. Expanding the area as

$$
\left|S_{\lambda}\right|=4 \pi R_{\hat{q}}^{2} \lambda^{2}+\left(\int_{\hat{S}} \theta_{k}^{(1)} \eta_{\hat{q}}\right) \lambda+\hat{\Theta}
$$

(7.24) becomes, after some cancellations,

$$
\left(\int_{\hat{S}} \theta_{k}^{(1)} \boldsymbol{\eta}_{\hat{q}}\right)^{2}+\left(8 \pi R_{\hat{q}}^{2} \lambda+\int_{\hat{S}} \theta_{k}^{(1)} \boldsymbol{\eta}_{\hat{q}}\right) \frac{d \hat{\Theta}}{d \lambda} \geq 16 \pi R_{\hat{q}}^{2} \hat{\Theta} .
$$

This alternative form of Proposition 7.4.1 will be used in Section 7.7 below.

GAB foliations have the property that (7.24) is always true. It is natural to ask whether the constancy of $\theta_{k}^{(1)}$ can be relaxed and still obtain sufficiently general conditions under which (7.24) holds. The issue, however, appears to be difficult. In the next subsection we study the behaviour of the derivative of $F\left(S_{\lambda}\right)$ near infinity and show that both cases of $F\left(S_{\lambda}\right)$ being monotonically increasing or monotonically decreasing near infinity are possible. 


\subsubsection{On the monotonicity of $F\left(S_{\lambda}\right)$ for large $\lambda$}

A necessary condition for (7.14) to be non-negative for all $\lambda$ is, of course, that its leading term at infinity is non-negative. To determine the asymptotic behaviour at infinity requires one extra term in the expansion of $\theta_{k}$ as compared to (7.6). To make sure this is possible we need a slightly stronger definition of asymptotic flatness.

Definition 7.4.3 (Strong past asymptotic flatness). A null hypersurface $\Omega$ in a spacetime $\left(M^{4}, g\right)$ is strong past asymptotically flat if it is past asymptotically flat with (i) in Definition 6.3.4 in Chapter 6 replaced by the stronger condition

(i)' There exist symmetric 2-covariant transversal and Lie constant tensor fields $\hat{q}$ (positive definite), $h$ and $\Psi_{0}$ such that $\tilde{\gamma}$ defined by $\gamma=\lambda^{2} \hat{q}+\lambda h+\Psi_{0}+\tilde{\gamma}$ is $\tilde{\gamma}=o_{1}(1) \cap o_{2}^{X}(1)$.

Remark 7.4.4. In strong asymptotically flat null hypersurfaces, all affine foliations $\left\{S_{\lambda}\right\}$ automatically satisfy item (i)' in the definition. Also, there always exist affine foliations $\left\{S_{\lambda}\right\}$ for which the asymptotic metric $\hat{q}$ is the round metric of unit radius on $\mathbb{S}^{2}$ (see Chapter 6 for a proof of both facts in the context of asymptotically flat null hypersurfaces, which carries over immediately to the strong asymptotically flat case).

A first consequence of strong asymptotic flatness is that the function $\Theta$ defined by

$$
\boldsymbol{\eta}_{S_{\lambda}}=\left(\lambda^{2}+\theta_{k}^{(1)} \lambda+\Theta\right) \boldsymbol{\eta}_{\hat{q}}
$$

is of the form $\Theta=\Theta_{0}+\tilde{\Theta}$, with $\Theta_{0}$ Lie constant and $\tilde{\Theta}=o_{1}(1)$. A second consequence, which follows from (B.12), is that $\theta_{k}$ admits the expansion

$$
\theta_{k}=\frac{-2}{\lambda}+\frac{\theta_{k}^{(1)}}{\lambda^{2}}+\frac{\theta_{k}^{(2)}}{\lambda^{3}}+o\left(\lambda^{-3}\right)
$$

with $\theta_{k}^{(2)}$ Lie constant. The following proposition relates $\Theta_{0}$ with $\theta_{k}^{(1)}$ and $\theta_{k}^{(2)}$ and provides a universal bound for $\theta_{k}^{(2)}$.

Proposition 7.4.5. Let $\Omega$ be a strong past asymptotically flat null hypersurface and $\left\{S_{\lambda}\right\}$ an affine foliation. Then

$$
\Theta_{0}:=\lim _{\lambda \rightarrow \infty} \Theta=\frac{1}{2}\left(\left(\theta_{k}^{(1)}\right)^{2}+\theta_{k}^{(2)}\right) .
$$

If in addition the spacetime satisfies the dominant energy condition then we will also have

$$
\theta_{k}^{(2)} \leq-\frac{1}{2}\left(\theta_{k}^{(1)}\right)^{2} \leq 0 .
$$




\subsection{On the inequality $D\left(S_{\lambda}, \ell\right) \leq \lim _{\lambda \rightarrow \infty} D\left(S_{\lambda}, \ell\right)$}

Proof. Inserting (7.27) and (7.28) into the evolution equation

$$
£_{-k} \boldsymbol{\eta}_{\boldsymbol{S}_{\lambda}}=-\theta_{k} \boldsymbol{\eta}_{\boldsymbol{S}_{\lambda}}
$$

gives

$$
\begin{aligned}
\left(2 \lambda+\theta_{k}^{(1)}+\frac{d \tilde{\Theta}}{d \lambda}\right) \boldsymbol{\eta}_{\hat{\boldsymbol{q}}} & =-\left(\frac{-2}{\lambda}+\frac{\theta_{k}^{(1)}}{\lambda^{2}}+\frac{\theta_{k}^{(2)}}{\lambda^{3}}+o\left(\lambda^{-3}\right)\right)\left(\lambda^{2}+\theta_{k}^{(1)} \lambda+\Theta_{0}+o(1)\right) \boldsymbol{\eta}_{\hat{\boldsymbol{q}}} \\
& =\left(2 \lambda+\theta_{k}^{(1)}+\left(2 \Theta_{0}-\left(\theta_{k}^{(1)}\right)^{2}-\theta_{k}^{(2)}\right) \frac{1}{\lambda}+o\left(\lambda^{-1}\right)\right) \boldsymbol{\eta}_{\hat{\boldsymbol{q}}} .
\end{aligned}
$$

Since $\frac{d \tilde{\Theta}}{d \lambda}=o\left(\lambda^{-1}\right)$ we conclude $2 \Theta_{0}-\left(\theta_{k}^{(1)}\right)^{2}-\theta_{k}^{(2)}=0$, which proves (7.29). For the universal bound (7.30), let us define $\hat{i}(\lambda)=\frac{\eta_{S_{\lambda}}}{\left(\theta_{k}^{(1)}+2 \lambda\right)^{2}}$. Its Lie derivative is

$$
£_{-k} \hat{I}(\lambda)=\frac{1}{\left(\theta_{k}^{(1)}+2 \lambda\right)^{2}}\left(-\theta_{k}-\frac{4}{\theta_{k}^{(1)}+2 \lambda}\right) \eta_{s_{\lambda}} \geq 0,
$$

where in the last inequality we used (7.16) (here is where the DEC is used). $\hat{\imath}(\lambda)$ has limit at infinity $\frac{1}{4} \boldsymbol{\eta}_{\hat{\boldsymbol{q}}}$. In combination with the fact that $\hat{l}$ is monotonically increasing we conclude

$$
\hat{\imath}(\lambda)=\frac{\eta_{S_{\lambda}}}{\left(\theta_{k}^{(1)}+2 \lambda\right)^{2}} \leq \frac{1}{4} \boldsymbol{\eta}_{\hat{\boldsymbol{q}}}
$$

Inserting (7.27), a direct computation gives

$$
\left(-\frac{1}{16}\left(\theta_{k}^{(1)}\right)^{2}+\frac{1}{4} \Theta_{0}\right) \frac{1}{\lambda^{2}}+o\left(\lambda^{-2}\right) \leq 0 \quad \Longrightarrow \quad \Theta_{0} \leq \frac{1}{4}\left(\theta_{k}^{(1)}\right)^{2},
$$

which is simply (7.30) after using the explicit form of $\Theta_{0}$.

Let us now find the asymptotic expansion of the right hand side of (7.14). Plugging the asymptotic expansion (17.28) gives, after a straightforward computation,

$$
\begin{aligned}
\frac{d}{d \lambda} F\left(S_{\lambda}\right)= & \frac{1}{\left(8 \pi R_{\hat{q}}^{2}\right)^{3}}\left(\left(\int_{\hat{S}} \theta_{k}^{(1)} \boldsymbol{\eta}_{\hat{q}}\right)^{2}-8 \pi R_{\hat{q}}^{2} \int_{\hat{S}}\left(\theta_{k}^{(1)}\right)^{2} \boldsymbol{\eta}_{\hat{q}}-8 \pi R_{\hat{q}}^{2} \int_{\hat{S}} \theta_{k}^{(2)} \boldsymbol{\eta}_{\hat{q}}\right) \frac{1}{\lambda^{3}} \\
& +o\left(\lambda^{-3}\right) .
\end{aligned}
$$

The leading coefficient can be rewritten as $\frac{F_{\infty}}{\left(8 \pi R_{a}^{2}\right)^{3}}$, where

$$
F_{\infty}=-4 \pi R_{\hat{q}}^{2} \int_{\hat{S}}\left(\left(\theta_{k}^{(1)}\right)^{2}+2 \theta_{k}^{(2)}\right) \boldsymbol{\eta}_{\hat{\boldsymbol{q}}}+\left[\left(\int_{\hat{\mathcal{S}}} \theta_{k}^{(1)} \boldsymbol{\eta}_{\hat{\boldsymbol{q}}}\right)^{2}-4 \pi R_{\hat{\mathfrak{q}}}^{2} \int_{\hat{\mathrm{S}}}\left(\theta_{k}^{(1)}\right)^{2} \boldsymbol{\eta}_{\hat{\boldsymbol{q}}}\right],
$$




\section{On the Penrose inequality along null hypersurfaces}

which is a difference of positive quantities. Indeed, the first term is non-negative because of (7.30), while the term in brackets is non-positive because

$$
\left(\int_{\hat{S}} \theta_{k}^{(1)} \boldsymbol{\eta}_{\hat{q}}\right)^{2} \leq 4 \pi R_{\hat{q}}^{2} \int_{\hat{S}}\left(\theta_{k}^{(1)}\right)^{2} \boldsymbol{\eta}_{\hat{q}}
$$

by the Hölder inequality. Depending on which term dominates, the functional $F\left(S_{\lambda}\right)$ will be increasing or decreasing near infinity. Non-negativity of the leading term (7.32) is obviously a necessary condition for the hypothesis of Proposition 7.4.1 to hold. However, even when (7.32) has the right sign, it is not at all obvious how to ensure that $F\left(S_{\lambda}\right)$ is monotonic for all $\lambda$ when the foliation is, in addition, assumed to approach large spheres. We have attempted (and failed) finding sufficient condition ensuring $\frac{d^{2}}{d \lambda^{2}} F\left(S_{\lambda}\right) \leq 0$, as this would immediately imply that $F\left(S_{\lambda}\right)$ is increasing (because $F^{\prime}\left(S_{\lambda}\right) \rightarrow 0$ at infinity). Despite the lack of success so far, approaching the null Penrose inequality using the monotonic functional $F\left(S_{\lambda}\right)$ remains an interesting open problem, specially in view of the fact that $F\left(S_{\lambda}\right)$ is always monotonic for GAB foliations.

\subsection{Renormalized area method for the Penrose inequa- lity}

Monotonicity of $F\left(S_{\lambda}\right)$ along affine foliations approaching large spheres is an interesting sufficient condition for the Penrose inequality along null hypersurfaces. However, as discussed in the previous subsection, it appears to be difficult to find general situations where $F^{\prime}\left(S_{\lambda}\right) \geq 0$ can be guaranteed. In this section we consider an a priori different setup which implies the validity of (7.22) and hence of the Penrose inequality whenever the foliations also satisfies the restriction of approaching large spheres. Let us assume from now on that $\hat{q}$ is a round metric on the sphere. Without loss of generality we can then assume that $\hat{q}$ is a round metric of radius one, which we denote by $\stackrel{q}{q}$. Then $R_{\hat{q}}=1$ and $\ell^{\star}=\ell$. We want to investigate the condition

$$
\frac{d}{d \lambda} D\left(S_{\lambda}, \ell^{\star}\right) \geq 0
$$

which indeed implies the validity of (7.22) and hence the validity of the Penrose inequality.

Note that this condition has already been used in Section 4.5 of Chapter 4 in the Minkowski context. The positive results obtained there suggest that studying (7.33) in a general setup may be also useful to tackle the Penrose inequality for arbitrary spacetimes. Indeed, under $D^{\prime} \geq 0$, (7.22) holds, which in turn is sufficient for (7.23). Applying this inequality in the Minkowski case for foliations approaching large spheres we find

$$
\sqrt{\frac{\left|S_{0}\right|}{16 \pi}}-\frac{1}{16 \pi} \int_{S_{0}} \theta_{\ell} \boldsymbol{\eta}_{S_{0}} \leq 0
$$




\subsection{Renormalized area method for the Penrose inequality}

after using that $\lim _{\lambda \rightarrow \infty} m_{H}\left(S_{\lambda}\right)=0$, because the Bondi energy of the Minkowski spacetime is identically zero. This argument shows in just a few steps how the shell Penrose inequality follows from $D^{\prime}(\lambda) \geq 0$.

Going back to the general setting, since $\left|S_{\lambda}\right|$ diverges at infinity like $4 \pi \lambda^{2}$, the functional $D\left(S_{\lambda}, \ell\right)$ can be regarded as a renormalization of the area functional, in order to make it bounded. We thus call the approach to the null Penrose inequality via (7.33) the renormalized area method. It is interesting that this method is, in fact, a subcase of the general setup involving monotonicity of $F\left(S_{\lambda}\right)$.

Proposition 7.5.1. Let $\Omega$ be a strong past asymptotically flat null hypersurface and $\left\{S_{\lambda}\right\}$ an affine foliation approaching large spheres. Then

$$
\frac{d}{d \lambda} D\left(S_{\lambda}, \ell\right) \geq 0 \Longrightarrow \frac{d}{d \lambda} F\left(S_{\lambda}\right) \geq 0 .
$$

Proof. Let $L:=\frac{1}{16 \pi} \int_{\mathbb{S}^{2}} \theta_{k}^{(1)} \boldsymbol{\eta}_{\tilde{q}}>0$ be the limit of $D\left(S_{\lambda}, \ell\right)$ at infinity. Since $\left|S_{\lambda}\right|=$ $16 \pi\left(D\left(S_{\lambda}, \ell\right)+\frac{\lambda}{2}\right)^{2}$ we can rewrite $F\left(S_{\lambda}\right)$ as

$$
F\left(S_{\lambda}\right)=\frac{\left|S_{\lambda}\right|}{\left(8 \pi \lambda+\int_{\mathbb{S}^{2}} \theta_{k}^{(1)} \eta_{\dot{q}}\right)^{2}}=\frac{\left(D_{\lambda}+\frac{\lambda}{2}\right)^{2}}{16 \pi\left(L+\frac{\lambda}{2}\right)^{2}},
$$

where $D_{\lambda}$ is a short-hand for $D\left(S_{\lambda}, \ell\right)$. Let

$$
f(\lambda):=\sqrt{16 \pi F\left(S_{\lambda}\right)}=\frac{D_{\lambda}+\frac{\lambda}{2}}{L+\frac{\lambda}{2}}
$$

so that

$$
f^{\prime}(\lambda)\left(L+\frac{\lambda}{2}\right)=\frac{d D_{\lambda}}{d \lambda}+\frac{1}{2}(1-f(\lambda)) .
$$

If $\frac{d D_{\lambda}}{d \lambda} \geq 0$ it follows $D_{\lambda} \leq \lim _{\lambda \rightarrow \infty} D_{\lambda}=L$ so that $f(\lambda)=\frac{D_{\lambda}+\frac{\lambda}{2}}{L+\frac{\lambda}{2}} \leq 1$ and we conclude from (7.34) that $f^{\prime}(\lambda) \geq 0$, which is is equivalent to $F^{\prime}\left(S_{\lambda}\right) \geq 0$.

The derivative of $D\left(S_{\lambda}, \ell\right)$ is

$$
\begin{aligned}
\frac{d}{d \lambda} D\left(S_{\lambda}, \ell\right) & =\frac{1}{2 \sqrt{16 \pi S_{\lambda}}}\left(\frac{d}{d \lambda}\left|S_{\lambda}\right|-\sqrt{16 \pi S_{\lambda}}\right) \\
& =\frac{1}{2 \sqrt{16 \pi S_{\lambda}}}\left(\int_{S_{\lambda}}\left(-\theta_{k}\right) \eta_{S_{\lambda}}-\sqrt{16 \pi S_{\lambda}}\right) .
\end{aligned}
$$

Given that $\theta_{k}<0$, the inequality $\frac{d}{d \lambda} D\left(S_{\lambda}, \ell\right) \geq 0$ can be equivalently written in a slightly more convenient form as $G(\lambda) \geq 0$, where

$$
G(\lambda):=\left(\int_{S_{\lambda}}\left(-\theta_{k}\right) \eta_{S_{\lambda}}\right)^{2}-16 \pi\left|S_{\lambda}\right|
$$

We start by computing the limit of $G(\lambda)$ at infinity. 


\section{On the Penrose inequality along null hypersurfaces}

Proposition 7.5.2. With the same assumptions as in Proposition 7.5.1,

$$
\lim _{\lambda \rightarrow \infty} G(\lambda)=F_{\infty}=\left(\int_{\mathbb{S}^{2}} \theta_{k}^{(1)} \boldsymbol{\eta}_{\dot{q}}\right)^{2}-8 \pi \int_{\mathbb{S}^{2}}\left(\theta_{k}^{(1)}\right)^{2} \boldsymbol{\eta}_{\dot{q}}-8 \pi \int_{\mathbb{S}^{2}} \theta_{k}^{(2)} \boldsymbol{\eta}_{\dot{\boldsymbol{q}}}
$$

Proof. We have shown in Proposition 7.4.5 that

$$
\boldsymbol{\eta}_{\boldsymbol{S}_{\lambda}}=\left(\lambda^{2}+\theta_{k}^{(1)} \lambda+\frac{1}{2}\left(\left(\theta_{k}^{(1)}\right)^{2}+\theta_{k}^{(2)}\right)+o(1)\right) \boldsymbol{\eta}_{\dot{q}}
$$

From expansion (7.6), we have $\theta_{k} \boldsymbol{\eta}_{\boldsymbol{s}_{\lambda}}=-2 \lambda-\theta_{k}^{(1)}+o(1)$, so that

$$
\left(\int_{S_{\lambda}} \theta_{k} \boldsymbol{\eta}_{S_{\lambda}}\right)^{2}=64 \pi^{2} \lambda^{2}+16 \pi \lambda \int_{\mathbb{S}^{2}} \theta_{k}^{(1)} \boldsymbol{\eta}_{\dot{q}}+\left(\int_{\mathbb{S}^{2}} \theta_{k}^{(1)} \boldsymbol{\eta}_{\dot{q}}\right)^{2}+o(1) .
$$

Also form (7.36),

$$
\left|S_{\lambda}\right|=4 \pi \lambda^{2}+\lambda \int_{\mathbb{S}^{2}} \theta_{k}^{(1)} \boldsymbol{\eta}_{\grave{q}}+\int_{\mathbb{S}^{2}} \frac{1}{2}\left(\left(\theta_{k}^{(1)}\right)^{2}+\theta_{k}^{(2)}\right) \boldsymbol{\eta}_{\grave{q}}+o(1) .
$$

Inserting both into $G(\lambda)$ the divergent terms cancel out and we are left with (7.35).

Remark 7.5.3. The limit of $G(\lambda)$ is directly related to the leading term in the asymptotic expansion of $F\left(S_{\lambda}\right)$ so that the inequality "at infinity" $F_{\infty} \geq 0$ is necessary for both methods. Thus, for sufficiently large $\lambda$, the renormalized area method does not only imply $F^{\prime}\left(S_{\lambda}\right) \geq 0$, but it is in fact equivalent to it (possibly excluding the case $F_{\infty}=0$ where higher order terms dominate). However, we do not expect this to be true for all $\lambda$, as it appears that $D^{\prime}\left(S_{\lambda}, \ell\right) \geq 0$ should be a proper subset of $F^{\prime}\left(S_{\lambda}\right) \geq 0$.

Assuming we are in the situation where $\lim _{\lambda \rightarrow \infty} G(\lambda) \geq 0$, we can ensure $G(\lambda) \geq 0$ by the condition $G^{\prime}(\lambda) \leq 0$. This derivative is, from the Raychaudhuri equation (B.18),

$$
\begin{aligned}
G^{\prime}(\lambda) & =2\left(\int_{S_{\lambda}} \theta_{k} \boldsymbol{\eta}_{S_{\lambda}}\right)\left(\frac{d}{d \lambda}\left(\int_{S_{\lambda}} \theta_{k} \boldsymbol{\eta}_{S_{\lambda}}\right)+8 \pi\right) \\
& =2\left(\int_{S_{\lambda}} \theta_{k} \boldsymbol{\eta}_{S_{\lambda}}\right)\left(\int_{S_{\lambda}}\left(\operatorname{Ric}^{g}(k, k)-\frac{1}{2} \theta_{k}^{2}+\Pi_{A B}^{k} \Pi^{k}{ }^{A B}\right) \boldsymbol{\eta}_{S_{\lambda}}+8 \pi\right),
\end{aligned}
$$

with $\Pi_{A B}^{k}:=K_{A B}^{k}-\frac{1}{2} \theta_{k} \gamma_{A B}$ the trace-free part of $K_{A B}^{k}$. Since the first term is always negative, $G^{\prime}(\lambda) \leq 0$ is equivalent to $H(\lambda) \geq 0$, where we have defined

$$
H(\lambda):=\int_{S_{\lambda}}\left(\operatorname{Ric}^{g}(k, k)-\frac{1}{2} \theta_{k}^{2}+\Pi_{A B}^{k} \Pi^{k^{A B}}\right) \eta_{S_{\lambda}}+8 \pi .
$$

We proceed with the computation of the derivative of this function and of its limit at infinity. 
Proposition 7.5.4. With the same assumptions as in Proposition 7.5.1, $\lim _{\lambda \rightarrow \infty} H(\lambda)=0$ and the derivative of $H(\lambda)$ is

$$
H^{\prime}(\lambda)=\int_{S_{\lambda}}\left(-2 \theta_{k} \operatorname{Ric}^{g}(k, k)+2\left(\Pi^{k}\right)^{A B} R_{A B}+\frac{d}{d \lambda} \operatorname{Ric}^{g}(k, k)\right) \eta_{S_{\lambda}},
$$

where $R_{A B}:=\operatorname{Riem}^{g}\left(X_{A}, k, X_{B}, k\right)$.

Proof. For the limit, we split $H(\lambda)$ in three terms and show that each one tends to zero. We start with $\int_{S_{\lambda}} \Pi_{A B}^{k} \Pi^{k A B} \eta_{S_{\lambda}}$. From equation (B.12) and the expansion (i) in Definition 7.4 .3 for the metric $\gamma$, it follows

$$
K_{A B}^{k}=-\stackrel{\circ}{q}_{A B} \lambda-\frac{1}{2} h_{A B}+o(1)
$$

so that its trace-free part is $\Pi_{A B}^{k}=O(1)$. Since $\gamma(\lambda)_{A B}=\lambda^{2} \stackrel{\circ}{q}_{A B}+o(\lambda)$, its inverse is

$$
\gamma(\lambda)^{A B}=\frac{1}{\lambda^{2}} \stackrel{q}{q}^{A B}+o\left(\lambda^{-2}\right)
$$

and $\Pi_{A B}^{k} \Pi^{k A B}=O\left(\lambda^{-4}\right)$ so that

$$
\int_{S_{\lambda}}\left(\Pi_{A B}^{k} \Pi^{k A B}\right) \eta_{S_{\lambda}} \stackrel{\lambda \rightarrow \infty}{\longrightarrow} 0
$$

as a consequence of $\boldsymbol{\eta}_{\boldsymbol{S}_{\lambda}}=\lambda^{2} \boldsymbol{\eta}_{\mathfrak{q}}+O(\lambda)$. Concerning the term in $\operatorname{Ric}^{g}(k, k)$, we note that inserting the expansion (7.6) into the Raychaudhuri equation (B.18) yields $\Pi_{A B}^{k} \Pi^{k A B}+$ $\operatorname{Ric}^{g}(k, k)=O\left(\lambda^{-4}\right)$ which implies $\operatorname{Ric}^{g}(k, k)=O\left(\lambda^{-4}\right)$ and again $\int_{S_{\lambda}} \operatorname{Ric}^{g}(k, k) \eta_{S_{\lambda}} \stackrel{\lambda \rightarrow \infty}{\longrightarrow}$ 0 . Finally, $\theta_{k}^{2} \boldsymbol{\eta}_{\boldsymbol{s}_{\boldsymbol{\lambda}}}=(4+o(1)) \boldsymbol{\eta}_{\mathfrak{q}}$ from which

$$
\int_{S_{\lambda}}\left(-\frac{1}{2} \theta_{k}^{2}\right) \boldsymbol{\eta}_{S_{\lambda}}+8 \pi \stackrel{\lambda \rightarrow \infty}{\longrightarrow} 0 .
$$

We next compute the derivative of $H(\lambda)$. Using now equation (B.19) in Appendix B, we obtain

$$
\frac{d}{d \lambda}\left(\Pi_{B}^{k^{A}} \Pi_{A}^{k^{B}}\right)=2 \theta_{k} \operatorname{tr}\left(\left(\Pi^{k}\right)^{2}\right)+2 R^{A B} \Pi_{A B}^{k}
$$

Using this together with (7.31) and the Raychaudhuri equation, the derivative (7.37) is obtained after a number of cancellations.

We can combine the previous computations to find a set of sufficient conditions under which the renormalized area method applies.

Theorem 7.5.5 (Sufficient conditions for the renormalized area method). Let $\Omega$ be a strong past asymptotically flat null hypersurface and $\left\{S_{\lambda}\right\}$ an affine foliation approaching large spheres. Assume that the spacetime satisfies the dominant energy condition. If the two conditions 
(i) $\left(\int_{\mathbb{S}^{2}} \theta_{k}^{(1)} \boldsymbol{\eta}_{\dot{q}}\right)^{2}-8 \pi \int_{\mathbb{S}^{2}}\left(\theta_{k}^{(1)}\right)^{2} \boldsymbol{\eta}_{\dot{q}}-8 \pi \int_{\mathbb{S}^{2}} \theta_{k}^{(2)} \boldsymbol{\eta}_{\dot{q}} \geq 0$,

(ii) $\int_{S_{\lambda}}\left(-2 \theta_{k} \operatorname{Ric}^{g}(k, k)+2\left(\Pi^{k}\right)^{A B} R_{A B}+\frac{d}{d \lambda} \operatorname{Ric}^{g}(k, k)\right) \eta_{S_{\lambda}} \leq 0, \quad \forall \lambda \geq 0$

hold, then

$$
\sqrt{\frac{\left|S_{0}\right|}{16 \pi}}-\frac{1}{16 \pi} \int_{S_{0}} \theta_{\ell} \eta_{S_{0}} \leq E_{B}
$$

where $E_{B}$ is the Bondi energy associated to the foliation $\left\{S_{\lambda}\right\}$. In particular, if $S_{0}$ is a weakly outer trapped surface then the Penrose inequality $E_{B} \geq \sqrt{\frac{\left|S_{0}\right|}{16 \pi}}$ holds.

Proof. From (ii) we have $H^{\prime}(\lambda) \leq 0$ which implies $H(\lambda) \geq 0$, as this function tends to zero at infinity. Hence $G^{\prime}(\lambda) \leq 0$. From (i) and Proposition 7.5.2 we have $\lim _{\lambda \rightarrow \infty} G(\lambda) \geq 0$ and we conclude $G(\lambda) \geq 0$, or equivalently $D^{\prime}\left(S_{\lambda}, \ell\right) \geq 0$. The theorem follows from (7.23) using the fact that $\left\{S_{\lambda}\right\}$ approaches large spheres.

It is remarkable that $H^{\prime}(\lambda)$ only involves curvature terms. This makes checking the validity of $H^{\prime}(\lambda) \geq 0$ feasible, at least in some cases. In the next two sections we explore the validity of conditions (i) and (ii) in two simple, but relevant situations.

\subsection{Shear-free vacuum case}

In this section we consider whether the functional $M\left(S_{\lambda}, \ell\right)$ can be used to prove the Penrose inequality in the case of shear-free null hypersurfaces $\Omega$ (i.e. satisfying $K^{k}=$ $\left.\frac{1}{2} \theta_{k} \gamma\right)$ embedded in a vacuum spacetime. The Penrose inequality in this setup was proven by Sauter [93] in full generality exploiting properties of the Hawking energy. Our interest in analyzing the shear-free case is to gain insight on the range of applicability and limitations of the methods discussed above.

For instance, concerning the renormalized area method in subsection 7.5, the vacuum and shear-free conditions immediately imply that $H^{\prime}(\lambda)=0$, so condition (ii) in Theorem 7.5 .5 is always satisfied. Thus $H(\lambda)$ vanishes identically, which is equivalent to $G(\lambda)=$ const. The method works if and only if this constant is non-negative. It can be computed from its limit at infinity in Proposition 7.5 .2 as

$$
G(\lambda)=\lim _{\lambda \rightarrow \infty} G(\lambda)=\left(\int_{\mathbb{S}^{2}} \theta_{k}^{(1)} \boldsymbol{\eta}_{\dot{q}}\right)^{2}-8 \pi \int_{\mathbb{S}^{2}}\left(\theta_{k}^{(1)}\right)^{2} \boldsymbol{\eta}_{\dot{\boldsymbol{q}}}-8 \pi \int_{\mathbb{S}^{2}} \theta_{k}^{(2)} \boldsymbol{\eta}_{\dot{\boldsymbol{q}}} .
$$




\subsection{Shear-free vacuum case}

In the shear-free vacuum case, the Raychaudhuri equation (B.18) is simply $\frac{d \theta_{k}}{d \lambda}=-\frac{1}{2} \theta_{k}^{2}$, which integrates to

$$
\theta_{k}=-\frac{2}{\lambda+\alpha}
$$

where $\alpha>0$ (because $\theta_{k}<0$ all along $\Omega$ ) is a Lie constant function. Expanding near infinity

$$
\theta_{k}=-\frac{2}{\lambda}+\frac{2 \alpha}{\lambda}-\frac{2 \alpha^{2}}{\lambda^{2}}+O\left(\lambda^{-3}\right) \quad \Longrightarrow \quad \theta_{k}^{(1)}=2 \alpha, \quad \theta_{k}^{(2)}=-2 \alpha^{2},
$$

which inserted into (17.41) yields

$$
G(\lambda)=4\left(\left(\int_{\mathbb{S}^{2}} \alpha \boldsymbol{\eta}_{\tilde{q}}\right)^{2}-4 \pi \int_{\mathbb{S}^{2}} \alpha^{2} \boldsymbol{\eta}_{\tilde{q}}\right) .
$$

By the Hölder inequality this constant is always non-positive and vanishes only when $\alpha=$ const (i.e. when $\left\{S_{\lambda}\right\}$ is a GAB foliation). Except in this case (which corresponds in the present setup to $\left.\theta_{k}\right|_{S_{0}}=$ const) we have $G(\lambda)<0$ and $D\left(S_{\lambda}, \ell\right)$ is strictly monotonically decreasing, which makes the renormalized area method method fail. In fact, as discussed in Remark 7.5.3, the function $F\left(S_{\lambda}\right)$ is also monotonically decreasing, at least in a neighbourhood of infinity, so the approach discussed in Proposition 7.4.1 also fails in the present setup.

Despite all this, the method involving the functional $M\left(S_{\lambda}, \ell\right)$ is capable of establishing the Penrose inequality in the shear-free vacuum case. However, as we shall see next, the argument is not based on the monotonicity of $M\left(S_{\lambda}, \ell\right)$ (which fails in general, see below) but via an integration of (7.4), which in turn relies on the fact that all the geometric information along $\Omega$ can be computed explicitly in the shear-free vacuum case. From the shear-free condition and the expression for $\theta_{k}$, the metric $\gamma_{S_{\lambda}}$ can be obtained from (B.12)

$$
\frac{d \gamma_{S_{\lambda}}}{d \lambda}=-2 K^{k}=-\theta_{k} \gamma_{S_{\lambda}}=\frac{2}{\lambda+\alpha} \gamma_{S_{\lambda}} \quad \Longleftrightarrow \quad \gamma_{S_{\lambda}}=(\lambda+\alpha)^{2} \stackrel{q}{q}
$$

where we used the fact that the foliation $\left\{S_{\lambda}\right\}$ approaches large spheres. The volume form is $\boldsymbol{\eta}_{S_{\lambda}}=(\lambda+\alpha)^{2} \boldsymbol{\eta}_{\mathfrak{q}}$. As shown in Lemma 7.2.1, the derivative of $M_{b}\left(S_{\lambda}, \ell\right)$ involves the connection one-form $s_{\boldsymbol{\ell}}$. This object satisfies the following evolution equation along an arbitrary foliation defined by a null generator $k$ (see equation (B.39) in Appendix $B$ )

$$
k\left(s_{\ell}(X)\right)=-X\left(Q_{k}\right)-s_{\ell}(X) \theta_{k}+\left(\operatorname{div}_{S_{r}} K^{k}\right)(X)-D_{X} \theta_{k}-\operatorname{Ein}^{g}(k, X),
$$

where $X$ is tangent to $S_{\lambda}$ and satisfies $[k, X]=0$. In the vacuum, affine and shear-free case this equation becomes

$$
\frac{d s_{\ell}(X)}{d \lambda}=-\frac{2}{\lambda+\alpha} s_{\ell}(X)+\frac{1}{(\lambda+\alpha)^{2}} X(\alpha)
$$




\section{On the Penrose inequality along null hypersurfaces}

after using the explicit form of $\theta_{k}$. This equation can be integrated to

$$
s_{\ell}=\frac{1}{(\lambda+\alpha)^{2}}(\lambda d \alpha+\omega)
$$

where $\boldsymbol{\omega}$ is a Lie constant transversal one-form. In order to investigate the monotonicity of the functional $M\left(S_{\lambda}, \ell\right)$ we need to evaluate (7.4) and in particular $\left|s_{\boldsymbol{\ell}}\right|_{\gamma_{S_{\lambda}}}^{2} \boldsymbol{\eta}_{S_{\lambda}}$. Using (17.42) and the form of $\gamma_{S_{\lambda}}$ we have

$$
\left|\boldsymbol{s}_{\boldsymbol{\ell}}\right|_{\gamma_{S_{\lambda}}}^{2} \boldsymbol{\eta}_{S_{\lambda}}=\frac{1}{(\lambda+\alpha)^{4}}|\lambda d \alpha+\boldsymbol{\omega}|_{\dot{q}}^{2} \boldsymbol{\eta}_{\grave{q}}
$$

and identity (7.4) simplifies to

$$
\frac{d M\left(S_{\lambda}, \ell\right)}{d \lambda}=\frac{1}{8 \pi} \sqrt{\frac{4 \pi}{\int_{\mathbb{S}^{2}}(\lambda+\alpha)^{2} \boldsymbol{\eta}_{\grave{q}}}} \int_{\mathbb{S}^{2}}(\lambda+\alpha) \boldsymbol{\eta}_{\dot{q}}-\frac{1}{2}+\frac{1}{8 \pi} \int_{\mathbb{S}^{2}} \frac{1}{(\lambda+\alpha)^{4}}|\lambda d \alpha+\boldsymbol{\omega}|_{\grave{q}}^{2} \boldsymbol{\eta}_{\dot{q}}
$$

We want to bound this expression from below. The Lie constant one-form $\boldsymbol{\omega}$ can be uniquely split into

$$
\boldsymbol{\omega}=-\beta d \alpha+\boldsymbol{\omega}^{\perp}, \quad\left\langle\boldsymbol{\omega}^{\perp}, d \alpha\right\rangle_{\mathfrak{q}}=0,
$$

where $\beta$ is a Lie constant function on $\Omega$. Thus

$$
\frac{d M\left(S_{\lambda}, \ell\right)}{d \lambda}=\frac{1}{2}\left(\frac{\int_{\mathbb{S}^{2}}(\lambda+\alpha) \boldsymbol{\eta}_{\mathfrak{q}}}{\sqrt{4 \pi \int_{\mathbb{S}^{2}}(\lambda+\alpha)^{2} \boldsymbol{\eta}_{\mathfrak{q}}}}-1\right)+\frac{1}{8 \pi} \int_{\mathbb{S}^{2}} \frac{\left((\lambda-\beta)^{2}|d \alpha|_{\grave{q}}^{2}+\left|\boldsymbol{\omega}^{\perp}\right|_{\grave{q}}^{2}\right)}{(\lambda+\alpha)^{4}} \boldsymbol{\eta}_{\dot{q}} .
$$

The Hölder inequality implies that the term in parenthesis is non-positive and strictly negative unless $\alpha$ constant (which corresponds both to the GAB case and also to the $D^{\prime}\left(S_{\lambda}, \ell\right) \geq 0$ case in the present context). Since $\beta$ may be positive and constant and $\boldsymbol{\omega}^{\perp}$ is allowed to be zero, it follows that $\left.\frac{d M\left(S_{\lambda}, \ell\right)}{d \lambda}\right|_{\lambda=\beta}$ may have either sign. This shows that one cannot expect $M\left(S_{\lambda}, \ell\right)$ to be a monotonic functional on all cases. Nevertheless, the right-hand side in (7.43) is an explicit function in $\lambda$ that can be integrated explicitly

$$
\begin{aligned}
M\left(S_{\lambda_{1}}, \ell\right) & -M\left(S_{0}, \ell\right)=\left[\frac{1}{2}\left(\sqrt{\frac{\int_{\mathbb{S}^{2}}(\lambda+\alpha)^{2} \boldsymbol{\eta}_{\dot{q}}}{4 \pi}}-\lambda\right)\right. \\
& \left.+\frac{1}{8 \pi} \int_{\mathbb{S}^{2}} \frac{\left[-\frac{\alpha^{2}}{4}-\frac{1}{3}\left(\beta-\frac{\alpha}{2}\right)^{2}+\lambda(\beta-\alpha)-\lambda^{2}\right]|d \alpha|_{\mathscr{q}}^{2}-\frac{1}{3}\left|\boldsymbol{\omega}^{\perp}\right|_{\grave{q}}^{2}}{(\lambda+\alpha)^{3}} \boldsymbol{\eta}_{\dot{q}}\right]\left.\right|_{\lambda=0} ^{\lambda_{1}} .
\end{aligned}
$$


Sending $\lambda_{1}$ to infinity, evaluating at $\lambda=0$ and using that the flow approaches large spheres

$$
\begin{aligned}
& E_{B}=M\left(S_{0}, \ell\right) \\
& +\frac{1}{8 \pi}\left(\int_{\mathbb{S}^{2}} \alpha \boldsymbol{\eta}_{\dot{q}}-\sqrt{4 \pi \int_{\mathbb{S}^{2}} \alpha^{2} \boldsymbol{\eta}_{\dot{q}}}+\int_{\mathbb{S}^{2}}\left(\frac{|d \alpha|_{\grave{q}}^{2}}{4 \alpha}+\frac{\left(\beta-\frac{\alpha}{2}\right)^{2}|d \alpha|_{\grave{q}}^{2}+\left|\boldsymbol{\omega}^{\perp}\right|_{\grave{q}}^{2}}{3 \alpha^{3}}\right) \boldsymbol{\eta}_{\dot{q}}\right) \\
& =\sqrt{\frac{\left|S_{0}\right|}{16 \pi}}-\frac{1}{16 \pi} \int_{S_{0}} \theta_{\ell} \boldsymbol{\eta}_{S_{0}} \\
& +\frac{1}{8 \pi}(\underbrace{\int_{\mathbb{S}^{2}}\left(\alpha+\frac{|d \alpha|_{\grave{q}}^{2}}{4 \alpha}\right) \boldsymbol{\eta}_{\grave{q}}-\sqrt{4 \pi \int_{\mathbb{S}^{2}} \alpha^{2} \boldsymbol{\eta}_{\dot{q}}}}_{:=I_{1}}+\underbrace{\int_{\mathbb{S}^{2}} \frac{\left(\beta-\frac{\alpha}{2}\right)^{2}|d \alpha|_{\grave{q}}^{2}+\left|\boldsymbol{\omega}^{\perp}\right|_{\grave{q}}^{2}}{3 \alpha^{3}} \boldsymbol{\eta}_{\dot{q}}}_{:=I_{2}})
\end{aligned}
$$

This identity is valid for any spacelike cross section $S_{0}$ embedded in a shear-free and vacuum $\Omega$. We now use the Beckner inequality already used in Chapter 4 (formula (4.26) ), which for $q=4$ and $n=2$ reads

$$
\int_{\mathbb{S}^{2}}\left(F^{2}+|d F|_{\dot{q}}^{2}\right) \boldsymbol{\eta}_{\dot{q}} \geq \sqrt{4 \pi \int_{\mathbb{S}^{2}} F^{4} \boldsymbol{\eta}_{\dot{q}}}
$$

with equality only for the constant functions. Writing $F=\sqrt{\alpha}$ it follows

$$
\int_{\mathbb{S}^{2}}\left(\alpha+\frac{|d \alpha|_{\grave{q}}^{2}}{4 \alpha}\right) \boldsymbol{\eta}_{\mathfrak{q}} \geq \sqrt{4 \pi \int_{\mathbb{S}^{2}} \alpha^{2} \boldsymbol{\eta}_{\mathfrak{q}}}
$$

and $I_{1}$ is non-negative. The Penrose inequality in this case follows because $I_{2}$ is manifestly non-negative and on a weakly outer trapped surface $\theta_{\ell} \leq 0$.

The proof by Sauter [93] of this inequality in the vacuum, shear-free case involved computing the Hawking energy for a foliation $\left\{S_{s}\right\}$ with the property $\theta_{k}\left(S_{s}\right)=\frac{2}{s}$. This is in general a different foliation to the one used before (they only agree when $\alpha$ is constant). A fundamental step in Sauter's argument was also the Beckner inequality.

Note also, that the Penrose inequality in the shear-free case involves not only the gap given by the Beckner inequality, but a second gap given by $I_{2}$. The stronger Penrose inequality (7.44) is obviously sharp because if $S_{0}$ is a MOTS $\left(\theta_{\ell}=0\right)$ we have equality in (7.44). It is an interesting question whether one can give a physical interpretation to each of the two positive terms in (7.44). Note that

$$
\boldsymbol{\omega}=\left.\alpha^{2} s_{\ell}\right|_{s_{0}}, \quad \alpha=-\frac{2}{\theta_{k} \mid s_{0}}, \quad \stackrel{q}{q}=\frac{1}{\alpha^{2}} \gamma_{S_{0}},
$$

so that $\beta$ and $\boldsymbol{\omega}^{\perp}$ can be determined in terms of the data on $S_{0}$ and both $I_{1}$ and $I_{2}$ can be written fully in terms of the geometry of the initial surface. 


\subsection{Renormalized area method for the shell Penrose inequality in $\mathcal{M}^{1,3}$}

In Chapter 4 we studied the shell Penrose inequality

$$
\int_{S_{0}} \theta_{\ell} \boldsymbol{\eta}_{S_{0}} \geq \sqrt{16 \pi\left|S_{0}\right|}
$$

by imposing the condition $D^{\prime}(\lambda) \geq 0$. This corresponds to the renormalized area method developed in this chapter in full generality. It is therefore convenient to apply the general results to the specific case of Minkowski. By doing this we should (and will) recover the results in Chapter 4 . We will also see how the general tools developed here allow us to derive the results in Minkowski in a much easier and efficient way. In addition we analyze the size of the set of solutions obtained with the renormalized area method in comparison with the ones obtained under the Ludvigsen \& Vickers and Bergqvist assumptions (existence of a GAB foliation that also tended to large spheres) in the Minkowski spacetime, cf. Section 7.3. In Section 7.8 we study the GAB foliation in the Minkowski setting.

The renormalized area method is particularly well-suited to the Minkowski spacetime. Indeed, the curvature tensor vanishes identically in this spacetime, so from Proposition 7.5.4 we have that $H(\lambda)$ is constant and hence zero, as its limit at infinity always vanishes. Thus, as in the shear-free case, $G(\lambda)$ is constant and its sign can be decided by its asymptotic value (7.35). We need to determine $\theta_{k}^{(1)}$ and $\theta_{k}^{(2)}$. In the Minkowski spacetime this is simple because $R_{B}^{A}=0$ makes the Ricatti equation explicitly integrable.

The solution to the Ricatti equation for $K^{k}$ (4.7) and for the metric $\gamma$ (4.8) has already been obtained in Chapter 4 , in (4.9) and (4.10) respectively. There the initial data was given in terms of the geometry of the euclidean surface $\widehat{S}_{0}$ obtained when intersecting the past null cone of $S_{0}$ with a constant time hyperplane $\left\{t=t_{0}\right\}$. In this chapter it is more convenient to express the solutions in terms of initial data defined on the surface $S_{0}$, specifically in terms of its second fundamental form $K_{0}^{k} A B$ along $k$ and its metric, namely

$$
\begin{aligned}
& \left.\left(K^{k}\right)_{B}^{A}\right|_{p}=\left.\left(K_{0}^{k}\right)_{C}^{A}\right|_{\tilde{\pi}(p)}\left[\left(\boldsymbol{I d}-\left.\lambda(p) K_{0}^{k}\right|_{\tilde{\pi}(p)}\right)^{-1}\right]_{B}^{C}, \\
& \left.(\gamma)_{A B}\right|_{p}=\left.(\gamma)_{A C}\right|_{\tilde{\pi}(p)}\left[\left(\boldsymbol{I} \boldsymbol{d}-\left.\lambda(p) \boldsymbol{K}_{0}^{k}\right|_{\tilde{\pi}(p)}\right)^{2}\right]_{B}^{C},
\end{aligned}
$$

where $\tilde{\pi}(p)$ is the (unique) point on $S_{0}$ lying on the null geodesic containing $p$ and tangent to $\left.k\right|_{p}$. Here $K_{0}^{k}$ denotes the endomorphism with components $\left(K_{0}^{k}\right)_{B}^{A}$ and $K_{0}^{k} A B$ stands to the null second fundamental form of $S_{0}$ along $k$. Taking the trace of (7.46) we find $\left.\theta_{k}\right|_{p}=\left.\left(K_{0}^{k}\right)_{C}^{A}\left[\left(\boldsymbol{I d}-\lambda \boldsymbol{K}_{0}^{k}\right)^{-1}\right]_{A}^{C}\right|_{\tilde{\pi}(p)}$, which for the sake of simplicity we 
write simply as

$$
\theta_{k}(\lambda)=\operatorname{tr}\left[\boldsymbol{K}_{0}^{k} \circ\left(\boldsymbol{I} \boldsymbol{d}-\lambda \boldsymbol{K}_{0}^{k}\right)^{-1}\right]
$$

dropping all reference to the point $p$.

Let us expand (7.48) near infinity. In order to do so,

$$
\begin{aligned}
\boldsymbol{K}_{0}^{k} \circ\left(\boldsymbol{I} \boldsymbol{d}-\lambda \boldsymbol{K}_{0}^{k}\right)^{-1} & =\left(\left(\boldsymbol{K}_{0}^{k}\right)^{-1}-\lambda \boldsymbol{I d}\right)^{-1}=\frac{-1}{\lambda}\left(\boldsymbol{I} \boldsymbol{d}-\frac{1}{\lambda}\left(\boldsymbol{K}_{0}^{k}\right)^{-1}\right)^{-1} \\
& =\frac{-\boldsymbol{I d}}{\lambda}+\frac{-\left(\boldsymbol{K}_{0}^{k}\right)^{-1}}{\lambda^{2}}+\frac{-\left(\boldsymbol{K}_{0}^{k}\right)^{-2}}{\lambda^{3}}+\ldots
\end{aligned}
$$

where we have used the relation $(\boldsymbol{I} \boldsymbol{d}-\epsilon \boldsymbol{A})^{-1}=\boldsymbol{I} \boldsymbol{d}+\epsilon \boldsymbol{A}+\epsilon^{2} \boldsymbol{A}^{2}+\ldots$, for small $\epsilon$. Then the expansion of (7.48) becomes

$$
\theta_{k}=\frac{-2}{\lambda}+\frac{-\operatorname{tr}\left(\left(\boldsymbol{K}_{0}^{k}\right)^{-1}\right)}{\lambda^{2}}+\frac{-\operatorname{tr}\left(\left(\boldsymbol{K}_{0}^{k}\right)^{-2}\right)}{\lambda^{3}}+o\left(\lambda^{-3}\right)
$$

Thus,

$$
\theta_{k}^{(1)}=-\operatorname{tr}\left(\left(\boldsymbol{K}_{0}^{k}\right)^{-1}\right), \quad \theta_{k}^{(2)}=-\operatorname{tr}\left(\left(\boldsymbol{K}_{0}^{k}\right)^{-2}\right) .
$$

We want to express the leading coefficients in the expansion (7.49) in terms of the time height function $\tau_{0}$ of $S_{0}$ and the support function $h$ of the surface $\widehat{S}_{0}$ obtained as the intersection of the past null cone $\Omega$ with the constant time hyperplane $\Sigma_{t_{0}}$. We could use solution (7.46) to obtain an expression of $K_{0}^{k}$ in terms of the second fundamental form of $\widehat{S}_{0}$. Replacing $\lambda=\tau_{0}$ in (7.46), using that $\left.K^{k}\right|_{\widehat{S}_{0}}=-\left(K_{0}\right)$ and solving for $K_{0}^{k}$ we would obtain the desired expression. However we find more direct to use the expression of $K^{k}$ in terms of $K_{0}$ computed in Chapter 4 . In this case and since $\boldsymbol{K}_{0}^{k}=-\left(\boldsymbol{K}_{0}\right) \circ\left(\boldsymbol{I} \boldsymbol{d}-\tau_{0} \boldsymbol{K}_{0}\right)^{-1}$, we have

$$
\theta_{k}^{(1)}=-\operatorname{tr}\left(\left(\boldsymbol{K}_{0}^{k}\right)^{-1}\right)=\operatorname{tr}\left(\left(\boldsymbol{K}_{0}\right)^{-1} \circ\left(\boldsymbol{I} \boldsymbol{d}-\tau_{0} \boldsymbol{K}_{0}\right)\right) \text {. }
$$

We use now the relation $B:=\left(K_{0}\right)^{-1}$ that we saw in Chapter 4, where $B^{A}{ }_{B}=$ $\left(\stackrel{\circ}{q}^{-1}\right)^{A C} \stackrel{\circ}{\nabla}_{C} \stackrel{\circ}{\nabla}_{B} h+\delta_{B}^{A} h$, with $\stackrel{q}{q}$ the spherical metric, $\stackrel{\circ}{\nabla}$ the spherical connection and $h$ the support function of $\widehat{S}_{0}$ (cf. (4.19) in Chapter 4 ). Hence

$$
\theta_{k}^{(1)}=\operatorname{tr}\left(\boldsymbol{B}-\tau_{0} \boldsymbol{I} \boldsymbol{d}\right)=\triangle_{q} h+2\left(h-\tau_{0}\right):=u \text {. }
$$

This function $u$ is defined in exactly the same way as in Chapter 4.

As we already saw in Chapter 4 , any $2 \times 2$ matrix $\boldsymbol{A}$ satisfies

$$
\operatorname{tr}\left(\boldsymbol{A}^{2}\right)=\operatorname{tr}(\boldsymbol{A})^{2}-2 \operatorname{det}(\boldsymbol{A})
$$




\section{On the Penrose inequality along null hypersurfaces}

which applied to $K_{0}^{k}$ gives $\theta_{k}^{(2)}=2 \operatorname{det}\left(\left(K_{0}^{k}\right)^{-1}\right)-u^{2}$. Inserting this into (7.35) yields

$$
F_{\infty}=\lim _{\lambda \rightarrow \infty} G(\lambda)=\left(\int_{\mathbb{S}^{2}} u \boldsymbol{\eta}_{\tilde{q}}\right)^{2}-16 \pi \int_{\mathbb{S}^{2}}\left(\operatorname{det}\left(\left(\boldsymbol{K}_{0}^{k}\right)^{-1}\right)\right) \boldsymbol{\eta}_{\tilde{q}}
$$

This expression can be related to the area of $\left|S_{0}\right|$ as follows. From the definition

$$
\stackrel{\circ}{q}=\lim _{\lambda \rightarrow \infty} \frac{\gamma(\lambda)}{\lambda^{2}}=\lim _{\lambda \rightarrow \infty} \frac{\gamma\left(\boldsymbol{I} \boldsymbol{d}-\lambda \boldsymbol{K}_{0}^{k}\right)^{2}}{\lambda^{2}}=\gamma\left(\boldsymbol{K}_{0}^{k}\right)^{2},
$$

we can relate the volume forms at $S_{0}$ and "at infinity" by

$$
\boldsymbol{\eta}_{\boldsymbol{S}_{0}}=\operatorname{det}\left(\left(\boldsymbol{K}_{0}^{k}\right)^{-1}\right) \boldsymbol{\eta}_{\mathfrak{q}}
$$

and (7.51) becomes

$$
F_{\infty}=\lim _{\lambda \rightarrow \infty} G(\lambda)=\left(\int_{\mathbb{S}^{2}} u \boldsymbol{\eta}_{\dot{q}}\right)^{2}-16 \pi\left|S_{0}\right| .
$$

Summarizing, in the Minkowski spacetime $G(\lambda)=F_{\infty}$ and $F_{\infty} \geq 0$ implies (cf. Theorem 7.5.5) the validity of (7.40), which is exactly (7.45) because the Bondi energy of the Minkowski spacetime vanishes identically. We have thus proved that the shell Penrose inequality in Minkowski holds provided

$$
\left(\int_{\mathbb{S}^{2}} u \boldsymbol{\eta}_{\dot{q}}\right)^{2} \geq 16 \pi\left|S_{0}\right|
$$

The formula for the area of $S_{0}$ in terms of $h$ and $\tau_{0}$ is (cf. (4.44)

$$
\left|S_{0}\right|=\int_{\mathbb{S}^{2}}\left(\left(h-\tau_{0}\right)^{2}+\left(\triangle_{\dot{q}} h\right)\left(h-\tau_{0}\right)-\frac{1}{2} h \triangle_{\dot{q}} h\right) \boldsymbol{\eta}_{\dot{q}}
$$

As it happened in Theorem 4.5.3 in Chapter 4, when we insert this last expression into (7.54) and after some manipulations, (7.54) can be rewritten in the form

$$
4 \pi \int_{\mathbb{S}^{2}}\left(\left(\triangle_{\dot{q}} h\right)^{2}+2 h \triangle_{\dot{q}} h\right) \boldsymbol{\eta}_{\dot{q}} \geq 4 \pi \int_{\mathbb{S}^{2}} u^{2} \boldsymbol{\eta}_{\dot{q}}-\left(\int_{\mathbb{S}^{2}} u \boldsymbol{\eta}_{\dot{q}}\right)^{2} .
$$

This is precisely the sufficient condition for the shell Penrose inequality in Minkowski obtained in Chapter 4. This is not surprising since the method in Chapter 4 also involved a monotonicity condition for $D(\lambda)=\sqrt{\frac{\left|S_{\lambda}\right|}{16 \pi}}-\frac{1}{2} \lambda$. However, the general framework developed here leads to the result in a much more efficient way. In fact, there is an even more direct way of reaching this conclusion as a consequence of Proposition 7.4.1, or rather of its rewriting in Remark 7.4.2. Indeed, from (7.47) and (7.53),

$$
\begin{aligned}
& \boldsymbol{\eta}_{S_{\lambda}}=\operatorname{det}\left(\left(\boldsymbol{K}_{0}^{k}\right)^{-1}-\lambda \boldsymbol{I} \boldsymbol{d}\right) \boldsymbol{\eta}_{\dot{q}}=\left(\lambda^{2}+\theta_{k}^{(1)} \lambda+\operatorname{det}\left(\left(\boldsymbol{K}_{0}^{k}\right)^{-1}\right)\right) \boldsymbol{\eta}_{\dot{q}} \quad \Longrightarrow \\
& \left|S_{\lambda}\right|=4 \pi \lambda^{2}+\left(\int_{\mathbb{S}^{2}} \theta_{k}^{(1)} \boldsymbol{\eta}_{\mathfrak{q}}\right) \lambda+\int_{\mathbb{S}^{2}} \operatorname{det}\left(\left(\boldsymbol{K}_{0}^{k}\right)^{-1}\right) \boldsymbol{\eta}_{\mathfrak{q}},
\end{aligned}
$$




\subsection{Renormalized area method for the shell Penrose inequality in $\mathcal{M}^{1,3}$}

where in the second equality we used the first expression in (7.50). Comparing with (7.25) it follows that $\hat{\Theta}$ is Lie constant and takes the value $\hat{\Theta}=\left|S_{0}\right|$, so that the necessary condition (7.26) becomes precisely (7.54).

In Corollary 4.5.7 of Chapter 4, we saw that among all the solutions that the renormalized area method gave in Minkowski, we could find a relatively small two-parameter family of solutions of the form

$$
\tau_{0}=\alpha\left(\triangle_{q} h+2 h\right)-\beta
$$

with $\alpha \in[0,1], \beta>2 \frac{H\left(\widehat{S}_{0}\right)}{\operatorname{Scal}\left(\widehat{S}_{0}\right)}-\frac{1}{\max _{1 \leq A \leq n}\left\{\kappa_{A}\right\}}$, where $H\left(\widehat{S}_{0}\right)$ and Scal $\left(\widehat{S}_{0}\right)$ are, respectively, the mean curvature and scalar curvature of $\widehat{S}_{0}$ as a hypersurface of the Euclidean hyperplane $\left\{t=t_{0}\right\}$. Bergqvist proves the validity of the Penrose inequality when he uses flows that correspond in our case to GAB foliations, and that also tend to large spheres. As $\theta_{k}^{(1)}=u=2 \beta$, with constant $\beta>0$, this corresponds, after solving for $\tau_{0}$ to

$$
\tau_{0}=\frac{1}{2}\left(\triangle_{q} h+2 h\right)-\beta,
$$

which is clearly a subcase of the family of solutions defined by (7.56). This indicates that the family of solutions obtained when applying the Bergqvist method to the Minkowski spacetime is rather modest.

The following proposition summarizes the results for the shell Penrose inequality in Minkowski obtained so far and shows, in addition, that in the Minkowski case monotonicity of $D\left(S_{\lambda}, \ell\right)$ is in fact equivalent to the a priori more general conditions (17.22), or $F^{\prime}\left(S_{\lambda}\right) \geq 0$.

Proposition 7.7.1 (Equivalence of the monotonicity methods in $\mathcal{M}^{\mathbf{1 , 3}}$ ). Let $\Omega$ be a past asymptotically flat null hypersurface in $\mathcal{M}^{1,3}$ and $\left\{S_{\lambda}\right\}$ an affine foliation approaching large spheres. The following conditions are equivalent:
(i) $\left(\int_{\mathbb{S}^{2}} u \boldsymbol{\eta}_{\dot{q}}\right)^{2} \geq 16 \pi\left|S_{0}\right|$,
(ii) $\frac{d}{d \lambda} D\left(S_{\lambda}, \ell\right) \geq 0$ (Renormalized area method),
(iii) $\frac{d}{d \lambda}\left|S_{\lambda}\right| \geq \frac{16 \pi\left|S_{\lambda}\right|}{8 \pi \lambda+\int_{\mathbb{S}^{2}} u \boldsymbol{\eta}_{\dot{q}}}\left(F^{\prime}\left(S_{\lambda}\right) \geq 0\right.$ method $)$,
(iv) $D\left(S_{\lambda}, \ell\right) \leq \lim _{\lambda \rightarrow \infty} D\left(S_{\lambda}, \ell\right)$,

where $u=-\operatorname{tr}\left(\left(K_{0}^{k}\right)^{-1}\right)$. The shell Penrose inequality for $S_{0}$ holds if one (and hence any) of these conditions holds. 
Proof. The implications (ii) $\Longrightarrow($ iii) and (ii) $\Longrightarrow$ (iv) are generally true. The equivalence of $(i)$ and (ii) is a consequence of $G(\lambda)=F_{\infty}$ and (7.51), as discussed above. We have also seen before that (iii) is equivalent to $(i)$ as a consequence of Remark 7.4.2. It only remains to show that $(i v) \Longrightarrow(i i i)$. Expression (7.55) for the area $\left|S_{\lambda}\right|$ yields

$$
\begin{aligned}
\frac{d}{d \lambda}\left|S_{\lambda}\right| \geq \frac{16 \pi\left|S_{\lambda}\right|}{8 \pi \lambda+\int_{\mathbb{S}^{2}} \theta_{k}^{(1)} \boldsymbol{\eta}_{\mathfrak{q}}} & \Longleftrightarrow\left(8 \pi \lambda+\int_{\mathbb{S}^{2}} \theta_{k}^{(1)} \boldsymbol{\eta}_{\dot{q}}\right)^{2} \geq 16 \pi\left|S_{\lambda}\right| \\
& \Longleftrightarrow \sqrt{\frac{\left|S_{\lambda}\right|}{16 \pi}}-\frac{\lambda}{2} \leq \frac{1}{16 \pi} \int_{\mathbb{S}^{2}} \theta_{k}^{(1)} \boldsymbol{\eta}_{\dot{q}}
\end{aligned}
$$

which establishes (iv)

\subsection{GAB foliations in $\mathcal{M}^{1,3}$. Applications to the shell Penrose inequality}

In the previous section we studied the renormalized area method for the shell Penrose inequality in Minkowski. In this section we investigate in the same setting the consequences of the general Penrose-type inequality obtained in Theorem 7.3.8. To that aim we need information on the limit of the Hawking energy along GAB foliations. In Chapter 6 we have studied the limit of the Hawking energy at infinity for a large class of foliations $\left\{S_{\lambda}\right\}$ along asymptotically flat null hypersurfaces. The results we need from that chapter can be summarized as follows:

Let $\left\{S_{\lambda}\right\}$ be an affine background foliation approaching large spheres and define $\theta_{k}^{(1)}$, $\theta_{\ell}^{(1)}$ and $s_{\ell}^{(1)}$ as in Definition 6.3.4. Consider any other affine foliation $\left\{S_{\lambda^{\prime}}\right\}$ starting on the same cross-section $S_{0}$. The level-set functions $\lambda$ and $\lambda^{\prime}$ are necessarily related by $\lambda=f \lambda^{\prime}$, with $f>0$ and Lie constant on $\Omega$. Then the limit of the Hawking energy along $\left\{S_{\lambda^{\prime}}\right\}$ is (see Theorem 6.5.3 in Chapter 6)

$$
\begin{array}{r}
\lim _{\lambda^{\prime} \rightarrow \infty} m_{H}\left(S_{\lambda^{\prime}}\right)=\frac{1}{8 \pi \sqrt{16 \pi}}\left(\sqrt{\int_{\mathbb{S}^{2}} f^{2} \boldsymbol{\eta}_{\dot{q}}}\right) \int_{\mathbb{S}^{2}}\left(\triangle_{\dot{q}} \theta_{k}^{(1)}-\left(\theta_{k}^{(1)}+\theta_{\ell}^{(1)}\right)\right. \\
\left.-4 \operatorname{div}_{\dot{q}}\left(s_{\ell}^{(1)}\right)\right) \frac{1}{f} \boldsymbol{\eta}_{\dot{q}} .
\end{array}
$$

In order to apply this result in the Minkowski context, we need to compute $\theta_{k}^{(1)}, \theta_{\ell}^{(1)}$ and $s_{\ell}^{(1)}$ for the background foliation, which we fix exactly as in Chapter 4 as follows: choose a Minkowskian coordinate system $\left(t, x^{i}\right)$ and define the unit Killing $\xi=\partial_{t}$. The null generator $k$ of $\Omega$ is then uniquely selected by the condition $\langle k, \xi\rangle=-1$ and 


\subsection{GAB foliations in $\mathcal{M}^{1,3}$. Applications to the shell Penrose inequality}

$\left\{S_{\lambda}\right\}$ is defined to be the level-set foliation of $\lambda \in C^{\infty}(\Omega, \mathbb{R})$ defined by $\left.\lambda\right|_{S_{0}}=0$ and $k(\lambda)=-1$. It is immediate to check that $\left\{S_{\lambda}\right\}$ approaches large spheres.

The time-height function $\tau_{\lambda}$ of the level set $S_{\lambda}$ with respect to the hyperplane $\{t=0\}$ is defined to be

$$
\tau_{\lambda}:=\left.t\right|_{S_{\lambda}}
$$

For simplicity and without loss of generality we have chosen the hyperplane $\{t=0\}$, although any other $\Sigma_{t_{0}}$ would have been valid. In particular $\tau_{0}=\left.t\right|_{s_{0}}$ and, in fact, $\left.\tau_{\lambda}\right|_{p}=\left.\tau_{0}\right|_{\pi(p)}-\lambda$ as a consequence of our choice of normalization for $k$.

Lemma 7.8.1 (Asymptotic expansion at $\lambda=+\infty$ ). Let $\Omega$ be a past asymptotically flat null hypersurface in $\mathcal{M}^{1,3}$ and $\left\{S_{\lambda}\right\}$ an affine foliation associated to a choice of Minkowskian coordinate system $\left\{t, x^{i}\right\}$ as described above. Let $\ell$ be orthogonal to $\left\{S_{\lambda}\right\}$ and satisfying $\langle\ell, k\rangle=-2$. Then the following asymptotic expansions hold

$$
\begin{array}{rlrl}
\theta_{k} & =\frac{-2}{\lambda}+\frac{u}{\lambda^{2}}+o\left(\lambda^{-2}\right), & u=-\operatorname{tr}\left(\left(K_{0}^{k}\right)^{-1}\right) \\
\theta_{\ell} & =\frac{2}{\lambda}+\frac{-u+2 \triangle_{\tilde{q}} \tau_{0}}{\lambda^{2}}+o\left(\lambda^{-2}\right), & \tau_{0}:=\left.t\right|_{S_{0}} \\
s_{\ell A} & =\frac{-\stackrel{\nabla}{\nabla}_{A} \tau_{0}}{\lambda}+o\left(\lambda^{-1}\right), & &
\end{array}
$$

where $K_{0}^{k}$ is the second fundamental form of $S_{0}$ along $k$.

Proof. In the previous section we already proved (7.58). For $\theta_{\ell}$ we exploit the identity

$$
\theta_{\ell}+\left(1+|D \tau|_{\gamma}^{2}\right) \theta_{k}-2 \triangle_{\gamma} \tau=0,
$$

valid for any spacelike surface $S$ in Minkowski whenever $\tau:=\left.t\right|_{S}$. This identity is a simple consequence of the fact that $\xi$ is a covariantly constant vector field and it has been used several times in this thesis (see Chapter 3, Lemma 3.3.4 for a proof). We apply this identity to $S_{\lambda}$ and expand for large $\lambda$ up to order $\lambda^{-2}$. In particular, we can neglect all terms of order $O\left(\lambda^{-3}\right)$ or higher. Since $\tau_{\lambda}=\tau_{0}-\lambda$ and $\gamma_{S_{\lambda}}$ has the expansion (7.39), the gradient term is $|D \tau|_{\gamma}^{2}=\gamma_{S_{\lambda}}^{-1 A B} \tau_{0, A} \tau_{0, B}=O\left(\lambda^{-2}\right)$ and the term $|D \tau|_{\gamma}^{2} \theta_{k}$ is $O\left(\lambda^{-3}\right)$ so that it can be ignored. Concerning the Laplacian term, since $\Delta_{\gamma} \tau=\Delta_{\gamma} \tau_{0}$ we have, in local coordinates $\left\{\lambda, y^{A}\right\}$ adapted to the foliation $\left\{S_{\lambda}\right\}$ (i.e. such that $k=-\partial_{\lambda}$ )

$$
\begin{aligned}
& \triangle_{\gamma} \tau_{\lambda}=\frac{1}{\sqrt{\operatorname{det}(\gamma)}} \partial_{A}\left(\sqrt{\operatorname{det}(\gamma)}\left(\gamma^{-1}\right)^{A B} \partial_{B} \tau\right) \triangle_{\gamma} \tau_{0} \\
& =\frac{1}{\sqrt{\operatorname{det}(\dot{q})}} \partial_{A}\left(\sqrt{\operatorname{det}(\stackrel{q}{q})}\left(\stackrel{q}{q}^{-1}\right)^{A B} \tau_{0, B}\right) \frac{1}{\lambda^{2}}+O\left(\lambda^{-3}\right)=\left(\triangle_{\dot{q}} \tau_{0}\right) \frac{1}{\lambda^{2}}+O\left(\lambda^{-3}\right),
\end{aligned}
$$




\section{On the Penrose inequality along null hypersurfaces}

where we have used $\gamma(\lambda)=\stackrel{q}{ } \lambda^{2}+O(\lambda)$. Inserting $\theta_{\ell}=\frac{2}{\lambda}+\frac{\theta_{\ell}^{(1)}}{\lambda^{2}}+o\left(\lambda^{-2}\right)$ and (7.58) into (7.61) and keeping only the terms in $\lambda^{-2}$ we obtain $\theta_{\ell}^{(1)}+u-2 \triangle_{\mathfrak{q}} \tau_{0}=0$, which gives (7.59).

It only remains to compute $\boldsymbol{s}_{\boldsymbol{\ell}}^{(1)}$ in the expansion $\boldsymbol{s}_{\boldsymbol{\ell}}=\frac{\boldsymbol{s}_{\boldsymbol{\ell}}^{(1)}}{\lambda}+o\left(\lambda^{-1}\right)$. Using formula (4.48) in Chapter 4, the expansion (7.60) follows directly after taking into account (7.39) and (7.38).

Lemma 7.8.1 allows us to compute the limit of the Hawking energy along very general foliations by exploiting the results in Chapter 6 . For affine foliations $\lambda=f \lambda^{\prime}$ we simply need to evaluate (7.57), which becomes

$$
\lim _{\lambda^{\prime} \rightarrow \infty} m_{H}\left(S_{\lambda^{\prime}}\right)=\frac{1}{8 \pi \sqrt{16 \pi}}\left(\sqrt{\int_{\mathbb{S}^{2}} f^{2} \boldsymbol{\eta}_{\dot{q}}}\right) \int_{\mathbb{S}^{2}} \triangle_{\dot{q}}\left(u+2 \tau_{0}\right) \frac{1}{f} \boldsymbol{\eta}_{\dot{q}} .
$$

In particular, the GAB foliation associated to $S_{0}$ has rescaling function $f:=\frac{\theta_{k}^{(1)}}{c}=$ $\frac{u}{c}, \quad c>0$ so that, along this GAB foliation,

$$
\lim _{\lambda^{\prime} \rightarrow \infty} m_{H}\left(S_{\lambda^{\prime}}\right)=\frac{1}{8 \pi \sqrt{16 \pi}}\left(\sqrt{\int_{\mathbb{S}^{2}} u^{2} \boldsymbol{\eta}_{\dot{q}}}\right) \int_{\mathbb{S}^{2}} \triangle_{\dot{q}}\left(u+2 \tau_{0}\right) \frac{1}{u} \boldsymbol{\eta}_{\dot{q}} .
$$

Thus, the particularization of Theorem 7.3 .8 to the Minkowski setting reads

Theorem 7.8.2. Let $\Omega$ be a past asymptotically flat null hypersurface in $\mathcal{M}^{1,3}$ and $S_{0}$ a spacelike cross section of $\Omega$. Then the following inequality holds:

$$
\sqrt{\frac{\left|S_{0}\right|}{16 \pi}} \leq \frac{1}{16 \pi} \int_{S_{0}} \theta_{\ell^{\star}} \boldsymbol{\eta}_{S_{0}}+\frac{1}{8 \pi \sqrt{16 \pi}}\left(\sqrt{\int_{\mathbb{S}^{2}} u^{2} \boldsymbol{\eta}_{\dot{q}}}\right) \int_{\mathbb{S}^{2}} \triangle_{\dot{q}}\left(u+2 \tau_{0}\right) \frac{1}{u} \boldsymbol{\eta}_{\dot{q}},
$$

where $u=-\operatorname{tr}\left(\left(K_{0}^{k}\right)^{-1}\right), \tau_{0}=\left.t\right|_{s_{0}}$ with $t$ a Minkowskian time coordinate. The round asymptotic metric $q$ is defined by (7.52) and $\{k, \ell\}$ are the future directed null normals to $S_{0}$ with $k$ tangent to $\Omega$ and satisfying $k(t)=1$ and $\langle k, \ell\rangle=-2$.

We can now rewrite the first term of the right-hand side of the inequality (7.62) in terms of $\theta_{\ell}$. To do so we need to relate the vector $\ell^{\star}=R_{\hat{q}} \ell^{\prime}$ with vector $\ell$, where $\ell^{\prime}$ is the transverse vector to $\Omega$ orthogonal to each $S_{\lambda^{\prime}}$ and satisfying $\left\langle k^{\prime}, \ell^{\prime}\right\rangle=-2$. Since $k^{\prime}=\frac{u}{c} k$, then $\ell^{\prime}=\frac{c}{u} \ell$ necessarily. Using now that $\lambda=\frac{u}{c} \lambda^{\prime}$, the expansion of the first fundamental form of $\Omega$ can be expressed as

$$
\gamma=\stackrel{q}{\lambda^{2}}+o\left(\lambda^{2}\right)=\hat{q}\left(\lambda^{\prime}\right)^{2}+o\left(\left(\lambda^{\prime}\right)^{2}\right)=\hat{q} \frac{c^{2}}{u^{2}} \lambda^{2}+o\left(\lambda^{2}\right),
$$


which shows that

$$
\hat{q}=\frac{u^{2}}{c^{2}} \stackrel{\circ}{q}
$$

and therefore $\boldsymbol{\eta}_{\hat{q}}=\frac{u^{2}}{c^{2}} \boldsymbol{\eta}_{\tilde{q}}$. This implies

$$
R_{\hat{q}}=\sqrt{\frac{1}{4 \pi} \int_{\mathbb{S}^{2}} \frac{u^{2}}{c^{2}} \boldsymbol{\eta}_{\dot{q}}}
$$

We finally conclude

$$
\ell^{\star}=R_{\hat{q}} \ell^{\prime}=R_{\hat{q}} \frac{c}{u} \ell=\frac{c}{u} \sqrt{\frac{1}{4 \pi} \int_{\mathbb{S}^{2}} \frac{u^{2}}{c^{2}} \boldsymbol{\eta}_{\dot{q}} \ell}=\left(\sqrt{\frac{1}{4 \pi u^{2}} \int_{\mathbb{S}^{2}} u^{2}}\right) \ell,
$$

and using in (7.62) that $\left.\theta_{F \ell}\right|_{s_{0}}=\left.F \theta_{\ell}\right|_{s_{0}}$ for any $F: S_{0} \rightarrow \mathbb{R}$, we obtain

$$
\begin{aligned}
\sqrt{\frac{\left|S_{0}\right|}{16 \pi} \leq} & \frac{1}{16 \pi} \int_{S_{0}}\left(\sqrt{\frac{1}{4 \pi u^{2}} \int_{\mathbb{S}^{2}} u^{2}}\right) \theta_{\ell} \boldsymbol{\eta}_{\boldsymbol{S}_{0}} \\
& +\frac{1}{8 \pi \sqrt{16 \pi}}\left(\sqrt{\int_{\mathbb{S}^{2}} u^{2} \boldsymbol{\eta}_{\dot{q}}}\right) \int_{\mathbb{S}^{2}} \triangle_{\dot{q}}\left(u+2 \tau_{0}\right) \frac{1}{u} \boldsymbol{\eta}_{\dot{q}}
\end{aligned}
$$

which, after factorizing the right-hand side, can be rewritten as

$$
\sqrt{\frac{\left|S_{0}\right|}{16 \pi}} \leq \frac{1}{32 \pi \sqrt{\pi}}\left(\sqrt{\int_{\mathbb{S}^{2}} u^{2} \boldsymbol{\eta}_{\tilde{q}}}\right)\left(\int_{S_{0}} \frac{1}{u} \theta_{\ell} \boldsymbol{\eta}_{S_{0}}+\int_{\mathbb{S}^{2}} \triangle_{\dot{q}}\left(u+2 \tau_{0}\right) \frac{1}{u} \boldsymbol{\eta}_{\dot{q}}\right),
$$

where $u=-\operatorname{tr}\left(\left(K_{0}^{k}\right)^{-1}\right)=\triangle_{q} h+2\left(h-\tau_{0}\right)$ (note that we write $u$ in two different ways: in terms of the geometry of the surface $S_{0}$ for the first term of the right-hand side, and in terms of the spherical geometry for the second term).

\subsection{An upper bound for the area of $S_{\lambda}$ along $\Omega$}

We close the chapter returning to the general setup of asymptotically flat null hypersurfaces in spacetimes satisfying the dominant energy condition. We also return to affine foliations not necessarily approaching large spheres. In this section we provide a general upper bound for the area $\left|S_{\lambda}\right|$ in terms of asymptotic quantities intrinsic to $\Omega$. We find an inequality which is weaker than the inequality $D\left(S_{\lambda}, \ell^{\star}\right) \leq \lim _{\lambda \rightarrow \infty} D\left(S_{\lambda}, \ell^{\star}\right)$, the difference between both being a Hölder inequality term.

The general idea behind the inequality in the present section is the observation that one possible method to approach the condition $D\left(S_{\lambda}, \ell^{\star}\right) \leq \lim _{\lambda \rightarrow \infty} D\left(S_{\lambda}, \ell^{\star}\right)$ it to obtain 


\section{On the Penrose inequality along null hypersurfaces}

an interpolating function $P(\lambda)$ satisfying $D\left(S_{\lambda}, \ell^{\star}\right) \leq P(\lambda) \leq \lim _{\lambda \rightarrow \infty} D\left(S_{\lambda}, \ell^{\star}\right)$. While this is hard (as finding such a $P(\lambda)$ would prove the Penrose inequality), we have been able to find a $P(\lambda)$ satisfying only the first inequality $D\left(S_{\lambda}, \ell^{\star}\right) \leq P(\lambda)$, from which a general inequality bounding $\left|S_{0}\right|$ from above in terms of asymptotic quantities follows.

Proposition 7.9.1. Let $\Omega$ be a past asymptotically flat null hypersurface embedded in a spacetime that satisfies the dominant energy condition, $S_{0}$ a cross section and $\left\{S_{\lambda}\right\}$ an affine foliation starting at $S_{0}$. Let $\theta_{k}^{(1)}$ be the asymptotic coefficient defined in (7.6) and $\hat{q}$ the asymptotic metric associated to $\left\{S_{\lambda}\right\}$. Then,

$$
\left|S_{\lambda}\right| \leq \frac{1}{4} \int_{\hat{S}}\left(\theta_{k}^{(1)}+2 \lambda\right)^{2} \boldsymbol{\eta}_{\hat{\boldsymbol{q}}}
$$

and in particular $\left|S_{0}\right| \leq \frac{1}{4} \int_{\hat{S}}\left(\theta_{k}^{(1)}\right)^{2} \boldsymbol{\eta}_{\hat{\boldsymbol{q}}}$.

Proof. Let us fix any $\lambda_{0}>0$ and consider the volume form on $S_{\lambda}(\lambda \geq 0)$ defined by

$$
\hat{\boldsymbol{\eta}}_{S_{\lambda}}:=\frac{1}{\left(\lambda+\lambda_{0}\right)^{2}} \boldsymbol{\eta}_{S_{\lambda}}
$$

Using the evolution equation $£_{-k} \boldsymbol{\eta}_{S_{\lambda}}=-\theta_{k} \boldsymbol{\eta}_{S_{\lambda}}$, the Lie derivative of $\hat{\boldsymbol{\eta}}_{S_{\lambda}}$ is

$$
£_{-k} \hat{\boldsymbol{\eta}}_{S_{\lambda}}=-\left(\theta_{k}+\frac{2}{\lambda+\lambda_{0}}\right) \hat{\boldsymbol{\eta}}_{S_{\lambda}} .
$$

Writing $\hat{\boldsymbol{\eta}}_{S_{\lambda}}=\hat{f}(\lambda) \boldsymbol{\eta}_{\hat{\boldsymbol{q}}}$, (7.64) becomes a differential equation for $\hat{f}$, which can be integrated as

$$
\hat{f}(\lambda)=\hat{f}(0) e^{-\int_{0}^{\lambda}\left(\theta_{k}+\frac{2}{s+\lambda_{0}}\right) d s} .
$$

The initial value $\hat{f}(0)$ can be computed "at infinity" as a consequence of $\hat{\boldsymbol{\eta}}_{S_{\lambda}} \longrightarrow \boldsymbol{\eta}_{\hat{q}}$ when $\lambda \rightarrow \infty$. Thus $\hat{f}(0)=e^{\int_{0}^{\infty}\left(\theta_{k}+\frac{2}{s+\lambda_{0}}\right) d s}$ and therefore

$$
\hat{f}(\lambda)=e^{\int_{\lambda}^{\infty}\left(\theta_{k}+\frac{2}{s+\lambda_{0}}\right) d s} .
$$

We aim at finding an upper bound for $\hat{f}(\lambda)$. We use inequality (7.16), which implies $\theta_{k}+\frac{2}{\lambda+\lambda_{0}} \leq \frac{-4}{\theta_{k}^{(1)}+2 \lambda}+\frac{2}{\lambda+\lambda_{0}}$ and then

$$
\int_{\lambda}^{\infty}\left(\theta_{k}+\frac{2}{s+\lambda_{0}}\right) d s \leq \int_{\lambda}^{\infty}\left(\frac{-4}{2 s+\theta_{k}^{(1)}}+\frac{2}{s+\lambda_{0}}\right) d s=\log \left(\frac{2 \lambda+\theta_{k}^{(1)}}{2\left(\lambda+\lambda_{0}\right)}\right)^{2} .
$$

Finally,

$$
\begin{aligned}
& \left|S_{\lambda}\right|=\int_{S_{\lambda}} \boldsymbol{\eta}_{S_{\lambda}}=\int_{\hat{S}}\left(\lambda+\lambda_{0}\right)^{2} \hat{f}(\lambda) \boldsymbol{\eta}_{\hat{\boldsymbol{q}}}=\int_{\hat{S}}\left(\lambda+\lambda_{0}\right)^{2} e^{\int_{\lambda}^{\infty}\left(\theta_{k}+\frac{2}{s+\lambda_{0}}\right) d s} \boldsymbol{\eta}_{\hat{\boldsymbol{q}}} \\
& \leq\left(\lambda+\lambda_{0}\right)^{2} \int_{\hat{S}} e^{\log \left(\frac{2 \lambda+\theta_{k}^{(1)}}{2\left(\lambda+\lambda_{0}\right)}\right)^{2}} \boldsymbol{\eta}_{\hat{\boldsymbol{q}}}=\frac{1}{4} \int_{\hat{S}}\left(\theta_{k}^{(1)}+2 \lambda\right)^{2} \boldsymbol{\eta}_{\hat{\boldsymbol{q}}} .
\end{aligned}
$$


Remark 7.9.2. The condition $D\left(S_{\lambda}, \ell^{\star}\right) \leq \lim _{\lambda \rightarrow \infty} D\left(S_{\lambda}, \ell^{\star}\right)$, namely

$$
\sqrt{\frac{\left|S_{\lambda}\right|}{16 \pi}}-\frac{R_{\hat{q}}}{2} \lambda \leq \frac{1}{16 \pi R_{\hat{q}}} \int_{\hat{S}} \theta_{k}^{(1)} \boldsymbol{\eta}_{\hat{q}}
$$

is equivalent to

$$
\left|S_{\lambda}\right| \leq \frac{1}{16 \pi R_{\hat{q}}^{2}}\left(\int_{\hat{S}}\left(\theta_{k}^{(1)}+2 \lambda\right) \boldsymbol{\eta}_{\hat{q}}\right)^{2} .
$$

As mentioned above, this inequality is stronger than (7.63), the difference being a Hölder inequality term. Indeed, a direct application of the Hölder inequality yields

$$
\left|S_{\lambda}\right| \leq \frac{1}{16 \pi R_{\hat{q}}^{2}}\left(\int_{\hat{S}}\left(\theta_{k}^{(1)}+2 \lambda\right) \boldsymbol{\eta}_{\hat{q}}\right)^{2} \leq \frac{1}{4} \int_{\hat{S}}\left(\theta_{k}^{(1)}+2 \lambda\right)^{2} \boldsymbol{\eta}_{\hat{\boldsymbol{q}}} .
$$




\section{Conclusions}

In this thesis we have addressed the null Penrose inequality conjecture and we have obtained several results that support both the validity of the general version of the inequality and its respective shell version. Projections in static spacetimes, graphs over hypersurfaces in the euclidean space and flows of surfaces along null hypersurfaces have been the main tools that we have used to address the problem. The following list summarizes the main results of this thesis.

1. Given a spacelike surface $S$ embedded in a static spacetime, we have obtained the expressions for its intrinsic and extrinsic geometry in terms of the geometry of its projection along the Killing direction onto a constant time hyperplane. We have also computed the analogous expressions when the projection is performed in a purely Riemannian context.

2. We have analyzed in full detail the gap in Gibbons' attempt [37] to prove the shell Penrose inequality in the Minkowski spacetime by using the projection along the Killing direction, and we have computed the correct expression for the total outer null expansion $\int_{S} \theta_{\ell} \boldsymbol{\eta}_{S}$ in terms of the geometry of the projected surface $\bar{S}$. The correct expression for the mean curvature $\bar{H}$ of $\bar{S}$ involving the null expansions of the original surface $S$ is also obtained.

3. We have proved the validity of the shell Penrose inequality in Minkowski for convex surfaces embedded in a constant time hyperplane. This result was obtained simultaneously and independently by Brendle and Wang [15].

4. We have obtained an expression for the shell Penrose inequality in the Minkowski spacetime of any dimension in terms of the so called time-height function $\tau$, which describes how far the initial surface $S$ lies from the constant time hyperplane, and in terms of the euclidean geometry of the surface obtained by intersecting the past null cone $\Omega$ of the initial surface $S$ with a constant time hyperplane. This has 
been possible by formulating and solving the equations for the quotient second fundamental form (Ricatti equation) and the metric of $\Omega$ in Minkowski, which allow us to rewrite the geometry of the initial surface $S$ in terms of its projection along its past null cone.

5. With the previous result at hand, and since the geometry of convex bodies is fully determined by the so-called support function $h$ associated to the projection of $S$ along its past null cone, we have obtained an expression for the shell Penrose inequality in Minkowski in terms of $h$ and the time height function $\tau$ of $S$. Using this form for the shell Penrose inequality, we have proved its validity in the particular case that the surfaces $S$ lies in the past null cone of a point in the arbitrary dimensional Minkowski spacetime.

6. The expression for the shell Penrose inequality in terms of the support function involves the inverse of endomorphisms represented by matrices of size $n \times n$. In the particular case $n=2$ (spacetime dimension four), we have computed explicitly this inverse and have obtained an explicit expression for the shell inequality in terms of the support function and the time height function.

7. In an euclidean context, and given an orientable hypersurface $\widehat{S}_{0}$, we have studied the geometry of a graph over $\widehat{S}_{0}$, and we have obtained the expressions that relate its first and second fundamental forms of the graph with those of the base and the graph function. This result has allowed us to relate the two projections of $S$ in the Minkowski spacetime: the projection in the Killing direction $\bar{S}$ and the projection $\widehat{S}_{0}$ along the past null cone $\Omega$ onto a constant time hyperplane. This has been applied to rewrite the convexity condition on $\bar{S}$ in terms of the geometry of the convex surface $\widehat{S}_{0}$ and the time-height function $\tau$.

8. In a general spacetime that admits a null hypersurface $\Omega$, we have obtained the expressions for the metric, extrinsic null curvatures and connection one-form of any cross section of $\Omega$ in terms of geometric elements defined on the leaves of a background foliation associated to a given future null vector field $k$ tangent to $\Omega$.

9. We have obtained a formula that gives the limit of the Hawking mass along flows of general character in a past asymptotically flat null hypersurface $\Omega$ in terms of the geometry of an affinely parametrized background foliation that tends to large spheres. The expression of the Bondi four momentum energy vector $P_{B}$ associated to the background geometry has also been computed in terms of the background geometry.

10. We have introduced the notion of GAB foliations along a null hypersurface $\Omega$ in an AF spacetime satisfying the dominant energy condition, and we have obtained a Penrose-type inequality for any cross section $S$ of $\Omega$ which involves the limit of the Hawking energy along such foliation. In the particular case when $S$ is WOTS, the 


\section{Conclusions}

inequality takes the same form as the null Penrose inequality but with the Bondi energy replaced by the limit of the Hawking energy along the GAB foliation. In addition, if the GAB foliation tends to large spheres, our inequality becomes the standard null Penrose inequality.

11. Combining the previous result with the expression for the limit of the Hawking energy along general flows on a null hypersurface $\Omega$, we have proved the validity of a shell Penrose type inequality in the four dimensional Minkowski spacetime.

12. Finally, we have developed a method to address the null Penrose inequality in AF spacetimes satisfying the dominant energy condition, and which is called renormalized area method. We have found two sufficient conditions for the method to apply. In the particular case of the four dimensional Minkowski spacetime, this method proves the validity of the shell Penrose inequality for the same class of surfaces as in Chapter 4, which were obtained by exploiting properties of the Minkowski spacetime. Thus the renormalized area method can be considered a generalization of the approach in Chapter 4 to general spacetimes satisfying the dominant energy condition.

The results in this thesis represent a substantial step forward in our understanding of the null Penrose inequality. In fact, some of our results (definition of asymptotically flat null hypersurface and general limit of the Hawking energy) have played a central role in another very recent approach to the null Penrose inequality put forward by $\mathrm{H}$. Roesch [92]. 



\section{Geometry of non-degenerate submanifolds}

In this appendix we compile elementary results on the relation between the geometry of an ambient manifold and an embedded submanifold. Specifically, the relation between the connections, the second fundamental forms, the Hessians and the curvature is given explicitly.

\section{A.1 Induced connection and second fundamental form}

Let $M$ and $N$ be pseudo-Riemannian manifolds of dimension $m$ and $n$ respectively, with $m \geq n$. A differentiable map $\phi: N \rightarrow M$ is called an immersion, if for any $p \in N$,

$$
d \phi: T_{p} N \longrightarrow T_{\phi(p)} M
$$

is injective. If an immersion $\phi: N \rightarrow M$ maps $N$ homeomorphically onto its image $\phi(N)$ in $M, \phi$ is called differentiable embedding. Any immersion is locally a differentiable embedding.

Let $N$ be an $n$-dimensional submanifold embedded in $(M, g)$. The pullback of the metric $g$ on $N$ gives a symmetric tensor $\gamma$ called first fundamental form, that depending on the nature of $N$, can be degenerate or not. When the pullback is positive definite, we will say that $N$ is spacelike. A submanifold will be called degenerate (respectively non-degenerate) provided the first fundamental form has this property.

When $N$ is non degenerate, we can define its Levi-Civita connection. The connection of $M$ (the ambient connection) will be denoted by $\nabla$ and the connection on $N$ (induced connection) by $\nabla^{N}$. For any point $p \in N$, consider the tangent space $T_{p} N$ and choose an orthonormal basis of vectors $B_{N}=\left\{e_{1}, \ldots, e_{n}\right\}$. Since $T_{p} N \subset T_{p} M$, let us extend $B_{N}$ to a basis $B=\left\{e_{1}, \ldots, e_{n}, u_{n+1}, \ldots, u_{m}\right\}$ of $T_{p} M$. By using the Gram-Schmidt orthogonalization method we obtain an orthonormal basis $B^{\prime}=\left\{e_{1}, \ldots, e_{n}, e_{n+1}, \ldots, e_{m}\right\}$ 


\section{A.1. Induced connection and second fundamental form}

of $T_{p} M$, where

$$
\bar{B}^{\prime}=\left\{e_{n+1}, \ldots, e_{m}\right\} \subset\left(T_{p} N\right)^{\perp}=\left\{v \in T_{p} M /\langle v, x\rangle_{g}=0 \forall x \in T_{p} N\right\} .
$$

We have that $T_{p} N \cap\left(T_{p} N\right)^{\perp}=\emptyset$, since otherwise there would be a degenerate vector for $N$, against hypothesis. Since the set of vectors of $B_{N}$ and $\bar{B}^{\prime}$ form a basis of $T_{p} M$, we have that

$$
T_{p} M=T_{p} N \oplus\left(T_{p} N\right)^{\perp} .
$$

According to (A.1), we can write the orthogonal decomposition

$$
\nabla_{X} Y=\left(\nabla_{X} Y\right)^{\top}+\left(\nabla_{X} Y\right)^{\perp}
$$

where the operators ' $T$ ' and ' $\perp$ ' give the tangent part and orthogonal part to $N$ of any vector respectively.

The following proposition shows the relation between the ambient and the induced connection.

Proposition A.1.1 (Induced connection). Let $N$ be a non-degenerate $n$-dimensional submanifold embedded in a pseudo-Riemannian m-dimensional manifold $(M, g)$. Then

$$
\nabla_{X}^{N} Y=\left(\nabla_{X} Y\right)^{\top}, \quad \text { with } \quad X, Y \in \mathfrak{X}(N) \text {, }
$$

where $\mathfrak{X}(N)$ is the set of vector fields tangent to $N$, and $T: T_{p} M \rightarrow T_{p} N$ with $p \in N$ is the orthogonal projection.

Proof. Let us define the mapping $\bar{\nabla}: \mathfrak{X}(M) \times \mathfrak{X}(M) \rightarrow \mathfrak{X}(M)$, by

$$
\bar{\nabla}(X, Y):=\bar{\nabla}_{X} Y=\left(\nabla_{X} Y\right)^{\top} .
$$

It is immediate to see that $\bar{\nabla}$ satisfies

(i) $\bar{\nabla}_{f X+g Y} Z=f \bar{\nabla}_{X} Z+g \bar{\nabla}_{Y} Z$,

(ii) $\bar{\nabla}_{X}(Y+Z)=\bar{\nabla}_{X} Y+\bar{\nabla}_{X} Z$,

(iii) $\bar{\nabla}_{X}(f Y)=X(f) Y+f \bar{\nabla}_{X} Y$,

where $X, Y, Z \in \mathfrak{X}(N)$, and $f, g: N \rightarrow \mathbb{R}$ are $C^{\infty}$. Hence $\bar{\nabla}$ is an affine connection. To see that $\bar{\nabla}$ is the Levi-Civita connection of $N$, we check that it is torsion-free $\left(T_{\bar{\nabla}}(X, Y)=\bar{\nabla}_{X} Y-\bar{\nabla}_{Y} X-[X, Y]=0\right)$ and metric $(\bar{\nabla} \gamma=0)$. For the first, recall that the ambient connection $\nabla$ is torsion free

$$
T_{\nabla}(X, Y)=\nabla_{X} Y-\nabla_{Y} X-[X, Y]=0
$$




\section{A. Geometry of non-degenerate submanifolds}

Applying the operator ' $T$ ' and using $[X, Y]^{\top}=[X, Y]$ the torsion-free property of $\bar{\nabla}$ follows immediately. For the second, we compute, for $X, Y, Z \in \mathfrak{X}(N)$,

$$
\begin{aligned}
\left(\bar{\nabla}_{Z} \gamma\right)(X, Y) & =\bar{\nabla}_{Z}(\gamma(X, Y))-\gamma\left(\bar{\nabla}_{Z} X, Y\right)-\gamma\left(X, \bar{\nabla}_{Z} Y\right) \\
& =Z(\gamma(X, Y))-\gamma\left(\left(\nabla_{Z} X\right)^{\top}, Y\right)-\gamma\left(X,\left(\nabla_{Z} Y\right)^{\top}\right) \\
& =\nabla_{Z}(g(X, Y))-g\left(\nabla_{Z} X, Y\right)-g\left(X, \nabla_{Z} Y\right)=\left(\nabla_{Z} g\right)(X, Y)=0,
\end{aligned}
$$

where in the last inequality we have used that $\nabla$ is metric. Since the Levi-Civita connection is the unique torsion-free and metric connection, it must be $\bar{\nabla}=\nabla^{N}$.

From (A.3), (A.2) becomes

$$
\nabla_{X} Y=\nabla_{X}^{N} Y+\left(\nabla_{X} Y\right)^{\perp}
$$

The second term of the right-hand side of (A.4) defines the so called second fundamental form vector, namely

Definition A.1.2 (Second fundamental form vector). The second fundamental form vector of $N$ as an embedded manifold in $M$ is

$$
\vec{K}(X, Y)=-\left(\nabla_{X} Y\right)^{\perp} .
$$

The second fundamental form vector is symmetric because

$$
\vec{K}(X, Y)=-\left(\nabla_{X} Y\right)^{\perp}=-\left(\nabla_{Y} X+[X, Y]\right)^{\perp}=-\left(\nabla_{Y} X\right)^{\perp}=\vec{K}(Y, X),
$$

where we have used that ' $\perp$ ' is linear and $[X, Y] \in \Gamma(T N)$, since $X, Y \in \Gamma(T N)$.

It is convenient to have the relation among several second fundamental forms when we have more than two manifolds, and successive embeddings:

Proposition A.1.3 (Successive embeddings). Let $S, \Sigma, M$ be three pseudo-Riemannian manifolds, with $S$ embedded in $\Sigma$ and $\Sigma$ embedded in $M$. Let $g$ be the metric on $M$. Assume that the metrics on $S$ and $\Sigma$ are induced by $g$. Let $\vec{K}_{S \rightarrow \Sigma}$ be the second fundamental form vector of $S$ as a submanifold embedded in $\Sigma, \vec{K}_{\Sigma \rightarrow M}$ the second fundamental form vector of $\Sigma$ as a submanifold in $M$, and $\vec{K}_{S \rightarrow M}$ the second fundamental form vector of $S$ as a submanifold in $M$. Then

$$
\vec{K}_{S \rightarrow M}(X, Y)=\vec{K}_{S \rightarrow \Sigma}(X, Y)+\vec{K}_{\Sigma \rightarrow M}(X, Y) \text { with } X, Y \in \Gamma(T S) \text {. }
$$

Proof. Given $X, Y \in \Gamma(T S)$, and by the definition of second fundamental form, we have

$$
\begin{array}{cc}
S \hookrightarrow M: & \nabla_{X}^{M} Y=\nabla_{X}^{S} Y-\vec{K}_{S \rightarrow M}(X, Y), \\
S \hookrightarrow \Sigma: & \nabla_{X}^{\Sigma} Y=\nabla_{X}^{S} Y-\vec{K}_{S \rightarrow \Sigma}(X, Y), \\
\Sigma \hookrightarrow M: & \nabla_{X}^{M} Y=\nabla_{X}^{\Sigma} Y-\vec{K}_{\Sigma \rightarrow M}(X, Y) .
\end{array}
$$

Computing (A.7) $-(\mathrm{A} .8)-(\mathrm{A} .9)$, we obtain $-\nabla_{X}^{\Sigma} Y=-\nabla_{X}^{\Sigma} Y-\vec{K}_{S \rightarrow M}(X, Y)+$ $\vec{K}_{S \rightarrow \Sigma}(X, Y)+\vec{K}_{\Sigma \rightarrow M}(X, Y)$, which is (A.6) after rearranging. 
Remark A.1.4. If the dimension of the embedded manifold is the same as the dimension of the ambient manifold, then the second fundamental form vector is by definition identically zero.

Remark A.1.5. The second fundamental form vector of $S$ as a submanifold of $\sum$ satisfies $\vec{K}_{S \rightarrow \Sigma} \in T_{p} \Sigma \cap\left(T_{p} S\right)^{\perp}$, and on the other hand $\vec{K}_{\Sigma \rightarrow M} \in\left(T_{p} \Sigma\right)^{\perp}$. The vector space tangent to $M$ splits in the direct sum $T_{p} M=T_{p} S \oplus\left(T_{p} S\right)^{\perp}$. Likewise we split $\left(T_{p} S\right)^{\perp}=$ $\left[T_{p} \Sigma \cap\left(T_{p} S\right)^{\perp}\right] \oplus\left(T_{p} \Sigma\right)^{\perp}$. For the sake of simplicity let us call $\left(T_{p}^{\Sigma} S\right)^{\perp}=\left[T_{p} \Sigma \cap\left(T_{p} S\right)^{\perp}\right]$, so that we have

$$
\left(T_{p} S\right)^{\perp}=\left(T_{p}^{\Sigma} S\right)^{\perp} \oplus\left(T_{p} \Sigma\right)^{\perp} .
$$

Relation (A.6) corresponds in fact to the decomposition associated to this direct sum.

\section{A.2 Induced Hessian}

We want to find relations between objects defined on the ambient manifold and the respective induced ones on the embedded submanifold. Let us compute the explicit relation between the Hessian of any function defined on the ambient manifold and the Hessian of the function obtained by restricting the original one to the embedded manifold:

Proposition A.2.1 (Hessian relation in an embedding). Let $N$ be an $n$-dimensional submanifold embedded in a pseudo-Riemannian m-dimensional manifold $M$ so that the pullback of the metric on $N$ is non-degenerate. Let $\phi$ be the embedding. Let $f: M \longrightarrow$ $\mathbb{R}$ a smooth function defined on $M$. If we denote by Hess $\nabla$ the Hessian of the ambient manifold $M$ and Hess $_{\nabla^{N}}$ the induced one on the submanifold $N$, we have

$$
\operatorname{Hess}_{\nabla} f(d \phi(X), d \phi(Y))=\operatorname{Hess}_{\nabla^{N}}(f \circ \phi)(X, Y)+\langle\operatorname{gradf}, \vec{K}(X, Y)\rangle,
$$

where $X, Y \in \Gamma(T N)$.

Proof. By definition, the Hessian is

$$
\operatorname{Hess} f(X, Y)=X(Y(f))-d f\left(\nabla_{X} Y\right) \text {. }
$$

In $M$ and $N$ we have the following relations

$$
\begin{aligned}
& \operatorname{Hess}_{\nabla} f(d \phi(X), d \phi(Y))=d \phi(X)(d \phi(Y)(f))-d f\left(\nabla_{d \phi(X)} d \phi(Y)\right), \\
& \operatorname{Hess}_{\nabla^{N}}(f \circ \phi)(X, Y)=X(Y(f \circ \phi))-d(f \circ \phi)\left(\nabla_{X}^{N} Y\right) .
\end{aligned}
$$

In (A.4) and (A.5) we identified the vectors tangent to the abstract submanifold with the tangent vectors to the embedded one. Adding explicitly the embedding $\phi$, the Gauss formula reads

$$
\nabla_{d \phi(X)} d \phi(Y)=d \phi\left(\nabla_{X}^{N} Y\right)-\vec{K}(X, Y)
$$




\section{A. Geometry of non-degenerate submanifolds}

Therefore it follows

$d f\left(\nabla_{d \phi(X)} d \phi(Y)\right)=d f\left(d \phi\left(\nabla_{X}^{N} Y\right)-\vec{K}(X, Y)\right)=d(f \circ \phi)\left(\nabla_{X}^{N} Y\right)-\langle\operatorname{grad} f, \vec{K}(X, Y)\rangle$.

Inserting (A.13) into (A.10) we have

$\operatorname{Hess}_{\nabla} f(d \phi(X), d \phi(Y))=d \phi(X)(d \phi(Y)(f))-d(f \circ \phi)\left(\nabla_{X}^{N} Y\right)+\langle\operatorname{grad} f, \vec{K}(X, Y)\rangle$.

Since $\left.X(f \circ \phi)\right|_{p}=\left.\phi_{*}(X) f\right|_{\phi(p)}$, we finally obtain

$$
d \phi(X)(d \phi(Y)(f))=X(Y(f \circ \phi)) .
$$

The two first terms of the right-hand side of (A.14) are precisely (A.11), which proves the result.

\section{A.3 Gauss, Ricci and Codazzi identities}

The following aim is to relate the curvature of the ambient manifold $M$ and the embedded manifold $N$. In our notation the curvature tensor is defined as

$$
R(X, Y) Z=\nabla_{X} \nabla_{Y} Z-\nabla_{Y} \nabla_{X} Z-\nabla_{[X, Y]} Z, \quad X, Y, Z \in \Gamma(T N)
$$

Theorem A.3.1 (Gauss equation). Let $N$ be an n-dimensional non-degenerate submanifold in a pseudo-Riemannian m-dimensional manifold $(M, g)$. Let $R$ be the curvature tensor of $M$ and $R^{N}$ the curvature tensor of $N$. Then

$$
\langle R(X, Y) Z, T\rangle=\left\langle R^{N}(X, Y) Z, T\right\rangle-\langle\vec{K}(Y, Z), \vec{K}(X, T)\rangle+\langle\vec{K}(X, Z), \vec{K}(Y, T)\rangle,
$$

where $X, Y, Z, T \in \Gamma(T N)$.

Proof. Using $\nabla_{X} Y=\nabla_{X}^{N} Y-\vec{K}(X, Y)$, we compute

$$
\begin{aligned}
& R(X, Y) Z=\nabla_{X} \nabla_{Y} Z-\nabla_{Y} \nabla_{X} Z-\nabla_{[X, Y]} Z= \\
& \nabla_{X}\left(\nabla_{Y}^{N} Z-\vec{K}(Y, Z)\right)-\nabla_{Y}\left(\nabla_{X}^{N} Z-\vec{K}(X, Z)\right)-\nabla_{[X, Y]}^{N} Z+\vec{K}([X, Y], Z)= \\
& \nabla_{X}^{N} \nabla_{Y}^{N} Z-\vec{K}\left(X, \nabla_{Y}^{N} Z\right)-\nabla_{X} \vec{K}(Y, Z)-\nabla_{Y}^{N} \nabla_{X}^{N} Z+\vec{K}\left(Y, \nabla_{X}^{N} Z\right)+ \\
& \nabla_{Y} \vec{K}(X, Z)-\nabla_{[X, Y]}^{N} Z+\vec{K}([X, Y], Z)= \\
& R^{N}(X, Y) Z-\vec{K}\left(X, \nabla_{Y}^{N} Z\right)+\vec{K}\left(Y, \nabla_{X}^{N} Z\right)+\vec{K}([X, Y], Z)-\nabla_{X} \vec{K}(Y, Z)+ \\
& \nabla_{Y} \vec{K}(X, Z) .
\end{aligned}
$$

Multiplying by a tangent vector $T$, and using that $\vec{K}$ is orthogonal to $N$ we obtain

$$
\langle R(X, Y) Z, T\rangle=\left\langle R^{N}(X, Y) Z, T\right\rangle-\left\langle\nabla_{X} \vec{K}(Y, Z), T\right\rangle+\left\langle\nabla_{Y} \vec{K}(X, Z), T\right\rangle .
$$




\section{A.3. Gauss, Ricci and Codazzi identities}

Using the orthogonality between $\vec{K}$ and $T$ we have

$$
\left\langle\nabla_{X} \vec{K}(Y, Z), T\right\rangle=-\left\langle\vec{K}(Y, Z), \nabla_{X} T\right\rangle=\langle\vec{K}(Y, Z), \vec{K}(X, T)\rangle .
$$

Likewise $\left\langle\nabla_{Y} \vec{K}(X, Z), T\right\rangle=\langle\vec{K}(X, Z), \vec{K}(Y, T)\rangle$. Combining (A.17) in (A.16) gives the result.

Given $X \in \Gamma(T N)$ and $\eta \in \Gamma\left((T N)^{\perp}\right)$, the normal connection $\nabla^{\perp}$ of the embedding is defined by

$$
\nabla_{x}^{\perp} \eta:=\left(\nabla_{x} \eta\right)^{\perp}=\nabla_{x} \eta-\left(\nabla_{x} \eta\right)^{\top} .
$$

It defines a connection because it is linear in $X$, additive in $\eta$, and satisfies

$$
\nabla_{X}^{\perp}(f \eta)=X(f) \eta+f \nabla_{X}^{\perp} \eta
$$

The curvature tensor associated to the connection is denoted by $R^{\perp}$ and is given, as usual, by

$$
R^{\perp}(X, Y) \eta=\nabla_{X}^{\perp} \nabla_{Y}^{\perp} \eta-\nabla_{Y}^{\perp} \nabla_{X}^{\perp} \eta-\nabla_{[X, Y]}^{\perp} \eta . \quad X, Y \in \Gamma(T N), \eta \in \Gamma\left((T N)^{\perp}\right) .
$$

The following result gives a relation between the ambient curvature tensor and the curvature tensor associated to the normal connection. The identity involves the socalled Weingarten map defined as

$$
\mathcal{W}_{\eta}: T_{p} N \longrightarrow T_{p} N, \quad \mathcal{W}_{\eta}(X)=\left(\nabla_{X} \eta\right)^{\top}
$$

Theorem A.3.2 (Ricci equation). Let $N$ be an n-dimensional non-degenerate submanifold in a pseudo-Riemannian m-dimensional manifold $(M, g)$. Let $R$ be the Riemann tensor of $M$ and $R^{\perp}$ the normal curvature of the embedding. Then

$$
\langle R(X, Y) \eta, \zeta\rangle=\left\langle R^{\perp}(X, Y) \eta, \zeta\right\rangle+\left\langle\left[\mathcal{W}_{\zeta}, \mathcal{W}_{\eta}\right](X), Y\right\rangle,
$$

with $\left[\mathcal{W}_{\zeta}, \mathcal{W}_{\eta}\right](X):=\left(\mathcal{W}_{\zeta} \mathcal{W}_{\eta}-\mathcal{W}_{\eta} \mathcal{W}_{\zeta}\right)(X)$, and where $X, Y \in \Gamma(T N)$, and $\eta, \zeta \in$ $\Gamma\left((T N)^{\perp}\right)$.

Proof. By definition,

$$
\begin{aligned}
& R(X, Y) \eta=\nabla_{X} \nabla_{Y} \eta-\nabla_{Y} \nabla_{X} \eta-\nabla_{[X, Y]} \eta= \\
& \nabla_{X}\left(\mathcal{W}_{\eta}(Y)+\nabla_{Y}^{\perp} \eta\right)-\nabla_{Y}\left(\mathcal{W}_{\eta}(X)+\nabla_{X}^{\perp} \eta\right)-\mathcal{W}_{\eta}([X, Y])-\nabla_{[X, Y]}^{\perp} \eta= \\
& \nabla_{X}^{N} \mathcal{W}_{\eta}(Y)-\vec{K}\left(X, \mathcal{W}_{\eta}(Y)\right)+\mathcal{W}_{\nabla_{Y}^{\perp} \eta}(X)+\nabla_{X}^{\perp} \nabla_{Y}^{\perp} \eta-\nabla_{Y}^{N} \mathcal{W}_{\eta}(X)+ \\
& \vec{K}\left(Y, \mathcal{W}_{\eta}(X)\right)-\mathcal{W}_{\nabla_{\bar{X}} \eta}(Y)-\nabla_{Y}^{\perp} \nabla_{X}^{\perp} \eta-\mathcal{W}_{\eta}([X, Y])-\nabla_{[X, Y]}^{\perp} \eta .
\end{aligned}
$$

Using (A.18) and multiplying by the orthogonal vector $\zeta$, all the terms tangent to $N$ cancel out and we obtain

$$
\langle R(X, Y) \eta, \zeta\rangle=\left\langle R^{\perp}(X, Y) \eta, \zeta\right\rangle-\left\langle\vec{K}\left(X, \mathcal{W}_{\eta}(Y)\right), \zeta\right\rangle+\left\langle\vec{K}\left(Y, \mathcal{W}_{\eta}(X)\right), \zeta\right\rangle .
$$




\section{A. Geometry of non-degenerate submanifolds}

Observe that

$$
\langle\vec{K}(X, Y), \eta\rangle=\left\langle-\nabla_{X} Y, \eta\right\rangle=\left\langle Y, \nabla_{X} \eta\right\rangle=\left\langle\mathcal{W}_{\eta}(X), Y\right\rangle
$$

The symmetry of $\vec{K}$ implies that the operator $\mathcal{W}_{\eta}$ is self-adjoint, i.e.

$$
\left\langle\mathcal{W}_{\eta}(X), Y\right\rangle=\left\langle X, \mathcal{W}_{\eta}(Y)\right\rangle
$$

A consequence of $(\mathrm{A} .20)$ and $(\mathrm{A} .21)$ is

$$
\left\langle\vec{K}\left(X, \mathcal{W}_{\eta}(Y)\right), \zeta\right\rangle=\left\langle\mathcal{W}_{\zeta}(X), \mathcal{W}_{\eta}(Y)\right\rangle=\left\langle\mathcal{W}_{\eta} \mathcal{W}_{\zeta}(X), Y\right\rangle
$$

The same reasoning applies to $\left\langle\vec{K}\left(Y, \mathcal{W}_{\eta}(X)\right), \zeta\right\rangle$. Inserting this and (A.22) in (A.19) gives the result.

The last theorem of the appendix relates the ambient curvature tensor contracted with three tangent vectors and one normal vector with the covariant derivatives of the second fundamental form vector of the embedded submanifold. Since this result requires the use of covariant derivatives of the second fundamental form vector, we introduce the operator $\tilde{\nabla}$ as

$$
\left(\tilde{\nabla}_{X} \vec{K}\right)(Y, Z):=\nabla_{X} \vec{K}(Y, Z)-\vec{K}\left(\nabla_{X}^{N} Y, Z\right)-\vec{K}\left(Y, \nabla_{X}^{N} Z\right), \quad X, Y, Z \in \Gamma(T N) .
$$

Theorem A.3.3 (Codazzi equation). Let $N$ be an $n$-dimensional non-degenerate submanifold in a pseudo-Riemannian m-dimensional manifold $(M, g)$. Let $R$ be the Riemann tensor of $M$. Then:

$$
\langle R(X, Y) Z, \eta\rangle=\left\langle\left(\tilde{\nabla}_{Y} \vec{K}\right)(X, Z), \eta\right\rangle-\left\langle\left(\tilde{\nabla}_{X} \vec{K}\right)(Y, Z), \eta\right\rangle
$$

where $X, Y, Z \in \Gamma(T N)$ and $\eta \in \Gamma\left((T N)^{\perp}\right)$.

Proof. Recall expression (A.15) for $R(X, Y) Z$. If we multiply both sides by $\eta$, and use that $[X, Y]=\nabla_{X} Y-\nabla_{Y} X$ we obtain

$$
\begin{aligned}
& \langle R(X, Y) Z, \eta\rangle=-\left\langle\vec{K}\left(X, \nabla_{Y}^{N} Z\right), \eta\right\rangle+\left\langle\vec{K}\left(Y, \nabla_{X}^{N} Z\right), \eta\right\rangle+\langle\vec{K}([X, Y], Z), \eta\rangle- \\
& \left\langle\nabla_{X} \vec{K}(Y, Z), \eta\right\rangle+\left\langle\nabla_{Y} \vec{K}(X, Z), \eta\right\rangle=\left\langle\nabla_{Y} \vec{K}(X, Z), \eta\right\rangle-\left\langle\vec{K}\left(\nabla_{Y}^{N} X, Z\right), \eta\right\rangle- \\
& \left\langle\vec{K}\left(X, \nabla_{Y}^{N} Z\right), \eta\right\rangle-\left(\left\langle\nabla_{X} \vec{K}(Y, Z), \eta\right\rangle-\left\langle\vec{K}\left(\nabla_{X}^{N} Y, Z\right), \eta\right\rangle-\left\langle\vec{K}\left(Y, \nabla_{X}^{N} Z\right), \eta\right\rangle\right)= \\
& \left\langle\left(\tilde{\nabla}_{Y} \vec{K}\right)(X, Z), \eta\right\rangle-\left\langle\left(\tilde{\nabla}_{X} \vec{K}\right)(Y, Z), \eta\right\rangle .
\end{aligned}
$$




\section{A.3. Gauss, Ricci and Codazzi identities}

The curvature identities above can also be written in terms of the Riemann tensor, which is defined as

$$
\operatorname{Riem}(X, Y, Z, T)=\langle R(Z, T) Y, X\rangle
$$

The corresponding expressions for the identities are:

- Gauss equation:

$$
\operatorname{Riem}^{g}(X, Y, Z, T)=\operatorname{Riem}^{N}(X, Y, Z, T)-\langle\vec{K}(X, Z), \vec{K}(Y, T)\rangle+\langle\vec{K}(Y, Z), \vec{K}(X, T)\rangle,
$$

- Ricci equation:

$$
\operatorname{Riem}^{g}(X, Y, \eta, \zeta)=\operatorname{Riem}^{\perp}(X, Y, \eta, \zeta)+\left\langle\left[\mathcal{W}_{\eta}, \mathcal{W}_{\zeta}\right](X), Y\right\rangle
$$

- Codazzi equation:

$$
\operatorname{Riem}^{g}(X, Y, Z, \eta)=\left\langle\left(\tilde{\nabla}_{X} \vec{K}\right)(Y, Z), \eta\right\rangle-\left\langle\left(\tilde{\nabla}_{Y} \vec{K}\right)(X, Z), \eta\right\rangle
$$

We conclude this appendix by taking the double trace of the Gauss equation when the ambient manifold $M$ is Lorentzian and $N$ is codimension-two and spacelike. This calculation is easier in index notation. Let $\left\{X_{A}\right\}$ be a basis of $N$. The Gauss equation is expressed in index notation as

$$
\operatorname{Riem}_{A B C D}^{g}=\operatorname{Riem}_{A B C D}^{N}-\left\langle\vec{K}_{A C}, \vec{K}_{B D}\right\rangle+\left\langle\vec{K}_{B C}, \vec{K}_{A D}\right\rangle
$$

For every $p \in N$, let us complete the basis $\left\{\left.X_{A}\right|_{p}\right\}$ to a basis of $T_{p} M$ with two null vectors $k_{p}$ and $\ell_{p}$ orthogonal to $N$ normalized as $\langle k, \ell\rangle=-\varphi$ for a given positive function $\varphi: N \rightarrow \mathbb{R}$. Decomposing $\vec{K}=-\frac{1}{\varphi}\left(K^{k} \ell+K^{\ell} k\right)$ we have

$$
\begin{aligned}
\operatorname{Riem}_{A B C D}^{g}= & \operatorname{Riem}_{A B C D}^{N}-\frac{1}{\varphi}\left(K_{B C}^{k} K_{A D}^{\ell}+K_{A D}^{k} K_{B C}^{\ell}\right) \\
& +\frac{1}{\varphi}\left(K_{A C}^{k} K_{B D}^{\ell}+K_{B D}^{k} K_{A C}^{\ell}\right)
\end{aligned}
$$

Taking the trace once on the first and third indices we obtain

$$
\gamma^{A C} \operatorname{Riem}_{A B C D}^{g}=\operatorname{Ric}_{B D}^{N}-\frac{1}{\varphi}\left(K^{k A}{ }_{B} K_{A D}^{\ell}+K^{k C}{ }_{D} K_{B C}^{\ell}\right)+\frac{1}{\varphi}\left(\theta_{k} K_{B D}^{\ell}+\theta_{\ell} K_{B D}^{k}\right) .
$$

Taking the trace again we find

$$
\gamma^{B D} \gamma^{A C} \operatorname{Riem}_{A B C D}^{g}=\operatorname{Scal}^{N}-\frac{2}{\varphi} K^{k A B} K_{A B}^{\ell}+\frac{2}{\varphi} \theta_{k} \theta_{\ell}
$$




\section{A. Geometry of non-degenerate submanifolds}

In the basis $\left\{X_{A}, k, \ell\right\}$, the contravariant version of the metric $g$ of $M$ reads

$$
g^{\#}=\gamma^{A B} X_{A} \otimes X_{B}-\frac{1}{\varphi} k \otimes \ell-\frac{1}{\varphi} \ell \otimes k .
$$

A straightforward computation shows that

$$
\gamma^{B D} \gamma^{A C} \operatorname{Riem}_{A B C D}^{g}=\operatorname{Scal}^{g}+\frac{4}{\varphi} \operatorname{Ric}^{g}(k, \ell)-\frac{2}{\varphi^{2}} \operatorname{Riem}^{g}(k, \ell, k, \ell) .
$$

Inserting (A.29) into (A.28) finally yields

$$
\mathrm{Scal}^{g}+\frac{4}{\varphi} \operatorname{Ric}^{g}(k, \ell)-\frac{2}{\varphi^{2}} \operatorname{Riem}^{g}(k, \ell, k, \ell)=\operatorname{Scal}^{N}+\frac{2}{\varphi} \theta_{k} \theta_{\ell}-\frac{2}{\varphi} K_{A B}^{k} K^{\ell^{A B}}
$$

Using the definition of the Einstein tensor, the relation above also becomes

$$
\frac{4}{\varphi} \operatorname{Ein}^{g}(k, \ell)-\operatorname{Scal}^{g}-\frac{2}{\varphi^{2}} \operatorname{Riem}^{g}(k, \ell, k, \ell)=\operatorname{Scal}^{N}+\frac{2}{\varphi} \theta_{k} \theta_{\ell}-\frac{2}{\varphi} K_{A B}^{k} K^{\ell A B} .
$$





\section{Geometry of null hypersurfaces}

In this appendix we present a self-contained description of the geometry of null hypersurfaces (for a detailed work on null hypersurfaces see e.g. [32, 38]). In particular we obtain the evolution equations along general flows by spacelike cross sections of the geometric elements of the leaves.

\section{B.1 Hypersurfaces and null geodesics}

Definition B.1.1 (Null hypersurface). Let $(M, g)$ be an $m$-dimensional spacetime. A manifold $\Omega$ embedded in $M$ is a null hypersurface when it has codimension one, and the pullback of $g$ on $\Omega$ is a degenerate tensor.

As we said before, the nature of this kind of submanifolds makes impossible to define a unique second fundamental form vector, since in this case $\forall p \in \Omega, T_{p} \Omega \cap\left(T_{p} \Omega\right)^{\perp} \neq 0$. Any vector in this intersection is automatically lightlike and defines a degeneration direction of the pullback of $g$.

Let us first see that on a null hypersurface $\Omega$ there is just one degenerate direction. Assume that there are two degenerate directions $\left\langle\ell_{1}\right\rangle$ and $\left\langle\ell_{2}\right\rangle$, where $\left\langle v_{1}, \ldots, v_{k}\right\rangle$ denotes the minimal subspace generated by $\left\{v_{1}, \ldots, v_{k}\right\}$. Without loss of generality we can consider $\ell_{1}$ and $\ell_{2}$ to be future. Then $\ell_{1}+\ell_{2}$ is a timelike vector whose inner product with $\ell_{1}$ and $\ell_{2}$ is zero (since they are degenerate directions), but this is impossible as the product of a timelike vector with a null vector can never be zero. Let us call this unique degenerate direction $k$. The rest of the directions $X \in T_{p} \Omega$ satisfy $\langle X, X\rangle \geq 0$. If not, there would be a timelike direction $X_{0}\left(\left\langle X_{0}, X_{0}\right\rangle<0\right)$ satisfying $\left\langle k, X_{0}\right\rangle=0$, which cannot happen. Moreover, if $X \notin<k>$ and $X \neq 0$, then $\langle X, X\rangle>0$, because otherwise, $X$ would be lightlike and orthogonal to $k$, which can only occur if $X$ is parallel to $k$, against hypothesis. 


\section{B.1. Hypersurfaces and null geodesics}

Let us next prove that $\left(k_{p}\right)^{\perp}=T_{p} \Omega$. Since $k_{p} \in T_{p} \Omega \cap\left(T_{p} \Omega\right)^{\perp}$ we have $\left(k_{p}\right)^{\perp} \supset T_{p} \Omega$. To prove the reverse inclusion, consider $t_{p}$ a timelike vector of $T_{p} M$. Since $\left\langle t_{p}\right\rangle$ $\cap T_{p} \Omega=\emptyset$, we have $T_{p} M=<t_{p}>\oplus T_{p} \Omega$. Then for $X_{p} \in\left(k_{p}\right)^{\perp}$, and decomposing $X_{p}=\alpha t_{p}+Y_{p}$ for $\alpha \in \mathbb{R}$ and $Y_{p} \in T_{p} \Omega$, yields

$$
0=\left\langle k_{p}, X_{p}\right\rangle=\left\langle k_{p}, \alpha t_{p}+Y_{p}\right\rangle=\alpha\left\langle k_{p}, t_{p}\right\rangle
$$

Since $\left\langle k_{p}, t_{p}\right\rangle \neq 0$, it follows necessarily that $\alpha=0$, and hence $X_{p} \in T_{p} \Omega$.

We can check the consistency of the previous results by analyzing the validity of the Grassman identity, which reads

$$
\operatorname{dim}\left(T_{p} \Omega+\left(T_{p} \Omega\right)^{\perp}\right)=\operatorname{dim} T_{p} \Omega+\operatorname{dim}\left(T_{p} \Omega\right)^{\perp}-\operatorname{dim}\left[T_{p} \Omega \cap\left(T_{p} \Omega\right)^{\perp}\right] .
$$

Given that $\left(W^{\perp}\right)^{\perp}=W$ for any subspace of a vector space with metric $g$, we have

$$
<k_{p}>=\left[\left(k_{p}\right)^{\perp}\right]^{\perp}=\left(T_{p} \Omega\right)^{\perp} \quad \forall p \in \Omega,
$$

so that $\operatorname{dim} T_{p} \Omega=m-1, \operatorname{dim}\left(T_{p} \Omega\right)^{\perp}=1$, and $\operatorname{dim}\left[T_{p} \Omega \cap\left(T_{p} \Omega\right)^{\perp}\right]=1$, and (B.1) holds.

Proposition B.1.2 (Null geodesics). Let $(M, g)$ be an m-dimensional spacetime. Consider a null hypersurface $\Omega$ embedded in $M$. The integral curves of $k$ are null geodesics (and they are also called null generators of $\Omega$ ).

Proof. It suffices to prove:

$$
\nabla_{k} k=\lambda k
$$

Let us consider $p \in \Omega$. We want to prove that $\left.\nabla_{k} k\right|_{p} \in\left(T_{p} \Omega\right)^{\perp}=<k_{p}>$, or equivalently $\left\langle\left.\nabla_{k} k\right|_{p}, X\right\rangle=0 \quad \forall X \in T_{p} \Omega$. To this purpose extend $X \in T_{p} \Omega$ making it invariant under the flux generated by $k$,

$$
[k, X]=\nabla_{k} X-\nabla_{X} k=0 .
$$

The system (B.2) always has a unique solution because in a suitable coordinate system rectifying $k$, this equation becomes an ordinary differential equation of first order with $X_{p}$ as initial data. By construction, the extended vector $X$ remains tangent to $\Omega$, so that $\langle k, X\rangle=0$ holds. Differentiating along $k$ we obtain

$$
\nabla_{k}\langle k, X\rangle=\left\langle\nabla_{k} k, X\right\rangle+\left\langle k, \nabla_{k} X\right\rangle=0
$$

Therefore:

$$
\left\langle\nabla_{k} k, X\right\rangle=-\left\langle k, \nabla_{k} X\right\rangle=-\left\langle k, \nabla_{X} k\right\rangle=-\frac{1}{2} X\langle k, k\rangle=0
$$




\section{B. Geometry of null hypersurfaces}

\section{B.2 Quotient structure on a null hypersurface}

The difficulty that arises with null hypersurfaces is that they do not inherit a metric from the ambient manifold. Hence it is not possible to raise and lower indices of tensor fields on $\Omega$. We cannot define a natural connection on $\Omega$ either. In order to address this difficulty, it is useful to introduce the following equivalence relation in $T_{p} \Omega$ :

For $X, Y \in \Gamma(T \Omega)$, we define

$$
X \sim Y \Longleftrightarrow X-Y=\lambda k \text {, with } \lambda \in \mathbb{R} \text {. }
$$

This endows $T_{p} \Omega$ with a quotient structure (see Figure B.1). The quotient vector space, denoted by $T_{p} \Omega / k$, is the set of equivalence classes of each $X_{p} \in T_{p} \Omega$, denoted by $\bar{X}_{p}$, namely

$$
T_{p} \Omega / k=\left\{\bar{X}_{p}: X_{p} \in T_{p} \Omega\right\} .
$$

The fiber bundle on $\Omega$ is the collection of all quotient spaces

$$
T \Omega / k=\bigcup_{p \in \Omega} T_{p} \Omega / k
$$

The dimension of the fibers is $m-2$. From now on and for the sake of simplicity, we will simply write $\bar{X}$ instead of $\bar{X}_{p}$.

\section{B.3 Positive definite metric on $\mathrm{T} \Omega / \mathrm{k}$}

It is convenient to construct a metric on the quotient bundle. Define the map:

$$
\begin{aligned}
\gamma^{\Omega}: T_{p} \Omega / k \times T_{p} \Omega / k & \longrightarrow \mathbb{R} \\
\gamma^{\Omega}(\bar{X}, \bar{Y}) & =\langle X, Y\rangle .
\end{aligned}
$$

The first thing to check is that the map is well defined, i.e., that it does not depend on the chosen representatives: let $X^{\prime} \sim X$, and $Y^{\prime} \sim Y$. This means there exist two real numbers $\alpha, \beta$ so that $X^{\prime}=X+\alpha k, Y^{\prime}=Y+\beta k$. Then $\gamma^{\Omega}\left(\overline{X^{\prime}}, \overline{Y^{\prime}}\right)=\left\langle X^{\prime}, Y^{\prime}\right\rangle=$ $\langle X+\alpha k, Y+\beta k\rangle=\langle X, Y\rangle+\beta\langle X, k\rangle+\alpha\langle k, Y\rangle+\alpha \beta\langle k, k\rangle=\langle X, Y\rangle=\gamma^{\Omega}(\bar{X}, \bar{Y})$, where we used $\left(k_{p}\right)^{\perp}=T_{p} \Omega$.

The next thing to see is that $\gamma^{\Omega}$ is in fact a metric. Consider $\bar{X} \in T_{p} \Omega / k, \bar{X} \neq 0$. This means that $X \in T_{p} \Omega$ is not parallel to $k_{p}$, i.e. $X$ is a spacelike direction. In other words

$$
\gamma^{\Omega}(\bar{X}, \bar{X})=\langle X, X\rangle>0,
$$

which directly implies that $\gamma^{\Omega}$ is a metric. 


\section{B.4 Second fundamental form on $T \Omega / k$}

We next define on $T_{p} \Omega / k$ the Weingarten map (see for instance [32]), which will be useful later to define the second fundamental form tensor. Consider the map

$$
\begin{aligned}
\boldsymbol{K}^{\Omega}: T_{p} \Omega / k & \longrightarrow T_{p} \Omega / k \\
\boldsymbol{K}^{\Omega}(\bar{X}) & =\overline{\nabla_{X} k} .
\end{aligned}
$$

This definition is consistent because $\left(\nabla_{x} k\right)_{p} \in T_{p} \Omega$. Indeed $\langle k, k\rangle=0 \Rightarrow 0=$ $\nabla_{x}\langle k, k\rangle=2\left\langle\nabla_{x} k, k\right\rangle \Rightarrow\left\langle\nabla_{x} k, k\right\rangle=0 \Rightarrow\left(\nabla_{x} k\right)_{p} \in\left(k_{p}\right)^{\perp}=T_{p} \Omega$.

We also need to check that $\overline{\nabla_{X} k}$ is independent of the representative $X \in \bar{X}$. Let $X^{\prime} \sim X$. Then $K^{\Omega}\left(\overline{X^{\prime}}\right)=\overline{\nabla_{X^{\prime}} k}=\overline{\nabla_{X+\alpha k} k}=\overline{\nabla_{X} k+\alpha \nabla_{k} k}=\overline{\nabla_{X} k+\alpha \lambda k}=\overline{\nabla_{X} k}=$ $K^{\Omega}(\bar{X})$

Using this map we can define the second fundamental form tensor in the quotient space:

Definition B.4.1 (Second fundamental form tensor on $T_{p} \Omega / k$ ). The second fundamental form tensor in the quotient space $T_{p} \Omega / k$ is the map:

$$
\begin{aligned}
K^{\Omega}: T_{p} \Omega / k \times T_{p} \Omega / k & \longrightarrow \mathbb{R} \\
K^{\Omega}(\bar{X}, \bar{Y}) & =\gamma^{\Omega}\left(K^{\Omega}(\bar{X}), \bar{Y}\right)=\left\langle\nabla_{X} k, Y\right\rangle .
\end{aligned}
$$

Proposition B.4.2. $K^{\Omega}$ is symmetric, i.e. $K^{\Omega}(\bar{X}, \bar{Y})=K^{\Omega}(\bar{Y}, \bar{X}), \forall \bar{X}, \bar{Y} \in T_{p} \Omega / k$, and hence the map $\boldsymbol{K}^{\Omega}$ is self-adjoint with respect to $\gamma^{\Omega}$.

Proof. Given $X_{p}, Y_{p} \in T_{p} \Omega$, extend them as vector fields $X, Y$ tangent to $\Omega$ in a neighbourhood of $p$ in $\Omega$. Using $X(\langle k, Y\rangle)=0$ and $Y(\langle k, X\rangle)=0$, it follows

$$
\begin{aligned}
K^{\Omega}(\bar{X}, \bar{Y}) & =\left\langle\nabla_{X} k, Y\right\rangle=-\left\langle k, \nabla_{X} Y\right\rangle \\
& =-\left\langle k, \nabla_{Y} X\right\rangle+\langle k,[X, Y]\rangle \\
& =\left\langle\nabla_{Y} k, X\right\rangle=K^{\Omega}(\bar{Y}, \bar{X}),
\end{aligned}
$$

where we used $[X, Y]_{p} \in T_{p} \Omega$, since both $X_{p}$ and $Y_{p}$ are tangent to $\Omega$.

Let us build now a basis $B$ of vectors of $T_{p} \Omega$, with the property that the first vector of the basis is the null direction $k_{p}$. To obtain the rest of vectors, we consider the intersection $S$ of another spacelike hypersurface $\Sigma$ with $\Omega$, i.e. $S=\Omega \cap \Sigma$. Under these conditions $T_{p} \Omega=\left\langle k_{p}\right\rangle \oplus T_{p}(\Omega \cap \Sigma)=\left\langle k_{p}\right\rangle \oplus T_{p} S$. In addition $\left.k\right|_{S} \in \Gamma\left((T S)^{\perp}\right)$. Choose $m-2$ linearly independent additional spacelike vectors $\left\{X_{1}, \ldots, X_{m-2}\right\}$ which form a basis $B^{\prime}$ of $T_{p} S \subset T_{p} \Omega$. It is clear that $B=\{k\} \cup B^{\prime}$ is a basis of $T_{p} \Omega$.

The elements of $B^{\prime}$ are in $T_{p} \Omega$. We can take its equivalence classes, and obtain a set of quotient vectors $\overline{B^{\prime}}=\left\{\bar{X}_{1}, \ldots, \bar{X}_{m-2}\right\}$, whose cardinal equals the dimension of 


\section{B. Geometry of null hypersurfaces}

$T_{p} \Omega / k$. Moreover they are linearly independent. Indeed if a linear combination $a^{A} \bar{X}_{A}$ vanishes, then there is $a^{0} \in \mathbb{R}$ such that $a^{A} X_{A}=a^{0} k$. But $\left\{k, X_{A}\right\}$ is a basis, so all coefficients $a^{A}$ and $a^{0}$ must be zero. Thus $\overline{B^{\prime}}$ is a basis of $T_{p} \Omega / k$.

We next define the concept of mean null curvature, also called null expansion:

Definition B.4.3 (Null expansion). Let $(M, g)$ be an m-dimensional spacetime and $\Omega \subset M$ an embedded null hypersurface. $\forall p \in T_{p} \Omega$, we can consider the basis $\overline{B^{\prime}}=$ $\left\{\bar{X}_{1}, \ldots, \bar{X}_{m-2}\right\}$ of $T_{p} \Omega / k$. Let $K^{\Omega}: T_{p} \Omega / k \times T_{p} \Omega / k \longrightarrow \mathbb{R}$ be the second fundamental form tensor. The null expansion is the scalar

$$
\theta_{k}=\operatorname{trace}_{\gamma \Omega}\left(K^{\Omega}\right)=\left(\gamma^{\Omega}\right)^{A B} K_{A B}^{\Omega},
$$

where $K_{A B}^{\Omega}:=K^{\Omega}\left(\bar{X}_{A}, \bar{X}_{B}\right)$, and $\left(\gamma^{\Omega}\right)^{A B}$ is the inverse of $\gamma_{A B}^{\Omega}:=\gamma^{\Omega}\left(\bar{X}_{A}, \bar{X}_{B}\right)$.

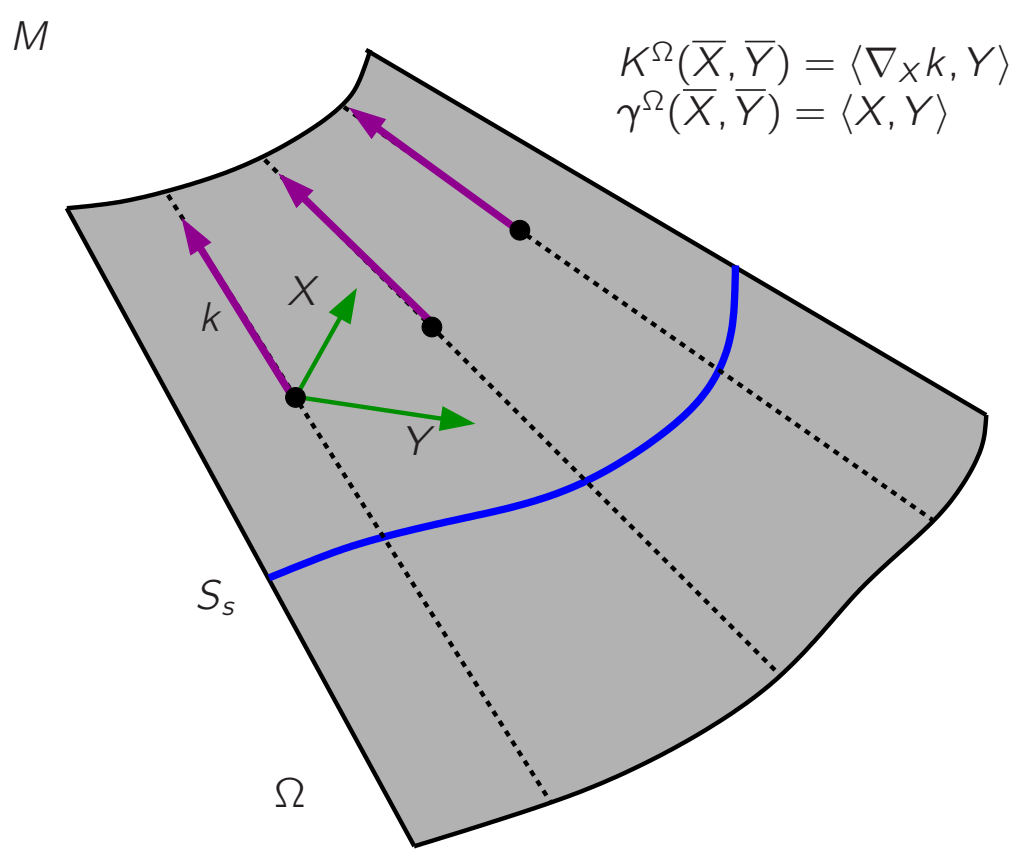

Figure B.1: A null hypersurface $\Omega$ embedded in a spacetime $M$. The tangent vector field $k$ to the null generators of $\Omega$ is unique up to reparametrization, and every tangent vector $X$ to the hypersurface is orthogonal to $k$. A canonical equivalence relation between vectors tangent to $\Omega$ can be defined, and a quotient bundle can be constructed. The quotient metric $\gamma^{\Omega}$ and second fundamental form tensor $K^{\Omega}$ are independent of the chosen representatives of the equivalence classes. The level sets of a function $s: \Omega \rightarrow \mathbb{R}$ satisfying $k(s)=1$ define a foliation $\left\{S_{s}\right\}$ along $\Omega$.

For any smooth nowhere zero function $f: \Omega \rightarrow \mathbb{R}$, the vector field $\widetilde{k}=f k$ is also a degeneration vector. Denote by $\boldsymbol{K}^{\Omega_{k}}$ and $\boldsymbol{K}^{\Omega_{\widetilde{k}}}$ the corresponding Weingarten 


\section{B.5. Evolution equations on $\Omega$. Ricatti and Raychaudhuri equation}

endomorphisms and $K_{k}^{\Omega}$ and $K_{\widetilde{k}}^{\Omega}$ the associated second fundamental forms. Then

$$
\boldsymbol{K}_{\widetilde{k}}(\bar{X})=\overline{\nabla_{X} \widetilde{k}}=\overline{\nabla_{X}(f k)}=\overline{X(f) k+f \nabla_{X} k}=\overline{f \nabla_{X} k}=f \overline{\nabla_{X} k}=f \boldsymbol{K}^{\Omega_{k}}(\bar{X}) .
$$

From here we conclude

$$
\begin{aligned}
\theta_{\widetilde{k}} & \left.=\left(\gamma^{\Omega}\right)^{A B}\left(K_{\widetilde{k}}^{\Omega}\right)_{A B}=\left(\gamma^{\Omega}\right)^{A B} \boldsymbol{\gamma}^{\Omega}\left(\boldsymbol{K}^{\Omega} \overline{\mathrm{X}}_{A}\right), \bar{X}_{B}\right) \\
& =\left(\gamma^{\Omega}\right)^{A B} \boldsymbol{\gamma}^{\Omega}\left(f \boldsymbol{K}^{\Omega}{ }_{k}\left(\bar{X}_{A}\right), \bar{X}_{B}\right)=f\left(\boldsymbol{\gamma}^{\Omega}\right)^{A B}\left(K_{k}^{\Omega}\right)_{A B}=f \theta_{k} .
\end{aligned}
$$

Remark B.4.4. Notice that, as a consequence of (B.4) , the Weingarten endomorphism $K^{\Omega}{ }_{k}$ depends only on the value of the vector field $k$ on $p$.

\section{B.5 Evolution equations on $\Omega$. Ricatti and Raychaud- huri equation}

Definition B.5.1 (Null generator of $\Omega$ ). Let $(M, g)$ be a spacetime of dimension $m$ and $\Omega \subset M$ an embedded null hypersurface. A null generator of $\Omega$ is any of the integral curves of the degeneration direction $\langle k\rangle$.

On each null generator $\alpha(s)$ we consider the Weingarten map at $p=\alpha(s)$, along the vector $\alpha^{\prime}$

$$
K^{\Omega}(s):=K_{\alpha^{\prime}(s)} .
$$

As already mentioned, there is no natural connection on $\Gamma(T \Omega)$, and even more so on the quotient bundle $\Gamma(T \Omega / k)$. However it is possible to give a natural definition of directional derivative along the null generators of $\Omega$ for tensor fields on $\Gamma(T \Omega / k)$. For any smooth function $f: \Omega \rightarrow \mathbb{R}$ the derivative of $f$ along $\alpha(s)$ can be defined as $f^{\prime}:=\frac{d f(\alpha(s))}{d s}$. For convenience the notation $f^{\prime}:=k(f)$ is also often used. The derivative of $X \in \Gamma(T \Omega)$ along $k$ is naturally defined as $X^{\prime}:=\nabla_{k} X$. This vector field is tangent to $\Omega$ because, as shown after (B.3), $\nabla_{X} k \in \Gamma(T \Omega)$ and $[X, k]$ is also tangent to $\Omega$. For the derivative of the class we simply put

$$
(\bar{X})^{\prime}:=\overline{X^{\prime}} .
$$

We need to check that this operation is well-defined, i.e. that it does not depend on the representative. To this purpose we consider $X \sim Y$, i.e., there is a function $\lambda: \Omega \rightarrow \mathbb{R}$ so that $Y=X+\lambda k$. Given that $\nabla_{k} k$ is proportional to $k$, then

$$
\begin{aligned}
& (\bar{Y})^{\prime}=\overline{\nabla_{k}(X+\lambda k)}=\overline{\nabla_{k} X+k(\lambda) k+\lambda \nabla_{k} k}= \\
& =\overline{\nabla_{k} X}=\overline{X^{\prime}}=(\bar{X})^{\prime} .
\end{aligned}
$$




\section{B. Geometry of null hypersurfaces}

A fundamental property of any covariant derivative is the Leibniz rule with respect to contraction. More specifically, if $\omega$ is any one-form and $X, Y$ are vector fields then

$$
\nabla_{Y}[\omega(X)]=\left(\nabla_{Y} \omega\right) X+\omega\left(\nabla_{Y} X\right)
$$

Such a rule can be used to define the directional derivative we are seeking. For the specific case of the Weingarten map we define $\left(\boldsymbol{K}^{\Omega}\right)^{\prime}$ by

$$
\left(\boldsymbol{K}^{\Omega}\right)^{\prime}(\bar{X}):=\left[\boldsymbol{K}^{\Omega}(\bar{X})\right]^{\prime}-\boldsymbol{K}^{\Omega}\left(\bar{X}^{\prime}\right), \quad \bar{X} \in \Gamma(T \Omega / k) .
$$

Differential equations of the form $\frac{d y}{d x}=A(x) y^{2}+B(x) y+C(x)$ are called Ricatti equations. Such equations make sense not only for real functions, but also for endomorphisms. As we next see, the Weingarten map $\boldsymbol{K}^{\Omega}$ satisfies a Ricatti equation:

Theorem B.5.2 (Ricatti equation for the Weingarten map). Consider $\Omega$ a null hypersurface embedded in a spacetime $(M, g)$ of dimension $m$. Let $k$ be a degeneration vector of $\Omega, Q_{k}$ the function that satisfies $\nabla_{k} k=Q_{k} k$, and $\alpha(s)$ the corresponding null generators of $\Omega$. Then the family of Weingarten maps $K^{\Omega}=K^{\Omega}(s)$ obeys the equation

$$
\left(\boldsymbol{K}^{\Omega}\right)^{\prime}+\left(\boldsymbol{K}^{\Omega}\right)^{2}+R-Q_{k} \boldsymbol{K}^{\Omega}=0,
$$

where' denotes the directional derivative along $\alpha(s)$, and $\left(\boldsymbol{K}^{\Omega}\right)^{2}=\boldsymbol{K}^{\Omega} \circ \boldsymbol{K}^{\Omega}$ (composition of endomorphisms), with

$$
\begin{aligned}
(\bar{Y})^{\prime} & :=\overline{Y^{\prime}}, \text { differentiation of a vector field in } \Gamma(T \Omega / k), \\
\left(K^{\Omega}\right)^{\prime}(\bar{X}) & :=\left[K^{\Omega}(\bar{X})\right]^{\prime}-K^{\Omega}\left(\overline{X^{\prime}}\right), \text { definition of endomorphism } b^{\prime}, \\
R(\bar{X}) & :=\overline{R\left(X, \alpha^{\prime}\right) \alpha^{\prime},} \text { with } R \text { the curvature tensor on } M .
\end{aligned}
$$

Proof. The first step is to check that $(\bar{B} .7)$ is well defined. Assume $Y=X+\lambda k$. Then

$$
\begin{aligned}
& R(\bar{Y})=\overline{R(Y, k) k}=\overline{R(X+\lambda k, k) k}=\overline{R(X, k) k+\lambda R(k, k) k}= \\
& =\overline{R(X, k) k}=R(\bar{X}),
\end{aligned}
$$

where linearity and the antisymmetric properties of the curvature tensor have been used.

Consider now any vector $X_{p} \in T_{p} \Omega$, and extend it along $\alpha$ making it invariant under the flux generated by $k$ as in (B.2). The defining property is

$$
\nabla_{k} X=\nabla_{X} k
$$

and will be used several times below without further notice. The definition of curvature tensor implies

$$
R(X, k) k=\nabla_{X} \nabla_{k} k-\nabla_{k} \nabla_{X} k-\nabla_{[X, k]} k=X\left(Q_{k}\right) k+Q_{k} \nabla_{k} X-\nabla_{k} \nabla_{k} X
$$




\section{B.5. Evolution equations on $\Omega$. Ricatti and Raychaudhuri equation}

where in the last equality both $(\overline{\mathrm{B} .8})$ and $\nabla_{k} k=Q_{k} k$ have been used. Notice that each term in equation (B.9) is in $T_{p} \Omega$. In fact, this equation can be rewritten as

$$
X^{\prime \prime}=X\left(Q_{k}\right) k+Q_{k} X^{\prime}-R\left(X, \alpha^{\prime}\right) \alpha^{\prime}
$$

Finally,

$$
\begin{aligned}
& \left(\boldsymbol{K}^{\Omega}\right)^{\prime}(\bar{X})=\overline{\left(\nabla_{X} k\right)^{\prime}}-\boldsymbol{K}^{\Omega}\left(\overline{\nabla_{k} X}\right)=\overline{\left(\nabla_{k} X\right)^{\prime}}-\boldsymbol{K}^{\Omega}\left(\overline{\nabla_{X} k}\right)= \\
& =\bar{X}^{\prime \prime}-\boldsymbol{K}^{\Omega}\left(\boldsymbol{K}^{\Omega}(\bar{X})\right)=Q_{k} \bar{X}^{\prime}-\overline{R\left(X, \alpha^{\prime}\right) \alpha^{\prime}}-\left(\boldsymbol{K}^{\Omega}\right)^{2}(\bar{X})= \\
& =Q_{k} \boldsymbol{K}^{\Omega}(\bar{X})-R(\bar{X})-\left(\boldsymbol{K}^{\Omega}\right)^{2}(\bar{X}) .
\end{aligned}
$$

Given that $X_{p}$ is arbitrary, this proves (B.6).

Our next aim is to find the expression in components of (B.6). Let $B_{p}=\left\{k_{p},\left(X_{1}\right)_{p}\right.$, $\left.\ldots,\left(X_{m-2}\right)_{p}\right\}$ be a basis for $T_{p} \Omega$ and define functions $\left(K^{\Omega}\right)^{L}{ }_{A}$ by

$$
K^{\Omega}\left(\bar{X}_{A}\right)=\left(K^{\Omega}\right)_{A}^{L} \bar{X}_{L} .
$$

From the definition of second fundamental form tensor,

$$
\begin{aligned}
\left(K^{\Omega}\right)_{A B} & =\gamma^{\Omega}\left(K^{\Omega}\left(\bar{X}_{A}\right), \bar{X}_{B}\right) \\
& =\gamma^{\Omega}\left[\left(K^{\Omega}\right)^{L}{ }_{A} \bar{X}_{L}, \bar{X}_{B}\right] \\
& =\left(K^{\Omega}\right)^{L}{ }_{A} \gamma^{\Omega}\left(\bar{X}_{L}, \bar{X}_{B}\right) \\
& =\left(K^{\Omega}\right)^{L}{ }_{A}\left(\gamma^{\Omega}\right)_{L B} \\
& =\left(K^{\Omega}\right)_{A B},
\end{aligned}
$$

which is simply the statement in components that the second fundamental form is obtained from $K^{\Omega}$ by lowering indices with $\gamma^{\Omega}$. Extend now every $X_{A}$ in $B_{p}$ along $\alpha(s)$ as in (B.2). Then

$$
\overline{\nabla_{k} X_{A}}=\overline{\nabla_{X_{A}} k}=K^{\Omega}\left(\bar{X}_{A}\right)=\left(\boldsymbol{K}^{\Omega}\right)^{L}{ }_{A} \bar{X}_{L} .
$$

The endomorphism $\left(\boldsymbol{K}^{\Omega}\right)^{\prime}$ acting on the elements of the basis reads

$$
\begin{aligned}
\left(\boldsymbol{K}^{\Omega}\right)^{\prime}\left(\bar{X}_{A}\right) & =\left[\left(\boldsymbol{K}^{\Omega}\right)^{\prime}\right]_{A} \bar{X}_{B} \\
& =\left[\boldsymbol{K}^{\Omega}\left(\bar{X}_{A}\right)\right]^{\prime}-\boldsymbol{K}^{\Omega}\left(\overline{X_{A}^{\prime}}\right) \\
& =\left(\left(\boldsymbol{K}^{\Omega}\right)_{A}{ }_{A} \bar{X}_{B}\right)^{\prime}-\boldsymbol{K}^{\Omega}\left(\overline{X_{A}^{\prime}}\right) \\
& =\left(\left(\boldsymbol{K}^{\Omega}\right)^{B}{ }_{A}\right)^{\prime} \bar{X}_{B}+\underbrace{\left(\boldsymbol{K}^{\Omega}\right)_{A}^{B}\left(\bar{X}_{B}\right)^{\prime}-\boldsymbol{K}^{\Omega}\left(\overline{X_{A}^{\prime}}\right)}_{(* *)},
\end{aligned}
$$

and the term $(* *)$ vanishes because

$$
\begin{aligned}
(* *) & =\left(\boldsymbol{K}^{\Omega}\right)^{B}{ }_{A} \overline{\nabla_{k} X_{B}}-\boldsymbol{K}^{\Omega}\left(\overline{\nabla_{k} X_{A}}\right) \\
& =\left(\boldsymbol{K}^{\Omega}\right)^{B}{ }_{A}\left(\boldsymbol{K}^{\Omega}\right)^{L}{ }_{B} \bar{X}_{L}-\boldsymbol{K}^{\Omega}\left(\left(\boldsymbol{K}^{\Omega}\right)^{L}{ }_{A} \bar{X}_{L}\right) \\
& =\left(\boldsymbol{K}^{\Omega}\right)^{B}{ }_{A}\left(\boldsymbol{K}^{\Omega}\right)^{L}{ }_{B} \bar{X}_{L}-\left(\boldsymbol{K}^{\Omega}\right)^{L}{ }_{A}\left(\boldsymbol{K}^{\Omega}\right)^{T}{ }_{L} \bar{X}_{T}=0 .
\end{aligned}
$$




\section{B. Geometry of null hypersurfaces}

Since $\left(\boldsymbol{K}^{\Omega}\right)^{B}{ }_{A}$ are functions, $(\underline{B .10})$ is simply

$$
\left(\boldsymbol{K}^{\Omega}\right)^{\prime}\left(\bar{X}_{A}\right)=\left[\left(\boldsymbol{K}^{\Omega}\right)^{B}{ }_{A}\right]^{\prime} \bar{X}_{B}=\frac{d\left(\boldsymbol{K}^{\Omega}\right)^{B}{ }_{A}}{d s} \bar{X}_{B} .
$$

The endomorphism $\left(\boldsymbol{K}^{\Omega}\right)^{2}$ in components is

$$
\left(\boldsymbol{K}^{\Omega}\right)^{2}\left(\bar{X}_{A}\right)=\left[\left(\boldsymbol{K}^{\Omega}\right)^{2}\right]_{A}^{B} \bar{X}_{B}=\left(\boldsymbol{K}^{\Omega}\right)^{S}{ }_{A}\left(\boldsymbol{K}^{\Omega}\right)^{B}{ }_{S} \bar{X}_{B},
$$

and we define $R^{B}$ by means of

$$
R\left(\bar{X}_{A}\right)=R_{A}^{B} \bar{X}_{B}
$$

Putting things together, the Ricatti equation becomes

$$
\begin{aligned}
0 & =\left[\left(\boldsymbol{K}^{\Omega}\right)^{\prime}+\left(\boldsymbol{K}^{\Omega}\right)^{2}+R-Q_{k} \boldsymbol{K}^{\Omega}\right]\left(\bar{X}_{A}\right)= \\
& =\left(\frac{d\left(\boldsymbol{K}^{\Omega}\right)^{B}{ }_{A}}{d s}+\left(\boldsymbol{K}^{\Omega}\right)_{A}^{S}\left(\boldsymbol{K}^{\Omega}\right)_{S}^{B}+R_{A}^{B}-Q_{k}\left(\boldsymbol{K}^{\Omega}\right)_{A}^{B}\right) \bar{X}_{B},
\end{aligned}
$$

that is to say

$$
\frac{d\left(\boldsymbol{K}^{\Omega}\right)^{B}}{d s}+\left(\boldsymbol{K}^{\Omega}\right)^{S}{ }_{A}\left(\boldsymbol{K}^{\Omega}\right)^{B}{ }_{S}+R^{B}{ }_{A}-Q_{k}\left(\boldsymbol{K}^{\Omega}\right)^{B}{ }_{A}=0
$$

It is also necessary to obtain $\left(\gamma_{A B}^{\Omega}\right)^{\prime}$ :

$$
\begin{aligned}
\frac{d\left(\gamma^{\Omega}\right)_{A B}}{d s} & =\alpha^{\prime}(s)\left[\left(\gamma^{\Omega}\right)_{A B}\right]=\nabla_{k}\left[\gamma^{\Omega}\left(\bar{X}_{A}, \bar{X}_{B}\right)\right] \\
& =\nabla_{k}\left\langle X_{A}, X_{B}\right\rangle=\left\langle\nabla_{k} X_{A}, X_{B}\right\rangle+\left\langle X_{A}, \nabla_{k} X_{B}\right\rangle \\
& =\left\langle\nabla_{X_{A}} k, X_{B}\right\rangle+\left\langle X_{A}, \nabla_{X_{B}} k\right\rangle \\
& =\gamma^{\Omega}\left(\bar{\nabla}_{X_{A}} k, \bar{X}_{B}\right)+\gamma^{\Omega}\left(\bar{X}_{A}, \bar{\nabla}_{X_{B}} k\right)=\left(K^{\Omega}\right)_{A B}+\left(K^{\Omega}\right)_{B A} \\
& =2\left(K^{\Omega}\right)_{A B}=2\left(K^{\Omega}\right)_{A B},
\end{aligned}
$$

where the symmetry of $\left(K^{\Omega}\right)_{A B}$, and $\left(K^{\Omega}\right)_{A B}=\left(K^{\Omega}\right)_{A B}$ have been used. Hence

$$
\frac{d\left(\gamma^{\Omega}\right)_{A B}}{d s}=2\left(K^{\Omega}\right)_{A B}
$$

Combining (B.11) and ( $(\overline{B .12})$, the derivative of $\left(\boldsymbol{K}^{\Omega}\right)_{A B}$ along $k=\alpha^{\prime}(s)$ is computed to be

$$
\begin{aligned}
k\left(\left(\boldsymbol{K}^{\Omega}\right)_{A B}\right) & =k\left(\left(\boldsymbol{K}^{\Omega}\right)_{B}{ }_{B}\left(\boldsymbol{\gamma}^{\Omega}\right)_{L A}\right) \\
& =\left(Q_{k}\left(\boldsymbol{K}^{\Omega}\right)_{B}-\left(\boldsymbol{K}^{\Omega}\right)_{B}^{S}\left(\boldsymbol{K}^{\Omega}\right)^{L}{ }_{S}-R_{B}^{L}{ }_{B}\left(\boldsymbol{\gamma}^{\Omega}\right)_{L A}+2\left(\boldsymbol{K}^{\Omega}\right)_{B}^{L_{B}}\left(\boldsymbol{K}^{\Omega}\right)_{L A}\right. \\
& =Q_{k}\left(\boldsymbol{K}^{\Omega}\right)_{A B}+\left(\boldsymbol{K}^{\Omega}\right)_{A S}\left(\boldsymbol{K}^{\Omega}\right)_{B}{ }_{B}-R_{A B},
\end{aligned}
$$




\section{B.6. Flow of surfaces along $\Omega$. Evolution equations}

where $R_{A B}:=\operatorname{Riem}^{g}\left(X_{A}, k, X_{B}, k\right)$.

Our next aim is to compute the derivative of the null expansion $\theta_{k}$. To this purpose we use

$$
k\left(\left(\gamma^{\Omega}\right)^{A B}\right)=-2\left(\boldsymbol{K}^{\Omega}\right)^{A B},
$$

which is an immediate consequence of (B.12). Combining $(\overline{B .13})$ and (B.14),

$$
\begin{aligned}
k\left(\theta_{k}\right) & =k\left(\left(\gamma^{\Omega}\right)^{A B}\left(\boldsymbol{K}^{\Omega}\right)_{A B}\right)= \\
& =-2\left(\boldsymbol{K}^{\Omega}\right)^{A B}\left(\boldsymbol{K}^{\Omega}\right)_{A B}+\left(\boldsymbol{\gamma}^{\Omega}\right)^{A B}\left(Q_{k}\left(\boldsymbol{K}^{\Omega}\right)_{A B}+\left(\boldsymbol{K}^{\Omega}\right)_{A S}\left(\boldsymbol{K}^{\Omega}\right)^{S}{ }_{B}-R_{A B}\right) \\
& =Q_{k} \theta_{k}-\left(\boldsymbol{K}^{\Omega}\right)_{A B}\left(\boldsymbol{K}^{\Omega}\right)^{A B}-\left(\boldsymbol{\gamma}^{\Omega}\right)^{A B} R_{A B} .
\end{aligned}
$$

For every $p \in \Omega$ and given a positive function $\varphi: \Omega \rightarrow \mathbb{R}$, the basis $B_{p}=\left\{k_{p},\left(X_{A}\right)_{p}\right\}$ can be extended to a basis of $T_{p} M$ by adding the unique future null vector $\ell_{p}$ orthogonal to each $X_{A}$ and satisfying $\left\langle k_{p}, \ell_{p}\right\rangle=-\varphi(p)$. In the basis $\left\{X_{A}, k, \ell\right\}$, the contravariant version of the metric $g$ of $M$ reads

$$
g^{\#}=\left(\gamma^{\Omega}\right)^{A B} X_{A} \otimes X_{B}-\frac{1}{\varphi} k \otimes \ell-\frac{1}{\varphi} \ell \otimes k .
$$

Therefore

$$
\left(\gamma^{\Omega}\right)^{A B} R_{A B}=\left(g^{\#}+\frac{1}{\varphi} k \otimes \ell+\frac{1}{\varphi} \ell \otimes k\right)\left(\operatorname{Riem}^{g}(\cdot, k, \cdot, k)\right)=\operatorname{Ric}^{g}(k, k),
$$

and (B.15) becomes the Raychaudhuri equation in standard form, namely

$$
k\left(\theta_{k}\right)=Q_{k} \theta_{k}-\left(\boldsymbol{K}^{\Omega}\right)_{A B}\left(\boldsymbol{K}^{\Omega}\right)^{A B}-\operatorname{Ric}^{g}(k, k) .
$$

This equation can also be expressed as

$$
k\left(\theta_{k}\right)=Q_{k} \theta_{k}-\frac{1}{2}\left(\theta_{k}\right)^{2}-\left(\Pi^{k}\right)_{A B}\left(\Pi^{k}\right)^{A B}-\operatorname{Ric}^{g}(k, k),
$$

where $\Pi_{A B}^{k}:=\left(\boldsymbol{K}^{\Omega}\right)_{A B}-\frac{1}{2} \theta_{k}\left(\boldsymbol{\gamma}^{\Omega}\right)_{A B}$ is the trace-free part of $\left(\boldsymbol{K}^{\Omega}\right)_{A B}$. Note finally that $(\overline{B .11})$ and $(\overline{B .18})$ yield

$$
k\left(\left(\Pi^{k}\right)_{B}^{A}\right)=\left(Q_{k}-\theta_{k}\right)\left(\Pi^{k}\right)_{B}^{A}-R_{B}^{A}+\frac{1}{2} \operatorname{Ric}^{g}(k, k) \delta_{B}^{A} .
$$

\section{B.6 Flow of surfaces along $\Omega$. Evolution equations}

A remarkable property of the geometry of a null hypersurface $\Omega$ is that, given any point $p \in \Omega$ and any embedded spacelike surface $S_{p}$ in $\Omega$ containing $p$, the induced metric 


\section{B. Geometry of null hypersurfaces}

$\gamma_{S_{p}}(X, Y)$ of $S_{p}$ and the second fundamental form $K_{S_{p}}^{k}(X, Y)=\left\langle\nabla_{X} k, Y\right\rangle$ of $S_{p}$ along the null normal $\left.k\right|_{p}$ satisfy

$$
\gamma^{\Omega}(\bar{X}, \bar{Y})=\langle X+\alpha k, Y+\beta k\rangle=\langle X, Y\rangle=\gamma_{S_{p}}(X, Y),
$$

and

$$
K^{\Omega}(\bar{X}, \bar{Y})=\gamma^{\Omega}\left(K^{\Omega}(\bar{X}), \bar{Y}\right)=\gamma^{\Omega}\left(\overline{\nabla_{X} k}, \bar{Y}\right)=\left\langle\nabla_{X} k, Y\right\rangle=K_{S_{p}}^{k}(X, Y),
$$

where $X, Y \in T_{p} S_{p}$. In other words, the induced metric and the extrinsic geometry along $k$ of any embedded spacelike surface in $\Omega$ depends only on $p$ and not on the details of how $S_{p}$ is embedded in $\Omega$. For this reason we use from now on $K^{\Omega}$ or $K^{k}$, and $\gamma^{\Omega}$ or $\gamma_{S}=\gamma$ indistinctly.

Let $s: \Omega \rightarrow \mathbb{R}$ be a function satisfying $k(s)=1$. The level sets of this function determine a foliation $\left\{S_{s}\right\}$ of spacelike surfaces in $\Omega$. The choice of future null normal $\ell$ transverse to $S_{s}$ and satisfying $\langle k, \ell\rangle=-\varphi$ is unique. Let us consider on each $S_{s}$ the tensor $K^{\ell}(X, Y)=\left\langle\nabla_{X} \ell, Y\right\rangle$, its trace $\theta_{\ell}$, and the connection one-form

$$
s_{\ell}(X)=\frac{1}{\varphi}\left\langle\nabla_{X} k, \ell\right\rangle .
$$

The values of $\boldsymbol{s}_{\boldsymbol{\ell}}$ determine completely the normal connection $\nabla^{\perp}$ and viceversa. Indeed, the normal connection is completely defined when both the values of $\nabla_{X}^{\frac{1}{X}} k$ and $\nabla_{X}^{\frac{1}{X}} \ell$ are given for any $X \in \Gamma\left(T S_{s}\right)$. Moreover, since $k$ is null, it necessarily follows that $\left\langle\nabla \frac{1}{X} k, k\right\rangle_{g}=0$ and hence $\nabla^{\frac{1}{X}} k$ must be a multiple of $k$. Using now $\langle k, \ell\rangle_{g}=-\varphi$, we directly have from the definition of $s_{\boldsymbol{\ell}}(X)$

$$
\nabla_{X}^{\perp} k=-s_{\ell}(X) k \text {. }
$$

An analogous reasoning for $\ell$ yields

$$
\nabla_{X}^{\perp} \ell=\left(\frac{X(\varphi)}{\varphi}+s_{\ell}(X)\right) \ell
$$

Note that the connection one-form $s_{\boldsymbol{\ell}}$ plays the role of the Christoffel symbols of the connection.

Unlike $\boldsymbol{K}^{\Omega}, \theta_{k}$ and $\boldsymbol{\gamma}^{\Omega}$, the tensors $K^{\ell}, \theta_{\boldsymbol{\ell}}$ and $\boldsymbol{s}_{\boldsymbol{\ell}}$ are not intrinsic properties of the null hypersurface $\Omega$, i.e. their values depend on how the spacelike surface $S_{s}$ is embedded in $\Omega$. The next task is to compute the derivatives of these tensors along $k$. Let us begin with the extrinsic curvature $K_{A B}^{\ell}$ :

$$
k\left(K_{A B}^{\ell}\right)=k\left(\left\langle\nabla_{X_{A}} \ell, X_{B}\right\rangle\right)=\left\langle\nabla_{k} \nabla_{X_{A}} \ell, X_{B}\right\rangle+\left\langle\nabla_{X_{A}} \ell, \nabla_{k} X_{B}\right\rangle .
$$

Using now that $\left[k, X_{A}\right]=0$ and $R\left(k, X_{A}\right) \ell=\nabla_{k} \nabla_{X_{A}} \ell-\nabla_{X_{A}} \nabla_{k} \ell$ we obtain

$$
k\left(K_{A B}^{\ell}\right)=\left\langle\nabla_{X_{A}} \nabla_{k} \ell+R\left(k, X_{A}\right) \ell, X_{B}\right\rangle+\left\langle\nabla_{X_{A}} \ell, \nabla_{X_{B}} k\right\rangle .
$$




\section{B.6. Flow of surfaces along $\Omega$. Evolution equations}

From the definition of $s_{\boldsymbol{\ell}}$ and $K_{A B}^{k}, \nabla_{X_{B}} k=-\left(s_{\ell}\right)_{B} k+\left(K^{k}\right)^{L}{ }_{B} X_{L}$, which inserted into (B.21) gives

$k\left(K_{A B}^{\ell}\right)=\left\langle\nabla_{X_{A}} \nabla_{k} \ell, X_{B}\right\rangle-\operatorname{Riem}^{g}\left(X_{A}, k, X_{B}, \ell\right)-\left(s_{\ell}\right)_{B}\left\langle\nabla_{X_{A}} \ell, k\right\rangle+\left(K^{k}\right)_{B}^{L}\left(K^{\ell}\right)_{L A}$.

Since $\langle k, \ell\rangle=-\varphi$, then

$$
\left\langle\nabla_{X_{A}} \ell, k\right\rangle=-\varphi_{, A}-\left\langle\nabla_{X_{A}} k, \ell\right\rangle=-\varphi_{, A}-\varphi\left(s_{\ell}\right)_{A}
$$

and consequently

$$
\begin{aligned}
k\left(K_{A B}^{\ell}\right)= & \left\langle\nabla_{X_{A}} \nabla_{k} \ell, X_{B}\right\rangle-\operatorname{Riem}^{g}\left(X_{A}, k, X_{B}, \ell\right)+\left(s_{\ell}\right)_{B} \varphi_{, A}+\varphi\left(s_{\ell}\right)_{A}\left(s_{\ell}\right)_{B} \\
& +\left(K^{k}\right)^{L}{ }_{B}\left(K^{\ell}\right)_{L A} .
\end{aligned}
$$

We next work out the term $\left\langle\nabla_{X_{A}} \nabla_{k} \ell, X_{B}\right\rangle$. To compute $\nabla_{k} \ell$ we simply note that $\left\langle k, \nabla_{k} \ell\right\rangle=\nabla_{k}\langle k, \ell\rangle-\left\langle\nabla_{k} k, \ell\right\rangle=-k(\varphi)+\varphi Q_{k}$ so that, combined with (B.20) and the fact that $\ell$ is null,

$$
\nabla_{k} \ell=\left(\frac{k(\varphi)}{\varphi}-Q_{k}\right) \ell-\varphi\left(s_{\ell}\right)^{L} X_{L}
$$

and then

$$
\begin{aligned}
\left\langle\nabla_{X_{A}} \nabla_{k} \ell, X_{B}\right\rangle= & \left\langle\nabla_{X_{A}}\left(\left(\frac{k(\varphi)}{\varphi}-Q_{k}\right) \ell-\varphi\left(s_{\ell}\right)^{L} X_{L}\right), X_{B}\right\rangle \\
= & \left(\frac{k(\varphi)}{\varphi}-Q_{k}\right) K_{A B}^{\ell}-\left\langle\varphi, A\left(s_{\ell}\right)^{L} X_{L}, X_{B}\right\rangle-\left\langle\varphi X_{A}\left(\left(s_{\ell}\right)^{L}\right) X_{L}, X_{B}\right\rangle \\
& \left.-\left\langle\nabla_{X_{A}} X_{L}, X_{B}\right\rangle\right) .
\end{aligned}
$$

Denoting by $\nabla^{S}$ the induced connection on each $S_{s}$, we have

$$
\left\langle\nabla_{X_{A}} X_{L}, X_{B}\right\rangle=\left\langle\nabla_{X_{A}}^{S} X_{L}, X_{B}\right\rangle=\Gamma_{A L}^{D} \gamma_{D B}
$$

where $\Gamma_{A L}^{D}$ are the Christoffel symbols of $\gamma_{S}$. This allows us to rewrite (B.24) as

$$
\left\langle\nabla_{X_{A}} \nabla_{k} \ell, X_{B}\right\rangle=\left(\frac{k(\varphi)}{\varphi}-Q_{k}\right) K_{A B}^{\ell}-\varphi_{, A}\left(s_{\ell}\right)_{B}-\varphi \nabla_{A}^{S}\left(s_{\ell}\right)_{B}
$$

Inserting (B.25) into (B.22) finally yields

$$
\begin{aligned}
k\left(K_{A B}^{\ell}\right)= & \left(\frac{k(\varphi)}{\varphi}-Q_{k}\right) K_{A B}^{\ell}+\left(K^{k}\right)_{B}^{L}\left(K^{\ell}\right)_{L A}-\operatorname{Riem}^{g}\left(X_{A}, k, X_{B}, \ell\right) \\
& +\varphi\left(\left(s_{\ell}\right)_{A}\left(s_{\ell}\right)_{B}-\nabla_{A}^{S}\left(s_{\ell}\right)_{B}\right),
\end{aligned}
$$

which is the evolution equation for the null second fundamental form along $\ell$. 


\section{B. Geometry of null hypersurfaces}

We next compute the derivative of $\theta_{\ell}$ along $k$. This follows directly from (B.14) and (B.26):

$$
\begin{aligned}
k\left(\theta_{\ell}\right)= & k\left(\gamma^{A B} K_{A B}^{\ell}\right) \\
= & -\left(K^{k}\right)^{A B}\left(K^{\ell}\right)_{A B}+\left(\frac{k(\varphi)}{\varphi}-Q_{k}\right) \theta_{\ell}-\gamma^{A B} \operatorname{Riem}^{g}\left(X_{A}, k, X_{B}, \ell\right) \\
& +\varphi\left(\left|s_{\ell}\right|_{\gamma}^{2}-\nabla_{A}^{N}\left(s_{\ell}\right)^{A}\right) .
\end{aligned}
$$

Expression $g^{\#}$ in $(\overline{B .16})$ can be used to compute the third term in the above expression as

$$
\begin{aligned}
\gamma^{A B} \operatorname{Riem}^{g}\left(X_{A}, k, X_{B}, \ell\right)= & \left(g^{\#}+\frac{1}{\varphi} k \otimes \ell+\frac{1}{\varphi} \ell \otimes k\right)\left(\operatorname{Riem}^{g}(\cdot, k, \cdot, \ell)\right)= \\
& =\operatorname{Ric}^{g}(k, \ell)-\frac{1}{\varphi} \operatorname{Riem}^{g}(k, \ell, k, \ell),
\end{aligned}
$$

and (B.27) becomes

$$
\begin{aligned}
k\left(\theta_{\ell}\right)= & \left(\frac{k(\varphi)}{\varphi}-Q_{k}\right) \theta_{\ell}-\left(K^{k}\right)^{A B}\left(K^{\ell}\right)_{A B}-\operatorname{Ric}^{g}(k, \ell)+\frac{1}{\varphi} \operatorname{Riem}^{g}(k, \ell, k, \ell) \\
& +\varphi\left(\left|s_{\ell}\right|_{\gamma}^{2}-\nabla_{A}^{N}\left(s_{\ell}\right)^{A}\right) .
\end{aligned}
$$

Solving for $\operatorname{Riem}^{g}(k, \ell, k, \ell)$ in the contracted Gauss identity (A.30), which relates the ambient scalar curvature $\mathrm{Scal}^{g}$ and the induced scalar curvature $\mathrm{Scal}^{S}$, and substituting in (B.28) finally yields

$$
k\left(\theta_{\ell}\right)=\left(\frac{k(\varphi)}{\varphi}-Q_{k}\right) \theta_{\ell}+\operatorname{Ein}^{g}(k, \ell)-\theta_{k} \theta_{\ell}-\frac{\varphi}{2} \operatorname{Scal}^{S}+\varphi\left(\left|s_{\ell}\right|_{\gamma}^{2}-\nabla_{A}^{N}\left(s_{\ell}\right)^{A}\right) .
$$

The last evolution equation we compute is the derivative along $k$ of the one-form $\boldsymbol{s}_{\boldsymbol{\ell}}$ :

$$
k\left(\left(s_{\ell}\right)_{A}\right)=k\left(\frac{1}{\varphi}\left\langle\nabla_{X_{A}} k, \ell\right\rangle\right)=\frac{-k(\varphi)}{\varphi}\left(s_{\ell}\right)_{A}+\frac{1}{\varphi}\left(\left\langle\nabla_{k} \nabla_{X_{A}} k, \ell\right\rangle+\left\langle\nabla_{X_{A}} k, \nabla_{k} \ell\right\rangle\right)(\text { B }
$$

Using $\nabla_{k} \nabla_{X_{A}} k=\nabla_{X_{A}} \nabla_{k} k+R\left(k, X_{A}\right) k$ in the second derivative term, and the geodesic relation $\nabla_{k} k=Q_{k} k$, implies

$$
\left\langle\nabla_{k} \nabla_{X_{A}} k, \ell\right\rangle=-\varphi\left(Q_{k}\right)_{, A}+\varphi Q_{k}\left(s_{\ell}\right)_{A}+R\left(\ell, k, k, X_{A}\right) .
$$

Using again expression (B.23),

$$
\left\langle\nabla_{X_{A}} k, \nabla_{k} \ell\right\rangle=\left(\frac{k(\varphi)}{\varphi}-Q_{k}\right) \varphi\left(s_{\ell}\right)_{A}-\varphi\left(s_{\ell}\right)^{L} K_{L A}^{k}
$$

Inserting $(\mathrm{B} .31)$ and $(\mathrm{B} .32)$ into $(\mathrm{B} .30)$ gives

$$
k\left(\left(s_{\ell}\right)_{A}\right)=-\left(Q_{k}\right)_{A}+\frac{1}{\varphi} R\left(\ell, k, k, X_{A}\right)-\left(s_{\ell}\right)^{L} K_{L A}^{k} .
$$




\section{B.6. Flow of surfaces along $\Omega$. Evolution equations}

As before, the curvature term in this expansion can be rewritten in terms of the Einstein tensor. To do so, we use the Codazzi equation (A.26), with $\eta=k$

$$
\operatorname{Riem}^{g}(Y, X, Z, k)=\left\langle\left(\tilde{\nabla}_{Y} \vec{K}\right)(X, Z), k\right\rangle-\left\langle\left(\tilde{\nabla}_{X} \vec{K}\right)(Y, Z), k\right\rangle .
$$

We need to compute the derivative of the second fundamental form vector. We first decompose $\vec{K}$ in the null basis $\{k, \ell\}$ as $\vec{K}=\frac{-1}{\varphi}\left(K^{\ell} k+K^{k} \ell\right)$. From the definition of the covariant derivative $\tilde{\nabla}$ in (A.23), where $\nabla^{N}$ is replaced by the connection $\nabla^{S}$ of $S_{s}$, and using that $\langle\vec{K}, k\rangle=K^{k}$, we have

$$
\left\langle\left(\tilde{\nabla}_{X} \vec{K}\right)(Y, Z), k\right\rangle=\left\langle\nabla_{X} \vec{K}(Y, Z), k\right\rangle-K^{k}\left(\nabla_{X}^{S} Y, Z\right)-K^{k}\left(Y, \nabla_{X}^{S} Z\right) .
$$

Working out the first term, we obtain

$$
\begin{aligned}
\left\langle\nabla_{X} \vec{K}(Y, Z), k\right\rangle & =\left\langle\nabla_{X}\left(-\frac{1}{\varphi}\left(K^{\ell}(Y, Z) k+K^{k}(Y, Z) \ell\right)\right), k\right\rangle \\
& =\left\langle\nabla_{X}\left(-\frac{1}{\varphi}\left(K^{k}(Y, Z) \ell\right)\right), k\right\rangle \\
& =\frac{-X(\varphi)}{\varphi} K^{k}(Y, Z)+X\left(K^{k}(Y, Z)\right)-\frac{1}{\varphi} K^{k}(Y, Z)\left\langle\nabla_{X} \ell, k\right\rangle \\
& =X\left(K^{k}(Y, Z)\right)+K^{k}(Y, Z) s_{\ell}(X),
\end{aligned}
$$

where we used $\left\langle\nabla_{X} \ell, k\right\rangle=-X(\varphi)-\varphi s_{\ell}(X)$. Inserting (B.36) into (B.35) and using $\left(\nabla_{X} K^{k}\right)(Y, Z):=X\left(K^{k}(Y, Z)\right)-K^{k}\left(\nabla_{X}^{S} Y, Z\right)-K^{k}\left(Y, \nabla_{X}^{S} Z\right)$, it follows

$$
\left\langle\left(\tilde{\nabla}_{X} \vec{K}\right)(Y, Z), k\right\rangle=\left(\nabla_{X} K^{k}\right)(Y, Z)+K^{k}(Y, Z) s_{\ell}(X)
$$

so that (B.34) becomes

$$
\begin{aligned}
\operatorname{Riem}^{g}(Y, X, Z, k)= & \left(\nabla_{Y} K^{k}\right)(X, Z)-\left(\nabla_{X} K^{k}\right)(Y, Z)+K^{k}(X, Z) s_{\boldsymbol{\ell}}(Y) \\
& -K^{k}(Y, Z) s_{\boldsymbol{\ell}}(X),
\end{aligned}
$$

or, in index notation

$$
\operatorname{Riem}^{g}\left(X_{A}, X_{B}, X_{C}, k\right)=\nabla_{A}^{S} K_{B C}^{k}-\nabla_{B}^{S} K_{A C}^{k}+K_{B C}^{k}\left(s_{\ell}\right)_{A}-K_{A C}^{k}\left(s_{\ell}\right)_{B} .
$$

Taking the trace we obtain

$$
\operatorname{Ein}^{g}\left(X_{A}, k\right)+\frac{1}{\varphi} \operatorname{Riem}^{g}\left(I, k, k, X_{A}\right)=\nabla_{L}^{S}\left(K^{k}\right)^{L}{ }_{A}-\left(\theta_{k}\right)_{, A}+K_{A L}^{k}\left(s_{\ell}\right)^{L}-\theta_{k}\left(s_{\ell}\right)_{A},
$$

and solving for $\frac{1}{\varphi} \operatorname{Riem}^{g}\left(I, k, k, X_{A}\right)$ and substituting it in (B.33) gives the final form for the evolution equation of $s_{\boldsymbol{\ell}}$

$$
k\left(\left(s_{\ell}\right)_{A}\right)=-\left(Q_{k}\right)_{A}-\left(s_{\ell}\right)_{A} \theta_{k}+\nabla_{L}^{S}\left(K^{k}\right)_{A}^{L}-\left(\theta_{k}\right)_{A}-\operatorname{Ein}^{g}\left(X_{A}, k\right) .
$$




\section{B. Geometry of null hypersurfaces}

We finally compute the Lie derivative $£_{k} \boldsymbol{\eta}_{S_{s}}$. Let us choose a system of coordinates $\left\{x^{1}, \ldots, x^{n}\right\}$ on each $S_{s}$, such that the volume form gets the usual form

$$
\eta_{S_{s}}=\sqrt{\operatorname{det} \gamma(s)} d x^{1} \ldots d x^{n}
$$

Using now the Jacobi identity for the derivative of the determinant of a uniparametric family of matrices, that says that

$$
\frac{d}{d s} \operatorname{det} A(s)=\operatorname{det} A(s) \operatorname{tr}\left(A^{-1}(s) \frac{d A(s)}{d s}\right)
$$

and considering as well relation (B.12), we obtain

$$
\frac{d}{d s} \operatorname{det} \gamma(s)=\operatorname{det} \gamma(s) \operatorname{tr}\left(\gamma^{-1}(s)^{A L} \partial_{s} \gamma(s)_{L B}\right)=\operatorname{det} \gamma(s) \gamma^{-1}(s)^{A B} 2 K_{A B}^{k}=2 \operatorname{det} \gamma(s) \theta_{k} .
$$

We finally conclude, using (B.40)

$$
£_{k} \boldsymbol{\eta}_{S_{s}}=\frac{d}{d s} \sqrt{\operatorname{det} \gamma(s)} d x^{1} \ldots d x^{n}=\frac{1}{2 \sqrt{\operatorname{det} \gamma(s)}} 2 \operatorname{det} \gamma(s) \theta_{k} d x^{1} \ldots d x^{n}=\theta_{k} \boldsymbol{\eta}_{S_{s}} .
$$





\section{Geometry of convex bodies. The support function}

This appendix is devoted to the study of the relation of the geometry of a convex body in $\mathbb{R}^{n}$ with the geometry of the standard sphere. Using the Gauss map as the link that connects both geometries, and studying some of the main properties of convex functions and in particular using the so-called support function of a convex body, we will be able to rewrite the first and second fundamental form of the convex body in terms of the support function and geometric objects naturally defined on the sphere. The main reference for this appendix is Schneider [94].

\section{C.1 Mapping a convex body onto the unit sphere}

Let $\mathcal{C}^{n}$ be the set of convex bodies (non-empty, compact, convex subsets) of $\mathbb{R}^{n}$. A set $A \subset \mathbb{R}^{n}$ is convex if together with any two points $x, y$ it contains the segment $[x, y]$, that is to say, $(1-\lambda) x+\lambda y \in A$ for $x, y \in A, 0 \leq \lambda \leq 1$. Let us now introduce some differentiability assumptions. A convex body $C \in \mathcal{C}^{n}$ is said to be of class $C^{k}$, for some $k \in \mathbb{N}$, if $\partial C$ (the boundary of $C$ ) is a regular submanifold of $\mathbb{R}^{n} k$-times continuously differentiable. $C$ is of class $C^{\infty}$ if it is of class $C^{k}$ for each $k \in \mathbb{N}$. $C$ is of class $C^{1}$ if and only if it has a unique tangent plane at each boundary point. The following theorem will be proved at the end of this appendix and requires the use of the support function of $C, h_{C}: \mathbb{R}^{n} \rightarrow \mathbb{R}$ (and in particular the support function of $\partial C, h: \partial C \rightarrow \mathbb{R}$, defined in terms of the previous) which will be defined later. The theorem states the following:

Theorem C.1.1. Let $C \in \mathcal{C}^{n}$ be of class $C^{2}$. Consider $\gamma_{A B}$ and $K_{A B}$ the first and second fundamental form of $\partial C$ as an embedded hypersurface of $\mathbb{R}^{n}$, and $h: \partial C \rightarrow \mathbb{R}$ the support function of $\partial C$. Then $\gamma_{A B}$ and $K_{A B}$ can be rewritten in terms of $h$ as 


\section{C.1. Mapping a convex body onto the unit sphere}

follows:

$$
\begin{aligned}
K_{A B} & =\stackrel{\circ}{\nabla}_{A} \stackrel{\circ}{\nabla}_{B} h+\stackrel{\circ}{q}_{A B} h, \\
\gamma_{A B} & =\left(\stackrel{\circ}{q}^{-1}\right)^{L M} K_{L A} K_{M B},
\end{aligned}
$$

where $\stackrel{9}{A B}_{A B}$ denotes the pullback of the standard metric of the unit sphere onto $\partial C$ via de Gauss map, and $\stackrel{\circ}{\nabla}$ its corresponding Levi-Civita connection.

Remark C.1.2. Throughout this appendix we will use for tensors the index notation (e.g. $\left.K_{A B}\right)$ and the alternative notation $K(X, Y)$ indistinctly.

Recall that for an embedded manifold $N$ in $M, p \in N$, and an orthogonal vector field $\eta$ along $N$, the Weingarten map $\mathcal{W}_{\eta}: T_{p} N \rightarrow T_{p} N$ is defined as the endomorphism

$$
\mathcal{W}_{\eta}(X)=\left(\nabla_{X} \eta\right)^{\top}
$$

Let $C \in \mathcal{C}^{n}$ be of class $C^{2}$. For $p \in \partial C$, let $\nu(p)$ be the outward unit normal vector of $\partial C$ at $p$. In this particular case where $\eta:=\nu$ has constant norm $(|\nu|=1)$, we have

$$
\mathcal{W}_{\nu}(X)=\left(\nabla_{X} \nu\right)^{\top}=\nabla_{X} \nu
$$

Consider the embedding $\phi$ of the abstract sphere $\mathbb{S}^{n-1}$ in $\mathbb{R}^{n}$. Since $C \in \mathcal{C}^{n}$ is of class $C^{2}$, the map $\nu: \partial C \rightarrow \phi\left(S^{n-1}\right)$, that assigns to each $p \in \partial C$ the unique point $\phi(u) \in \phi\left(\mathbb{S}^{n-1}\right)$ with $u \in \mathbb{S}^{n-1}$ so that the vector $\nu(p)$ and the one defined by the origin and $\phi(u)$ are parallel in the standard way in the Euclidean space, is well-defined and is in fact of class $C^{1}$. This is called spherical image map or Gauss map of $\partial C$. Observe that in this context $\nu$ will be used both to denote the unit normal to $\partial C$ and to denote the map going from $\partial C$ to the embedded unit sphere. In spite of this duality no confusion arises since the situation will be clear by the context. The map $\nu$ induces another map $\nu_{C}: \partial C \rightarrow \mathbb{S}^{n-1}$ to the abstract sphere. We will refer to $\nu_{C}$ as the abstract Gauss map, and it is defined by $\nu_{C}(p)=u$, or equivalently $\nu=\phi \circ \nu_{C}$.

As in Chapter [5, given two points $p_{1}$ and $p_{2}$ of $\mathbb{R}^{n}$, we denote by $\mathcal{T}_{p_{1} \rightarrow p_{2}}$ the map that transports parallely a vector from $T_{p_{1}} \mathbb{R}^{n}$ to $T_{p_{2}} \mathbb{R}^{n}$. The differential $d \nu$ of the Gauss map $\nu$ is the Weingarten map $\mathcal{W}_{\nu}$ when the vectors of $T_{\phi(u)} \phi\left(\mathbb{S}^{n-1}\right)$ and $T_{p} \partial C$ are identified by parallel transport. In other words, the Weingarten map $\mathcal{W}_{\nu}$ acting on $X$ at $p \in \partial C$ can also be expressed in the following way

$$
\left.\mathcal{W}_{\nu}(X)\right|_{p}=\mathcal{T}_{\phi(u) \rightarrow p}\left(d \nu_{p}(X)\right)
$$

The equivalence of this expression with (C.1) is direct in Cartesian coordinates $\left\{x^{A}\right\}$, where $\left(\nabla_{\partial_{x^{A}}} \nu\right)^{B}=d \nu^{B}\left(\partial_{x^{A}}\right)$. The second fundamental form of $\partial C$ at $p$ is defined in terms of the Weingarten map as

$$
K_{p}(X, Y)=\left.\left\langle\mathcal{W}_{\nu}(X), Y\right\rangle\right|_{p}
$$




\section{Geometry of convex bodies. The support function}

with $X, Y \in T_{p} \partial C$.

In local coordinates, we can describe the constructions above as follows. Let $U \subset \mathbb{R}^{n-1}$ be an open set, and $X: U \rightarrow \partial C \subset \mathbb{R}^{n}$ a local parametrization of class $C^{2}$ of a neighbourhood of $p$ on $\partial C$, where $p=X(y)$ with $y \in U$. Then $N=\nu \circ X: U \rightarrow$ $\phi\left(S^{n-1}\right) \subset \mathbb{R}^{n}$ is a local parametrization of a neighbourhood of $\phi(u)$ on $\phi\left(S^{n-1}\right)$, and is a map of class $C^{1}$ (see Figure C.1). Therefore

$$
d N_{y}=\left.d \nu_{p} \circ d X_{y} \Rightarrow \mathcal{W}_{\nu}\right|_{p} \equiv d \nu_{p}=d N_{y} \circ d X_{y}^{-1}
$$

Consider a basis $\left\{e_{A}\right\}$ of $T_{y} U$. Let us define $X_{A}:=d X\left(e_{A}\right)$ and $N_{A}:=d N\left(e_{A}\right)$. From (C.3) we have

$$
\mathcal{W}_{p}\left(X_{A}\right)=\mathcal{T}_{\phi(u) \rightarrow p} N_{A}
$$

For the sake of simplicity we will simply write $\mathcal{W}_{p}\left(X_{A}\right)=N_{A}$ when it is clear from the context. The second fundamental form and the metric of $\partial C$ read respectively

$$
\begin{aligned}
K_{A B} & =K\left(X_{A}, X_{B}\right)=\left\langle\mathcal{W}_{\nu}\left(X_{A}\right), X_{B}\right\rangle=\left\langle N_{A}, X_{B}\right\rangle \\
\gamma_{A B} & =\left\langle X_{A}, X_{B}\right\rangle .
\end{aligned}
$$

Since $\left.\mathcal{W}_{\nu}\right|_{p}$ is an endomorphism on $T_{p} \partial C, \mathcal{W}_{\nu}\left(X_{A}\right)$ can be expressed in terms of functions $\mathcal{W}_{\nu}{ }^{L}{ }_{A}$ defined on $\partial C$ and the basis $X_{A}$, namely

$$
\mathcal{W}_{\nu}\left(X_{A}\right)=\mathcal{W}_{\nu}{ }_{A}{ } X_{L}
$$

For this reason

$$
K_{A B}=\left\langle\mathcal{W}_{\nu}\left(X_{A}\right), X_{B}\right\rangle=\left\langle\mathcal{W}_{\nu}{ }^{L}{ }_{A} X_{L}, X_{B}\right\rangle=\mathcal{W}_{\nu}{ }^{L}{ }_{A} \gamma_{L B},
$$

which is just the rewriting of (C.2) in components.

The following assumption, stronger that $C^{k}$, will also be important. We say that $C$ is of class $C_{+}^{k}$ (for $k \geq 2$ ) if $C$ is of class $C^{k}$ and the Gauss map $\nu: \partial C \rightarrow \phi\left(\mathbb{S}^{n-1}\right)$ is a diffeomorphism (of class $C^{1}$, and hence $C^{k-1}$ ). This is equivalent to the assumption that its differential, the Weingarten map $\mathcal{W}_{\nu}$, is everywhere of maximal rank, and thus to the assumption that all the principal curvatures (the eigenvalues of the Weingarten map at a point) are non-zero.

Let $C$ be of class $C_{+}^{2}$. Then the map $\nu$ has an inverse $\nu^{-1}$ of class $C^{1}$ :

$$
\nu^{-1}: \phi\left(\mathbb{S}^{n-1}\right) \rightarrow \partial C
$$

which is known as the inverse Gauss map. Using its differential

$$
d \nu^{-1}: T_{\phi(u)} \phi\left(\mathbb{S}^{n-1}\right) \rightarrow T_{p} \partial C
$$




\section{C.1. Mapping a convex body onto the unit sphere}

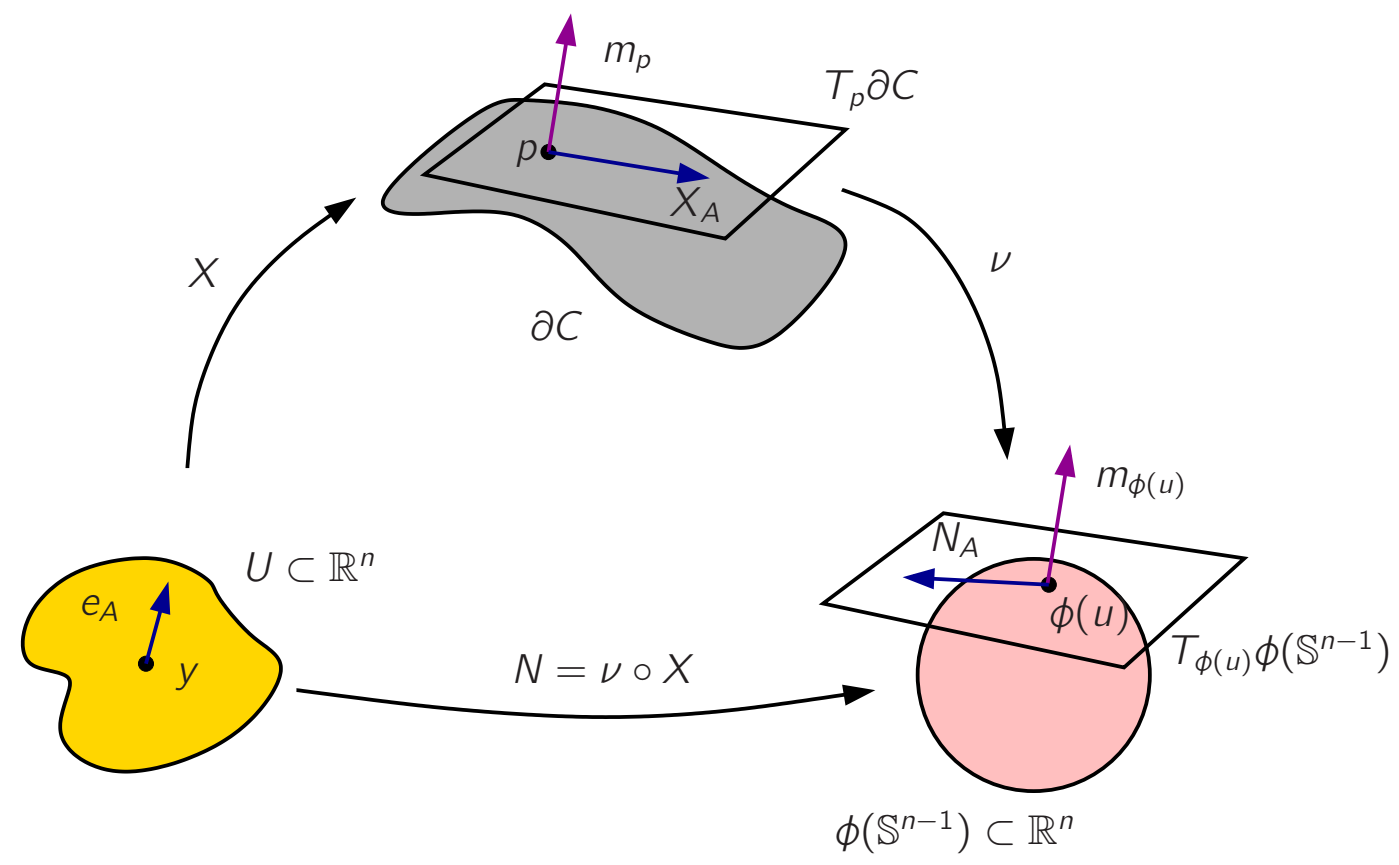

Figure C.1: Schematic figure representing the construction above, where a local parametrization of the boundary of a convex body $C$ and of the embedded sphere $\phi\left(\mathbb{S}^{n-1}\right)$ is given, as well as the Gauss map $\nu$ that maps diffeomorphically a neighbourhood of $p \in \partial C$ to a neighbourhood of $\phi(u) \in \phi\left(\mathbb{S}^{n-1}\right) . U \subset \mathbb{R}^{n}$ is an open set, $X: U \rightarrow \partial C \subset \mathbb{R}^{n}$ is a local parametrization of class $C^{2}$ of a neighbourhood of $p \in \partial C$, with $p=X(y)$ and $y \in U . N: U \rightarrow \phi\left(\mathbb{S}^{n-1}\right) \subset \mathbb{R}^{n}$ is a local parametrization of a neighbourhood of $\phi(u)$ on $\phi\left(\mathbb{S}^{n-1}\right)$, and is a map of class $C^{1}$. The map $\nu: \partial C \rightarrow \phi\left(\mathbb{S}^{n-1}\right) \subset \mathbb{R}^{n}$ is the Gauss map of $\partial C$. The image of $p$ by $\nu$ is $\phi(u)$, so that the respective outer unit normals $m_{p}$ and $m_{\phi(u)}$ are parallel in the standard way in $\mathbb{R}^{n}$.

we can define the reverse Weingarten map endomorphism $\mathcal{B}$ acting on vectors of $T_{\phi(u)} \phi\left(\mathbb{S}^{n-1}\right)$ as

$$
\mathcal{B}_{\phi(u)}(v):=\left.\mathcal{T}_{p \rightarrow \phi(u)} d \nu^{-1}\right|_{\phi(u)}(v),
$$

from where $\mathcal{B}_{\phi(u)}\left(N_{A}\right)=\left.\mathcal{T}_{p \rightarrow \phi(u)} d \nu^{-1}\right|_{\phi(u)}\left(N_{A}\right)=\mathcal{T}_{p \rightarrow \phi(u)} X_{A}$. For the sake of simplicity and for the same reasons as before we will simply write

$$
\mathcal{B}\left(N_{A}\right)=X_{A}
$$

The reverse second fundamental form $B$ of $\partial C$ is defined in terms of this map as

$$
B(v, w)=\langle\mathcal{B}(v), w\rangle, \quad v, w \in T_{\phi(u)} \phi\left(\mathbb{S}^{n-1}\right) .
$$

Applying this tensor on vectors of the basis $\left\{N_{A}\right\}$ we obtain

$$
B\left(N_{A}, N_{B}\right)=\left\langle\mathcal{B}\left(N_{A}\right), N_{B}\right\rangle=\left\langle X_{A}, N_{B}\right\rangle=K\left(X_{B}, X_{A}\right)=K\left(X_{A}, X_{B}\right),
$$




\section{Geometry of convex bodies. The support function}

where in the last equality the symmetry of the second fundamental form has been used. Since $N_{A}=d \nu\left(X_{A}\right)$, it follows

$$
K=\nu^{*}(B) .
$$

Consider the spherical metric $\stackrel{\circ}{q}_{A B}=\left\langle N_{A}, N_{B}\right\rangle$ and the functions $\mathcal{B}_{A}^{L}$ defined by

$$
X_{A}=\mathcal{B}\left(N_{A}\right)=\mathcal{B}^{L}{ }_{A} N_{L} \text {. }
$$

Then

$$
B_{A B}=\left\langle\mathcal{B}\left(N_{A}\right), N_{B}\right\rangle=\left\langle X_{A}, N_{B}\right\rangle=\left\langle\mathcal{B}^{L}{ }_{A} N_{L}, N_{B}\right\rangle=\mathcal{B}^{L}{ }_{A} \stackrel{\circ}{q B}_{L B}
$$

which means that the spherical metric raises and lowers the indices of the reverse Weingarten map.

\section{C.2 Convex functions. The support function}

There is an interesting relation between convex sets and convex functions. Let us first define what a convex function is:

Definition C.2.1 (Convex function). A function $f: \mathbb{R}^{n} \rightarrow \mathbb{R}$ is convex if

$$
f((1-\lambda) x+\lambda y) \leq(1-\lambda) f(x)+\lambda f(y),
$$

for all $x, y \in \mathbb{R}^{n}$ and $0 \leq \lambda \leq 1$.

The following theorems are general analytic results of convex functions and can be found for instance in [94]:

Theorem C.2.2. Let $f: \mathbb{R}^{n} \rightarrow \mathbb{R}$ be a convex function. Consider $x \in \mathbb{R}^{n}$. If $f$ has partial derivatives (of first order) at $x$, then $f$ is differentiable at $x$.

Theorem C.2.3. Let $f: \mathbb{R}^{n} \rightarrow \mathbb{R}$ be a convex function. Consider $x \in \mathbb{R}^{n}$. Then there exists

$$
f^{\prime}(x, u):=\lim _{\lambda \rightarrow 0^{+}} \frac{f(x+\lambda u)-f(x)}{\lambda} .
$$

We call $f^{\prime}(x, \cdot)$ the semidirectional derivative function of $f$ at $x$.

Remark C.2.4. The difference between semidirectional and the more standard directional derivative is that in the later $\lambda \rightarrow 0$ without restriction while in the former the limit is one sided $\lambda \rightarrow 0^{+}$.

A function $f: \mathbb{R}^{n} \rightarrow \mathbb{R}$ is called positively homogeneous if

$$
f(\lambda x)=\lambda f(x)
$$

for all $\lambda \geq 0$ and all $x \in \mathbb{R}^{n}$, and $f$ is called subadditive if

$$
f(x+y) \leq f(x)+f(y)
$$

for all $x, y \in \mathbb{R}^{n}$. 


\section{C.2. Convex functions. The support function}

Definition C.2.5 (Sublinear function). A sublinear function is a function that is positively homogeneous and subadditive. Every sublinear function is clearly convex.

The following result clarifies the relation between the semidirectional derivative functions of the convex functions and sublinear functions:

Lemma C.2.6. Let $f: \mathbb{R}^{n} \rightarrow \mathbb{R}$ be convex, and $x \in \mathbb{R}^{n}$. Then the semidirectional derivative function

$$
f^{\prime}(x ; \cdot): \mathbb{R}^{n} \rightarrow \mathbb{R}
$$

is sublinear.

Proof. Let $u \in \mathbb{R}^{n} \backslash\{0\}$. Consider $\lambda, \tau>0$. We may write

$$
\frac{f(x+\tau \lambda u)-f(x)}{\tau}=\lambda \frac{f(x+\tau \lambda u)-f(x)}{\tau \lambda} .
$$

When we take the limit $\tau \rightarrow 0^{+}$, we obtain

$$
f^{\prime}(x ; \lambda u)=\lambda f^{\prime}(x ; u) .
$$

Using the convexity of $f$, for $u, v \in \mathbb{R}^{n}$ we have

$$
f(x+\tau(u+v))=f\left(\frac{1}{2}(x+2 \tau u)+\frac{1}{2}(x+2 \tau v)\right) \leq \frac{1}{2} f(x+2 \tau u)+\frac{1}{2} f(x+2 \tau v) .
$$

Hence

$$
\lim _{\tau \rightarrow 0^{+}} \frac{f(x+\tau(u+v))-f(x)}{\tau} \leq \lim _{\tau \rightarrow 0^{+}} \frac{f(x+2 \tau u)-f(x)}{2 \tau}+\lim _{\tau \rightarrow 0^{+}} \frac{f(x+2 \tau v)-f(x)}{2 \tau},
$$

that is to say, $f^{\prime}(x ; u+v) \leq f^{\prime}(x ; u)+f^{\prime}(x ; v)$.

The following lemma shows that the semidirectional derivative function of a sublinear function is always upper bounded by the function itself:

Lemma C.2.7. Let $f: \mathbb{R}^{n} \rightarrow \mathbb{R}$ be sublinear. Let $x \in \mathbb{R}^{n}$. Then $f^{\prime}(x ; \cdot) \leq f$.

Proof. For $u \in \mathbb{R}^{n}$ and $\tau>0$, the sublinearity of $f$ yields

$$
f(x+\tau u) \leq f(x)+\tau f(u),
$$

which is equivalent to

$$
\frac{f(x+\tau u)-f(x)}{\tau} \leq f(u) .
$$

Taking the limit when $\tau \rightarrow 0^{+}$, we obtain $f^{\prime}(x ; u) \leq f(u)$. 


\section{Geometry of convex bodies. The support function}

A convex body can be described by real functions. One of them is the so-called support function. As we will see, the support function combined with the geometry of the standard sphere will determine the first and second fundamental forms of a convex body. Let us first introduce the definition of support function:

Definition C.2.8 (Support function). Let $C$ be a convex body. The support function $h(C, \cdot)=h_{C}$ of $C$ is:

$$
h(C, u)=\sup \{\langle x, u\rangle: x \in C\} \quad \text { for all } u \in \mathbb{R}^{n} .
$$

Remark C.2.9. The support function is a convex function. In fact it is sublinear, because it is both positively homogeneous

$$
h_{C}(\alpha u)=\sup \{\langle x, \alpha u\rangle: x \in C\}=\alpha \sup \{\langle x, u\rangle: x \in C\}=\alpha h_{C}(u), \quad \alpha \geq 0,
$$

and subadditive, since

$$
\begin{aligned}
h_{C}(u+v) & =\sup \{\langle x, u+v\rangle: x \in C\} \leq \sup \{\langle x, u\rangle: x \in C\}+\sup \{\langle x, v\rangle: x \in C\} \\
& =h_{C}(u)+h_{C}(v) .
\end{aligned}
$$

For a convex body $C$ (which recall is by definition compact), the supremum in the definition of $h(C, u)$ is attained and finite for each $u$.

The support function allows us to describe the geometry of a convex body in $\mathbb{R}^{n}$ (see Figure (C.2). Given any vector $u \in \mathbb{R}^{n} \backslash\{0\}$, there is just one plane orthogonal to $u$ which makes contact with $C$ for the first time and that leaves the body $C$ on the side where $u$ does not point. This plane is the support plane, denoted by $H(C, u)$, and is defined as

$$
H(C, u):=\left\{x \in \mathbb{R}^{n}:\langle x, u\rangle=h(C, u)\right\} .
$$

This plane separates the Euclidean space in two halfspaces. The one where the convex body $C$ lies is the supporting halfspace and will be denoted by $H^{-}(C, u)$. This halfspace is defined as

$$
H^{-}(C, u):=\left\{x \in \mathbb{R}^{n}:\langle x, u\rangle \leq h(C, u)\right\} .
$$

The intersection set of the support plane and the convex body will be denoted by $F(C, u)$ and called support set of $C$. Namely

$$
F(C, u):=H(C, u) \cap C .
$$

As described above, the support function specifies the position of the support planes, which determine the corresponding supporting halfspaces, and consequently the convex body, since such an object is always the intersection of all the supporting halfspaces.

The intuitive meaning of the support function is simple. Indeed for a unit vector $m \in \phi\left(\mathbb{S}^{n-1}\right)$, the number $h(C, m)$ is the signed distance of the support plane to $C$ 


\section{C.2. Convex functions. The support function}

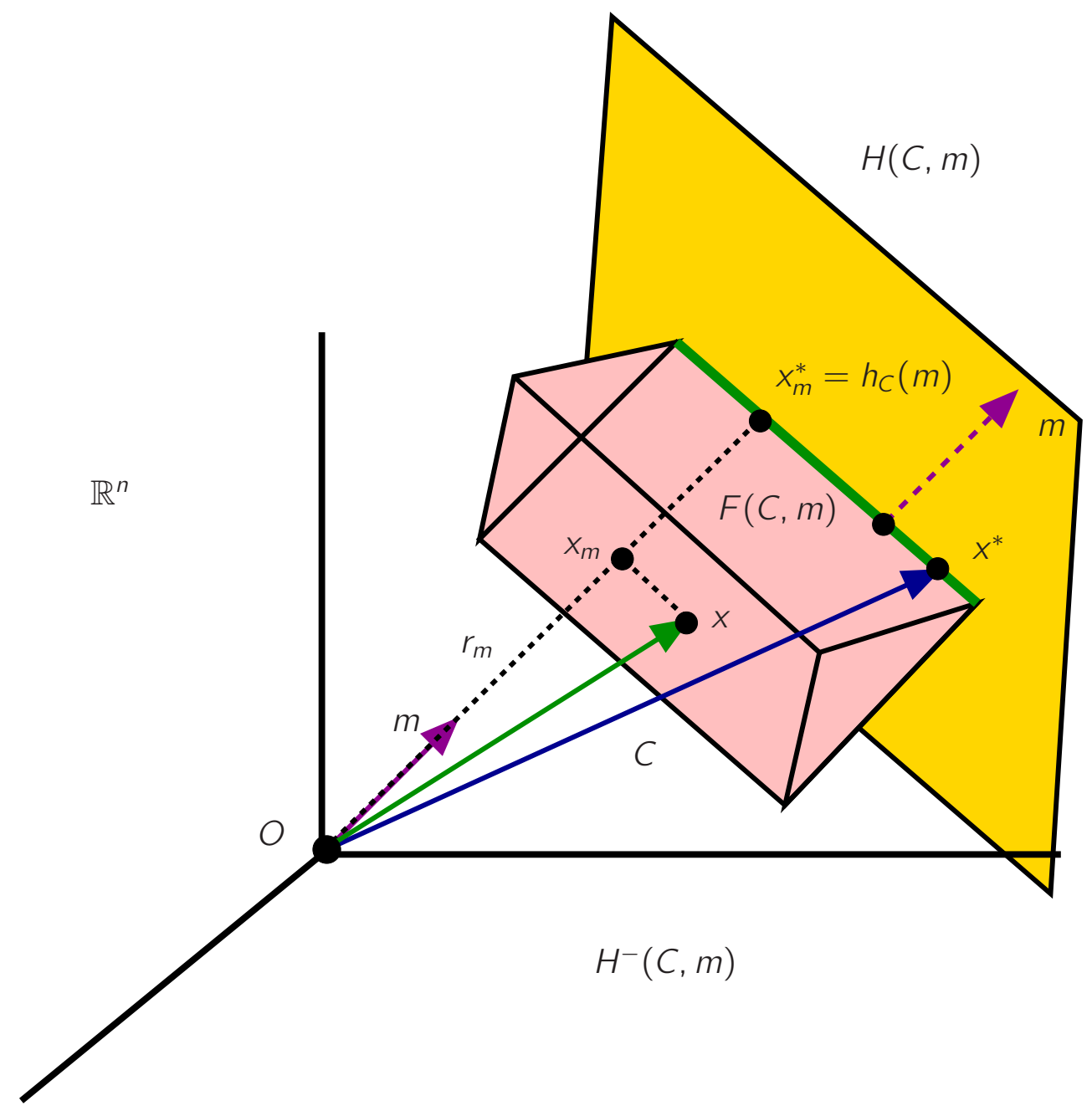

Figure C.2: Schematic figure representing the support function $h_{C}$ of the convex set $C$ not necessarily of class $C^{2}$ evaluated on a unit vector $m$. By definition, $h_{C}(m)=$ $\sup \{\langle x, m\rangle: x \in C\}=\sup \left\{x_{m}: x \in C\right\}$, where $x_{m}$ is the signed distance from the origin $O$ to the projection of $x$ onto the line $r_{m}$ generated by $m$. In this case, the supremum is attained at the points $x^{*} \in \partial C$ (it is not necessarily unique), and $F(C, m)=H(C, m) \cap C=\left\{x^{*} \in \partial C\right\}$. The signed distance $x_{m}^{*}$ is defined by $x_{m}^{*}:=$ $\left\langle x^{*}, m\right\rangle$ for any $x^{*} \in F(C, m)$ and coincides with $h_{C}(m)$. The support plane $H(C, m)$ is the first plane orthogonal to $m$ that touches $C$. We denote by $H^{-}(C, m)$ the halfspace defined by the support plane and that contains $C$.

with outer normal vector $m$ from the origin; the distance is negative if and only if $m$ points into the open halfspace containing the origin. Let us prove this. Let $r_{m}$ be the line generated by $m$ and containing the origin, and let us denote by $x_{m}:=\langle x, m\rangle$, i.e. the signed distance from the origin to the projection of $x$ onto $r_{m}$. The signed distance 


\section{Geometry of convex bodies. The support function}

$x_{m}$ will be $\geq 0$ if and only if $m$ points out of the supporting halfspace $\mathrm{H}^{-}(C, m)$, and $x_{m} \leq 0$ when the vector $m$ points in the direction of $H^{-}(C, m)$. Then

$$
h_{C}(m)=\sup \{\langle x, m\rangle: x \in C\}=\sup \left\{x_{m}: x \in C\right\} .
$$

This supremum will always be attained by the points $x^{*} \in C \cap H(C, m)=F(C, m)$, which are always contained in $\partial C$. Thus,

$$
h_{C}(m)=\sup \left\{x_{m}: x \in C\right\}=x_{m}^{*}=\left\langle x^{*}, m\right\rangle, \quad x^{*} \in F(C, m) \subset \partial C .
$$

From $x^{*} \in H(C, m) \cap C$, it follows that $x_{m}^{*}=\left\langle x^{*}, m\right\rangle$ is the signed distance from the tangent plane $H(C, m)$ to $C$ to the origin, as we wanted to prove.

The support function has the following properties:

Proposition C.2.10. For $C \in \mathcal{C}^{n}$ and $u \in \mathbb{R}^{n} \backslash\{0\}$, the following properties hold

(i) $h(C, \cdot)=\langle z, \cdot\rangle$ if and only if $C=\{z\}$,

(ii) $h(C+t, u)=h(C, u)+\langle t, u\rangle$ for $t \in \mathbb{R}^{n}$,

(iii) $h(\lambda C, \cdot)=\lambda h(C, \cdot)$ for $\lambda \geq 0$ and $h(-C, u)=h(C,-u)$,

(iv) $h_{C} \leq h_{L}$ if and only if $C \subset L$.

Proof. The first three properties are direct consequence of the definition of support function. For property (iv), assume first that $C \subset L$. Then for any $u \in \mathbb{R}^{n}$,

$$
h_{C}(u)=\sup \{\langle x, u\rangle: x \in C\} \leq \sup \{\langle x, u\rangle: x \in L\}=h_{L}(u),
$$

since the set where the second supremum is taken is larger than for the first one. Conversely assume that $C$ is not included in $L$. Consider the sets $\partial C \backslash L$ and $\partial L$, and note that $\partial C \backslash L$ is non-empty since $C$ is not contained in $L$. Define the function $d(\cdot, \partial L): \partial C \backslash L \rightarrow \mathbb{R}$, which gives the euclidean distance of a point $p \in \partial C \backslash L$ to $\partial L$. This function attains its maximum at some (possibly non-unique) point $p^{*} \in \partial C \backslash L$. Let $q^{*} \in \partial L$ satisfy $d\left(p^{*}, q^{*}\right)=d\left(p^{*}, \partial L\right)$ and consider the vector $\overline{q^{*} p^{*}}$, and the unit vector $m^{*}=\frac{\bar{q}^{*} p^{*}}{\left|q^{*} p^{*}\right|}$. By construction, $p^{*}$ is a contact point of $C$ with $H\left(C, m^{*}\right)$, i.e. $h_{C}\left(m^{*}\right)=\left\langle p^{*}, m^{*}\right\rangle$. It also holds $h_{L}\left(m^{*}\right)=\left\langle q^{*}, m^{*}\right\rangle$ since $q^{*}$ belongs to the set of contact points of $H\left(L, m^{*}\right)$ with $L$. Multiplying the relation $p^{*}=q^{*}+\overline{q^{*} p^{*}}$ by $m^{*}$, we obtain

$$
h_{C}\left(m^{*}\right)=h_{L}\left(m^{*}\right)+\left\langle\overline{q^{*} p^{*}}, m^{*}\right\rangle=h_{L}\left(m^{*}\right)+\left\langle\left|\overline{q^{*} p^{*}}\right| m^{*}, m^{*}\right\rangle=h_{L}\left(m^{*}\right)+\left|\overline{q^{*} p^{*}}\right|,
$$

where $\left|\overline{q^{*} p^{*}}\right|>0$. Thus, $h_{C}\left(m^{*}\right)>h_{L}\left(m^{*}\right)$. 


\section{C.2. Convex functions. The support function}

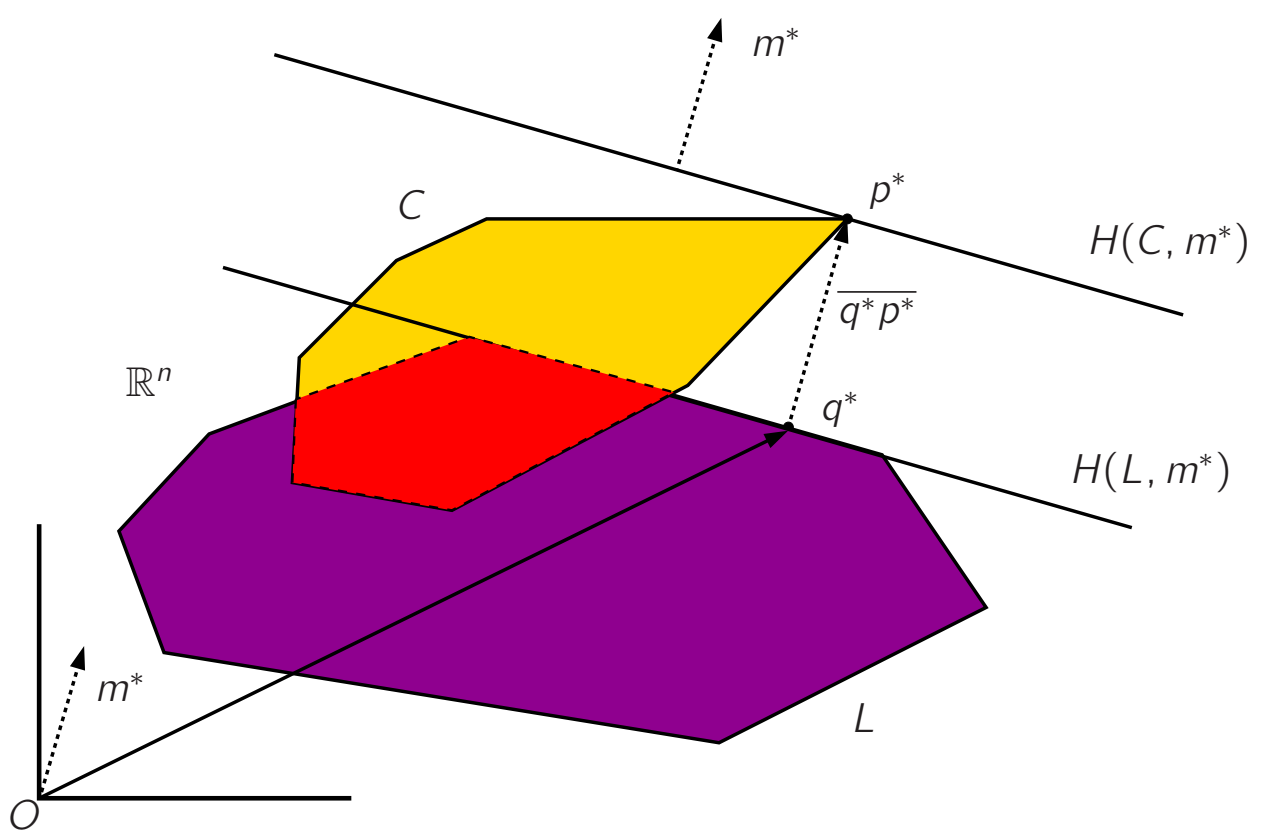

Figure C.3: Relation between the support functions of the convex sets $C$ and $L$ when $C$ is not included in $L$. The function $d(\cdot, \partial L): \partial C \backslash L \rightarrow \mathbb{R}$ attains its maximum at a point $p^{*} \in \partial C \backslash L$ and $q^{*} \in \partial L$ satisfies $d\left(p^{*}, q^{*}\right)=d\left(p^{*}, \partial L\right)$. The unit vector $m^{*}=\frac{\overline{q^{*} p^{*}}}{\left|q^{*} p^{*}\right|}$ define the support planes $H\left(C, m^{*}\right)$ and $H\left(L, m^{*}\right)$, which first touch $C$ at $p^{*}$ and $L$ at $q^{*}$, not being these points necessarily unique. The support functions satisfy in this case $h_{C}\left(m^{*}\right)>h_{L}\left(m^{*}\right)$.

Since the points $x^{*}$ always lie on $\partial C$, it makes sense to define the support function of a closed, convex and connected hypersurface $S \subset \mathbb{R}^{n}$ which is the boundary of a convex body $C$ of class $C_{+}^{2}$, i.e. where $S=\partial C$. In this case $S$ is of class $C^{1}$ and diffeomorphic to the unit sphere and given a unit direction $m$, the tangent plane $H(C, m)$ to $S$ touches the hypersurface in a single point $x^{*}$, i.e. $F(C, m)=\left\{x^{*}\right\}$ (see Figure C.4). In this case $m$ is also the outer unit normal of $S$ at $x^{*}$ (as well as the unit normal to the tangent plane $H(C, m))$. We have

$$
h_{C}(m)=\sup \{\langle x, m\rangle: x \in C\}=\left\langle x^{*}, m\right\rangle .
$$

By homogeneity, the support function $h_{C}$ is fully determined when it is defined on each unit direction. Since in the present case $\mathbb{S}^{n-1}$ and $\partial C$ are diffeomorphic, we can naturally define a function $h$ on $\partial C$ as

$$
h:=h_{C} \circ \nu
$$

This function $h: S \rightarrow \mathbb{R}$ will be called the support function of $S$ and can be explicitly computed by

$$
h(p)=\left(h_{C} \circ \nu\right)(p)=h_{C}(m)=\sup \{\langle x, m\rangle: x \in C\}=\langle x(p), m(p)\rangle,
$$




\section{Geometry of convex bodies. The support function}

where $m(p)$ is the unit normal at $p$ pointing towards the exterior of $S$ and $x(p)$ is the position vector of the point $p \in S$.

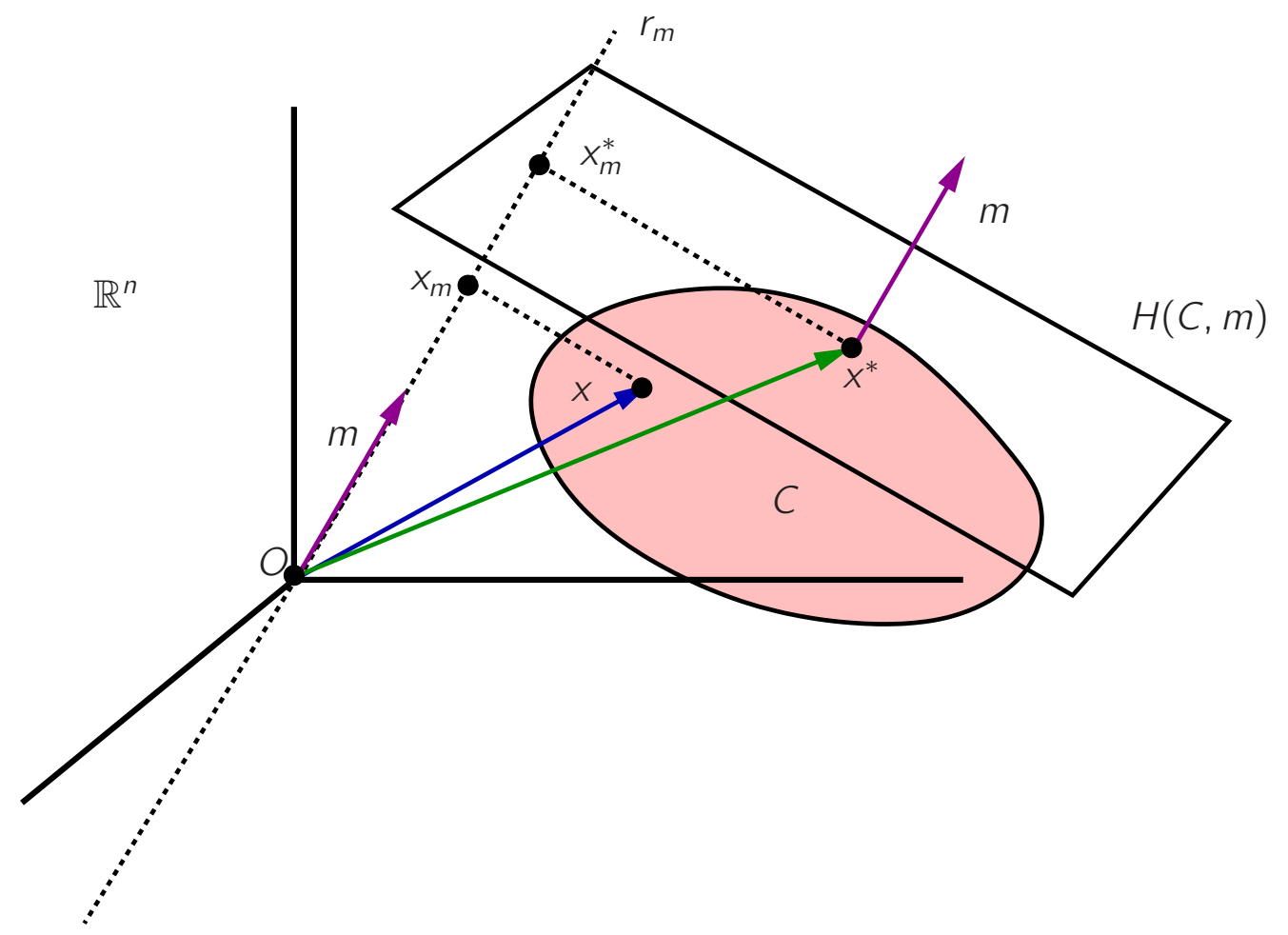

Figure C.4: Schematic figure representing the support function $h_{C}$ of the convex set $C$ of class $C^{2}$ evaluated on a unit vector $m$. By definition, $h_{C}(m)=\sup \{\langle x, m\rangle: x \in C\}=$ $\sup \left\{x_{m}: x \in C\right\}$, where $x_{m}$ is the signed distance from the origin $O$ to the projection of $x$ onto the line $r_{m}$ generated by $m$. When $C$ is of class $C_{+}^{2}$, the supremum is attained at a unique point $x^{*} \in \partial C$, and $\left\{x^{*}\right\}=F(C, m)=H(C, m) \cap C$. The signed distance $x_{m}^{*}$ is defined by $x_{m}^{*}:=\left\langle x^{*}, m\right\rangle=h_{C}(m)$.

\section{C.3 Geometry of Euclidean hypersurfaces in terms of the support function}

The following result is necessary for the proof of Theorem C.1.1. It shows that the set of sublinear functions is a subset of the support functions of convex bodies. The proof is somewhat delicate and can be found in [94].

Theorem C.3.1. If $f: \mathbb{R}^{n} \rightarrow \mathbb{R}$ is a sublinear function, then there is a unique convex body $C \in \mathcal{C}^{n}$ with support function $f$. 


\section{C.3. Geometry of Euclidean hypersurfaces in terms of the support function}

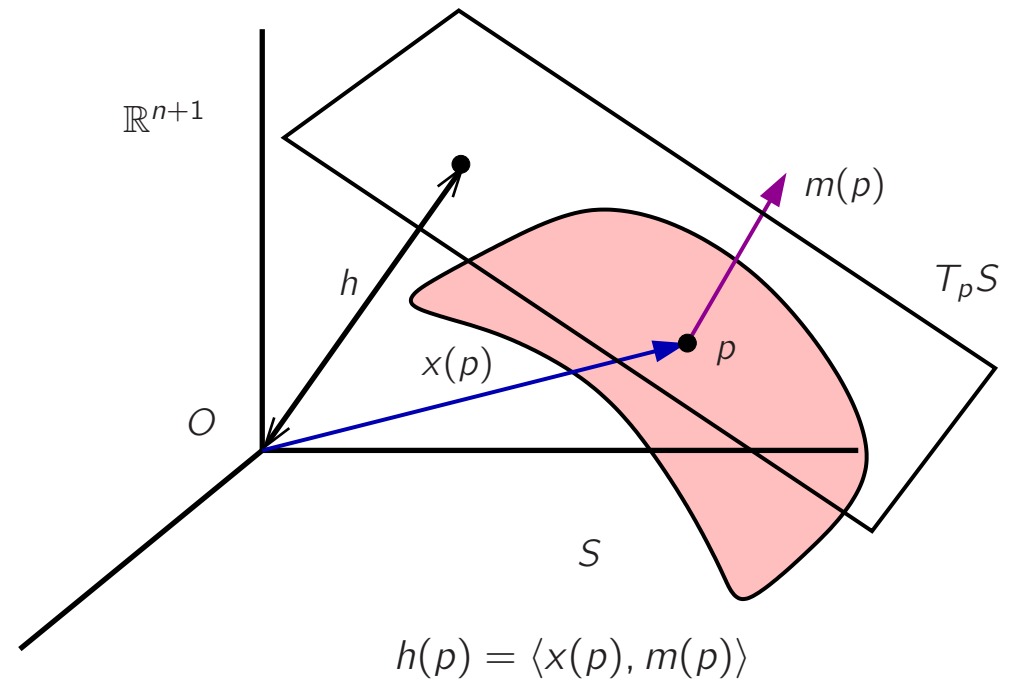

Figure C.5: Definition of the support function $h$ of a convex surface $S$. This function at a point $p \in S$ measures the signed distance of the tangent plane $T_{p} S$ to the origin of coordinates. Equivalently $h(p)$ is the product of the position vector $x(p)$ with the outer unit normal $m(p)$ to $S$. The support function describes the geometry of a convex body in the euclidean space.

The semidirectional derivatives of support functions are closely related to the support function of the corresponding support sets. This is the content of the following theorem:

Theorem C.3.2. For $C \in \mathcal{C}^{n}$ and $u \in \mathbb{R}^{n} \backslash\{0\}$,

$$
h_{C}^{\prime}(u ; x)=h(F(C, u) ; x) \text { for } \quad x \in \mathbb{R}^{n} .
$$

Proof. Applying Lemma C.2.6 we obtain that $h_{C}^{\prime}\left(u_{;} \cdot\right)$ is sublinear. Hence using Theorem C.3.1, $h_{C}^{\prime}\left(u_{;} \cdot\right)$ is the support function of a convex body $C^{\prime}$ that satisfies $C^{\prime} \subset C$ because $h_{C}^{\prime}(u ; \cdot) \leq h_{C}$ (see Lemma C.2.7 and item (iv) in Proposition C.2.10). Let $y \in C^{\prime}$. By the definition of support function, $\langle y, u\rangle \leq h(C, u)$. Let us next show the reverse inequality. Since $y \in C^{\prime}$, we can apply items (i) and (iv) to the sets $\{y\}$ and $C^{\prime}$ to find that their respective support functions satisfy $\langle y, \cdot\rangle \leq h_{C}^{\prime}\left(u_{;} \cdot\right)$. Evaluating at $-u$ we obtain $\langle y,-u\rangle \leq h_{C}^{\prime}(u ;-u)=-h(C, u)$, where in the last equality we have used the definition of semidirectional derivative and the homogeneity properties of the support function, namely

$$
h_{C}^{\prime}(u ;-u)=\lim _{\lambda \rightarrow 0^{+}} \frac{h_{C}(u+\lambda(-u))-h_{C}(u)}{\lambda}=\lim _{\lambda \rightarrow 0^{+}} \frac{(1-\lambda) h_{C}(u)-h_{C}(u)}{\lambda}=-h(C, u) .
$$

Therefore $\langle y, u\rangle=h(C, u)$ and hence $y \in F(C, u)$. We conclude $C^{\prime} \subset F(C, u)$.

For the reverse inclusion we consider $y \in F(C, u)$. From the definition of $F(C, u)$, it follows $\langle y, u\rangle=h(C, u)$, and since $y \in C$, we have $\langle y, v\rangle \leq h(C, v)$ for all $v \in \mathbb{R}^{n}$, 


\section{Geometry of convex bodies. The support function}

where we have used the definition of support function. Choosing $v=u+\lambda x(\lambda>0$, $x \in \mathbb{R}^{n}$ ) we get

$$
h(C, u+\lambda x) \geq\langle y, u+\lambda x\rangle=\langle y, u\rangle+\lambda\langle y, x\rangle=h(C, u)+\lambda\langle y, x\rangle .
$$

and then

$$
\langle y, x\rangle \leq \frac{h(C, u+\lambda x)-h(C, u)}{\lambda} .
$$

Taking the limit $\lambda \rightarrow 0^{+}$we conclude $\langle y, x\rangle \leq h_{C}^{\prime}(u ; x)$. In other words, the support function of the set $\{y\}$ is less than or equal to the support function of $C^{\prime}$, so from item (i) in Proposition C.2.10 it necessarily follows that $y \in C^{\prime}$. This proves $F(C, u) \subset C^{\prime}$ and hence $F(C, u)=C^{\prime}$ and the equality (C.5).

In the following theorem, we will prove that when the support function $h_{C}$ is differentiable at $u \in \mathbb{R}^{n}$, then its gradient at this point exists and is given by the unique contact point $x$ of the hyperplane $H(C, u)$ orthogonal to $u$ with the convex body $C$.

Theorem C.3.3. Let $C \in \mathcal{C}^{n}$ and $u \in \mathbb{R}^{n} \backslash\{0\}$. The support function $h_{C}$ is differentiable at $u$ if and only if the support set $F(C, u)$ contains only one point $x$. In this case,

$$
x=\operatorname{grad} h_{C}(u)
$$

Proof. The support function is a convex function and consequently the semidirectional derivative function $h_{C}^{\prime}(u ;)$ exists. To show differentiability, it is hence sufficient by Theorem C.2.2 to prove that the partial derivatives exist. Fixing an orthonormal basis $e_{1}, \ldots, e_{n}$ and expressed in terms of semidirectional derivatives, this happens if and only if

$$
h_{C}^{\prime}\left(u ; e_{A}\right)=-h_{C}^{\prime}\left(u ;-e_{A}\right) \quad \forall A=1, \ldots, n .
$$

We can rewrite the above condition by using formula (‥5) as

$$
h\left(F(C, u), e_{A}\right)=-h\left(F(C, u),-e_{A}\right) \quad \forall A=1, \ldots, n .
$$

Condition (C.6) describes the situation where the unique support plane $H\left(F(C, u), e_{A}\right)$ orthogonal to $e_{A}$ and tangent to $F(C, u)$ lies at the opposite signed distance from the origin than the plane $H\left(F(C, u),-e_{A}\right)$ orthogonal to $-e_{A}$ and also tangent to $F(C, u)$. This can only happen if the two tangent planes coincide $\left(H\left(F(C, u), e_{A}\right)=\right.$ $\left.H\left(F(C, u),-e_{A}\right)\right)$ and the set $F(C, u)$ is contained within it, i.e. if and only if

$$
F(C, u) \subset H\left(F(C, u), e_{A}\right),
$$

This must happen for every $A=1, \ldots, n$, which means that $F(C, u)$ is included in $n$ different hyperplanes, each one perpendicular to one of the vectors $e_{A}$ in the orthonormal basis $\left\{e_{A}\right\}$. Thus, these hyperplanes are pairwise orthogonal, and given that $F(C, u)$ is included in all of them, it must be $F(C, u)=\{x\}$ for some $x$ in the boundary of $C$. 


\section{C.3. Geometry of Euclidean hypersurfaces in terms of the support function}

By property (i) in Proposition C.2.10, the support function of a single point $\{x\}$ is $\langle x, \cdot\rangle$. Then

$$
\langle x, v\rangle=h(F(C, u), v)=h_{C}^{\prime}(u ; v) \quad \text { for all } \quad v \in \mathbb{R}^{n}
$$

In particular

$$
\left\langle x, e_{A}\right\rangle=h_{C}^{\prime}\left(u ; e_{A}\right)=\partial_{A} h_{C}(u), \quad \text { for } \quad A=1, \cdots, n .
$$

In other words, $x=\operatorname{grad} h_{C}(u)$.

Remark C.3.4. Note that $\{x\}=F(C, u)$ is the unique contact point of $H(C, u)$ with $C$. In particular $x$ lies in the boundary of $C$.

Let us now consider the relation between Theorem C.3.3 and the construction at the beginning of this appendix where we introduced the Gauss map $\nu$ of the boundary of a convex body $C$. Let us assume that $C \in C_{+}^{2}$. Then the map $\nu$ has an inverse $\nu^{-1}$ of class $C^{1}$. The support function $h_{C}$ can be rewritten in terms of $\nu^{-1}$. Recall that $\nu^{-1}: \phi\left(\mathbb{S}^{n-1}\right) \rightarrow \partial C$, and when we consider $m \in \phi\left(\mathbb{S}^{n-1}\right)$, then $\nu^{-1}(m) \in \partial C$ is the point whose outer unit normal coincides with $m$. For this reason given $p \in \partial C$, there exists $m \in \phi\left(\mathbb{S}^{n-1}\right)$ so that $\nu^{-1}(m)=x(p)$, where $x(p)$ is the position vector of $p$, and since $m$ is the outer unit vector of $\partial C$ at $p$, then (C.4) can be expressed as

$$
h_{C}(m)=\left\langle\nu^{-1}(m), m\right\rangle \text {. }
$$

Note that since $\nu^{-1}$ is of class $C^{1}$, then $h_{C}$ is differentiable on $\phi\left(\mathbb{S}^{n-1}\right)$ and by homogeneity on $\mathbb{R}^{n} \backslash\{0\}$. Given $u \in \mathbb{R}^{n} \backslash\{0\}$, the unit vector $m=\frac{u}{|u|}$ lies in $\phi\left(\mathbb{S}^{n-1}\right)$, and there is a point $p \in \partial C$ satisfying $\nu^{-1}\left(\frac{u}{|u|}\right)=x(p)$, so we finally obtain

$$
\nu^{-1}\left(\frac{u}{|u|}\right)=x(p)=\operatorname{grad} h_{C}(u), \quad u \in \mathbb{R}^{n} \backslash\{0\}
$$

The following definition extends to all Euclidean space in a natural way the reverse Gauss map $\nu^{-1}$ :

Definition C.3.5. For $u \in \mathbb{R}^{n} \backslash\{0\}$, let $\overline{\nu^{-1}}(u) \in \partial C$ the unique point of $\partial C$ at which $u$ is an outward normal vector. From (C.7) this map can be written explicitly as

$$
\begin{aligned}
\overline{\nu^{-1}}: \mathbb{R}^{n} \backslash\{0\} & \longrightarrow \frac{\partial C}{u} \\
u & \longrightarrow \overline{\nu^{-1}}(u)=\operatorname{grad} h_{C}(u)
\end{aligned}
$$

Remark C.3.6. With this definition, $\overline{\nu^{-1}}$ is a positively homogeneous function of degree zero. Note that the restriction to the embedded unit sphere $\left.\overline{\nu^{-1}}\right|_{\phi\left(\mathbb{S}^{n-1}\right)}=\nu^{-1}$ : $\phi\left(\mathbb{S}^{n-1}\right) \longrightarrow \partial C$ is the reverse Gauss map. Its differential $d \nu^{-1}: T_{\phi(u)} \phi\left(\mathbb{S}^{n-1}\right) \rightarrow T_{p} \partial C$ is used to construct the reverse Weingarten endomorphism $\mathcal{B}$ when the two tangent planes $T_{\phi(u)} \phi\left(\mathbb{S}^{n-1}\right)$ and $T_{p} \partial C$ are identified. 


\section{Geometry of convex bodies. The support function}

So far we have studied the maps that link the geometry of convex bodies with the geometry of the sphere. Besides we have analyzed the main properties of the support function of a convex body, but we have not yet related both of them. The next Theorem C.3.9 will provide the relation between the reverse second fundamental form and the support function of a convex body C (see Figure C.6). Before stating the result, we need the following analytic Lemmas:

Lemma C.3.7. Let $f: \mathbb{R}^{n} \rightarrow \mathbb{R}$ be an homogeneous function of degree one. Then

$$
\partial_{A} \partial_{B} f(x) x^{A}=0 .
$$

Proof. The function $f$ satisfies the relation $f(\lambda x)=\lambda f(x)$. If we differentiate with respect to $\lambda$ and evaluate at $\lambda=1$ we recover Euler's Theorem

$$
\partial_{A} f(x) x^{A}=f(x) .
$$

Differentiating with respect to $x^{B}$ :

$$
\partial_{B} \partial_{A} f(x) x^{A}+\partial_{A} f(x) \partial_{B} x^{A}=\partial_{B} f(x) .
$$

Since $\partial_{B} X^{A}=\delta^{A}{ }_{B}$, relation (C.8) follows immediately.

Lemma C.3.8. Let $f: \mathbb{R}^{n} \rightarrow \mathbb{R}$ be a homogeneous function of degree one and $x \in$ $\mathbb{R}^{n} \backslash\{0\}$. Then

$$
\left(\operatorname{Hess}_{\mathbb{R}^{n}} f\right)_{x}(a, b)=\frac{1}{|x|}\left(\operatorname{Hess}_{\mathbb{R}^{n}} f\right)_{\frac{x}{|x|}}\left(\mathcal{T}_{x \rightarrow \frac{x}{|x|}} a, \mathcal{T}_{x \rightarrow \frac{x}{|x|}} b\right), \quad a, b \in \mathbb{R}^{n} .
$$

Proof. We use the form $\left.(\operatorname{Hess} f)\right|_{x}(v, w)=\left.\left\langle\nabla_{v} \operatorname{grad} f, w\right\rangle\right|_{x}$ for the Hessian of $f$. Note that the Hessian of $f$ becomes zero when is contracted with any direction parallel to $x$ because

$$
\left.(\operatorname{Hess} f)\right|_{x}(x, \cdot)=\left\langle\nabla_{x} \operatorname{grad} f, \cdot\right\rangle,
$$

and using Euclidean coordinates

$$
\nabla_{x} \operatorname{grad} f=\nabla_{\left(x^{A} \partial_{x^{A}}\right)}\left(\frac{\partial f}{\partial x^{B}} \partial_{x^{B}}\right)=x^{A}\left(\partial_{A} \partial_{B} f\right) \partial_{x^{B}}=0,
$$

from Lemma C.3.7. It only remains to evaluate the Hessian of $f$ at $x$ along vectors perpendicular to $x$.

Let us consider the map $\theta: \mathbb{R}^{n} \backslash\{0\} \rightarrow \phi\left(\mathbb{S}^{n-1}\right) \subset \mathbb{R}^{n}$ defined by $\theta(x)=\frac{x}{|x|}$. Note that when it is restricted to $\mathbb{S}^{n-1}(|x|)$, i.e. the $(n-1)$-sphere of radius $|x|$, then it is precisely the Gauss map of this surface. Using the homogeneity of $f$, we have

$$
f(x)=|x| f\left(\frac{x}{|x|}\right)=|x|(f \circ \theta)(x),
$$




\section{C.3. Geometry of Euclidean hypersurfaces in terms of the support function}

An immediate computation gives $\partial_{A}(|x|)=\frac{x^{A}}{|x|}$ and

$$
\frac{\partial \theta^{C}(x)}{\partial x^{A}}=\frac{\partial}{\partial x^{A}}\left(\frac{x^{C}}{|x|}\right)=\frac{\delta^{C} A}{|x|}-\frac{x^{A} x^{C}}{|x|^{3}},
$$

which yields

$$
\begin{aligned}
\left(\partial_{A} f\right)(x) & =\partial_{A}(|x|(f \circ \theta)(x))=\frac{x^{A}}{|x|}(f \circ \theta)(x)+|x| \sum_{C=1}^{n} \frac{\partial f}{\partial x^{C}}(\theta(x)) \frac{\partial \theta^{C}(x)}{\partial x^{A}} \\
& =\frac{x^{A}}{|x|}(f \circ \theta)(x)+\frac{\partial f}{\partial x^{A}}(\theta(x))-\left\langle\left.\operatorname{grad} f\right|_{\theta(x)}, \theta(x)\right\rangle \frac{x^{A}}{|x|}=\frac{\partial f}{\partial x^{A}}(\theta(x)),
\end{aligned}
$$

where we used $\left\langle\left.\operatorname{grad} f\right|_{\theta(x)}, \theta(x)\right\rangle=f(\theta(x))$ (see ([C.9) ) in the last equality. In vector notation, (C.11) states that $\left.\operatorname{grad} f\right|_{x}=\mathcal{T}_{\theta(x) \rightarrow x}\left(\left.\operatorname{grad} f\right|_{\theta(x)}\right)$. If we restrict $\operatorname{grad} f$ to $\mathbb{S}^{n-1}(|x|)$, we can write

$$
\mathcal{T}\left(\left.\operatorname{grad} f\right|_{\mathbb{S}^{n-1}(|x|)}\right)=\left.\operatorname{grad} f\right|_{\mathbb{S}^{n-1}}
$$

where $\left.(\mathcal{T} Z)\right|_{\theta(x)}:=\mathcal{T}_{x \rightarrow \theta(x)}\left(\left.Z\right|_{x}\right)$ for any vector field $Z$ along $\mathbb{S}^{n-1}(|x|)$. The two spheres $\mathbb{S}^{n-1}(|x|)$ and $\mathbb{S}^{n-1}$ are diffeomorphic by $\theta$, so we can apply Lemma 5.2.1 in Chapter 5 , to obtain

$$
\mathcal{T}_{x \rightarrow \theta(x)}\left(\left.\left(\left.\nabla_{a} \operatorname{grad} f\right|_{\mathbb{S}^{n-1}(|x|)}\right)\right|_{x}\right)=\left.\left(\left.\nabla_{d \theta(a)} \operatorname{grad} f\right|_{\mathbb{S}^{n-1}}\right)\right|_{\theta(x)} .
$$

Moreover,

$$
\left.d \theta\right|_{x}(a)=\frac{1}{|x|} \mathcal{T}_{x \rightarrow \theta(x)} a
$$

as a consequence of (C.10) and the orthogonality of $a$ and $x$. Substituting in (C.12) gives

$$
\mathcal{T}_{x \rightarrow \theta(x)}\left(\left.\left(\left.\nabla_{a} \operatorname{grad} f\right|_{\mathbb{S}^{n-1}(|x|)}\right)\right|_{x}\right)=\left.\frac{1}{|x|}\left(\nabla_{\mathcal{T}_{x \rightarrow \theta(x)}}\left(\left.\operatorname{grad} f\right|_{\mathbb{S}^{n-1}}\right)\right)\right|_{\theta(x)} .
$$

We finally use the obvious fact $\left.\langle v, w\rangle\right|_{p}=\left.\left\langle\mathcal{T}_{p \rightarrow q} v, \mathcal{T}_{p \rightarrow q} w\right\rangle\right|_{q}$ for any $p, q \in \mathbb{R}^{n}$ and $v, w \in T_{p} \mathbb{R}^{n}$, to conclude

$$
\begin{aligned}
\left.\left(\operatorname{HesS}_{\mathbb{R}^{n}} f\right)\right|_{x}(a, b) & =\left.\left\langle\nabla_{a} \operatorname{grad} f, b\right\rangle\right|_{x}=\left.\left\langle\mathcal{T}_{x \rightarrow \theta(x)}\left(\left.\left(\left.\nabla_{a} \operatorname{grad} f\right|_{\mathbb{S}^{n-1}(|x|)}\right)\right|_{x}\right), \mathcal{T}_{x \rightarrow \theta(x)} b\right\rangle\right|_{\theta(x)} \\
& =\left.\frac{1}{|x|}\left\langle\nabla_{\mathcal{T}_{x \rightarrow \theta(x)}}\left(\left.\operatorname{grad} f\right|_{\mathbb{S}^{n-1}}\right), \mathcal{T}_{x \rightarrow \theta(x)} b\right\rangle\right|_{\theta(x)} \\
& =\frac{1}{|x|}\left(\operatorname{Hess}_{\mathbb{R}^{n}} f\right)_{\theta(x)}\left(\mathcal{T}_{x \rightarrow \theta(x)} a, \mathcal{T}_{x \rightarrow \theta(x)} b\right),
\end{aligned}
$$

as claimed. 


\section{Geometry of convex bodies. The support function}

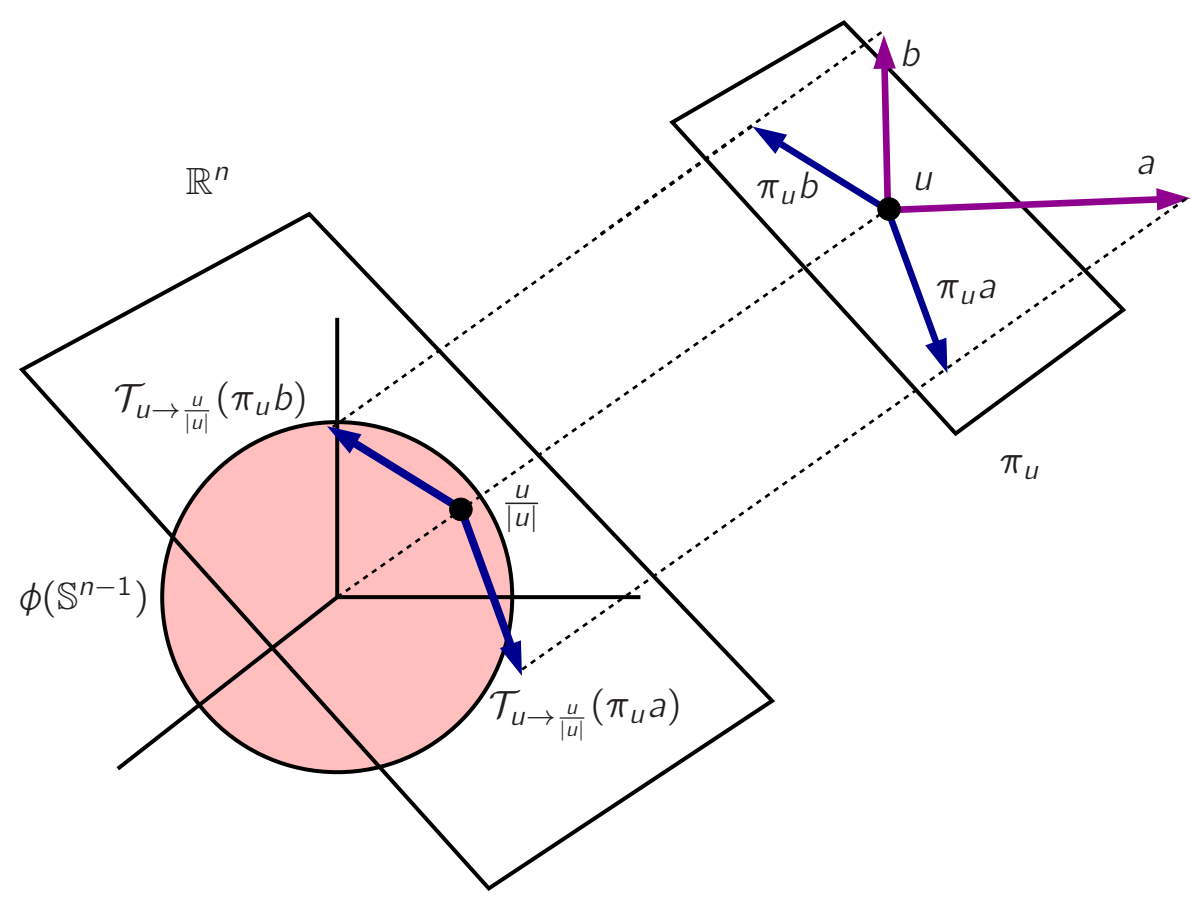

Figure C.6: The Hessian of the support function $h_{C}$ on $\mathbb{R}^{n}$ corresponding to a convex body $C$ can be expressed in terms of the reverse Weingarten map $\mathcal{B}$, which is an endomorphism acting on vectors tangent to the embedded unit sphere $\phi\left(\mathbb{S}^{n-1}\right)$, and providing extrinsic curvature information of the convex body. For any point $u \in \mathbb{R}^{n}$ and for any $a, b \in T_{u} \mathbb{R}^{n}$, the map $\pi_{u}: T_{u} \mathbb{R}^{n} \rightarrow T_{u} \mathbb{S}^{n-1}(|u|)$ projects any vector on $T_{u} \mathbb{R}^{n}$ to the plane $T_{u} \mathbb{S}^{n-1}(|u|)$ tangent to the embedded $(n-1)$-sphere $\mathbb{S}^{n-1}(|u|)$ of radius $|u|$. The Hessian of $h_{C}$ satisfies the relation $\left(\operatorname{Hess}_{\mathbb{R}^{n}} h_{C}\right)_{u}(a, b)=\frac{1}{|u|}\left\langle\mathcal{B}\left(\mathcal{T}_{u \rightarrow \frac{u}{|u|}}\left(\pi_{u} a\right)\right), \mathcal{T}_{u \rightarrow \frac{u}{|u|}}\left(\pi_{u} b\right)\right\rangle$.

We are ready to state the relation between the Hessian of the support function and the reverse Weingarten map:

Theorem C.3.9. Let $C \in \mathcal{C}^{n}$ and $u \in \mathbb{R}^{n} \backslash\{0\}$. Then

$$
\left(\operatorname{Hess}_{\mathbb{R}^{n}} h_{C}\right)_{u}(a, b)=\frac{1}{|u|}\left\langle\mathcal{B}\left(\mathcal{T}_{u \rightarrow \frac{u}{|u|}}\left(\pi_{u} a\right)\right), \mathcal{T}_{u \rightarrow \frac{u}{|u|}}\left(\pi_{u} b\right)\right\rangle
$$

for all $a, b \in \mathbb{R}^{n}$, where $\pi_{u}$ is the orthogonal projection onto the orthogonal plane to $u$ (as a vector) and that contains $u$ (as a point).

Proof. Let $u \in \mathbb{R}^{n} \backslash\{0\}$ be a unit vector $(|u|=1)$ and $\left\{e_{1}, \ldots, e_{n}\right\}$ an orthonormal basis of vectors of $\mathbb{R}^{n}$ with $e_{n}=u$. We write $\left(x^{1}, \ldots, x^{n}\right)$ for the corresponding Cartesian coordinates. Observe that in these coordinates $u \equiv(0, \ldots, 1)$.

From Lemma C.3.7 we know that $\partial_{A} \partial_{B} h_{C}(x) x^{A}=0$, which evaluated at $x=u$ gives

$$
0=\partial_{A} \partial_{B} h_{C}(u) u^{A}=\partial_{B} \partial_{n} h_{C}(u) \text { for all } B=1, \ldots, n \text {. }
$$




\section{C.3. Geometry of Euclidean hypersurfaces in terms of the support function}

Then

$$
\left(\operatorname{Hess}_{\mathbb{R}^{n}} h_{C}\right)_{u}(a, b)=\sum_{A, B=1}^{n} \partial_{A} \partial_{B} h_{C}(u) a^{A} b^{B}=\sum_{A, B=1}^{n-1} \partial_{A} \partial_{B} h_{C}(u) a^{A} b^{B}
$$

We also know that $\overline{\nu^{-1}}(u)=\operatorname{grad} h_{C}(u)$ (see Definition C.3.5). The differential of $\overline{\nu^{-1}}$ can be represented by the matrix

$$
d \overline{\nu^{-1}} \equiv\left(\begin{array}{ccc}
\partial_{1} \partial_{1} h_{C} & \ldots & \partial_{1} \partial_{n} h_{C} \\
\vdots & \ddots & \vdots \\
\partial_{1} \partial_{n} h_{C} & \ldots & \partial_{n} \partial_{n} h_{C}
\end{array}\right)
$$

so that $\partial_{A} \partial_{B} h_{C}(u)=\left\langle\left. d \overline{\nu^{-1}}\right|_{u}\left(e_{A}\right), e_{B}\right\rangle$. Restricting to $A, B=1, \ldots, n-1$ we have

$$
\partial_{A} \partial_{B} h_{C}(u)=\left\langle\left. d \overline{\nu^{-1}}\right|_{u}\left(e_{A}\right), e_{B}\right\rangle=\left\langle\left. d \nu^{-1}\right|_{u}\left(e_{A}\right), e_{B}\right\rangle=\left\langle\mathcal{B}_{u}\left(\pi_{u}\left(e_{A}\right)\right), \pi_{u}\left(e_{B}\right)\right\rangle,
$$

where in the last equality we have used that $e_{A} \in T_{u} \phi\left(\mathbb{S}^{n-1}\right)$ for $A=1, \ldots, n-1$ and consequently $e_{A}=\pi_{u}\left(e_{A}\right)$. Hence

$$
\sum_{A, B=1}^{n-1} \partial_{A} \partial_{B} h_{C}(u) a^{A} b^{B}=\sum_{A, B=1}^{n-1}\left\langle\mathcal{B}_{u}\left(\pi_{u}\left(e_{A}\right)\right), \pi_{u}\left(e_{B}\right)\right\rangle a^{A} b^{B}=\left\langle\mathcal{B}_{u}\left(\pi_{u} a\right), \pi_{u} b\right\rangle .
$$

Combining (C.14) and (C.15) gives

$$
\left(\operatorname{Hess}_{\mathbb{R}^{n}} h_{C}\right)_{u}(a, b)=\left\langle\mathcal{B}_{u}\left(\pi_{u} a\right), \pi_{u} b\right\rangle \quad \forall u \in \phi\left(\mathbb{S}^{n-1}\right) .
$$

We can extend this formula to all $u \in \mathbb{R}^{n} \backslash\{0\}$ by using Lemma C.3.8 and the fact that $h_{C}$ is homogeneous of degree one:

$$
\begin{aligned}
\left.\operatorname{Hess}_{\mathbb{R}^{n}} h_{C}\right)_{u}(a, b) & =\frac{1}{|u|}\left(\operatorname{Hess}_{\mathbb{R}^{n}} h_{C}\right)_{\frac{u}{|u|}}\left(\mathcal{T}_{u \rightarrow \frac{u}{|u|}} a, \mathcal{T}_{u \rightarrow \frac{u}{|u|}} b\right) \\
& =\frac{1}{|u|}\left\langle\mathcal{B}\left(\mathcal{T}_{u \rightarrow \frac{u}{|u|}}\left(\pi_{u} a\right)\right), \mathcal{T}_{u \rightarrow \frac{u}{|u|}}\left(\pi_{u} b\right)\right\rangle
\end{aligned}
$$

which is (C.13).

We have all the ingredients to find the expression for the second fundamental form of the boundary $\partial C$ of the convex body $C$ in terms of the support function $h$ associated to $\partial C$. In order to do so, let us consider $u \in \mathbb{S}^{n-1},\left\{u_{A}\right\}$ a basis of $T_{u} \mathbb{S}^{n-1}$ and $N_{A}=d \phi\left(u_{A}\right)$ (recall that $\phi$ is the embedding of the abstract sphere into $\mathbb{R}^{n}$ ). Applying Theorem C.3.9 we obtain

$$
\begin{aligned}
\left(\operatorname{Hess}_{\mathbb{R}^{n}} h_{C}\right)_{\phi(u)}\left(N_{A}, N_{B}\right) & =\left\langle\mathcal{B}\left(\pi_{\phi(u)}\left(N_{A}\right)\right), \pi_{\phi(u)}\left(N_{B}\right)\right\rangle=\left\langle\mathcal{B}\left(N_{A}\right), N_{B}\right\rangle \\
& =\left\langle X_{A}, N_{B}\right\rangle=\left\langle X_{A}, \mathcal{W}\left(X_{B}\right)\right\rangle=K\left(X_{A}, X_{B}\right) .
\end{aligned}
$$




\section{Geometry of convex bodies. The support function}

This last result determines the second fundamental form of $\partial C$ in terms of the Hessian of its support function with respect to the euclidean metric. In Proposition A.2.1 in Appendix A we have proved that if if $\phi: N \rightarrow M$ is an embedding of $N$ in $M$, and $f: M \rightarrow \mathbb{R}$ is a smooth function, the following identity relating the ambient Hessian to the induced Hessian holds,

$$
\operatorname{Hess}_{M} f(d \phi(X), d \phi(Y))=\operatorname{Hess}_{N}(f \circ \phi)(X, Y)+\langle\operatorname{grad} f, \vec{K}(X, Y)\rangle
$$

where $X, Y \in \Gamma(T N)$. We apply this result in our present context where $M=\mathbb{R}^{n}, N=$ $\mathbb{S}^{n-1}, f=h_{C}$, and $\phi$ is the embedding of the abstract sphere into $\mathbb{R}^{n}$. We get

$$
\left.\left(\operatorname{Hess}_{\mathbb{R}^{n}} h_{C}\right)\right|_{\phi(u)}\left(N_{A}, N_{B}\right)=\left.\operatorname{Hess}_{\mathbb{S}^{n-1}}\left(h_{C} \circ \phi\right)\right|_{u}\left(u_{A}, u_{B}\right)+\left.\left\langle\operatorname{grad} h_{C}, \vec{K}\left(N_{A}, N_{B}\right)\right\rangle\right|_{\phi(u)} .
$$

Let us compute now the last term of this expression. The sphere is totally umbilical and in fact

$$
\left.\vec{K}\right|_{\phi(u)}\left(N_{A}, N_{B}\right)=K_{\phi(u)}\left(N_{A}, N_{B}\right) \phi(u)=\left\langle N_{A}, N_{B}\right\rangle \phi(u)=\stackrel{q}{q}\left(u_{A}, u_{B}\right) \phi(u),
$$

where recall $\stackrel{q}{q}$ is the metric of the abstract standard sphere. Using that $h_{C}$ is homogeneous of degree one, we obtain

$$
\left.\left\langle\operatorname{grad} h_{C}, \vec{K}\left(N_{A}, N_{B}\right)\right\rangle\right|_{\phi(u)}=\left.\stackrel{q}{q}\left(u_{A}, u_{B}\right) \nabla_{\phi(u)} h_{C}\right|_{\phi(u)}=\stackrel{q}{q}\left(u_{A}, u_{B}\right) h_{C}(\phi(u))
$$

after applying Euler's Theorem (C.9). As a consequence (C.17) becomes

$$
\left(\operatorname{Hess}_{\mathbb{R}^{n}} h_{C}\right)_{\phi(u)}\left(N_{A}, N_{B}\right)=\operatorname{Hess}_{\mathbb{S}^{n-1}}\left(h_{C} \circ \phi\right)_{u}\left(u_{A}, u_{B}\right)+\stackrel{q}{q}\left(u_{A}, u_{B}\right)\left(h_{C} \circ \phi\right)(u),
$$

which in combination with (C.16) gives

$$
K\left(X_{A}, X_{B}\right)=\operatorname{Hess}_{\mathbb{S}^{n-1}}\left(h_{C} \circ \phi\right)_{u}\left(u_{A}, u_{B}\right)+\stackrel{q}{q}\left(u_{A}, u_{B}\right)\left(h_{C} \circ \phi\right)(u) .
$$

The second fundamental form $K$ is naturally defined on $\partial C$, whereas the right-hand side of the above inequality is defined on the abstract sphere. We want to find the expression for the equality where every tensor and function is defined on $\partial C$. To do so, we recall that $d \nu_{C}\left(X_{A}\right)=u_{A}$. This implies

$$
\begin{aligned}
\operatorname{Hess}_{\left(\mathbb{S}^{n-1}, \mathfrak{q}\right)}\left(h_{C} \circ \phi\right)_{u}\left(u_{A}, u_{B}\right) & =\operatorname{Hess}_{\left(\mathbb{S}^{n-1}, \mathfrak{q}\right)}\left(h_{C} \circ \phi\right)_{u}\left(d \nu_{C}\left(X_{A}\right), d \nu_{C}\left(X_{B}\right)\right) \\
& =\left.\nu_{C}^{*}\left(\operatorname{Hess}_{\left(\mathbb{S}^{n-1}, \mathfrak{q}\right)}\left(h_{C} \circ \phi\right)\right)\right|_{p}\left(X_{A}, X_{B}\right) \\
& =\left.\operatorname{Hess}_{\left(\partial C, \nu_{C}^{*}(\mathfrak{q})\right)}\left(\nu_{C}^{*}\left(h_{C} \circ \phi\right)\right)\right|_{p}\left(X_{A}, X_{B}\right) .
\end{aligned}
$$

Recalling now that $\nu_{C}^{*}\left(h_{C} \circ \phi\right)(p)=h_{C} \circ \phi \circ \nu_{C}(p)=h(p)$, where $h: \partial C \rightarrow \mathbb{R}^{n}$ is the support function of $\partial C$, and writing the pullback $\nu_{C}^{*}(\stackrel{\circ}{q})$ of the spherical metric still as $\stackrel{\circ}{q}$ (no confusion arises since both are defined on different manifolds), expression ([C.18) finally becomes

$$
K_{p}\left(X_{A}, X_{B}\right)=\left.\operatorname{Hess}_{(\partial C, \mathfrak{q})} h\right|_{p}\left(X_{A}, X_{B}\right)+\stackrel{q}{q}\left(X_{A}, X_{B}\right) h(p),
$$




\section{C.4. The support function of the sphere}

which expressed in index notation gives the expression stated in Theorem C.1.1 at the beginning of the appendix, namely

$$
K_{A B}=\stackrel{\circ}{\nabla}_{A} \stackrel{\circ}{\nabla}_{B} h+\stackrel{\circ}{q}_{A B} h .
$$

All objects in this expression are defined on the hypersurface $\partial C$ ( $\partial C$ has been endowed with the spherical metric $\stackrel{q}{q}$ via $\nu_{C}$, and $\stackrel{\circ}{\nabla}$ is the connection defined by such metric). The metric $\gamma$ of $\partial C$ can also be expressed in terms of the spherical geometry as

$$
\gamma_{A B}=\left\langle X_{A}, X_{B}\right\rangle=\left\langle X_{A}, X_{B}\right\rangle=\left\langle\mathcal{B}\left(N_{A}\right), \mathcal{B}\left(N_{B}\right)\right\rangle=\left(\stackrel{\circ}{q}^{-1}\right)^{L M} B_{L A} B_{M B},
$$

which upon using $K_{A B}=B_{A B}$, can be written as

$$
\gamma_{A B}=\left(\stackrel{\circ}{q}^{-1}\right)^{L M} K_{L A} K_{M B} .
$$

This completes the proof of Theorem C.1.1.

\section{C.4 The support function of the sphere}

In Subsection 4.4.1 of Chapter 4, we need the support function of the compact ball $B\left(c, R_{0}\right)=\left\{x \in \mathbb{R}^{n}:|x-c| \leq R_{0}\right\}$ of radius $R_{0}$ and center $c \in \mathbb{R}^{n}$. In this particular case $\partial C=\mathbb{S}^{n-1}\left(c, R_{0}\right)$, i.e. the $(n-1)$-sphere of radius $R_{0}$ centered on $c$. Given any point $p$ of the sphere, the position vector can be written as

$$
x(p)=c+R_{0} m(p)
$$

for some unit vector $m(p)$. It also happens that the exterior unit normal vector at $p$ is $m(p)$. Then

$$
h(p)=\langle x(p), m(p)\rangle=\left\langle c+R_{0} m(p), m(p)\right\rangle=R_{0}+\langle c, m(p)\rangle .
$$

Restricting ourselves to $\mathbb{R}^{3}, c=\left(c^{x}, c^{y}, c^{z}\right)$, and formula (C.21) reduces to

$$
h(p)=R_{0}+c^{x} m^{x}(p)+c^{y} m^{y}(p)+c^{z} m^{z}(p) .
$$

The components of the vector $m(p)$ decompose in terms of the usual spherical coordinates as

$$
m^{x}=\sin \theta \cos \phi, \quad m^{y}=\sin \theta \sin \phi, \quad m^{z}=\cos \theta,
$$

which are the three linear independent spherical harmonics solution to the equation $\triangle_{\dot{q}} f=-2 f$.

Remark C.4.1. The support function of a sphere whose center lies at the origin of coordinates is the constant function defined by the radius of the sphere. 


\section{Bibliography}

[1] S. Alexakis, "The Penrose inequality on perturbations of the Schwarzschild exterior", arXiv:1506.06400v1 [gr-qc] (2015). 1

[2] S. Aretakis, "Stability and Instability of Extreme Reissner-Nordström Black Hole Spacetimes for Linear Scalar Perturbations I", Commun. Math. Phys. 307, 17-63 (2007). 1

[3] R. Arnowitt, S. Deser, C.W. Misner, "The Dynamics of General Relativity" in Gravitation: An Introduction to Current Research, L. Witten. J. Wiley \& Sons Inc. (Editors) (1962) 2.3

[4] S. Aretakis, "Stability and Instability of Extreme Reissner-Nordström Black Hole Spacetimes for Linear Scalar Perturbations II", Annals Henri Poincaré 12, 14911538 (2011). [1]

[5] C. Barrabés, W. Israel, "Thin shells in general relativity and cosmology: the lightlike limit", Phys. Rev. D 43, 1129-1142 (1991). 1, 2.6, 2.6

[6] R. Bartnik, "Bondi mass in the NQS gauge", Class. Quantum Grav. 21, 59-71 (2004). 1, 2.7, 6.7.5

[7] W. Beckner, "Sharp Sobolev inequalities on the sphere and the Moser-Trudinger inequality", Ann. Math. 138, 213-242 (1993). 1, 4.1, 4.4.1

[8] G. Bergqvist, "On the Penrose inequality and the role of auxiliary spinor fields", Class. Quantum Grav. 14, 2577-2583 (1997). 1, 1, 4.1, 4.4.2, 4.5, 4.5, 4.5.2, $7.1,7.3,7.3 .5$ 
[9] H. Bondi, M.G.J. van der Burg, A.W.K. Metzner, "Gravitational Waves in General Relativity. VII. Waves from Axi-Symmetric Isolated Systems", Proc. Roy. Soc. London A. 269, 1364-5021 (1962). 2.3

[10] T.Bonnesen, W.Fenchel, Theory of Convex Bodies, BCS Associates (1987). 1, 3.3 .1

[11] W.B. Bonnor, P.A. Vickers, "Junction conditions in General Relativity", Gen. Rel. Grav. 13, 29-36 (1981). 2.6

[12] H.L. Bray, "Proof of the Riemannian Penrose inequality using the positive mass theorem", J. Diff. Geom. 59, 177-267 (2001). 1]

[13] H.L. Bray, M.A. Khuri, "P.D.E.'s which imply the Penrose Conjecture", Asian J. Math 15, 557-610 (2011). 1, 1]

[14] H.L. Bray, D.A. Lee, "On the Riemannian Penrose inequality in dimensions less than eight", Duke Math. J. 148, 81-106 (2009).

[15] S. Brendle, M.T. Wang, "A Gibbons-Penrose inequality for surfaces in Schwarzschild Spacetime", Commun. Math. Phys. 330, 33-43 (2014). 1, 3.1] 3.3, 3.3.2, 8

[16] Y.S. Cha, M.A. Khuri, A. Sakovich, "Reduction arguments for geometric inequalities associated with asymptotically hyperboloidal slices", Class. Quantum Grav. 33, 035009 (2016). 1

[17] Y. Choquet-Bruhat, General Relativity and the Einstein Equations, Oxford University Press (2009). 1

[18] D. Christodoulou, S. Kleinerman, "The Global Nonlinear Stability of the Minkowski Space" in Princeton Mathematical series, volume 41, Princeton NJ, Princeton University Press (1993).

[19] P.T. Chruściel, J. Lopes Costa, "On uniqueness of stationary vacuum black holes", Astérisque 321, 195-265 (2008). 2.5

[20] P.T. Chruściel, E. Delay, G.J. Galloway, R. Howard, "Regularity of horizons and the area theorem", Annals Henri Poincaré 2, 109-178 (2001). 2.5, 2.5.1

[21] P.T. Chruściel, G.J. Galloway, D. Solis, “Topological censorship for Kaluza-Klein space-times", Annals Henri Poincaré 9, 893-912 (2009). 2.5

[22] P.T. Chruściel, J. Jezierski, S. Leski, “The Trautman-Bondi mass of hyperboloidal initial data sets", Adv. Theor. Math. Phys. 8, 83-139 (2004). 1 
[23] P.T. Chruściel, T-T. Paetz, "The mass of light-cones", Class. Quantum Grav. 31, 102001 (2014). 6.3

[24] C.J.S. Clarke, T. Dray, "Junction conditions for null hypersurfaces", Class. Quantum Grav. 4, 265-275 (1987). 2.6, 2.6.6

[25] M. Dafermos, G. Holzegel, I. Rodniansky, "The linear stability of the Schwarzschild solution to gravitational perturbations", arXiv:1601.06467v1 [gr-qc] (2016). 1]

[26] M. Dafermos, I. Rodniansky, "The black hole stability problem for linear scalar perturbations" in Proceedings of the Twelfth Marcel Grossmann Meeting, T. Damour, R.T. Jantzen and R. Ruffini (Editors) (2012). 1]

[27] M. Dafermos, I. Rodniansky, "A proof of the uniform boundedness of solutions to the wave equation on slowly rotating Kerr backgrounds", Invent. math. 185, 467-559 (2011).

[28] M. Dafermos, I. Rodniansky, Y. Shlapentokh-Rothman, "Decay for solutions of the wave equation on Kerr exterior spacetimes III: The full subextremal case $|a|<M^{\prime \prime}$, Ann. Math. 183, 787-913 (2016). [1

[29] M. Dahl, R. Gicquaud, A. Sakovich, "Penrose type inequalities for asymptotically hyperbolic graphs", Annals Henri Poincaré 14, 1135-1168 (2013). 1

[30] S. Dain, I. Gentile de Austria, "On the linear stability of the extreme Kerr black hole under axially symmetric perturbations", Class. Quantum Grav. 31, 195009 (2014). 1

[31] A. Einstein, "Die Feldgleichungen der Gravitation", Sitzungsberichte der Königlich Preußischen Akademie der Wissenschaften (Berlin), 844-847 (1915) 1

[32] G.J. Galloway, "Null Geometry and the Einstein Equations" in The Einstein Equations and the Large Scale Behaviour of Gravitational Fields, P.T. Chruściel, H. Friedrich (Editors) (2000). 4.2, B, B.4

[33] R. Geroch, "Energy Extraction", Ann. N. Y. Acad. Sci. 224, 108-117 (1973).

[34] R. Geroch, J. Traschen, "Strings and other distributional sources in general relativity", Phys. Rev. D 36, 1017-1031 (1987). 2.6

[35] R. Geroch, J. Winicour, "Linkages in General Relativity", J. Math. Phys. 22, 803812 (1981). 2.3

[36] G.W. Gibbons, Ph.D. thesis: Some aspects of gravitational radiation and gravitational collapse, Cambridge University (1973). 1 
[37] G.W. Gibbons, "Collapsing shells and the isoperimetric inequality for black holes", Class. Quantum Grav. 14, 2905-2915 (1997). 1, 3.3, 3.3, 3.3.1, 3.3.1, 3.3.1, 3.3.1, 3.3.8, 8

[38] E. Gourgoulhon, J.L. Jaramillo, "A $3+1$ perspective on null hypersurfaces and isolated horizons", Phys. Rep. 423, 159-294 (2006). B

[39] P. Guan, J. Li, "The Quermassintegral for k-convex starshaped domains", Advan. Math. 221, 1725-1732 (2009). 3.3.1

[40] S.W. Hawking, "The occurrence of singularities in cosmology. III. Causality and singularities", Proc. Roy. Soc. London A. A300, 187-201 (1967). 2.5

[41] S.W. Hawking, "Gravitational radiation in an expanding universe", J. Math. Phys. 9, 598-604 (1968). 1

[42] S.W. Hawking, "Gravitational Radiations from colliding black holes", Phys. Rev. Lett. 26, 1344-1346 (1971). 2.5

[43] S.W. Hawking, "Black holes in General Relativity", Commun. Math. Phys. 25, 152-166 (1972). 2.5

[44] S.W. Hawking, G.F.R. Ellis, The large scale structure of space-time, Cambridge University Press (1973). 1, 2.4

[45] S.W. Hawking, R. Penrose, "The singularities of gravitational collapse and cosmology", Proc. Roy. Soc. London A. 314, 529-548 (1970). 1

[46] S.A. Hayward, "Quasi-localization of Bondi-Sachs energy-loss", Class. Quantum Grav. 11, 3037 (1994). 1]

[47] S.A. Hayward, "Gravitational energy in spherical symmetry", Phys. Rev. D 53, 1938-1949 (1996). [1]

[48] M. Heusler, Black hole uniqueness theorems, Cambridge Lecture Notes in Physics, Cambridge University Press, Cambridge (1996). 2.5

[49] G.T. Horowitz, M.J. Perry, "Gravitational energy cannot become negative", Phys. Rev. Lett. 48, 371-374 (1982). 2.3

[50] L-H. Huang, D. Wu, "The equality case of the Penrose inequality for asymptotically flat graphs", Trans. Amer. Math. Soc. 367, 31-47 (2015). 1

[51] G. Huisken, "Flow by mean curvature of convex surfaces into spheres", J. Diff. Geom. 20, 237-266 (1984). 4.4 
[52] G. Huisken, T. Ilmanen, "The inverse mean curvature flow and the Riemannian Penrose inequality", J. Diff. Geom. 59, 353-437 (2001). 1, 1

[53] P-K. Hung, J. Keller, M-T. Wang, "Linear Stability of Schwarzschild Spacetime: The Cauchy Problem of Metric Coefficients", arXiv:1702.02843v2 [gr-qc] (2017). 1

[54] W. Israel, "Singular hypersurfaces and thin shells in general relativity", Nuovo Cimento B44, 1-14 (erratum B48 463) (1967). 2.6

[55] P.S. Jang, R.M. Wald, "The positive energy conjecture and the cosmic censor hypothesis", J. Math. Phys. 18, 41 (1977). 1]

[56] B.S. Kay, R.M. Wald, "Linear stability of Schwarzschild under perturbations which are non-vanishing on the bifurcation 2-sphere", Class. Quantum Grav. 4, 893 (1987). [1

[57] M-K.G. Lam, Ph.D. thesis: The graphs cases of the Riemannian positive mass and Penrose inequalities in all dimensions, Duke University (2010). 1

[58] K. Lanczos, "Bemerkung zur de Sitterschen Welt", Physikalische Zeitschrift 23, 539-547 (1922). 2.6

[59] K. Lanczos, "Flächenhafte Verteilung der Materie in der Einsteinschen Gravitationstheorie", Annalen der Physik (Leipzig) 74, 518-540 (1924). 2.6

[60] P.G. LeFloch, C. Mardare, "Definition and weak stability of spacetimes with distributional curvature", Portugaliae Mathematica 64, 535-573 (2007). 2.6

[61] A. Lichnerowicz, Théories Relativistes de la Gravitation et de l'Electromagnétisme, Paris, Masson (1955). 2.6

[62] LIGO Scientific Collaboration, "Observation of Gravitational Waves from a Binary Black Hole Merger", Phys. Rev. Lett. 116, 061102 (2016). 1

[63] E.L. Lima, "The Jordan-Brouwer separation theorem for smooth hypersurfaces", Amer. Math. Month. 95, 39-42 (1988). 4.3

[64] L. Lopes de Lima, F. Girão, "Positive mass and Penrose type inequalities for asymptotically hyperbolic hypersurfaces" in General Relativity and Gravitation, volume 47, Springer US (2015). [1

[65] L. Lopes de Lima, F. Girão, "An Alexandrov-Fenchel-type inequality in hyperbolic space with an application to a Penrose inequality", Annals Henri Poincaré 17, 979-1002 (2016). 1 
[66] M. Ludvigsen, J.A.G. Vickers, "A simple proof of the positivity of Bondi mass", J. Phys. A: Math. Gen. 15, L67-L70 (1982). 2.3

[67] M. Ludvigsen, J.A.G. Vickers, "An inequality relating the total mass and the area of a trapped surface in general relativity", J. Phys. A: Math. Gen. 16, 3349-3353 (1983). 1, 1, 4.1, 4.4.2, 4.5, 7.1, 7.3, 7.3.5

[68] E. Malec, N. Ó Murchadha, "Trapped surfaces and the Penrose inequality in spherically symmetric geometries", Phys. Rev. D 49, 6931-6934 (1994). 1

[69] M. Mars, "Present status of the Penrose inequality", Class. Quantum Grav. 26, 193001 (2009). 1, 1, 1, 2.7

[70] M. Mars, "Introduction to the Penrose inequality for null shells", Seminar at University of Málaga (Spain) , (13-04-2011). 2.6

[71] M. Mars, "Constraint equations for general hypersurfaces and applications to shells", Gen. Rel. Grav. 45, 2175-2221 (2013). 1, 2.6, 2.6, 2.6, 2.6, 2.6, 2.6, 2.6, 6.3

[72] M. Mars, J.M.M. Senovilla, "Geometry of general hypersurfaces in spacetime: junction conditions", Class. Quantum Grav. 10, 1865-1897 (1993). 2.6, 2.6, 2.6.6, 2.6, 2.6

[73] M. Mars, J.M.M. Senovilla, R. Vera, "Lorentzian and signature changing branes", Phys. Rev. D 76, 044029 (2007). 2.6, 2.6.6

[74] M. Mars, A. Soria, "On the Penrose inequality for dust null shells in the Minkowski spacetime of arbitrary dimension", Class. Quantum Grav. 29, 135005 (2012). 1, 1, 4.1, 4.3, 4.4.1, 4.4.2

[75] M. Mars, A. Soria, "Geometry of normal graphs in Euclidean space and applications to the Penrose inequality in Minkowski", Annales Henri Poincaré 15, 1903-1918 (2014). 1, 3.1, 3.3.2, 5.1

[76] M. Mars, A. Soria, "The asymptotic behaviour of the Hawking energy along null asymptotically flat hypersurfaces", Class. Quantum Grav. 32, 185020 (2015). 1, 6.1

[77] M. Mars, A. Soria, "On the Penrose inequality along null hypersurfaces", Class. Quantum Grav. 33, 115019 (2016). 7.1

[78] C.W. Misner, D.H. Sharp, "Relativistic Equations for Adiabatic, Spherically Symmetric Gravitational Collapse", Phys. Rev. 136, B571-B576 (1964). [

[79] V. Moncrief, "Gauge-invariant perturbations of Reisner-Nordstrom black holes", Phys. Rev. D 12, 1526-1537 (1975). 1 
[80] A. Neves, "Insufficient convergence of inverse mean curvature flow on asymptotically hyperbolic manifolds", J. Diff. Geom. 84, 191-229 (2010). 11 1

[81] J.R. Oppenheimer, H. Snyder, "On Continued Gravitational Contraction", Phys. Rev. 56, 455-459 (1939). [1

[82] J.R. Oppenheimer, G.M. Volkoff, "On Massive Neutron Cores", Phys. Rev. 55, 374-381 (1939). [1]

[83] R. Penrose, "Gravitational collapse and space-time singularities", Phys. Rev. Lett. 14, 57 (1965). 1, 2.5

[84] R. Penrose, "Gravitational collapse: The role of General Relativity", Nuovo Cimento 1, 252-276 (1969). [1

[85] R. Penrose, "Naked singularities", Ann. N. Y. Acad. Sci. 224, 125-134 (1973). [1, 1, 2.6, 4.4

[86] R. Penrose, "Singularities and Time-Asymmetry", in General Relativity: An Einstein Centenary Survey, S.W. Hawking and W. Israel (Editors), Cambridge, Cambridge University Press (1979). [1

[87] R. Penrose, W. Rindler, Spinors and space-time, Cambridge University Press (1987). 1, 2.7, 6.7, 6.7, 6.7, 6.7.5

[88] R. Price, "Nonspherical Perturbations of Relativistic Gravitational Collapse. I. Scalar and Gravitational Perturbations", Phys. Rev. D 5, 2419 (1972). [1]

[89] R. Price, "Nonspherical Perturbations of Relativistic Gravitational Collapse. II. Integer-Spin, Zero-Rest-Mass Fields", Phys. Rev. D 5, 2439 (1972). [1]

[90] T. Regge, J.A. Wheeler, "Stability of a Schwarzshild singularity", Phys. Rev. 108, 1063-1069 (1957). [1

[91] O. Reula, P. Tod, "Positivity of the Bondi energy", J. Math. Phys. 25, 1004 (1984). 2.3

[92] H. Roesch, "Proof of a Null Penrose Conjecture using a new Quasi-local Mass", arXiv:1609.02875v1 [gr-qc] (2016). 1, 8

[93] J. Sauter, Ph.D. thesis: Foliations of null hypersurfaces and the Penrose inequality, ETH Zürich (2008). 1, 6.2, 6.4, 6.7.5, 7.6, 7.6

[94] R. Schneider, Convex Bodies: the Brunn-Minkowski theory, Cambridge University Press, Pg. 37-41, 103-113 (1993). C, C.2, C.3 
[95] R. Schoen, S.T. Yau, "On the proof of the positive mass conjecture in general relativity", Commun. Math. Phys. 65, 45 (1979). 2.3

[96] R. Schoen, S.T. Yau, "Proof that the Bondi mass is positive", Phys. Rev. Lett. 48, 369-371 (1982). 2.3

[97] J. A. Schouten, Ricci Calculus, Springer, Berlin (1954). 2.6, 2.6.1

[98] J.M.M Senovilla, "Singularity theorems and their consequences", Gen. Rel. Grav. 30, 701-848 (1998). 1, 2.5

[99] J. Tafel, "A lower bound of the Trautman-Bondi energy", Class. Quantum Grav. 33, 025002 (2016). 1

[100] A.H. Taub, "Space-times with distribution valued curvature tensors", J. Math. Phys. 21, 1423-1431 (1980). 2.6

[101] K.P. Tod, "Penrose quasi-local mass and the isoperimetric inequality for static black holes", Class. Quantum Grav. 2, L65-L68 (1985). 1, 4.1, 4.4, 4.4.5, 4.5.5, 5.3

[102] K.P. Tod, "More on Penrose's quasi-local mass", Class. Quantum Grav. 3, 11691189 (1986). 4.4.5

[103] K.P. Tod, "The hoop conjecture and the Gibbons-Penrose construction of trapped surfaces”, Class. Quantum Grav. 9, 1581-1591 (1992). 1, 4.4

[104] N. Trudinger, "Isoperimetric inequalities for quermassintegrals", Ann. Inst. H. Poincaré Anal. Non Linéare 11, 411-425 (1994). 3.3.1

[105] C.V. Vishveshwara, "Stability of the Schwarzschild Metric", Phys. Rev. D 1, 2870 (1970).

[106] R.M. Wald, General Relativity, The University of Chicago Press (1984). 1, 2.2, 2.5, 3.3, 6.3

[107] X. Wang, "Mass for asymptotically hyperbolic manifolds", J. Diff. Geom. 57, 273-299 (2001). 1

[108] M-T. Wang, "Quasilocal mass and surface Hamiltonian in spacetime" in XVIIth International Congress on Mathematical Physics, Arne Jensen (Editor) (2013). 1

[109] M-T. Wang, S-T. Yau, "Quasilocal mass in General Relativity", Phys. Rev. Lett. 102, 021101 (2009).

[110] M-T. Wang, S-T. Yau, "Isometric embeddings into the Minkowski space and new quasi-local mass", Commun. Math. Phys. 288, 919-942 (2009). 1, 3.3 
[111] E. Witten, "A new proof of the positive energy theorem", Commun. Math. Phys. 80, 381-402 (1981). 2.3

[112] B.F. Whiting, "Mode stability of the Kerr black hole", J. Math. Phys. 30, 13011305 (1989). [1

[113] F.J. Zerilli, "Effective potential for even parity Regge-Wheeler gravitational perturbation equations", Phys. Rev. Lett. 24, 737-738 (1970). 1

[114] X. Zhang, "On the relation between ADM and Bondi energy-momenta", Adv. Theor. Math. Phys. 10, 261-282 (2006). 2.3 